\title{
A General, Multimetallic Cross-Ullmann Biheteroaryl Synthesis from Heteroaryl Halides and Heteroaryl Triflates
}

\author{
Kai Kang ${ }^{1}$, Nathan L. Loud ${ }^{1}$, Tarah A. DiBenedetto ${ }^{2}$ and Daniel J. Weix *,I \\ ${ }^{1}$ University of Wisconsin-Madison, Madison, WI 53706, USA \\ ${ }^{2}$ University of Rochester, Rochester, NY 14627, USA \\ Supporting Information
}

1. General Information

S2

2. Reaction Optimization............................................................... S5

3. Synthesis of Starting Materials................................................................... S12

3.1 Synthesis of heteroaryl triflates

3.2 Synthesis of heteroaryl halides

4. Cross-Electrophile Couplings of Heteroaryl Halides with Heteroaryl Triflates.

4.1 General procedure for reaction setup in scope investigations

4.2 Structures and characterizations of the cross-coupled products in scope investigations

4.3 Bench setup of the synthesis of compound 3ao on $5.0 \mathrm{mmol}$ scale

4.4 Mechanistic studies on the difference between Conditions A and B

5. High-Throughput Experimentation.

S60

5.1 Application of HTE in scope investigations

5.2 Application of HTE in condition optimizations

5.3 Synthesis of products $\mathbf{B 3}, \mathbf{C 6}$, and $\mathbf{H 2}$ under re-optimized conditions

6. References. 


\section{General Information}

\section{Reagents}

Catalysts and Reductants: All catalysts and metal reductants, unless otherwise noted, were stored and handled in a nitrogen-filled glovebox and used as-is. Nickel(II) bromide ethylene glycol dimethyl ether complex $\left(\mathrm{NiBr}_{2}(\mathrm{dme})\right)$ was purchased from Sigma-Aldrich. Palladium(II) chloride $\left(\mathrm{PdCl}_{2}\right)$ was purchased from Alfa Aesar. The reductant zinc powder (-325 mesh, 97\%) and manganese powder (-325 mesh) were purchased from Alfa Aesar.

Ligands: Amine ligands and phosphine ligands used in reaction optimizations and scope investigations were purchased from Sigma-Aldrich, TCI, or Ambeed and used as-is.

Salt Additives: All the salt additives were purchased from Alfa-Aesar, TCI or Sigma-Aldrich and used as-is. $\mathrm{MgI}_{2}$ was stored in a $-20{ }^{\circ} \mathrm{C}$ fridge in a $\mathrm{N}_{2}$ filled glovebox.

ChemBeads: Solid reagent coated ChemBeads were prepared according to reported procedures. ${ }^{1}$ Solvents for Reactions: Anhydrous $N, N$-dimethylformamide (DMF, 99.9\%) was purchased from Sigma-Aldrich. It was opened and stored in a nitrogen-filled glovebox and used as-is. Dry solvents used for synthesis of starting materials were purchased from Fisher Chemicals and purified by inert solvent purification system (PureSolve MD 5, Inert Corp.) before use.

Starting Materials: Heteroaryl phenols, heteroaryl bromides, and heteroaryl chlorides used in this study were purchased from Sigma-Aldrich or Ambeed and used as-is. Preparations and characterizations of synthesized starting materials were described in 3. Synthesis of Starting Materials of this document.

\section{Methods}

NMR Spectroscopy: ${ }^{1} \mathrm{H}$ nuclear magnetic resonance (NMR) spectroscopy chemical shifts are reported in ppm and referenced to TMS (tetramethylsilane) in solvent (for $\mathrm{CDCl}_{3}, \delta=0 \mathrm{ppm}$ ) or the residual solvent peaks (for $\mathrm{CDCl}_{3}, \delta=7.26 \mathrm{ppm}$ ). For ${ }^{13} \mathrm{C} \mathrm{NMR}$ chemical shifts, the residual solvent peak (for $\mathrm{CDCl}_{3}, \delta=77.20 \mathrm{ppm}$ ) was used as references. For ${ }^{19} \mathrm{~F}$ NMR chemical shifts, the external standard, $\alpha, \alpha, \alpha$-trifluorotoluene $(\delta=0 \mathrm{ppm})$, were used as references. NMR spectra were recorded on Avance Bruker NMR spectrometers operating at either $400 \mathrm{MHz}$ or $500 \mathrm{MHz}$ and data analysis was performed with MestreNova. Chemical shifts are reported in parts per million (ppm), multiplicities are indicated by s (singlet), $\mathrm{d}$ (doublet), $\mathrm{t}$ (triplet), q (quartet), $\mathrm{m}$ (multiplet) and br (broad). Coupling constants $(J)$ are reported in Hertz $(\mathrm{Hz})$.

Gas Chromatography: GC analyses were performed on an Agilent 7890A GC equipped with dual DB-5 columns $(20 \mathrm{~m} \times 180 \mu \mathrm{m} \times 0.18 \mu \mathrm{m})$, dual FID detectors, and hydrogen as the carrier 
gas. A sample volume of $1 \mu \mathrm{L}$ was injected at a temperature of $300{ }^{\circ} \mathrm{C}$ and a $100: 1$ split ratio. The initial inlet pressure was 20.3 psi but varied as the column flow was held constant at $1.8 \mathrm{~mL} / \mathrm{min}$ for the duration of the run. The initial oven temperature of $50{ }^{\circ} \mathrm{C}$ was held for 0.46 min followed by a temperature ramp of $65^{\circ} \mathrm{C} / \mathrm{min}$ up to $300^{\circ} \mathrm{C}$. The temperature was held at $300{ }^{\circ} \mathrm{C}$ for $3 \mathrm{~min}$. The total run time was $\sim 7.3 \mathrm{~min}$ and the FID temperature was $325^{\circ} \mathrm{C}$.

GC/MS Analysis: GC/MS analyses were performed on a Shimadzu GCMS-QP2010 equipped with an RTX-XLB column $(30 \mathrm{~m} \times 0.25 \mathrm{~mm} \times 0.28 \mu \mathrm{m})$ with a quadrupole mass analyzer using helium as the carrier gas. The analysis method used in all cases was $5 \mu \mathrm{L}$ injection of sample, an injection temp of $225^{\circ} \mathrm{C}$, and a 25:1 split ratio. The initial inlet pressure was 7.8 psi, but varied as the column flow was held constant at $1.0 \mathrm{~mL} / \mathrm{min}$ for the duration of the run. The interface temperature was held at $250^{\circ} \mathrm{C}$, and the ion source $(\mathrm{EI}+, 30 \mathrm{eV})$ was held at $250{ }^{\circ} \mathrm{C}$. The initial oven temperature was held at $50{ }^{\circ} \mathrm{C}$ for $2 \mathrm{~min}$ with the detector off, followed by a temperature ramp, with the detector on, to $280^{\circ} \mathrm{C}$ at $40^{\circ} \mathrm{C} / \mathrm{min}$. The temperature was held at $280{ }^{\circ} \mathrm{C}$ for $3 \mathrm{~min}$. Total run time was $11.75 \mathrm{~min}$.

Supercritical Fluid Chromatography Mass Spectrometry (SFC-MS): SFC-MS analyses were performed on a Waters ACQUITY UPC2 equipped with ACQUITY UPC2 PDA and ACQUITY QDa Detector. A Daicel Dcpack SFC-A column (3mm ID $\times 150 \mathrm{~mm} \mathrm{L,} 3 \mu \mathrm{m}$ PS) was used for separations. The eluent was a mixture $\left(97: 3 \mathrm{CO}_{2} / \mathrm{MeOH}\right)$ with a flow rate of $2 \mathrm{~mL} / \mathrm{min}$ at $40{ }^{\circ} \mathrm{C}$ with a ABPR at 1500 psi.

Infrared Spectroscopy (IR): IR spectra were recorded on a Shimadzu IRAffinity-1 Fourier Transform Infrared Spectrophotometer and are reported in wavenumbers $\left(\mathrm{cm}^{-1}\right)$.

Flash Chromatography for Isolations: Chromatography was performed on silica gel (EMD, silica gel 60, particle size 0.040-0.063 mm) using standard flash techniques or on 40-100 g HP Silica column using a Teledyne Isco Rf-200 (detection at 254 and $280 \mathrm{~nm}$ ).

Melting Point (mp): Measurements of melting points were performed on DigiMelt M160 Melting Point Apparatus.

High Throughput Screening: HTE screens were performed in $1 \mathrm{~mL}$ glass shell vials (Analytical Sales 84001) in a 96-well photoredox and parallel synthesis reactor block (Analytical Sales 96973) using a modified Torrey-Pines Echotherm SC25XT orbital mixing chilling/heating dry bath. Filtrations were performed with a $2 \mathrm{~mL}$ filter plate with a $0.20 \mu \mathrm{m}$ polypropylene membrane (Analytical Sales 96222) and a $340 \mu \mathrm{L}$ V-bottomed polypropylene 96-well collection plate (Analytical Sales 96340) on a vacuum manifold filtration system (Analytical Sales 968444). The collection plate was sealed with an adhesive sealing film (Analytical Sales 961802) and then the samples were analyzed by SFC-MS 
using the standard method. See 5. High-Throughput Experimentation of this document for detailed procedure. 


\section{Reaction Optimization}

\section{General procedure for optimizations}

The following procedures were conducted in a $\mathrm{N}_{2}$ filled glovebox for convenience.

Preparation of nickel catalyst stock solution $(0.050 \mathrm{M}$ with respect to nickel)

An oven-dried 1-dram vial equipped with a PTFE-coated stir-bar was charged with $\mathrm{NiBr}_{2}(\mathrm{dme})(30.8 \mathrm{mg}, 0.100 \mathrm{mmol})$ and the listed amine ligand $(0.110 \mathrm{mmol})$. DMF $(2.0 \mathrm{~mL})$ was then added before the dram vial was capped with a screw cap fitted with a PTFE-faced silicone septum. The mixture was stirred (1000 rpm) at $60{ }^{\circ} \mathrm{C}$ for $3 \mathrm{~h}$ before being used (vide infra). Attention: $4.0 \mathrm{~mL}$ of DMF was used for the preparation of a stock solution with a nickel concentration of $0.025 \mathrm{M}$, which was used in 5. High-Throughput Experimentation of this document.

Preparation of palladium catalyst stock solution ( $0.025 \mathrm{M}$ with respect to palladium)

An oven-dried 1-dram vial equipped with a PTFE-coated stir-bar was charged with $\mathrm{PdCl}_{2}$ $(17.7 \mathrm{mg}, 0.100 \mathrm{mmol})$ and the listed amine ligand $(0.110 \mathrm{mmol})$. DMF $(4.0 \mathrm{~mL})$ was then added before the dram vial was capped with a screw cap fitted with a PTFE-faced silicone septum. The mixture was stirred $(1000 \mathrm{rpm})$ at $60{ }^{\circ} \mathrm{C}$ for $3 \mathrm{~h}$ before being used (vide infra).

\section{Catalytic reaction setup}

An oven-dried 1-dram vial equipped with a PTFE-coated stir-bar was charged sequentially with $1 \mathbf{a}(0.25 \mathrm{mmol}, 1.0$ equiv), $2 \mathrm{a}(0.25$ or $0.38 \mathrm{mmol}, 1.0$ or 1.5 equiv $)$, the listed salt additive ( 0.25 or $0.50 \mathrm{mmol}, 1.0$ or 2.0 equiv), and the listed reductant ( $0.50 \mathrm{mmol}, 2.0$ equiv). Aliquots from the $0.050 \mathrm{M}$ nickel (50,100 or $250 \mu \mathrm{L}$ for $1.0,2.0$ or $5.0 \mathrm{~mol} \%$ catalyst loading, respectively) and $0.025 \mathrm{M}$ palladium (100, 200 or $500 \mu \mathrm{L}$ for $1.0,2.0$ or $5.0 \mathrm{~mol} \%$ catalyst loading, respectively) stock solutions (vide supra) were then added, followed by 1,3,5-trimethoxybenzene (10-20 mg) as an internal standard. Sufficient DMF was then added to bring the total volume of solvent to 0.75 $\mathrm{mL}$. The vial was capped with a screw cap fitted with a PTFE-faced silicone septum, and the mixture was stirred $(1000 \mathrm{rpm})$ for $10 \mathrm{~min}$ in the glovebox at rt. The reaction vial was then removed from the glovebox and heated in a reaction block set to the desired temperature with stirring (1250 rpm) for $12 \mathrm{~h}$.

\section{Reaction workup}


The reaction mixture was cooled to $\mathrm{rt}$ before being transferred to a $20 \mathrm{~mL}$ scintillation vial equipped with a PTFE-coated stir-bar with a Pasteur pipette. Three portions of dichloromethane $(0.5 \mathrm{~mL} \times 3)$ were used to further rinse the reaction vial. $\mathrm{Na}_{4}$ EDTA aqueous solution $(25 \% \mathrm{wt}, 2.0$ $\mathrm{mL}$ ) was then added to the $20 \mathrm{~mL}$ scintillation vial. The vial was capped with a screw cap fitted with a PTFE-faced silicone septum, and the mixture was stirred $(1000 \mathrm{rpm})$ for $1 \mathrm{~h}$ at rt. After that, the quenched mixture was transferred to a $100 \mathrm{~mL}$ separatory funnel containing dichloromethane $(10 \mathrm{~mL})$ and water $(10 \mathrm{~mL})$. The dichloromethane layer was set aside, and the aqueous layer was washed with dichloromethane $(2 \times 10 \mathrm{~mL})$. The dichloromethane layers were combined and dried over anhydrous $\mathrm{Na}_{2} \mathrm{SO}_{4}$. The resulting mixture was filtered through a disposable filter funnel (20 $\mathrm{mL}, 10$-micron polyethylene frit) to remove the solid, and the filtrate was collected for GC analysis.

\section{GC analysis}

An aliquot $(200 \mu \mathrm{L})$ of the filtrate from the reaction workup was further diluted with ethyl acetate $(2.0 \mathrm{~mL})$. The resulting solution was filtered through a syringe filter (PVDF membrane, $0.22 \mu \mathrm{m}$ pore size, $13 \mathrm{~mm}$ diameter), and the filtrate was collected in a GC vial. The sample was analyzed by GC, and the yields were determined based on the peak area of the analyte compared to 1,3,5-trimethoxybezene as an internal standard.

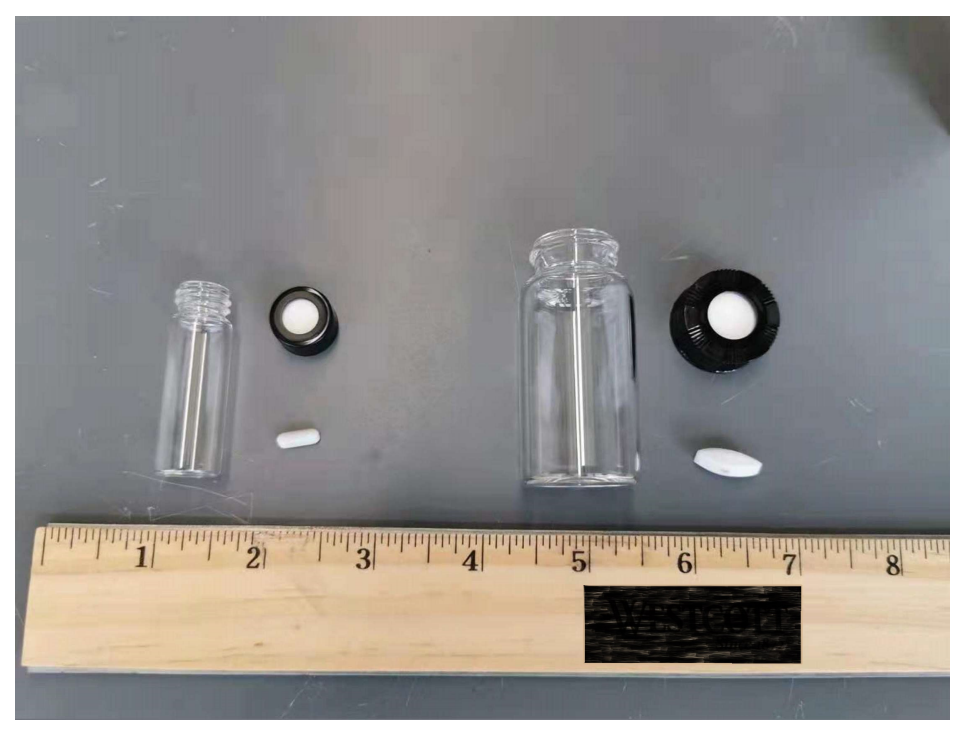

Figure S1. (Left) Screw cap fitted with a PTFE-faced silicone septum, 1-dram vial, and PTFEcoated stir-bar used in the preparation of catalyst stock solution and catalytic reaction setup; (Right) Screw cap fitted with a PTFE-faced silicone septum, $20 \mathrm{~mL}$ scintillation vial, and PTFE-coated stir-bar used in the reaction workup. 
Table S1. Control reactions

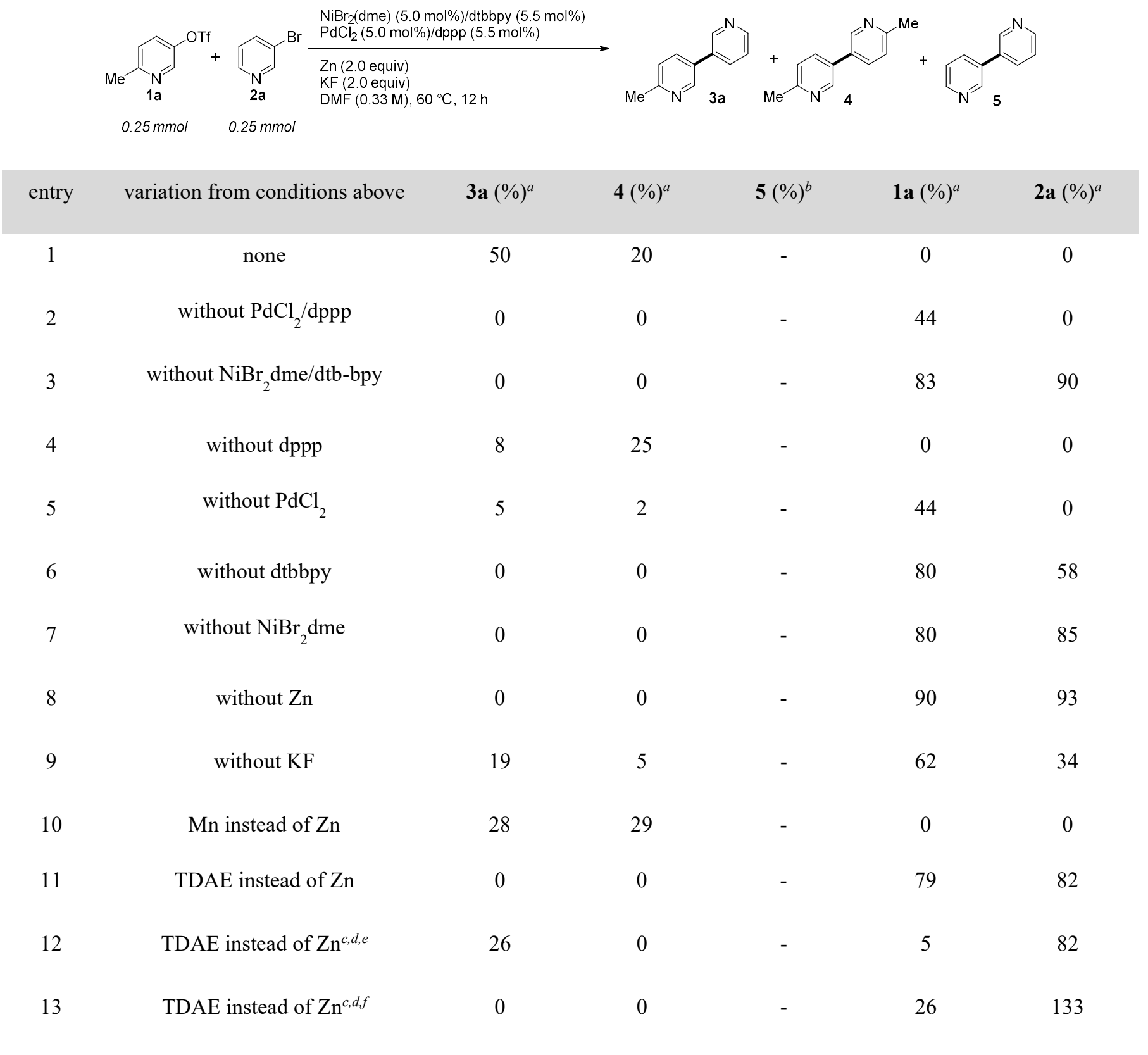

(a) Corrected GC yields using 1,3,5-trimethoxybenzene as an internal standard. (b) The GC peaks for compound $\mathbf{5}$ are wide and flat, which are not suitable for integration. Therefore, the yield of compound 5 was not calculated. (c) 1.0 Equivalent of $\mathrm{KBr}$ was used as salt additive instead of KF. (d) 1.5 Equivalents of 2a were used. (e) 0.2 Equivalent of Zn was added. (f) 0.2 Equivalent of $\mathrm{ZnBr}_{2}$ was added. 
Table S2. Effect of salt additives

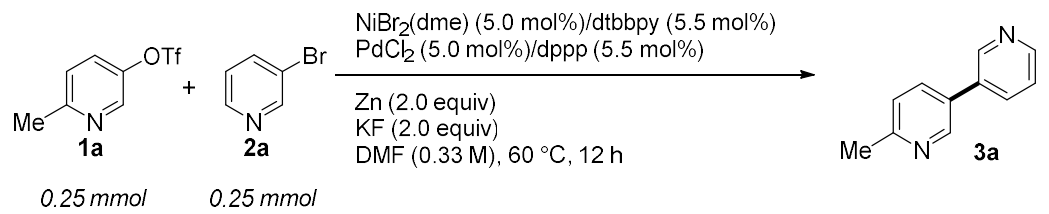

\begin{tabular}{|c|c|c|c|c|}
\hline entry & variation from conditions above & $3 \mathbf{a}(\%)^{a}$ & $1 \mathbf{a}(\%)^{a}$ & $2 \mathbf{a}(\%)^{a}$ \\
\hline 1 & none & 50 & 0 & 0 \\
\hline 2 & 1.0 equiv $\mathrm{KF}$ as additive & 44 & 45 & 0 \\
\hline 3 & 2.0 equiv $\mathrm{CsF}$ as additive & 0 & 76 & 83 \\
\hline 4 & 2.0 equiv $\mathrm{LiF}$ as additive & 31 & 59 & 18 \\
\hline 5 & 2.0 equiv $\mathrm{LiCl}$ as additive & 32 & 0 & 0 \\
\hline 6 & 2.0 equiv $\mathrm{LiBr}$ as additive & 29 & 0 & 0 \\
\hline 7 & 2.0 equiv $\mathrm{MgI}_{2}$ as additive & 22 & 0 & 0 \\
\hline 8 & 2.0 equiv $\mathrm{MgBr}_{2}$ as additive & 7 & 0 & 0 \\
\hline 9 & 2.0 equiv $\mathrm{KI}$ as additive & 40 & 0 & 0 \\
\hline 10 & 2.0 equiv $\mathrm{NaBr}$ as additive & 31 & 0 & 0 \\
\hline 11 & 2.0 equiv $\mathrm{NaI}$ as additive & 8 & 0 & 0 \\
\hline 12 & 2.0 equiv $\mathrm{KCl}$ as additive & 46 & 0 & 0 \\
\hline 13 & 2.0 equiv $\mathrm{KBr}$ as additive & 47 & 0 & 0 \\
\hline 14 & 1.0 equiv $\mathrm{KBr}$ as additive & 50 & 0 & 0 \\
\hline
\end{tabular}

(a) Corrected GC yields using 1,3,5-trimethoxybenzene as an internal standard. 
Table S3. Effect of amine ligands on nickel

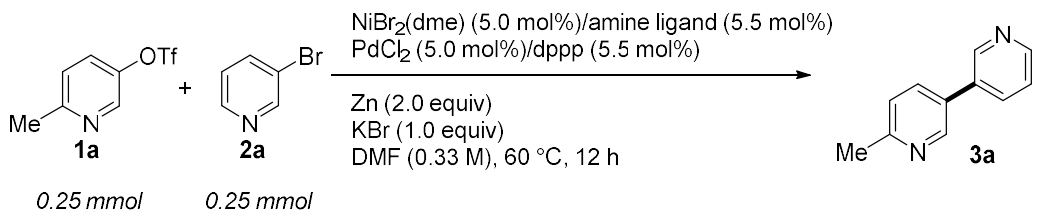

\begin{tabular}{|c|c|c|c|c|c|}
\hline entry & amine ligand on nickel & $\mathbf{3 a}(\%)^{a}$ & entry & amine ligand on nickel & 3a $(\%)^{a}$ \\
\hline 1 & $4,4^{\prime}$-di-t-butyl-2,2'-bipyridine & 50 & 8 & 4,4'-di-phenyl-2,2'-bipyridine & 33 \\
\hline 2 & 2,2'-bipyridine & 28 & 9 & 1,10-phenanthroline & 40 \\
\hline 3 & 4,4'-di-methyl-2,2'-bipyridine & 43 & 10 & bathophenanthroline & 27 \\
\hline 4 & 5,5'-di-methyl-2,2'-bipyridine & 29 & 11 & neocuproine & 24 \\
\hline 5 & 6,6'-di-methyl-2,2'-bipyridine & 22 & 12 & $2,2^{\prime} ; 6^{\prime}, 2^{\prime \prime}$-terpyridine & 39 \\
\hline 6 & $\begin{array}{l}\text { dimethyl 2,2'-bipyridine- } 4,4^{\prime} \text { - } \\
\text { dicarboxylate }\end{array}$ & 25 & 13 & $\begin{array}{c}4,4^{\prime}, 4^{\prime \prime} \text {-tri- } t \text {-butyl-2, } 2^{\prime}: 6^{\prime}, 2^{\prime \prime}- \\
\text { terpyridine }\end{array}$ & 43 \\
\hline 7 & 6,6'-di-bromo-2,2'-bipyridine & 26 & 14 & $4^{\prime}$-chloro-2,2':6',2"-terpyridine & 42 \\
\hline
\end{tabular}

(a) Corrected GC yields using 1,3,5-trimethoxybenzene as an internal standard.

Table S4. Effect of phosphine ligands on palladium

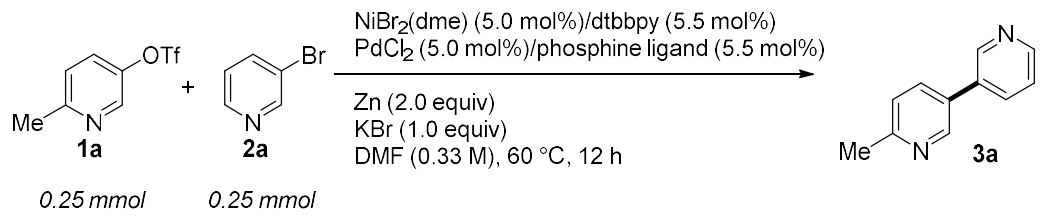

\begin{tabular}{ccc|ccc} 
entry & phosphine ligand on palladium & $\mathbf{3 a}(\%)^{a}$ & entry & phosphine ligand on palladium & 3a $(\%)^{a}$ \\
1 & dppp & 50 & 8 & $\mathrm{Ph}_{3} \mathrm{P}^{b}$ & 38 \\
2 & dppb & 32 & 9 & $\mathrm{Cy}_{3} \mathrm{P}^{b}$ & 73 \\
3 & dppe & 27 & 10 & $\mathrm{dppp}^{c}$ & 70 \\
4 & Xantphos & 39 & 11 & DPEphos $^{c}$ & \\
5 & DPEphos & 52 & & & \\
7 & dppbz & 45 & & & \\
\hline
\end{tabular}

(a) GC corrected yields using 1,3,5-trimethoxybenzene as an internal standard. (b) $11 \mathrm{~mol} \%$ phosphine ligands were used. (c) 1.5 equivalents of $2 \mathbf{a}$ were used. 
Table S5. Final screenings

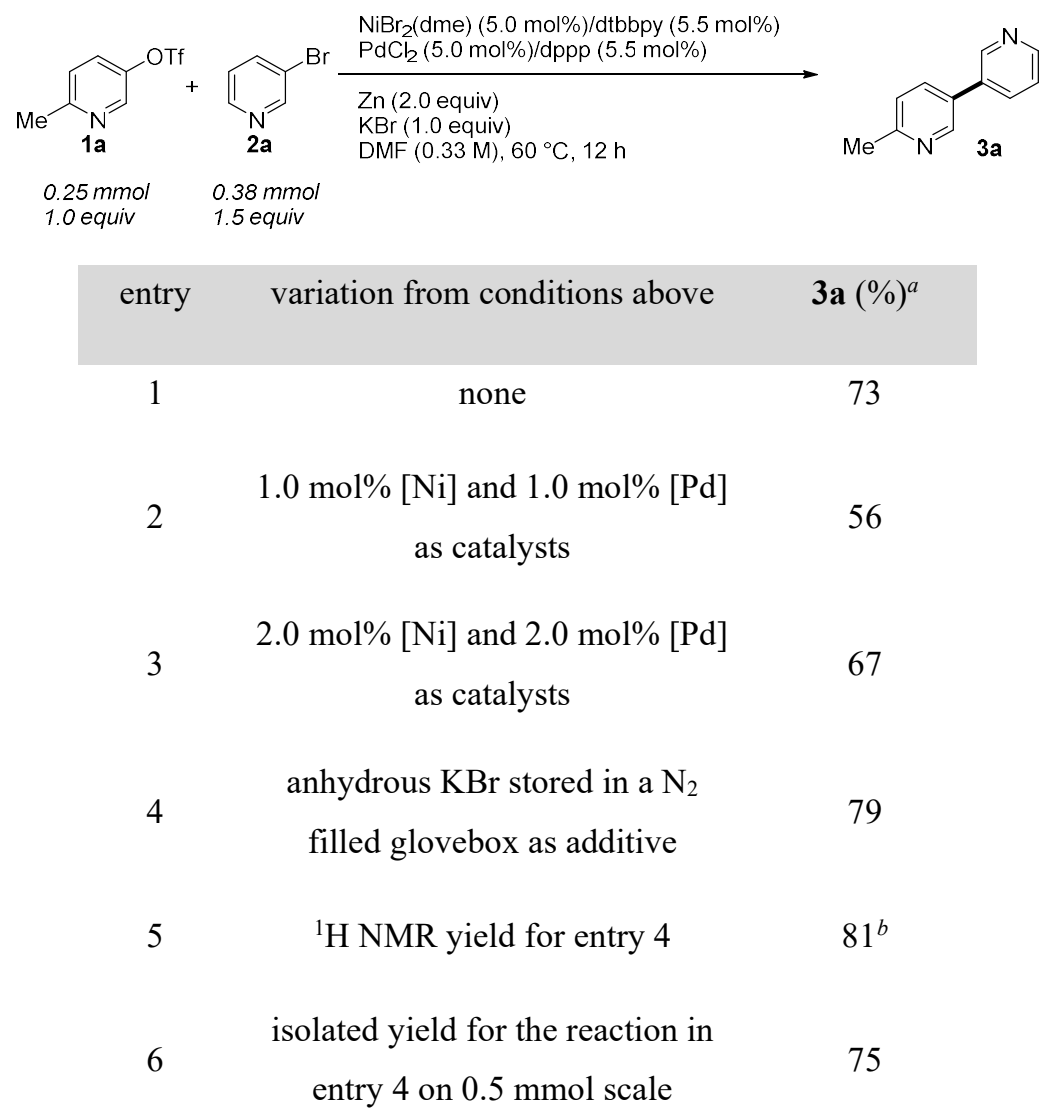

(a) Corrected GC yields using 1,3,5-trimethoxybenzene as an internal standard. (b) 1,3,5-Trimethoxybenzene was used as an internal standard. 
Table S6. Optimization of model reaction ${ }^{a}$
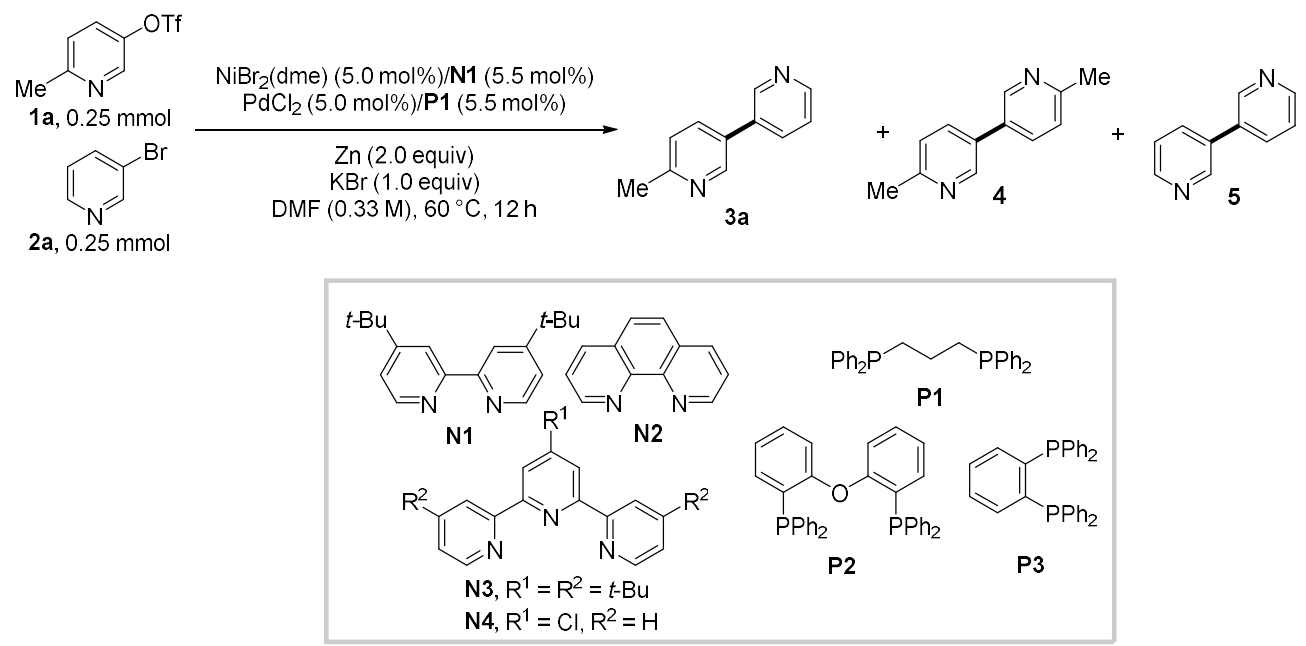

\begin{tabular}{|c|c|c|c|c|c|c|}
\hline entry & variations from above conditions & $3 a(\%)$ & $4(\%)$ & $5(\%)^{c}$ & $1 \mathrm{a}(\%)$ & $2 a(\%)$ \\
\hline 1 & $\mathrm{KF}$ (2.0 equiv) as additive & 50 & 20 & - & 0 & 0 \\
\hline 2 & none & 50 & 26 & - & 0 & 0 \\
\hline 3 & 1.5 equiv of $2 \mathrm{a}$ was used & 73 & 11 & - & 0 & 0 \\
\hline 4 & $\mathrm{~N} 2$ instead of N1 & 40 & 20 & - & 0 & 0 \\
\hline 5 & $\mathrm{~N} 3$ instead of $\mathrm{N} 1$ & 43 & 20 & - & 0 & 0 \\
\hline 6 & N4 instead of N1 & 42 & 19 & - & 0 & 0 \\
\hline 7 & P2 instead of P1 & 52 & 13 & - & 0 & 0 \\
\hline 8 & $\mathrm{P} 2$ instead of $\mathrm{P} 1,1.5$ equiv of $2 \mathrm{a}$ was used & 70 & 10 & - & 0 & 0 \\
\hline 9 & P3 instead of P1 & 45 & 17 & - & 0 & 0 \\
\hline 10 & rigorously anhydrous $\mathrm{KBr}, 1.5$ equiv of $2 \mathrm{a}$ was used & $79\left(81^{b}\right)$ & 8 & - & 0 & 0 \\
\hline
\end{tabular}

(a) Reactions on a $0.25 \mathrm{mmol}$ scale in $0.75 \mathrm{~mL}$ of DMF. Calibrated GC yield using 1,3,5-trimethoxybenzene as an internal standard. $\mathrm{KBr}$ was stored in air unless otherwise noted. (b) ${ }^{1} \mathrm{H}$ NMR yield using 1,3,5-trimethoxybenzene as an internal standard. (c) The GC peaks for compound 5 are wide and flat, which are not suitable for integration. Therefore, the yield of compound $\mathbf{5}$ was not calculated. 


\section{Synthesis of Starting Materials}

\subsection{Synthesis of heteroaryl triflates}

Scheme S1. Aryl triflates synthesized in this study

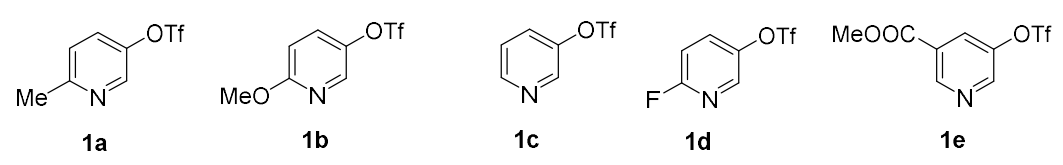

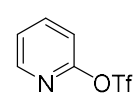

$1 f$

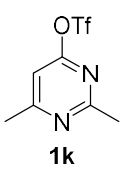

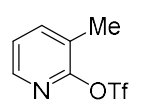

$1 \mathrm{~g}$

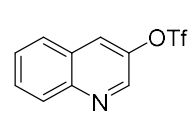

11

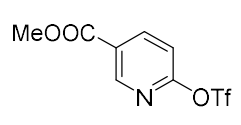

$1 \mathrm{~h}$

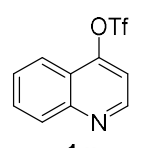

$1 \mathrm{~m}$

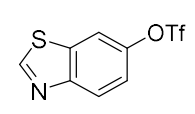

$1 \mathbf{i}$

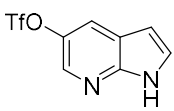

Known compounds $1 \mathbf{a},{ }^{2} \mathbf{1 b},{ }^{3} \mathbf{1 c},{ }^{4} \mathbf{1 e},{ }^{5} \mathbf{1 f},{ }^{6} \mathbf{1 g},{ }^{7} \mathbf{1 h},{ }^{8} \mathbf{1 i},{ }^{9} \mathbf{1 j},{ }^{10} \mathbf{1 l},{ }^{11}$ and $\mathbf{1 n}{ }^{12}$ were prepared according to the reported procedures. The preparations of unknown compounds $\mathbf{1 d}, \mathbf{1 k}$, and $\mathbf{1} \mathbf{~ m}$ were described below.

\section{Procedure for the synthesis of $1 d$}

Scheme S2. Synthesis of aryl triflate 1d

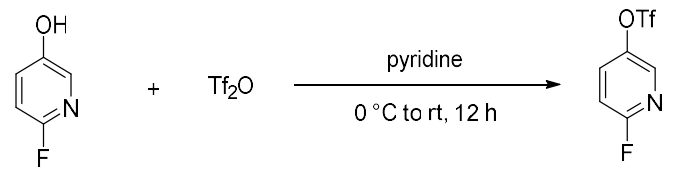

A two-neck $50 \mathrm{~mL}$ round bottom flask equipped with a PTFE-coated stir-bar was charged with 6-fluoropyridin-3-ol (1131 mg, $10.0 \mathrm{mmol}, 1.00$ equiv) and pyridine (10 mL). The flask was then connected to a $\mathrm{N}_{2}$ Schlenk line via a needle through a rubber septum (center neck) and a second rubber septum was fitted to the side-neck. The mixture was stirred $(600 \mathrm{rpm})$ in an icewater bath for 10 min under positive $\mathrm{N}_{2}$ pressure, and then trifluoromethanesulfonic anhydride (4230 $\mathrm{mg}, 15.0 \mathrm{mmol}, 1.50$ equiv) was added dropwise ( $\sim 30 \mathrm{drops} / \mathrm{min}$ ) by a syringe through the rubber septum in the side neck with cooling. After the addition, the reaction mixture was allowed to recover to $\mathrm{rt}$ with stirring $(600 \mathrm{rpm})$ over $12 \mathrm{~h}$. The reaction was judged complete by TLC (hexanes/ethyl acetate $=10 / 1, R_{\mathrm{f}}$ of $\mathbf{1 d}=0.6$ ), and the mixture was poured into a $250 \mathrm{~mL}$ separatory funnel containing dichloromethane $(30 \mathrm{~mL})$ and water $(30 \mathrm{~mL})$. The organic layer was set aside, and the aqueous layer was further washed with dichloromethane $(30 \mathrm{~mL} \times 3)$. The organic layers were combined and dried over anhydrous $\mathrm{Na}_{2} \mathrm{SO}_{4}$. The mixture was filtered to remove the solids, and the filtrate was concentrated by rotary evaporation. The residue was purified by flash chromatography on silica gel (hexanes/ethyl acetate $=50 / 1$ to $20 / 1$ ) to afford the desired product 
as a colorless oil (2009.8 mg, 82\%). ${ }^{1} \mathrm{H}$ NMR (400 MHz, $\left.\mathrm{CDCl}_{3}\right) \delta 8.22(\mathrm{dd}, J=3.1,1.5 \mathrm{~Hz}, 1 \mathrm{H})$, $7.79-7.70(\mathrm{~m}, 1 \mathrm{H}), 7.06(\mathrm{dd}, J=8.9,3.5 \mathrm{~Hz}, 1 \mathrm{H}) ;{ }^{19} \mathrm{~F}\left\{{ }^{1} \mathrm{H}\right\} \mathrm{NMR}\left(376 \mathrm{MHz}, \mathrm{CDCl}_{3}\right) \delta-66.29$ $(\mathrm{s}, 1 \mathrm{~F}),-72.39(\mathrm{~s}, 3 \mathrm{~F}) ;{ }^{13} \mathrm{C}\left\{{ }^{1} \mathrm{H}\right\} \mathrm{NMR}\left(126 \mathrm{MHz}, \mathrm{CDCl}_{3}\right) \delta 162.1(\mathrm{~d}, J=242.9 \mathrm{~Hz}), 144.4(\mathrm{~d}, J=$ $5.2 \mathrm{~Hz}), 140.9(\mathrm{~d}, J=16.6 \mathrm{~Hz}), 134.6(\mathrm{~d}, J=9.2 \mathrm{~Hz}), 118.9(\mathrm{q}, J=321.0 \mathrm{~Hz}), 111.4(\mathrm{~d}, J=40.8$ $\mathrm{Hz}$ ) ppm. HRMS (ESI) m/z calcd for $[\mathrm{M}+\mathrm{H}]^{+} 245.9843$, found 245.9841. IR ( $\left.\mathrm{cm}^{-1}\right): 3110,3063$, $1474,1426,1207,1134,884,840,608$.

\section{Procedure for the synthesis of $1 \mathrm{k}$}

Scheme S3. Synthesis of aryl triflate $\mathbf{1 k}$

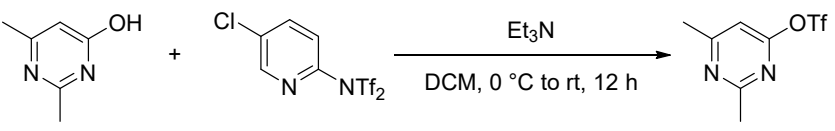

A two-neck $100 \mathrm{~mL}$ round bottom flask equipped with a PTFE-coated stir-bar was connected to a $\mathrm{N}_{2}$ Schlenk line with a needle through a rubber septum in the center neck. The flask was charged with 2,6-dimethylpyrimidin-4-ol (1000 mg, $8.06 \mathrm{mmol}, 1.00$ equiv) and $\mathrm{N}$-(5-chloro2-pyridyl)bis(trifluoromethanesulfonimide) (3798 mg, $9.67 \mathrm{mmol}, 1.20$ equiv) through the side neck under positive $\mathrm{N}_{2}$ flow. The side neck was then capped with a rubber septum. The mixture was cooled in an ice-water bath under positive $\mathrm{N}_{2}$ flow. Dry dichloromethane $(16 \mathrm{~mL})$ and triethyl amine (3256 mg, $32.24 \mathrm{mmol}, 4.00$ equiv) were added to the flask by syringes through the rubber septum in the side neck. The mixture was stirred $(600 \mathrm{rpm})$ in the ice-water bath, and the temperature was allowed to naturally recover to $\mathrm{rt}$ within $12 \mathrm{~h}$. When the reaction was judged complete after $12 \mathrm{~h}$ by TLC (hexanes/ethyl acetate $=10 / 1, \mathrm{R}_{\mathrm{f}}$ of $\mathbf{3 k}=0.1$ ), the reaction mixture was poured into a 250 separatory funnel containing dichloromethane $(20 \mathrm{~mL})$ and water $(20 \mathrm{~mL})$. The dichloromethane layer was set aside, and the aqueous layer was further washed with dichloromethane $(20 \mathrm{~mL} \times 2)$. The organic layers were combined and dried over anhydrous $\mathrm{Na}_{2} \mathrm{SO}_{4}$. The mixture was filtered to remove the solids, and the filtrate was concentrated by rotary evaporation. The residue was purified by flash chromatography on silica gel (hexanes/ethyl acetate $=20 / 1$ to $6 / 1)$ to afford the desired product as a yellow oil $(1189.4 \mathrm{mg}, 58 \%) .{ }^{1} \mathrm{H} \mathrm{NMR}(500 \mathrm{MHz}$, $\left.\mathrm{CDCl}_{3}\right) \delta 6.83(\mathrm{~s}, 1 \mathrm{H}), 2.70(\mathrm{~s}, 3 \mathrm{H}), 2.57(\mathrm{~s}, 3 \mathrm{H}) ;{ }^{19} \mathrm{~F}\left\{{ }^{1} \mathrm{H}\right\}$ NMR $\left(376 \mathrm{MHz}, \mathrm{CDCl}_{3}\right) \delta-73.01$; ${ }^{13} \mathrm{C}\left\{{ }^{1} \mathrm{H}\right\} \mathrm{NMR}\left(126 \mathrm{MHz}, \mathrm{CDCl}_{3}\right) \delta 172.5,169.4,163.2,118.7$ (q, $\left.J=320.6 \mathrm{~Hz}\right), 107.4,25.8,24.5$ ppm. HRMS (ESI) m/z calcd for $[\mathrm{M}+\mathrm{H}]^{+}$257.0202, found 257.0199. IR $\left(\mathrm{cm}^{-1}\right): 2974,2927,1590$, $1505,1210,1180,982,816,625$.

\section{Procedure for the synthesis of $1 \mathrm{~m}$}

Scheme S4. Synthesis of aryl triflate $\mathbf{1 m}$ 
(c)

Aryl triflate $\mathbf{1 m}$ was synthesized according to procedure for the synthesis of $\mathbf{1 k}$ using quinoline-4-ol (435 $\mathrm{mg}, \quad 3.00 \mathrm{mmol}, \quad 1.00 \quad$ equiv) and $N$-(5-chloro-2pyridyl)bis(trifluoromethanesulfonimide) (1413 mg, $3.60 \mathrm{mmol}, 1.20$ equiv) as starting materials. The reaction was quenched according to the procedure and the crude product was purified by flash chromatography on silica gel (hexanes/ethyl acetate $=20 / 1$ ) to afford the desired product as a colorless oil (385.0 mg, 46\%). ${ }^{1} \mathrm{H}$ NMR (400 MHz, $\left.\mathrm{CDCl}_{3}\right) \delta 8.98(\mathrm{~d}, J=4.9 \mathrm{~Hz}, 1 \mathrm{H}), 8.21(\mathrm{~d}, J$ $=8.5 \mathrm{~Hz}, 1 \mathrm{H}), 8.08(\mathrm{~d}, J=8.2 \mathrm{~Hz}, 1 \mathrm{H}), 7.85(\mathrm{ddd}, J=8.5,6.9,1.4 \mathrm{~Hz}, 1 \mathrm{H}), 7.72(\mathrm{ddd}, J=8.2$, 6.9, $1.2 \mathrm{~Hz}, 1 \mathrm{H}), 7.42(\mathrm{~d}, J=5.0 \mathrm{~Hz}, 1 \mathrm{H}) ;{ }^{19} \mathrm{~F}\left\{{ }^{1} \mathrm{H}\right\} \mathrm{NMR}\left(377 \mathrm{MHz}, \mathrm{CDCl}_{3}\right) \delta-73.05 ;{ }^{13} \mathrm{C}\left\{{ }^{1} \mathrm{H}\right\}$ NMR $\left(126 \mathrm{MHz}, \mathrm{CDCl}_{3}\right) \delta 153.0,150.8,150.6,131.3,130.0,128.6,121.4,120.8,118.8(\mathrm{q}, J=$ 320.6 Hz), $112.0 \mathrm{ppm}$. HRMS (ESI) m/z calcd for $[\mathrm{M}+\mathrm{H}]^{+} 278.0093$, found 278.0090. IR $\left(\mathrm{cm}^{-1}\right)$ : 3076, 1602, 1499, 1421, 1211, 1134, 1026, 896, 761, 596.

\subsection{Synthesis of heteroaryl halides}

Scheme S5. Aryl halides synthesized in this study

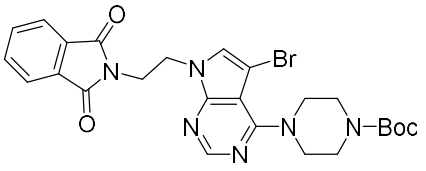

$2 \mathrm{~b}$

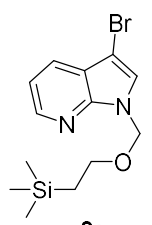

$2 \mathrm{~g}$

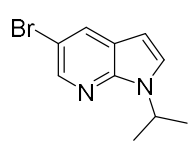

2c

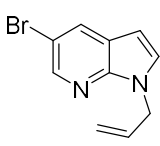

$2 \mathrm{~d}$

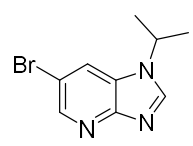

$2 e$

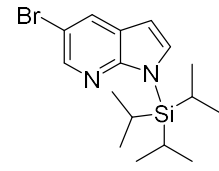

$2 f$

Known compounds $\mathbf{2 c},{ }^{13} \mathbf{2 f},{ }^{14}$ and $\mathbf{2} \mathbf{g}^{15}$ were prepared according to the reported procedures. The preparations of unknown compound $\mathbf{2} \mathbf{b}, \mathbf{2} \mathbf{d}$, and $\mathbf{2 e}$ were described below.

\section{Procedure for the synthesis of $2 \mathrm{~b}$}

Scheme S6. Synthesis of aryl bromide $\mathbf{2 b}$
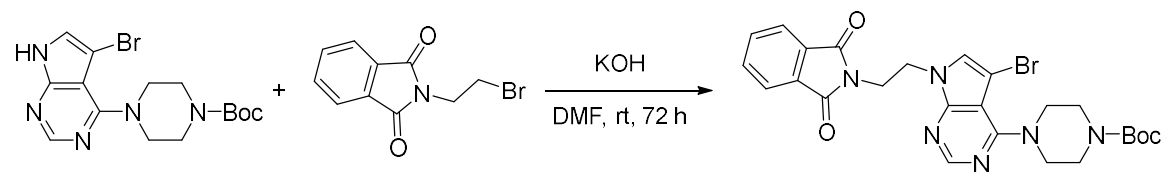

$2 \mathbf{b}$

A $20 \mathrm{~mL}$ scintillation vial with a PTFE-coated stir-bar was charged with tert-butyl 4-(5bromo-7H-pyrrolo[2,3-d]pyrimidin-4-yl)piperazine-1-carboxylate $(382 \mathrm{mg}, 1.00 \mathrm{mmol}, 1.00$ equiv), 2-(2-bromoethyl)isoindoline-1,3-dione (508 mg, $2.00 \mathrm{mmol}, 2.00$ equiv), and $\mathrm{KOH}$ (120 
$\mathrm{mg}, \geq 85 \%$, pellets). DMF (6 mL) was then added, and the vial was capped with a screw cap fitted with a PTFE-faced silicone septum. The mixture was stirred (1000 rpm) at $\mathrm{rt}$ for $72 \mathrm{~h}$, and the reaction was judged complete by TLC (hexanes/ethyl acetate $=1 / 1, \mathrm{R}_{\mathrm{f}}$ of $\mathbf{2 b}=0.4$ ). The reaction mixture was poured into a 250 separatory funnel containing dichloromethane $(20 \mathrm{~mL})$ and water $(20 \mathrm{~mL})$. The dichloromethane layer was set aside, and the aqueous layer was further washed with dichloromethane $(20 \mathrm{~mL} \times 2)$. The organic layers were combined and dried over anhydrous $\mathrm{Na}_{2} \mathrm{SO}_{4}$. The mixture was filtered to remove the solids, and the filtrate was concentrated by rotary evaporation. The residue was purified by flash chromatography on silica gel (hexanes/ethyl acetate $=3 / 1$ to $1 / 1)$ to afford the desired product as a white solid $(293.2 \mathrm{mg}, 53 \%)$. mp $116-117{ }^{\circ} \mathrm{C} .{ }^{1} \mathrm{H}$ NMR (500 MHz, $\left.\mathrm{CDCl}_{3}\right) \delta 8.08(\mathrm{~s}, 1 \mathrm{H}), 7.75(\mathrm{dd}, J=5.5,3.1 \mathrm{~Hz}, 2 \mathrm{H}), 7.67(\mathrm{dd}, J=5.5,3.1 \mathrm{~Hz}$, $2 \mathrm{H}), 7.02(\mathrm{~s}, 1 \mathrm{H}), 4.48(\mathrm{t}, J=5.0 \mathrm{~Hz}, 2 \mathrm{H}), 4.06(\mathrm{t}, J=5.0 \mathrm{~Hz}, 2 \mathrm{H}), 3.61-3.58(\mathrm{~m}, 4 \mathrm{H}), 3.55-$ $3.53(\mathrm{~m}, 4 \mathrm{H}), 1.47(\mathrm{~s}, 9 \mathrm{H}) ;{ }^{13} \mathrm{C}\left\{{ }^{1} \mathrm{H}\right\} \mathrm{NMR}\left(126 \mathrm{MHz}, \mathrm{CDCl}_{3}\right) \delta 167.9,160.0,155.0,151.5,151.2$, 134.2, 132.0, 125.6, 123.4, 105.6, 87.6, 80.1, 50.1, 44.1, 43.3, 43.3, 37.9, 28.6 ppm. Attention: the four carbons in the piperazine structure is responsible for three different signals in the ${ }^{13} \mathrm{C} \mathrm{NMR}$ spectrum with one peak being sharp and strong (should be the peak at 50.1 ppm according to the ${ }^{13}$ C NMR spectrum of compound 3ae) and the other two wide and low (44.1 and 43.3 ppm). This is consistent with the reported ${ }^{13}$ C NMR spectrum (see Supporting Information page S147 of ref. 16) of a similar structure, ${ }^{16}$ tert-butyl 4-(7H-pyrrolo[2,3-d]pyrimidin-4-yl)piperazine-1carboxylate. HRMS (ESI) $\mathrm{m} / \mathrm{z}$ calcd for $[\mathrm{M}+\mathrm{H}]^{+}$555.1350, found 555.1344. IR $\left(\mathrm{cm}^{-1}\right): 2927$, $1712,1690,1556,1393,1237,1166,998,719$.

\section{Procedure for the synthesis of $2 d$}

Scheme S7. Synthesis of aryl bromide 2d

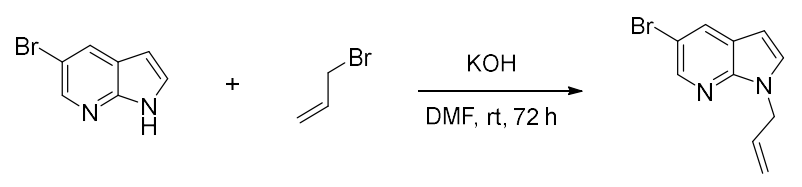

Compound 2c was synthesized following procedure for the synthesis of $\mathbf{2} \boldsymbol{b}$ using 5-bromo$1 H$-pyrrolo[2,3-b]pyridine (394 mg, $2.00 \mathrm{mmol}, 1.00$ equiv), 3-bromoprop-1-ene (484 mg, 4.00 mmol, 2.00 equiv) and $\mathrm{KOH}(240 \mathrm{mg}, \geq 85 \%$, pellets $)$ as starting materials and DMF (12 mL) as solvent. After stirring at $\mathrm{rt}$ for $72 \mathrm{~h}$, the reaction was judged complete by TLC (hexanes/ethyl acetate $=5 / 1, R_{\mathrm{f}}$ of $\mathbf{2 d}=0.8$ ). The reaction was quenched following the procedure, and the crude material was purified by flash chromatography on silica gel (hexanes/ethyl acetate $=10 / 1$ ) to afford the desired product as a colorless oil (380.3 mg, 80\% yield). ${ }^{1} \mathrm{H} \mathrm{NMR}\left(500 \mathrm{MHz}, \mathrm{CDCl}_{3}\right) \delta 8.33$ $(\mathrm{d}, J=2.1 \mathrm{~Hz}, 1 \mathrm{H}), 8.01(\mathrm{~d}, J=2.2 \mathrm{~Hz}, 1 \mathrm{H}), 7.21(\mathrm{~d}, J=3.5 \mathrm{~Hz}, 1 \mathrm{H}), 6.41(\mathrm{~d}, J=3.5 \mathrm{~Hz}, 1 \mathrm{H})$, 
$6.06-5.98(\mathrm{~m}, 1 \mathrm{H}), 5.20(\mathrm{dq}, J=10.2,1.4 \mathrm{~Hz}, 1 \mathrm{H}), 5.08$ (dq, $J=17.1,1.6 \mathrm{~Hz}, 1 \mathrm{H}), 4.87$ (dt, $J$ $=5.5,1.6 \mathrm{~Hz}, 2 \mathrm{H}) ;{ }^{13} \mathrm{C}\left\{{ }^{1} \mathrm{H}\right\} \operatorname{NMR}\left(126 \mathrm{MHz}, \mathrm{CDCl}_{3}\right) \delta 145.9,143.5,133.5,130.9,129.4,122.2$, 117.7, 111.8, 99.5, $46.9 \mathrm{ppm}$. HRMS (ESI) $\mathrm{m} / \mathrm{z}$ calcd for $[\mathrm{M}+\mathrm{H}]^{+} 237.0022$, found 237.0022. IR $\left(\mathrm{cm}^{-1}\right): 3082,2920,1505,1467,1405,1200,912,884,763,719$.

\section{Procedure for the synthesis of $2 \mathrm{e}$}

Scheme S8. Synthesis of aryl bromide 2e

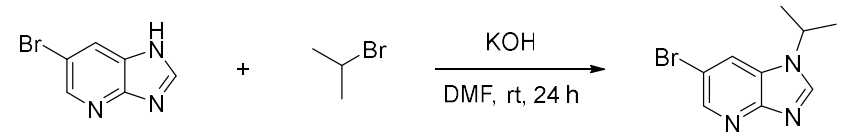

Compound $\mathbf{2 e}$ was synthesized following procedure for the synthesis of $\mathbf{2 b}$ using 6-bromo$1 H$-imidazo[4,5-b]pyridine (198 mg, $1.00 \mathrm{mmol}, 1.00$ equiv), 2-bromopropane (369 mg, 3.00 mmol, 3.00 equiv) and $\mathrm{KOH}$ (168 $\mathrm{mg}, \geq 85 \%$, pellets) as starting materials and DMF (6 mL) as solvent. After stirring at $\mathrm{rt}$ for $24 \mathrm{~h}$, the reaction was judged complete by TLC (hexanes (containing triethyl amine, $5 \% \mathrm{v} / \mathrm{v}) /$ ethyl acetate $=1 / 1, \mathrm{R}_{\mathrm{f}}$ of $\mathbf{2 e}=0.4$ ). The reaction was quenched following the procedure, and the crude material was purified by flash chromatography on silica gel (hexanes (containing triethyl amine, $5 \% \mathrm{v} / \mathrm{v}$ )/ethyl acetate $=3 / 2$ ) to afford the desired product as a colorless oil (140.0 mg, 58\% yield). ${ }^{1} \mathrm{H}$ NMR (500 MHz, $\left.\mathrm{CDCl}_{3}\right) \delta 8.42(\mathrm{~d}, J=2.1 \mathrm{~Hz}, 1 \mathrm{H}), 8.18(\mathrm{~d}, J=$ $2.0 \mathrm{~Hz}, 1 \mathrm{H}), 8.10(\mathrm{~s}, 1 \mathrm{H}), 4.93$ (hept, $J=6.8 \mathrm{~Hz}, 1 \mathrm{H}), 1.63(\mathrm{~d}, J=6.8 \mathrm{~Hz}, 6 \mathrm{H}) ;{ }^{13} \mathrm{C}\left\{{ }^{1} \mathrm{H}\right\} \mathrm{NMR}$ $\left(126 \mathrm{MHz}, \mathrm{CDCl}_{3}\right) \delta 145.5,144.9,142.8,137.0,130.4,114.0,47.2,22.7 \mathrm{ppm}$. HRMS (ESI) m/z calcd for $[\mathrm{M}+\mathrm{H}]^{+} 240.0131$, found 240.0130. IR $\left(\mathrm{cm}^{-1}\right): 2976,2933,1485,1388,1223,914,882$, $799,640,521$. 


\section{Cross-electrophile Couplings of Heteroaryl Halides with Heteroaryl Triflates}

\subsection{General procedure for reaction setup in scope investigations}

The following procedures were conducted in a $\mathrm{N}_{2}$ filled glovebox for convenience. All the reactions were conducted under Conditions A unless otherwise noted. Conditions A and B use different amine ligands on nickel, but they share the same procedures for preparation of palladium catalyst stock solution, catalytic reaction setup, SFC-MS analysis, reaction workup, and product purification.

Preparation of nickel catalyst stock solution for Conditions A (0.050 M with respect to nickel)

An oven-dried 1-dram vial equipped with a PTFE-coated stir-bar was charged with $\mathrm{NiBr}_{2}(\mathrm{dme})(30.8 \mathrm{mg}, 0.100 \mathrm{mmol})$ and 4,4'-di-tert-butyl-2,2'-bipyridine (N1) (29.5 mg, 0.110 mmol). DMF $(2.0 \mathrm{~mL})$ was then added before the dram vial was capped with a screw cap fitted with a PTFE-faced silicone septum. The mixture was stirred $(1000 \mathrm{rpm})$ at $60{ }^{\circ} \mathrm{C}$ for $3 \mathrm{~h}$ before being used (vide infra).

Preparation of nickel catalyst stock solution for Conditions B (0.050 M with respect to nickel)

An oven-dried 1-dram vial equipped with a PTFE-coated stir-bar was charged with $\mathrm{NiBr}_{2}(\mathrm{dme})(30.8 \mathrm{mg}, 0.100 \mathrm{mmol})$ and 4,4',4"-tri-tert-butyl-2,2':6',2"-terpyridine (N3) (44.2 mg, $0.110 \mathrm{mmol})$. DMF $(2.0 \mathrm{~mL})$ was then added before the dram vial was capped with a screw cap fitted with a PTFE-faced silicone septum. The mixture was stirred $(1000 \mathrm{rpm})$ at $60{ }^{\circ} \mathrm{C}$ for $3 \mathrm{~h}$ before being used (vide infra).

\section{Preparation of palladium catalyst stock solution $(0.025 \mathrm{M}$ with respect to palladium)}

An oven-dried 1-dram vial equipped with a PTFE-coated stir-bar was charged with $\mathrm{PdCl}_{2}$ (17.7 $\mathrm{mg}, 0.100 \mathrm{mmol})$ and dppp (P1) (45.4 mg, 0.110 mmol). DMF (4.0 mL) was then added before the dram vial was capped with a screw cap fitted with a PTFE-faced silicone septum. The mixture was stirred $(1000 \mathrm{rpm})$ at $60{ }^{\circ} \mathrm{C}$ for $3 \mathrm{~h}$ before being used (vide infra).

\section{Catalytic reaction setup}

An oven-dried 1-dram vial equipped with a PTFE-coated stir-bar was charged sequentially with heteroaryl triflate $(0.50 \mathrm{mmol}, 1.00$ equiv), heteroaryl halide $(0.75 \mathrm{mmol}, 1.50$ equiv), $\mathrm{KBr}$ (59.5 mg, $0.50 \mathrm{mmol}, 1.00$ equiv), and $\mathrm{Zn}(65.4 \mathrm{mg}, 1.00 \mathrm{mmol}, 2.00$ equiv). Aliquots from both 
the $0.050 \mathrm{M}$ nickel (500 $\mu \mathrm{L}$ for $5.0 \mathrm{~mol} \%$ catalyst loading) and $0.025 \mathrm{M}$ palladium (1000 $\mu \mathrm{L}$ for $5.0 \mathrm{~mol} \%$ catalyst loading) stock solutions (vide supra) were then added. The vial was capped with a screw cap fitted with a PTFE-faced silicone septum, and the mixture was stirred (1000 rpm) for $10 \mathrm{~min}$ in the glovebox at rt. The reaction vial was then removed from the glovebox and heated in a reaction block set to the desired temperature with stirring (1250 rpm) for $24 \mathrm{~h}$.

\section{SFC-MS analysis \#1}

After $24 \mathrm{~h}$, the reaction mixture was analyzed by SFC-MS. A $20 \mu \mathrm{L}$ aliquot of the reaction mixture was further diluted with $\mathrm{MeOH}(1 \mathrm{~mL})$, and the resulting mixture was transferred into a 1 $\mathrm{mL}$ syringe and then filtered into an SFC-MS vial through a syringe filter (PVDF membrane, 0.22 $\mu \mathrm{m}$ pore size, $13 \mathrm{~mm}$ diameter). The sample was analyzed by our standard method (see $\underline{1 . \text { General }}$ Information of this document for details) on SFC-MS. The reaction was judged complete when either the heteroaryl triflate or heteroaryl halide was fully consumed as determined by SFC-MS. Attention: Most heteroaryl triflates and halides used in this study are soluble in DMF and MeOH. However, because in SFC-MS analysis \#1 the aliquot from the reaction mixture was not quenched by Na $a_{4}$ DTA aqueous solution, the SFC-MS analysis results obtained here should NOT be used to determine the formation of biheteroaryl product in the reaction. We found that in some cases, the biheteroaryl products (especially 2,2'-bipyridine-like compounds) could form ligand-metal complexes which are insoluble in DMF or MeOH, and thus could not be detected by SFC-MS if the aliquot was not properly quenched.

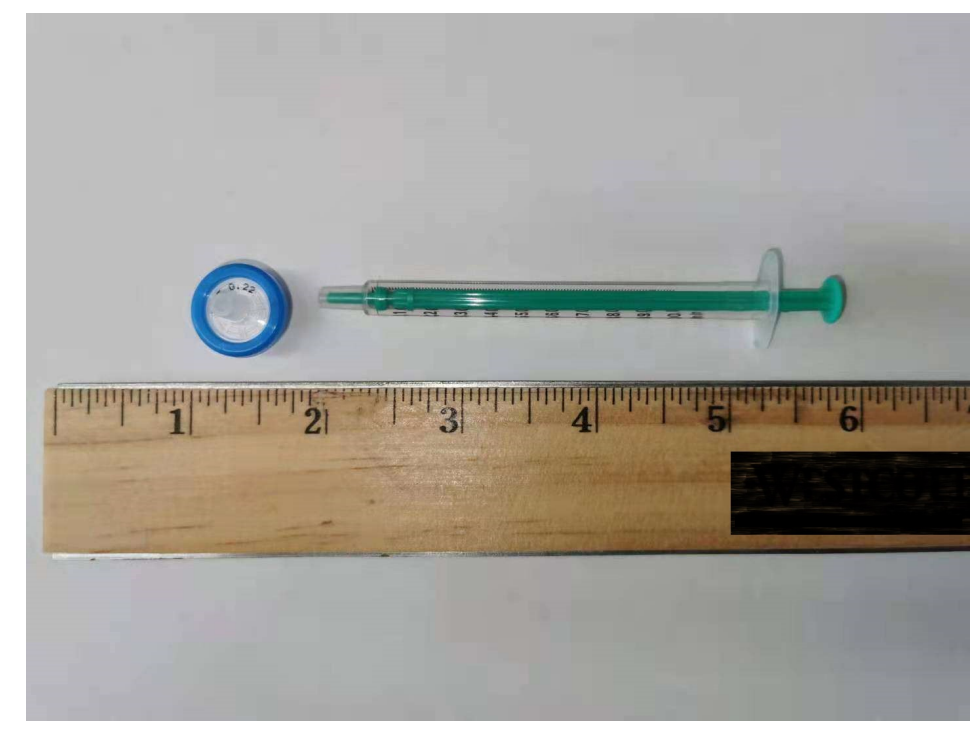

Figure S2. A $1 \mathrm{~mL}$ syringe and a syringe filter (PVDF membrane, $0.22 \mu \mathrm{m}$ pore size, $13 \mathrm{~mm}$ diameter) used in SFC-MS analysis \#1. 


\section{Reaction workup}

The reaction mixture was cooled to $\mathrm{rt}$ before being transferred to a $20 \mathrm{~mL}$ scintillation vial that was equipped with a PTFE-coated stir-bar with a Pasteur pipette. Three portions of dichloromethane $(1.0 \mathrm{~mL} \times 3)$ were used to further rinse the reaction vial. $\mathrm{Na}_{4} \mathrm{EDTA}$ aqueous solution $(25 \% \mathrm{wt}, 4.0 \mathrm{~mL})$ was then added to the $20 \mathrm{~mL}$ scintillation vial. The vial was capped with a screw cap fitted with a PTFE-faced silicone septum, and the mixture was stirred (1000 rpm) for $1 \mathrm{~h}$ at $\mathrm{rt}$. After that, the quenched mixture was transferred to a $250 \mathrm{~mL}$ separatory funnel containing dichloromethane $(20 \mathrm{~mL})$ and water $(20 \mathrm{~mL})$. The dichloromethane layer was set aside, and the aqueous layer was washed with dichloromethane $(20 \mathrm{~mL} \times 2)$. The dichloromethane layers were combined and dried over anhydrous $\mathrm{Na}_{2} \mathrm{SO}_{4}$. The resulting mixture was filtered through a disposable filter funnel $(20 \mathrm{~mL}, 10$-micron polyethylene frit) to remove the solid, and the filtrate was collected for SFC-MS analysis and purification. Attention: we found that in some cases, filtration through a short plug of silica gel or even Celite could result in loss of biheteroaryl product.

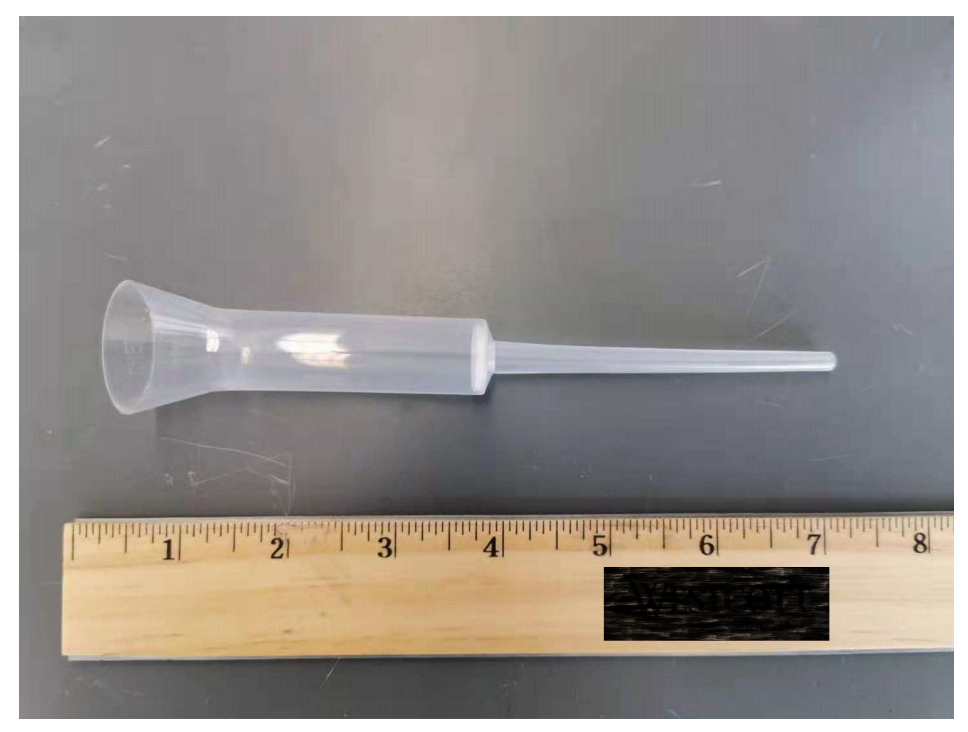

Figure S3. A disposable filter funnel (20 mL, 10-micron polyethylene frit) used in the reaction workup.

\section{SFC-MS analysis \#2}

SFC-MS analysis was conducted again to detect the formation of biheteroaryl product. An aliquot $(50 \mu \mathrm{L})$ of the solution from the reaction workup was further diluted with $\mathrm{MeOH}(1 \mathrm{~mL})$, and the resulting mixture was transferred into a $1 \mathrm{~mL}$ syringe and then filtered into an SFC-MS vial through a syringe filter (PVDF membrane, $0.22 \mu \mathrm{m}$ pore size, $13 \mathrm{~mm}$ diameter). The sample 
was analyzed by our standard method (see 1. General Information of this document for details) on SCF-MS.

\section{Purification}

Silica gel $(\sim 2 \mathrm{~g})$ was added to the resulting filtrate from the reaction workup, and the volatile solvents were removed by rotary evaporation. The dry-loaded material was purified by flash chromatography on silica gel to provide the desired product. 


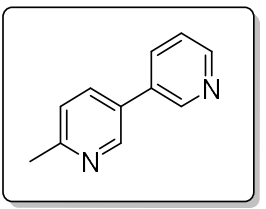

6-Methyl-3,3'-bipyridine (3a) [CAS: 78210-83-0] was synthesized according to the general procedure using 6-methylpyridin-3-yl trifluoromethanesulfonate $(0.50 \mathrm{mmol}, 120.6 \mathrm{mg}, 1.00$ equiv) and 3-bromopyridine ( $0.75 \mathrm{mmol}, 118.5 \mathrm{mg}, 1.50$ equiv) as starting materials. The reaction was quenched according to the general procedure after stirring at $60{ }^{\circ} \mathrm{C}$ for $24 \mathrm{~h}$, and the crude material was purified by flash chromatography on silica gel (hexanes (containing triethyl amine, $5 \% \mathrm{v} / \mathrm{v}) /$ ethyl acetate $=3 / 1)$ to afford a colorless oil $(80.8 \mathrm{mg})$ containing $\mathbf{3 a}(63.6 \mathrm{mg}, 75 \%$ yield $)$, 3,3'-bipyridine (11.7 mg, 15\% yield), and 6,6'-dimethyl-3,3'-bipyridine (5.5 mg, 6\% yield). ${ }^{1} \mathrm{H}$ NMR $\left(500 \mathrm{MHz}, \mathrm{CDCl}_{3}\right) \delta 8.77(\mathrm{~d}, J=1.8 \mathrm{~Hz}, 1 \mathrm{H}), 8.66(\mathrm{~d}, J=2.3 \mathrm{~Hz}, 1 \mathrm{H}), 8.57$ (dd, $J=4.8$, $1.6 \mathrm{~Hz}, 1 \mathrm{H}), 7.80$ (ddd, $J=7.9,2.0,1.6 \mathrm{~Hz}, 1 \mathrm{H}), 7.71$ (dd, $J=8.1,2.5 \mathrm{~Hz}, 1 \mathrm{H}), 7.33$ (ddd, $J=$ 8.0, 4.8, 0.9 Hz, $1 \mathrm{H}), 7.20(\mathrm{~d}, J=8.0 \mathrm{~Hz}, 1 \mathrm{H}), 2.56(\mathrm{~s}, 3 \mathrm{H}) ;{ }^{13} \mathrm{C}\left\{{ }^{1} \mathrm{H}\right\} \mathrm{NMR}\left(126 \mathrm{MHz}, \mathrm{CDCl}_{3}\right)$ $\delta 158.3,149.1,148.1,147.5,134.7,134.4,134.2,130.6,123.8,123.4,24.2$ ppm. HRMS (ESI) m/z calcd for $[\mathrm{M}+\mathrm{H}]^{+}$171.0917, found 171.0914. For 3,3'-bipyridine, ${ }^{17} \mathrm{HRMS}$ (ESI) $\mathrm{m} / \mathrm{z}$ calcd for $[\mathrm{M}+\mathrm{H}]^{+}$157.0760, found 157.0759. For 6,6'-dimethyl-3,3'-bipyridine, ${ }^{18} \mathrm{HRMS}$ (ESI) m/z calcd for $[\mathrm{M}+\mathrm{H}]^{+}$185.1073, found 185.1072 .

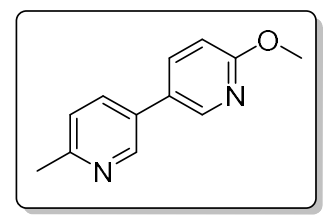

6-Methoxy-6'-methyl-3,3'-bipyridine (3b) was synthesized according to the general procedure using 6-methylpyridin-3-yl trifluoromethanesulfonate $(0.50 \mathrm{mmol}, 120.6 \mathrm{mg}, 1.00$ equiv) and 5bromo-2-methoxypyridine $(0.75 \mathrm{mmol}, 141.0 \mathrm{mg}, 1.50 \mathrm{equiv})$ as starting materials. The reaction was quenched according to the general procedure after stirring at $60{ }^{\circ} \mathrm{C}$ for $24 \mathrm{~h}$, and the crude material was purified by flash chromatography on silica gel (hexanes/ethyl acetate $=9 / 1$ to $1 / 1$ ) to afford the desired product as a white solid (78.7 mg, 79\% yield). mp $70-71{ }^{\circ} \mathrm{C} .{ }^{1} \mathrm{H}$ NMR $(500$ $\left.\mathrm{MHz} \mathrm{CDCl}_{3}\right) \delta 8.65(\mathrm{~d}, J=2.4 \mathrm{~Hz}, 1 \mathrm{H}), 8.34(\mathrm{~d}, J=2.6 \mathrm{~Hz}, 1 \mathrm{H}), 7.74(\mathrm{dd}, J=8.6,2.6 \mathrm{~Hz}, 1 \mathrm{H})$, $7.68(\mathrm{dd}, J=8.0,2.4 \mathrm{~Hz}, 1 \mathrm{H}), 7.20(\mathrm{~d}, J=8.0 \mathrm{~Hz}, 1 \mathrm{H}), 6.82(\mathrm{dd}, J=8.5,0.8 \mathrm{~Hz}, 1 \mathrm{H}), 3.96(\mathrm{~s}, 3$ $\mathrm{H}), 2.58(\mathrm{~s}, 3 \mathrm{H}) ;{ }^{13} \mathrm{C}\left\{{ }^{1} \mathrm{H}\right\} \operatorname{NMR}\left(126 \mathrm{MHz} \mathrm{CDCl}_{3}\right) \delta 164.1,157.5,147.2,145.1,137.3,134.4$, 130.8, 127.0, 123.4, 111.3, 53.7, 24.3 ppm. HRMS (ESI) $\mathrm{m} / \mathrm{z}$ calcd for $[\mathrm{M}+\mathrm{H}]^{+} 201.1022$, found 201.1021. IR (cm $\left.{ }^{-1}\right): 3011,2921,1598,1477,1282,1020,820,732$. 


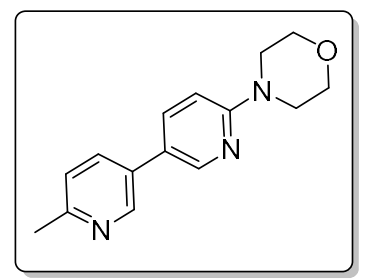

4-(6'-Methyl-[3,3'-bipyridin]-6-yl)morpholine (3c) was synthesized according to the general procedure using 6-methylpyridin-3-yl trifluoromethanesulfonate $(0.50 \mathrm{mmol}, 120.6 \mathrm{mg}, 1.00$ equiv) and 4-(5-bromopyridin-2-yl)morpholine ( $0.75 \mathrm{mmol}, 182.3 \mathrm{mg}, 1.50$ equiv) as starting materials. The reaction was quenched according to the general procedure after stirring at $60{ }^{\circ} \mathrm{C}$ for $24 \mathrm{~h}$, and the crude material was purified by flash chromatography on silica gel (hexanes/ethyl acetate $=5 / 1$ to $1 / 1)$ to afford the desired product as a white solid (100.5 $\mathrm{mg}, 79 \%$ yield). $\mathrm{mp}$ $160{ }^{\circ} \mathrm{C}$ dec. ${ }^{1} \mathrm{H}$ NMR $\left(500 \mathrm{MHz}, \mathrm{CDCl}_{3}\right) \delta 8.64(\mathrm{~d}, J=2.4 \mathrm{~Hz}, 1 \mathrm{H}), 8.40(\mathrm{~d}, J=2.6 \mathrm{~Hz}, 1 \mathrm{H})$, $7.69(\mathrm{dd}, J=4.4,2.5 \mathrm{~Hz}, 1 \mathrm{H}), 7.67(\mathrm{dd}, J=3.6,2.5 \mathrm{~Hz}, 1 \mathrm{H}), 7.18(\mathrm{~d}, J=8.0 \mathrm{~Hz}, 1 \mathrm{H}), 6.69(\mathrm{~d}, J$ $=8.7 \mathrm{~Hz}, 1 \mathrm{H}), 3.82(\mathrm{t}, J=5.0 \mathrm{~Hz}, 4 \mathrm{H}), 3.54(\mathrm{t}, J=5.0 \mathrm{~Hz}, 4 \mathrm{H}), 2.56(\mathrm{~s}, 3 \mathrm{H}) ;{ }^{13} \mathrm{C}\left\{{ }^{1} \mathrm{H}\right\} \mathrm{NMR}$ $\left(126 \mathrm{MHz}, \mathrm{CDCl}_{3}\right) \delta 159.1,156.9,146.8,146.2,136.0,133.9,131.1,123.6,123.4,106.9,66.8$, 45.7, $24.2 \mathrm{ppm}$. HRMS (ESI) $\mathrm{m} / \mathrm{z}$ calcd for $[\mathrm{M}+\mathrm{H}]^{+} 256.1444$, found 256.1441. IR $\left(\mathrm{cm}^{-1}\right): 2964$, 2922, 2855, 1598, 1476, 1237, 1116, 942, 809, 734.

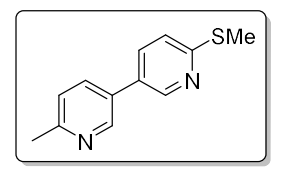

6-Methyl-6'-(methylthio)-3,3'-bipyridine (3d) was synthesized according to the general procedure using 6-methylpyridin-3-yl trifluoromethanesulfonate $(0.50 \mathrm{mmol}, 120.6 \mathrm{mg}, 1.00$ equiv) and 5-bromo-2-(methylthio)pyridine (0.75 mmol, $153.1 \mathrm{mg}, 1.50$ equiv) as starting materials. The reaction was quenched according to the general procedure after stirring at $60{ }^{\circ} \mathrm{C}$ for $24 \mathrm{~h}$, and the crude material was purified by flash chromatography on silica gel (hexanes (containing triethyl amine, $5 \% \mathrm{v} / \mathrm{v}$ ) $/$ ethyl acetate $=5 / 1$ to $1 / 1$ ) to afford the desired product as a white solid (25.3 mg, 23\% yield). mp $69-70{ }^{\circ} \mathrm{C} .{ }^{1} \mathrm{H}$ NMR $\left(500 \mathrm{MHz}, \mathrm{CDCl}_{3}\right) \delta 8.62$ (d, J=2.1 $\mathrm{Hz}, 1 \mathrm{H}), 8.58(\mathrm{~d}, J=1.6 \mathrm{~Hz}, 1 \mathrm{H}), 7.66(\mathrm{dd}, J=8.0,2.4 \mathrm{~Hz}, 1 \mathrm{H}), 7.60(\mathrm{dd}, J=8.3,2.4 \mathrm{~Hz}, 1 \mathrm{H})$, $7.20(\mathrm{dd}, J=8.3,0.9 \mathrm{~Hz}, 1 \mathrm{H}), 7.17(\mathrm{~d}, J=8.0 \mathrm{~Hz}, 1 \mathrm{H}), 2.534(\mathrm{~s}, 3 \mathrm{H}), 2.532(\mathrm{~s}, 3 \mathrm{H}) ;{ }^{13} \mathrm{C}\left\{{ }^{1} \mathrm{H}\right\}$ NMR $\left(126 \mathrm{MHz}, \mathrm{CDCl}_{3}\right) \delta 159.8,158.0,147.6,147.2,134.5,134.2,130.6,129.2,123.6,121.7$, 24.3, $13.5 \mathrm{ppm}$. HRMS (ESI) $\mathrm{m} / \mathrm{z}$ calcd for $[\mathrm{M}+\mathrm{H}]^{+} 217.0794$, found 217.0793. IR $\left(\mathrm{cm}^{-1}\right): 2853$, $1588,1457,1118,816,730$. 


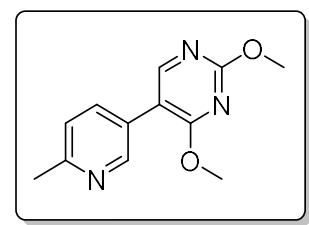

2,4-Dimethoxy-5-(6-methylpyridin-3-yl)pyrimidine (3e) was synthesized according to the general procedure using 6-methylpyridin-3-yl trifluoromethanesulfonate $(0.50 \mathrm{mmol}, 120.6 \mathrm{mg}$, 1.00 equiv) and 5-bromo-2,4-dimethoxypyrimidine ( $0.75 \mathrm{mmol}, 164.2 \mathrm{mg}, 1.50$ equiv) as starting materials. The reaction was quenched according to the general procedure after stirring at $60{ }^{\circ} \mathrm{C}$ for $24 \mathrm{~h}$, and the crude material was purified by flash chromatography on silica gel (hexanes (containing triethyl amine, $5 \% \mathrm{v} / \mathrm{v}$ ) $/$ ethyl acetate $=5 / 1$ to $4 / 1$ ) to afford the desired product as a white solid (87.3 mg, 76\% yield). mp $112-113{ }^{\circ} \mathrm{C} .{ }^{1} \mathrm{H}$ NMR $\left(500 \mathrm{MHz}, \mathrm{CDCl}_{3}\right) \delta 8.58(\mathrm{~d}, J=$ $1.6 \mathrm{~Hz}, 1 \mathrm{H}), 8.23$ (s, $1 \mathrm{H}), 7.69$ (dd, $J=8.0,2.3 \mathrm{~Hz}, 1 \mathrm{H}), 7.19$ (d, J=8.0 Hz, $1 \mathrm{H}), 4.01$ (s, $3 \mathrm{H})$, $4.00(\mathrm{~s}, 3 \mathrm{H}), 2.57(\mathrm{~s}, 3 \mathrm{H}) ;{ }^{13} \mathrm{C}\left\{{ }^{1} \mathrm{H}\right\} \mathrm{NMR}\left(126 \mathrm{MHz}, \mathrm{CDCl}_{3}\right) \delta 168.4,165.1,157.8,157.5,148.8$, 136.5, 126.4, 123.0, 113.2, 55.1, 54.3, $24.3 \mathrm{ppm}$. HRMS (ESI) m/z calcd for $[\mathrm{M}+\mathrm{H}]^{+} 232.1081$, found 232.1079. IR ( $\left.\mathrm{cm}^{-1}\right)$ : 2957, 2922, 1565, 1466, 1376, 1082, 1007, 763, 750.

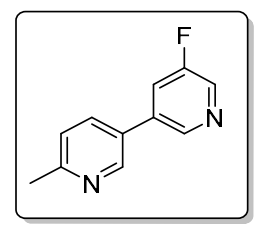

5-Fluoro-6'-methyl-3,3'-bipyridine (3f) was synthesized according to the general procedure under Conditions B using 6-methylpyridin-3-yl trifluoromethanesulfonate $(0.50 \mathrm{mmol}, 120.6 \mathrm{mg}$, 1.00 equiv) and 3-bromo-5-fluoropyridine ( $0.75 \mathrm{mmol}, 132.0 \mathrm{mg}, 1.50$ equiv) as starting materials. The reaction was quenched according to the general procedure after stirring at $60{ }^{\circ} \mathrm{C}$ for $24 \mathrm{~h}$, and the crude material was purified by flash chromatography on silica gel (hexanes (containing triethyl amine, $5 \% \mathrm{v} / \mathrm{v}) /$ ethyl acetate $=5 / 1$ to $3 / 1)$ to afford the desired product as a white solid $(70.2 \mathrm{mg}$, $75 \%$ yield). mp $116-117{ }^{\circ} \mathrm{C} .{ }^{1} \mathrm{H}$ NMR $\left(500 \mathrm{MHz}, \mathrm{CDCl}_{3}\right) \delta 8.68(\mathrm{~d}, J=2.4 \mathrm{~Hz}, 1 \mathrm{H}), 8.61(\mathrm{t}, J$ $=1.8 \mathrm{~Hz}, 1 \mathrm{H}), 8.45(\mathrm{~d}, J=2.7 \mathrm{~Hz}, 1 \mathrm{H}), 7.74(\mathrm{dd}, J=8.1,2.5 \mathrm{~Hz}, 1 \mathrm{H}), 7.54(\mathrm{dt}, J=9.3,2.2 \mathrm{~Hz}$, $1 \mathrm{H}), 7.25(\mathrm{~d}, J=8.0 \mathrm{~Hz}, 1 \mathrm{H}), 2.59(\mathrm{~s}, 3 \mathrm{H}) ;{ }^{19} \mathrm{~F}\left\{{ }^{1} \mathrm{H}\right\} \mathrm{NMR}\left(377 \mathrm{MHz}, \mathrm{CDCl}_{3}\right) \delta-126.38(\mathrm{~s}, 1 \mathrm{~F})$; ${ }^{13} \mathrm{C}\left\{{ }^{1} \mathrm{H}\right\} \operatorname{NMR}\left(126 \mathrm{MHz}, \mathrm{CDCl}_{3}\right) \delta 159.8(\mathrm{~d}, J=257.6 \mathrm{~Hz}), 159.1,147.6,144.0(\mathrm{~d}, J=4.1 \mathrm{~Hz})$, $137.4(\mathrm{~d}, J=23.1 \mathrm{~Hz}), 135.3(\mathrm{~d}, J=3.9 \mathrm{~Hz}), 134.9,129.3$ (d, $J=1.6 \mathrm{~Hz}), 123.6,121.0(\mathrm{~d}, J=18.7$ $\mathrm{Hz}$ ), $24.3 \mathrm{ppm}$. HRMS (ESI) $\mathrm{m} / \mathrm{z}$ calcd for $[\mathrm{M}+\mathrm{H}]^{+}$189.0822, found 189.0821. IR $\left(\mathrm{cm}^{-1}\right): 3018$, 2921, 1600, 1498, 1427, 1371, 1192, 1021, 895, 879, 832, 705. 


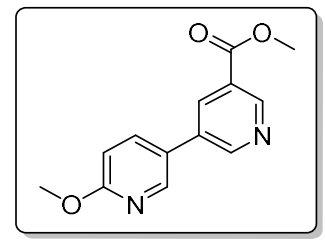

Methyl 6'-methoxy-[3,3'-bipyridine]-5-carboxylate (3g) was synthesized according to the general procedure under Conditions B using 6-methoxylpyridin-3-yl trifluoromethanesulfonate (0.50 mmol, $128.6 \mathrm{mg}, 1.00$ equiv) and methyl 5-bromonicotinate $(0.75 \mathrm{mmol}, 162.0 \mathrm{mg}, 1.50$ equiv) as starting materials. The reaction was quenched according to the general procedure after stirring at $60{ }^{\circ} \mathrm{C}$ for $24 \mathrm{~h}$, and the crude material was purified by flash chromatography on silica gel (hexanes/ethyl acetate $=20 / 1$ to $4 / 1)$ to afford the desired product as a white solid $(89.4 \mathrm{mg}$, $73 \%$ yield). mp $131-132{ }^{\circ} \mathrm{C} .{ }^{1} \mathrm{H}$ NMR $\left(500 \mathrm{MHz}, \mathrm{CDCl}_{3}\right) \delta 9.14(\mathrm{~d}, J=2.0 \mathrm{~Hz}, 1 \mathrm{H}), 8.91(\mathrm{~d}, J$ $=2.4 \mathrm{~Hz}, 1 \mathrm{H}), 8.38(\mathrm{~m}, 2 \mathrm{H}), 7.78(\mathrm{dd}, J=8.6,2.6 \mathrm{~Hz}, 1 \mathrm{H}), 6.83(\mathrm{~d}, J=8.5 \mathrm{~Hz}, 1 \mathrm{H}), 3.96(\mathrm{~s}, 3$ $\mathrm{H}), 3.95(\mathrm{~s}, 3 \mathrm{H}) ;{ }^{13} \mathrm{C}\left\{{ }^{1} \mathrm{H}\right\} \mathrm{NMR}\left(126 \mathrm{MHz}, \mathrm{CDCl}_{3}\right) \delta 165.7,164.6,151.3,149.5,145.4,137.4$, 134.8, 133.6, 126.2 , 125.8, 111.6, 53.8, 52.7 ppm. HRMS (ESI) m/z calcd for $[\mathrm{M}+\mathrm{H}]^{+} 245.0921$, found 245.0919. IR $\left(\mathrm{cm}^{-1}\right): 3035,2959,1724,1608,1501,1288,1262,1114,1020,828,768$.

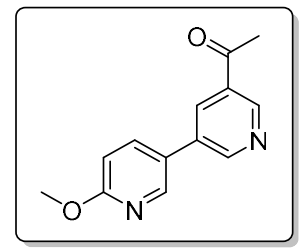

1-(6'-methoxy-[3,3'-bipyridin]-5-yl)ethan-1-one (3h) was synthesized according to the general procedure under Conditions B using 6-methoxylpyridin-3-yl trifluoromethanesulfonate $(0.50$ mmol, $128.6 \mathrm{mg}, 1.00$ equiv) and 1-(5-bromopyridin-3-yl)ethan-1-one (0.75 mmol, $150.0 \mathrm{mg}, 1.50$ equiv) as starting materials. The reaction was quenched according to the general procedure after stirring at $60^{\circ} \mathrm{C}$ for $24 \mathrm{~h}$, and the crude material was purified by flash chromatography on silica gel (hexanes (containing triethyl amine, $5 \% \mathrm{v} / \mathrm{v}$ )/ethyl acetate $=5 / 1$ ) to afford the desired product as a white solid (56.6 mg, 50\% yield). mp $140-141{ }^{\circ} \mathrm{C} .{ }^{1} \mathrm{H}$ NMR $\left(500 \mathrm{MHz}, \mathrm{CDCl}_{3}\right) \delta 9.10(\mathrm{~d}, J$ $=2.1 \mathrm{~Hz}, 1 \mathrm{H}), 8.94(\mathrm{~d}, J=2.4 \mathrm{~Hz}, 1 \mathrm{H}), 8.40(\mathrm{~d}, J=2.6 \mathrm{~Hz}, 1 \mathrm{H}), 8.33(\mathrm{t}, J=2.2 \mathrm{~Hz}, 1 \mathrm{H}), 7.80$ $(\mathrm{dd}, J=8.6,2.6 \mathrm{~Hz}, 1 \mathrm{H}), 6.85(\mathrm{~d}, J=8.6 \mathrm{~Hz}, 1 \mathrm{H}), 3.97(\mathrm{~s}, 3 \mathrm{H}), 2.67(\mathrm{~s}, 3 \mathrm{H}) ;{ }^{13} \mathrm{C}\left\{{ }^{1} \mathrm{H}\right\} \mathrm{NMR}$ $\left(126 \mathrm{MHz}, \mathrm{CDCl}_{3}\right) \delta 196.7,164.6,151.5,148.6,145.4,137.4,134.0,133.2,132.5,125.8,111.6$, 53.9, 27.0 ppm. HRMS (ESI) m/z calcd for $[\mathrm{M}+\mathrm{H}]^{+}$229.0972, found 229.0972. IR $\left(\mathrm{cm}^{-1}\right): 3034$, 2923, 1688, 1605, 1502, 1363, 1291, 1247, 1021, 843, 709, 599. 


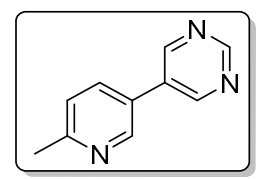

5-(6-Methylpyridin-3-yl)pyrimidine (3i) was synthesized according to the general procedure under Conditions B using 6-methylpyridin-3-yl trifluoromethanesulfonate $(0.50 \mathrm{mmol}, 120.6 \mathrm{mg}$, 1.00 equiv) and 5-bromopyrimidine ( $0.75 \mathrm{mmol}, 119.2 \mathrm{mg}, 1.50$ equiv) as starting materials. The reaction was quenched according to the general procedure after stirring at $60{ }^{\circ} \mathrm{C}$ for $24 \mathrm{~h}$, and the crude material was purified by flash chromatography on silica gel (hexanes (containing triethyl amine, $5 \% \mathrm{v} / \mathrm{v}) /$ ethyl acetate $=3 / 1$ to $3 / 2)$ to afford a white solid $(65.5 \mathrm{mg})$ containing $\mathbf{3 i}(57.8 \mathrm{mg}$, 67\% yield) and 5,5'-bipyrimidine (7.7 mg, 10\% yield). ${ }^{1} \mathrm{H} \mathrm{NMR}\left(500 \mathrm{MHz}, \mathrm{CDCl}_{3}\right) \delta 9.23$ (s, 1 H), $8.94(\mathrm{~s}, 2 \mathrm{H}), 8.72(\mathrm{~d}, J=2.1 \mathrm{~Hz}, 1 \mathrm{H}), 7.78(\mathrm{dd}, J=8.1,2.5 \mathrm{~Hz}, 1 \mathrm{H}), 7.31$ (d, $J=8.0 \mathrm{~Hz}, 1$ $\mathrm{H}), 2.63(\mathrm{~s}, 3 \mathrm{H}) ;{ }^{13} \mathrm{C}\left\{{ }^{1} \mathrm{H}\right\} \mathrm{NMR}\left(126 \mathrm{MHz}, \mathrm{CDCl}_{3}\right) \delta 159.6,158.1,154.9,147.4,134.7,131.7$, 127.4, 123.8, $24.5 \mathrm{ppm}$. HRMS (ESI) m/z calcd for $[\mathrm{M}+\mathrm{H}]^{+}$172.0869, found 172.0869. IR $\left(\mathrm{cm}^{-}\right.$ $\left.{ }^{1}\right): 3037,2920,1550,1414,1375,998,725$. For 5,5'-bipyrimidine, ${ }^{19}$ HRMS (ESI) m/z calcd for $[\mathrm{M}+\mathrm{H}]^{+} 159.0665$, found 159.0665 .

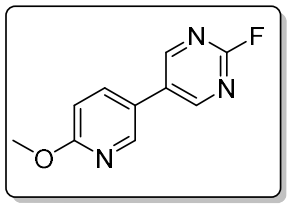

2-Fluoro-5-(6-methoxypyridin-3-yl)pyrimidine (3j) was synthesized according to the general procedure under Conditions B using 6-methoxylpyridin-3-yl trifluoromethanesulfonate (0.50 mmol, $128.6 \mathrm{mg}, 1.00$ equiv) and 5-bromo-2-fluoropyrimidine ( $0.75 \mathrm{mmol}, 132.7 \mathrm{mg}, 1.50$ equiv) as starting materials. The reaction was quenched according to the general procedure after stirring at $60{ }^{\circ} \mathrm{C}$ for $24 \mathrm{~h}$, and the crude material was purified by flash chromatography on silica gel (hexanes/ethyl acetate $=5 / 1$ ) to afford the desired product as a white solid $(86.2 \mathrm{mg}, 74 \%$ yield). mp $136-137{ }^{\circ} \mathrm{C} .{ }^{1} \mathrm{H}$ NMR $\left(500 \mathrm{MHz}, \mathrm{CDCl}_{3}\right) \delta 8.77$ (d, $\left.J=1.6 \mathrm{~Hz}, 1 \mathrm{H}\right), 7.74(\mathrm{dd}, J=8.6,2.6$ $\mathrm{Hz}, 1 \mathrm{H}), 6.89(\mathrm{~d}, J=8.6 \mathrm{~Hz}, 1 \mathrm{H}), 3.99(\mathrm{~s}, 2 \mathrm{H}) ;{ }^{19} \mathrm{~F}\left\{{ }^{1} \mathrm{H}\right\} \mathrm{NMR}\left(376 \mathrm{MHz}, \mathrm{CDCl}_{3}\right) \delta-47.38(\mathrm{~s}, 1$ $\mathrm{F}) ;{ }^{13} \mathrm{C}\left\{{ }^{1} \mathrm{H}\right\} \mathrm{NMR}\left(126 \mathrm{MHz}, \mathrm{CDCl}_{3}\right) \delta 164.9,162.7(\mathrm{~d}, J=220.5 \mathrm{~Hz}), 158.6(\mathrm{~d}, J=12.1 \mathrm{~Hz})$, 145.3, 137.1, 130.3 (d, $J=5.4 \mathrm{~Hz}), 122.4$ (d, $J=1.7 \mathrm{~Hz}), 112.0,54.0 \mathrm{ppm}$. HRMS (ESI) m/z calcd for $[\mathrm{M}+\mathrm{H}]^{+}$206.0724, found 206.0725. IR $\left(\mathrm{cm}^{-1}\right)$ : 3050, 2923, 1614, 1574, 1449, 1299, 1014, 828,785 . 


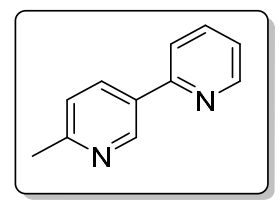

6'-methyl-2,3'-bipyridine (3k) [CAS: 340026-65-5] was synthesized according to the general procedure using 6-methylpyridin-3-yl trifluoromethanesulfonate $(0.50 \mathrm{mmol}, 120.6 \mathrm{mg}, 1.00$ equiv) and 2-bromopyridine ( $0.75 \mathrm{mmol}, 118.5 \mathrm{mg}, 1.50$ equiv) as starting materials. The reaction was quenched according to the general procedure after stirring at $60{ }^{\circ} \mathrm{C}$ for $24 \mathrm{~h}$, and the crude material was purified by flash chromatography on silica gel (hexanes (containing triethyl amine, $5 \% \mathrm{v} / \mathrm{v}) /$ ethyl acetate $=5 / 1$ to $3 / 1)$ to afford the desired product as a colorless oil $(49.1 \mathrm{mg}, 58 \%$ yield). ${ }^{1} \mathrm{H}$ NMR (500 MHz, $\left.\mathrm{CDCl}_{3}\right) \delta 9.07$ (d, $\left.J=2.3 \mathrm{~Hz}, 1 \mathrm{H}\right), 8.71$ (dt, $\left.J=4.8,1.4 \mathrm{~Hz}, 1 \mathrm{H}\right), 8.22$ $(\mathrm{dd}, J=8.1,2.4 \mathrm{~Hz}, 1 \mathrm{H}), 7.77$ (td, $J=7.7,1.8 \mathrm{~Hz}, 1 \mathrm{H}), 7.76-7.70$ (m, $1 \mathrm{H}), 7.29-7.23$ (m, 2 $\mathrm{H}), 2.62(\mathrm{~s}, 3 \mathrm{H}) ;{ }^{13} \mathrm{C}\left\{{ }^{1} \mathrm{H}\right\} \mathrm{NMR}\left(126 \mathrm{MHz}, \mathrm{CDCl}_{3}\right) \delta 159.1,155.2,150.2,147.7,137.1,134.9$, 132.3, 123.4, 122.7, 120.5, $24.5 \mathrm{ppm}$. The NMR data are consistent with the reported ones in literature. $^{20}$

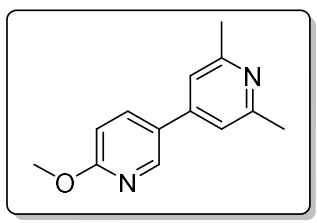

6-Methoxy-2',6'-dimethyl-3,4'-bipyridine (3l) was synthesized according to the general procedure using 6-methoxypyridin-3-yl trifluoromethanesulfonate $(0.50 \mathrm{mmol}, 128.6 \mathrm{mg}, 1.00$ equiv) and 4-bromo-2,6-dimethylpyridine ( $0.75 \mathrm{mmol}, 139.5 \mathrm{mg}, 1.50$ equiv) as starting materials. The reaction was quenched according to the general procedure after stirring at $60{ }^{\circ} \mathrm{C}$ for $24 \mathrm{~h}$, and the crude material was purified by flash chromatography on silica gel (hexanes (containing triethyl amine, $5 \% \mathrm{v} / \mathrm{v}) /$ ethyl acetate $=100 / 1$ to $50 / 1)$ to afford the desired product as a white solid $(89.3$ mg, 83\% yield). mp $130-131{ }^{\circ} \mathrm{C} .{ }^{1} \mathrm{H}$ NMR (500 MHz, $\left.\mathrm{CDCl}_{3}\right) \delta 8.35(\mathrm{~d}, J=1.9 \mathrm{~Hz}, 1 \mathrm{H}), 7.72$ $(\mathrm{dd}, J=8.6,2.6 \mathrm{~Hz}, 1 \mathrm{H}), 7.04(\mathrm{~s}, 2 \mathrm{H}), 6.75(\mathrm{dd}, J=8.7,0.7 \mathrm{~Hz}, 1 \mathrm{H}), 3.92(\mathrm{~s}, 3 \mathrm{H}), 2.51(\mathrm{~s}, 6 \mathrm{H})$; ${ }^{13} \mathrm{C}\left\{{ }^{1} \mathrm{H}\right\}$ NMR $\left(126 \mathrm{MHz}, \mathrm{CDCl}_{3}\right) \delta 164.6,158.4,145.9,145.3,137.2,127.5,117.7,111.1,53.7$, $24.6 \mathrm{ppm}$. HRMS (ESI) $\mathrm{m} / \mathrm{z}$ calcd for $[\mathrm{M}+\mathrm{H}]^{+} 215.1179$, found 215.1178 . IR $\left(\mathrm{cm}^{-1}\right): 2947,2923$, $1603,1496,1360,1283,1023,827,625$.

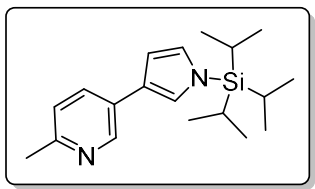


2-Methyl-5-(1-(triisopropylsilyl)-1H-pyrrol-3-yl)pyridine (3m) was synthesized according to the general procedure using 6-methylpyridin-3-yl trifluoromethanesulfonate $(0.50 \mathrm{mmol}, 120.6$ mg, 1.00 equiv) and 3-bromo-1-(triisopropylsilyl)- $1 H$-pyrrole ( $0.75 \mathrm{mmol}, 226.7 \mathrm{mg}, 1.50$ equiv) as starting materials. The reaction was quenched according to the general procedure after stirring at $60{ }^{\circ} \mathrm{C}$ for $24 \mathrm{~h}$, and the crude material was purified by flash chromatography on silica gel (hexanes/ethyl acetate $=5 / 1$ to $4 / 1)$ to afford the desired product as a brown oil $(112.4 \mathrm{mg}, 72 \%$ yield). ${ }^{1} \mathrm{H}$ NMR (500 MHz, $\left.\mathrm{CDCl}_{3}\right) \delta 8.69(\mathrm{~d}, J=2.3 \mathrm{~Hz}, 1 \mathrm{H}), 7.68(\mathrm{dd}, J=8.0,2.4 \mathrm{~Hz}, 1 \mathrm{H})$, $7.08(\mathrm{~d}, J=8.0 \mathrm{~Hz}, 1 \mathrm{H}), 7.05(\mathrm{t}, J=1.8 \mathrm{~Hz}, 1 \mathrm{H}), 6.82(\mathrm{t}, J=2.4 \mathrm{~Hz}, 1 \mathrm{H}), 6.59$ (dd, $J=2.8,1.5$ $\mathrm{Hz}, 1 \mathrm{H}), 2.53(\mathrm{~s}, 3 \mathrm{H}), 1.47$ (hept, $J=7.4 \mathrm{~Hz}, 3 \mathrm{H}), 1.12(\mathrm{~d}, J=7.6 \mathrm{~Hz}, 18 \mathrm{H}) ;{ }^{13} \mathrm{C}\left\{{ }^{1} \mathrm{H}\right\} \mathrm{NMR}$ $\left(126 \mathrm{MHz} \mathrm{CDCl}_{3}\right) \delta 154.9,146.1,132.9,129.0,125.7,123.5,123.1,120.7,108.6,24.1,17.9,11.8$ ppm. HRMS (ESI) m/z calcd for $[\mathrm{M}+\mathrm{H}]^{+}$315.2251, found 315.2246. IR $\left(\mathrm{cm}^{-1}\right): 2945,2866,1491$, $1462,1132,1086,882,782,658$.

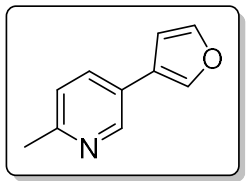

5-(Furan-3-yl)-2-methylpyridine (3n) was synthesized according to the general procedure using 6-methylpyridin-3-yl trifluoromethanesulfonate (0.50 mmol, $120.6 \mathrm{mg}, 1.00$ equiv) and 3bromofuran ( $0.75 \mathrm{mmol}, 110.2 \mathrm{mg}, 1.50$ equiv) as starting materials. The reaction was quenched according to the general procedure after stirring at $60{ }^{\circ} \mathrm{C}$ for $24 \mathrm{~h}$, and the crude material was purified by flash chromatography on silica gel (hexanes/ethyl acetate $=5 / 1$ to $2 / 1$ ) to afford the desired product as a colorless oil (42.3 mg, 53\% yield). ${ }^{1} \mathrm{H} \mathrm{NMR}\left(500 \mathrm{MHz}, \mathrm{CDCl}_{3}\right) \delta 8.63(\mathrm{~d}, J$ $=2.4 \mathrm{~Hz}, 1 \mathrm{H}), 7.73(\mathrm{~s}, 1 \mathrm{H}), 7.63(\mathrm{dd}, J=8.0,2.4 \mathrm{~Hz}, 1 \mathrm{H}), 7.49(\mathrm{t}, J=1.7 \mathrm{~Hz}, 1 \mathrm{H}), 7.14(\mathrm{~d}, J=$ $8.0 \mathrm{~Hz}, 1 \mathrm{H}), 6.68(\mathrm{~s}, 1 \mathrm{H}), 2.55(\mathrm{~s}, 3 \mathrm{H}) ;{ }^{13} \mathrm{C}\left\{{ }^{1} \mathrm{H}\right\} \mathrm{NMR}\left(126 \mathrm{MHz}, \mathrm{CDCl}_{3}\right) \delta 157.0,146.6,144.1$, 138.7, 133.6, 125.6, 123.44, 123.35, 108.7, 24.2 ppm. HRMS (ESI) m/z calcd for $[\mathrm{M}+\mathrm{H}]^{+}$ 160.0757, found 160.0755. IR ( $\left.\mathrm{cm}^{-1}\right): 2923.2855,1761,1744,1302,1124,1037,954,873$.

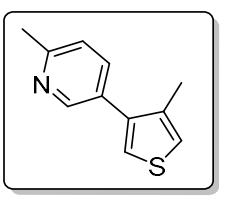

2-Methyl-5-(4-methylthiophen-3-yl)pyridine (3o) was synthesized according to the general procedure using 6-methylpyridin-3-yl trifluoromethanesulfonate $(0.50 \mathrm{mmol}, 120.6 \mathrm{mg}, 1.00$ equiv) and 3-bromo-4-methylthiophene (0.75 mmol, $132.8 \mathrm{mg}, 1.50$ equiv) as starting materials. The reaction was quenched according to the general procedure after stirring at $60{ }^{\circ} \mathrm{C}$ for $24 \mathrm{~h}$, and the crude material was purified by flash chromatography on silica gel (hexanes/ethyl acetate $=9 / 1)$ 
to afford the desired product as a colorless oil $\left(78.7 \mathrm{mg}, 83 \%\right.$ yield). ${ }^{1} \mathrm{H} \mathrm{NMR}\left(500 \mathrm{MHz}, \mathrm{CDCl}_{3}\right)$ $\delta 8.54(\mathrm{~d}, J=2.1 \mathrm{~Hz}, 1 \mathrm{H}), 7.58(\mathrm{dd}, J=7.9,2.4 \mathrm{~Hz}, 1 \mathrm{H}), 7.21(\mathrm{~d}, J=3.2 \mathrm{~Hz}, 1 \mathrm{H}), 7.19(\mathrm{~d}, J=$ $7.9 \mathrm{~Hz}, 1 \mathrm{H}), 7.07-7.02(\mathrm{~m}, 1 \mathrm{H}), 2.59(\mathrm{~s}, 3 \mathrm{H}), 2.25(\mathrm{~s}, 3 \mathrm{H}) ;{ }^{13} \mathrm{C}\left\{{ }^{1} \mathrm{H}\right\} \mathrm{NMR}\left(126 \mathrm{MHz}, \mathrm{CDCl}_{3}\right)$ $\delta 157.0,148.8,139.6,136.24,136.22,130.0,123.8,122.9,122.7,24.3,15.5$ ppm. HRMS (ESI) $\mathrm{m} / \mathrm{z}$ calcd for $[\mathrm{M}+\mathrm{H}]^{+} 190.0685$, found 190.0685. IR $\left(\mathrm{cm}^{-1}\right): 2923,1492,1264,791,734,703$.

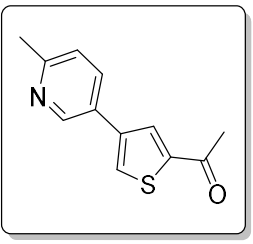

1-(4-(6-Methylpyridin-3-yl)thiophen-2-yl)ethan-1-one (3p) was synthesized according to the general procedure using 6-methylpyridin-3-yl trifluoromethanesulfonate $(0.50 \mathrm{mmol}, 120.6 \mathrm{mg}$, 1.00 equiv) and 1-(4-bromothiophen-2-yl)ethan-1-one (0.75 mmol, $153.8 \mathrm{mg}, 1.50 \mathrm{equiv})$ as starting materials. The reaction was quenched according to the general procedure after stirring at $60{ }^{\circ} \mathrm{C}$ for $24 \mathrm{~h}$, and the crude material was purified by flash chromatography on silica gel (hexanes/ethyl acetate $=2 / 1$ ) to afford the desired product as a white solid (53.6 $\mathrm{mg}$, 49\% yield). mp $102-103{ }^{\circ} \mathrm{C} .{ }^{1} \mathrm{H}$ NMR $\left(500 \mathrm{MHz}, \mathrm{CDCl}_{3}\right) \delta 8.73$ (d, $\left.J=2.2 \mathrm{~Hz}, 1 \mathrm{H}\right), 7.91$ (d, $J=1.5 \mathrm{~Hz}, 1$ $\mathrm{H}), 7.76-7.74(\mathrm{~m}, 2 \mathrm{H}), 7.21(\mathrm{~d}, J=8.0 \mathrm{~Hz}, 1 \mathrm{H}), 2.61(\mathrm{~s}, 3 \mathrm{H}), 2.59(\mathrm{~s}, 3 \mathrm{H}) ;{ }^{13} \mathrm{C}\left\{{ }^{1} \mathrm{H}\right\} \mathrm{NMR}(126$ $\left.\mathrm{MHz}_{,} \mathrm{CDCl}_{3}\right) \delta 190.7,158.0,146.9,145.7,140.2,134.1,130.7,128.8,128.0,123.5,27.1,24.4$ ppm. HRMS (ESI) m/z calcd for $[\mathrm{M}+\mathrm{H}]^{+}$218.0634, found 218.0633. IR $\left(\mathrm{cm}^{-1}\right): 3040,2917,1650$, $1419,1283,1025,813$.

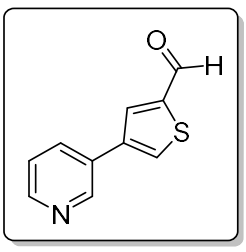

4-(Pyridin-3-yl)thiophene-2-carbaldehyde (3q) [CAS: 166450-82-4] was synthesized according to the general procedure using pyridin-3-yl trifluoromethanesulfonate $(0.50 \mathrm{mmol}$, $113.8 \mathrm{mg}, 1.00$ equiv) and 4-bromothiophene-2-carbaldehyde ( $0.75 \mathrm{mmol}, 143.3 \mathrm{mg}, 1.50$ equiv) as starting materials. The reaction was quenched according to the general procedure after stirring at $60{ }^{\circ} \mathrm{C}$ for $24 \mathrm{~h}$, and the crude material was purified by flash chromatography on silica gel (hexanes/ethyl acetate $=1 / 1$ to $1 / 4)$ to afford the desired product as a white solid $(34.3 \mathrm{mg}, 36 \%$ yield). ${ }^{1} \mathrm{H}$ NMR (500 MHz, $\left.\mathrm{CDCl}_{3}\right) \delta 9.98(\mathrm{~d}, J=1.3 \mathrm{~Hz}, 1 \mathrm{H}), 8.87$ (d, $\left.J=2.4 \mathrm{~Hz}, 1 \mathrm{H}\right), 8.59$ (dd, $J=4.8,1.6 \mathrm{~Hz}, 1 \mathrm{H}), 8.03(\mathrm{~d}, J=1.5 \mathrm{~Hz}, 1 \mathrm{H}), 7.91(\mathrm{~s}, 1 \mathrm{H}), 7.87$ (dt, $J=7.9,2.0 \mathrm{~Hz}, 1 \mathrm{H}), 7.37$ 
$(\mathrm{dd}, J=7.9,4.9 \mathrm{~Hz}, 1 \mathrm{H}) ;{ }^{13} \mathrm{C} \mathrm{NMR}\left(126 \mathrm{MHz}, \mathrm{CDCl}_{3}\right) \delta 182.9,149.4,147.7,145.2,140.3,134.2$, 133.7, 130.6, 130.4, 124.0 ppm. The NMR data are consistent with the ones reported in literature. ${ }^{21}$

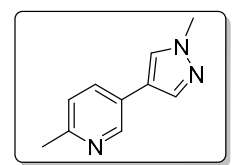

2-methyl-5-(1-methyl-1H-pyrazol-4-yl)pyridine (3r) was synthesized according to the general procedure using 6-methylpyridin-3-yl trifluoromethanesulfonate $(0.50 \mathrm{mmol}, 120.6 \mathrm{mg}, 1.00$ equiv) and 4-bromo-1-methyl-1 $H$-pyrazole $(0.75 \mathrm{mmol}, 120.8 \mathrm{mg}, 1.50$ equiv) as starting materials. The reaction was quenched according to the general procedure after stirring at $60{ }^{\circ} \mathrm{C}$ for $24 \mathrm{~h}$, and the crude material was purified by flash chromatography on silica gel (hexanes (containing triethyl amine, $5 \% \mathrm{v} / \mathrm{v}$ )/ethyl acetate $=3 / 1$ to $1 / 1$ ) to afford the desired product as a colorless oil (37.2 mg, 43\% yield). ${ }^{1} \mathrm{H} \mathrm{NMR}\left(500 \mathrm{MHz}, \mathrm{CDCl}_{3}\right) \delta 8.58(\mathrm{~d}, J=2.4 \mathrm{~Hz}, 1 \mathrm{H}), 7.70$ (s, $1 \mathrm{H}), 7.60-7.57(\mathrm{~m}, 2 \mathrm{H}), 7.09(\mathrm{~d}, J=8.0 \mathrm{~Hz}, 1 \mathrm{H}), 3.90(\mathrm{~s}, 3 \mathrm{H}), 2.51(\mathrm{~s}, 3 \mathrm{H}) ;{ }^{13} \mathrm{C}\left\{{ }^{1} \mathrm{H}\right\} \mathrm{NMR}$ $\left(126 \mathrm{MHz} \mathrm{CDCl}_{3}\right) \delta 156.2,146.2,136.7,133.2,127.0,125.8,123.3,119.9,39.2,24.1 \mathrm{ppm}$. HRMS (ESI) m/z calcd for $[\mathrm{M}+\mathrm{H}]^{+} 174.1026$, found174.1025. IR $\left(\mathrm{cm}^{-1}\right): 2923,1571,1445,1403$, $1210,953,830,664$.

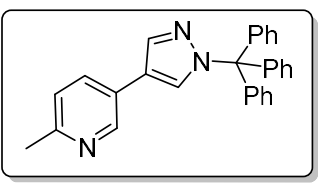

2-methyl-5-(1-trityl-1H-pyrazol-4-yl)pyridine (3s) was synthesized according to the general procedure using 6-methylpyridin-3-yl trifluoromethanesulfonate $(0.50 \mathrm{mmol}, 120.6 \mathrm{mg}, 1.00$ equiv) and 4-bromo-1-trityl-1 $H$-pyrazole $(0.75 \mathrm{mmol}, 291.8 \mathrm{mg}, 1.50$ equiv) as starting materials. The reaction was quenched according to the general procedure after stirring at $60{ }^{\circ} \mathrm{C}$ for $24 \mathrm{~h}$, and the crude material was purified by flash chromatography on silica gel (hexanes/ethyl acetate $=1 / 1)$ to afford the desired product as a white solid (170.6 mg, 85\% yield). mp $227-228{ }^{\circ} \mathrm{C}$. ${ }^{1} \mathrm{H}$ NMR $\left(500 \mathrm{MHz} \mathrm{CDCl}_{3}\right) \delta 8.59(\mathrm{~d}, J=2.3 \mathrm{~Hz}, 1 \mathrm{H}), 7.94(\mathrm{~s}, 1 \mathrm{H}), 7.64(\mathrm{~s}, 1 \mathrm{H}), 7.59(\mathrm{dd}, J=8.0,2.4$ $\mathrm{Hz}, 1 \mathrm{H}), 7.35-7.32(\mathrm{~m}, 9 \mathrm{H}), 7.23-7.19(\mathrm{~m}, 6 \mathrm{H}), 7.10(\mathrm{~d}, J=8.0 \mathrm{~Hz}, 1 \mathrm{H}), 2.54(\mathrm{~s}, 3 \mathrm{H}) ;{ }^{13} \mathrm{C}\left\{{ }^{1} \mathrm{H}\right\}$ NMR $\left(126 \mathrm{MHz}, \mathrm{CDCl}_{3}\right) \delta 156.4,146.4,143.1,137.2,133.3,130.3,129.2,128.01,127.96,125.8$, 123.2, 118.5, 79.2, 24.2 ppm. HRMS (ESI) m/z calcd for $[\mathrm{M}+\mathrm{Na}]^{+} 424.1784$, found 424.1780. IR (cm-1): 3057, 2922, 1568, 1488, 1444, 1160, 747, 699. 


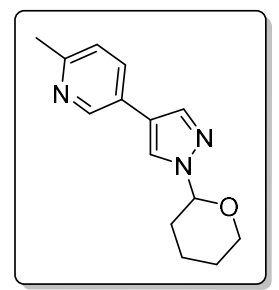

2-methyl-5-(1-(tetrahydro-2H-pyran-2-yl)-1H-pyrazol-4-yl)pyridine (3t) was synthesized according to the general procedure using 6-methylpyridin-3-yl trifluoromethanesulfonate $(0.50$ mmol, $120.6 \mathrm{mg}, 1.00$ equiv) and 4-bromo-1-(tetrahydro- $2 H$-pyran-2-yl)-1H-pyrazole ( $0.75 \mathrm{mmol}$, $173.3 \mathrm{mg}, 1.50$ equiv) as starting materials. The reaction was quenched according to the general procedure after stirring at $60{ }^{\circ} \mathrm{C}$ for $24 \mathrm{~h}$, and the crude material was purified by flash chromatography on silica gel (hexanes (containing triethyl amine, $5 \% \mathrm{v} / \mathrm{v}$ ) $/$ ethyl acetate $=3 / 1$ to $1 / 1)$ to afford the desired product as a colorless oil (76.2 $\mathrm{mg}, 63 \%$ yield). ${ }^{1} \mathrm{H}$ NMR (500 MHz, $\left.\mathrm{CDCl}_{3}\right) \delta 8.62(\mathrm{~d}, J=2.3 \mathrm{~Hz}, 1 \mathrm{H}), 7.86(\mathrm{~s}, 1 \mathrm{H}), 7.79(\mathrm{~s}, 1 \mathrm{H}), 7.62(\mathrm{dd}, J=8.0,2.4 \mathrm{~Hz}, 1 \mathrm{H}), 7.11$ $(\mathrm{d}, J=8.0 \mathrm{~Hz}, 1 \mathrm{H}), 5.40(\mathrm{dd}, J=9.0,3.4 \mathrm{~Hz}, 1 \mathrm{H}), 4.06(\mathrm{dd}, J=11.2,2.8 \mathrm{~Hz}, 1 \mathrm{H}), 3.71(\mathrm{td}, J=$ 11.2, $2.8 \mathrm{~Hz}, 1 \mathrm{H}), 2.53$ (s, $3 \mathrm{H}), 2.16-2.07$ (m, $2 \mathrm{H}), 2.06$ - 1.99 (m, $1 \mathrm{H}), 1.75-1.66$ (m, $2 \mathrm{H})$, $1.62-1.58(\mathrm{~m}, 1 \mathrm{H}) ;{ }^{13} \mathrm{C}\left\{{ }^{1} \mathrm{H}\right\} \mathrm{NMR}\left(126 \mathrm{MHz}, \mathrm{CDCl}_{3}\right) \delta 156.5,146.4,137.1,133.4,125.7,124.6$, 123.3, 120.2, 88.0, 68.0, 30.7, 25.1, 24.2, $22.5 \mathrm{ppm}$. HRMS (ESI) $\mathrm{m} / \mathrm{z}$ calcd for $[\mathrm{M}+\mathrm{H}]^{+} 244.1444$, found 244.1442. IR ( $\left.\mathrm{cm}^{-1}\right): 2941,2854,1731,1569,1440,1374,1082,1042,832$.

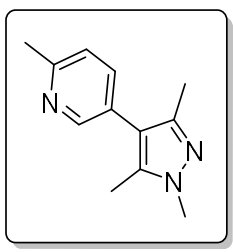

2-methyl-5-(1,3,5-trimethyl-1H-pyrazol-4-yl)pyridine (3u) was synthesized according to the general procedure using 6-methylpyridin-3-yl trifluoromethanesulfonate $(0.50 \mathrm{mmol}, 120.6 \mathrm{mg}$, 1.00 equiv) and 4-bromo-1,3,5-trimethyl- $1 H$-pyrazole ( $0.75 \mathrm{mmol}, 141.8 \mathrm{mg}, 1.50 \mathrm{equiv})$ as starting materials. The reaction was quenched according to the general procedure after stirring at $60{ }^{\circ} \mathrm{C}$ for $24 \mathrm{~h}$, and the crude material was purified by flash chromatography on silica gel (hexanes (containing triethyl amine, $5 \% \mathrm{v} / \mathrm{v}$ )/ethyl acetate $=4 / 1$ to $3 / 7$ ) to afford the desired product as a colorless oil (88.5 mg, 86\% yield). ${ }^{1} \mathrm{H} \mathrm{NMR}\left(500 \mathrm{MHz}, \mathrm{CDCl}_{3}\right) \delta 8.37$ (d, J=1.9 Hz, $\left.1 \mathrm{H}\right), 7.43$ $(\mathrm{dd}, J=8.0,2.3 \mathrm{~Hz}, 1 \mathrm{H}), 7.16(\mathrm{~d}, J=7.9 \mathrm{~Hz}, 1 \mathrm{H}), 3.75(\mathrm{~s}, 3 \mathrm{H}), 2.56(\mathrm{~s}, 3 \mathrm{H}), 2.201(\mathrm{~s}, 3 \mathrm{H})$, $2.197(\mathrm{~s}, 3 \mathrm{H}) ;{ }^{13} \mathrm{C}\left\{{ }^{1} \mathrm{H}\right\} \mathrm{NMR}\left(126 \mathrm{MHz}, \mathrm{CDCl}_{3}\right) \delta 156.1,149.6,145.4,137.1,136.7,127.2,123.1$, 115.7, 36.2, 24.2, 12.4, 10.3 ppm. HRMS (ESI) m/z calcd for $[\mathrm{M}+\mathrm{H}]^{+}$202.1339, found 202.1338. IR ( $\left.\mathrm{cm}^{-1}\right): 2923,1561,1461,1381,1008,764,737$. 


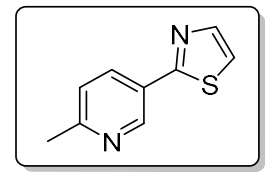

2-(6-methylpyridin-3-yl)thiazole (3v) [CAS: 1823923-11-0] was synthesized according to the general procedure using 6-methylpyridin-3-yl trifluoromethanesulfonate $(0.50 \mathrm{mmol}, 120.6 \mathrm{mg}$, 1.00 equiv) and 2-bromothiazole $(0.75 \mathrm{mmol}, 123.0 \mathrm{mg}, 1.50$ equiv) as starting materials. The reaction was quenched according to the general procedure after stirring at $60{ }^{\circ} \mathrm{C}$ for $24 \mathrm{~h}$, and the crude material was purified by flash chromatography on silica gel (hexanes/ethyl acetate $=5 / 1$ to $3 / 1$ ) to afford the desired product as a brown solid (72.8 $\mathrm{mg}, 83 \%$ yield). ${ }^{1} \mathrm{H}$ NMR (500 $\mathrm{MHz}$, $\left.\mathrm{CDCl}_{3}\right) \delta 9.06(\mathrm{~d}, J=2.4 \mathrm{~Hz}, 1 \mathrm{H}), 8.13(\mathrm{dd}, J=8.1,2.4 \mathrm{~Hz}, 1 \mathrm{H}), 7.89(\mathrm{~d}, J=3.3 \mathrm{~Hz}, 1 \mathrm{H}), 7.36$ $(\mathrm{d}, J=3.3 \mathrm{~Hz}, 1 \mathrm{H}), 7.24(\mathrm{~d}, J=8.1 \mathrm{~Hz}, 1 \mathrm{H}), 2.61(\mathrm{~s}, 3 \mathrm{H}) ;{ }^{13} \mathrm{C}\left\{{ }^{1} \mathrm{H}\right\} \mathrm{NMR}\left(126 \mathrm{MHz}, \mathrm{CDCl}_{3}\right) \delta$ 165.4, 160.2, 147.3, 144.1, 134.2, 127.2, 123.6, 119.3, 24.6 ppm. HRMS (ESI) m/z calcd for [M $+\mathrm{H}^{+}$177.0481, found 177.0480. The NMR data are consistent with the ones reported in literature. ${ }^{22}$

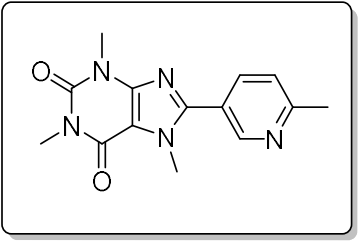

1,3,7-trimethyl-8-(6-methylpyridin-3-yl)-3,7-dihydro-1H-purine-2,6-dione (3w) was synthesized according to the general procedure using 6-methylpyridin-3-yl trifluoromethanesulfonate $(0.50 \mathrm{mmol}, 120.6 \mathrm{mg}, 1.00$ equiv) and 8-bromocaffein ( $0.75 \mathrm{mmol}$, $204.8 \mathrm{mg}, 1.50$ equiv) as starting materials. The reaction was quenched according to the general procedure after stirring at $60{ }^{\circ} \mathrm{C}$ for $24 \mathrm{~h}$, and the crude material was purified by flash chromatography on silica gel (methanol/dichloromethane $=1 / 100$ to $1 / 20$ ) to afford the desired product as yellow solid (89.6 mg, 63\% yield). mp $150-152{ }^{\circ} \mathrm{C} .{ }^{1} \mathrm{H}$ NMR $\left(500 \mathrm{MHz}, \mathrm{CDCl}_{3}\right) \delta$ $8.82(\mathrm{~d}, J=3.1 \mathrm{~Hz}, 1 \mathrm{H}), 7.92(\mathrm{dd}, J=8.0,2.4 \mathrm{~Hz}, 1 \mathrm{H}), 7.32(\mathrm{~d}, J=8.1 \mathrm{~Hz}, 1 \mathrm{H}), 4.07$ (s, $3 \mathrm{H})$, $3.61(\mathrm{~s}, 3 \mathrm{H}), 3.42$ (s, $3 \mathrm{H}), 2.65(\mathrm{~s}, 3 \mathrm{H}) ;{ }^{13} \mathrm{C}\left\{{ }^{1} \mathrm{H}\right\} \mathrm{NMR}\left(126 \mathrm{MHz}, \mathrm{CDCl}_{3}\right) \delta 160.8,155.7,151.8$, 149.6, 149.2, 148.5, 136.9, 123.4, 122.2, 109.0, 34.0, 30.0, 28.2, 24.7 ppm. HRMS (ESI) m/z calcd for $[\mathrm{M}+\mathrm{H}]^{+}$286.1299, found 286.1295. IR $\left(\mathrm{cm}^{-1}\right)$ : 2935, 1700, 1659, 1541, 1431, 1035, 735 . 


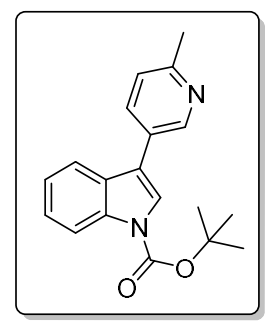

Tert-butyl 3-(6-methylpyridin-3-yl)-1H-indole-1-carboxylate (3x) was synthesized according to the general procedure using 6-methylpyridin-3-yl trifluoromethanesulfonate $(0.50 \mathrm{mmol}, 120.6$ $\mathrm{mg}, 1.00$ equiv) and tert-butyl 3-bromo- $1 H$-indole-1-carboxylate $(0.75 \mathrm{mmol}, 222.0 \mathrm{mg}, 1.50$ equiv) as starting materials. The reaction was quenched according to the general procedure after stirring at $60{ }^{\circ} \mathrm{C}$ for $24 \mathrm{~h}$, and the crude material was purified by flash chromatography on silica gel (hexanes/ethyl acetate $=1 / 20$ to $1 / 5)$ to afford the desired product as colorless oil $(88.5 \mathrm{mg}$, $57 \%$ yield). ${ }^{1} \mathrm{H}$ NMR $\left(500 \mathrm{MHz}, \mathrm{CDCl}_{3}\right) \delta 8.70(\mathrm{~d}, J=1.4 \mathrm{~Hz}, 1 \mathrm{H}), 8.14(\mathrm{~d}, J=8.0 \mathrm{~Hz}, 1 \mathrm{H}), 7.74$ $(\mathrm{dd}, J=8.0,2.3 \mathrm{~Hz}, 1 \mathrm{H}), 7.67-7.65(\mathrm{~m}, 2 \mathrm{H}), 7.30(\mathrm{td}, J=8.3,1.3 \mathrm{~Hz}, 1 \mathrm{H}), 7.22(\mathrm{td}, J=8.1$, $1.1 \mathrm{~Hz}, 1 \mathrm{H}), 7.16(\mathrm{~d}, J=7.9 \mathrm{~Hz}, 1 \mathrm{H}), 2.54(\mathrm{~s}, 3 \mathrm{H}), 1.61(\mathrm{~s}, 9 \mathrm{H}) ;{ }^{13} \mathrm{C}\left\{{ }^{1} \mathrm{H}\right\} \mathrm{NMR}(126 \mathrm{MHz}$, $\left.\mathrm{CDCl}_{3}\right) \delta 157.2,149.8,148.3,136.0,135.6,128.9,127.0,125.0,123.4,123.3,123.2,119.7,118.9$, 115.7, 84.3, 28.4, 24.4 ppm. HRMS (ESI) $\mathrm{m} / \mathrm{z}$ calcd for $[\mathrm{M}+\mathrm{H}]^{+} 309.1598$, found 309.1594. IR $\left(\mathrm{cm}^{-1}\right): 2977,2926,1730,1450,1368,1150,744$.

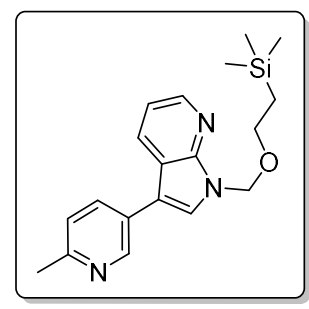

\section{3-(6-Methylpyridin-3-yl)-1-((2-(trimethylsilyl)ethoxy)methyl)-1 $H$-pyrrolo[2,3-b]pyridine}

(3y) was synthesized according to the general procedure using 6-methylpyridin-3-yl trifluoromethanesulfonate $\quad(0.50 \mathrm{mmol}, 120.6 \mathrm{mg}, 1.00$ equiv $)$ and 3-bromo-1-((2(trimethylsilyl)ethoxy)methyl)-1H-pyrrolo[2,3-b]pyridine (0.75 mmol, $245.5 \mathrm{mg}, 1.50$ equiv) as starting materials. The reaction was quenched according to the general procedure after stirring at $60{ }^{\circ} \mathrm{C}$ for $24 \mathrm{~h}$, and the crude material was purified by flash chromatography on silica gel (hexanes/ethyl acetate $=1 / 3$ to $1 / 1)$ to afford the desired product as a yellow oil $(152.2 \mathrm{mg}, 90 \%$ yield). ${ }^{1} \mathrm{H}$ NMR (500 MHz, $\left.\mathrm{CDCl}_{3}\right) \delta 8.77(\mathrm{~d}, J=2.5 \mathrm{~Hz}, 1 \mathrm{H}), 8.36(\mathrm{dd}, J=4.7,1.5 \mathrm{~Hz}, 1 \mathrm{H})$, $8.10(\mathrm{dd}, J=7.9,1.6 \mathrm{~Hz}, 1 \mathrm{H}), 7.77$ (dd, $J=8.0,2.4 \mathrm{~Hz}, 1 \mathrm{H}), 7.52$ (s, $1 \mathrm{H}), 7.19$ (d, $J=8.0 \mathrm{~Hz}, 1$ H), $7.13(\mathrm{dd}, J=7.9,4.7 \mathrm{~Hz}, 1 \mathrm{H}), 5.70(\mathrm{~s}, 2 \mathrm{H}), 3.57$ (t, $J=4.0 \mathrm{~Hz}, 2 \mathrm{H}), 2.57$ (s, $3 \mathrm{H}), 0.91$ (t, $J$ $=4.0 \mathrm{~Hz}, 2 \mathrm{H}),-0.09(\mathrm{~s}, 9 \mathrm{H}) ;{ }^{13} \mathrm{C}\left\{{ }^{1} \mathrm{H}\right\} \mathrm{NMR}\left(126 \mathrm{MHz}, \mathrm{CDCl}_{3}\right) \delta 156.3,148.7,147.4,143.9$, $134.6,128.0,127.7,125.1,123.4,118.7,117.0,113.2$, 73.0, 66.5, 24.2, 17.8, -1.4 ppm. HRMS 
(ESI) $\mathrm{m} / \mathrm{z}$ calcd for $[\mathrm{M}+\mathrm{H}]^{+} 340.1840$, found 340.1835 . IR $\left(\mathrm{cm}^{-1}\right): 3053,2952,1559,1435,1247$, $1071,833,771$.

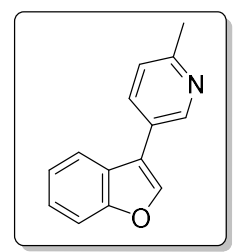

5-(Benzofuran-3-yl)-2-methylpyridine (3z) was synthesized according to the general procedure using 6-methylpyridin-3-yl trifluoromethanesulfonate $(0.50 \mathrm{mmol}, 120.6 \mathrm{mg}, 1.00$ equiv) and 3bromobenzofuran $(0.75 \mathrm{mmol}, 146.8 \mathrm{mg}, 1.50$ equiv) as starting materials. The reaction was quenched according to the general procedure after stirring at $60^{\circ} \mathrm{C}$ for $24 \mathrm{~h}$, and the crude material was purified by flash chromatography on silica gel (hexanes/ethyl acetate $=5 / 1$ to $2 / 1$ ) to afford the desired product as a white solid $\left(78.4 \mathrm{mg}, 75 \%\right.$ yield). mp $65-67{ }^{\circ} \mathrm{C} .{ }^{1} \mathrm{H}$ NMR $(500 \mathrm{MHz}$, $\left.\mathrm{CDCl}_{3}\right) \delta 8.80(\mathrm{~d}, J=2.3 \mathrm{~Hz}, 1 \mathrm{H}), 7.83(\mathrm{dd}, J=8.0,2.3 \mathrm{~Hz}, 1 \mathrm{H}), 7.81(\mathrm{~s}, 1 \mathrm{H}), 7.77$ (d, $J=6.9$ $\mathrm{Hz}, 1 \mathrm{H}), 7.56(\mathrm{~d}, J=8.0 \mathrm{~Hz}, 1 \mathrm{H}), 7.37(\mathrm{td}, J=8.2,7.8,1.4 \mathrm{~Hz}, 1 \mathrm{H}), 7.32(\mathrm{td}, J=7.5,1.2 \mathrm{~Hz}, 1$ H), $7.26(\mathrm{~d}, J=8.0 \mathrm{~Hz}, 1 \mathrm{H}), 2.63(\mathrm{~s}, 3 \mathrm{H}) ;{ }^{13} \mathrm{C}\left\{{ }^{1} \mathrm{H}\right\} \mathrm{NMR}\left(126 \mathrm{MHz}, \mathrm{CDCl}_{3}\right) \delta$ 157.6, 155.9, 147.9, 141.6, 135.2, 126.3, 125.3, 125.0, 123.5, 123.4, 120.2, 119.2, 112.1, 24.4 ppm. HRMS (ESI) $\mathrm{m} / \mathrm{z}$ calcd for $[\mathrm{M}+\mathrm{H}]^{+}$210.0913, found 210.0912. IR $\left(\mathrm{cm}^{-1}\right): 3084,2925,1452,1264,1110,730$, 702.

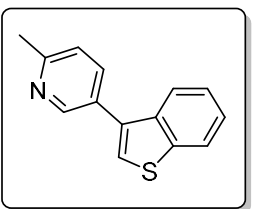

5-(Benzo[b]thiophen-3-yl)-2-methylpyridine (3aa) was synthesized according to the general procedure using 6-methylpyridin-3-yl trifluoromethanesulfonate $(0.50 \mathrm{mmol}, 120.6 \mathrm{mg}, 1.00$ equiv) and 3-bromobenzo[b]thiophene $(0.75 \mathrm{mmol}, 159.8 \mathrm{mg}, 1.50$ equiv) as starting materials. The reaction was quenched according to the general procedure after stirring at $60^{\circ} \mathrm{C}$ for $24 \mathrm{~h}$, and the crude material was purified by flash chromatography on silica gel (hexanes/ethyl acetate $=$ $50 / 1$ to $5 / 1)$ to afford the desired product as a white solid ( $88.1 \mathrm{mg}, 78 \%$ yield). $\mathrm{mp} 78-89^{\circ} \mathrm{C} .{ }^{1} \mathrm{H}$ NMR (400 MHz, $\left.\mathrm{CDCl}_{3}\right) \delta 8.73(\mathrm{~d}, J=3.2 \mathrm{~Hz}, 1 \mathrm{H}), 7.94-7.91(\mathrm{~m}, 1 \mathrm{H}), 7.86-7.82(\mathrm{~m}, 1 \mathrm{H})$, $7.79(\mathrm{dd}, J=8.0,2.3 \mathrm{~Hz}, 1 \mathrm{H}), 7.43$ (s, $1 \mathrm{H}), 7.42-7.40$ (m, $2 \mathrm{H}), 7.28$ (d, $J=8.0 \mathrm{~Hz}, 1 \mathrm{H}), 2.64$ (s, $3 \mathrm{H}) ;{ }^{13} \mathrm{C}\left\{{ }^{1} \mathrm{H}\right\}$ NMR $\left(101 \mathrm{MHz}, \mathrm{CDCl}_{3}\right) \delta 157.7,148.9,139.8,137.8,135.9,134.6,129.0,124.8$, $124.8,124.3,123.3,123.2,122.6,24.5 \mathrm{ppm}$. HRMS (ESI) $\mathrm{m} / \mathrm{z}$ calcd for $[\mathrm{M}+\mathrm{H}]^{+} 226.0685$, found 226.0682. IR ( $\left.\mathrm{cm}^{-1}\right): 3059,2919,1478,1029,759,732$. 


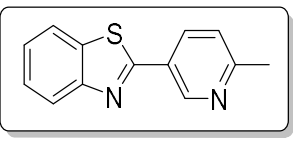

2-(6-Methylpyridin-3-yl)benzo $[\boldsymbol{d}]$ thiazole (3ab) was synthesized according to the general procedure using 6-methylpyridin-3-yl trifluoromethanesulfonate $(0.50 \mathrm{mmol}, 120.6 \mathrm{mg}, 1.00$ equiv) and 2-bromobenzo[d]thiazole ( $0.75 \mathrm{mmol}, 160.5 \mathrm{mg}, 1.50$ equiv) as starting materials. The reaction was quenched according to the general procedure after stirring at $60{ }^{\circ} \mathrm{C}$ for $24 \mathrm{~h}$, and the crude material was purified by flash chromatography on silica gel (hexanes/ethyl acetate $=20 / 1$ to $5 / 1)$ to afford the desired product as a white solid $(83.4 \mathrm{mg}, 74 \%$ yield $) . \mathrm{mp} 107-108{ }^{\circ} \mathrm{C} .{ }^{1} \mathrm{H}$ $\operatorname{NMR}\left(500 \mathrm{MHz}, \mathrm{CDCl}_{3}\right) \delta 9.07(\mathrm{~d}, J=2.3 \mathrm{~Hz}, 1 \mathrm{H}), 8.15(\mathrm{dd}, J=8.1,2.4 \mathrm{~Hz}, 1 \mathrm{H}), 7.98(\mathrm{~d}, J=$ $8.1 \mathrm{~Hz}, 1 \mathrm{H}), 7.81(\mathrm{~d}, J=7.9 \mathrm{~Hz}, 1 \mathrm{H}), 7.41(\mathrm{td}, J=8.2,7.7,1.2 \mathrm{~Hz}, 1 \mathrm{H}), 7.30$ (td, $J=7.7,7.2$, $1.2 \mathrm{~Hz}, 1 \mathrm{H}), 7.17(\mathrm{~d}, J=8.1 \mathrm{~Hz}, 1 \mathrm{H}), 2.54(\mathrm{~s}, 3 \mathrm{H}) ;{ }^{13} \mathrm{C}\left\{{ }^{1} \mathrm{H}\right\} \mathrm{NMR}\left(126 \mathrm{MHz}, \mathrm{CDCl}_{3}\right) \delta 165.1$, 161.2, 154.1, 148.1, 134.96, 134.95, 127.1, 126.6, 125.6, 123.5, 123.4, 121.8, 24.7 ppm. Attention: the ${ }^{13}$ C NMR peak at 134.96 overlaps with the peak at 134.95 and shows as a shoulder peak. See spectra copies for details. HRMS (ESI) m/z calcd for $[\mathrm{M}+\mathrm{H}]^{+} 227.0638$, found 227.0635. IR (cm ${ }^{-}$ )): 3051, 1600, 1263, 958, 731, 703.

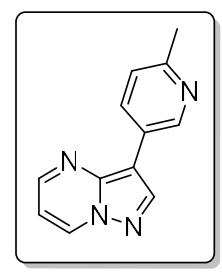

3-(6-methylpyridin-3-yl)pyrazolo[1,5-a]pyrimidine (3ac) was synthesized according to the general procedure using 6-methylpyridin-3-yl trifluoromethanesulfonate $(0.50 \mathrm{mmol}, 120.6 \mathrm{mg}$, 1.00 equiv) and 3-bromopyrazolo[1,5- $a$ ]pyrimidine ( $0.75 \mathrm{mmol}, 148.5 \mathrm{mg}, 1.50$ equiv) as starting materials. The reaction was quenched according to the general procedure after stirring at $60{ }^{\circ} \mathrm{C}$ for $24 \mathrm{~h}$, and the crude material was purified by flash chromatography on silica gel (hexanes (containing triethyl amine, $5 \% \mathrm{v} / \mathrm{v}$ ) $/$ ethyl acetate $=1 / 1$ ) to afford the desired product as a yellow solid (39.2 mg, 37\% yield). mp $158-159{ }^{\circ} \mathrm{C} .{ }^{1} \mathrm{H} \mathrm{NMR}\left(500 \mathrm{MHz}, \mathrm{CDCl}_{3}\right) \delta 9.07(\mathrm{~d}, J=2.3 \mathrm{~Hz}$, $1 \mathrm{H}), 8.69(\mathrm{dd}, J=7.0,1.8 \mathrm{~Hz}, 1 \mathrm{H}), 8.55(\mathrm{dd}, J=4.0,1.8 \mathrm{~Hz}, 1 \mathrm{H}), 8.44$ (s, $1 \mathrm{H}), 8.28(\mathrm{dd}, J=$ 8.1, 2.4 Hz, $1 \mathrm{H}), 7.22(\mathrm{~d}, J=8.1 \mathrm{~Hz}, 1 \mathrm{H}), 6.86(\mathrm{dd}, J=7.0,4.0 \mathrm{~Hz}, 1 \mathrm{H}), 2.58(\mathrm{~s}, 3 \mathrm{H}) ;{ }^{13} \mathrm{C}\left\{{ }^{1} \mathrm{H}\right\}$ NMR $\left(126 \mathrm{MHz}, \mathrm{CDCl}_{3}\right) \delta 156.3,149.6,146.9,145.3,142.5,135.5,134.0,125.2,123.3,108.4$, 108.0, 24.4 ppm. HRMS (ESI) m/z calcd for $[\mathrm{M}+\mathrm{H}]^{+}$211.0978, found 211.0977. IR $\left(\mathrm{cm}^{-1}\right): 3054$, 2917, 1613, 1520, 1400, 1302, 1263, 1196, 969, 776. 


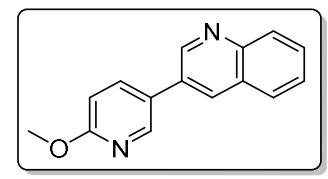

3-(6-Methoxypyridin-3-yl)quinoline (3ad) was synthesized according to the general procedure using 6-methoxypyridin-3-yl trifluoromethanesulfonate $(0.50 \mathrm{mmol}, 128.6 \mathrm{mg}, 1.00$ equiv) and 3bromoquinoline $(0.75 \mathrm{mmol}, 156.0 \mathrm{mg}, 1.50$ equiv) as starting materials. The reaction was quenched according to the general procedure after stirring at $60^{\circ} \mathrm{C}$ for $24 \mathrm{~h}$, and the crude material was purified by flash chromatography on silica gel (hexanes (containing triethyl amine, 5\% $\mathrm{v} / \mathrm{v}) /$ ethyl acetate $=20 / 1)$ to afford the desired product as a white solid (56.6 $\mathrm{mg}, 48 \%$ yield). $\mathrm{mp}$ $125-126{ }^{\circ} \mathrm{C} .{ }^{1} \mathrm{H}$ NMR $\left(500 \mathrm{MHz}, \mathrm{CDCl}_{3}\right) \delta 9.08(\mathrm{~d}, J=2.3 \mathrm{~Hz}, 1 \mathrm{H}), 8.47(\mathrm{~d}, J=2.7 \mathrm{~Hz}, 1 \mathrm{H})$, $8.17(\mathrm{~d}, J=2.3 \mathrm{~Hz}, 1 \mathrm{H}), 8.10(\mathrm{~d}, J=8.4 \mathrm{~Hz}, 1 \mathrm{H}), 7.85(\mathrm{dd}, J=8.6,2.6 \mathrm{~Hz}, 1 \mathrm{H}), 7.81(\mathrm{~d}, J=8.2$ $\mathrm{Hz}, 1 \mathrm{H}), 7.68$ (t, $J=7.7 \mathrm{~Hz}, 1 \mathrm{H}), 7.53$ (t, $J=7.5 \mathrm{~Hz}, 1 \mathrm{H}), 6.85$ (d, J=8.5 Hz, $1 \mathrm{H}), 3.98$ (s, 3 $\mathrm{H}) ;{ }^{13} \mathrm{C}\left\{{ }^{1} \mathrm{H}\right\}$ NMR $\left(126 \mathrm{MHz}, \mathrm{CDCl}_{3}\right) \delta 164.2,149.4,147.4,145.5,137.6,132.7,130.8,129.6$, 129.4, 128.0, 128.0, 127.2, 126.9, 111.4, $53.8 \mathrm{ppm}$. HRMS (ESI) $\mathrm{m} / \mathrm{z}$ calcd for $[\mathrm{M}+\mathrm{H}]^{+} 237.1022$, found 237.1020. IR ( $\left.\mathrm{cm}^{-1}\right)$ : 3051, 2950, 1606, 1505, 1315, 1025, 830, 741.

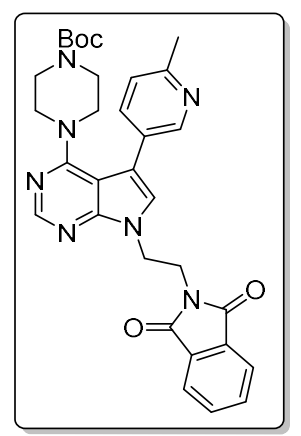

Tert-butyl 4-(7-(2-(1,3-dioxoisoindolin-2-yl)ethyl)-5-(6-methylpyridin-3-yl)-7H-pyrrolo[2,3d]pyrimidin-4-yl)piperazine-1-carboxylate (3ae) was synthesized according to the general procedure using 6-methylpyridin-3-yl trifluoromethanesulfonate $(0.50 \mathrm{mmol}, 120.6 \mathrm{mg}, 1.00$ equiv) and tert-butyl 4-(5-bromo-7-(2-(1,3-dioxoisoindolin-2-yl)ethyl)-7H-pyrrolo[2,3d]pyrimidin-4-yl)piperazine-1-carboxylate $(0.75 \mathrm{mmol}, 416.2 \mathrm{mg}, 1.50$ equiv) as starting materials. The reaction was quenched according to the general procedure after stirring at $60{ }^{\circ} \mathrm{C}$ for $24 \mathrm{~h}$, and the crude material was purified by flash chromatography on silica gel (hexanes (containing triethyl amine, $5 \% \mathrm{v} / \mathrm{v}) /$ ethyl acetate $=5 / 1$ to $2 / 1)$ to afford the desired product as a yellow solid (120.2 mg, 42\% yield). mp $189-191{ }^{\circ} \mathrm{C} .{ }^{1} \mathrm{H}$ NMR $\left(500 \mathrm{MHz}, \mathrm{CDCl}_{3}\right) \delta 8.51(\mathrm{~d}, J=2.3 \mathrm{~Hz}, 1 \mathrm{H}), 8.21$ $(\mathrm{s}, 1 \mathrm{H}), 7.73(\mathrm{~m}, 2 \mathrm{H}), 7.66(\mathrm{~m}, 2 \mathrm{H}), 7.58(\mathrm{dd}, J=7.9,2.4 \mathrm{~Hz}, 1 \mathrm{H}), 7.15(\mathrm{~d}, J=7.9 \mathrm{~Hz}, 1 \mathrm{H})$, $6.97(\mathrm{~s}, 1 \mathrm{H}), 4.55(\mathrm{t}, J=5.8 \mathrm{~Hz}, 2 \mathrm{H}), 4.11(\mathrm{t}, J=5.8 \mathrm{~Hz}, 2 \mathrm{H}), 3.20(\mathrm{~s}, 8 \mathrm{H}), 2.56(\mathrm{~s}, 3 \mathrm{H}), 1.40$ $(\mathrm{s}, 9 \mathrm{H}) ;{ }^{13} \mathrm{C}\left\{{ }^{1} \mathrm{H}\right\} \mathrm{NMR}\left(126 \mathrm{MHz}, \mathrm{CDCl}_{3}\right) \delta 167.9,160.4,156.8,154.8,152.9,151.0,148.5,136.0$, 
134.2, 132.0, 128.1, 124.4, 123.4, 123.0, 113.6, 104.0, 80.0, 49.5, 49.2, 43.5, 43.2, 42.6, 37.92, 28.51, $24.32 \mathrm{ppm}$. Attention: the four carbons in the piperazine structure are responsible for four different broad signals in the ${ }^{13} \mathrm{C} N M R$ spectrum (49.5, 49.2, 43.5 and $42.6 \mathrm{ppm}$ ). HRMS (ESI) $\mathrm{m} / \mathrm{z}$ calcd for $[\mathrm{M}+\mathrm{H}]^{+} 568.2667$, found 568.2666. IR $\left(\mathrm{cm}^{-1}\right): 2976,2926,1710,1689,1551,1391$, $1241,1168,1012,719$.

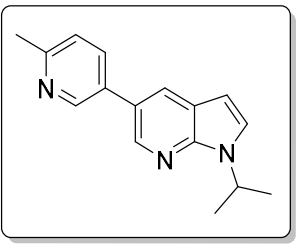

1-Isopropyl-5-(6-methylpyridin-3-yl)-1 $\boldsymbol{H}$-pyrrolo[2,3-b]pyridine (3af) was synthesized according to the general procedure using 6-methylpyridin-3-yl trifluoromethanesulfonate $(0.50$ mmol, $120.6 \mathrm{mg}, 1.00$ equiv) and 5-bromo-1-isopropyl-1 $H$-pyrrolo[2,3-b]pyridine ( $0.75 \mathrm{mmol}$, $179.3 \mathrm{mg}, 1.50$ equiv) as starting materials. The reaction was quenched according to the general procedure after stirring at $60{ }^{\circ} \mathrm{C}$ for $24 \mathrm{~h}$, and the crude material was purified by flash chromatography on silica gel (hexanes (containing triethyl amine, $5 \% \mathrm{v} / \mathrm{v}$ ) $/$ ethyl acetate $=20 / 1$ to $5 / 1)$ to afford the desired product as a white solid (92.5 mg, $74 \%$ yield). $\mathrm{mp} 116-117^{\circ} \mathrm{C} .{ }^{1} \mathrm{H}$ $\operatorname{NMR}\left(500 \mathrm{MHz}, \mathrm{CDCl}_{3}\right) \delta 8.75(\mathrm{~d}, J=3.3 \mathrm{~Hz}, 1 \mathrm{H}), 8.50(\mathrm{~d}, J=2.1 \mathrm{~Hz}, 1 \mathrm{H}), 8.04(\mathrm{~d}, J=2.2 \mathrm{~Hz}$, $1 \mathrm{H}), 7.78(\mathrm{dd}, J=8.0,2.4 \mathrm{~Hz}, 1 \mathrm{H}), 7.36(\mathrm{~d}, J=3.5 \mathrm{~Hz}, 1 \mathrm{H}), 7.23(\mathrm{~d}, J=8.0 \mathrm{~Hz}, 1 \mathrm{H}), 6.51$ (d, $J=3.6 \mathrm{~Hz}, 1 \mathrm{H}), 5.21$ (hept, $J=6.8 \mathrm{~Hz}, 1 \mathrm{H}), 2.60(\mathrm{~s}, 3 \mathrm{H}), 1.54(\mathrm{~d}, J=6.8 \mathrm{~Hz}, 9 \mathrm{H}) ;{ }^{13} \mathrm{C}\left\{{ }^{1} \mathrm{H}\right\}$ NMR $\left(126 \mathrm{MHz}, \mathrm{CDCl}_{3}\right) \delta 157.0,147.8,146.9,141.6,135.0,132.6,127.1,126.2,125.4,123.4$, 120.9, 100.0, 45.7, 24.2, $23.0 \mathrm{ppm}$. HRMS (ESI) $\mathrm{m} / \mathrm{z}$ calcd for $[\mathrm{M}+\mathrm{H}]^{+} 252.1495$, found 252.1493 . IR $\left(\mathrm{cm}^{-1}\right): 2977,2926,1594,1473,1405,1259,906,831,742$.

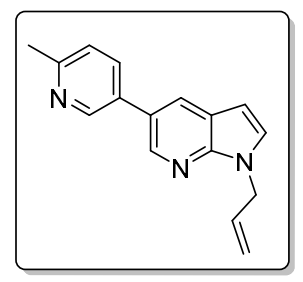

1-Allyl-5-(6-methylpyridin-3-yl)-1 H-pyrrolo[2,3-b]pyridine (3ag) was synthesized according to the general procedure using 6-methylpyridin-3-yl trifluoromethanesulfonate $(0.50 \mathrm{mmol}, 120.6$ $\mathrm{mg}, 1.00$ equiv) and 1 -allyl-5-bromo- $1 H$-pyrrolo[2,3-b]pyridine ( $0.75 \mathrm{mmol}, 177.8 \mathrm{mg}, 1.50$ equiv) as starting materials. The reaction was quenched according to the general procedure after stirring at $60{ }^{\circ} \mathrm{C}$ for $24 \mathrm{~h}$, and the crude material was purified by flash chromatography on silica gel (hexanes (containing triethyl amine, $5 \% \mathrm{v} / \mathrm{v}$ ) $/$ ethyl acetate $=10 / 1$ to $5 / 1$ ) to afford the desired product as a white solid (56.3 mg, 45\% yield). mp $90-91{ }^{\circ} \mathrm{C} .{ }^{1} \mathrm{H}$ NMR $\left(500 \mathrm{MHz}, \mathrm{CDCl}_{3}\right) \delta 8.76$ 
$(\mathrm{d}, J=2.3 \mathrm{~Hz}, 1 \mathrm{H}), 8.51(\mathrm{~d}, J=2.2 \mathrm{~Hz}, 1 \mathrm{H}), 8.06(\mathrm{~d}, J=2.1 \mathrm{~Hz}, 1 \mathrm{H}), 7.79(\mathrm{dd}, J=8.0,2.4 \mathrm{~Hz}$, $1 \mathrm{H}), 7.27(\mathrm{~d}, J=3.5 \mathrm{~Hz}, 1 \mathrm{H}), 7.24(\mathrm{~d}, J=8.0 \mathrm{~Hz}, 1 \mathrm{H}), 6.54(\mathrm{~d}, J=3.5 \mathrm{~Hz}, 1 \mathrm{H}), 6.07$ (ddd, $J=$ 22.6, 10.6, $5.5 \mathrm{~Hz}, 1 \mathrm{H}), 5.22(\mathrm{dd}, J=10.2,1.4 \mathrm{~Hz}, 1 \mathrm{H}), 5.11(\mathrm{dd}, J=17.0,1.5 \mathrm{~Hz}, 1 \mathrm{H}), 4.95$ (dt, $J=5.5,1.7 \mathrm{~Hz}, 2 \mathrm{H}), 2.61(\mathrm{~s}, 3 \mathrm{H}) ;{ }^{13} \mathrm{C}\left\{{ }^{1} \mathrm{H}\right\} \mathrm{NMR}\left(126 \mathrm{MHz}, \mathrm{CDCl}_{3}\right) \delta 157.1,147.9,147.3,142.1$, 135.1, 133.8, 132.5, 129.1, 127.2, 126.4, 123.4, 120.8, 117.6, 100.3, 46.9, 24.3 ppm. HRMS (ESI) $\mathrm{m} / \mathrm{z}$ calcd for $[\mathrm{M}+\mathrm{H}]^{+} 250.1339$, found $250.1337 . \mathrm{IR}\left(\mathrm{cm}^{-1}\right): 2921,1598,1476,1409,1275,912$, $829,733$.

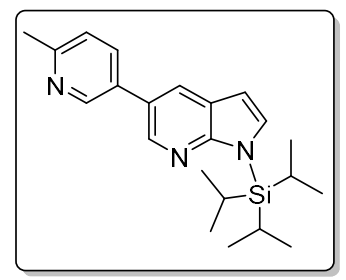

5-(6-Methylpyridin-3-yl)-1-(triisopropylsilyl)-1 $H$-pyrrolo[2,3-b]pyridine (3ah) was synthesized according to the general procedure using 6-methylpyridin-3-yl trifluoromethanesulfonate ( $0.50 \mathrm{mmol}, 120.6 \mathrm{mg}, 1.00$ equiv) and 5-bromo-1-(triisopropylsilyl)$1 H$-pyrrolo[2,3-b]pyridine $(0.75 \mathrm{mmol}, 265.0 \mathrm{mg}, 1.50$ equiv) as starting materials. The reaction was quenched according to the general procedure after stirring at $60{ }^{\circ} \mathrm{C}$ for $24 \mathrm{~h}$, and the crude material was purified by flash chromatography on silica gel (hexanes/ethyl acetate $=5 / 1$ ) to afford the desired product as a white solid (120.3 mg, 66\% yield). mp $143-144{ }^{\circ} \mathrm{C} .{ }^{1} \mathrm{H} \mathrm{NMR}(500 \mathrm{MHz}$, $\left.\mathrm{CDCl}_{3}\right) \delta 8.78(\mathrm{~d}, J=2.4 \mathrm{~Hz}, 1 \mathrm{H}), 8.47(\mathrm{~d}, J=2.3 \mathrm{~Hz}, 1 \mathrm{H}), 8.02(\mathrm{~d}, J=2.3 \mathrm{~Hz}, 1 \mathrm{H}), 7.81(\mathrm{dd}, J$ = 8.0, 2.4 Hz, $1 \mathrm{H}), 7.35(\mathrm{~d}, J=3.5 \mathrm{~Hz}, 1 \mathrm{H}), 7.23(\mathrm{~d}, J=8.0 \mathrm{~Hz}, 1 \mathrm{H}), 6.60(\mathrm{~d}, J=3.5 \mathrm{~Hz}, 1 \mathrm{H})$, $2.61(\mathrm{~s}, 3 \mathrm{H}), 1.88$ (hept, $J=7.6 \mathrm{~Hz}, 3 \mathrm{H}), 1.14(\mathrm{~d}, J=7.6 \mathrm{~Hz}, 18 \mathrm{H}) ;{ }^{13} \mathrm{C}\left\{{ }^{1} \mathrm{H}\right\} \mathrm{NMR}(126 \mathrm{MHz}$, $\left.\mathrm{CDCl}_{3}\right) \delta 156.8,153.8,147.8,141.4,135.0,132.7,132.4,126.3,126.2,123.4,122.5,103.3,24.2$, 18.3, 12.4 ppm. HRMS (ESI) $\mathrm{m} / \mathrm{z}$ calcd for $[\mathrm{M}+\mathrm{H}]^{+}$366.2360, found 366.2357. IR $\left(\mathrm{cm}^{-1}\right): 3053$, 2867, 1467, 1264, 895, 731, 703 .

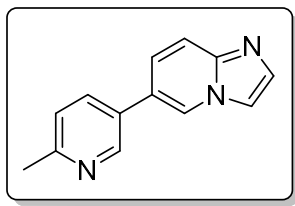

6-(6-Methylpyridin-3-yl)imidazo[1,2-a]pyridine (3ai) was synthesized according to the general procedure using 6-methylpyridin-3-yl trifluoromethanesulfonate $(0.50 \mathrm{mmol}, 120.6 \mathrm{mg}, 1.00$ equiv) and 6-bromoimidazo[1,2-a]pyridine (0.75 mmol, $147.8 \mathrm{mg}, 1.50$ equiv) as starting materials. The reaction was quenched according to the general procedure after stirring at $60{ }^{\circ} \mathrm{C}$ for $24 \mathrm{~h}$, and the crude material was purified by flash chromatography on silica gel 
(methanol/dichloromethane $=1 / 20$ to $1 / 10)$ to afford the desired product as a yellow solid $(63.1$ mg, 60\% yield). mp $156-157{ }^{\circ} \mathrm{C} .{ }^{1} \mathrm{H}$ NMR (500 MHz, $\left.\mathrm{CDCl}_{3}\right) \delta 8.64(\mathrm{~d}, J=2.5 \mathrm{~Hz}, 1 \mathrm{H}), 8.26$ (s, $1 \mathrm{H}), 7.69(\mathrm{dd}, J=8.0,2.5 \mathrm{~Hz}, 1 \mathrm{H}), 7.65$ (d, $J=9.4 \mathrm{~Hz}, 1 \mathrm{H}), 7.61$ (d, $J=7.3 \mathrm{~Hz}, 2 \mathrm{H}), 7.30$ $(\mathrm{dd}, J=9.4,1.9 \mathrm{~Hz}, 1 \mathrm{H}), 7.20(\mathrm{~d}, J=8.0 \mathrm{~Hz}, 1 \mathrm{H}), 2.56(\mathrm{~s}, 3 \mathrm{H}) ;{ }^{13} \mathrm{C}\left\{{ }^{1} \mathrm{H}\right\} \mathrm{NMR}\left(126 \mathrm{MHz}, \mathrm{CDCl}_{3}\right)$ $\delta 158.1,147.2,144.7,134.6,134.3,130.1,124.6,123.7,123.4,123.1,118.1,113.0,24.2$ ppm. HRMS (ESI) $\mathrm{m} / \mathrm{z}$ calcd for $[\mathrm{M}+\mathrm{H}]^{+}$210.1026, found 210.1023. IR $\left(\mathrm{cm}^{-1}\right): 3054,3004,1613$, $1520,1408,1303,1264,969,776$.

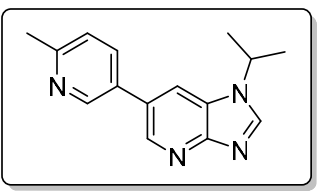

1-Isopropyl-6-(6-methylpyridin-3-yl)-1H-imidazo[4,5-b]pyridine (3aj) was synthesized according to the general procedure using 6-methylpyridin-3-yl trifluoromethanesulfonate $(0.50$ mmol, $120.6 \mathrm{mg}, 1.00$ equiv) and 6-bromo-1-isopropyl- $1 H$-imidazo[4,5- $b]$ pyridine $(0.75 \mathrm{mmol}$, $180.1 \mathrm{mg}, 1.50$ equiv) as starting materials. The reaction was quenched according to the general procedure after stirring at $60{ }^{\circ} \mathrm{C}$ for $24 \mathrm{~h}$, and the crude material was purified by flash chromatography on silica gel (hexanes (containing triethyl amine, $5 \% \mathrm{v} / \mathrm{v}$ ) $/$ ethyl acetate $=1 / 1$ to ethyl acetate/triethylamine $=9 / 1)$ to afford the desired product as a white solid $(91.4 \mathrm{mg}, 73 \%$ yield). mp $102-103{ }^{\circ} \mathrm{C} .{ }^{1} \mathrm{H}$ NMR $\left(500 \mathrm{MHz}, \mathrm{CDCl}_{3}\right) \delta 8.74(\mathrm{~d}, J=2.5 \mathrm{~Hz}, 1 \mathrm{H}), 8.57(\mathrm{~d}, J=2.1$ $\mathrm{Hz}, 1 \mathrm{H}), 8.18(\mathrm{~d}, J=2.0 \mathrm{~Hz}, 1 \mathrm{H}), 8.15(\mathrm{~s}, 1 \mathrm{H}), 7.78(\mathrm{dd}, J=8.0,2.4 \mathrm{~Hz}, 1 \mathrm{H}), 7.24(\mathrm{~d}, J=8.0$ $\mathrm{Hz}, 1 \mathrm{H}), 4.98$ (hept, $J=6.8 \mathrm{~Hz}, 1 \mathrm{H}), 2.59(\mathrm{~s}, 3 \mathrm{H}), 1.65(\mathrm{~d}, J=6.8 \mathrm{~Hz}, 6 \mathrm{H}) ;{ }^{13} \mathrm{C}\left\{{ }^{1} \mathrm{H}\right\} \mathrm{NMR}(126$ $\left.\mathrm{MHz}_{\mathrm{CDCl}}\right) \delta 157.6,147.9,146.7,143.1,142.6,136.1,135.2,131.8,129.2,126.2,123.5,47.1$, 24.3, $22.8 \mathrm{ppm}$. HRMS (ESI) $\mathrm{m} / \mathrm{z}$ calcd for $[\mathrm{M}+\mathrm{H}]^{+} 253.1448$, found 253.1444. IR $\left(\mathrm{cm}^{-1}\right): 2979$, $1602,1489,1394,1264,1222,732$.

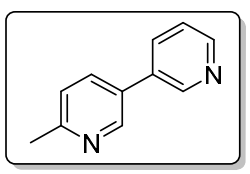

6-methyl-3,3'-bipyridine (3ak) was synthesized according to the general procedure using 6methylpyridin-3-yl trifluoromethanesulfonate $(0.50 \mathrm{mmol}, 120.6 \mathrm{mg}, 1.00$ equiv) and 3chloropyridine ( $0.75 \mathrm{mmol}, 85.2 \mathrm{mg}, 1.50$ equiv) as starting materials. The reaction was quenched according to the general procedure after stirring at $80{ }^{\circ} \mathrm{C}$ for $24 \mathrm{~h}$, and the crude material was purified by flash chromatography on silica gel (hexanes (containing triethyl amine, 5\% v/v)/ethyl acetate $=10 / 1$ to $2 / 1)$ to afford a colorless oil $(63.2 \mathrm{mg})$ containing 3ak (52.7 $\mathrm{mg}, 62 \%$ yield), 3,3'bipyridine ( $8.2 \mathrm{mg}, 11 \%$ yield), and 6,6'-dimethyl-3,3'-bipyridine (2.3 $\mathrm{mg}, 3 \%$ yield). ${ }^{1} \mathrm{H}$ NMR 
$\left(500 \mathrm{MHz}, \mathrm{CDCl}_{3}\right) \delta 8.78(\mathrm{~d}, J=1.8 \mathrm{~Hz}, 1 \mathrm{H}), 8.68(\mathrm{~d}, J=2.2 \mathrm{~Hz}, 1 \mathrm{H}), 8.58(\mathrm{dd}, J=4.9,1.6 \mathrm{~Hz}$, $1 \mathrm{H}), 7.81$ (dt, $J=7.9,1.9 \mathrm{~Hz}, 1 \mathrm{H}), 7.73$ (dd, $J=8.1,2.5 \mathrm{~Hz}, 1 \mathrm{H}), 7.34$ (ddd, $J=7.9,4.9,0.9 \mathrm{~Hz}$, $1 \mathrm{H}), 7.22(\mathrm{~d}, J=8.0 \mathrm{~Hz}, 1 \mathrm{H}), 2.57(\mathrm{~s}, 3 \mathrm{H}) \mathrm{ppm}$. The NMR data are consistent with the same compound 3a synthesized using 6-methylpyridin-3-yl trifluoromethanesulfonate and 3bromopyridine as starting materials. HRMS (ESI) $\mathrm{m} / \mathrm{z}$ calcd for $[\mathrm{M}+\mathrm{H}]^{+} 171.0917$, found 171.0914. For 3,3'-bipyridine, ${ }^{15} \mathrm{HRMS}(\mathrm{ESI}) \mathrm{m} / \mathrm{z}$ calcd for $[\mathrm{M}+\mathrm{H}]^{+} 157.0760$, found 157.0759 . For 6,6'-dimethyl-3,3'-bipyridine, ${ }^{16} \mathrm{HRMS}$ (ESI) $\mathrm{m} / \mathrm{z}$ calcd for $[\mathrm{M}+\mathrm{H}]^{+} 185.1073$, found 185.1072 .

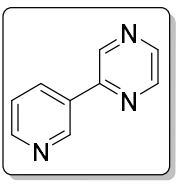

2-(Pyridin-3-yl)pyrazine (3al) [CAS: 93844-97-4] was synthesized according to the general procedure using pyridin-3-yl trifluoromethanesulfonate $(0.50 \mathrm{mmol}, 113.6 \mathrm{mg}, 1.00 \mathrm{equiv})$ and 2chloropyrazine ( $0.75 \mathrm{mmol}, 85.5 \mathrm{mg}, 1.50$ equiv) as starting materials. The reaction was quenched according to the general procedure after stirring at $60{ }^{\circ} \mathrm{C}$ for $24 \mathrm{~h}$, and the crude material was purified by flash chromatography on silica gel (hexanes (containing triethyl amine, 5\% v/v)/ethyl acetate $=3 / 1$ to $1 / 1)$ to afford the product as a yellow solid (31.1 mg, 40\% yield). ${ }^{1} \mathrm{H}$ NMR (500 $\left.\mathrm{MHz}, \mathrm{CDCl}_{3}\right) \delta 9.21(\mathrm{~d}, J=2.3 \mathrm{~Hz}, 1 \mathrm{H}), 9.02(\mathrm{~d}, J=1.5 \mathrm{~Hz}, 1 \mathrm{H}), 8.67(\mathrm{dd}, J=4.8,1.7 \mathrm{~Hz}, 1 \mathrm{H})$, $8.63(\mathrm{dd}, J=2.5,1.6 \mathrm{~Hz}, 1 \mathrm{H}), 8.54(\mathrm{~d}, J=2.5 \mathrm{~Hz}, 1 \mathrm{H}), 8.29$ (dt, $J=7.9,2.0 \mathrm{~Hz}, 1 \mathrm{H}), 7.40$ (ddd, $J=8.1,4.8,0.9 \mathrm{~Hz}, 1 \mathrm{H}) ;{ }^{13} \mathrm{C}\left\{{ }^{1} \mathrm{H}\right\} \mathrm{NMR}\left(126 \mathrm{MHz}, \mathrm{CDCl}_{3}\right) \delta 150.9,150.5,148.2,144.6,143.9$, 142.1, 134.4, 132.1, 123.9 ppm. The NMR data are consistent with those reported in the literature. $^{23}$

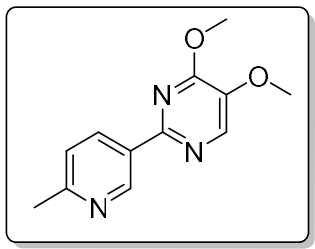

4,5-Dimethoxy-2-(6-methylpyridin-3-yl)pyrimidine (3am) was synthesized according to the general procedure using 6-methylpyridin-3-yl trifluoromethanesulfonate $(0.50 \mathrm{mmol}, 120.6 \mathrm{mg}$, 1.00 equiv) and 2-chloro-4,5-dimethoxypyrimidine ( $0.75 \mathrm{mmol}, 130.9 \mathrm{mg}, 1.50$ equiv) as starting materials. The reaction was quenched according to the general procedure after stirring at $60{ }^{\circ} \mathrm{C}$ for $24 \mathrm{~h}$, and the crude material was purified by flash chromatography on silica gel (hexanes/ethyl acetate $=5 / 1$ to $3 / 2)$ to afford the product as a white solid $(106.0 \mathrm{mg}, 92 \%$ yield $) . \mathrm{mp} 122-123{ }^{\circ} \mathrm{C}$. ${ }^{1} \mathrm{H}$ NMR $\left(500 \mathrm{MHz}, \mathrm{CDCl}_{3}\right) \delta 9.34(\mathrm{~d}, J=2.2 \mathrm{~Hz}, 1 \mathrm{H}), 8.37(\mathrm{dd}, J=8.2,2.3 \mathrm{~Hz}, 1 \mathrm{H}), 8.01(\mathrm{~s}, 1$ 
H), $7.13(\mathrm{~d}, J=8.0 \mathrm{~Hz}, 1 \mathrm{H}), 4.06(\mathrm{~s}, 3 \mathrm{H}), 3.87(\mathrm{~s}, 3 \mathrm{H}), 2.53(\mathrm{~s}, 3 \mathrm{H}) ;{ }^{13} \mathrm{C}\left\{{ }^{1} \mathrm{H}\right\} \mathrm{NMR}(126 \mathrm{MHz}$, $\left.\mathrm{CDCl}_{3}\right) \delta 159.7,159.5,154.4,148.8,141.3,137.1,135.1,130.2,122.8,56.4,54.1,24.4$ ppm. HRMS (ESI) $\mathrm{m} / \mathrm{z}$ calcd for $[\mathrm{M}+\mathrm{H}]^{+}$232.1080, found 232.1078. IR $\left(\mathrm{cm}^{-1}\right): 2944,2916,1581$, $1485,1418,1328,1230,1006,909,782$.

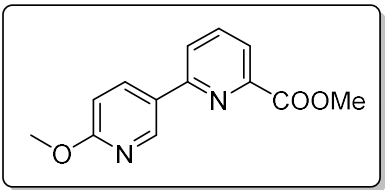

Methyl 6'-methoxy-[2,3'-bipyridine]-6-carboxylate (3an) was synthesized according to the general procedure using 6-methoxypyridin-3-yl trifluoromethanesulfonate $(0.50 \mathrm{mmol}, 128.6 \mathrm{mg}$, 1.00 equiv) and methyl 6-chloropicolinate $(0.75 \mathrm{mmol}, 128.7 \mathrm{mg}, 1.50$ equiv) as starting materials. The reaction was quenched according to the general procedure after stirring at $60{ }^{\circ} \mathrm{C}$ for $24 \mathrm{~h}$, and the crude material was purified by flash chromatography on silica gel (hexanes (containing triethyl amine, $5 \% \mathrm{v} / \mathrm{v}) /$ ethyl acetate $=3 / 1$ to $2 / 1)$ to afford the desired product as a white solid (90.3 $\mathrm{mg}$, $74 \%$ yield). mp $130-131{ }^{\circ} \mathrm{C} .{ }^{1} \mathrm{H}$ NMR $\left(500 \mathrm{MHz}, \mathrm{CDCl}_{3}\right) \delta 8.75(\mathrm{~d}, J=2.1 \mathrm{~Hz}, 1 \mathrm{H}), 8.31$ (dd, $J=8.6,2.5 \mathrm{~Hz}, 1 \mathrm{H}), 8.01(\mathrm{dd}, J=7.5,1.1 \mathrm{~Hz}, 1 \mathrm{H}), 7.86(\mathrm{t}, J=7.7 \mathrm{~Hz}, 1 \mathrm{H}), 7.81(\mathrm{dd}, J=7.9$, $1.1 \mathrm{~Hz}, 1 \mathrm{H}), 6.83(\mathrm{~d}, J=8.7 \mathrm{~Hz}, 1 \mathrm{H}), 3.99(\mathrm{~s}, 3 \mathrm{H}), 3.97(\mathrm{~s}, 3 \mathrm{H}) ;{ }^{13} \mathrm{C}\left\{{ }^{1} \mathrm{H}\right\} \mathrm{NMR}(126 \mathrm{MHz}$, $\left.\mathrm{CDCl}_{3}\right) \delta 166.0,165.1,155.5,148.3,145.9,138.0,137.8,128.0,123.4,123.0,111.2,53.8,53.0$ ppm. HRMS (ESI) m/z calcd for $[\mathrm{M}+\mathrm{H}]^{+}$245.0921, found 245.0917. IR $\left(\mathrm{cm}^{-1}\right): 2997,2951,1717$, $1608,1444,1287,1138,1031,827,773$.

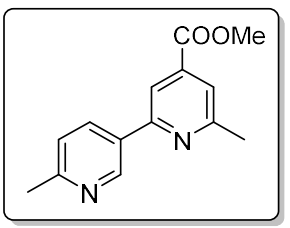

Methyl 6,6'-dimethyl-[2,3'-bipyridine]-4-carboxylate (3ao) was synthesized according to the general procedure using 6-methoxypyridin-3-yl trifluoromethanesulfonate $(0.50 \mathrm{mmol}, 128.6 \mathrm{mg}$, 1.00 equiv) and methyl 2-chloro-6-methylisonicotinate $(0.75 \mathrm{mmol}, 139.2 \mathrm{mg}, 1.50$ equiv) as starting materials. The reaction was quenched according to the general procedure after stirring at $60{ }^{\circ} \mathrm{C}$ for $24 \mathrm{~h}$, and the crude material was purified by flash chromatography on silica gel (hexanes (containing triethyl amine, $5 \% \mathrm{v} / \mathrm{v}$ ) $/$ ethyl acetate $=20 / 1$ ) to afford the desired product as a white solid (86.5 mg, 71\% yield). mp $89-90{ }^{\circ} \mathrm{C} .{ }^{1} \mathrm{H}$ NMR $\left(500 \mathrm{MHz}, \mathrm{CDCl}_{3}\right) \delta 9.09(\mathrm{~d}, J=2.1 \mathrm{~Hz}, 1$ H), $8.22(\mathrm{dd}, J=8.1,2.4 \mathrm{~Hz}, 1 \mathrm{H}), 8.04(\mathrm{~s}, 1 \mathrm{H}), 7.64(\mathrm{~s}, 1 \mathrm{H}), 7.23(\mathrm{~d}, J=8.1 \mathrm{~Hz}, 1 \mathrm{H}), 3.95(\mathrm{~s}, 3$ $\mathrm{H}), 2.66(\mathrm{~s}, 3 \mathrm{H}), 2.60(\mathrm{~s}, 3 \mathrm{H}) ;{ }^{13} \mathrm{C}\left\{{ }^{1} \mathrm{H}\right\} \mathrm{NMR}\left(126 \mathrm{MHz}, \mathrm{CDCl}_{3}\right) \delta 166.0,160.0,159.3,155.6$, 147.8, 138.7, 134.9, 131.7, 123.3, 121.3, 116.6, 52.8, 24.8, 24.5 ppm. HRMS (ESI) m/z calcd for 
$[\mathrm{M}+\mathrm{H}]^{+}$243.1128, found 243.1125. IR $\left(\mathrm{cm}^{-1}\right): 2952,2924,1729,1601,1571,1339,1254,1211$, 996, 767, 737.

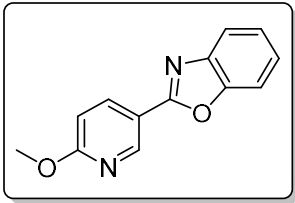

2-(6-Methoxypyridin-3-yl)benzo[d] oxazole (3ap) [CAS: 945375-84-8] was synthesized according to the general procedure using 6-methoxypyridin-3-yl trifluoromethanesulfonate $(0.50$ mmol, $128.6 \mathrm{mg}, 1.00$ equiv) and 2-chlorobenzo[ $d]$ oxazole ( $0.75 \mathrm{mmol}, 115.2 \mathrm{mg}, 1.50$ equiv) as starting materials. The reaction was quenched according to the general procedure after stirring at $60{ }^{\circ} \mathrm{C}$ for $24 \mathrm{~h}$, and the crude material was purified by flash chromatography on silica gel (hexanes (containing triethyl amine, $5 \% \mathrm{v} / \mathrm{v}$ ) $/$ ethyl acetate $=20 / 1$ ) to afford the desired product as a white solid (56.3 mg, 50\% yield). ${ }^{1} \mathrm{H}$ NMR (500 MHz, $\left.\mathrm{CDCl}_{3}\right) \delta 9.01(\mathrm{~d}, J=2.4 \mathrm{~Hz}, 1 \mathrm{H}), 8.34$ (dd, $J=$ 8.7, $2.4 \mathrm{~Hz}, 1 \mathrm{H}), 7.76-7.68$ (m, $1 \mathrm{H}), 7.58-7.50$ (m, $1 \mathrm{H}), 7.34-7.29$ (m, $1 \mathrm{H}), 6.84$ (d, $J=8.7$ $\mathrm{Hz}, 1 \mathrm{H}), 4.00(\mathrm{~s}, 3 \mathrm{H}) ;{ }^{13} \mathrm{C}\left\{{ }^{1} \mathrm{H}\right\} \mathrm{NMR}\left(126 \mathrm{MHz}, \mathrm{CDCl}_{3}\right) \delta 166.1,161.5,150.7,147.3,142.1$, $137.6,125.1,124.8,119.9,117.2,111.5,110.6,54.1 \mathrm{ppm}$. The NMR data are consistent with those reported in the literature. ${ }^{24}$

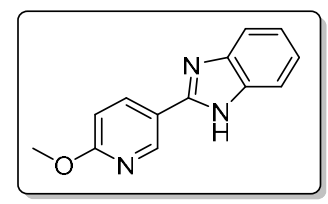

2-(6-Methoxypyridin-3-yl)-1 $H$-benzo[d]imidazole (3aq) [CAS: 1091605-91-2] was synthesized according to the general procedure using 6-methoxypyridin-3-yl trifluoromethanesulfonate $(0.50$ mmol, $128.6 \mathrm{mg}, 1.00$ equiv) and 2-chloro- $1 H$-benzo[ $d$ ]imidazole $(0.75 \mathrm{mmol}, 114.4 \mathrm{mg}, 1.50$ equiv) as starting materials. The reaction was quenched according to the general procedure after stirring at $60{ }^{\circ} \mathrm{C}$ for $24 \mathrm{~h}$, and the crude material was purified by flash chromatography on silica gel (methanol/dichloromethane $=1 / 20$ to $1 / 10)$ to afford the desired product as a white solid $(74.2$ mg, 66\% yield). ${ }^{1} \mathrm{H}$ NMR (500 MHz, DMSO- $\left.d_{6}\right) \delta 12.90(\mathrm{~s}, 1 \mathrm{H}), 8.96(\mathrm{~d}, J=2.4 \mathrm{~Hz}, 1 \mathrm{H}), 8.42$ (dd, $J=8.7,2.5 \mathrm{~Hz}, 1 \mathrm{H}), 7.66$ (d, $J=7.7 \mathrm{~Hz}, 1 \mathrm{H}), 7.53$ (d, $J=7.6 \mathrm{~Hz}, 1 \mathrm{H}), 7.20$ (t, $J=7.5 \mathrm{~Hz}$, $2 \mathrm{H}), 7.00(\mathrm{~d}, J=8.7 \mathrm{~Hz}, 1 \mathrm{H}), 3.94(\mathrm{~s}, 3 \mathrm{H}) ;{ }^{13} \mathrm{C}\left\{{ }^{1} \mathrm{H}\right\} \mathrm{NMR}\left(126 \mathrm{MHz}\right.$, DMSO- $\left.d_{6}\right) \delta$ 164.3, 149.2, $145.4,143.7,137.2,134.9,122.4,121.7,120.1,118.7,111.2,110.9,53.6 \mathrm{ppm}$. The NMR data are consistent with those reported in the literature. ${ }^{25}$ 


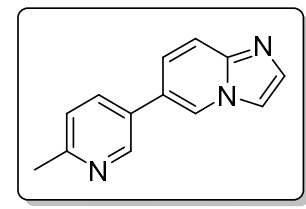

6-(6-Methylpyridin-3-yl)imidazo[1,2-a]pyridine (3ar) was synthesized according to the general procedure using 6-methylpyridin-3-yl trifluoromethanesulfonate $(0.50 \mathrm{mmol}, 120.6 \mathrm{mg}, 1.00$ equiv) and 6-chloroimidazo[1,2-a]pyridine ( $0.75 \mathrm{mmol}, 114.4 \mathrm{mg}, 1.50$ equiv) as starting materials. The reaction was quenched according to the general procedure after stirring at $60{ }^{\circ} \mathrm{C}$ for $24 \mathrm{~h}$, and the crude material was purified by flash chromatography on silica gel (methanol/dichloromethane $=1 / 20$ to $1 / 10)$ to afford the desired product as a yellow solid (72.2 mg, 69\% yield). ${ }^{1} \mathrm{H}$ NMR (500 $\left.\mathrm{MHz} \mathrm{CDCl}_{3}\right) \delta 8.66(\mathrm{~d}, J=2.5 \mathrm{~Hz}, 1 \mathrm{H}), 8.26(\mathrm{~s}, 1 \mathrm{H}), 7.70(\mathrm{dd}, J=8.0,2.5 \mathrm{~Hz}, 1 \mathrm{H}), 7.66(\mathrm{~d}, J$ $=9.3 \mathrm{~Hz}, 1 \mathrm{H}), 7.62(\mathrm{~d}, J=7.4 \mathrm{~Hz}, 2 \mathrm{H}), 7.32(\mathrm{dd}, J=9.3,1.8 \mathrm{~Hz}, 1 \mathrm{H}), 7.21(\mathrm{~d}, J=8.0 \mathrm{~Hz}, 1 \mathrm{H})$, $2.57(\mathrm{~s}, 3 \mathrm{H}) ;{ }^{13} \mathrm{C}\left\{{ }^{1} \mathrm{H}\right\} \mathrm{NMR}\left(126 \mathrm{MHz}, \mathrm{CDCl}_{3}\right) \delta 158.1,147.2,144.8,134.6,134.4,130.2,124.6$, 123.7, 123.5, 123.1, 118.2, 113.0, $24.2 \mathrm{ppm}$. The NMR data are consistent with those of the same compound 2ai synthesized using 6-methylpyridin-3-yl trifluoromethanesulfonate and 6bromoimidazo[1,2- $a]$ pyridine as starting materials.

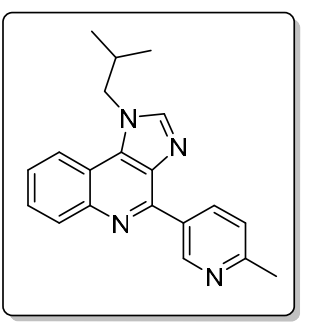

1-Isobutyl-4-(6-methylpyridin-3-yl)-1H-imidazo[4,5-c]quinoline (3as) was synthesized according to the general procedure using 6-methoxypyridin-3-yl trifluoromethanesulfonate $(0.50$ mmol, $128.6 \mathrm{mg}, 1.00$ equiv) and 4-chloro-1-isobutyl-1 $H$-imidazo[4,5-c]quinoline (0.75 mmol, $194.8 \mathrm{mg}, 1.50$ equiv) as starting materials. The reaction was quenched according to the general procedure after stirring at $60{ }^{\circ} \mathrm{C}$ for $24 \mathrm{~h}$, and the crude material was purified by flash chromatography on silica gel (hexanes (containing triethyl amine, $5 \% \mathrm{v} / \mathrm{v}$ ) $/$ ethyl acetate $=4 / 1$ to $2 / 3$ ) to afford the desired product as a white solid $\left(74.5 \mathrm{mg}, 43 \%\right.$ yield). mp $154-155{ }^{\circ} \mathrm{C} .{ }^{1} \mathrm{H}$ NMR (500 MHz, $\left.\mathrm{CDCl}_{3}\right) \delta 9.75(\mathrm{~d}, J=1.5 \mathrm{~Hz}, 1 \mathrm{H}), 8.88(\mathrm{dd}, J=8.1,2.3 \mathrm{~Hz}, 1 \mathrm{H}), 8.31(\mathrm{dd}, J=$ 8.4, $1.3 \mathrm{~Hz}, 1 \mathrm{H}), 8.07$ (dd, $J=8.4,1.4 \mathrm{~Hz}, 1 \mathrm{H}), 7.91$ (s, $1 \mathrm{H}), 7.67$ (ddd, $J=8.4,7.0,1.4 \mathrm{~Hz}, 1$ H), $7.58(\mathrm{ddd}, J=8.3,6.9,1.3 \mathrm{~Hz}, 1 \mathrm{H}), 7.32(\mathrm{~d}, J=8.0 \mathrm{~Hz}, 1 \mathrm{H}), 4.33$ (d, $J=7.4 \mathrm{~Hz}, 2 \mathrm{H}), 2.65$ $(\mathrm{s}, 3 \mathrm{H}), 2.35(\mathrm{~m}, 1 \mathrm{H}), 1.02(\mathrm{~d}, J=6.7 \mathrm{~Hz}, 6 \mathrm{H}) ;{ }^{13} \mathrm{C}\left\{{ }^{1} \mathrm{H}\right\} \mathrm{NMR}\left(126 \mathrm{MHz}, \mathrm{CDCl}_{3}\right) \delta 159.2,150.5$, $149.6,144.7,143.8,137.6,136.6,133.7,131.3,130.9,127.5,126.5,122.8,120.0,117.8,55.3$, 
28.9, 24.6, 19.9 ppm. HRMS (ESI) $\mathrm{m} / \mathrm{z}$ calcd for $[\mathrm{M}+\mathrm{H}]^{+}$317.1761, found 317.1757. IR $\left(\mathrm{cm}^{-1}\right)$ : 2960, 2925, 1620, 1568, 1517, 1349, 1310, 1028, 923, 756, 736, 642.

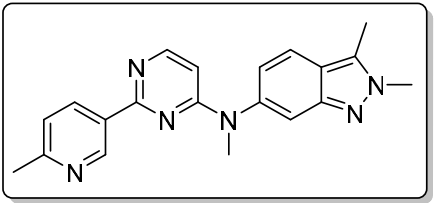

$N, 2,3-t r i m e t h y l-N$-(2-(6-methylpyridin-3-yl)pyrimidin-4-yl)-2H-indazol-6-amine (3at) was synthesized according to the general procedure using 6-methoxypyridin-3-yl trifluoromethanesulfonate $(0.50 \mathrm{mmol}, 128.6 \mathrm{mg}, 1.00$ equiv) and $\mathrm{N}$-(2-chloropyrimidin-4-yl)$N$,2,3-trimethyl-2H-indazol-6-amine ( $0.75 \mathrm{mmol}, 215.2 \mathrm{mg}, 1.50$ equiv) as starting materials. The reaction was quenched according to the general procedure after stirring at $60{ }^{\circ} \mathrm{C}$ for $24 \mathrm{~h}$, and the crude material was purified by flash chromatography on silica gel (hexanes (containing triethyl amine, $5 \% \mathrm{v} / \mathrm{v}$ ) $/$ ethyl acetate $=2 / 1$ to hexanes (containing triethyl amine, $5 \% \mathrm{v} / \mathrm{v}$ ) $/$ ethyl acetate/methanol $=4 / 16 / 1)$ to afford the desired product as a yellow solid (94.3 $\mathrm{mg}$, 55\% yield). mp $152-153{ }^{\circ} \mathrm{C} .{ }^{1} \mathrm{H}$ NMR $\left(500 \mathrm{MHz}, \mathrm{CDCl}_{3}\right) \delta 9.49(\mathrm{~d}, J=2.2 \mathrm{~Hz}, 1 \mathrm{H}), 8.53(\mathrm{dd}, J=8.0,2.3$ $\mathrm{Hz}, 1 \mathrm{H}), 8.08$ (d, $J=6.1 \mathrm{~Hz}, 1 \mathrm{H}), 7.59$ (d, $J=8.7 \mathrm{~Hz}, 1 \mathrm{H}), 7.47$ (s, $1 \mathrm{H}), 7.19$ (d, J=8.0 Hz, 1 H), $6.86(\mathrm{~d}, J=8.2 \mathrm{~Hz}, 1 \mathrm{H}), 6.23(\mathrm{~d}, J=6.1 \mathrm{~Hz}, 1 \mathrm{H}), 4.07(\mathrm{~s}, 3 \mathrm{H}), 3.60$ (s, $3 \mathrm{H}), 2.59$ (s, $6 \mathrm{H})$; ${ }^{13} \mathrm{C}\left\{{ }^{1} \mathrm{H}\right\} \mathrm{NMR}\left(126 \mathrm{MHz}, \mathrm{CDCl}_{3}\right) \delta 162.3,162.2,159.9,155.0,149.4,148.0,142.4,135.8,132.1$, 131.4, 122.8, 121.7, 120.2, 120.1, 114.6, 103.6, 37.9, 37.6, 24.5, 10.0 ppm. HRMS (ESI) m/z calcd for $[\mathrm{M}+\mathrm{H}]^{+}$345.1822, found 345.1818. IR $\left(\mathrm{cm}^{-1}\right)$ : 3033, 2921, 1580, 1481, 1402, 1377, 1139 , $978,732,669$.

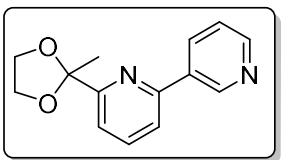

6-(2-Methyl-1,3-dioxolan-2-yl)-2,3'-bipyridine (3au) was synthesized according to the general procedure using pyridin-3-yl trifluoromethanesulfonate $(0.50 \mathrm{mmol}, 113.6 \mathrm{mg}, 1.00 \mathrm{equiv})$ and 2bromo-6-(2-methyl-1,3-dioxolan-2-yl)pyridine $(0.75 \mathrm{mmol}, 183.0 \mathrm{mg}, 1.50$ equiv) as starting materials. The reaction was quenched according to the general procedure after stirring at $60{ }^{\circ} \mathrm{C}$ for $24 \mathrm{~h}$, and the crude material was purified by flash chromatography on silica gel (hexanes/ethyl acetate $=9 / 1$ to $7 / 3$ ) to afford the desired product as a colorless oil (42.2 mg, 35\% yield). ${ }^{1} \mathrm{H} \mathrm{NMR}$ $\left(500 \mathrm{MHz} \mathrm{CDCl}_{3}\right) \delta 9.21(\mathrm{~d}, J=2.2 \mathrm{~Hz}, 1 \mathrm{H}), 8.62(\mathrm{dd}, J=4.8,1.7 \mathrm{~Hz}, 1 \mathrm{H}), 8.38(\mathrm{dt}, J=7.9$, $2.0 \mathrm{~Hz}, 1 \mathrm{H}), 7.78$ (t, $J=7.8 \mathrm{~Hz}, 1 \mathrm{H}), 7.68$ (dd, $J=7.8,1.0 \mathrm{~Hz}, 1 \mathrm{H}), 7.55$ (dd, $J=7.6,1.0 \mathrm{~Hz}, 1$ $\mathrm{H}), 7.38(\mathrm{dd}, J=8.0,4.8 \mathrm{~Hz}, 1 \mathrm{H}), 4.17-4.07(\mathrm{~m}, 2 \mathrm{H}), 4.02-3.92(\mathrm{~m}, 2 \mathrm{H}), 1.82(\mathrm{~s}, 3 \mathrm{H}) ;{ }^{13} \mathrm{C}$ NMR $\left(126 \mathrm{MHz}, \mathrm{CDCl}_{3}\right) \delta 161.4,154.5,150.0,148.5,137.7,134.9,134.8,123.7,119.8,118.5$, 
108.9, 65.3, $25.1 \mathrm{ppm}$. HRMS (ESI) $\mathrm{m} / \mathrm{z}$ calcd for $[\mathrm{M}+\mathrm{H}]^{+} 243.1128$, found 243.1126. IR $\left(\mathrm{cm}^{-1}\right)$ : $2985,2885,1576,1425,1195,1036,871,796,707$.

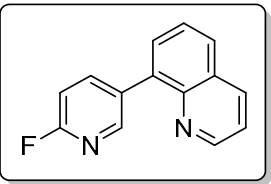

8-(6-fluoropyridin-3-yl)quinoline (3av) was synthesized according to the general procedure using 6-fluoropyridin-3-yl trifluoromethanesulfonate (0.50 mmol, $122.6 \mathrm{mg}, 1.00$ equiv) and 8bromoquinoline $(0.75 \mathrm{mmol}, 156.0 \mathrm{mg}, 1.50$ equiv) as starting materials. The reaction was quenched according to the general procedure after stirring at $60^{\circ} \mathrm{C}$ for $24 \mathrm{~h}$, and the crude material was purified by flash chromatography on silica gel (hexanes/ethyl acetate $=5 / 1$ ) to a white solid (76.1 mg) containing 3av (72.3 mg, 64\% yield) and 6,6'-difluoro-3,3'-bipyridine (3.8 mg, 5\% yield). ${ }^{1} \mathrm{H}$ NMR (500 MHz, $\left.\mathrm{CDCl}_{3}\right) \delta 8.92(\mathrm{dd}, J=4.1,1.8 \mathrm{~Hz}, 1 \mathrm{H}), 8.51(\mathrm{~d}, J=2.5 \mathrm{~Hz}, 1 \mathrm{H})$, $8.21(\mathrm{~m}, 2 \mathrm{H}), 7.88(\mathrm{dd}, J=8.2,1.5 \mathrm{~Hz}, 1 \mathrm{H}), 7.73(\mathrm{dd}, J=7.2,1.5 \mathrm{~Hz}, 1 \mathrm{H}), 7.62$ (dd, $J=8.2,7.1$ $\mathrm{Hz}, 1 \mathrm{H}), 7.44(\mathrm{dd}, J=8.3,4.2 \mathrm{~Hz}, 1 \mathrm{H}), 7.05(\mathrm{dd}, J=8.4,2.9 \mathrm{~Hz}, 1 \mathrm{H}) ;{ }^{19} \mathrm{~F}\left\{{ }^{1} \mathrm{H}\right\} \mathrm{NMR}(376 \mathrm{MHz}$, $\left.\mathrm{CDCl}_{3}\right) \delta-70.37 ;{ }^{13} \mathrm{C}\left\{{ }^{1} \mathrm{H}\right\} \mathrm{NMR}\left(126 \mathrm{MHz}, \mathrm{CDCl}_{3}\right) \delta 163.1(\mathrm{~d}, J=239.1 \mathrm{~Hz}), 150.6,148.5(\mathrm{~d}, J$ $=14.7 \mathrm{~Hz}), 145.9,143.7(\mathrm{~d}, J=7.9 \mathrm{~Hz}), 136.6,136.1,133.1$ (d, $J=4.6 \mathrm{~Hz}), 130.3,128.9,128.7$, 126.5, 121.6, $108.7\left(\mathrm{~d}, J=37.4 \mathrm{~Hz}\right.$ ) ppm. HRMS (ESI) $\mathrm{m} / \mathrm{z}$ calcd for $[\mathrm{M}+\mathrm{H}]^{+} 225.0823$, found 225.0821. IR (cm $\left.{ }^{-1}\right): 3055,3031,1584,1486,1466,1365,1246,1123,1029,817,791$. For 6,6'difluoro-3,3'-bipyridine, ${ }^{26} \mathrm{HRMS}$ (ESI) m/z calcd for $[\mathrm{M}+\mathrm{H}]^{+} 193.0572$, found 193.0572 .

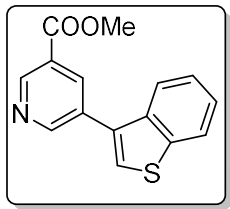

Methyl 5-(benzo[b]thiophen-3-yl)nicotinate (3aw) was synthesized according to the general procedure using methyl 5-(((trifluoromethyl)sulfonyl)oxy)nicotinate $(0.50 \mathrm{mmol}, 142.6 \mathrm{mg}, 1.00$ equiv) and 3-bromobenzo[b]thiophene $(0.75 \mathrm{mmol}, 159.8 \mathrm{mg}, 1.50$ equiv) as starting materials. The reaction was quenched according to the general procedure after stirring at $60{ }^{\circ} \mathrm{C}$ for $24 \mathrm{~h}$, and the crude material was purified by flash chromatography on silica gel (hexanes/ethyl acetate $=$ $10 / 1$ to $5 / 1)$ to afford the desired product as a white solid $(90.5 \mathrm{mg}, 67 \%)$. mp $129-130{ }^{\circ} \mathrm{C} .{ }^{1} \mathrm{H}$ $\operatorname{NMR}\left(500 \mathrm{MHz}, \mathrm{CDCl}_{3}\right) \delta 9.23(\mathrm{~d}, J=2.1 \mathrm{~Hz}, 1 \mathrm{H}), 8.98(\mathrm{~d}, J=2.2 \mathrm{~Hz}, 1 \mathrm{H}), 8.48(\mathrm{t}, J=2.1 \mathrm{~Hz}$, $1 \mathrm{H}), 7.95-7.87(\mathrm{~m}, 1 \mathrm{H}), 7.85$ - $7.77(\mathrm{~m}, 1 \mathrm{H}), 7.50(\mathrm{~s}, 1 \mathrm{H}), 7.44-7.36(\mathrm{~m}, 2 \mathrm{H}), 3.97$ (s, $3 \mathrm{H})$; ${ }^{13} \mathrm{C}\left\{{ }^{1} \mathrm{H}\right\} \mathrm{NMR}\left(126 \mathrm{MHz}, \mathrm{CDCl}_{3}\right) \delta 165.7,153.0,149.7,140.8,137.2,136.7,133.2,131.7,126.1$, 125.6, 124.97, 124.96, 123.2, 122.2, $52.6 \mathrm{ppm}$. HRMS (ESI) $\mathrm{m} / \mathrm{z}$ calcd for $[\mathrm{M}+\mathrm{H}]^{+} 270.0583$, 
found 270.0582. IR $\left(\mathrm{cm}^{-1}\right): 3082,2963,1719,1596,1457,1423,1318,1286,1222,1116,998,765$, 729.

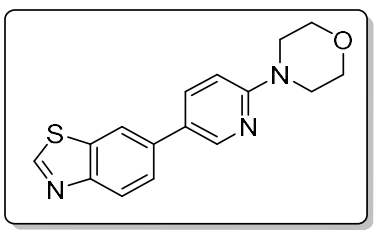

4-(5-(Benzo $[d]$ thiazol-6-yl)pyridin-2-yl)morpholine (3ax) was synthesized according to the general procedure using benzo[d]thiazol-6-yl trifluoromethanesulfonate $(0.50 \mathrm{mmol}, 141.6 \mathrm{mg}$, 1.00 equiv) and 4-(5-bromopyridin-2-yl)morpholine ( $0.75 \mathrm{mmol}, 182.2 \mathrm{mg}, 1.50$ equiv) as starting materials. The reaction was quenched according to the general procedure after stirring at $60{ }^{\circ} \mathrm{C}$ for $24 \mathrm{~h}$, and the crude material was purified by flash chromatography on silica gel (hexanes/ethyl acetate $=10 / 1$ to $5 / 1)$ to afford the desired product as a white solid $(84.8 \mathrm{mg}, 57 \%) . \mathrm{mp} 187-$ $188^{\circ} \mathrm{C} .{ }^{1} \mathrm{H}$ NMR $\left(500 \mathrm{MHz}, \mathrm{CDCl}_{3}\right) \delta 8.96(\mathrm{~s}, 1 \mathrm{H}), 8.51(\mathrm{~s}, 1 \mathrm{H}), 8.16(\mathrm{~d}, J=8.5 \mathrm{~Hz}, 1 \mathrm{H}), 8.05$ (s, $1 \mathrm{H}), 7.78$ (dd, $J=8.8,2.6 \mathrm{~Hz}, 1 \mathrm{H}), 7.66$ (d, $J=8.5 \mathrm{~Hz}, 1 \mathrm{H}), 6.72(\mathrm{~d}, J=8.8 \mathrm{~Hz}, 1 \mathrm{H}), 3.84$ (t, $J=4.8 \mathrm{~Hz}, 4 \mathrm{H}), 3.56(\mathrm{t}, J=4.8 \mathrm{~Hz}, 4 \mathrm{H}) ;{ }^{13} \mathrm{C}\left\{{ }^{1} \mathrm{H}\right\} \mathrm{NMR}\left(126 \mathrm{MHz}, \mathrm{CDCl}_{3}\right) \delta$ 159.0, 153.9, 152.4, 146.6, 136.5, 136.3, 134.9, 126.1, 125.2, 124.0, 119.2, 106.9, 66.9, 45.7 ppm. HRMS (ESI) $\mathrm{m} / \mathrm{z}$ calcd for $[\mathrm{M}+\mathrm{H}]^{+}$298.1009, found 298.1007. IR $\left(\mathrm{cm}^{-1}\right): 3034,2965,2852,1600,1504,1413$, $1242,1115,944,864,808$.

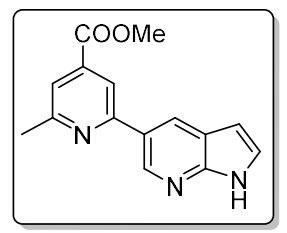

Methyl 2-methyl-6-(1H-pyrrolo[2,3-b]pyridin-5-yl)isonicotinate (3ay) was synthesized according to the general procedure using $1 H$-pyrrolo[2,3-b]pyridin-5-yl trifluoromethanesulfonate ( $0.50 \mathrm{mmol}, 133.1 \mathrm{mg}, 1.00$ equiv) and methyl 2-chloro-6-methylisonicotinate ( $0.75 \mathrm{mmol}, 139.2$ mg, 1.50 equiv) as starting materials. The reaction was quenched according to the general procedure after stirring at $60{ }^{\circ} \mathrm{C}$ for $24 \mathrm{~h}$, and the crude material was purified by flash chromatography on silica gel (hexanes (containing triethyl amine, $5 \% \mathrm{v} / \mathrm{v}$ )/ethyl acetate/methanol $=49 / 49 / 2)$ to afford the desired product as a white solid $(97.0 \mathrm{mg}, 73 \%)$. mp $228-229{ }^{\circ} \mathrm{C} .{ }^{1} \mathrm{H}$ NMR $\left(500 \mathrm{MHz}, \mathrm{DMSO}-d_{6}\right) \delta 11.80(\mathrm{~s}, 1 \mathrm{H}), 8.96(\mathrm{~d}, J=2.1 \mathrm{~Hz}, 1 \mathrm{H}), 8.64(\mathrm{~d}, J=2.1 \mathrm{~Hz}, 1 \mathrm{H})$, $8.14(\mathrm{~s}, 1 \mathrm{H}), 7.62(\mathrm{~d}, J=1.2 \mathrm{~Hz}, 1 \mathrm{H}), 7.53(\mathrm{~d}, J=2.2 \mathrm{~Hz}, 1 \mathrm{H}), 6.56(\mathrm{~d}, J=3.3 \mathrm{~Hz}, 1 \mathrm{H}), 3.92(\mathrm{~s}$, $3 \mathrm{H}), 2.63(\mathrm{~s}, 3 \mathrm{H}) ;{ }^{13} \mathrm{C}\left\{{ }^{1} \mathrm{H}\right\} \mathrm{NMR}\left(126 \mathrm{MHz}, \mathrm{DMSO}-d_{6}\right) \delta 165.4,159.3,156.4,149.0,141.8,138.3$, 127.2, 126.5, 125.9, 119.8, 119.5, 115.6, 100.8, 52.7, $24.3 \mathrm{ppm}$. HRMS (ESI) m/z calcd for [M + 
$\mathrm{H}]^{+}$268.1081, found 268.1076. IR $\left(\mathrm{cm}^{-1}\right): 3128,2993,2844,1727,1605,1567,1434,1334,1260$, $1213,763,740$.

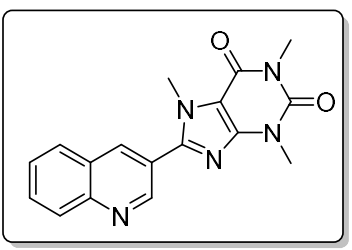

1,3,7-Trimethyl-8-(quinolin-3-yl)-3,7-dihydro-1H-purine-2,6-dione (3az) was synthesized according to the general procedure using quinolin-3-yl trifluoromethanesulfonate $(0.50 \mathrm{mmol}$, $138.6 \mathrm{mg}, 1.00$ equiv) and 8-bromo-1,3,7-trimethyl-3,7-dihydro- $1 H$-purine-2,6-dione ( $0.75 \mathrm{mmol}$, $204.8 \mathrm{mg}, 1.50$ equiv) as starting materials. The reaction was quenched according to the general procedure after stirring at $60{ }^{\circ} \mathrm{C}$ for $24 \mathrm{~h}$, and the crude material was purified by flash chromatography on silica gel (hexanes (containing triethyl amine, $5 \% \mathrm{v} / \mathrm{v}$ )/ethyl acetate $=4 / 1$ to hexanes (containing triethyl amine, $5 \% \mathrm{v} / \mathrm{v}$ )/ethyl acetate/methanol $=40 / 60 / 3$ ) to afford the product as a white solid (91.9 mg, 57\% yield). mp $199-200{ }^{\circ} \mathrm{C} .{ }^{1} \mathrm{H}$ NMR $\left(500 \mathrm{MHz}, \mathrm{CDCl}_{3}\right) \delta 9.24(\mathrm{~d}, J$ $=2.2 \mathrm{~Hz}, 1 \mathrm{H}), 8.48(\mathrm{~d}, J=2.4 \mathrm{~Hz}, 1 \mathrm{H}), 8.16(\mathrm{~d}, J=8.4 \mathrm{~Hz}, 1 \mathrm{H}), 7.92(\mathrm{~d}, J=8.1 \mathrm{~Hz}, 1 \mathrm{H}), 7.82$ (t, $J=7.7 \mathrm{~Hz}, 1 \mathrm{H}), 7.64$ (t, $J=7.5 \mathrm{~Hz}, 1 \mathrm{H}), 4.15(\mathrm{~s}, 3 \mathrm{H}), 3.64(\mathrm{~s}, 3 \mathrm{H}), 3.43(\mathrm{~s}, 3 \mathrm{H}) ;{ }^{13} \mathrm{C}\left\{{ }^{1} \mathrm{H}\right\}$ NMR $\left(126 \mathrm{MHz}, \mathrm{CDCl}_{3}\right) \delta 155.7,151.8,149.6,149.3,148.6,148.4,136.7,131.8,129.7,128.5$, 128.0, 127.1, 121.9, 109.1, 34.2, 30.0, $28.2 \mathrm{ppm}$. HRMS (ESI) $\mathrm{m} / \mathrm{z}$ calcd for $[\mathrm{M}+\mathrm{H}]^{+} 322.1299$, found 322.1295. IR ( $\left.\mathrm{cm}^{-1}\right)$ : 2938, 1695, 1652, 1541, 1424, 1288, 1225, 745.

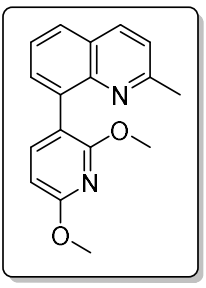

8-(2,6-Dimethoxypyridin-3-yl)-2-methylquinoline (3aaa) was synthesized according to the general procedure using 2-methylquinolin-8-yl trifluoromethanesulfonate $(0.50 \mathrm{mmol}, 145.6 \mathrm{mg}$, 1.00 equiv) and 3-bromo-2,6-dimethoxypyridine ( $0.75 \mathrm{mmol}, 163.5 \mathrm{mg}, 1.50$ equiv) as starting materials. The reaction was quenched according to the general procedure after stirring at $60{ }^{\circ} \mathrm{C}$ for $24 \mathrm{~h}$, and the crude material was purified by flash chromatography on silica gel (hexanes/ethyl acetate $=10 / 1)$ to afford the desired product as a white solid (134.6 mg, 96\% yield). ${ }^{1} \mathrm{H}$ NMR (500 $\left.\mathrm{MHz}, \mathrm{CDCl}_{3}\right) \delta 7.98(\mathrm{~d}, J=8.4 \mathrm{~Hz}, 1 \mathrm{H}), 7.73(\mathrm{~d}, J=7.9 \mathrm{~Hz}, 1 \mathrm{H}), 7.74-7.68(\mathrm{~m}, 3 \mathrm{H}), 7.46(\mathrm{t}$, $J=15.4 \mathrm{~Hz}, 1 \mathrm{H}), 7.20$ (d, $J=8.4 \mathrm{~Hz}, 1 \mathrm{H}), 6.44$ (d, $J=8.0 \mathrm{~Hz}, 1 \mathrm{H}), 3.97$ (s, $3 \mathrm{H}), 3.88(\mathrm{~s}, 3 \mathrm{H})$, $2.61(\mathrm{~s}, 3 \mathrm{H}) ;{ }^{13} \mathrm{C}\left\{{ }^{1} \mathrm{H}\right\} \mathrm{NMR}\left(126 \mathrm{MHz}, \mathrm{CDCl}_{3}\right) \delta 162.4,160.2,158.4,146.0,144.2,136.2,135.3$, 
131.1, 127.2, 126.9, 125.1, 121.8, 113.8, 100.3, 53.6, 53.4, 25.8 ppm. HRMS (ESI) m/z calcd for $[\mathrm{M}+\mathrm{H}]^{+}$281.1284, found 281.1282. IR $\left(\mathrm{cm}^{-1}\right): 3048,2946,1600,1578,1377,1312,1264,1229$, $1018,733,702$.

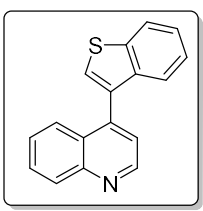

4-(Benzo $[\boldsymbol{b}]$ thiophen-3-yl)quinoline (3aab) was synthesized according to the general procedure using quinolin-4-yl trifluoromethanesulfonate $(0.50 \mathrm{mmol}, 138.6 \mathrm{mg}, 1.00 \mathrm{equiv})$ and 3 bromobenzo[b]thiophene $(0.75 \mathrm{mmol}, 159.8 \mathrm{mg}, 1.50$ equiv $)$ as starting materials. The reaction was quenched according to the general procedure after stirring at $60{ }^{\circ} \mathrm{C}$ for $24 \mathrm{~h}$, and the crude material was purified by flash chromatography on silica gel (hexanes/ethyl acetate $=10 / 1$ to $5 / 1$ ) to afford a yellow oil (30.3 mg) containing 3aab ( $28.4 \mathrm{mg}, 22 \%$ yield) and quinoline ( $1.9 \mathrm{mg}, 3 \%$ yield). ${ }^{1} \mathrm{H}$ NMR (500 MHz, $\left.\mathrm{CDCl}_{3}\right) \delta 8.92(\mathrm{~d}, J=4.4 \mathrm{~Hz}, 1 \mathrm{H}), 8.13(\mathrm{~d}, J=8.4 \mathrm{~Hz}, 1 \mathrm{H}), 7.89$ (d, $J=8.2 \mathrm{~Hz}, 1 \mathrm{H}), 7.69$ (dd, $J=8.6,1.4 \mathrm{~Hz}, 1 \mathrm{H}), 7.65$ (ddd, $J=8.4,6.8,1.4 \mathrm{~Hz}, 1 \mathrm{H}), 7.47$ (s, 1 $\mathrm{H}), 7.39-7.30(\mathrm{~m}, 4 \mathrm{H}), 7.23$ (ddd, $J=8.1,7.0,1.1 \mathrm{~Hz}, 1 \mathrm{H}) ;{ }^{13} \mathrm{C}\left\{{ }^{1} \mathrm{H}\right\} \mathrm{NMR}\left(126 \mathrm{MHz}, \mathrm{CDCl}_{3}\right)$ $\delta 150.3,148.9,142.6,140.3,138.8,133.7,130.1,129.8,127.6,126.9,126.4,126.2,125.0,124.8$, 123.2, 123.0, $122.3 \mathrm{ppm}$. HRMS (ESI) $\mathrm{m} / \mathrm{z}$ calcd for $[\mathrm{M}+\mathrm{H}]^{+} 262.0685$, found 262.0684. IR $\left(\mathrm{cm}^{-}\right.$ $\left.{ }^{1}\right)$ : 3059, 2922, 1701, 1658, 1584, 1427, 1259, 757, 733, 631. For quinoline, ${ }^{27}[\mathrm{M}+\mathrm{H}]^{+}$130.0651, found 130.0651 .

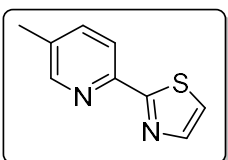

2-(5-Methylpyridin-2-yl)thiazole (3aac) [CAS: 120332-31-2] was synthesized according to the general procedure using 5-methylpyridin-2-yl trifluoromethanesulfonate $(0.50 \mathrm{mmol}, 120.6 \mathrm{mg}$, 1.00 equiv) and 2-bromothiazole ( $0.75 \mathrm{mmol}, 123.0 \mathrm{mg}, 1.50$ equiv) as starting materials. The reaction was quenched according to the general procedure after stirring at $60^{\circ} \mathrm{C}$ for $24 \mathrm{~h}$, and the crude material was purified by flash chromatography on silica gel (hexanes/ethyl acetate $=10 / 1$ ) to afford to a colorless oil $(63.3 \mathrm{mg}$ ) containing 3aac (60.5 mg, 69\% yield) and 4,4'-di-tert-butyl2,2'-bipyridine ( $2.7 \mathrm{mg}, 2 \%$ yield). ${ }^{1} \mathrm{H}$ NMR $\left(500 \mathrm{MHz}, \mathrm{CDCl}_{3}\right) \delta 8.43$ (s, $\left.1 \mathrm{H}\right), 8.08$ (d, $J=8.1$ $\mathrm{Hz}, 1 \mathrm{H}), 7.89(\mathrm{~d}, J=3.2 \mathrm{~Hz}, 1 \mathrm{H}), 7.59(\mathrm{dd}, J=8.1,2.2 \mathrm{~Hz}, 1 \mathrm{H}), 7.39(\mathrm{~d}, J=3.2 \mathrm{~Hz}, 1 \mathrm{H}), 2.36$ (s, $3 \mathrm{H}) ;{ }^{13} \mathrm{C}$ NMR $\left(126 \mathrm{MHz}, \mathrm{CDCl}_{3}\right) \delta 169.6,149.9,149.1,143.9,137.6,134.5,120.9,119.3$, $18.6 \mathrm{ppm}$. The NMR data are consistent with those reported in the literature. ${ }^{28} \mathrm{HRMS}$ (ESI) $\mathrm{m} / \mathrm{z}$ 
calcd for $[\mathrm{M}+\mathrm{H}]^{+}$177.0481, found 177.0481. For 4,4'-di-tert-butyl-2,2'-bipyridine, ${ }^{29} \mathrm{HRMS}$ (ESI) $\mathrm{m} / \mathrm{z}$ calcd for $[\mathrm{M}+\mathrm{H}]^{+}$269.2012, found 269.2008.

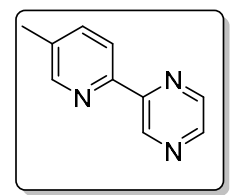

2-(5-Methylpyridin-2-yl)pyrazine (3aad) [CAS: 341503-04-6] was synthesized according to the general procedure using 5-methylpyridin-2-yl trifluoromethanesulfonate $(0.50 \mathrm{mmol}, 120.6 \mathrm{mg}$, 1.00 equiv) and 2-chloropyrazine ( $0.75 \mathrm{mmol}, 85.9 \mathrm{mg}, 1.50$ equiv) as starting materials. The reaction was quenched according to the general procedure after stirring at $60{ }^{\circ} \mathrm{C}$ for $24 \mathrm{~h}$, and the crude material was purified by flash chromatography on silica gel (hexanes (containing triethyl amine, $5 \% \mathrm{v} / \mathrm{v}) /$ ethyl acetate $=100 / 1$ to $20 / 1)$ to afford the product as a white solid $(30.1 \mathrm{mg}, 35 \%$ yield). ${ }^{1} \mathrm{H}$ NMR $\left(400 \mathrm{MHz}, \mathrm{CDCl}_{3}\right) \delta 9.60(\mathrm{~d}, J=1.6 \mathrm{~Hz}, 1 \mathrm{H}), 8.61-8.52(\mathrm{~m}, 3 \mathrm{H}), 8.24(\mathrm{~d}, J=$ $8.1 \mathrm{~Hz}, 1 \mathrm{H}), 7.65(\mathrm{dd}, J=8.2,2.2 \mathrm{~Hz}, 1 \mathrm{H}), 2.41(\mathrm{~s}, 3 \mathrm{H}) ;{ }^{13} \mathrm{C}\left\{{ }^{1} \mathrm{H}\right\} \mathrm{NMR}\left(126 \mathrm{MHz}, \mathrm{CDCl}_{3}\right) \delta$ $151.7,151.3,150.0,144.3,143.7,143.4,137.9,134.6,121.2,18.6 \mathrm{ppm}$. The NMR data are consistent with those reported in the literature. ${ }^{30}$

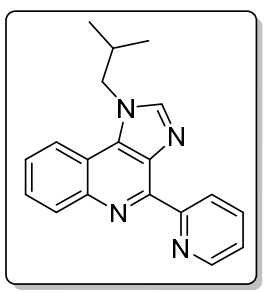

1-Isobutyl-4-(pyridin-2-yl)-1H-imidazo[4,5-c]quinoline (3aae) was synthesized according to the general procedure using pyridin-2-yl trifluoromethanesulfonate $(0.50 \mathrm{mmol}, 113.6 \mathrm{mg}, 1.00$ equiv) and 4-chloro-1-isobutyl- $1 H$-imidazo[4,5-c] quinoline (0.75 mmol, $194.8 \mathrm{mg}, 1.50$ equiv) as starting materials. The reaction was quenched according to the general procedure after stirring at $60{ }^{\circ} \mathrm{C}$ for $24 \mathrm{~h}$, and the crude material was purified by flash chromatography on silica gel (hexanes (containing triethyl amine, $5 \% \mathrm{v} / \mathrm{v}$ )/ethyl acetate/methanol $=50 / 50 / 1$ to $10 / 10 / 1$ ) to afford the desired product as a white solid (87.8 mg, 58\% yield). ${ }^{1} \mathrm{H} \mathrm{NMR}\left(400 \mathrm{MHz}, \mathrm{CDCl}_{3}\right) \delta 8.97$ (ddd, $J$ $=4.9,1.9,0.9 \mathrm{~Hz}, 1 \mathrm{H}), 8.65(\mathrm{~d}, J=8.0 \mathrm{~Hz}, 1 \mathrm{H}), 8.47(\mathrm{dd}, J=8.3,1.4 \mathrm{~Hz}, 1 \mathrm{H}), 8.13(\mathrm{dd}, J=8.3$, $1.5 \mathrm{~Hz}, 1 \mathrm{H}), 8.01(\mathrm{~s}, 1 \mathrm{H}), 7.92(\mathrm{td}, J=7.8,1.8 \mathrm{~Hz}, 1 \mathrm{H}), 7.70(\mathrm{ddd}, J=8.4,6.9,1.5 \mathrm{~Hz}, 1 \mathrm{H})$, 7.63 (ddd, $J=8.3,7.0,1.4 \mathrm{~Hz}, 1 \mathrm{H}), 7.40$ (ddd, $J=7.6,4.8,1.2 \mathrm{~Hz}, 1 \mathrm{H}), 4.38$ (d, $J=7.4 \mathrm{~Hz}, 2$ H), 2.37 (hept, $J=7.5 \mathrm{~Hz}, 1 \mathrm{H}), 1.03(\mathrm{~d}, J=8.4 \mathrm{~Hz}, 6 \mathrm{H}) ;{ }^{13} \mathrm{C}\left\{{ }^{1} \mathrm{H}\right\} \mathrm{NMR}\left(101 \mathrm{MHz}, \mathrm{CDCl}_{3}\right) \delta$ $155.7,150.4,150.1,144.51,144.48,136.9,136.6,134.3,131.8,127.6,127.0,125.6,123.9,120.0$, 
118.1, 55.4, 28.9, 20.0 ppm. HRMS (ESI) m/z calcd for $[\mathrm{M}+\mathrm{H}]^{+} 303.1604$, found 363.1602. IR $\left(\mathrm{cm}^{-1}\right): 3051,2924,1667,1546,1371,1063,1023,810,761,704$.

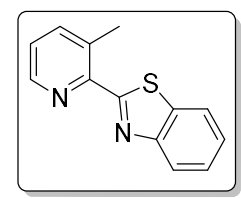

2-(3-Methylpyridin-2-yl)benzo[d]thiazole (3aaf) was synthesized according to the general procedure using 3-methylpyridin-2-yl trifluoromethanesulfonate $(0.50 \mathrm{mmol}, 120.6 \mathrm{mg}, 1.00$ equiv) and 2-bromobenzo[d]thiazole $(0.75 \mathrm{mmol}, 160.6 \mathrm{mg}, 1.50$ equiv) as starting materials. The reaction was quenched according to the general procedure after stirring at $60{ }^{\circ} \mathrm{C}$ for $24 \mathrm{~h}$, and the crude material was purified by flash chromatography on silica gel (hexanes/ethyl acetate $=50 / 1$ ) to afford the product as a yellow solid (88.3 mg, 78\% yield). mp $117-118{ }^{\circ} \mathrm{C} .{ }^{1} \mathrm{H}$ NMR $(500$ $\left.\mathrm{MHz} \mathrm{CDCl}_{3}\right) \delta 8.53(\mathrm{~d}, J=3.1 \mathrm{~Hz}, 1 \mathrm{H}), 8.08(\mathrm{~d}, J=8.1 \mathrm{~Hz}, 1 \mathrm{H}), 7.94(\mathrm{~d}, J=9.2 \mathrm{~Hz}, 1 \mathrm{H}), 7.63$ $(\mathrm{d}, J=6.4 \mathrm{~Hz}, 1 \mathrm{H}), 7.47$ (ddd, $J=8.2,7.1,1.3 \mathrm{~Hz}, 1 \mathrm{H}), 7.39(\mathrm{ddd}, J=8.2,7.1,1.3 \mathrm{~Hz}, 1 \mathrm{H})$, $7.24(\mathrm{dd}, J=7.8,4.6 \mathrm{~Hz}, 1 \mathrm{H}), 2.91(\mathrm{~s}, 3 \mathrm{H}) ;{ }^{13} \mathrm{C}\left\{{ }^{1} \mathrm{H}\right\} \mathrm{NMR}\left(126 \mathrm{MHz}, \mathrm{CDCl}_{3}\right) \delta 171.1,155.0$, 149.2, 146.9, 140.2, 136.0, 133.5, 126.0, 125.7, 124.4, 124.0, 121.8, 21.3 ppm. HRMS (ESI) m/z calcd for $[\mathrm{M}+\mathrm{H}]^{+}$227.0638, found 227.0636. IR $\left(\mathrm{cm}^{-1}\right): 3051,2924,1508,1445,1420,1312,967$, $763,730,699$.

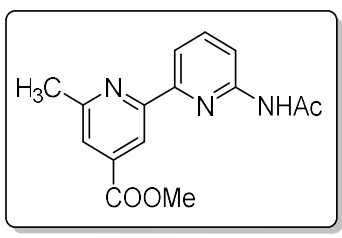

Methyl 6'-acetamido-6-methyl-[2,2'-bipyridine]-4-carboxylate (3aag) was synthesized according to the general procedure using 6-acetamidopyridin-2-yl trifluoromethanesulfonate $(0.50$ mmol, $142.1 \mathrm{mg}, 1.00$ equiv) and methyl 2-chloro-6-methylisonicotinate (0.75 mmol, $139.2 \mathrm{mg}$, 1.50 equiv) as starting materials. The reaction was quenched according to the general procedure after stirring at $60{ }^{\circ} \mathrm{C}$ for $24 \mathrm{~h}$, and the crude material was purified by flash chromatography on silica gel (hexanes (containing triethyl amine, $5 \% \mathrm{v} / \mathrm{v}$ )/ethyl acetate $=3 / 1$ ) to afford the product as a white solid (41.2 mg, 29\% yield). mp $198-200{ }^{\circ} \mathrm{C} .{ }^{1} \mathrm{H}$ NMR $\left(500 \mathrm{MHz}, \mathrm{CDCl}_{3}\right) \delta 8.57$ (s, $\left.1 \mathrm{H}\right)$, $8.27-8.19$ (m, $2 \mathrm{H}), 8.13$ (d, $J=7.6 \mathrm{~Hz}, 1 \mathrm{H}), 7.81$ (t, $J=7.9 \mathrm{~Hz}, 2 \mathrm{H}), 7.70$ (s, $1 \mathrm{H}), 3.96$ (s, 3 $\mathrm{H}), 2.68(\mathrm{~s}, 3 \mathrm{H}), 2.24(\mathrm{~s}, 3 \mathrm{H}) ;{ }^{13} \mathrm{C}\left\{{ }^{1} \mathrm{H}\right\} \mathrm{NMR}\left(126 \mathrm{MHz}, \mathrm{CDCl}_{3}\right) \delta 168.9,166.2,159.4,156.3$, $153.9,151.0,139.5,138.6,122.6,117.5,117.3,114.2,52.8,24.9,24.8$ ppm. HRMS (ESI) m/z calcd for $[\mathrm{M}+\mathrm{H}]^{+}$286.1186, found 286.1182. IR $\left(\mathrm{cm}^{-1}\right): 3233,3055,2949,2924,1726,1571$, $1546,1453,1298,1227,998,834,771,749$. 


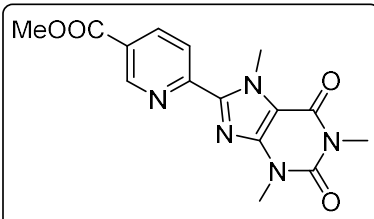

Methyl 6-(1,3,7-trimethyl-2,6-dioxo-2,3,6,7-tetrahydro-1H-purin-8-yl)nicotinate (3aah) was synthesized according to the general procedure using methyl 6(((trifluoromethyl)sulfonyl)oxy)nicotinate $(0.50 \mathrm{mmol}, 142.6 \mathrm{mg}, 1.00$ equiv) and 8-bromo-1,3,7trimethyl-3,7-dihydro- $1 H$-purine-2,6-dione (0.75 mmol, $204.8 \mathrm{mg}, 1.50$ equiv) as starting materials. The reaction was quenched according to the general procedure after stirring at $60{ }^{\circ} \mathrm{C}$ for $24 \mathrm{~h}$, and the crude material was purified by flash chromatography on silica gel (hexanes (containing triethyl amine, $5 \% \mathrm{v} / \mathrm{v}$ )/ethyl acetate $=5 / 1$ ) to afford the product as a yellow solid (110.3 mg, 67\% yield). mp $240-242{ }^{\circ} \mathrm{C} .{ }^{1} \mathrm{H} \mathrm{NMR}\left(400 \mathrm{MHz}, \mathrm{CDCl}_{3}\right) \delta 9.23(\mathrm{~d}, J=1.2 \mathrm{~Hz}, 1 \mathrm{H})$, $8.39(\mathrm{~m}, 1 \mathrm{H}), 8.32(\mathrm{~m}, 1 \mathrm{H}), 4.50(\mathrm{~s}, 3 \mathrm{H}), 3.98(\mathrm{~s}, 3 \mathrm{H}), 3.62(\mathrm{~s}, 3 \mathrm{H}), 3.42(\mathrm{~s}, 3 \mathrm{H}) ;{ }^{13} \mathrm{C}\left\{{ }^{1} \mathrm{H}\right\} \mathrm{NMR}$ $\left(126 \mathrm{MHz}, \mathrm{CDCl}_{3}\right) \delta 165.4,155.8,152.5,151.8,150.1,147.9,147.1,137.9,125.7,124.1,110.1$, 52.8, 35.6, 29.9, $28.2 \mathrm{ppm}$. HRMS (ESI) $\mathrm{m} / \mathrm{z}$ calcd for $[\mathrm{M}+\mathrm{H}]^{+}$330.1197, found 330.1194. IR $\left(\mathrm{cm}^{-1}\right):$ 2954, 2922, 1724, 1699, 1655, 1590, 1426, 1302, 1121, 734.

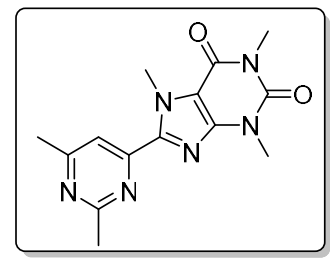

8-(2,6-Dimethylpyrimidin-4-yl)-1,3,7-trimethyl-3,7-dihydro-1 $H$-purine-2,6-dione (3aai) was synthesized according to the general procedure using methyl 2,6-dimethylpyrimidin-4-yl trifluoromethanesulfonate (0.50 mmol, $128.1 \mathrm{mg}, 1.00$ equiv) and 8-bromo-1,3,7-trimethyl-3,7dihydro-1H-purine-2,6-dione $(0.75 \mathrm{mmol}, 204.8 \mathrm{mg}, 1.50 \mathrm{equiv})$ as starting materials. The reaction was quenched according to the general procedure after stirring at $60{ }^{\circ} \mathrm{C}$ for $24 \mathrm{~h}$, and the crude material was purified by flash chromatography on silica gel (hexanes (containing triethyl amine, $5 \% \mathrm{v} / \mathrm{v}$ ) $/$ ethyl acetate $=4 / 1$ to hexanes (containing triethyl amine, 5\% v/v) $/$ ethyl acetate $/$ methanol $=35 / 15 / 4)$ to afford the product as a yellow solid $(76.8 \mathrm{mg}, 51 \%$ yield $) . \mathrm{mp} 240$ - $241{ }^{\circ} \mathrm{C} .{ }^{1} \mathrm{H}$ NMR $\left(500 \mathrm{MHz}, \mathrm{CDCl}_{3}\right) \delta 7.84(\mathrm{~s}, 1 \mathrm{H}), 4.52(\mathrm{~s}, 3 \mathrm{H}), 3.62(\mathrm{~s}, 3 \mathrm{H}), 3.41(\mathrm{~s}, 3 \mathrm{H})$, $2.74(\mathrm{~s}, 3 \mathrm{H}), 2.57(\mathrm{~s}, 3 \mathrm{H}) ;{ }^{13} \mathrm{C}\left\{{ }^{1} \mathrm{H}\right\} \mathrm{NMR}\left(126 \mathrm{MHz}, \mathrm{CDCl}_{3}\right) \delta 168.5,167.6,155.79,155.78$, 151.7, 147.8, 146.2, 116.6, 110.3, 35.6, 29.9, 28.2, 26.2, 24.6 ppm. HRMS (ESI) m/z calcd for [M $+\mathrm{H}]^{+}$301.1408, found 301.1405. IR $\left(\mathrm{cm}^{-1}\right):$ 2955, 2928, 1698, 1654, 1546, 1430, 744. 


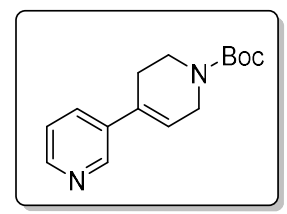

Tert-butyl 3',6'-dihydro-[3,4'-bipyridine]-1'(2'H)-carboxylate (3aaj) [CAS: 690261-74-6] was synthesized according to the general procedure using tert-butyl 4(((trifluoromethyl)sulfonyl)oxy)-3,6-dihydropyridine-1(2H)-carboxylate $(0.50 \mathrm{mmol}, 165.7 \mathrm{mg}$, 1.00 equiv) and 3-bromopyridine $(0.75 \mathrm{mmol}, 118.5 \mathrm{mg}, 1.50$ equiv) as starting materials. The reaction was quenched according to the general procedure after stirring at $60{ }^{\circ} \mathrm{C}$ for $24 \mathrm{~h}$, and the crude material was purified by flash chromatography on silica gel (hexanes (containing triethyl amine, $5 \% \mathrm{v} / \mathrm{v}) /$ ethyl acetate $=5 / 1$ to afford the desired product as a colorless oil $(78.2 \mathrm{mg}, 60 \%)$. ${ }^{1} \mathrm{H}$ NMR $\left(500 \mathrm{MHz}, \mathrm{CDCl}_{3}\right) \delta 8.62(\mathrm{~d}, J=2.4 \mathrm{~Hz}, 1 \mathrm{H}), 8.46(\mathrm{dd}, J=4.8,1.6 \mathrm{~Hz}, 1 \mathrm{H}), 7.61$ (dt, $J=8.0,2.1 \mathrm{~Hz}, 1 \mathrm{H}), 7.22(\mathrm{dd}, J=8.0,4.8 \mathrm{~Hz}, 1 \mathrm{H}), 6.06(\mathrm{~s}, 1 \mathrm{H}), 4.07(\mathrm{~s}, 2 \mathrm{H}), 3.63(\mathrm{t}, J=5.7$ $\mathrm{Hz}, 2 \mathrm{H}), 2.49$ (s, $2 \mathrm{H}), 1.46(\mathrm{~s}, 9 \mathrm{H}) \mathrm{ppm}$. The NMR data are consistent with those reported in the literature. ${ }^{31} \mathrm{HRMS}$ (ESI) $\mathrm{m} / \mathrm{z}$ calcd for $[\mathrm{M}+1] 261.1598$, found 261.1595 .

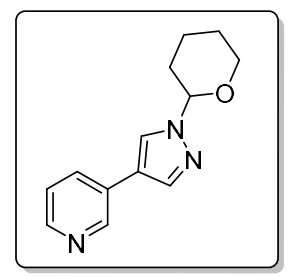

3-(1-(Tetrahydro-2H-pyran-2-yl)-1H-pyrazol-4-yl)pyridine (3aak) [CAS: 938066-19-4] was synthesized according to the general procedure using 3-pyridyl trifluoromethanesulfonate $(0.50$ mmol, $113.5 \mathrm{mg}, 1.00$ equiv) and 4-bromo-1-(tetrahydro-2H-pyran-2-yl)- $1 H$-pyrazole ( $0.75 \mathrm{mmol}$, $173.3 \mathrm{mg}, 1.50$ equiv) as starting materials. The reaction was quenched according to the general procedure after stirring at $60{ }^{\circ} \mathrm{C}$ for $24 \mathrm{~h}$, and the crude material was purified by flash chromatography on silica gel (hexanes (containing triethyl amine, $5 \% \mathrm{v} / \mathrm{v}$ ) $/$ ethyl acetate $=3 / 1$ to $2 / 1$ to afford a colorless oil (78.6 mg) containing 3aak (71.0 mg, 61\% yield) and 3,3'-bipyridine ${ }^{17}$ (7.6 mg, 11\%). ${ }^{1} \mathrm{H} \mathrm{NMR}\left(500 \mathrm{MHz}, \mathrm{CDCl}_{3}\right) \delta 8.69(\mathrm{~d}, J=2.2 \mathrm{~Hz}, 1 \mathrm{H}), 8.39$ (dd, $J=4.9,1.7 \mathrm{~Hz}$, $1 \mathrm{H}), 7.84$ (s, $1 \mathrm{H}), 7.77$ (s, $1 \mathrm{H}), 7.68$ (dt, $J=7.9,2.0 \mathrm{~Hz}, 1 \mathrm{H}), 7.20$ (dd, $J=7.8,4.9 \mathrm{~Hz}, 1 \mathrm{H})$, $5.35(\mathrm{dd}, J=8.6,3.8 \mathrm{~Hz}, 1 \mathrm{H}), 4.04-3.98(\mathrm{~m}, 1 \mathrm{H}), 3.65(\mathrm{td}, J=11.1,3.0 \mathrm{~Hz}, 1 \mathrm{H}), 2.12-1.93$ (m, $3 \mathrm{H}), 1.73-1.50(\mathrm{~m}, 3 \mathrm{H}) ;{ }^{13} \mathrm{C} \mathrm{NMR}\left(126 \mathrm{MHz}, \mathrm{CDCl}_{3}\right) \delta 147.8,147.1,137.2,132.9,128.6$, $124.9,123.8,120.1,88.0,68.0,30.7,25.0,22.4$ ppm. The NMR data are consistent with those reported in the literature. ${ }^{32}$ 


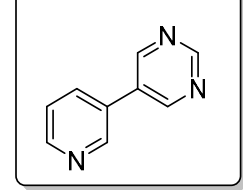

5-(Pyridin-3-yl)pyrimidine (3aal) [CAS: 73082-69-6] was synthesized under Conditions B according to the general procedure using 3-pyridyl trifluoromethanesulfonate $(0.50 \mathrm{mmol}, 113.5$ $\mathrm{mg}, 1.00$ equiv) and 5-bromopyrimidine ( $0.75 \mathrm{mmol}, 119.3 \mathrm{mg}, 1.50$ equiv) as starting materials. The reaction was quenched according to the general procedure after stirring at $60{ }^{\circ} \mathrm{C}$ for $24 \mathrm{~h}$, and the crude material was purified by flash chromatography on silica gel (hexanes (containing triethyl amine, $5 \% \mathrm{v} / \mathrm{v}) /$ ethyl acetate $=4 / 1$ to $1 / 1$ to afford a colorless oil $(55.3 \mathrm{mg})$ containing 3aal (44.0 mg, 56\% yield) and 5,5'-bipyrimidine ${ }^{19}(11.3 \mathrm{mg}, 13 \%) .{ }^{1} \mathrm{H}$ NMR (500 MHz, $\left.\mathrm{CDCl}_{3}\right) \delta 8.93$ (s, 1H), 8.90 (s, $2 \mathrm{H}), 8.79$ (d, $J=1.7 \mathrm{~Hz}, 1 \mathrm{H}), 8.66$ (dd, $J=4.8,1.7 \mathrm{~Hz}, 1 \mathrm{H}), 7.84$ (ddd, $J=7.9,2.3$, $1.6 \mathrm{~Hz}, 1 \mathrm{H}), 7.40(\mathrm{ddd}, J=7.9,4.9,0.9 \mathrm{~Hz}, 1 \mathrm{H}) ;{ }^{13} \mathrm{C} \mathrm{NMR}\left(126 \mathrm{MHz}, \mathrm{CDCl}_{3}\right) \delta 158.4,155.1$, $150.4,148.1,134.4,131.6,130.3,124.1 \mathrm{ppm}$. The NMR data are consistent with those reported in the literature. 33 


\subsection{Bench setup of the synthesis of compound 3ao on 5.0 mmol scale}

Scheme S9. Synthesis of compound 3ao on a $5.0 \mathrm{mmol}$ scale

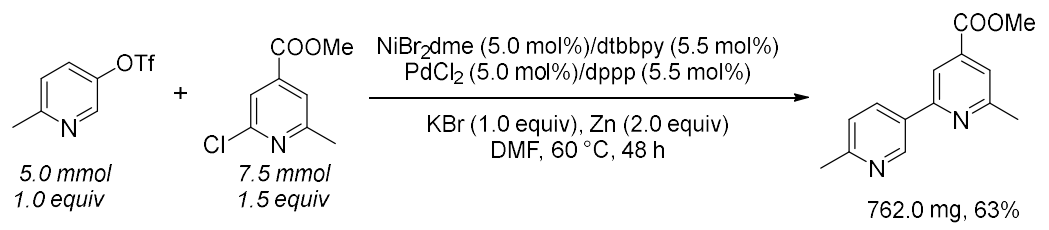

\section{Preparation of nickel catalyst stock solution (0.050 $\mathrm{M}$ with respect to nickel)}

A 2-dram vial equipped with a PTFE-coated stir-bar was charged with $\mathrm{NiBr}_{2}(\mathrm{dme})(77.2$ $\mathrm{mg}, 0.250 \mathrm{mmol}$ ) and 4,4'-di-tert-butyl-2,2'-bipyridine (N1) $(73.7 \mathrm{mg}, 0.275 \mathrm{mmol})$. The dram vial was capped with a screw cap fitted with a PTFE-faced silicone septum. The vial was then connected to a $\mathrm{N}_{2}$ Schlenk line via a needle through the septum. The vial was then connected to air via a second venting needle and purged under positive $\mathrm{N}_{2}$ flow for $5 \mathrm{~min}$. The venting needle was then removed. Anhydrous DMF $(5.0 \mathrm{~mL})$ was then added by a syringe under positive $\mathrm{N}_{2}$ pressure. The mixture was stirred $(1000 \mathrm{rpm})$ for $12 \mathrm{~h}$ under positive $\mathrm{N}_{2}$ pressure before being used.

\section{Preparation of palladium catalyst stock solution $(0.025 \mathrm{M}$ with respect to palladium)}

The palladium catalyst stock solution was prepared following the procedure of preparing the nickel stock solution using $\mathrm{PdCl}_{2}$ (44.2 mg, $0.250 \mathrm{mmol}$ ), dppp (P1) (113.3 mg, $0.275 \mathrm{mmol}$ ), and anhydrous DMF $(10 \mathrm{~mL})$ as the solvent in a $20 \mathrm{~mL}$ scintillation vial.
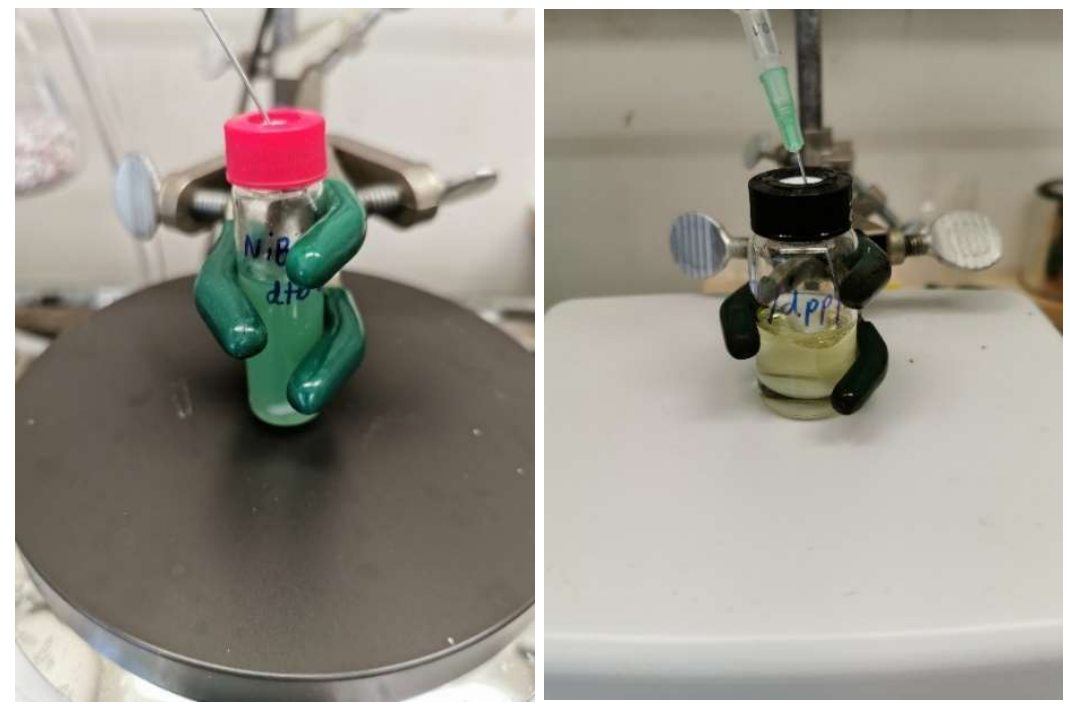

Figure S4. (Left) Pre-stirred nickel catalyst stock solution ready for use; (Right) Pre-stirred palladium catalyst stock solution ready for use. 


\section{Catalytic reaction setup}

A two-neck $50 \mathrm{~mL}$ round bottom flask equipped with a PTFE-coated stir-bar was charged with 6-methylpyridin-3-yl trifluoromethanesulfonate (1206 mg, $5.00 \mathrm{mmol}, 1.00$ equiv), methyl 2-chloro-6-methylisonicotinate (1392 mg, $7.50 \mathrm{mmol}, 1.50$ equiv), $\mathrm{KBr}$ (595 mg, $5.00 \mathrm{mmol}, 1.00$ equiv), and $\mathrm{Zn}$ (654 mg, $10.0 \mathrm{mmol}, 2.00$ equiv). The center neck of the flask was sealed with a rubber septum and the side neck was connected to a $\mathrm{N}_{2}$ Schlenk line. The flask was then evacuated and refilled with $\mathrm{N}_{2} 3$ times. The nickel $(5 \mathrm{~mL})$ and palladium $(10 \mathrm{~mL})$ catalyst stock solutions were then added via a syringe through the septum in the center neck under positive $\mathrm{N}_{2}$ pressure. The stopcock in the side neck was turned off and the side neck was disconnected from the $\mathrm{N}_{2}$ Schlenk line. The flask was again connected to the $\mathrm{N}_{2}$ Schlenk line with a needle through the septum in the center neck to keep a positive $\mathrm{N}_{2}$ pressure in the flask. The mixture was stirred (1000 $\mathrm{rpm}$ ) for $30 \mathrm{~min}$ and the flask was moved to an oil bath which was pre-heated to $60{ }^{\circ} \mathrm{C}$. The mixture was stirred $(1000 \mathrm{rpm})$ for another $48 \mathrm{~h}$ with heating before the reaction workup.

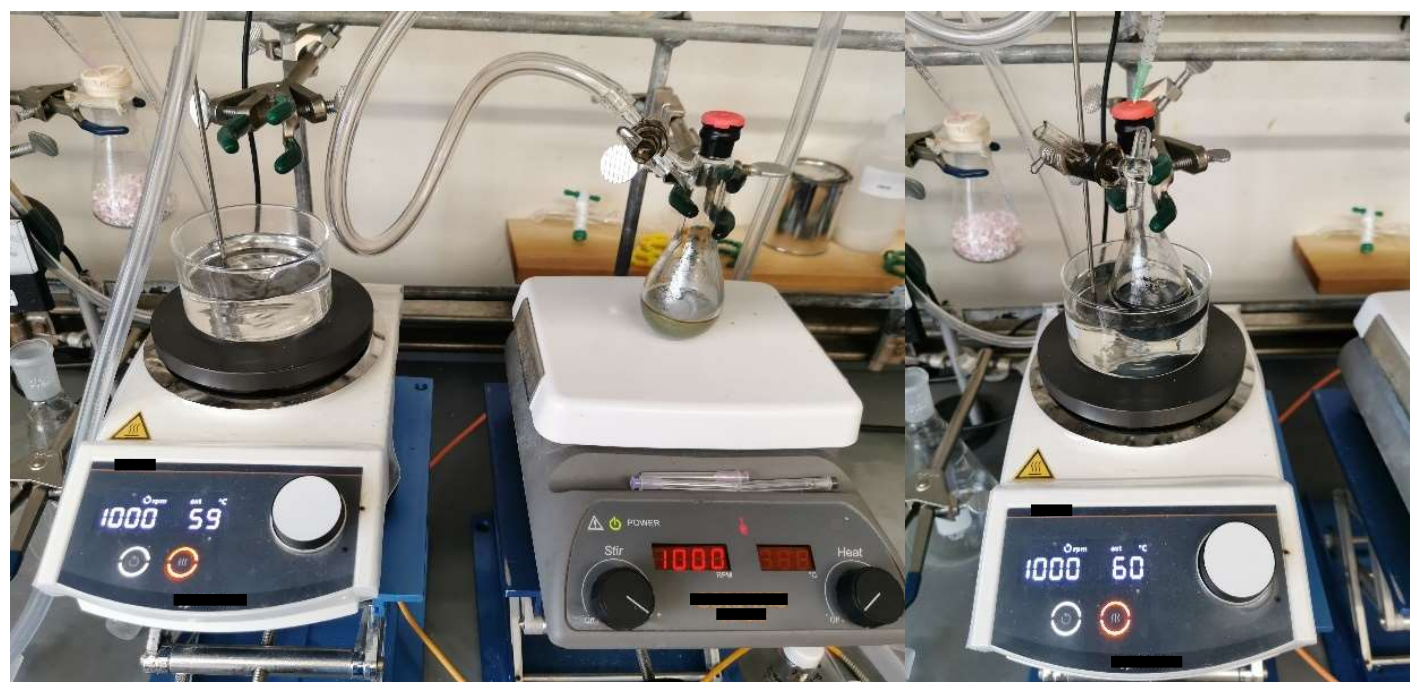

Figure S5. (Left) An oil bath pre-heated to $60{ }^{\circ} \mathrm{C}$; (Middle) The reaction mixture being stirred at rt; (Right) The reaction mixture being stirred at $60{ }^{\circ} \mathrm{C}$.

\section{Reaction workup}

The reaction was judged complete by TLC analysis (hexanes (containing triethyl amine, $5 \% \mathrm{v} / \mathrm{v}) /$ ethyl acetate $=5 / 1, \mathrm{R}_{\mathrm{f}}$ of $\left.\mathbf{3 a o}=0.3\right)$ after $48 \mathrm{~h}$. The reaction mixture was cooled to $\mathrm{rt}$ before being transferred to a $100 \mathrm{~mL}$ round bottom flask equipped with a PTFE-coated stir-bar. Three portions of dichloromethane $(5.0 \mathrm{~mL} \times 3)$ were used to further rinse the reaction flask. $\mathrm{Na}_{4}$ EDTA aqueous solution $(25 \% \mathrm{wt}, 40 \mathrm{~mL})$ was then added. The round bottom flask was capped 
with a rubber septum, and the mixture was stirred $(1000 \mathrm{rpm})$ for $3 \mathrm{~h}$ at $\mathrm{rt}$. After that, the quenched mixture was transferred to a $250 \mathrm{~mL}$ separatory funnel containing dichloromethane $(50 \mathrm{~mL})$ and water $(20 \mathrm{~mL})$. The dichloromethane layer was set aside, and the aqueous layer was further washed with dichloromethane $(50 \mathrm{~mL} \times 2)$. The dichloromethane layers were combined and dried over anhydrous $\mathrm{Na}_{2} \mathrm{SO}_{4}$. The resulting mixture was filtered through a disposable filter funnel $(20 \mathrm{~mL}$, 10-micron polyethylene frit) to remove the solid, and the filtrate was collected.

\section{Purification}

Silica gel ( $\sim 4 \mathrm{~g})$ was added to the resulting filtrate from the reaction workup, and the volatile solvents were removed by rotary evaporation. The dry-loaded material was purified by flash chromatography on silica gel (hexanes/ethyl acetate $=5 / 1$ then hexanes (containing triethyl amine, $5 \% \mathrm{v} / \mathrm{v}) /$ ethyl acetate $=9 / 1)$ to provide the desired product as a white solid $(762.0 \mathrm{mg}, 63 \%$ yield). ${ }^{1} \mathrm{H}$ NMR (400 MHz, $\left.\mathrm{CDCl}_{3}\right) \delta 9.07$ (d, $\left.J=2.1 \mathrm{~Hz}, 1 \mathrm{H}\right), 8.19(\mathrm{dd}, J=8.1,2.4 \mathrm{~Hz}, 1 \mathrm{H})$, $8.01(\mathrm{~s}, 1 \mathrm{H}), 7.62(\mathrm{~s}, 1 \mathrm{H}), 7.21(\mathrm{~d}, J=8.1 \mathrm{~Hz}, 1 \mathrm{H}), 3.93(\mathrm{~s}, 3 \mathrm{H}), 2.64(\mathrm{~s}, 3 \mathrm{H}), 2.57(\mathrm{~s}, 3 \mathrm{H}) ;{ }^{13} \mathrm{C}$ NMR $\left(101 \mathrm{MHz} \mathrm{CDCl}_{3}\right) \delta 165.9,160.0,159.3,155.5,147.8,138.6,134.8,131.6,123.2,121.3$, 116.6, 52.8, 24.8, $24.4 \mathrm{ppm}$. The NMR data are consistent with the same compound 3ao synthesized on a $0.5 \mathrm{mmol}$ scale.

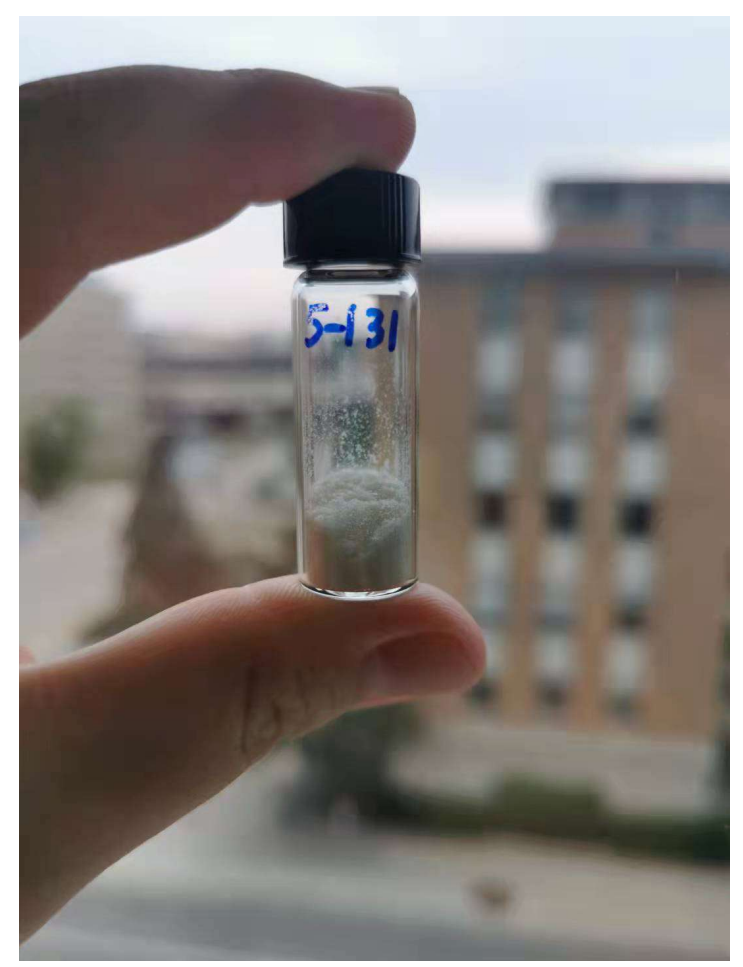

Figure S6. Compound 3ao isolated from the reaction as a crystalline white solid $(762.0 \mathrm{mg}, 63 \%$ yield). 
Scheme S10. Inhibitors, low yielding substrates, substrates incompatible with reaction conditions, and products that decomposed during isolation.
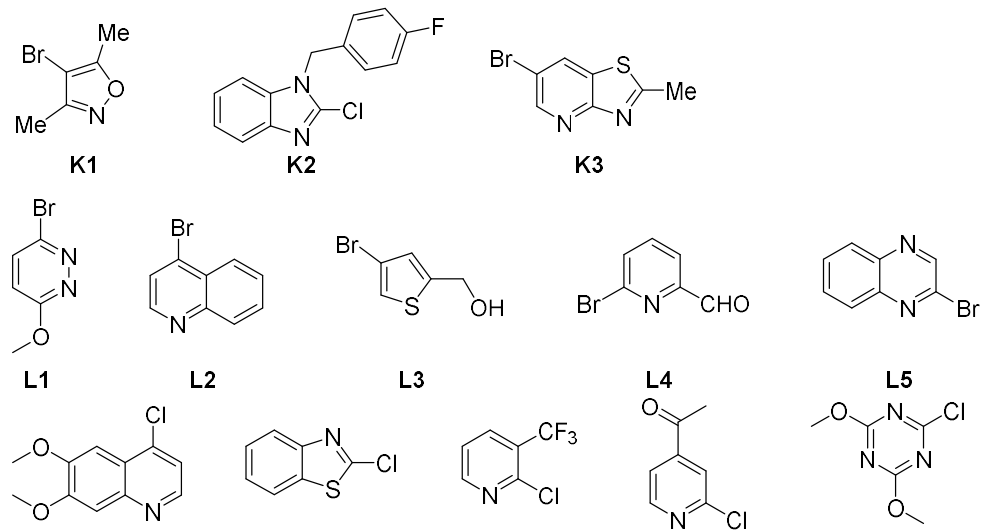

L2

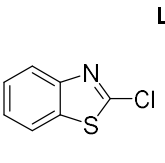

L3

L6

L7
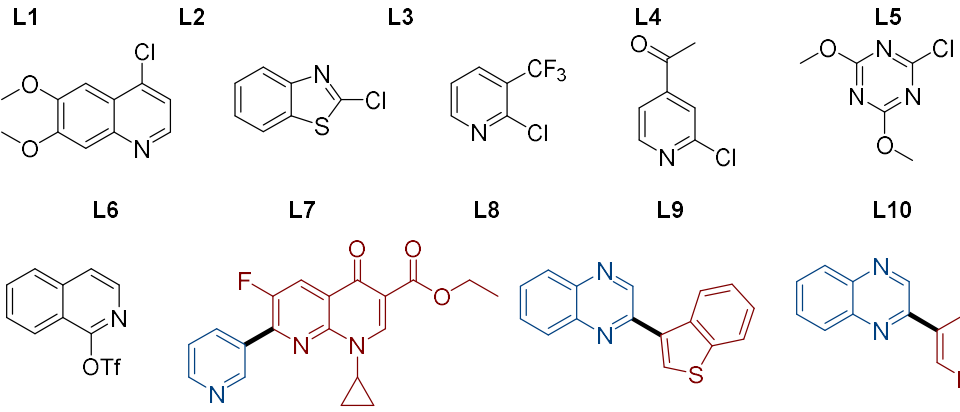

L8

L9

L10

T1

U1

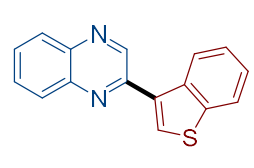

U2

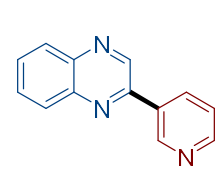

U3

Heteroaryl halides K1 - $\mathbf{3}$ shut down the reactivity completely and no conversion of either starting material was observed in reactions with these substrates. Reactions involving heteroaryl halides L1 - 10 gave low yields of cross-coupled products due to the dimerization or hydrodehalogenation side reactions of heteroaryl halides. Heteroaryl triflate $\mathbf{T 1}$ decomposed to the corresponding heteroaryl phenol under Conditions A. Biheteroaryl products $\mathbf{U} \mathbf{1}$ - $\mathbf{3}$ (aryls from heteroaryl triflates are blue and aryls from heteroaryl halides are red; for $\mathbf{U 1}$, halide $=\mathrm{Cl}$, for $\mathbf{U} \mathbf{2}$ and $\mathbf{U} \mathbf{3}$, halide $=\mathrm{Br}$ ) were all detected as one of the major products in each reaction by SFC-MS (with full conversion of starting materials), but no cross-coupled products were isolated after the purification by flash chromatography on silica gel. 


\subsection{Mechanistic studies on the difference between Conditions A and B}

\section{General procedures}

\section{Preparation of catalyst stock solutions}

See 4.1 General procedure for reaction setup in scope investigations of this document for preparations of nickel catalyst stock solution for Conditions A ( $0.050 \mathrm{M}$ with respect to nickel), nickel catalyst stock solution for Conditions B (0.050 M with respect to nickel), and palladium catalyst stock solution ( $0.025 \mathrm{M}$ with respect to palladium).

\section{Preparation of iodine quenching solution ${ }^{34}$}

In a $\mathrm{N}_{2}$ filled glovebox, $\mathrm{LiCl}(127 \mathrm{mg}, 3.00 \mathrm{mmol})$ and THF $(6.0 \mathrm{~mL})$ were added to an oven-dried scintillation vial equipped with a PTFE-coated stir-bar. The solution was stirred at rt overnight until all solids were dissolved, resulting in a $0.5 \mathrm{M}$ solution of LiCl. In a separate scintillation vial was weighed $\mathrm{I}_{2}(254 \mathrm{mg}, 1.00 \mathrm{mmol})$, and $4 \mathrm{~mL}$ of the $0.5 \mathrm{M}$ solution of $\mathrm{LiCl}$ was added. The resulting brown solution was stirred at $\mathrm{rt}$ for 1 hour before being used.

\section{Reaction setup}

In a $\mathrm{N}_{2}$ filled glovebox, an oven-dried 1-dram vial equipped with a PTFE-coated stir-bar was charged sequentially with methyl 5-bromonicotinate (1-Br) (0.25 mmol, $54.0 \mathrm{mg}, 1.00$ equiv), 1,3,5-trimethoxybenzene (50.4 mg, $0.30 \mathrm{mmol}), \mathrm{KBr}(29.8 \mathrm{mg}, 0.25 \mathrm{mmol}, 1.00$ equiv), and $\mathrm{Zn}$ (32.7 $\mathrm{mg}, 0.50 \mathrm{mmol}, 2.00$ equiv). Aliquots from the $0.050 \mathrm{M}$ nickel catalyst stock solution for Conditions A or B (250 $\mu \mathrm{L}$ for $5.0 \mathrm{~mol} \%$ catalyst loading) and $0.025 \mathrm{M}$ palladium stock solutions (500 $\mu \mathrm{L}$ for $5.0 \mathrm{~mol} \%$ catalyst loading) were then added. The vial was capped with a screw cap fitted with a PTFE-faced silicone septum, and the mixture was stirred $(1000 \mathrm{rpm})$ in a reaction block set to $60{ }^{\circ} \mathrm{C}$ in the glovebox.

\section{Procedures for iodine quenching and SFC-MS analysis}

In the glovebox, a $20 \mu \mathrm{L}$ aliquot of the reaction mixture was transferred into another ovendried 1-dram vial equipped with a PTFE-coated stir-bar. The iodine-LiCl solution $(80 \mu \mathrm{L})$ was added immediately, and the vial was capped with a screw cap fitted with a PTFE-faced silicone septum. The mixture was stirred at rt in the glovebox for $15 \mathrm{~min}$ before the vial was removed from the glovebox. Next, 25\% wt Na4EDTA aqueous solution $(100 \mu \mathrm{L})$ was added and the mixture was stirred at $\mathrm{rt}$ for another $15 \mathrm{~min}$. The quenched mixture was then diluted with dichloromethane (500 $\mu \mathrm{L})$. A $100 \mu \mathrm{L}$ aliquot of the mixture was further diluted with $\mathrm{MeOH}(1 \mathrm{~mL})$, and the resulting 
mixture was transferred into a $1 \mathrm{~mL}$ syringe and then filtered into an SFC-MS vial through a syringe filter (PVDF membrane, $0.22 \mu \mathrm{m}$ pore size, $13 \mathrm{~mm}$ diameter). The sample was analyzed by our standard method (see 1. General Information of this document for details) on SFC-MS. For each sample, the integrated peak area of the target compound (1-Br, 1-I, or 1-dimer) and the internal standard (TMB, 1,3,5-trimethoxybenzene) in SFC-MS chromatograms (UV $254 \mathrm{~nm}$ ) were recorded and the product/internal standard ratios were calculated.

Table S7. The ratios of target compound (1-Br, 1-I, or 1-dimer) to internal standard (TMB, 1,3,5trimethoxybenzene) under Conditions A and B

\begin{tabular}{|c|c|c|c|c|c|c|}
\hline \multirow[b]{2}{*}{ time $(\min )$} & \multicolumn{3}{|c|}{ Conditions A } & \multicolumn{3}{|c|}{ Conditions B } \\
\hline & $\mathrm{c}(1-\mathrm{Br}) / \mathrm{c}(\mathrm{TMB})$ & $c(1-\mathrm{I}) / \mathrm{c}(\mathrm{TMB})$ & $c(1$-dimer $) / c(T M B)$ & $\mathrm{c}(1-\mathrm{Br}) / \mathrm{c}(\mathrm{TMB})$ & $c(1-\mathrm{I}) / \mathrm{c}(\mathrm{TMB})$ & $\mathrm{c}(1$-dimer $) / \mathrm{c}(\mathrm{TMB})$ \\
\hline 0 & 4.4 & 0 & 0 & 4.4 & 0 & 0 \\
\hline 10 & 4.4 & 0.44 & 0 & 3.7 & 1.7 & 0.13 \\
\hline 20 & 4.1 & 0.29 & 0.35 & 1.5 & 2.3 & 0.29 \\
\hline 30 & 3.5 & 0.28 & 0.53 & 1.4 & 4.1 & 0.64 \\
\hline 60 & 2.9 & 0.31 & 0.92 & 0.53 & 5.1 & 1.4 \\
\hline 90 & 2.4 & 0.41 & 1.5 & 0.07 & 5.8 & 1.9 \\
\hline 120 & 2.6 & 0.57 & 2.4 & 0 & 4.9 & 2.5 \\
\hline 180 & 2.1 & 1.1 & 4.3 & 0 & 5.4 & 2.2 \\
\hline 240 & 1.7 & 1.6 & 5.6 & 0 & 5.1 & 2.4 \\
\hline 300 & 1.2 & 1.7 & 6.3 & 0 & 5.2 & 2.4 \\
\hline 360 & 0.84 & 2.3 & 7.6 & 0 & 4.7 & 2.3 \\
\hline 480 & 0.27 & $2.2(22 \%)^{a}$ & $9.1(24 \%)^{a}$ & 0 & $5.2(53 \%)^{a}$ & $2.4(6 \%)^{a}$ \\
\hline
\end{tabular}

(a) ${ }^{1} \mathrm{H}$ NMR with 1,3,5-trimethoxybenzene as the internal standard. 

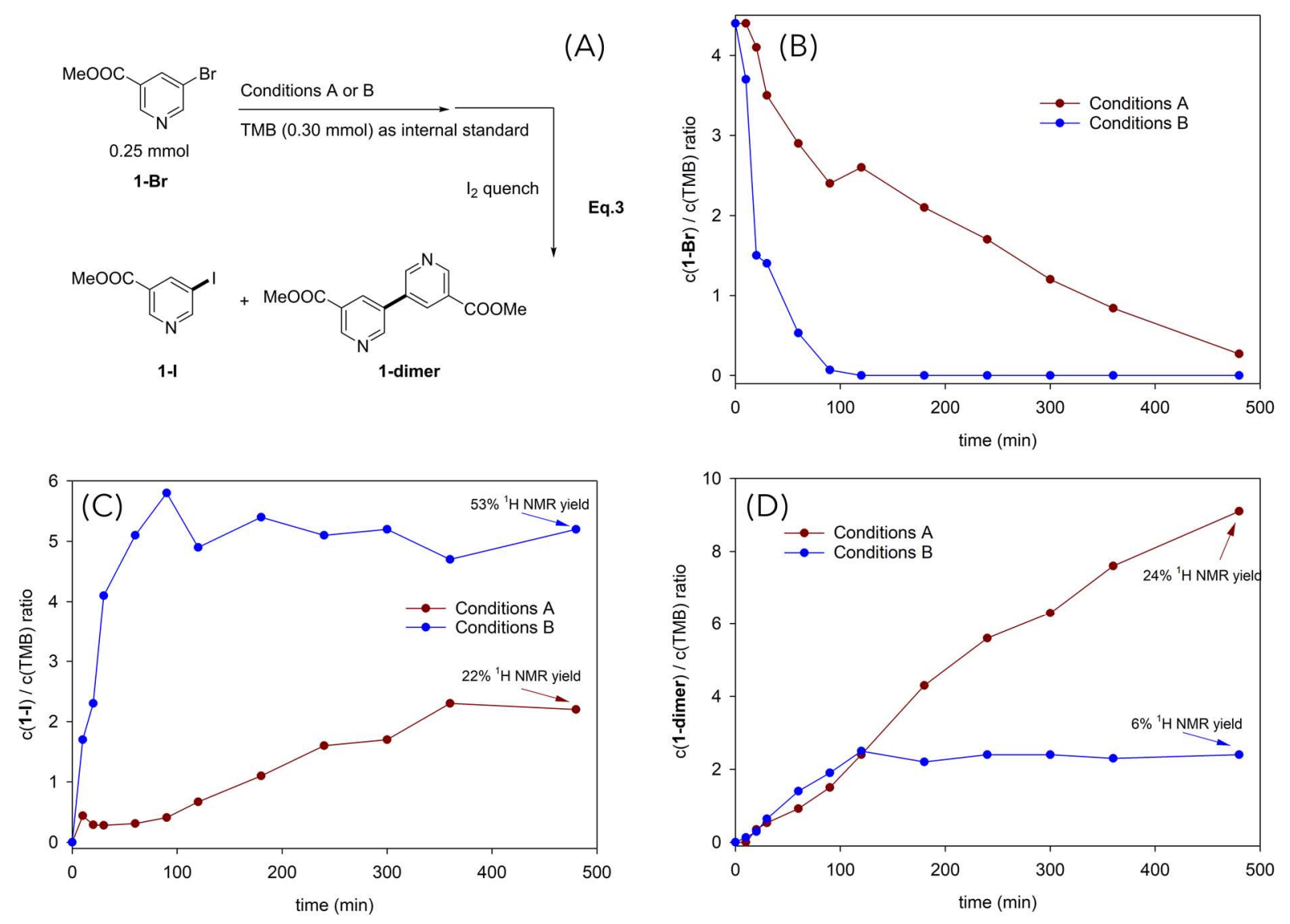

Figure S7. (A) Dimerization of 1-Br under Conditions A or B. (B) The ratio of the concentrations of 1-Br to internal standard (TMB) vs time. (C) The ratio of the concentrations of 1-I to internal standard (TMB) vs time. (D) The ratio of the concentrations of 1-dimer to internal standard (TMB) vs time. 


\section{High-Throughout Experimentation}
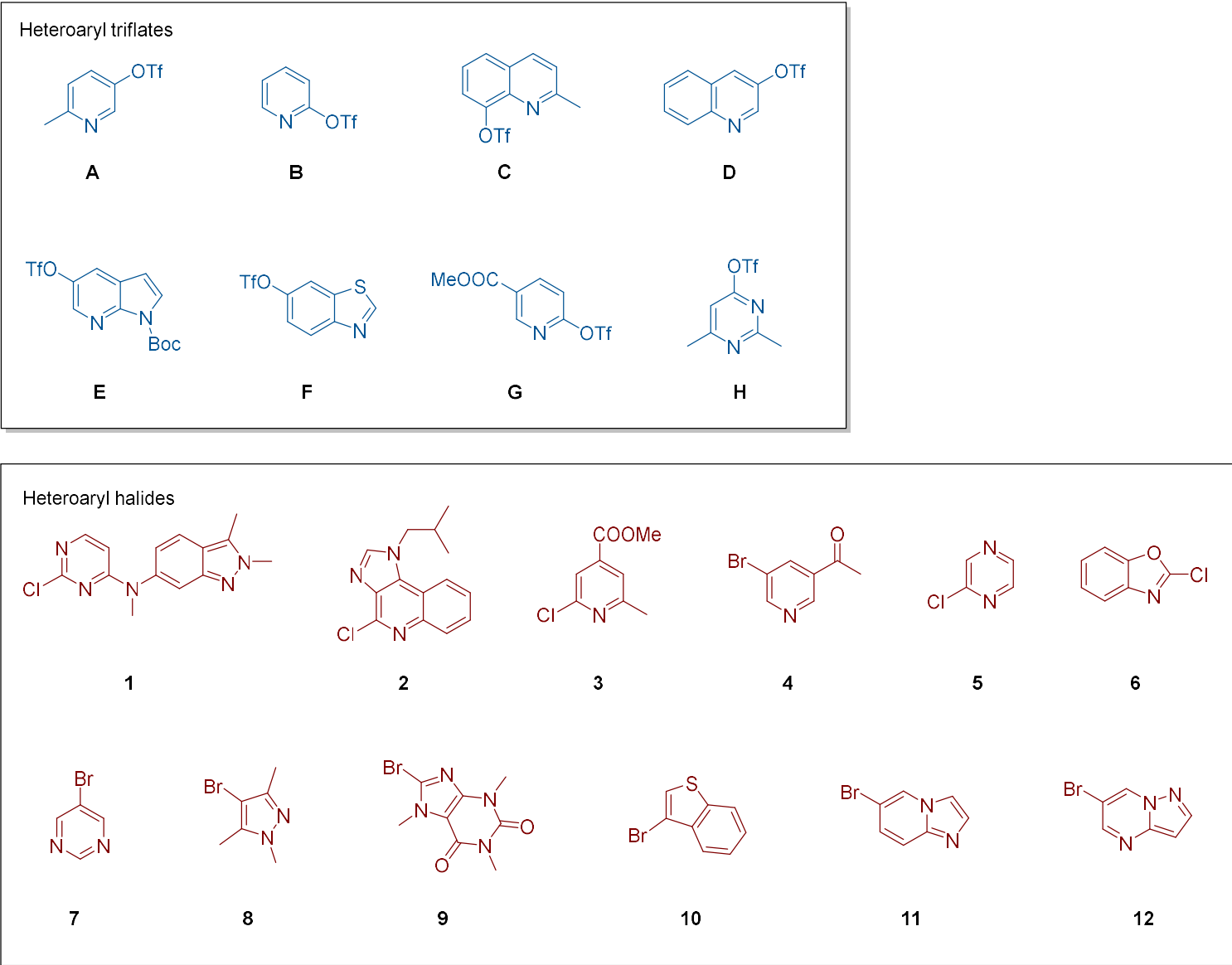

Figure S8. Structures of heteroaryl triflates and heteroaryl halides used in HTE. 


\subsection{Application of HTE in scope investigations}

Scheme S11. $10 \mu \mathrm{mol}$ scale reactions for HTE in scope investigations

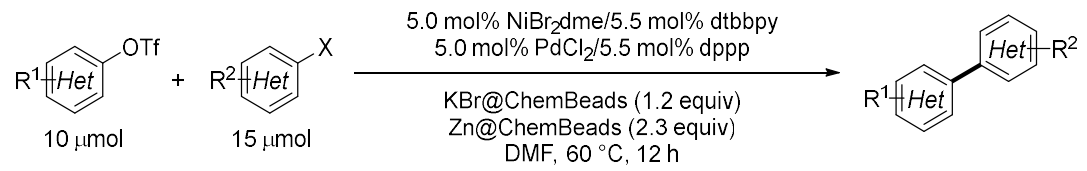

The following procedures were conducted in a $\mathrm{N}_{2}$ filled glovebox for convenience. For the preparation of nickel catalyst stock solution (0.025 $\mathrm{M}$ in DMF with respect to nickel) and palladium catalyst stock solution (0.025 $\mathrm{M}$ in DMF with respect to palladium), see $\underline{2 \text { Reaction }}$ Optimizations of this document. ChemBeads were dispensed to the 96-well plate vials with a 15 mg calibrated scoop and a non-static funnel. All the solutions were dispensed with a $5-120 \mu \mathrm{L}$ electronic 8-channel pipette.

\section{Preparation of heteroaryl triflate (A to $\mathrm{H}$ ) and halide (1 to 8 and 10 to 12) stock solutions}

An oven-dried 1-dram vial equipped with a PTFE-coated stir-bar was charged with the listed aryl triflate $(0.20 \mathrm{mmol})$ or aryl halide $(0.30 \mathrm{mmol})$. Anhydrous DMF was added to bring the total volume to $0.4 \mathrm{~mL}$ (for aryl triflates $\mathbf{A}-\mathbf{H}$ and aryl halides $\mathbf{2}-\mathbf{8}, \mathbf{1 0}-\mathbf{1 1}$ ) or $0.8 \mathrm{~mL}$ (for aryl halides 1 and 12). The vial was capped with a screw cap fitted with a PTFE-faced silicone septum. The mixture was stirred $(1000 \mathrm{rpm})$ at $\mathrm{rt}$ for $30 \mathrm{~min}$ before use. Attention:1) the concentration of heteroaryl triflate solutions is $0.50 \mathrm{M}$; 2) while most aryl halides are readily soluble in DMF (c=0.75 M), the concentration of the stock solutions of aryl halides 1 and 12 is $0.38 \mathrm{M}$ due to their poor solubilities in DMF at rt; 3) the solubility of aryl halide 9 in DMF is very low and glass ChemBeads coated with aryl halide $9(60 \mathrm{mg}, 7.5 \% \mathrm{w} / \mathrm{w}, 0.016 \mathrm{mmol}, 1.6$ equiv with respect to $0.010 \mathrm{mmol}$ heteroaryl triflate) was used, instead of the stock solution in DMF.

\section{Catalytic reaction setup}

One $8 \times 30 \mathrm{~mm}$ glass vial was placed into each of the 96 wells. Then, each of the $96-w e l l$ plate reaction vials was charged sequentially with Zn-coated ChemBeads $(5.0 \% \mathrm{w} / \mathrm{w}, 30 \mathrm{mg}, 0.023$ mmol, 2.3 equiv), KBr-coated ChemBeads (10.0\% w/w, $15 \mathrm{mg}, 0.012 \mathrm{mmol}, 1.2$ equiv), the listed aryl triflate stock solution $(0.50 \mathrm{M}, 20 \mu \mathrm{L}, 0.010 \mathrm{mmol}, 1.0$ equiv), the listed aryl halide stock solution ( $0.75 \mathrm{M}$ or $0.38 \mathrm{M}, 20$ or $40 \mu \mathrm{L}, 0.015 \mathrm{mmol}, 1.5$ equiv), nickel catalyst stock solution $\left(0.025 \mathrm{M}, 20 \mu \mathrm{L}, 5 \times 10^{-4} \mathrm{mmol}, 5 \mathrm{~mol} \%\right)$, and palladium catalyst stock solution $(0.025 \mathrm{M}, 20 \mu \mathrm{L}$, $5 \times 10^{-4} \mathrm{mmol}, 5 \mathrm{~mol} \%$ ). 1,3,5-trimethoxybenzene (4.0 M in DMF, $12.5 \mu \mathrm{L}, 0.050 \mathrm{mmol}, 5.0$ equiv) 
was then added as an internal standard. The reactor plate was capped and placed on a heater/shaker at $60{ }^{\circ} \mathrm{C}$ for $12 \mathrm{~h}$ with the shaking rate set at 8 .
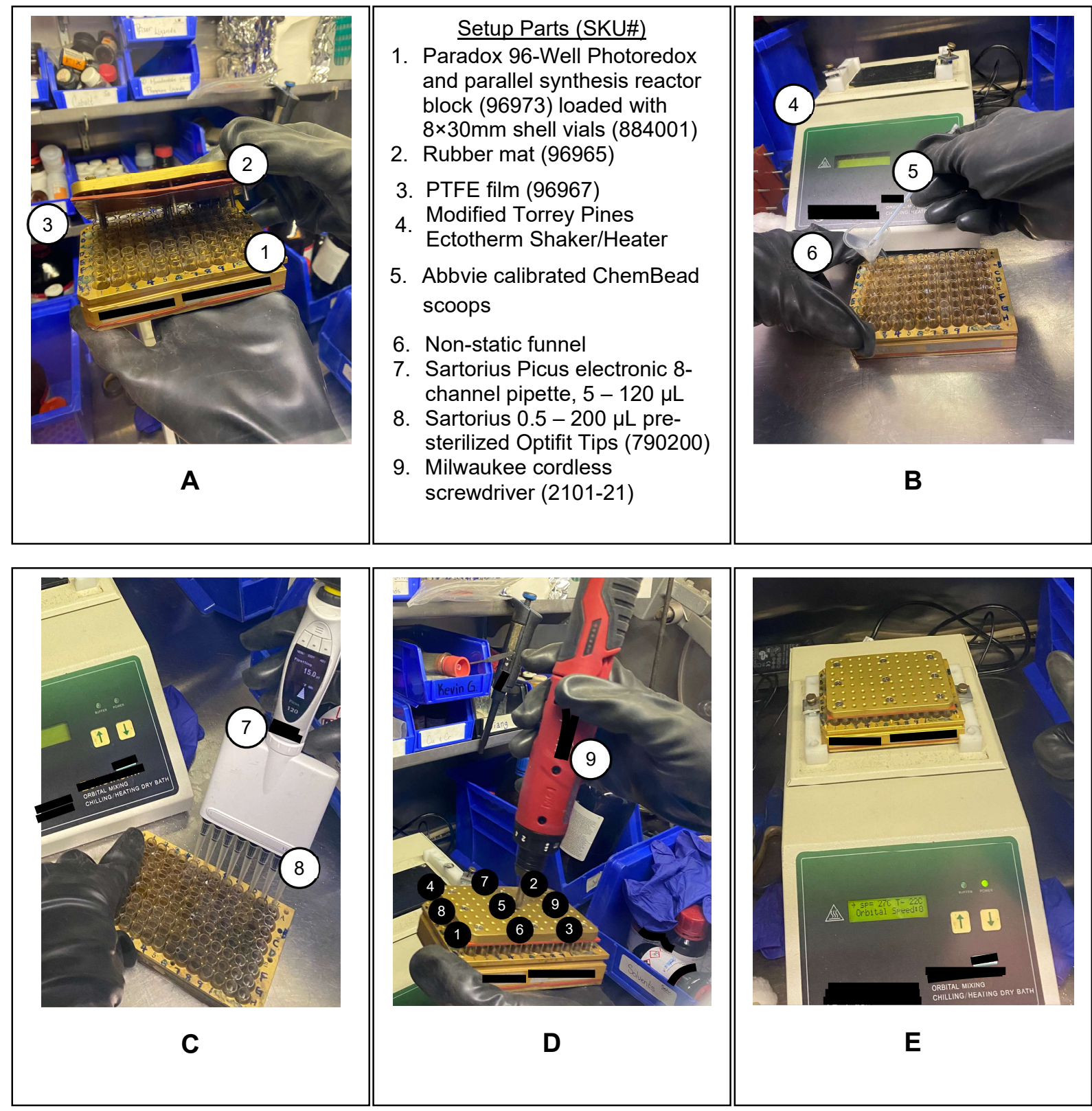

Figure S9. (A) An assembled lid and a 96-well reaction plate loaded with reaction vials. (B) The addition of solid reagent coated ChemBeads to the reaction vials with a calibrated scoop and a non-static funnel. (C) The addition of stock solutions to the reaction vials with an electronic 8channel pipette. (D) The assembled lid was screwed to the reaction plate with a cordless electronic screwdriver in the shown pattern with the torque set at 6. (E) Heating/shaking the block assembly in a $\mathrm{N}_{2}$ filled glovebox. 


\section{Reaction workup}

After $12 \mathrm{~h}$, the reaction plate was removed from the heater/shaker and cooled to rt. The cap plate was removed and ammonia solution $(2.0 \mathrm{M}$ in methanol, $100 \mu \mathrm{L}, 0.20 \mathrm{mmol}, 20$ equiv) was added to each reaction vial to quench the reaction. The reaction vials were re-sealed, and the plate was shaken at $\mathrm{rt}$ with the shaking rate set at 8 for $1 \mathrm{~h}$. The reaction plate was then removed from the glovebox. The cap plate was removed again, and each well was diluted with dichloromethane $(200 \mu \mathrm{L})$. For each reaction, a $30 \mu \mathrm{L}$ aliquot of the diluted mixtures was taken and further diluted with methanol $(200 \mu \mathrm{L})$, and then filtered through a 96-well filter plate on a vacuum manifold filtration system. The filtrates were collected in a $340 \mu \mathrm{L}$ V-bottom 96-well plate and sealed with a clear adhesive PTFE film. The resulting sample plate was placed on the SFC-MS for analysis by our standard method (see 1. General Information of this document).

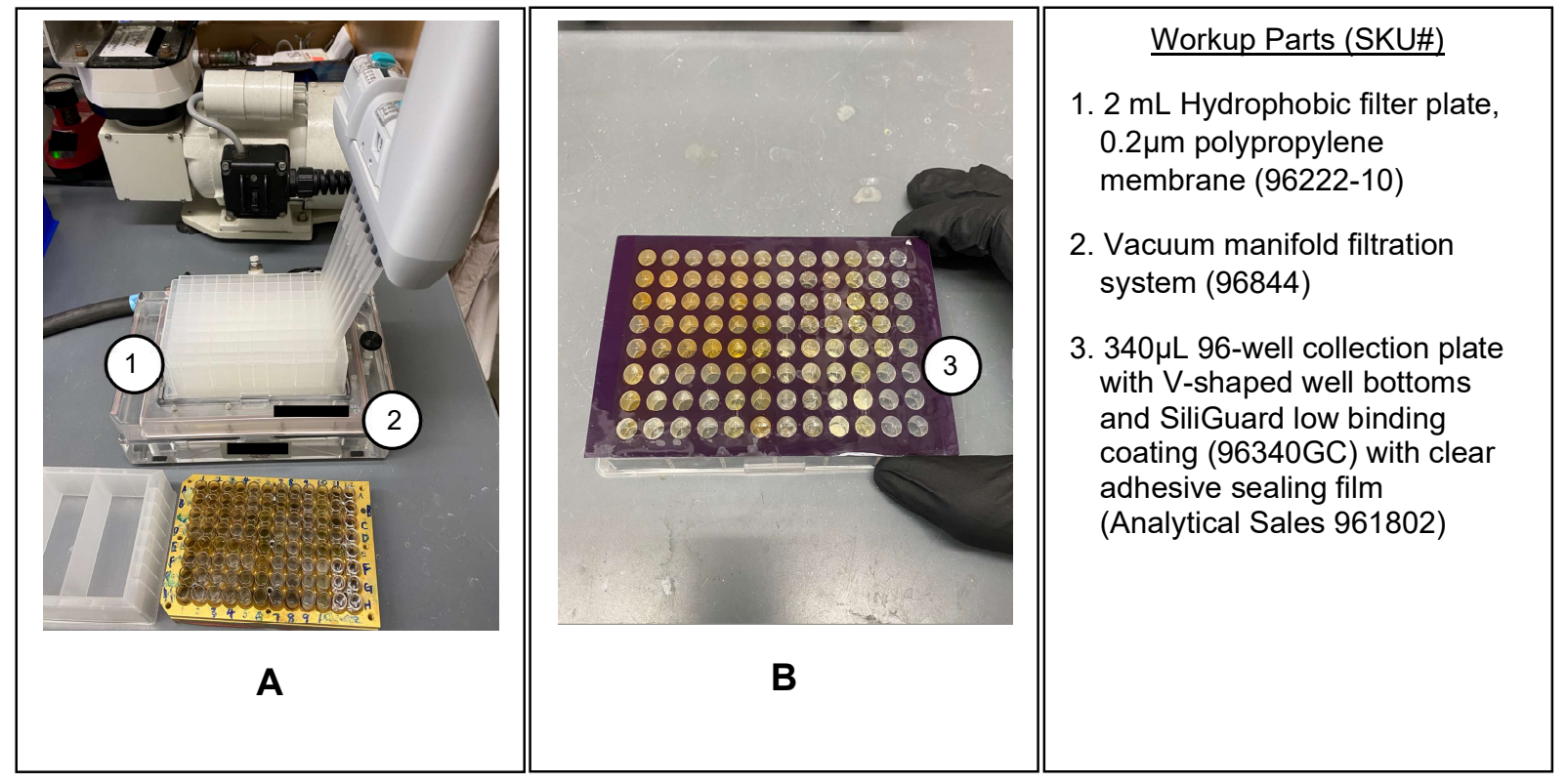

Figure S10. (A) The addition of diluted quenched reaction mixtures into a 96-well filter plate. (B) A $340 \mu \mathrm{L}$ V-bottom 96-well plate sealed with a clear adhesive PTFE film.

\section{Data collection}

For each sample, the integrated peak area of the cross-coupling product and the internal standard (TMB, 1,3,5-trimethoxybenzene) in SFC-MS chromatograms (UV $254 \mathrm{~nm}$ ) were recorded and the product/internal standard ratios were calculated.

\section{Heatmap generation}

The heatmaps with labels were generated based on the collected data in Origin 2020. 

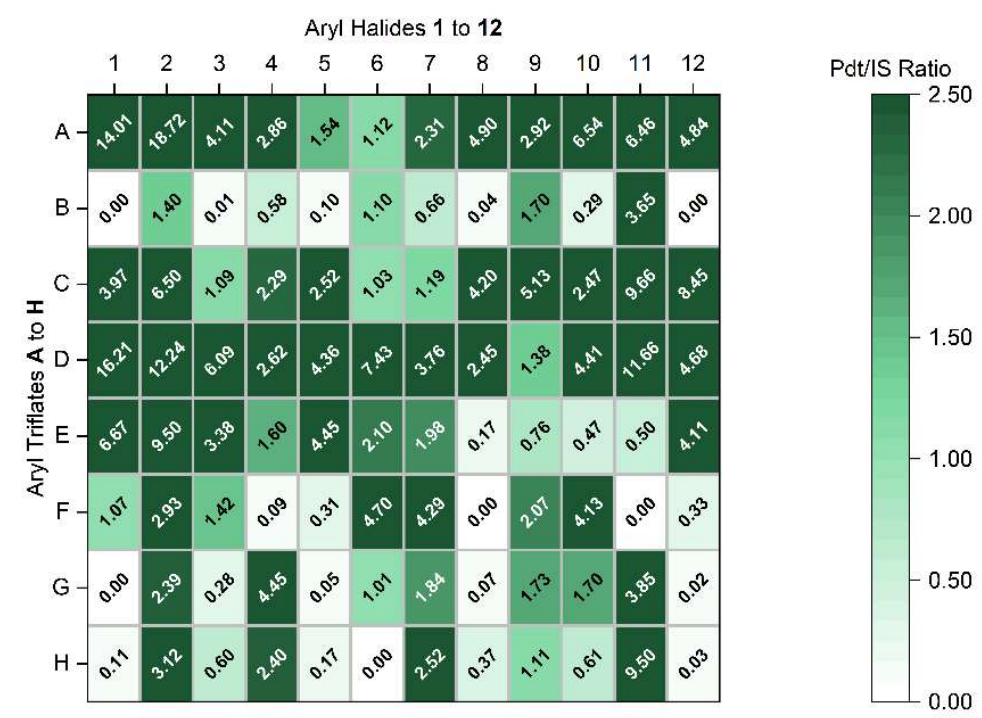

Figure S11. Heatmap generated based on the product/internal standard ratios in the scope investigations. 


\subsection{Application of HTE in condition optimizations}

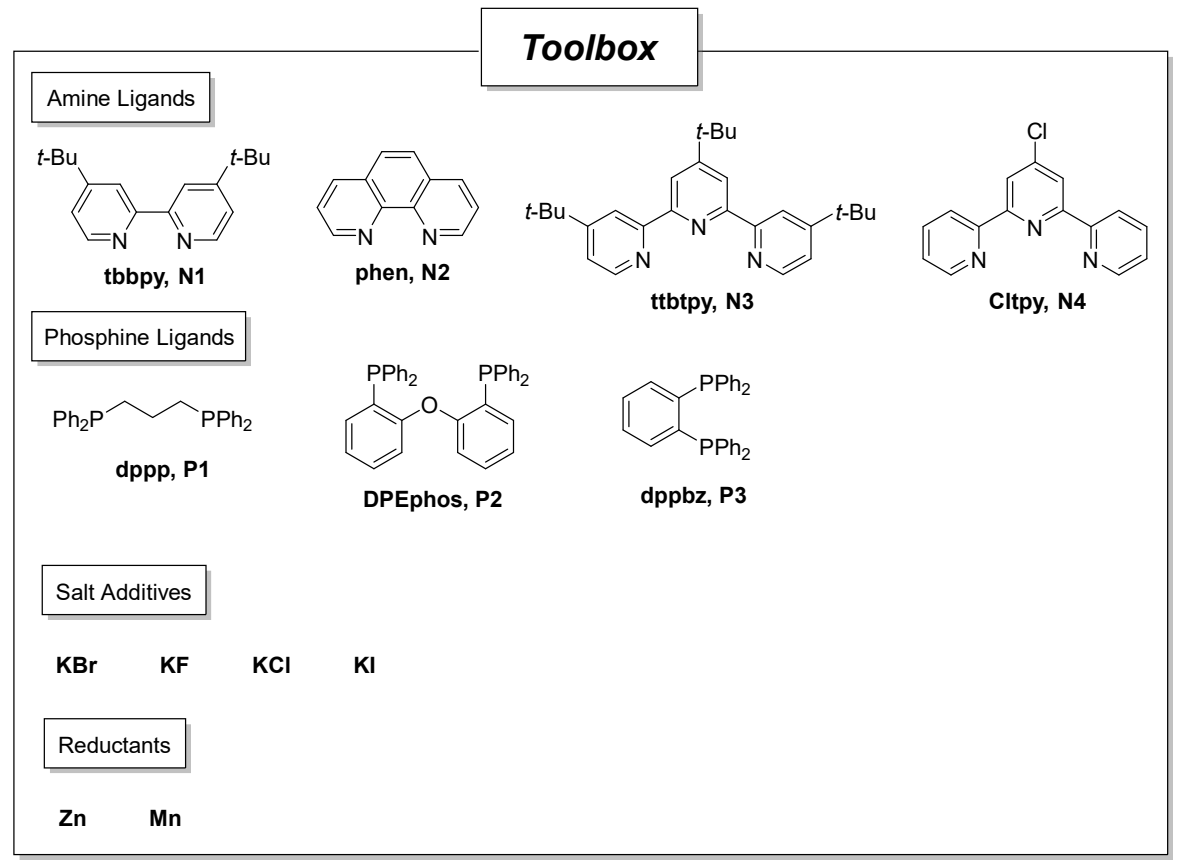

Figure S12. Amine ligands, phosphine ligands, salt additives, and reductants in Toolbox Plate.

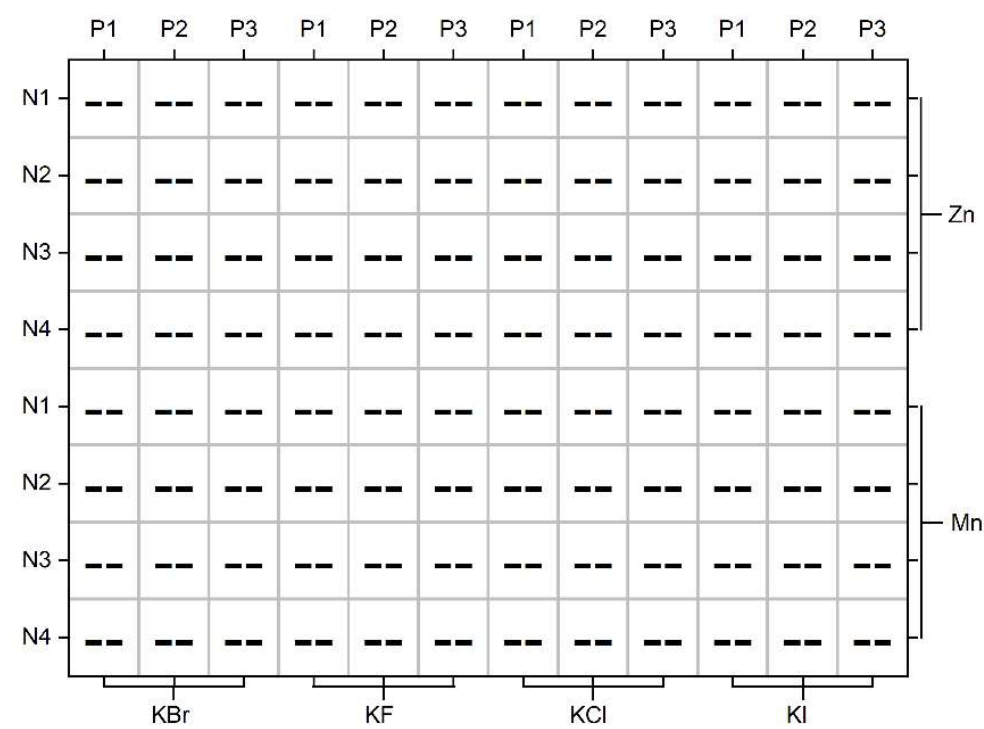

Figure S13. Layout for dosing a Toolbox Plate for condition optimizations. 


\subsubsection{Optimization for the synthesis of compound B3}

Scheme S12. Optimization for the synthesis of compound B3

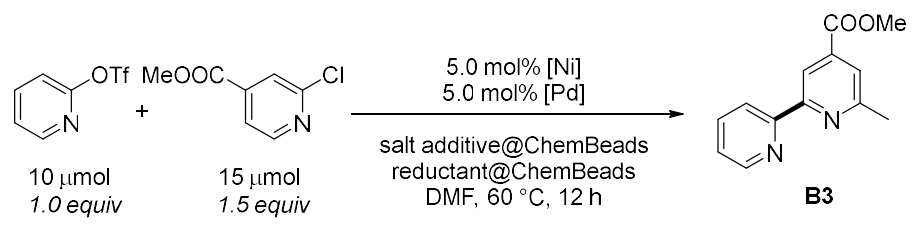

\section{Preparation of nickel and palladium catalyst stock solutions (0.025 M)}

The nickel and palladium catalyst stock solution (0.025 M in DMF with respect to nickel or palladium) were prepared according to the procedure in 2. Reaction Optimization section of this document using $\mathrm{NiBr}_{2}$ dme and the listed amine ligands or $\mathrm{PdCl}_{2}$ and the listed phosphine ligands as the starting materials.

\section{Catalytic reaction setup}

According to Figure S9, each of the reaction vial was charged sequentially with the listed reductant (Zn-coated ChemBeads, 5.0\% w/w, $30 \mathrm{mg}, 0.023 \mathrm{mmol}, 2.3$ equiv or Mn-coated ChemBeads, $5.0 \% \mathrm{w} / \mathrm{w}, 30 \mathrm{mg}, 0.027 \mathrm{mmol}, 2.7$ equiv), the listed salt additive ( $\mathrm{KBr}$-coated ChemBeads, 10.0\% w/w, $15 \mathrm{mg}, 0.012 \mathrm{mmol}, 1.2$ equiv, or KF-coated Chembeads, 10.0\% w/w, $15 \mathrm{mg}, 0.026 \mathrm{mmol}, 2.6$ equiv, or KCl-coated ChemBeads, 10.0\% w/w, $15 \mathrm{mg}, 0.020 \mathrm{mmol}, 2.0$ equiv, or KI-coated ChemBeads, $10.0 \% \mathrm{w} / \mathrm{w}, 30 \mathrm{mg}, 0.018 \mathrm{mmol}, 1.8$ equiv), pyridin-2-yl trifluoromethanesulfonate $(0.50 \mathrm{M}$ in $\mathrm{DMF}, 20 \mu \mathrm{L}, 0.010 \mathrm{mmol}, 1.0$ equiv), methyl 2chloroisonicotinate ( $0.75 \mathrm{M}$ in $\mathrm{DMF}, 20 \mu \mathrm{L}, 0.015 \mathrm{mmol}, 1.5$ equiv), nickel catalyst stock solution $\left(20 \mu \mathrm{L}, 5 \times 10^{-4} \mathrm{mmol}, 5 \mathrm{~mol} \%\right)$, and palladium catalyst stock solution $\left(20 \mu \mathrm{L}, 5 \times 10^{-4} \mathrm{mmol}, 5\right.$ mol\%). 1,3,5-trimethoxybenzene (4.0 M in DMF, $12.5 \mu \mathrm{L}, 0.050 \mathrm{mmol}, 5.0$ equiv) was then added as an internal standard. The vials were sealed according to 5.1 Application of HTE in scope investigations and placed on the heater/shaker. The plate was heated at $60{ }^{\circ} \mathrm{C}$ for $12 \mathrm{~h}$ with the shaking rate set at 8 .

\section{Reaction workup, data collection and heatmap generation}

See 5.1 Application of HTE in scope investigations of this document. 

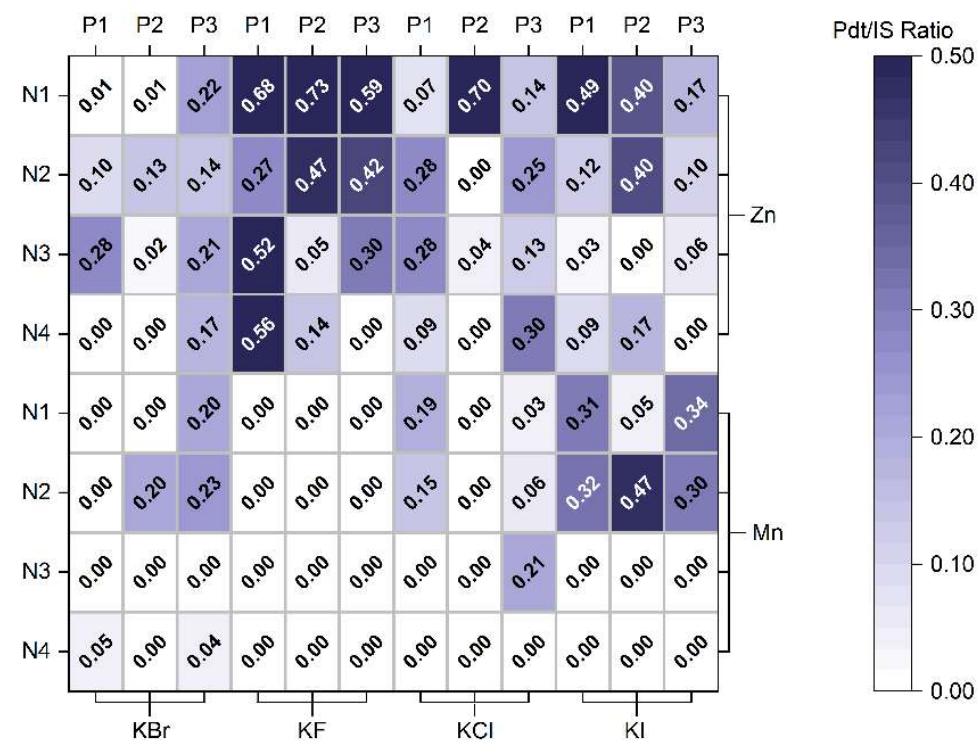

Figure S14. Heatmap generated based on the product/internal standard ratios in the reaction optimization of compound B3. 


\subsubsection{Optimization for the synthesis of compound C6}

Scheme S13. Optimization for the synthesis of compound C6

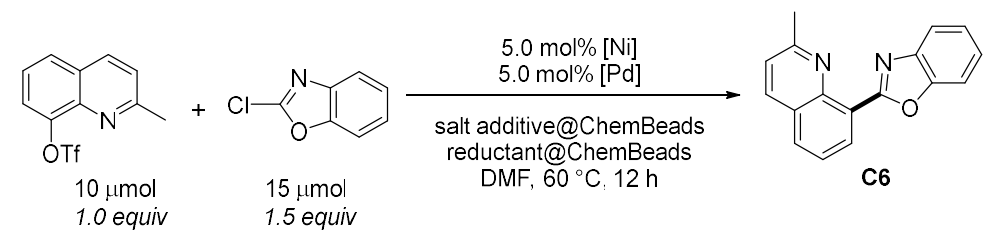

For procedures of reaction setup, reaction workup, data collection, and heatmap generation, see 5.2.1 Optimization for the synthesis of compound $\mathbf{B} 3$ of this document.
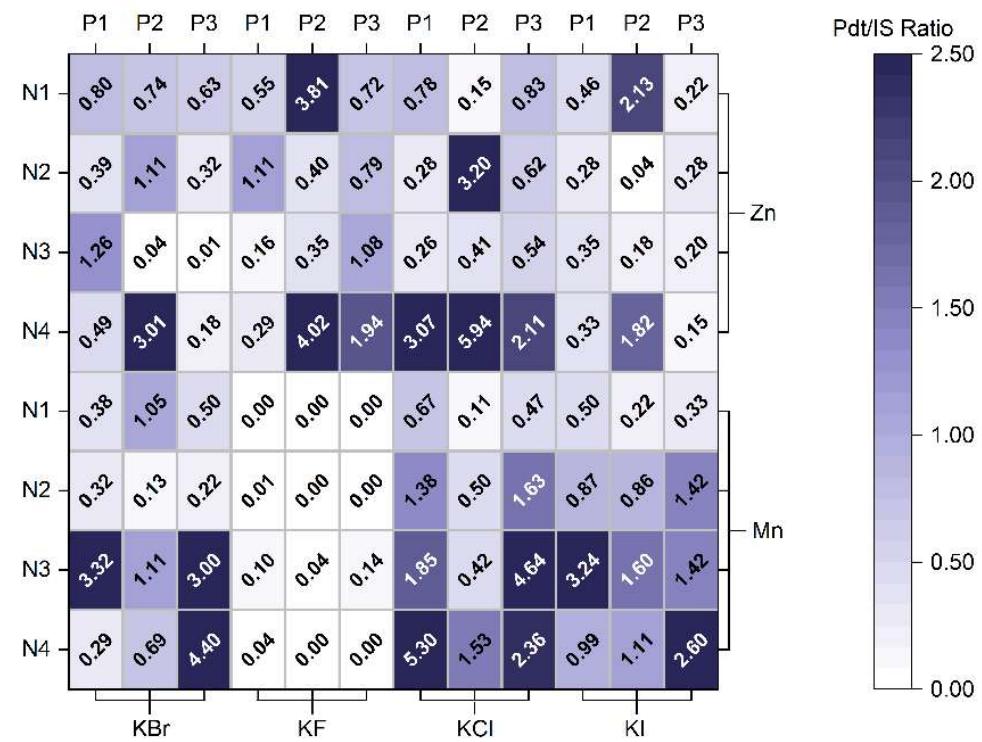

Figure S15. Heatmap generated based on the product/internal standard ratios in the reaction optimization of compound $\mathbf{C 6}$. 


\subsubsection{Optimization for the synthesis of compound $\mathrm{H} 2$}

Scheme S14. Optimization for the synthesis of compound $\mathbf{H 2}$
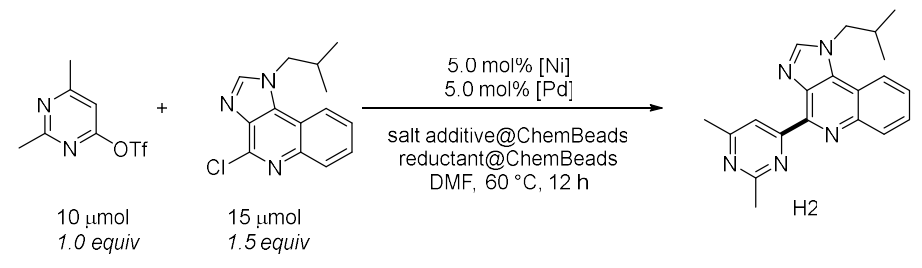

For procedures of reaction setup, reaction workup, data collection, and heatmap generation, see 5.2.1 Optimization for the synthesis of compound B3 of this document.
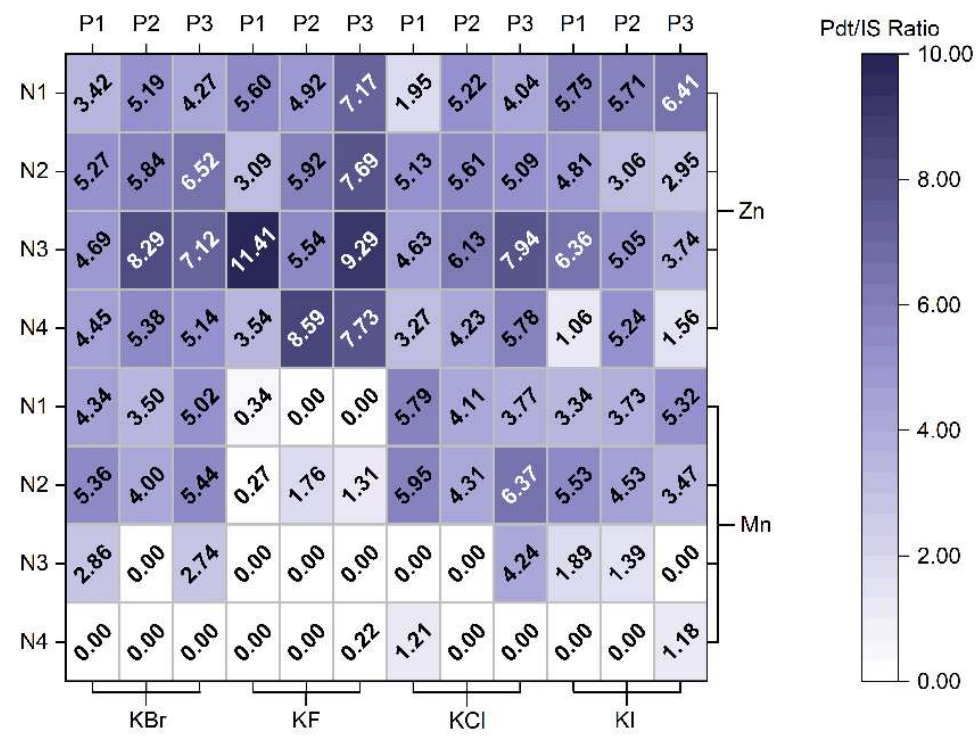

Figure S16. Heatmap generated based on the product/internal standard ratios in the reaction optimization of compound $\mathbf{H 2}$. 


\subsection{Synthesis of products B3, C6, and $\mathrm{H} 2$ under re-optimized conditions}

\subsubsection{Synthesis of compound B3 under optimized conditions}

Scheme S15. Synthesis of compound B3 on a $0.5 \mathrm{mmol}$ scale under optimized conditions

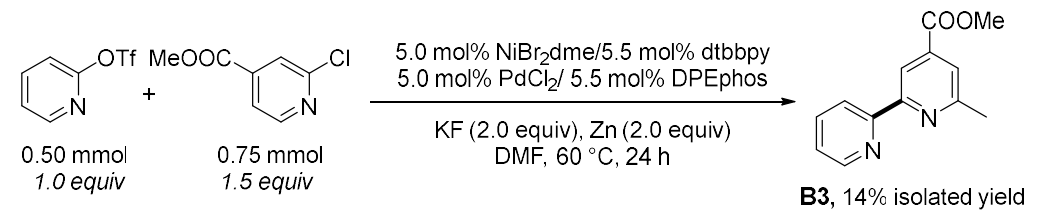

For procedures for preparation of nickel and palladium catalyst stock solutions, catalytic reaction setup, and SFC-MS analysis, see 4.1 General procedure for reaction setup in scope investigations of this document.

\section{Reaction workup}

For consistency, ammonium solution in methanol was used to quench the reaction, instead of $\mathrm{Na}_{4}$ EDTA aqueous solution, after the reaction completion (judged by SFC-MS analysis). The reaction vial was removed from the heat plate and cooled to rt. Ammonium solution (2.0 M in methanol, $2.5 \mathrm{~mL}, 5.0 \mathrm{mmol}, 10$ equiv) was added to the reaction mixture before the vial was recapped. The mixture was stirred (1000 rpm) at $\mathrm{rt}$ for $1 \mathrm{~h}$.

\section{Purification}

The quenched mixture was transferred to a $100 \mathrm{~mL}$ round bottom flask. The reaction vial was further rinsed by dichloromethane $(4 \mathrm{~mL} \times 3)$ to minimize product loss. Silica gel $(\sim 3 \mathrm{~g})$ was added to the round bottom flask, and the volatile solvents were removed by rotary evaporation. The dry-loaded material was purified by flash chromatography (hexanes (containing triethyl amine, $5 \% \mathrm{v} / \mathrm{v}) /$ ethyl acetate $=10 / 1)$ on silica gel to afford a white solid containing compound $\mathbf{B 3}$ (16.3 mg, 14\% yield) and dimethyl 6,6'-dimethyl-[2,2'-bipyridine]-4,4'-dicarboxylate (2.1 mg, 1.4\%). ${ }^{1} \mathrm{H}$ NMR $\left(500 \mathrm{MHz}, \mathrm{CDCl}_{3}\right) \delta 8.73-8.68(\mathrm{~m}, 2 \mathrm{H}), 8.42(\mathrm{dt}, J=8.0,1.1 \mathrm{~Hz}, 1 \mathrm{H}), 7.81(\mathrm{td}, J=$ 7.7, $1.8 \mathrm{~Hz}, 1 \mathrm{H}), 7.73(\mathrm{~d}, J=1.5 \mathrm{~Hz}, 1 \mathrm{H}), 7.31$ (ddd, $J=7.5,4.7,1.2 \mathrm{~Hz}, 1 \mathrm{H}), 3.96$ (s, $3 \mathrm{H}), 2.69$ $(\mathrm{s}, 3 \mathrm{H}) ;{ }^{13} \mathrm{C}\left\{{ }^{1} \mathrm{H}\right\} \mathrm{NMR}\left(126 \mathrm{MHz}, \mathrm{CDCl}_{3}\right) \delta 166.2,159.2,156.9,155.8,149.5,138.8,137.1,124.1$, 122.6, 121.4, 117.7, 52.7, $24.8 \mathrm{ppm}$. HRMS (ESI) m/z calcd for $[\mathrm{M}+\mathrm{H}]^{+} 229.0971$, found 229.0969. For dimethyl 6,6'-dimethyl-[2,2'-bipyridine]-4,4'-dicarboxylate ${ }^{35}$, HRMS (ESI) m/z calcd for $[\mathrm{M}+\mathrm{H}]^{+}$301.1183, found 301.1177. 


\subsubsection{Synthesis of compound C6 under optimized conditions}

Scheme S16. Synthesis of compound $\mathbf{C 6}$ on a $0.5 \mathrm{mmol}$ scale under optimized conditions
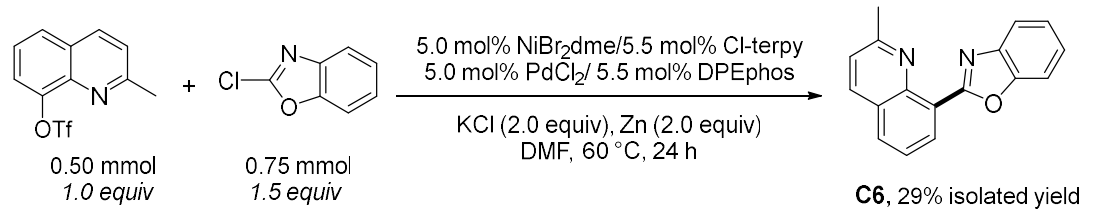

For procedures for preparation of nickel and palladium catalyst stock solutions, catalytic reaction setup, and SFC-MS analysis, see 4.1 General procedure for reaction setup in scope investigations of this document. For procedures for reaction workup and purification, see 5.2.1 Synthesis of compound B3 under optimized conditions of this document.

The product was purified by flash chromatography (hexanes/ethyl acetate $=10 / 1$ to $5 / 1$ ) on silica gel to afford the desired product compound $\mathbf{C 6}$ as a colorless oil (37.2 $\mathrm{mg}, 29 \%$ yield). Attention: compound $\mathbf{C 6}$ is unstable in $\mathrm{CDCl}_{3}$. The color of a $\mathrm{CDCl}_{3}$ solution of $\mathbf{C 6}$ turned from pale yellow to brown within $12 \mathrm{~h}$ at $r$ in an NMR tube. ${ }^{1} \mathrm{H} \mathrm{NMR}\left(500 \mathrm{MHz}, \mathrm{C}_{6} \mathrm{D}_{6}\right) \delta 8.33(\mathrm{dd}, J=$ $7.3,1.5 \mathrm{~Hz}, 1 \mathrm{H}), 7.86(\mathrm{dq}, J=8.1,0.6 \mathrm{~Hz}, 1 \mathrm{H}), 7.42(\mathrm{dt}, J=8.0,1.0 \mathrm{~Hz}, 1 \mathrm{H}), 7.41(\mathrm{~d}, J=8.4$ $\mathrm{Hz}, 1 \mathrm{H}), 7.36(\mathrm{dd}, J=8.1,1.5 \mathrm{~Hz}, 1 \mathrm{H}), 7.07$ (td, $J=7.6,0.9 \mathrm{~Hz}, 2 \mathrm{H}), 7.01$ (td, J = 8.0, $1.0 \mathrm{~Hz}$, $1 \mathrm{H}), 6.71(\mathrm{~d}, J=8.4 \mathrm{~Hz}, 1 \mathrm{H}), 2.48(\mathrm{~s}, 3 \mathrm{H}) ;{ }^{13} \mathrm{C} \mathrm{NMR}\left(126 \mathrm{MHz}, \mathrm{C}_{6} \mathrm{D}_{6}\right) \delta 164.5,160.5,152.7$, $146.9,143.5,136.2$, 133.3, 131.2, 128.0, 127.3, 125.4, 125.3, 124.7, 122.8, 121.1, 111.4, 26.0 ppm. HRMS (ESI) $\mathrm{m} / \mathrm{z}$ calcd for $[\mathrm{M}+\mathrm{H}]^{+} 261.1022$, found 261.1019 . 


\subsubsection{Synthesis of compound $\mathrm{H} 2$ under optimized conditions}

Scheme S17. Synthesis of compound $\mathbf{H} 2$ on a $0.5 \mathrm{mmol}$ scale under optimized conditions

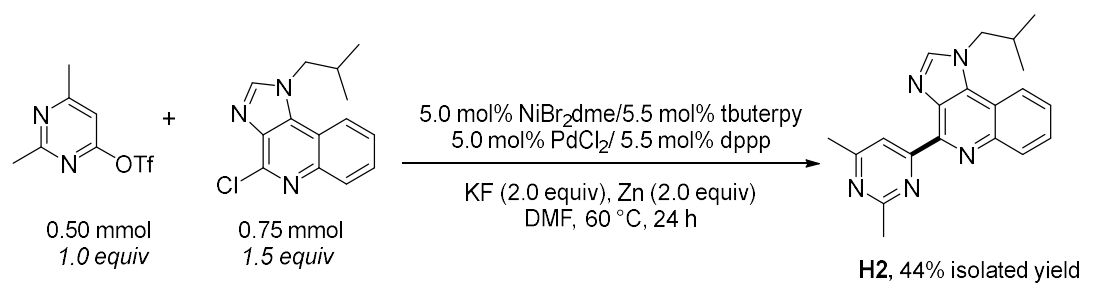

For procedures for preparation of nickel and palladium catalyst stock solutions, catalytic reaction setup, and SFC-MS analysis, see 4.1 General procedure for reaction setup in scope investigations of this document. For procedures for reaction workup and purification, see 5.2.1 Synthesis of compound B3 under optimized conditions of this document.

The product was purified by flash chromatography (hexanes/ethyl acetate/methanol $=$ 48/48/5) on silica gel to afford the desired product compound $\mathbf{H 2}$ as a yellow oil $(73.3 \mathrm{mg}, 44 \%$ yield). ${ }^{1} \mathrm{H}$ NMR (600 MHz, $\left.\mathrm{CDCl}_{3}\right) \delta 8.45(\mathrm{~d}, J=6.9 \mathrm{~Hz}, 1 \mathrm{H}), 8.24(\mathrm{~s}, 1 \mathrm{H}), 8.15(\mathrm{~d}, J=8.3 \mathrm{~Hz}$, $1 \mathrm{H}), 8.00$ (s, $1 \mathrm{H}$ ), 7.70 (dddd, $J=30.6,8.3,6.9,1.4 \mathrm{~Hz}, 2 \mathrm{H}$ ), 4.40 (d, $J=7.4 \mathrm{~Hz}, 2 \mathrm{H}), 2.91$ (s, $3 \mathrm{H}), 2.65$ (s, $3 \mathrm{H}), 1.05(\mathrm{~d}, J=6.6 \mathrm{~Hz}, 6 \mathrm{H}) ;{ }^{13} \mathrm{C}\left\{{ }^{1} \mathrm{H}\right\} \mathrm{NMR}\left(151 \mathrm{MHz}, \mathrm{CDCl}_{3}\right) \delta 168.5,167.8$, 162.4, 149.2, 144.59, 144.56, 136.8, 134.4, 132.2, 127.7, 127.6, 120.0, 118.9, 118.3, 55.4, 29.0, 26.8, 24.7, $20.0 \mathrm{ppm}$. HRMS (ESI) $\mathrm{m} / \mathrm{z}$ calcd for $[\mathrm{M}+\mathrm{Na}]^{+} 354.1689$, found 354.184 . 


\section{References}

(1) (a) Tu, N. P.; Dombrowski, A. W.; Goshu, G. M.; Vasudevan, A.; Djuric, S. W.; Wang, Y. High-Throughput Reaction Screening with Nanomoles of Solid Reagents Coated on Glass Beads. Angew. Chem. Int. Ed. 2019, 58, 7987-7991. (b) Aguirre, A. L.; Loud, N. L.; Johnson, K. A.; Weix, D. J.; Wang, Y. ChemBead Enabled High-Throughput Cross-Electrophile Coupling Reveals a New Complementary Ligand. Chem. Eur. J. 2021, 27, 12981-12986.

(2) Lee, S.; Song, J.-H.; Park, C. M.; Kim, J.-S.; Jeong, J.-H.; Cho, W.-Y.; Lim, D.-C. Discovery of Octahydroindenes as PAR1 Antagonists. ACS Med. Chem. Lett. 2013, 4, 1054-1058.

(3) Wilson, K.; Murray, J.; Jamieson, C.; Watson, A. Cyrene as a Bio-Based Solvent for the Suzuki-Miyaura Cross-Coupling. Synlett 2018, 29, 650-654.

(4) Seganish, W. M.; DeShong, P. Preparation and Palladium-Catalyzed Cross-Coupling of Aryl Triethylammonium Bis(Catechol) Silicates with Aryl Triflates. J. Org. Chem. 2004, 69, 11371143.

(5) Momose, Y.; Maekawa, T.; Odaka, H.; Kimura, H. 5-Membered N-heterocyclic Compounds with Hypoglycemic and Hypolipidemic Activity. WO 2001038325. May 31, 2001.

(5) Jana, N.; Nguyen, Q.; Driver, T. G. Development of a Suzuki Cross-Coupling Reaction between 2-Azidoarylboronic Pinacolate Esters and Vinyl Triflates To Enable the Synthesis of [2,3]-Fused Indole Heterocycles. J. Org. Chem. 2014, 79, 2781-2791.

(7) Hofmayer, M. S.; Lutter, F. H.; Grokenberger, L.; Hammann, J. M.; Knochel, P. Practical NiCatalyzed Cross-Coupling of Unsaturated Zinc Pivalates with Unsaturated Nonaflates and Triflates. Org. Lett. 2019, 21, 36-39.

(8) Torrado, A.; Lopez, S.; Alvarez, R.; de Lera, A. R. General Synthesis of Retinoids and Arotinoids via Palladium-Catalyzed Cross-Coupling of Boronic Acids with Electrophiles. Synthesis 1995, 285-293.

(9) Verheyen, T.; van Turnhout, L.; Vandavasi, J. K.; Isbrandt, E. S.; De Borggraeve, W. M.; Newman, S. G. Ketone Synthesis by a Nickel-Catalyzed Dehydrogenative Cross-Coupling of Primary Alcohols. J. Am. Chem. Soc. 2019, 141, 6869-6874.

(10) Huang, Z.; Liu, Z.; Zhou, J. An Enantioselective, Intermolecular $\alpha$-Arylation of Ester Enolates To Form Tertiary Stereocenters. J. Am. Chem. Soc. 2011, 133, 15882-15885

(11) Lee, H. G.; Milner, P. J.; Buchwald, S. L. An Improved Catalyst System for the Pd-Catalyzed Fluorination of (Hetero)Aryl Triflates. Org. Lett. 2013, 15, 5602-5605. 
(12) Smyth, L. A.; Phillips, E. M.; Chan, V. S.; Napolitano, J. G.; Henry, R.; Shekhar, S. PdCatalyzed Synthesis of Aryl and Heteroaryl Triflones from Reactions of Sodium Triflinate with Aryl (Heteroaryl) Triflates. J. Org. Chem. 2016, 81, 1285-1294.

(13) Wu, L.; Zhang, L.; Zhang, P.; Chen, Z.; Chen, S. $\alpha$-7 Nicotine Acetylcholine Receptor Agonist and Application Thereof. WO 2018210229. Nov 22, 2018.

(14) Waldmann, C.; Schober, O.; Haufe, G.; Kopka, K. A Closer Look at the Bromine-Lithium Exchange with Tert-Butyllithium in an Aryl Sulfonamide Synthesis. Org. Lett. 2013, 15, 2954 2957.

(15) Nimje, R. Y.; Vytla, D.; Kuppusamy, P.; Velayuthaperumal, R.; Jarugu, L. B.; Reddy, C. A.; Chikkananjaiah, N. K.; Rampulla, R. A.; Cavallaro, C. L.; Li, J.; Mathur, A.; Gupta, A.; Roy, A. Synthesis of Differentially Protected Azatryptophan Analogs via Pd2(Dba)3/XPhos Catalyzed Negishi Coupling of N-Ts Azaindole Halides with Zinc Derivative from Fmoc-Protected TertButyl (R)-2-Amino-3-Iodopropanoate. J. Org. Chem. 2020, 85, 11519-11530.

(16) Henderson, J. L.; McDermott, S. M.; Buchwald, S. L. Palladium-Catalyzed Amination of Unprotected Halo-7-Azaindoles. Org. Lett. 2010, 12, 4438-4441.

(17) Ye, M.; Gao, G.-L.; Edmunds, A. J. F.; Worthington, P. A.; Morris, J. A.; Yu, J.-Q. LigandPromoted C3-Selective Arylation of Pyridines with Pd Catalysts: Gram-Scale Synthesis of $( \pm)$ Preclamol. J. Am. Chem. Soc. 2011, 133, 19090-19093.

(18) Qi, C.; Sun, X.; Lu, C.; Yang, J.; Du, Y.; Wu, H.; Zhang, X.-M. Palladium Catalyzed Reductive Homocoupling Reactions of Aromatic Halides in Dimethyl Sulfoxide (DMSO) Solution. J. Organomet. Chem. 2009, 694, 2912-2916.

(19) Rahil, R.; Sengmany, S.; Gall, E. L.; Léonel, E. Nickel-Catalyzed Electrochemical Reductive Homocouplings of Aryl and Heteroaryl Halides: A Useful Route to Symmetrical Biaryls. Synthesis 2018, 50, 146-154.

(20) Duong, V. K.; Horan, A. M.; McGarrigle, E. M. Synthesis of Pyridylsulfonium Salts and Their Application in the Formation of Functionalized Bipyridines. Org. Lett. 2020, 22, 8451-8457. (21) Bathula, C.; Mamidala, R.; Thulluri, C.; Agarwal, R.; Jha, K. K.; Munshi, P.; Adepally, U.; Singh, A.; Chary, M. T.; Sen, S. Substituted Furopyridinediones as Novel Inhibitors of $\alpha$ Glucosidase. RSC Adv. 2015, 5, 90374-90385.

(22) Cross, J. B.; Zhang, J.; Yang, Q.; Mesleh, M. F.; Romero, J. A. C.; Wang, B.; Bevan, D.; Poutsiaka, K. M.; Epie, F.; Moy, T.; Daniel, A.; Shotwell, J.; Chamberlain, B.; Carter, N.; Andersen, O.; Barker, J.; Ryan, M. D.; Metcalf, C. A.; Silverman, J.; Nguyen, K.; Lippa, B.; Dolle, 
R. E. Discovery of Pyrazolopyridones as a Novel Class of Gyrase B Inhibitors Using Structure Guided Design. ACS Med. Chem. Lett. 2016, 7, 374-378.

(23) Shigeno, M.; Kai, Y.; Yamada, T.; Hayashi, K.; Nozawa-Kumada, K.; Denneval, C.; Kondo, Y. Construction of Biaryl Scaffolds from Iodoarenes and $\mathrm{C}-\mathrm{H}$ Heteroarenes Using an Amide Base Generated in Situ from Aminosilane and Fluoride Anion. Asian J. Org. Chem. 2018, 7, 2082-2086. (24) Tian, Z.-Y.; Lin, Z.-H.; Zhang, C.-P. Pd/Cu-Catalyzed C-H/C-H Cross Coupling of (Hetero)Arenes with Azoles through Arylsulfonium Intermediates. Org. Lett. 2021, 23, 44004405.

(25) Savall, B. M.; Fontimayor, J. R. Synthesis of 2-Arylbenzimidazoles via Microwave SuzukiMiyaura Reaction of Unprotected 2-Chlorobenzimidazoles. Tetrahedron Lett. 2008, 49, 66676669.

(26) Yuan, C.; Zheng, L.; Zhao, Y. Cu(II)-Catalyzed Homocouplings of (Hetero)Arylboronic Acids with the Assistance of 2-O-Methyl-d-Glucopyranose. Molecules 2019, 24, 3678.

( 27 ) Bhattacharjya, A.; Klumphu, P.; Lipshutz, B. H. Ligand-Free, Palladium-Catalyzed Dihydrogen Generation from TMDS: Dehalogenation of Aryl Halides on Water. Org. Lett. 2015, $17,1122-1125$.

(28) Yoshino, K.; Kohno, T.; Morita, T.; Tsukamoto, G. Organic Phosphorus Compounds. 2. Synthesis and Coronary Vasodilator Activity of (Benzothiazolylbenzyl)Phosphonate Derivatives. J. Med. Chem. 1989, 32, 1528-1532.

(29) Buonomo, J. A.; Everson, D. A.; Weix, D. J. Substituted 2,2'-Bipyridines by Nickel Catalysis: 4,4'-Di-Tert-Butyl-2,2'-Bipyridine. Synthesis 2013, 45, 3099-3102.

(30) Mathieu, J.; Gros, P.; Fort, Y. Straightforward Access to Methyl-Polyheterocycles from Direct Para-Lithiation of 3-Picoline. Tetrahedron Lett. 2001, 42, 1879-1881.

(31) Butora, G.; Pasternak, A.; Yang, L.; Zhou, C. Gamma-Aminoamide Modulators of Chemokine Receptor Activity. WO2004041279 (A1). May 21, 2004.

(32) Font-Sanchis, E.; Céspedes-Guirao, F. J.; Sastre-Santos, Á.; Fernández-Lázaro, F. IndiumMediated Synthesis of Heterobiaryls. J. Org. Chem. 2007, 72, 3589-3591.

(33) Chen, X.; Zhou, L.; Li, Y.; Xie, T.; Zhou, S. Synthesis of Heteroaryl Compounds through Cross-Coupling Reaction of Aryl Bromides or Benzyl Halides with Thienyl and Pyridyl Aluminum Reagents. J. Org. Chem. 2014, 79, 230-239.

(34) Krasovskiy, A.; Knochel, P. Convenient Titration Method for Organometallic Zinc, Magnesium, and Lanthanide Reagents. Synthesis 2006, 890-891. 
(35) Chen, S.; Hong, Y.; Liu, Y.; Xue, M.; Zheng, Y.; Shen, Q. Synthesis and Properties of Sodium and Europium (III) Cryptates Incorporating the 4,4'-Substituted-2,2'-bipyridine Units. Chin. J. Org. Chem. 2017, 5, $1198-1204$. 


\section{Copies of NMR Spectra}




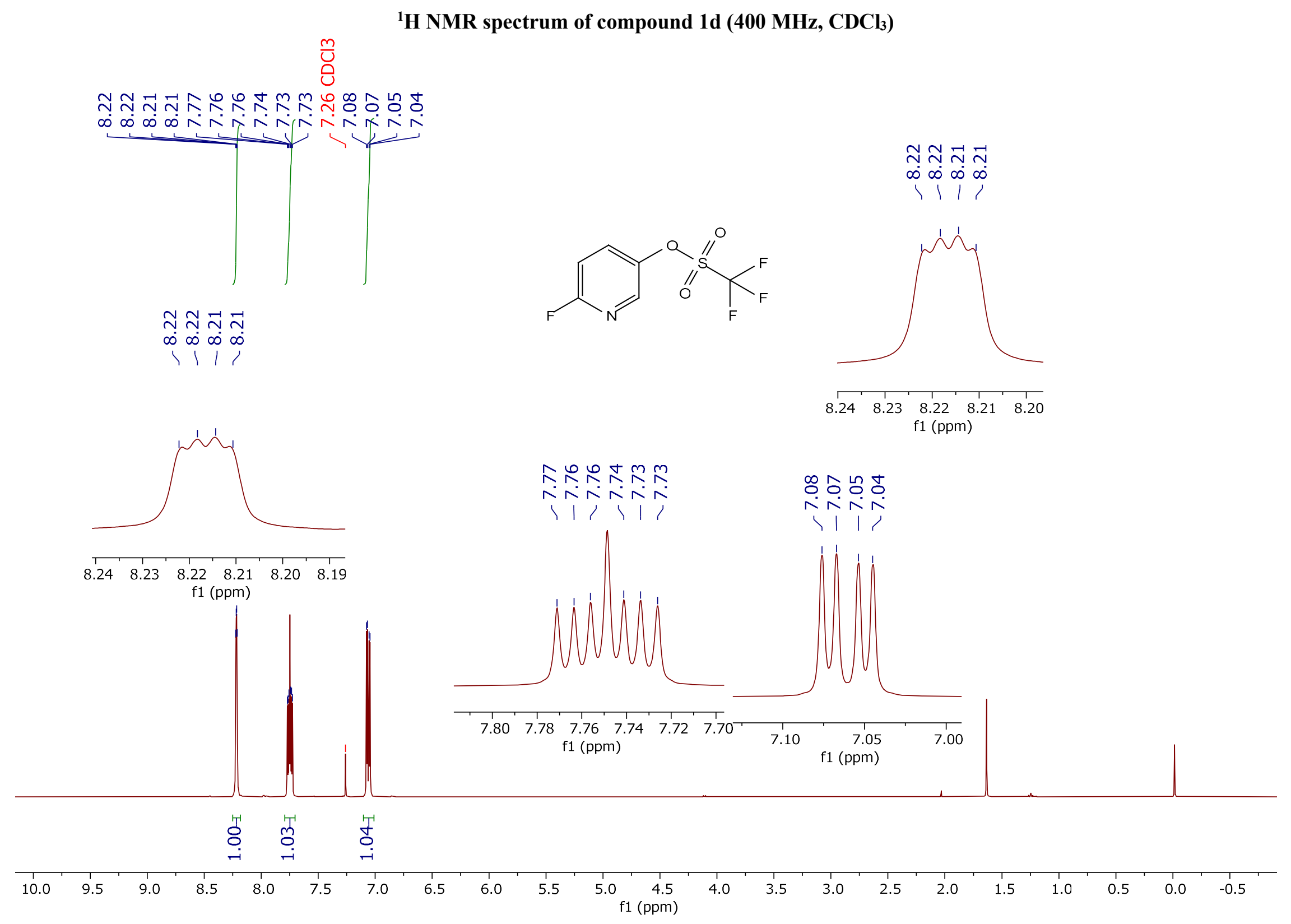


${ }^{19} \mathrm{~F}\left\{{ }^{1} \mathrm{H}\right\}$ NMR spectrum of compound $1 \mathrm{~d}\left(376 \mathrm{MHz}, \mathrm{CDCl}_{3}\right)$

จิ

ஜ

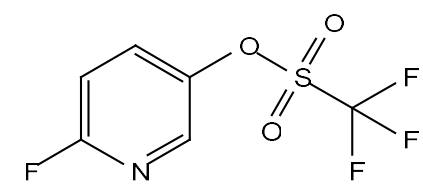

$i$

ồ

$\begin{array}{lllllllllllllllllllllllllllll}10 & 0 & -10 & -20 & -30 & -40 & -50 & -60 & -70 & -80 & -90 & -100 & -110 & -120 & -130 & -140 & -150 & -160 & -170 & -180 & -190 & -200 & -210 & 1\end{array}$




\section{${ }^{13} \mathrm{C}\left\{{ }^{1} \mathrm{H}\right\}$ NMR spectrum of compound $1 \mathrm{~d}\left(126 \mathrm{MHz}, \mathrm{CDCl}_{3}\right)$}

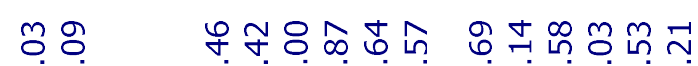

ஸिं

11

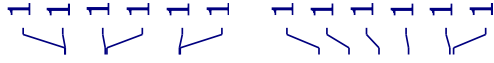

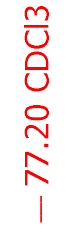

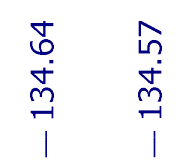

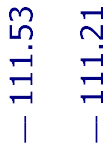

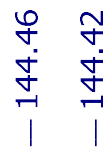

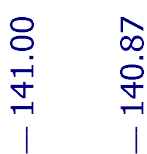
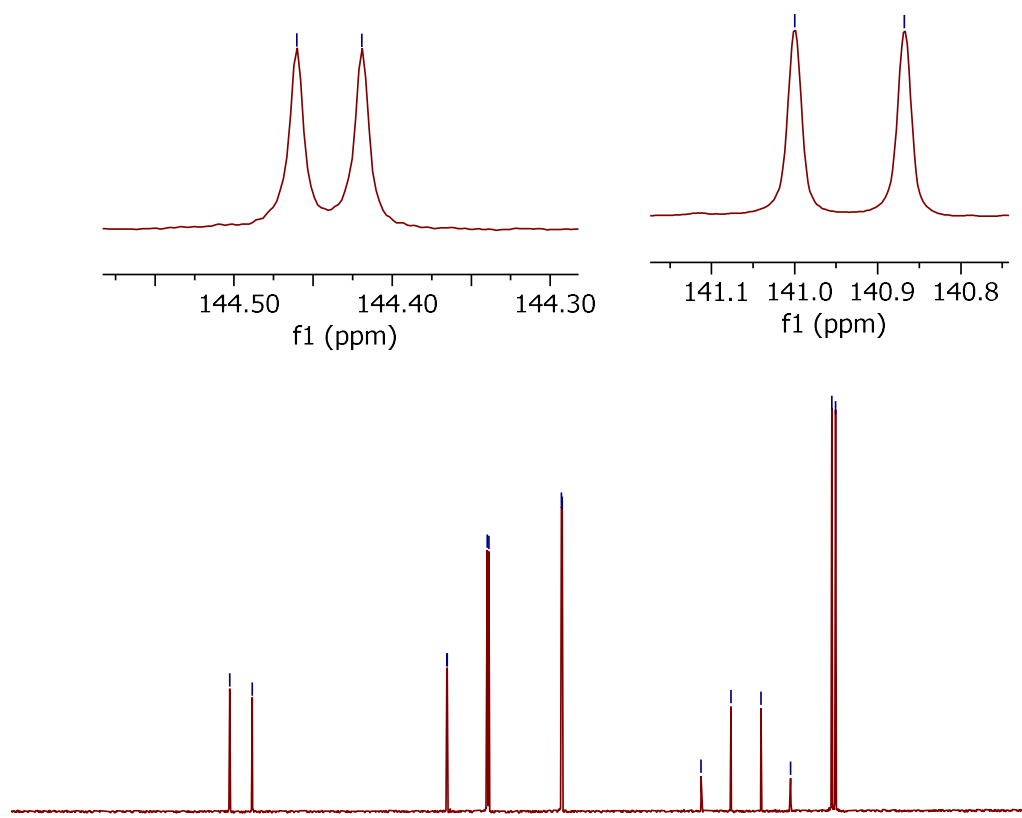

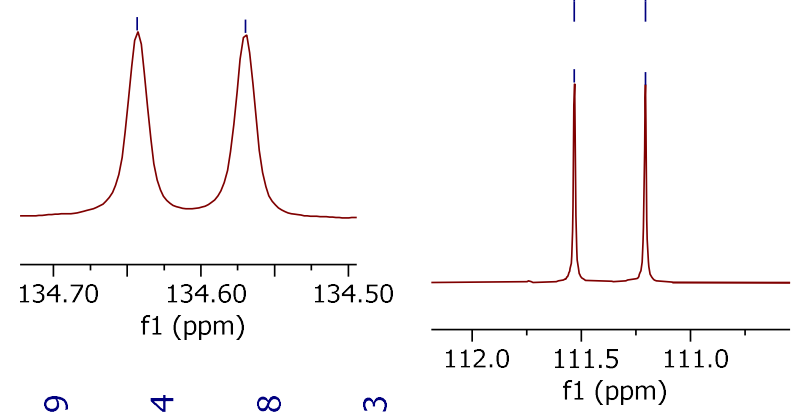

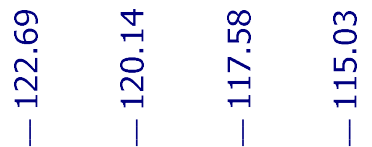

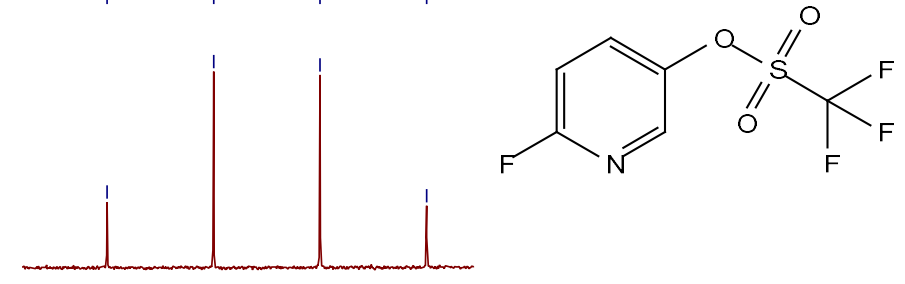
$\begin{array}{lllll}124 & 122 & \begin{array}{c}120 \\ \mathrm{f} 1(\mathrm{ppm})\end{array} & 118 & 114\end{array}$ 


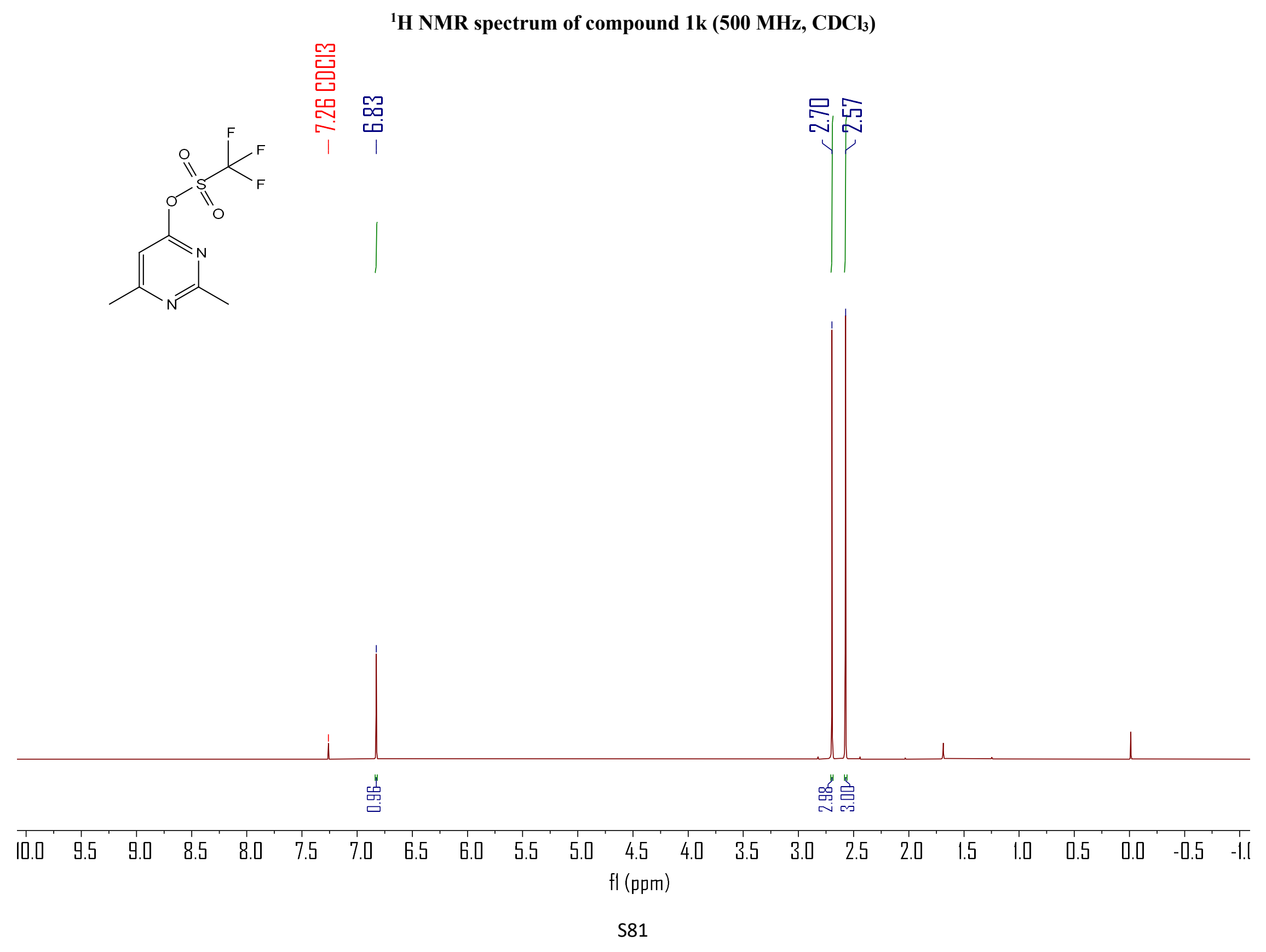


${ }^{19} \mathrm{~F}\left\{{ }^{1} \mathrm{H}\right\}$ NMR spectrum of compound $1 \mathrm{k}\left(376 \mathrm{MHz}, \mathrm{CDCl}_{3}\right)$

豆

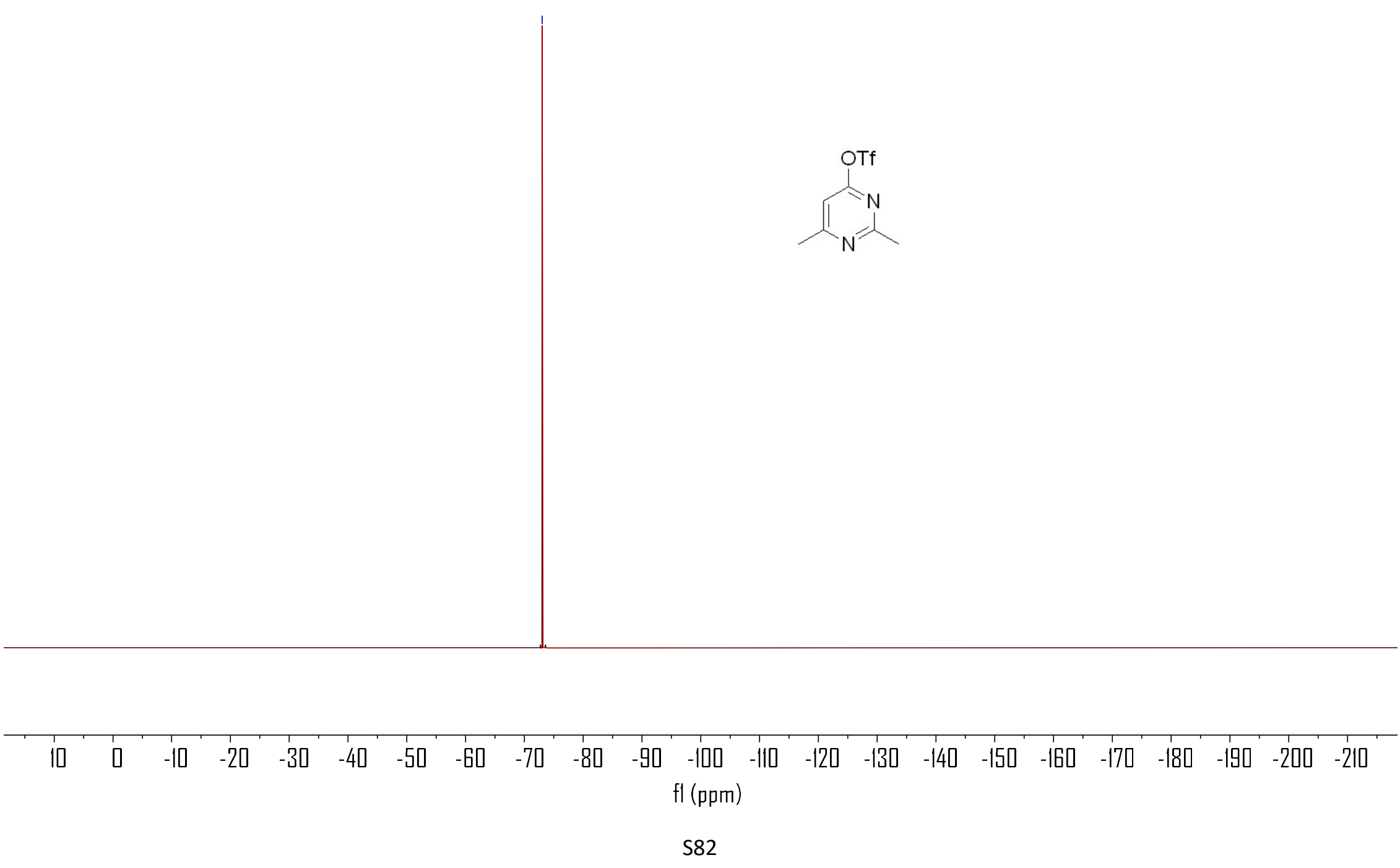




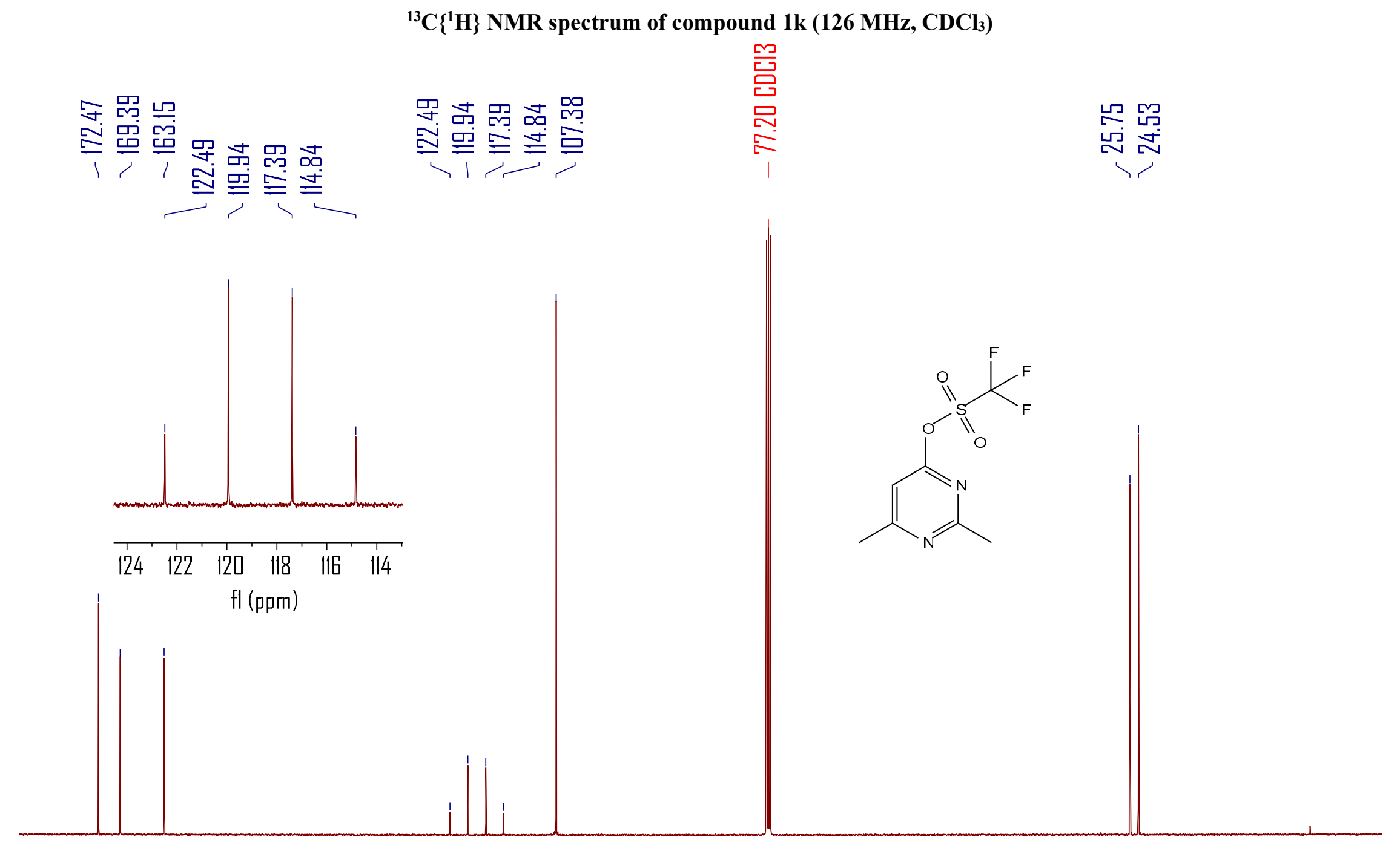

\begin{tabular}{|c|c|c|c|c|c|c|c|c|c|c|c|c|c|c|c|c|c|c|}
\hline 180 & 170 & 160 & 150 & 140 & 130 & 120 & $\| 0$ & I00 & & 80 & 70 & 60 & 50 & 40 & 30 & 20 & & 0 \\
\hline
\end{tabular}


${ }^{1} \mathrm{H}$ NMR spectrum of compound $1 \mathrm{~m}\left(500 \mathrm{MHz}, \mathrm{CDCl}_{3}\right)$

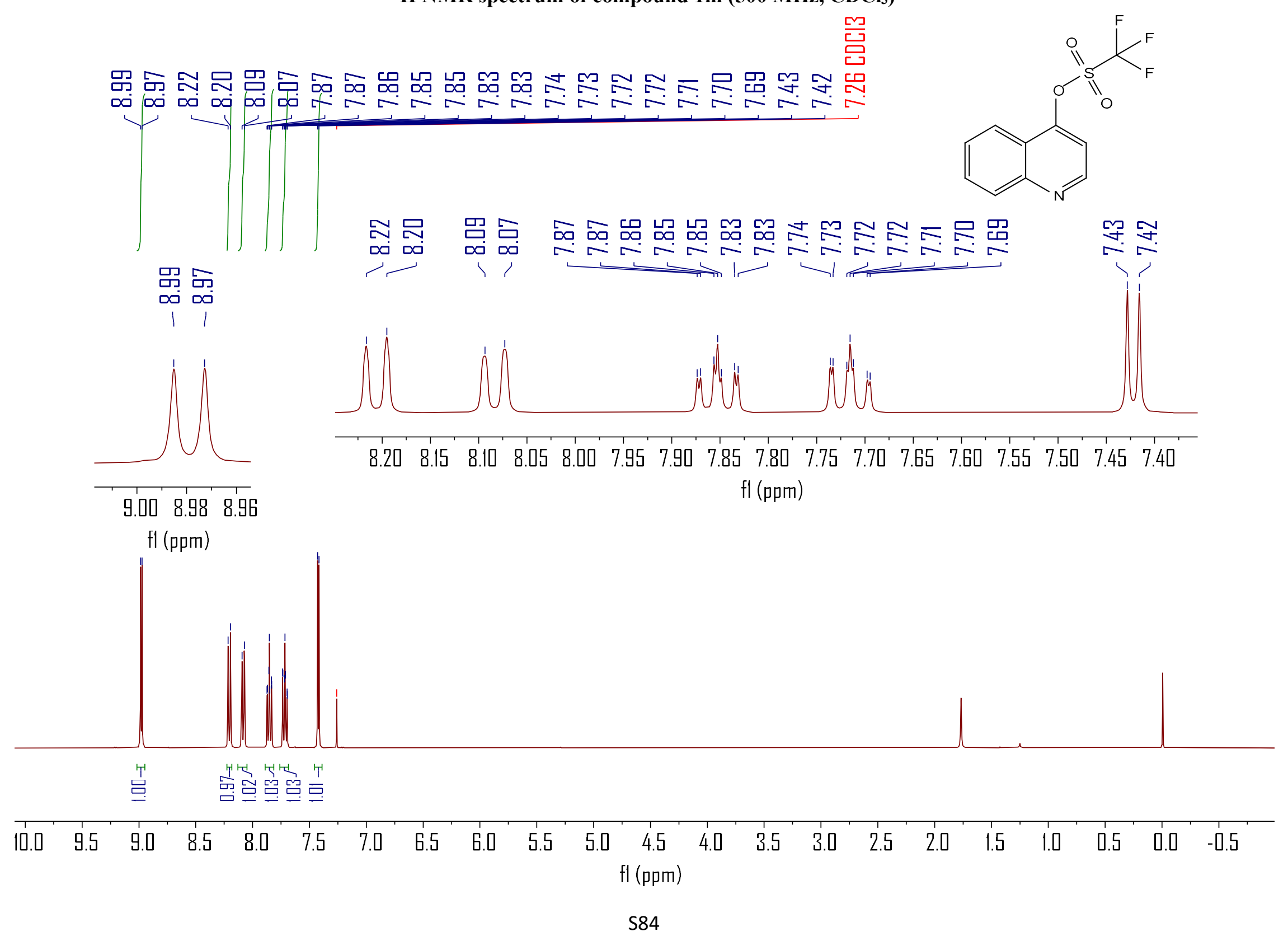


${ }^{19} \mathrm{~F}\left\{{ }^{1} \mathrm{H}\right\}$ NMR spectrum of compound $1 \mathrm{~m}\left(376 \mathrm{MHz}, \mathrm{CDCl}_{3}\right)$

吕
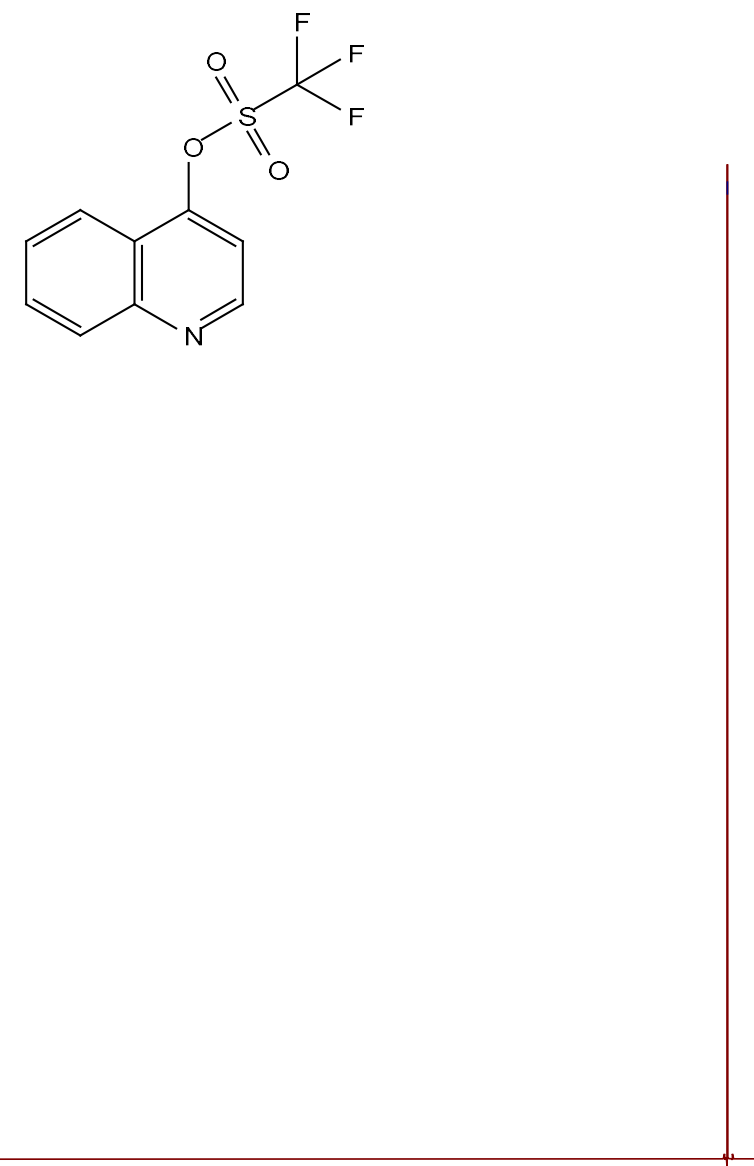

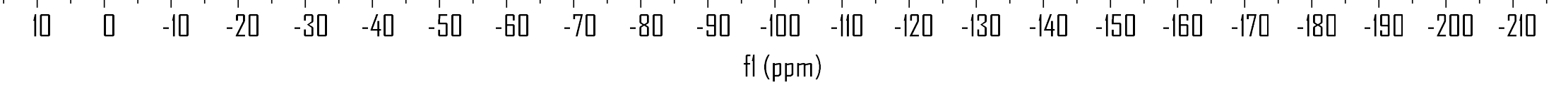


${ }^{13} \mathrm{C}\left\{{ }^{1} \mathrm{H}\right\}$ NMR spectrum of compound $1 \mathrm{~m}\left(126 \mathrm{MHz}, \mathrm{CDCl}_{3}\right)$

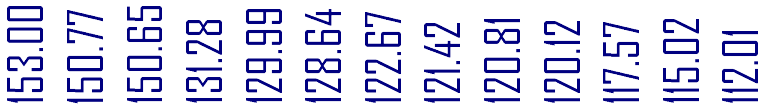

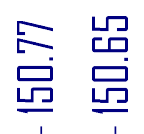

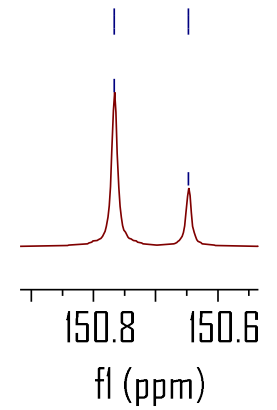

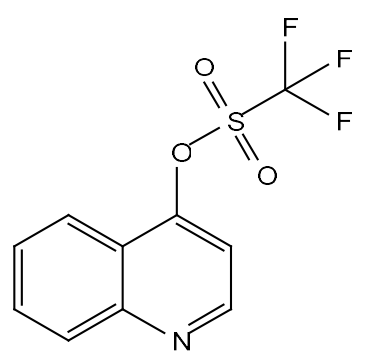

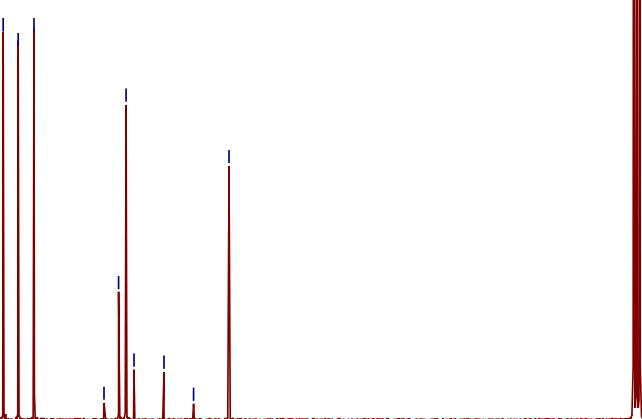

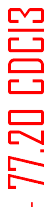
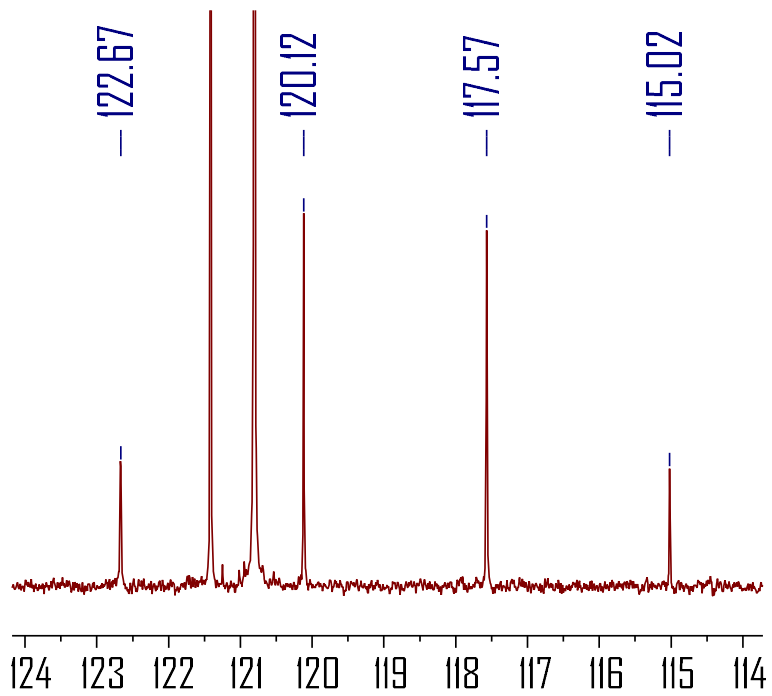

fl (ppm)

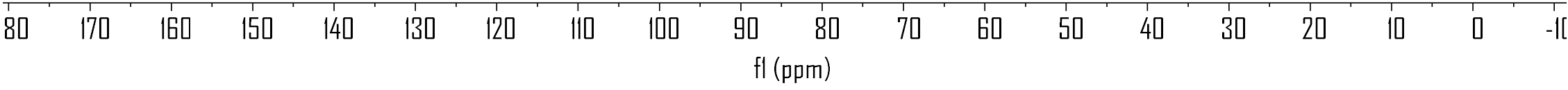


${ }^{1} \mathrm{H}$ NMR spectrum of compound $2 \mathrm{~b}\left(500 \mathrm{MHz}, \mathrm{CDCl}_{3}\right)$
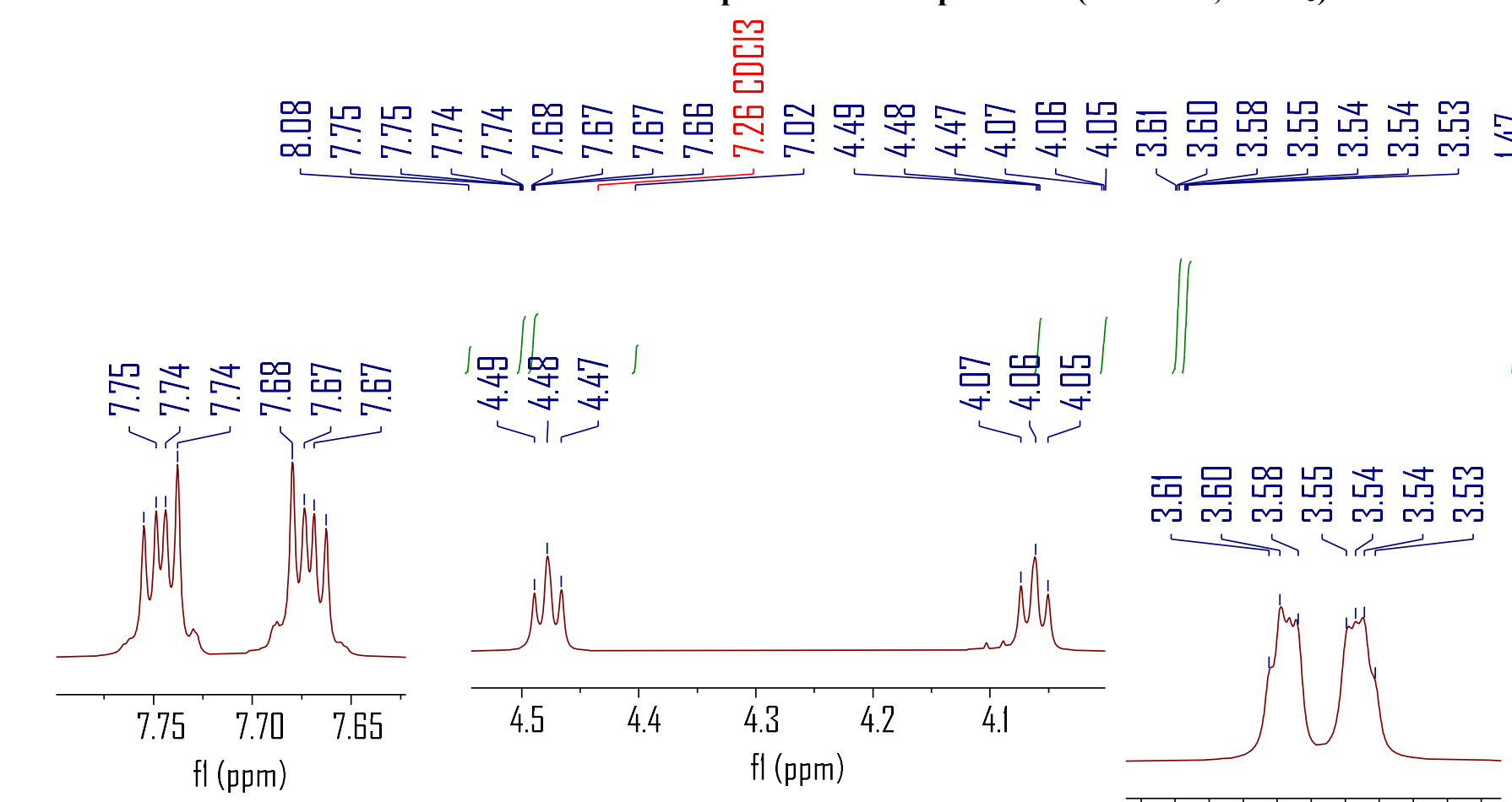

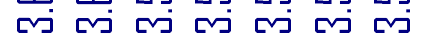
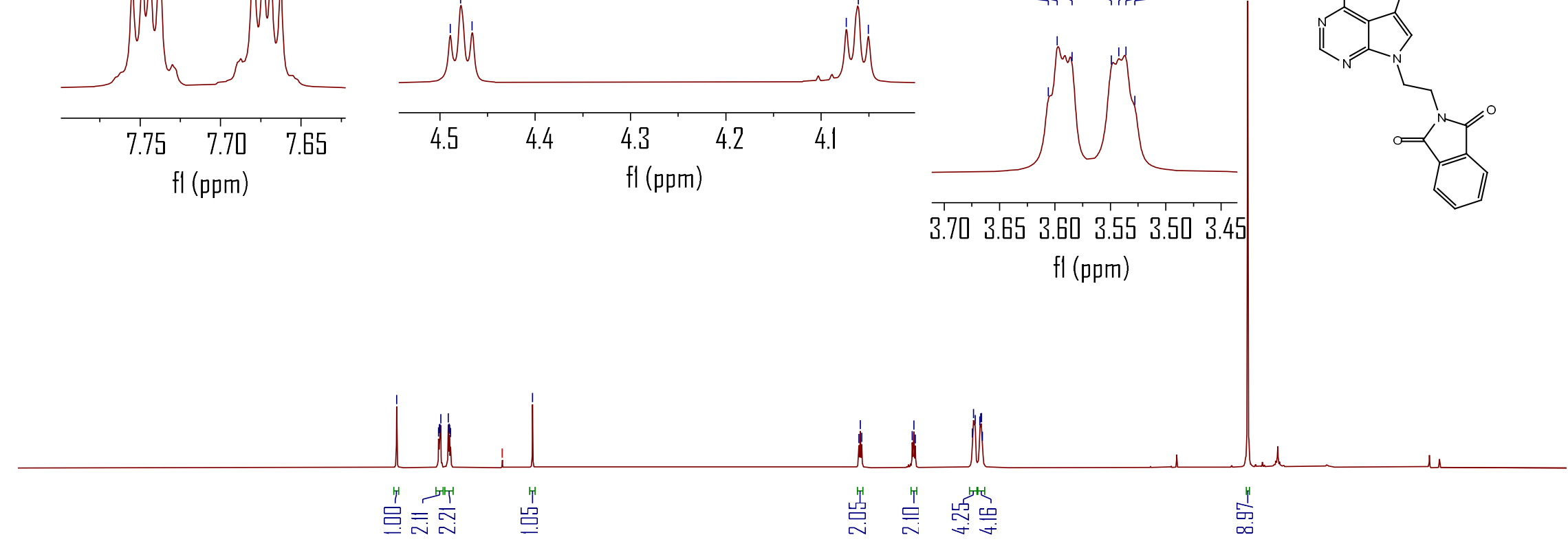

\begin{tabular}{lllllllllllllllllllllllllll}
\hline .0 & 10.5 & 10.0 & 9.5 & 9.0 & 8.5 & 8.0 & 7.5 & 7.0 & 6.5 & 6.0 & 5.5 & 5.0 & 4.5 & 4.0 & 3.5 & 3.0 & 2.5 & 2.0 & 1.5 & 1.0 & 0.5 & 0.0 & -0.5 & -1 \\
$\mathrm{fl}(\mathrm{ppm})$ & & & & &
\end{tabular} 
${ }^{13} \mathrm{C}\left\{{ }^{1} \mathrm{H}\right\}$ NMR spectrum of compound $2 \mathrm{~b}\left(126 \mathrm{MHz}, \mathrm{CDCl}_{3}\right)$

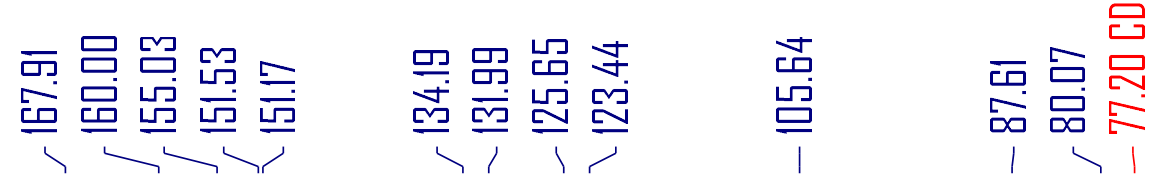

뭄 品 嵒

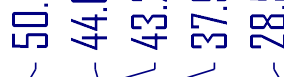

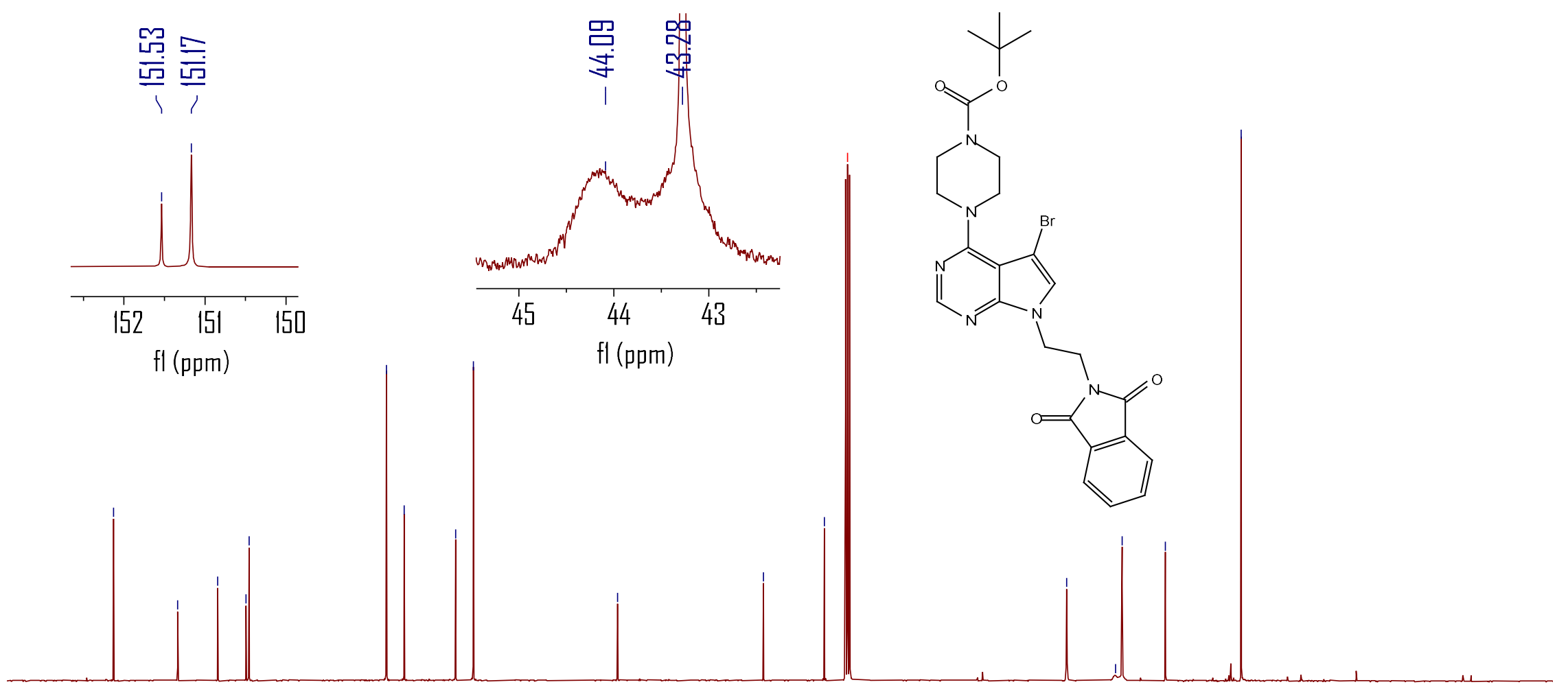

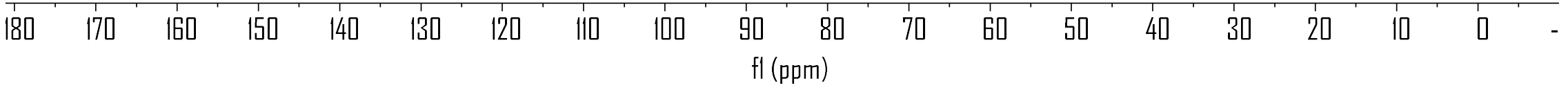


${ }^{1} \mathrm{H}$ NMR spectrum of compound $2 \mathrm{~d}\left(500 \mathrm{MHz}, \mathrm{CDCl}_{3}\right)$

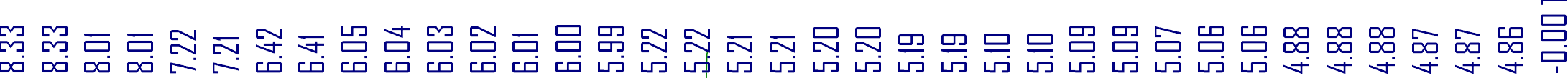

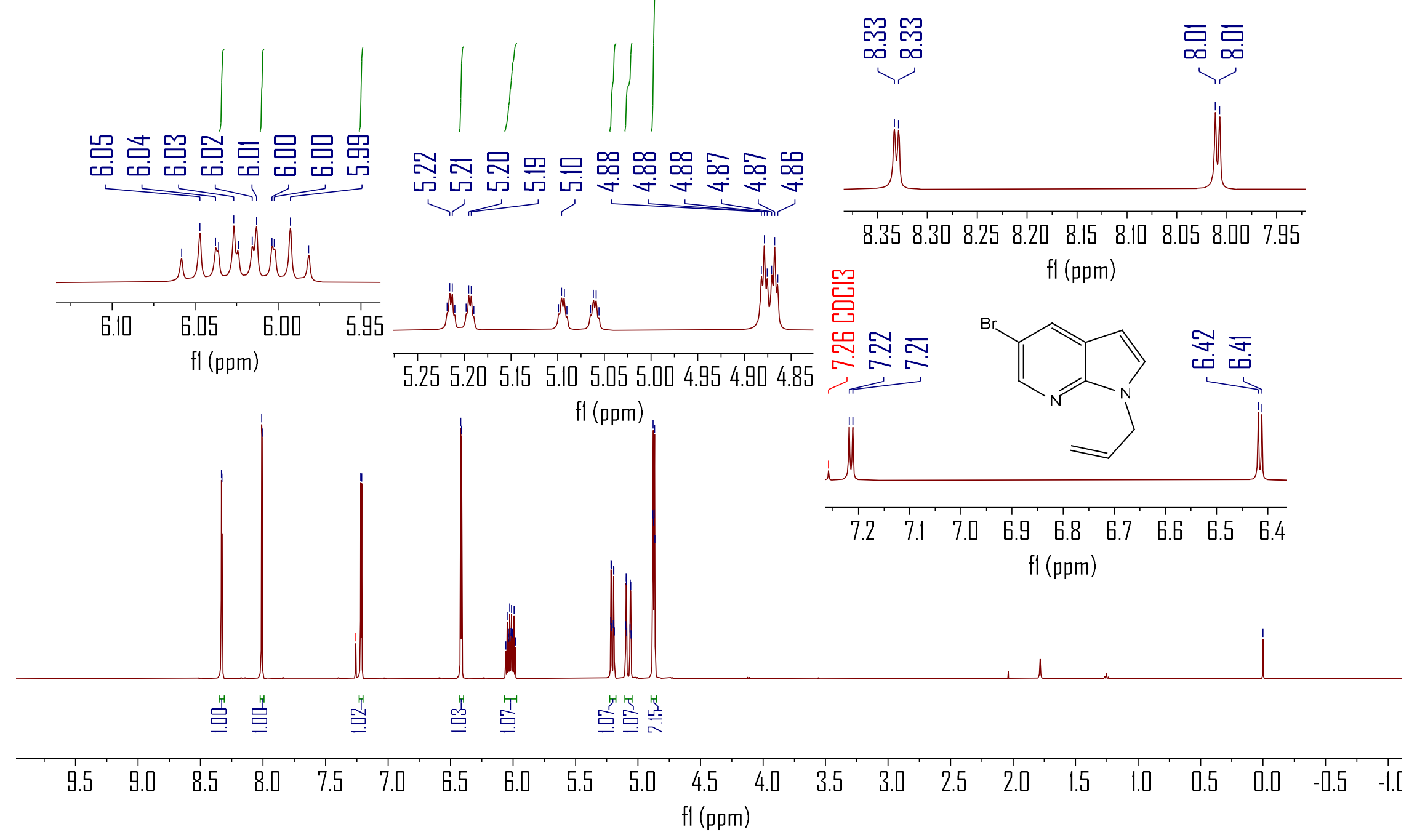


${ }^{13} \mathrm{C}\left\{{ }^{1} \mathrm{H}\right\}$ NMR spectrum of compound $2 \mathrm{~d}\left(126 \mathrm{MHz}, \mathrm{CDCl}_{3}\right)$

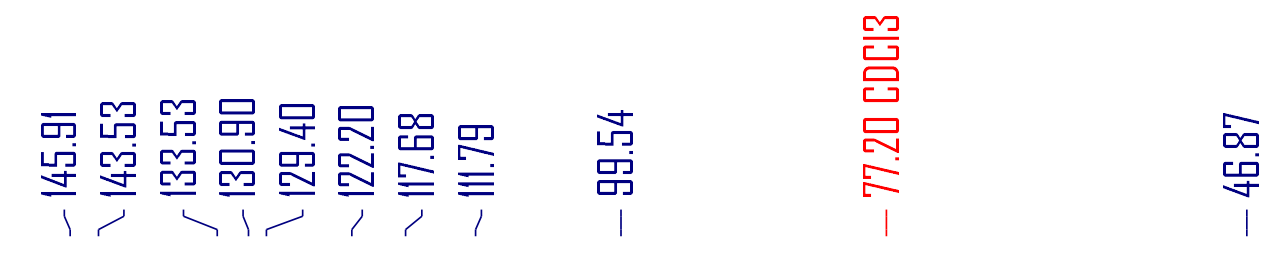

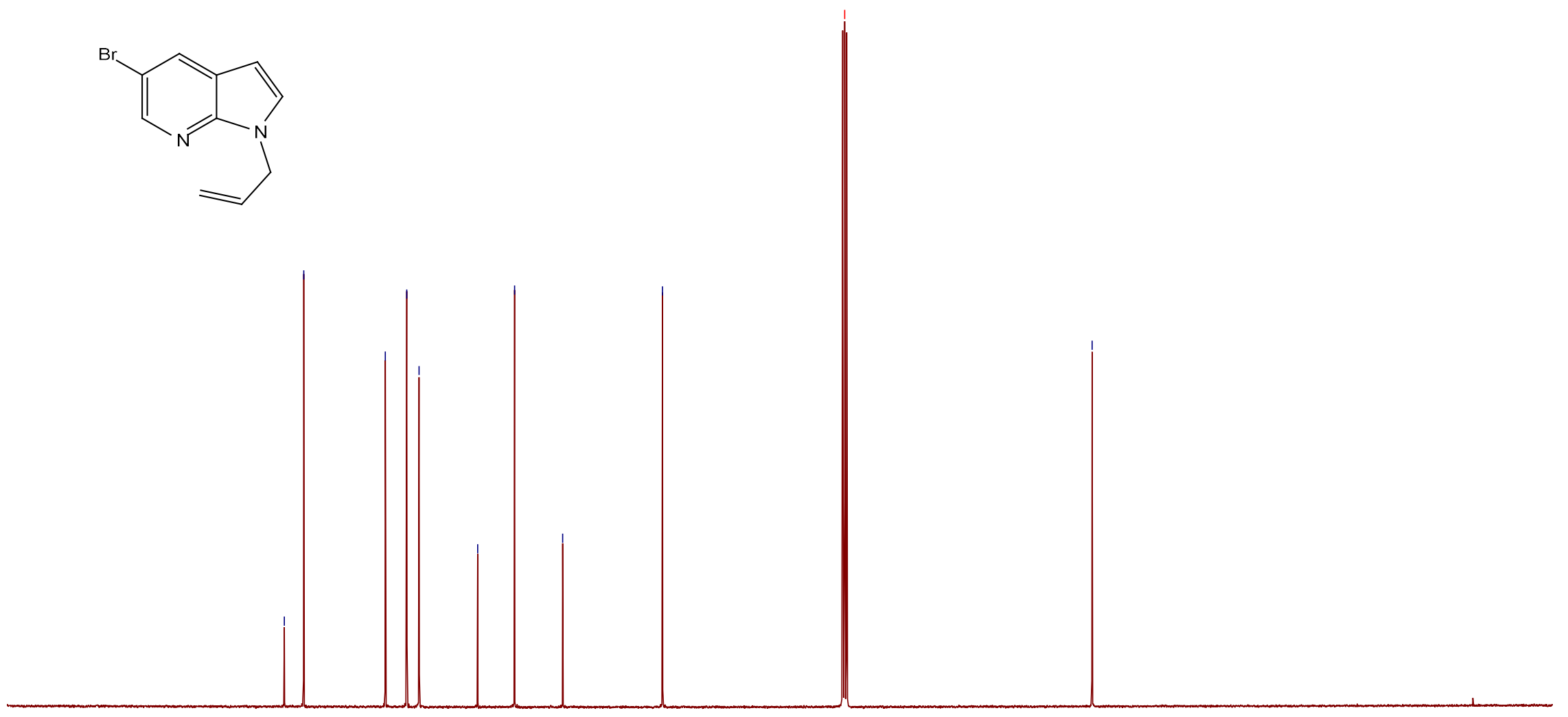

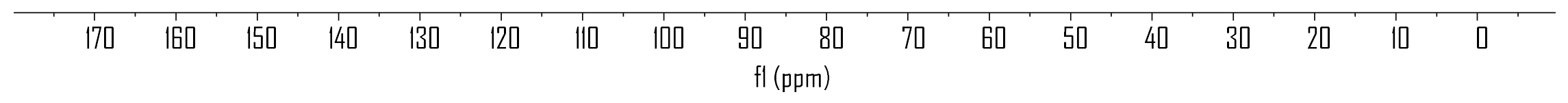




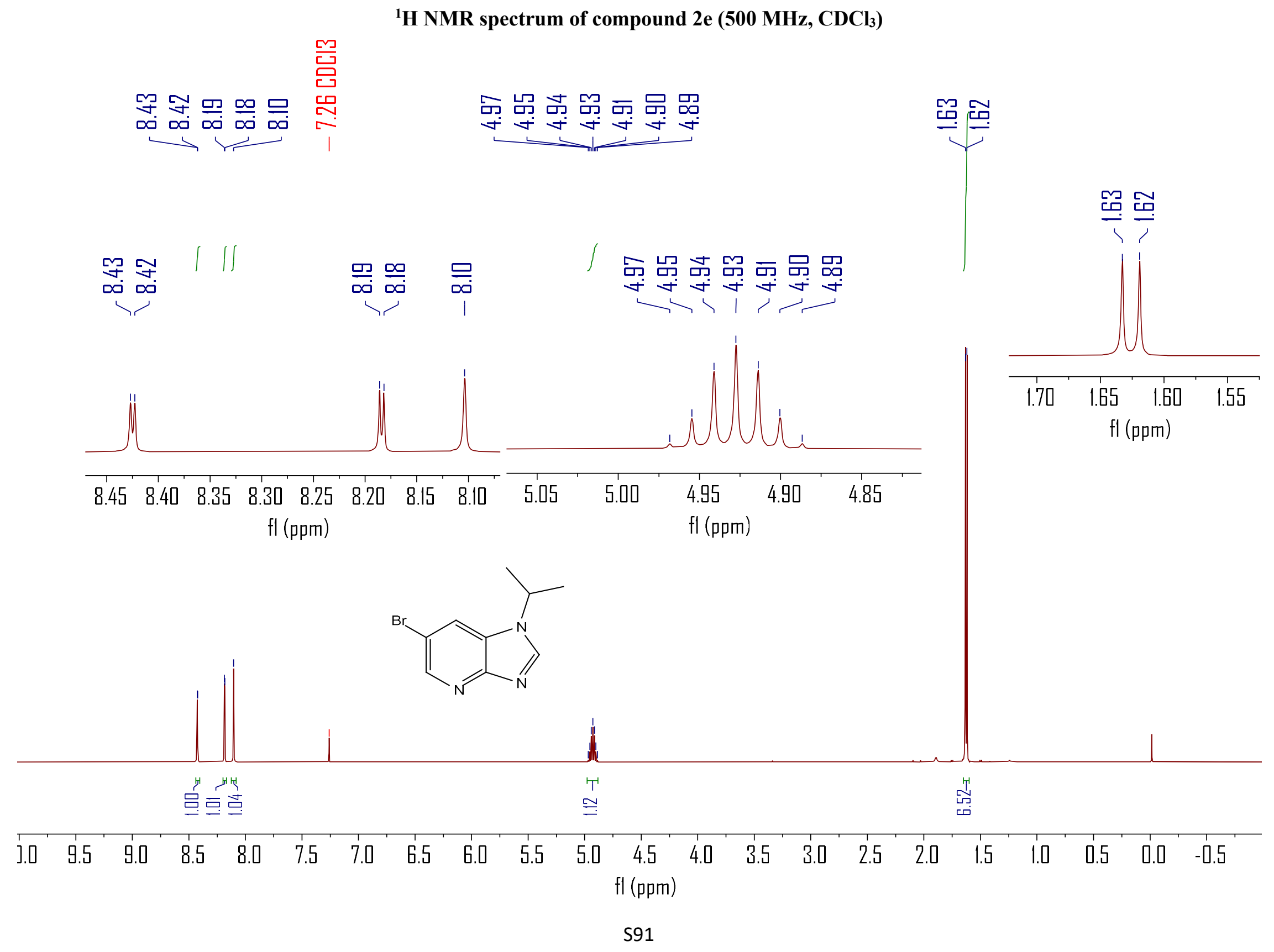




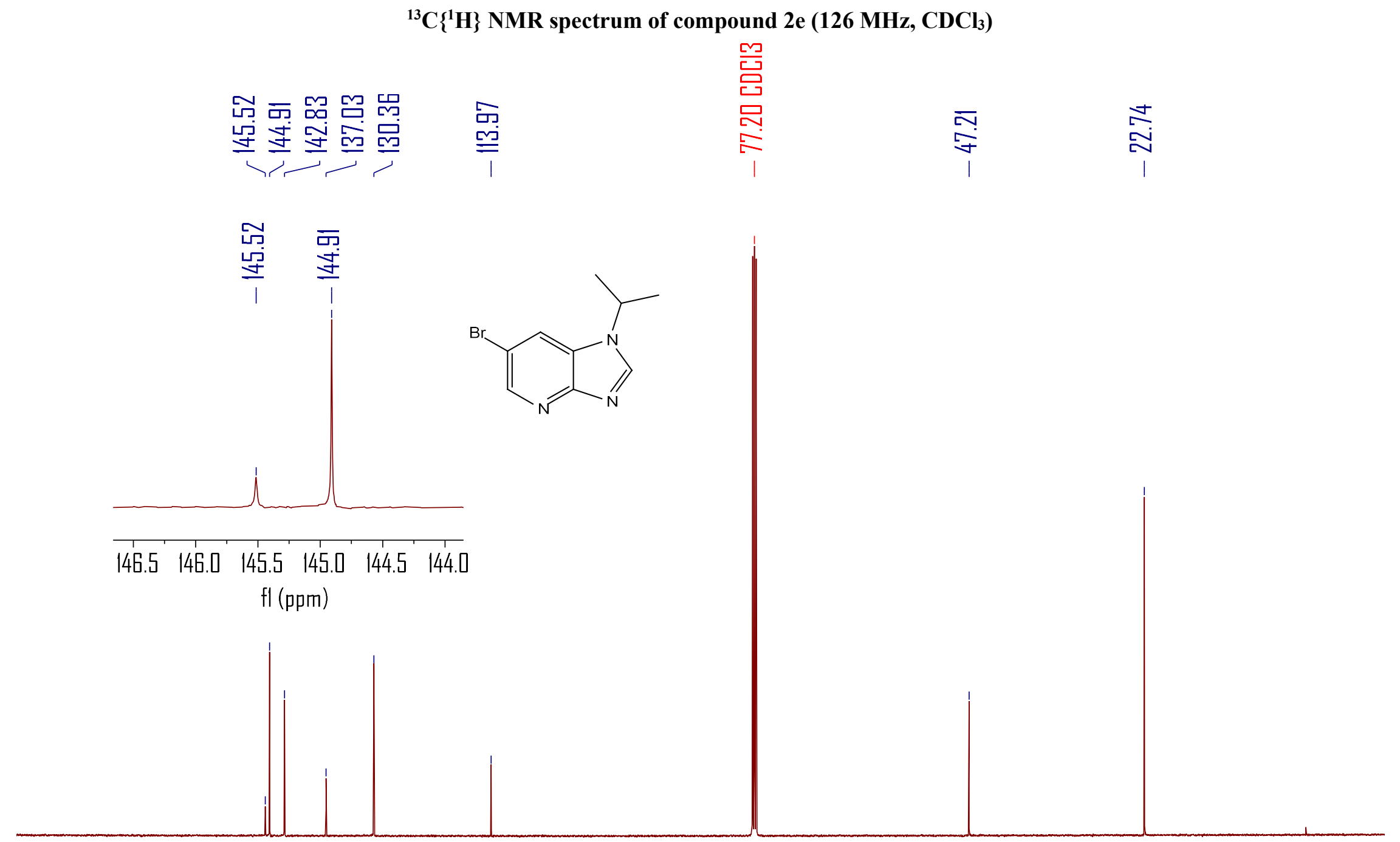

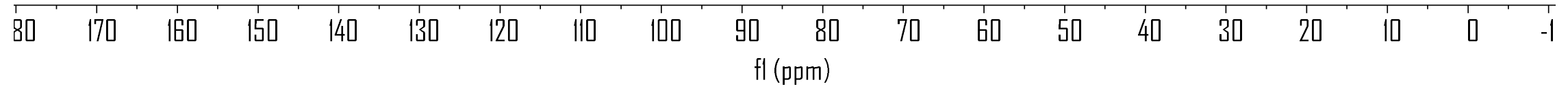


${ }^{1} \mathrm{H}$ NMR spectrum of compound 3a (500 $\left.\mathrm{MHz}, \mathrm{CDCl}_{3}\right)$

듬

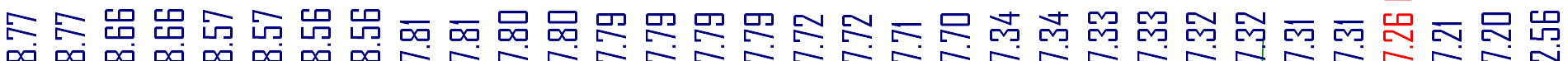

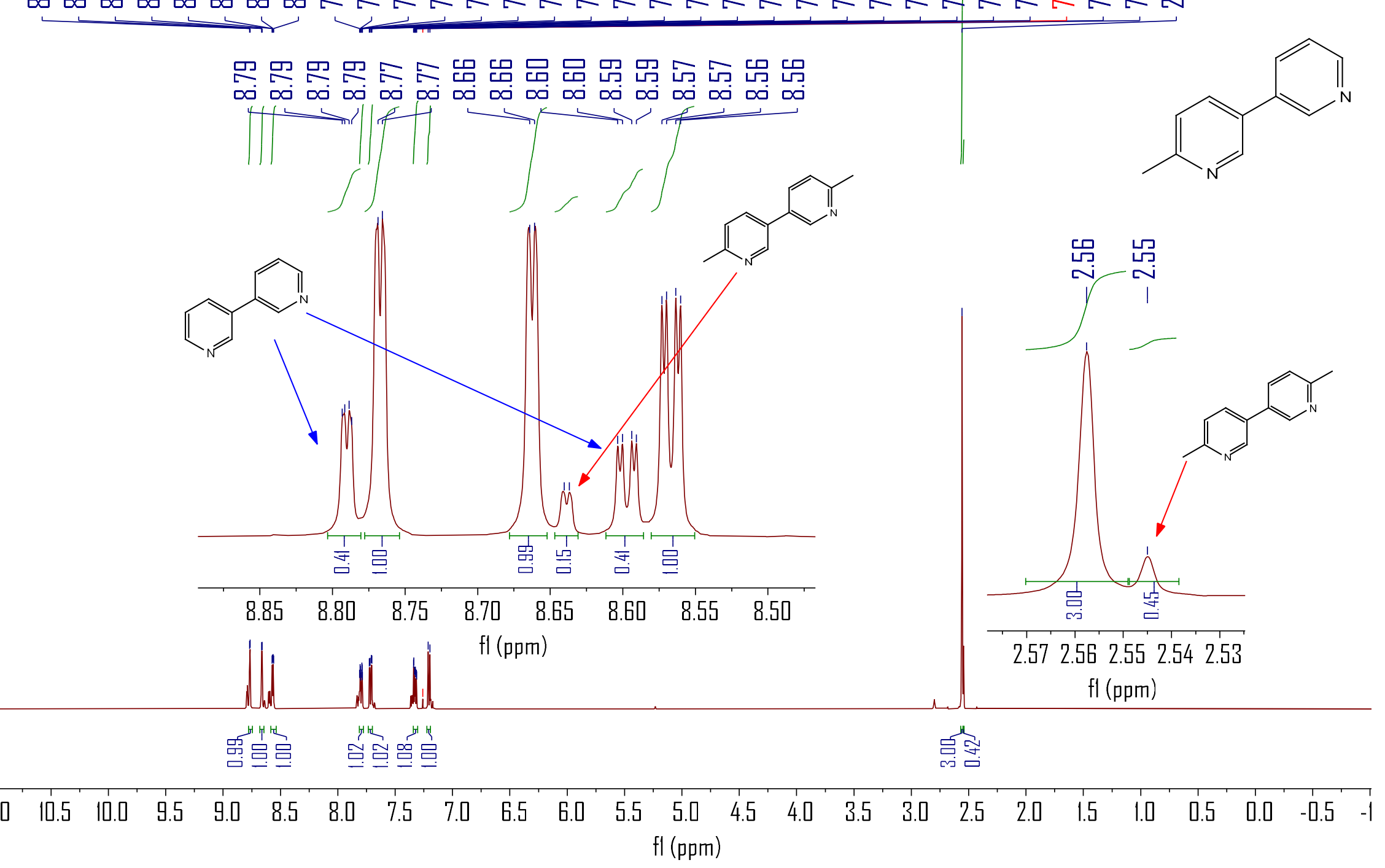




\section{${ }^{13} \mathrm{C}\left\{{ }^{1} \mathrm{H}\right\}$ NMR spectrum of compound 3a (126 MHz, $\left.\mathrm{CDCl}_{3}\right)$}

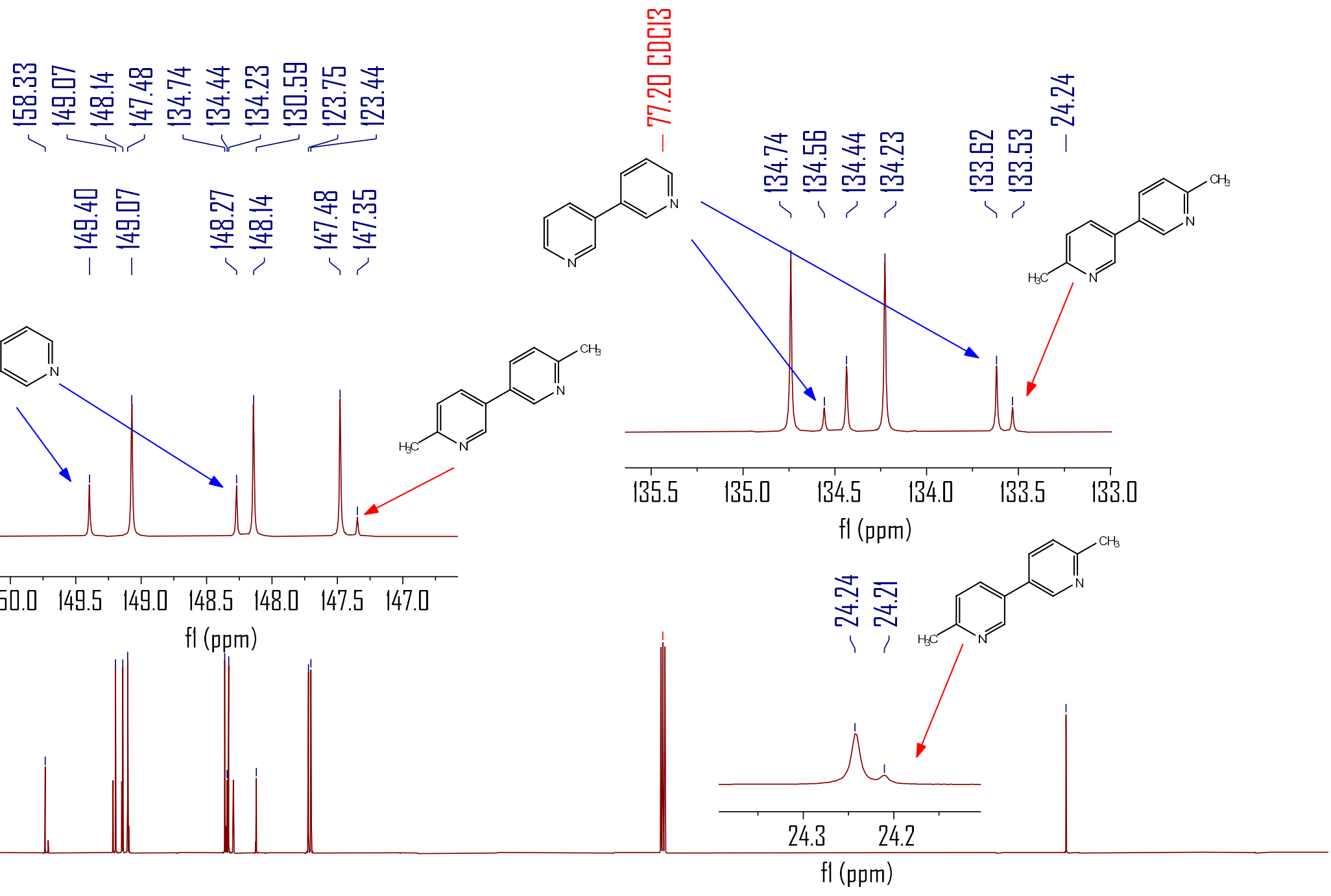

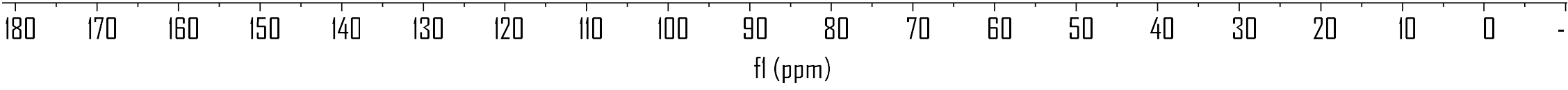


${ }^{1} \mathrm{H}$ NMR spectrum of compound $3 \mathrm{~b}\left(500 \mathrm{MHz}, \mathrm{CDCl}_{3}\right)$

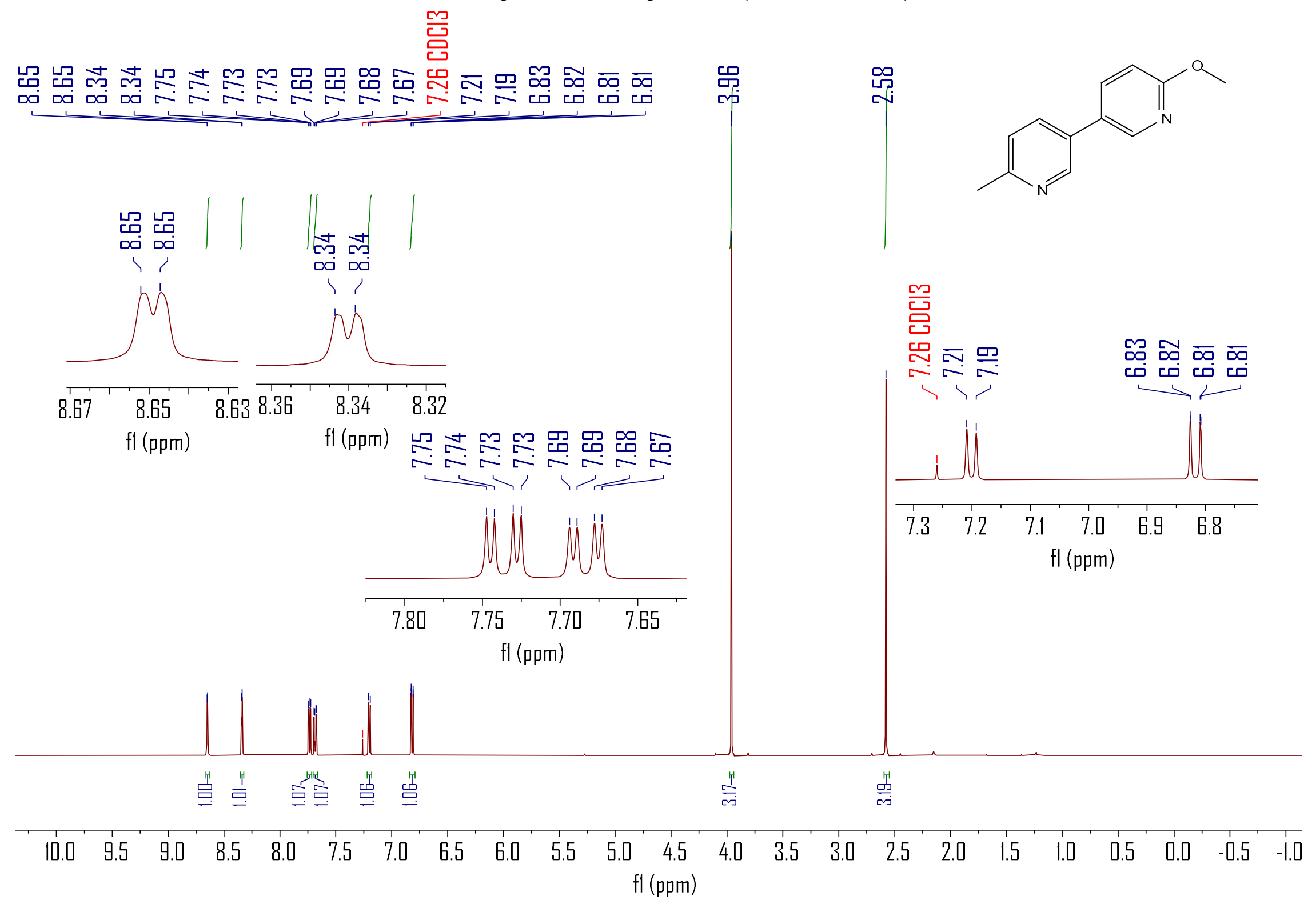




\section{${ }^{13} \mathrm{C}\left\{{ }^{1} \mathrm{H}\right\}$ NMR spectrum of compound $3 \mathrm{~b}\left(126 \mathrm{MHz}, \mathrm{CDCl}_{3}\right)$}

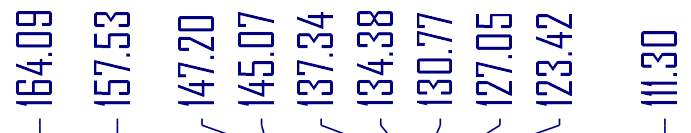

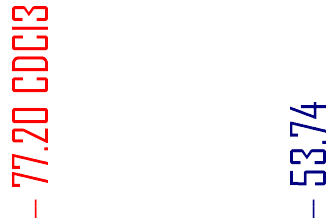

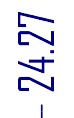
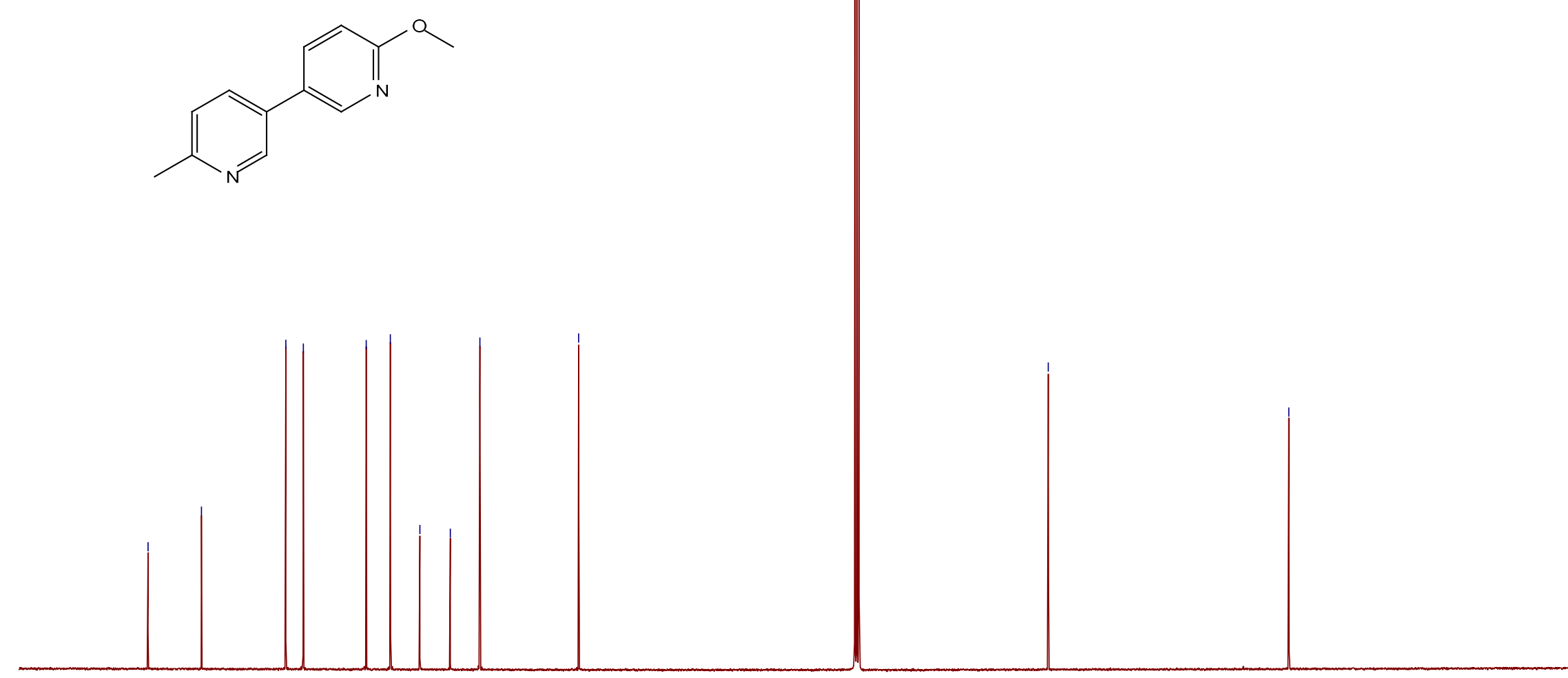

过

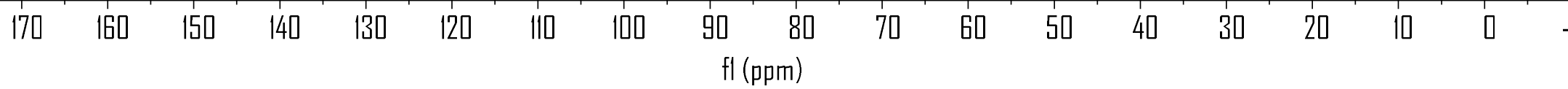


${ }^{1} \mathrm{H}$ NMR spectrum of compound $3 \mathrm{c}\left(500 \mathrm{MHz}, \mathrm{CDCl}_{3}\right)$

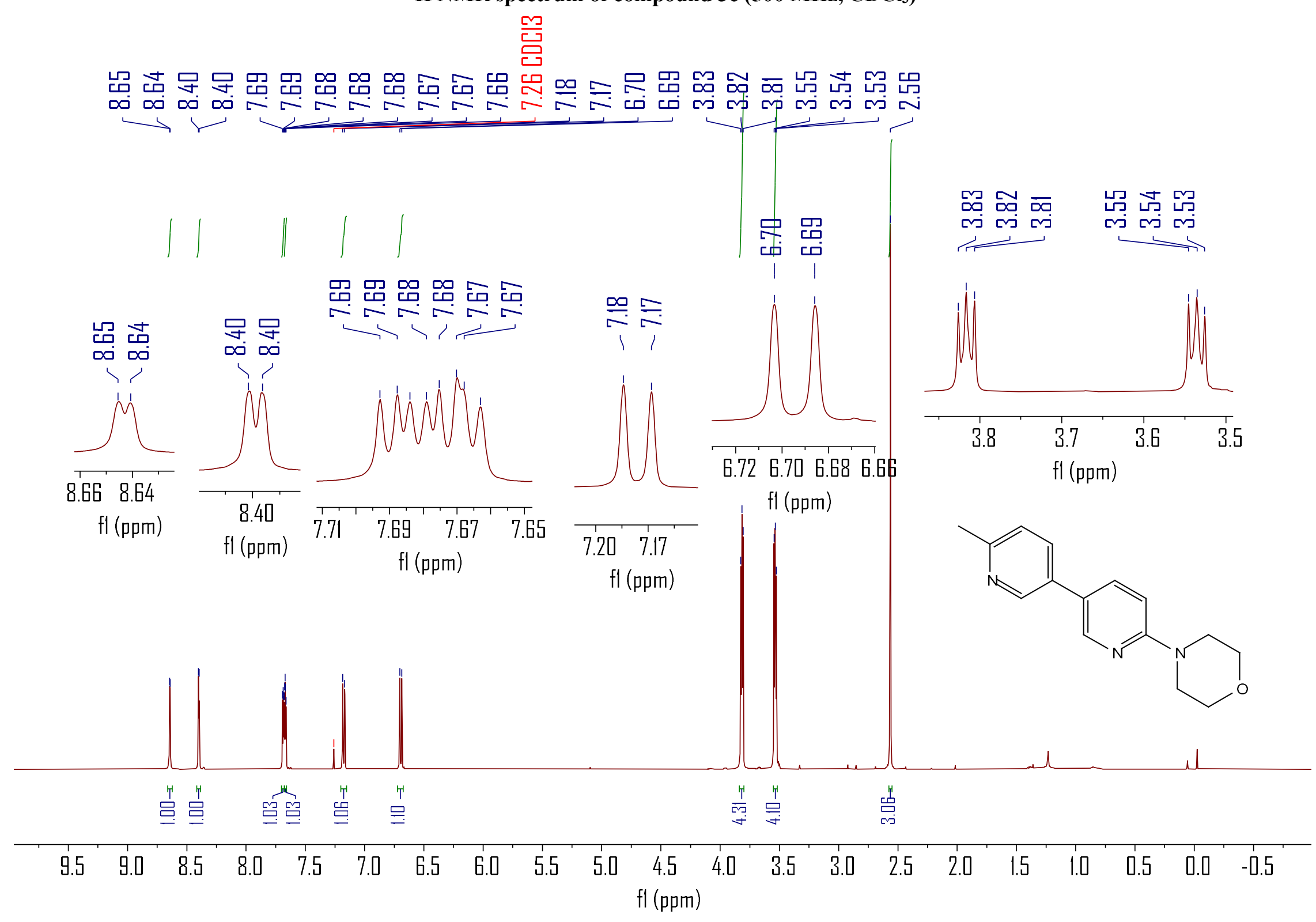


${ }^{13} \mathrm{C}\left\{{ }^{1} \mathrm{H}\right\}$ NMR spectrum of compound $3 \mathrm{c}\left(126 \mathrm{MHz}, \mathrm{CDCl}_{3}\right)$

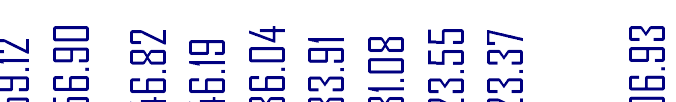

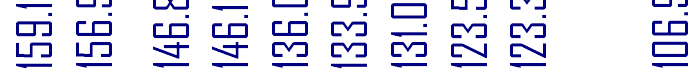

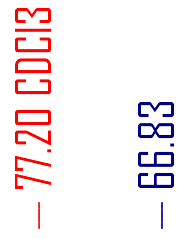

$\begin{array}{ll}\text { 品 } & \text { 号 } \\ \text { 号 } & \text { । }\end{array}$

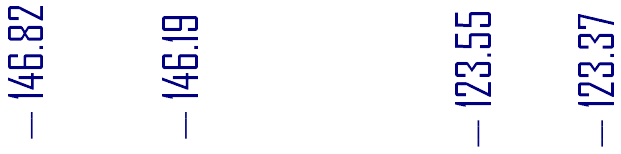
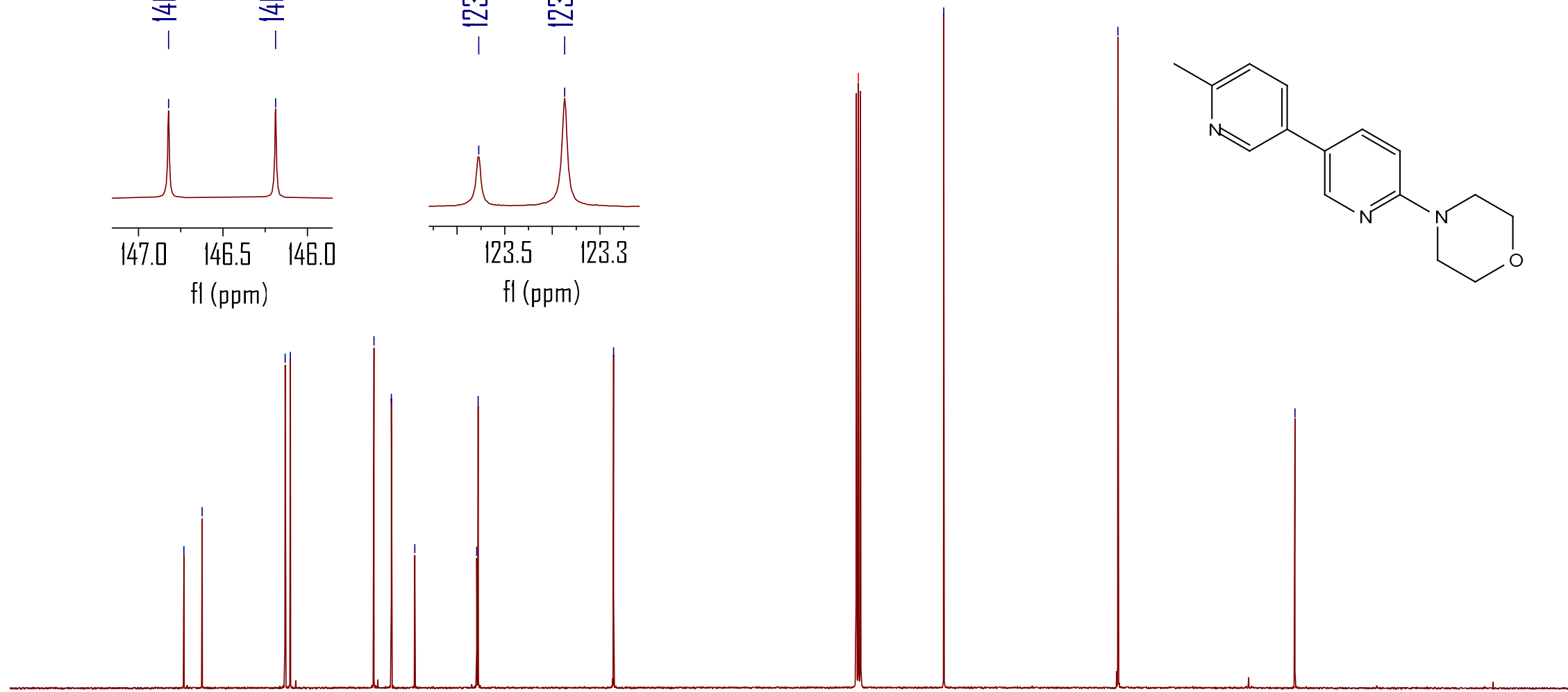

\section{0}

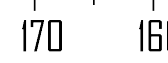

150

$140 \quad 130$

110

fl $(\mathrm{ppm})$

$70 \quad 60$ 


\section{${ }^{1} \mathrm{H}$ NMR spectrum of compound $3 \mathrm{~d}\left(500 \mathrm{MHz}, \mathrm{CDCl}_{3}\right)$}

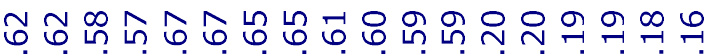
$\infty \infty^{\infty} \infty \pi N N$
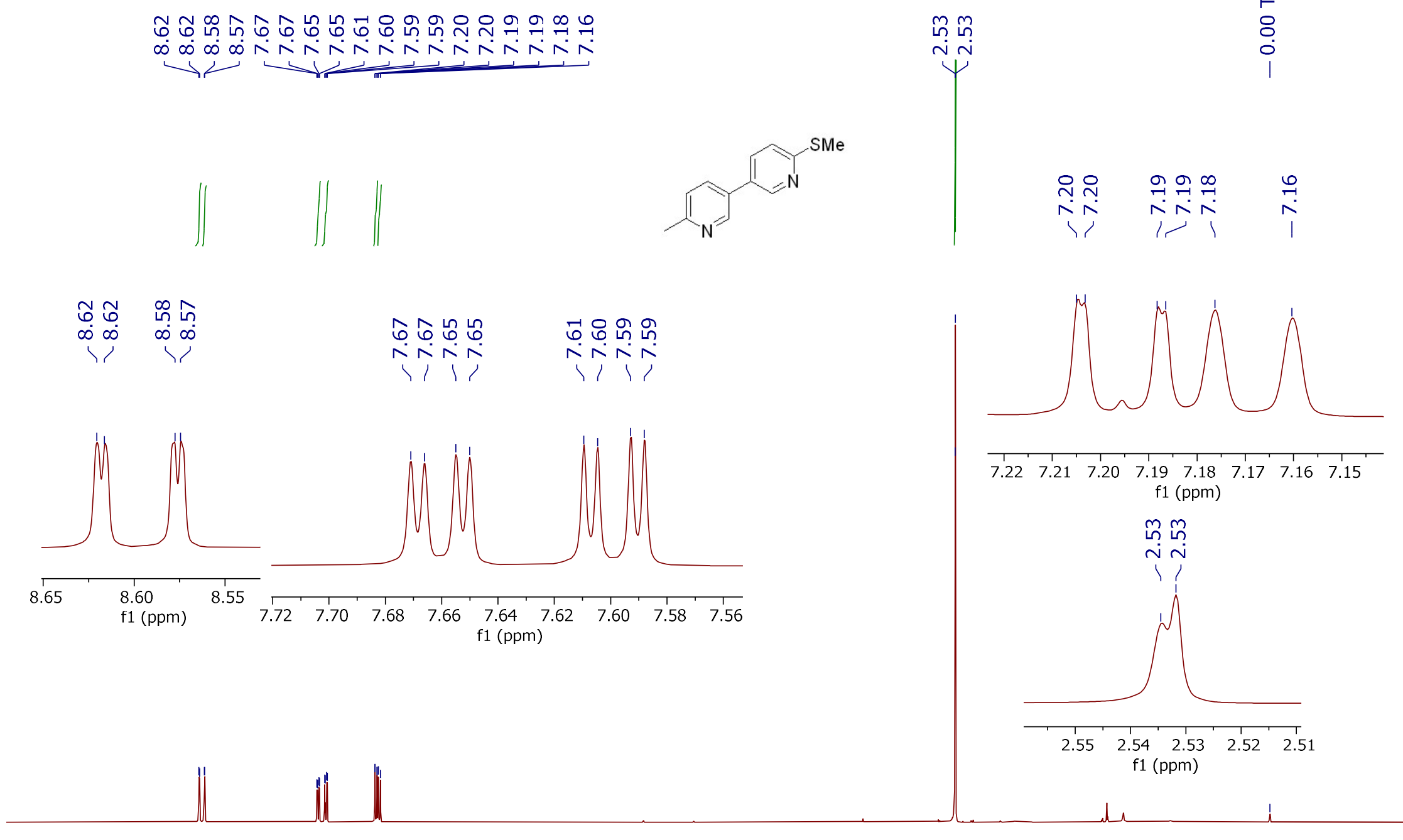

f1 (ppm)
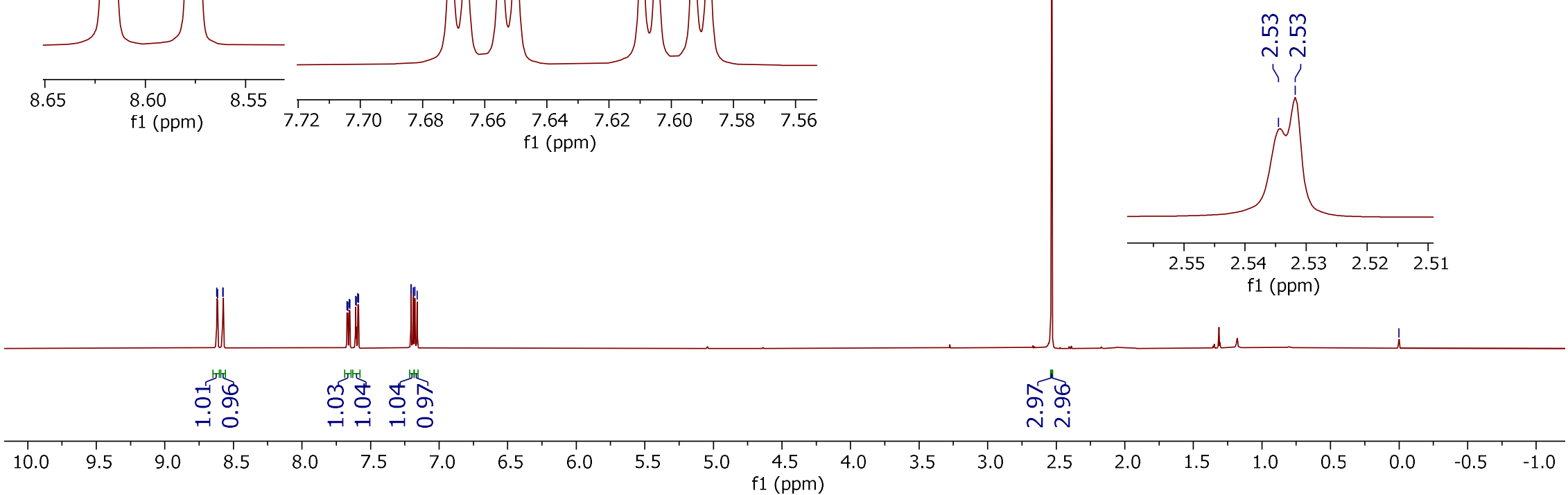


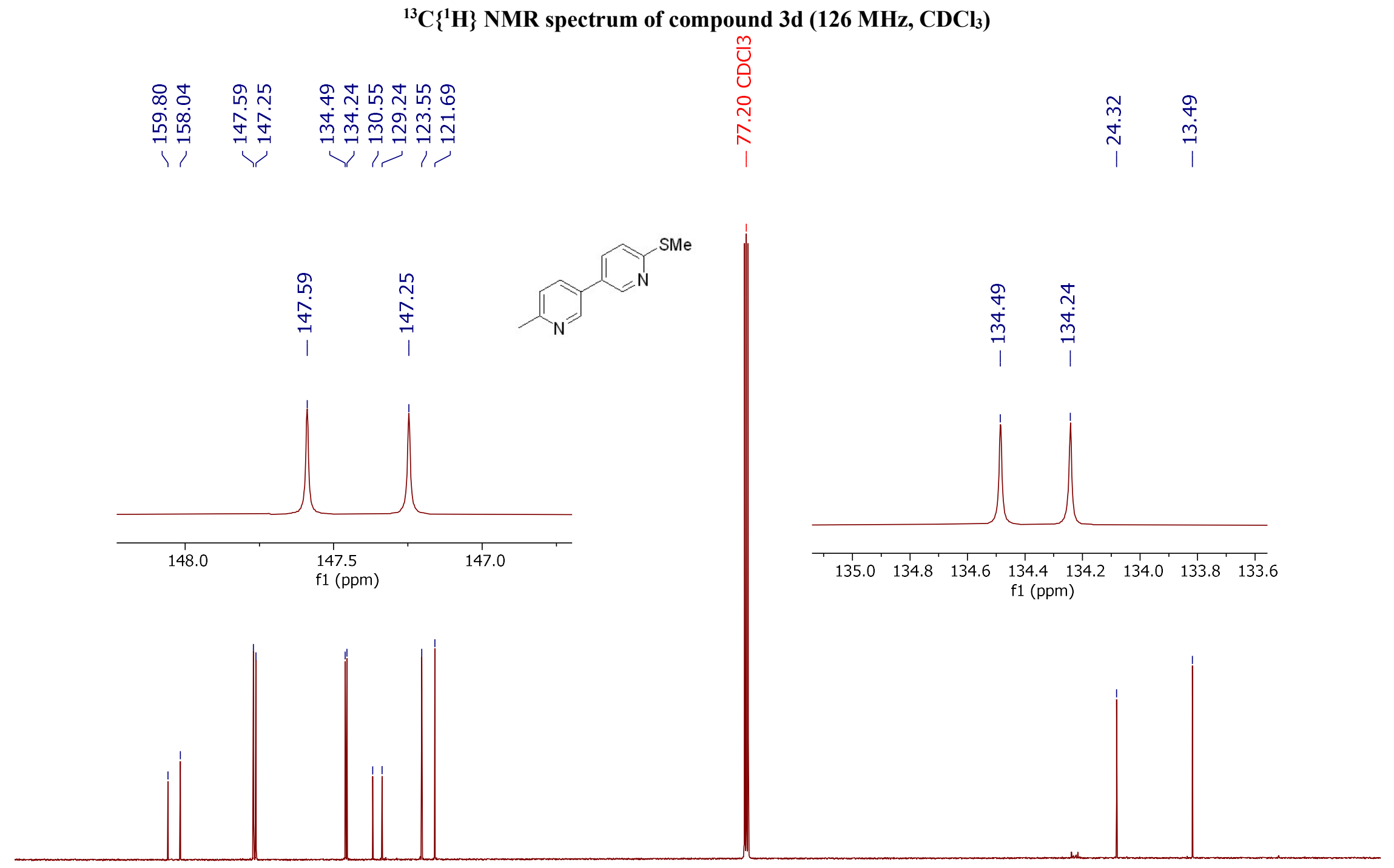




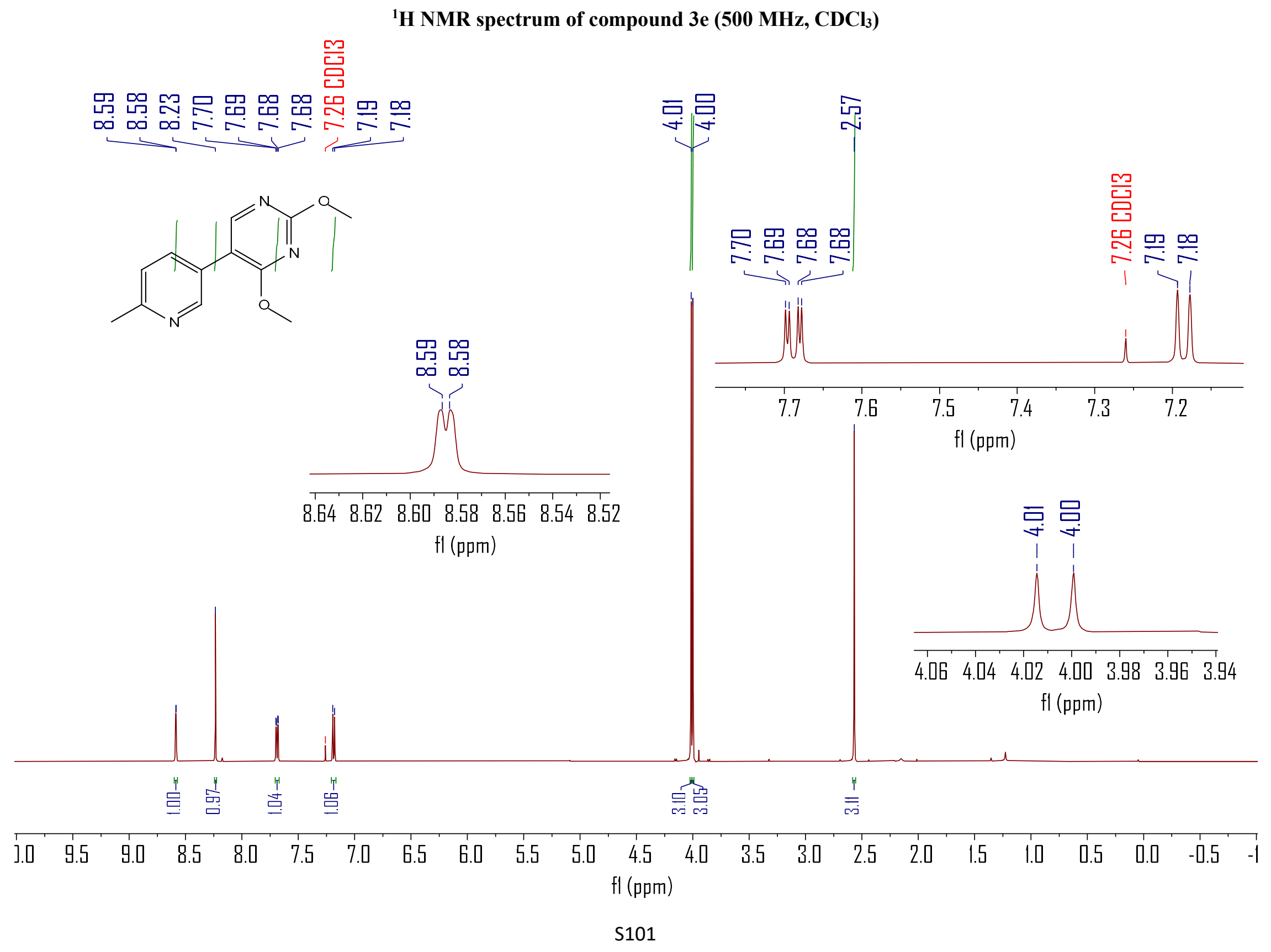




\section{${ }^{13} \mathrm{C}\left\{{ }^{1} \mathrm{H}\right\}$ NMR spectrum of compound $3 \mathrm{e}\left(126 \mathrm{MHz}, \mathrm{CDCl}_{3}\right)$}

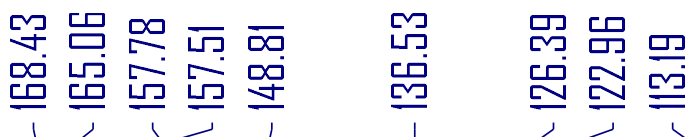
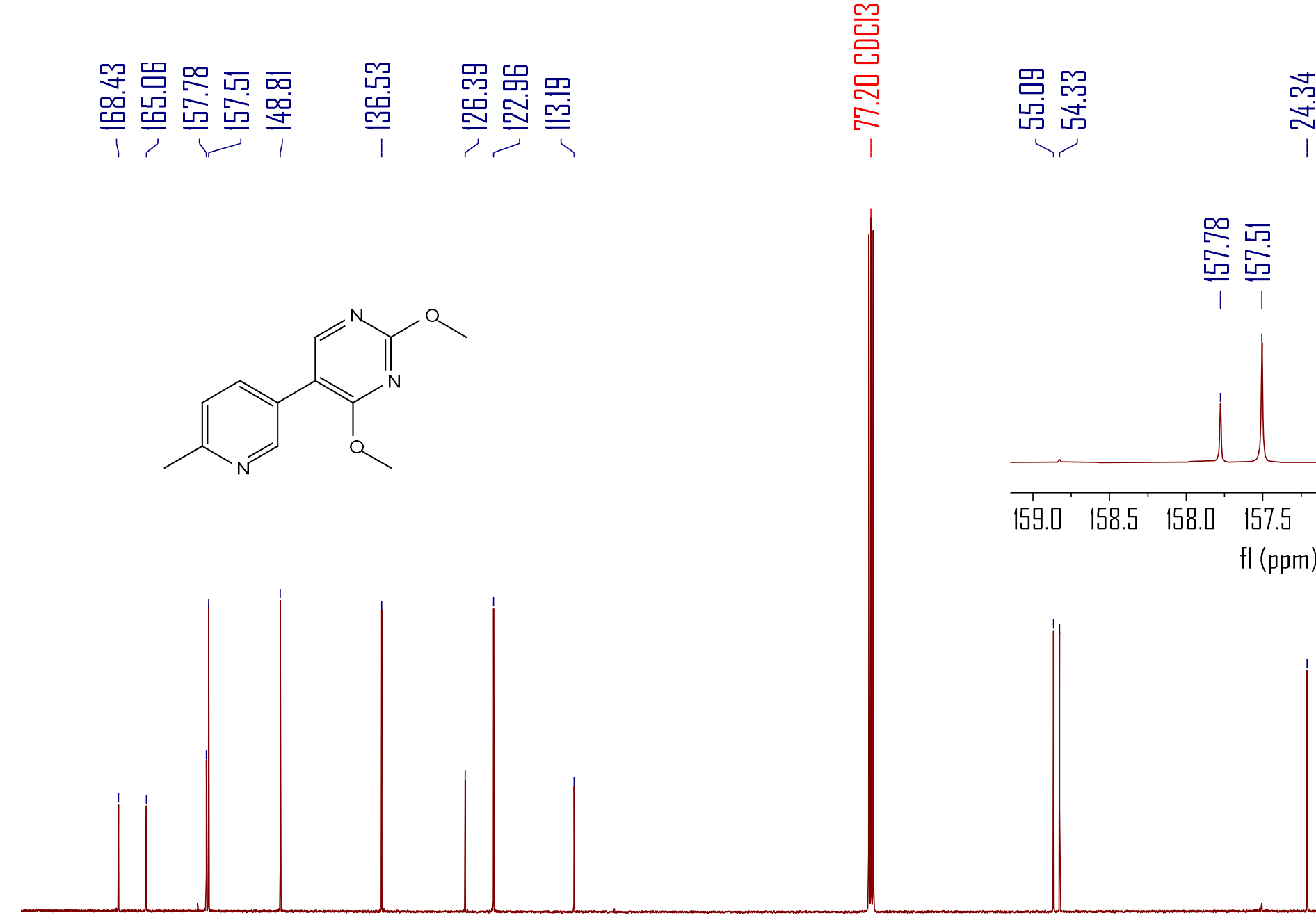

曲

돈도
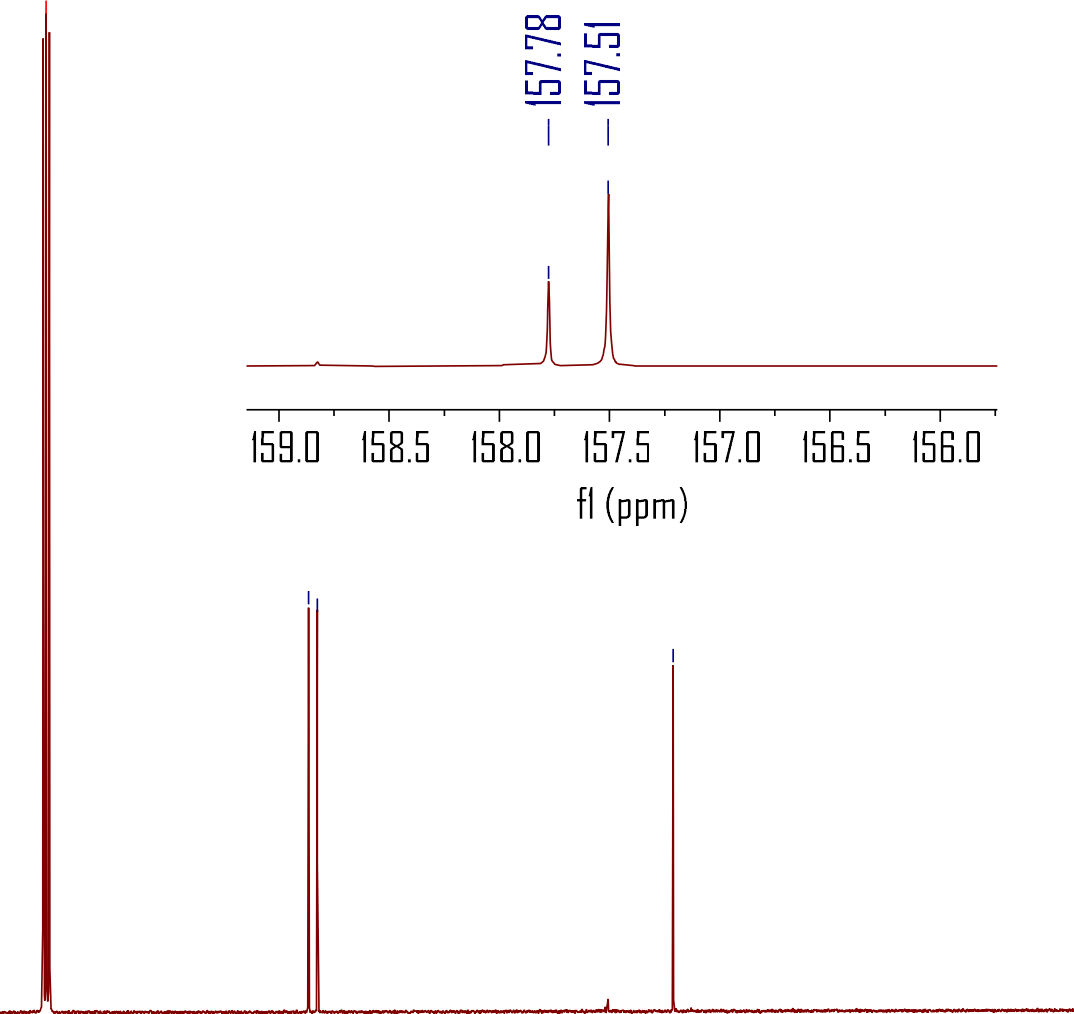

fl (ppm)

志

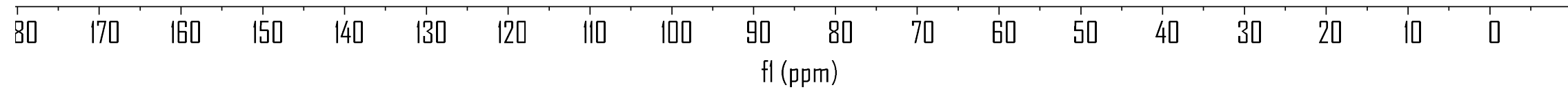


${ }^{1} \mathrm{H}$ NMR spectrum of compound $3 \mathrm{f}\left(500 \mathrm{MHz}, \mathrm{CDCl}_{3}\right)$

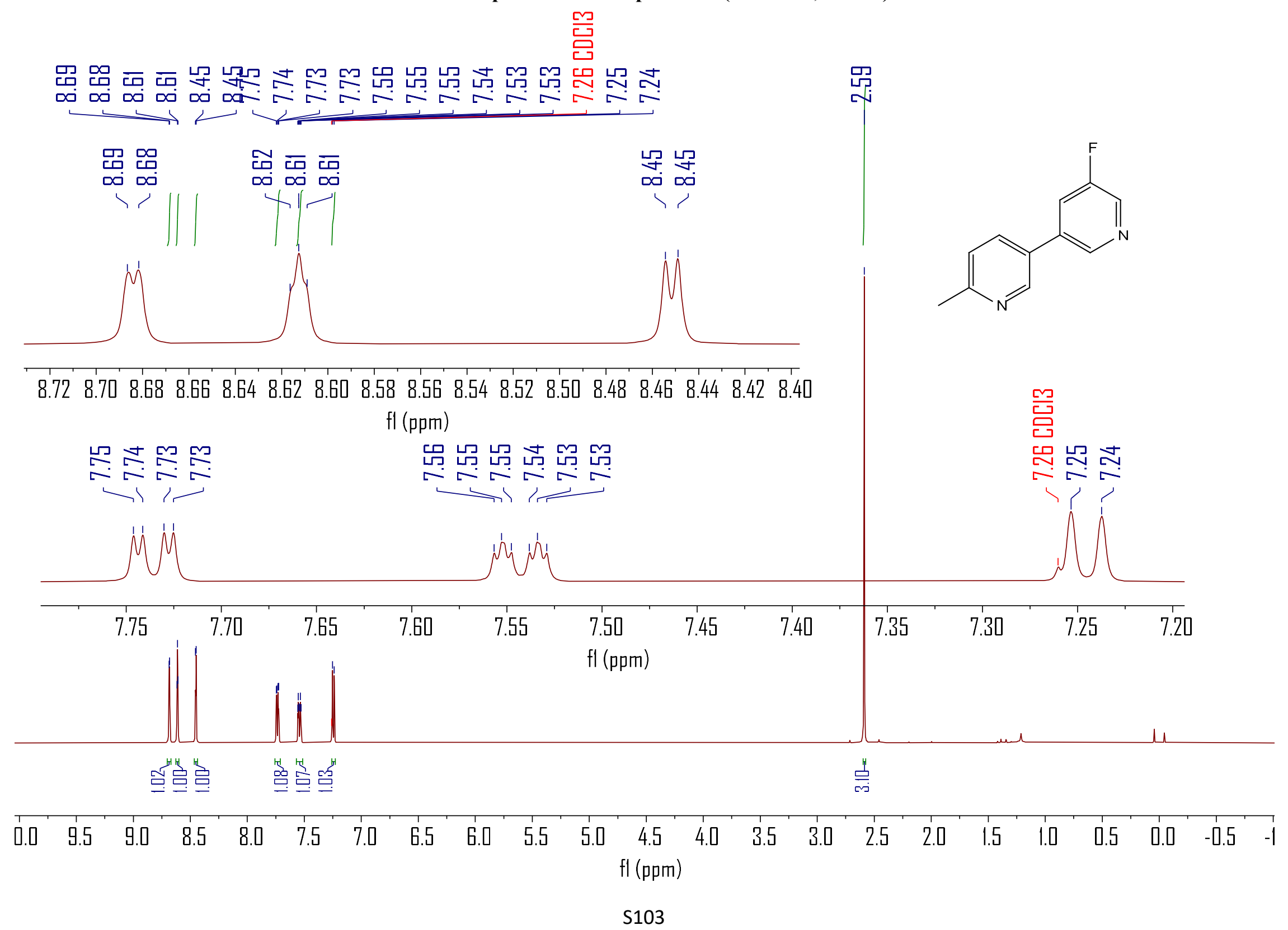


${ }^{19} \mathrm{~F}\left\{{ }^{1} \mathrm{H}\right\}$ NMR spectrum of compound $3 f\left(376 \mathrm{MHz}, \mathrm{CDCl}_{3}\right)$

品

뜸

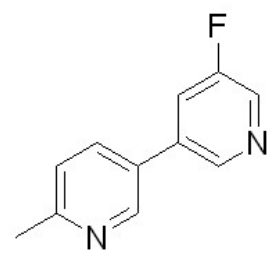

।

$10 \begin{array}{llllllllllllllllllllll}0 & -10 & -20 & -30 & -40 & -50 & -60 & -70 & -80 & -90 & -100 & -110 & -120 & -130 & -140 & -150 & -160 & -170 & -180 & -190 & -200 & -210\end{array}$ $\mathrm{fl}(\mathrm{ppm})$ 


\section{${ }^{13} \mathrm{C}\left\{{ }^{1} \mathrm{H}\right\}$ NMR spectrum of compound $3 \mathrm{f}\left(126 \mathrm{MHz}, \mathrm{CDCl}_{3}\right)$}

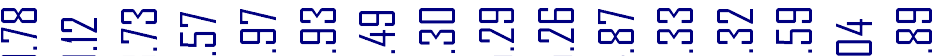

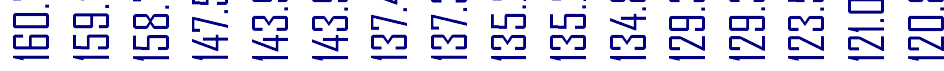

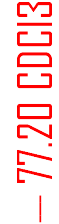
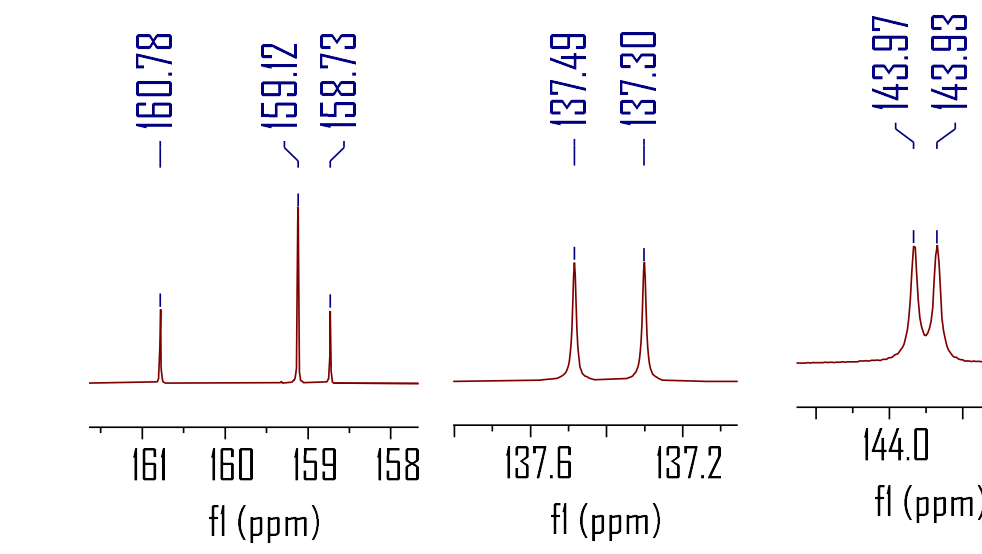

뜨

品品

泀 品
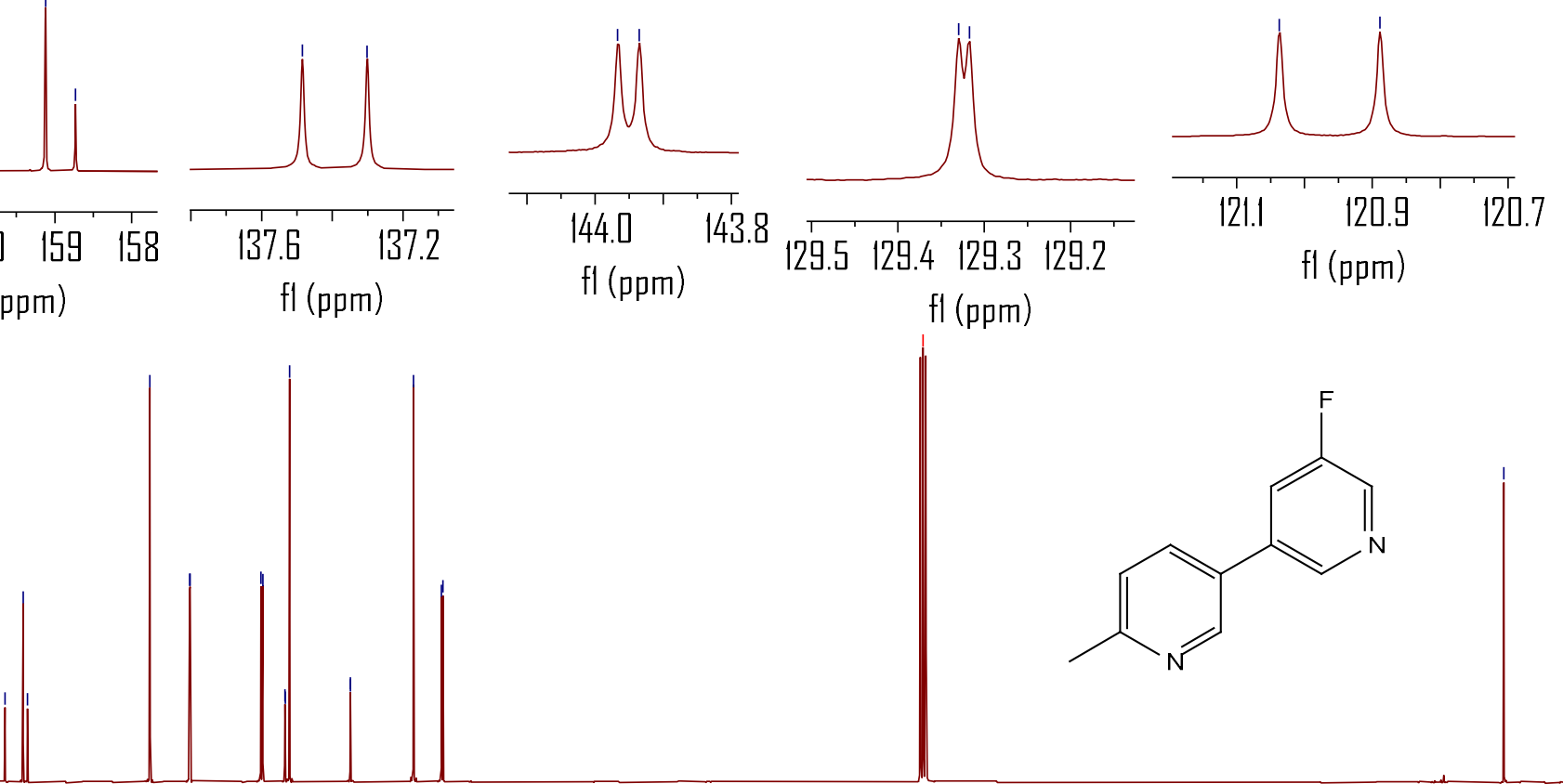

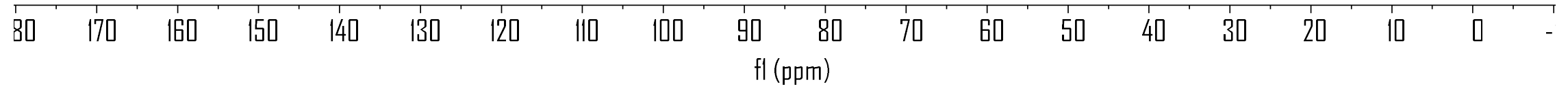




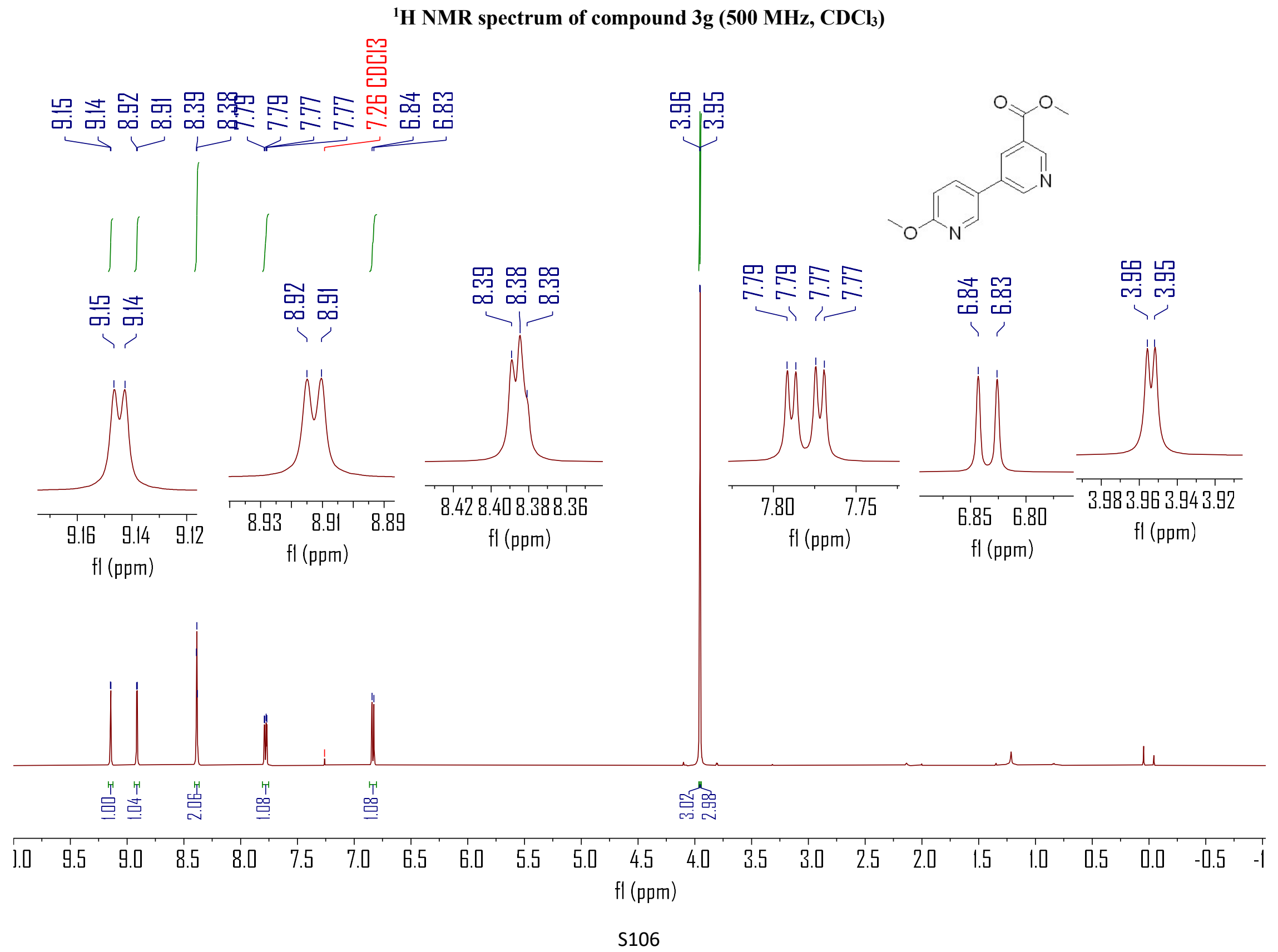




\section{${ }^{13} \mathrm{C}\left\{{ }^{1} \mathrm{H}\right\}$ NMR spectrum of compound $3 \mathrm{~g}\left(126 \mathrm{MHz}, \mathrm{CDCl}_{3}\right)$}

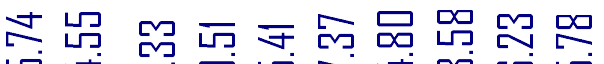

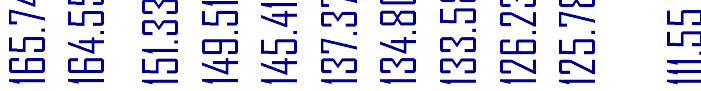

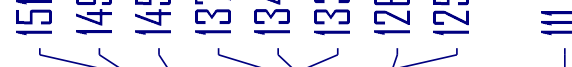

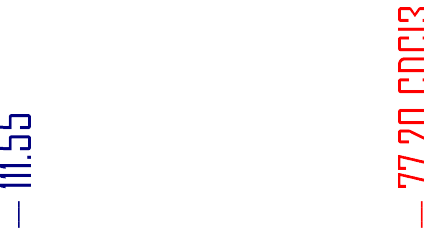

品

贸 出

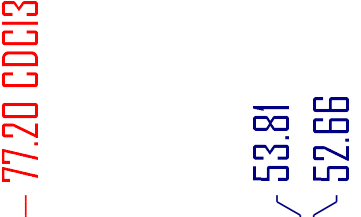

$\sqrt{x}$

뜸 㟧

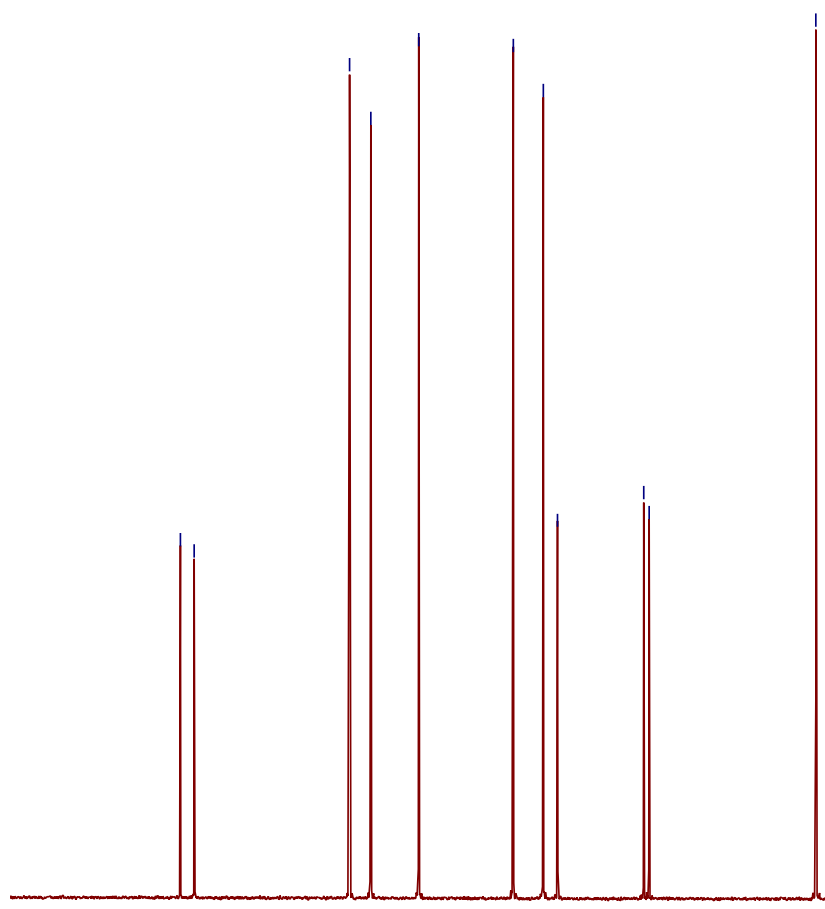

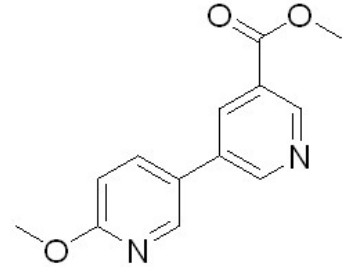

.

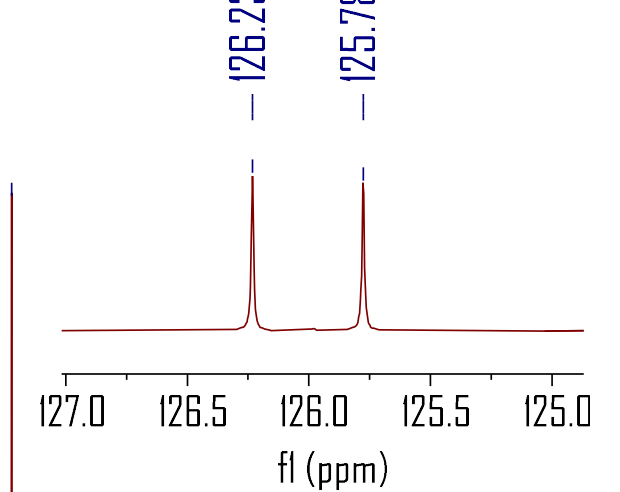


${ }^{1} \mathrm{H}$ NMR spectrum of compound $3 \mathrm{~h}\left(500 \mathrm{MHz}, \mathrm{CDCl}_{3}\right)$

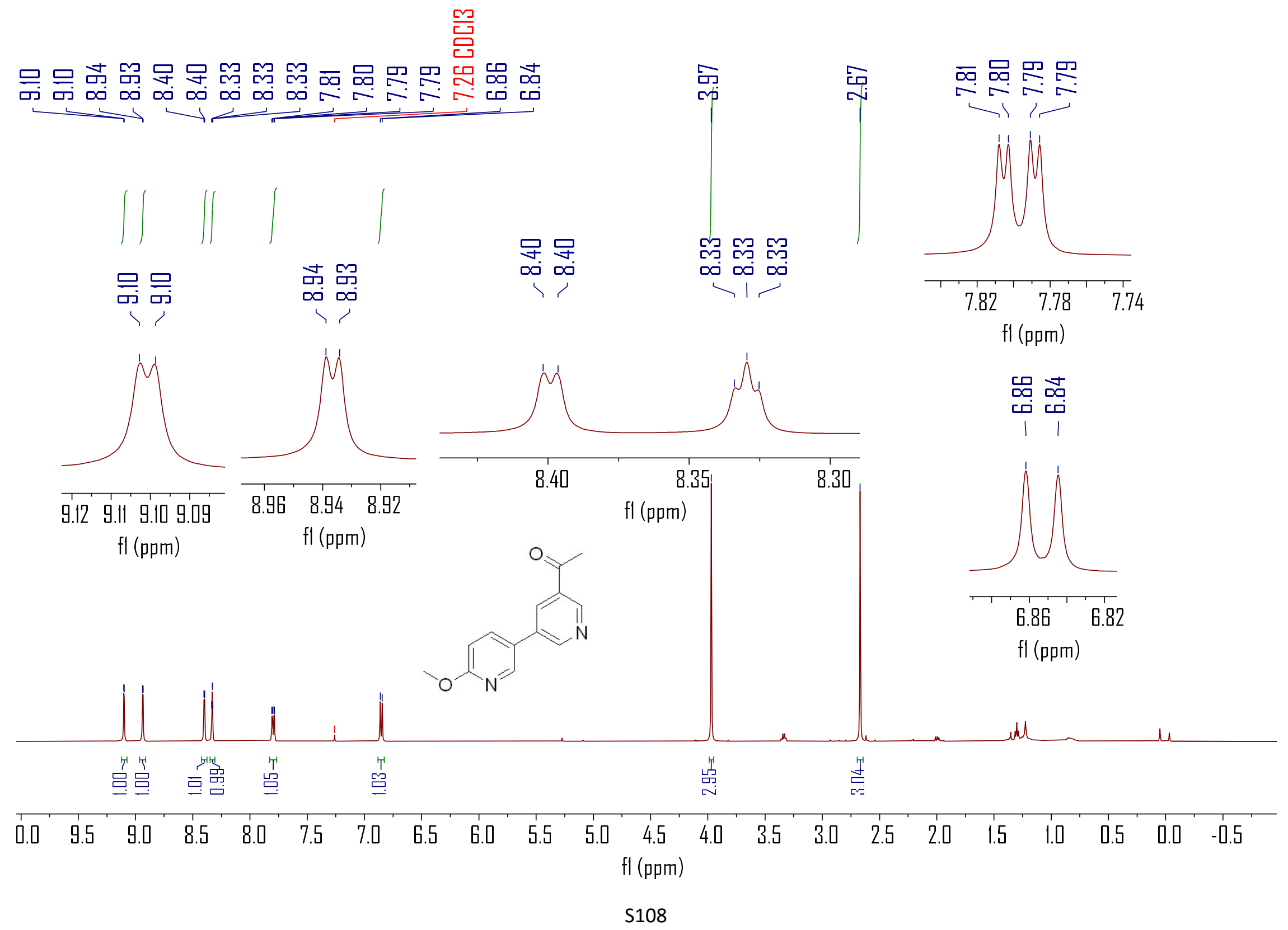




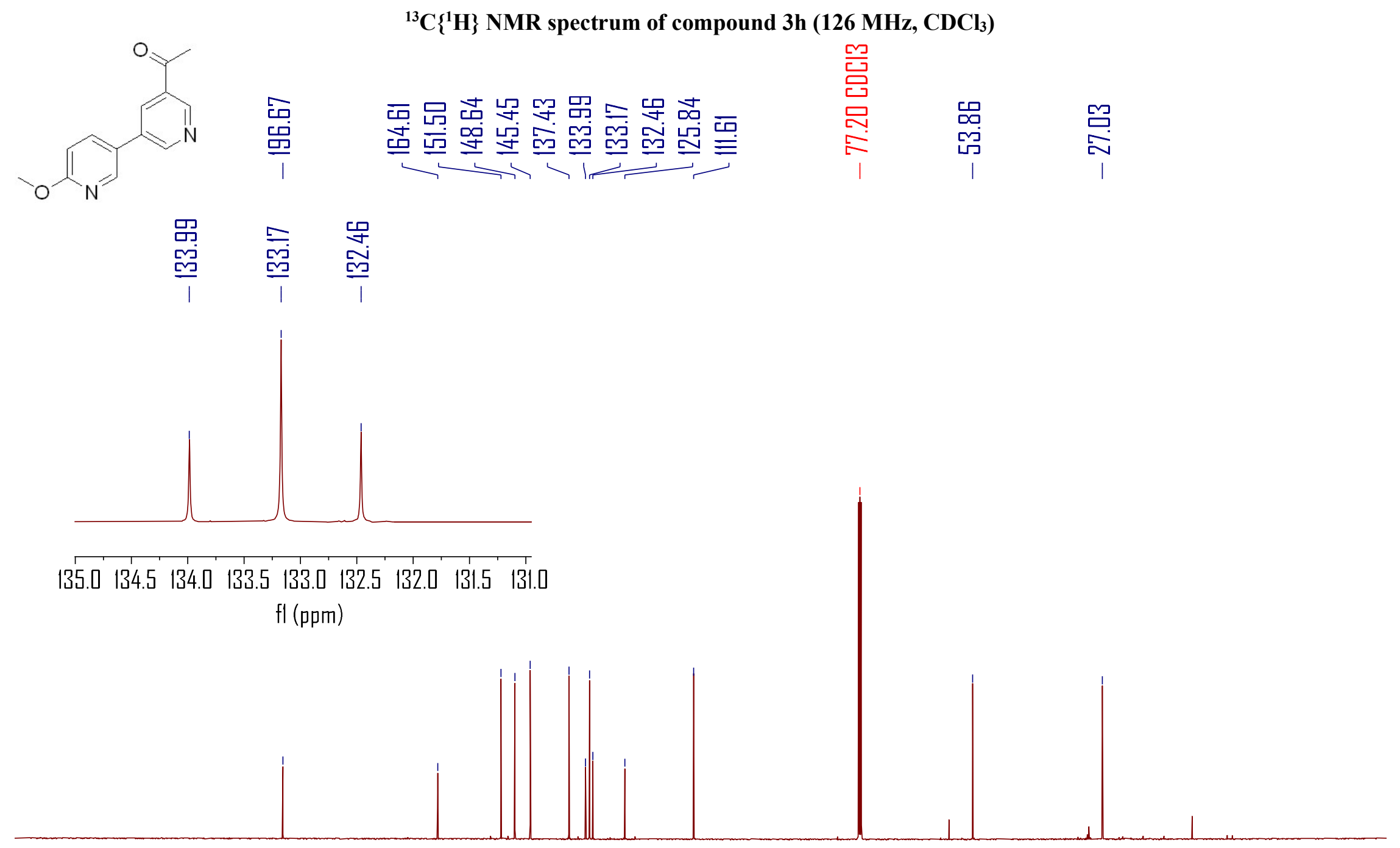

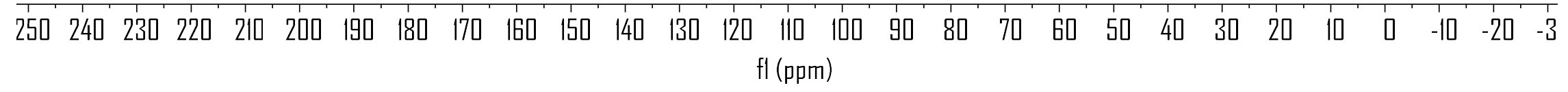
S109 
${ }^{1} \mathrm{H}$ NMR spectrum of compound $3 \mathrm{i}\left(126 \mathrm{MHz}, \mathrm{CDCl}_{3}\right)$

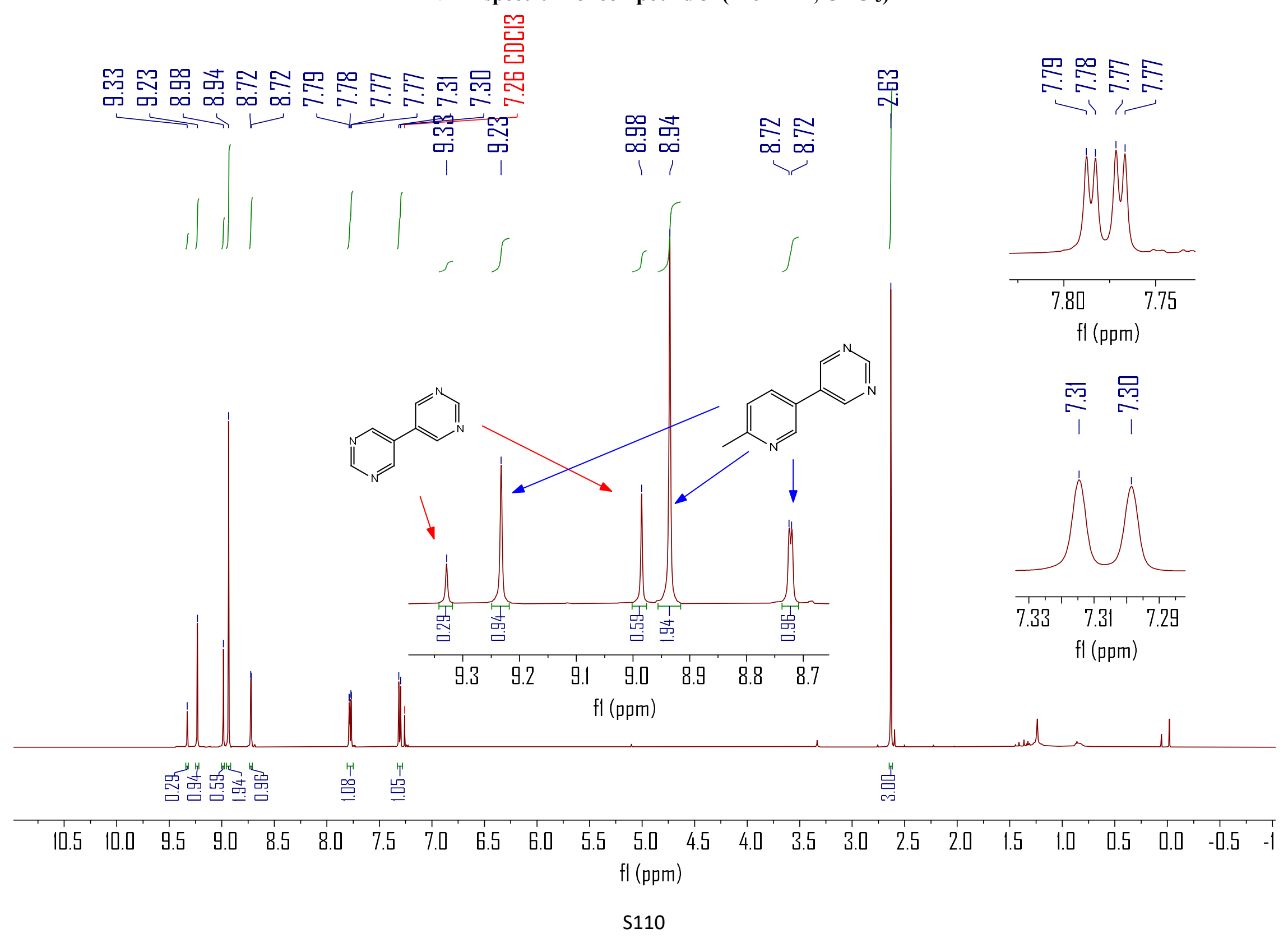




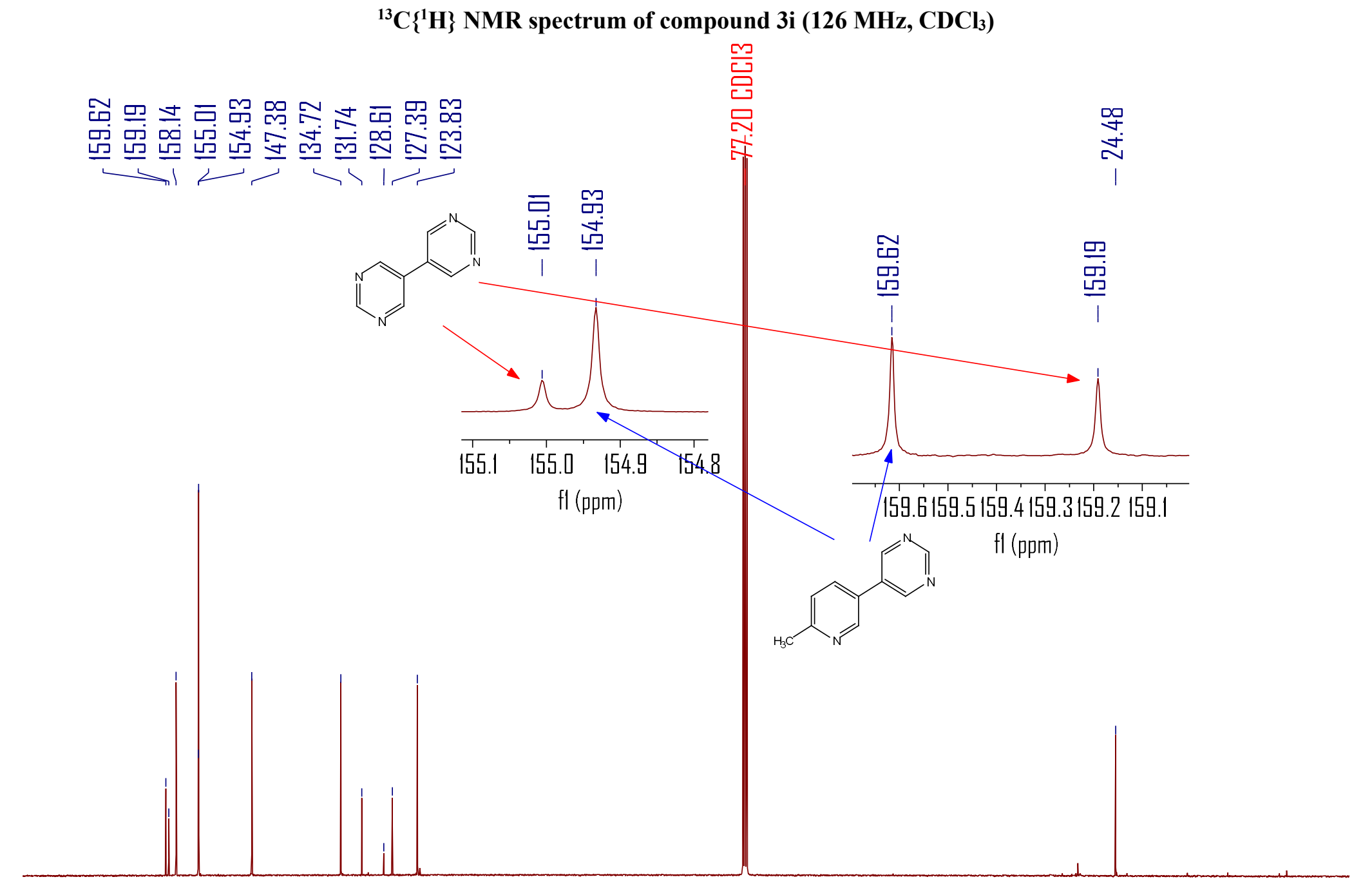

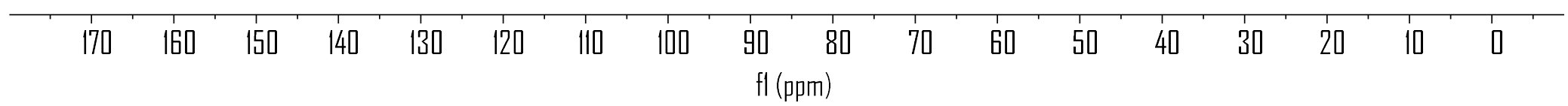




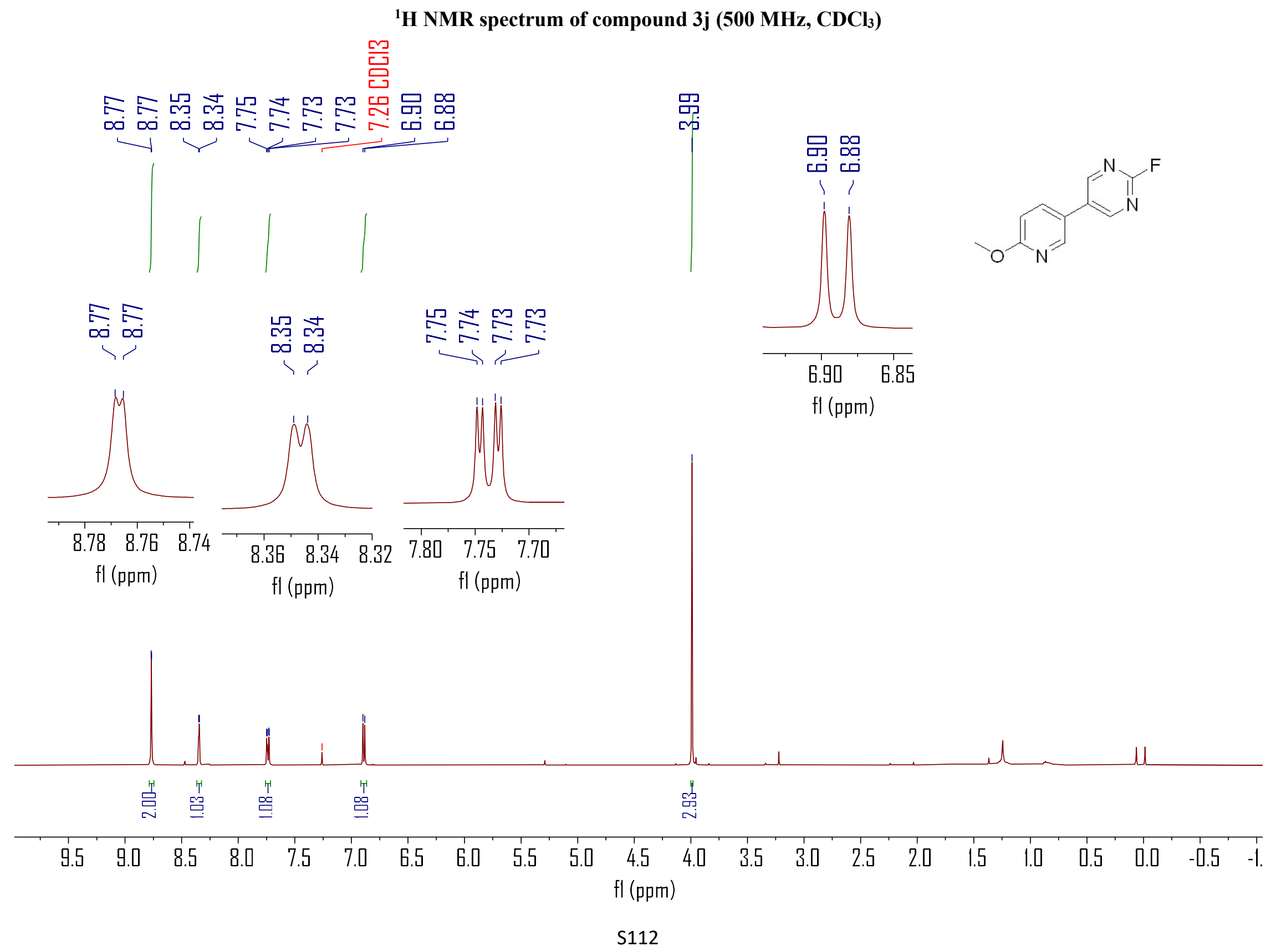


${ }^{19} \mathrm{~F}\left\{{ }^{1} \mathrm{H}\right\}$ NMR spectrum of compound $3 \mathrm{j}\left(376 \mathrm{MHz}, \mathrm{CDCl}_{3}\right)$

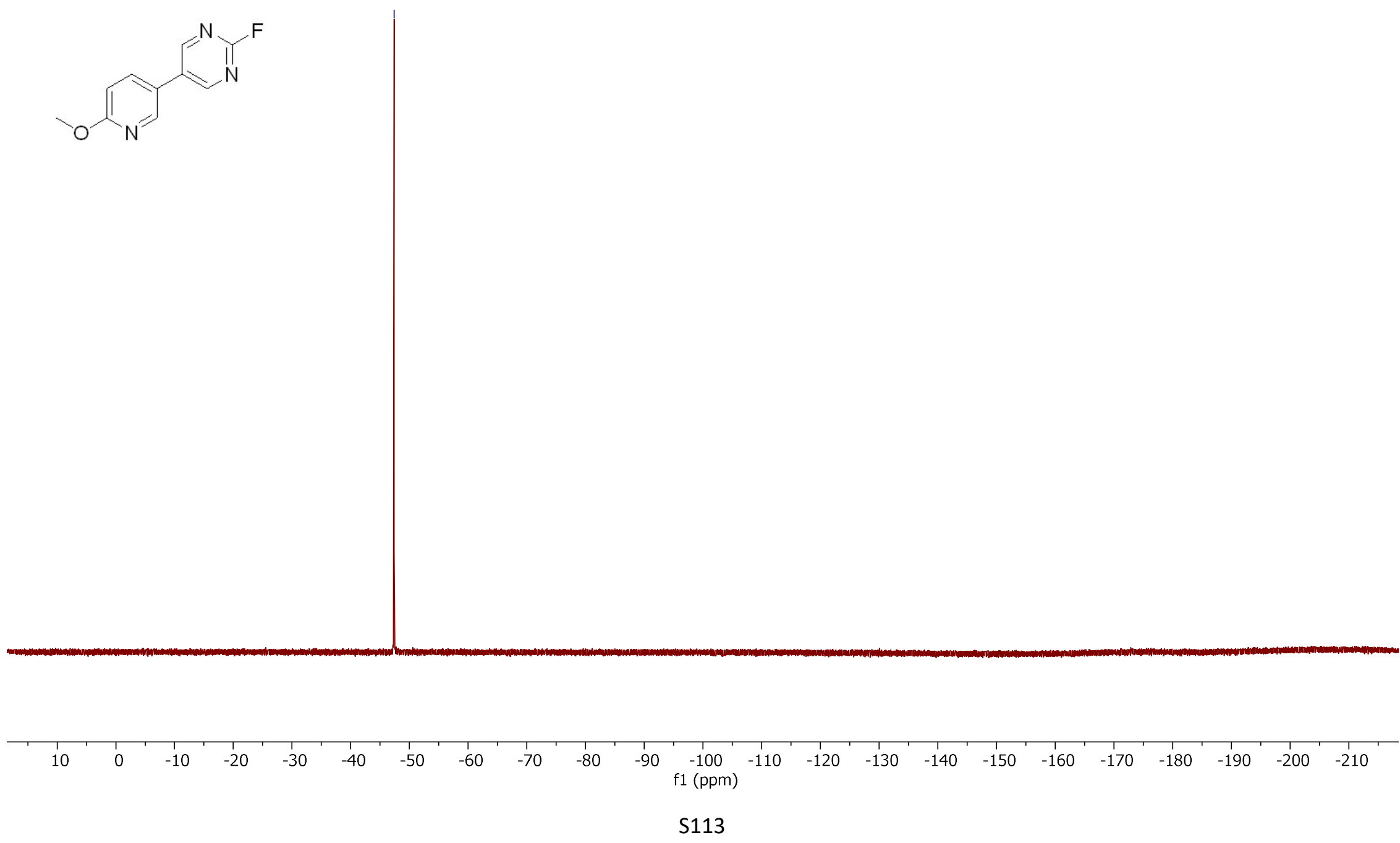




\section{${ }^{13} \mathrm{C}\left\{{ }^{1} \mathrm{H}\right\}$ NMR spectrum of compound $3 \mathrm{j}\left(126 \mathrm{MHz}, \mathrm{CDCl}_{3}\right)$}

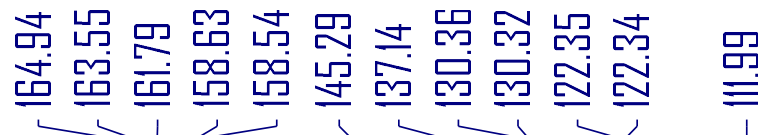
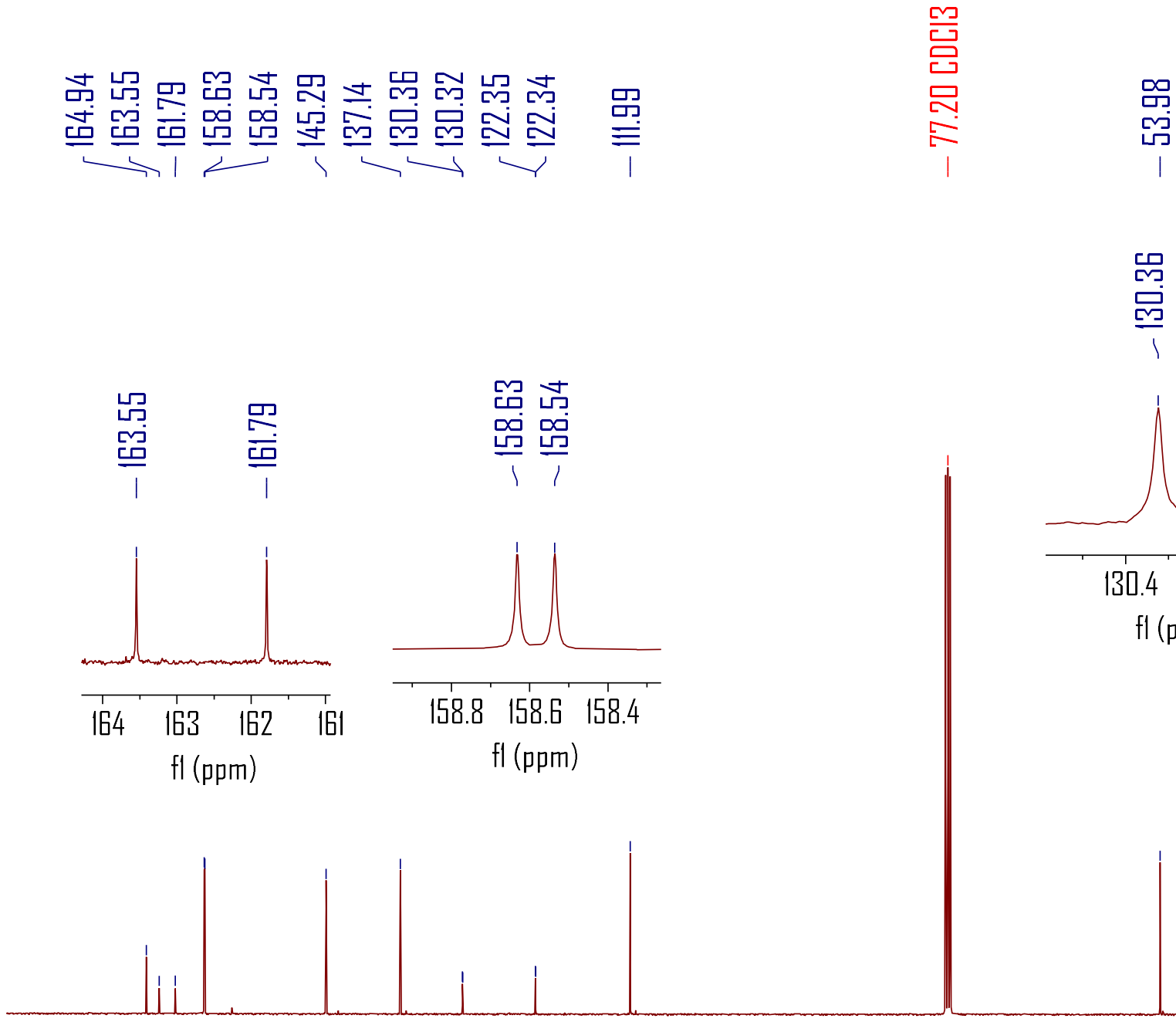
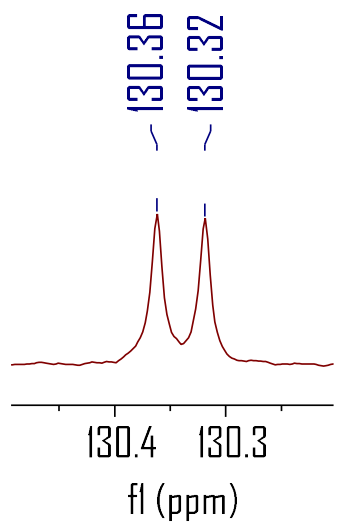

$\mathrm{fl}(\mathrm{ppm})$
品㟧

저
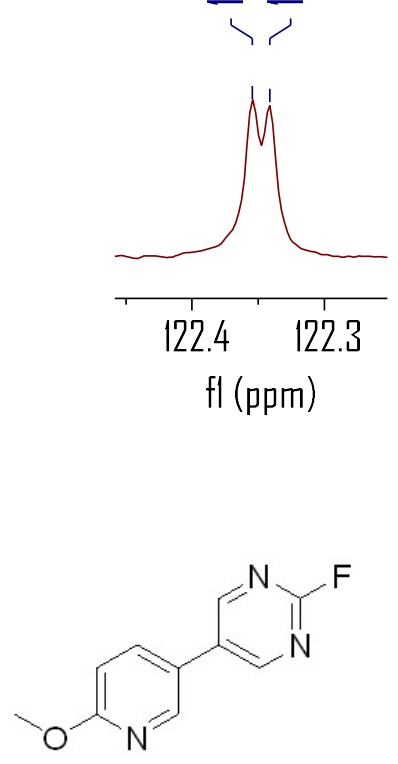

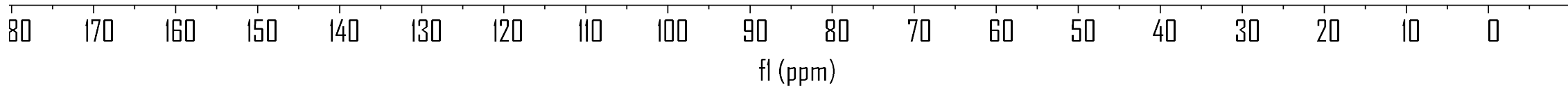




\section{${ }^{1} \mathrm{H}$ NMR spectrum of compound 3k (500 $\left.\mathrm{MHz}, \mathrm{CDCl}_{3}\right)$}

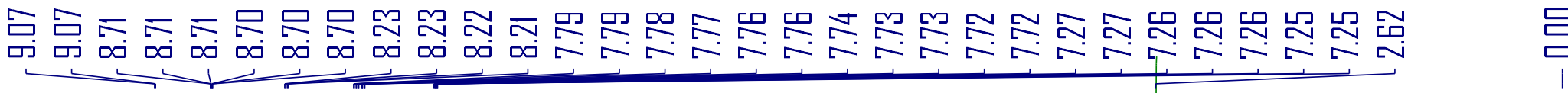

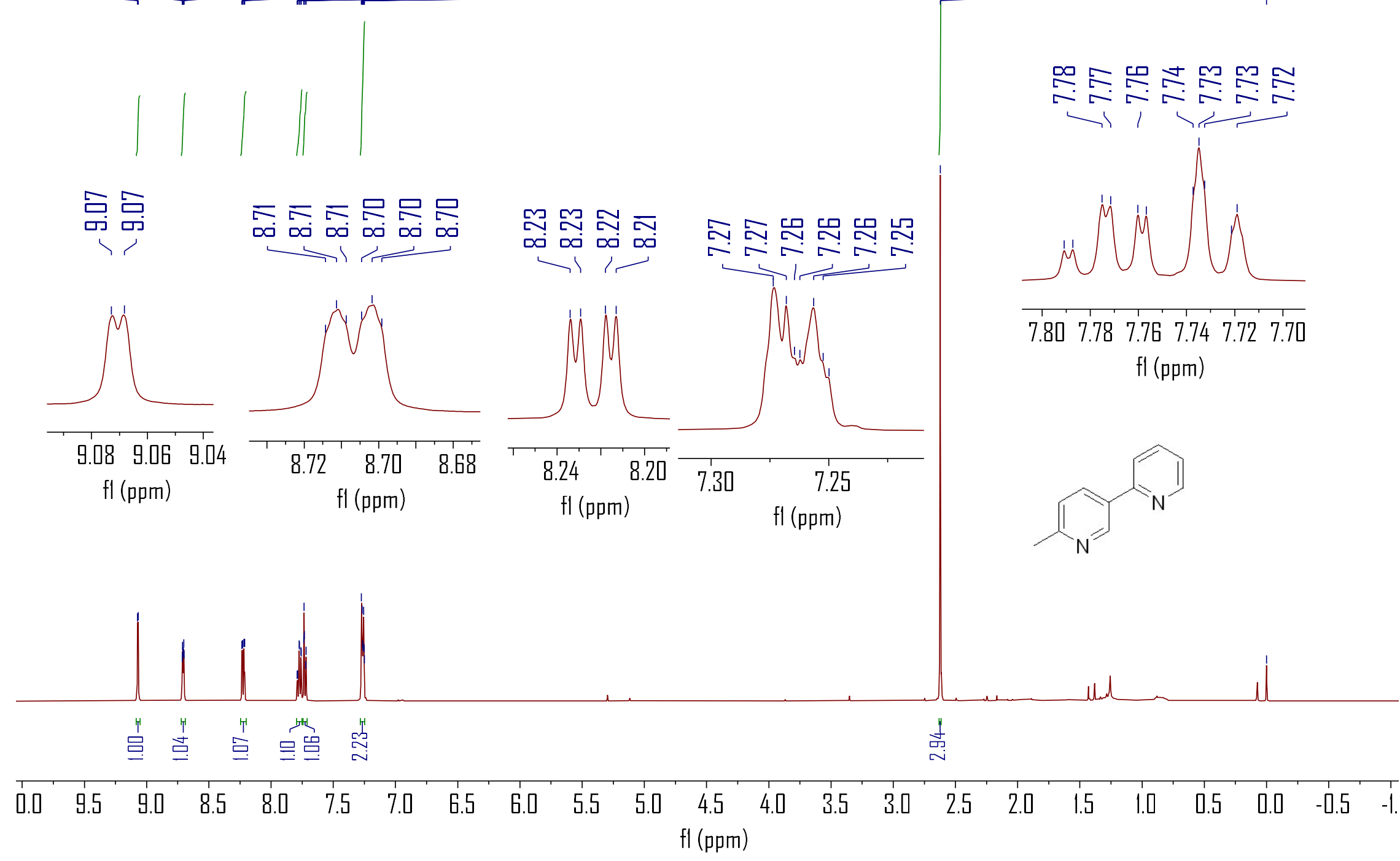




\section{${ }^{13} \mathrm{C}\left\{{ }^{1} \mathrm{H}\right\}$ NMR spectrum of compound 3k (126 $\left.\mathrm{MHz}, \mathrm{CDCl}_{3}\right)$}

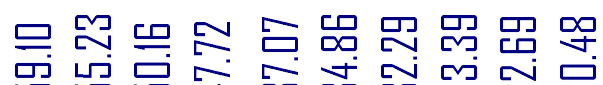

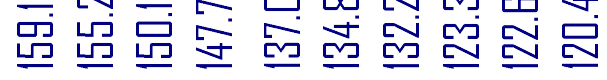

$111,51,12$
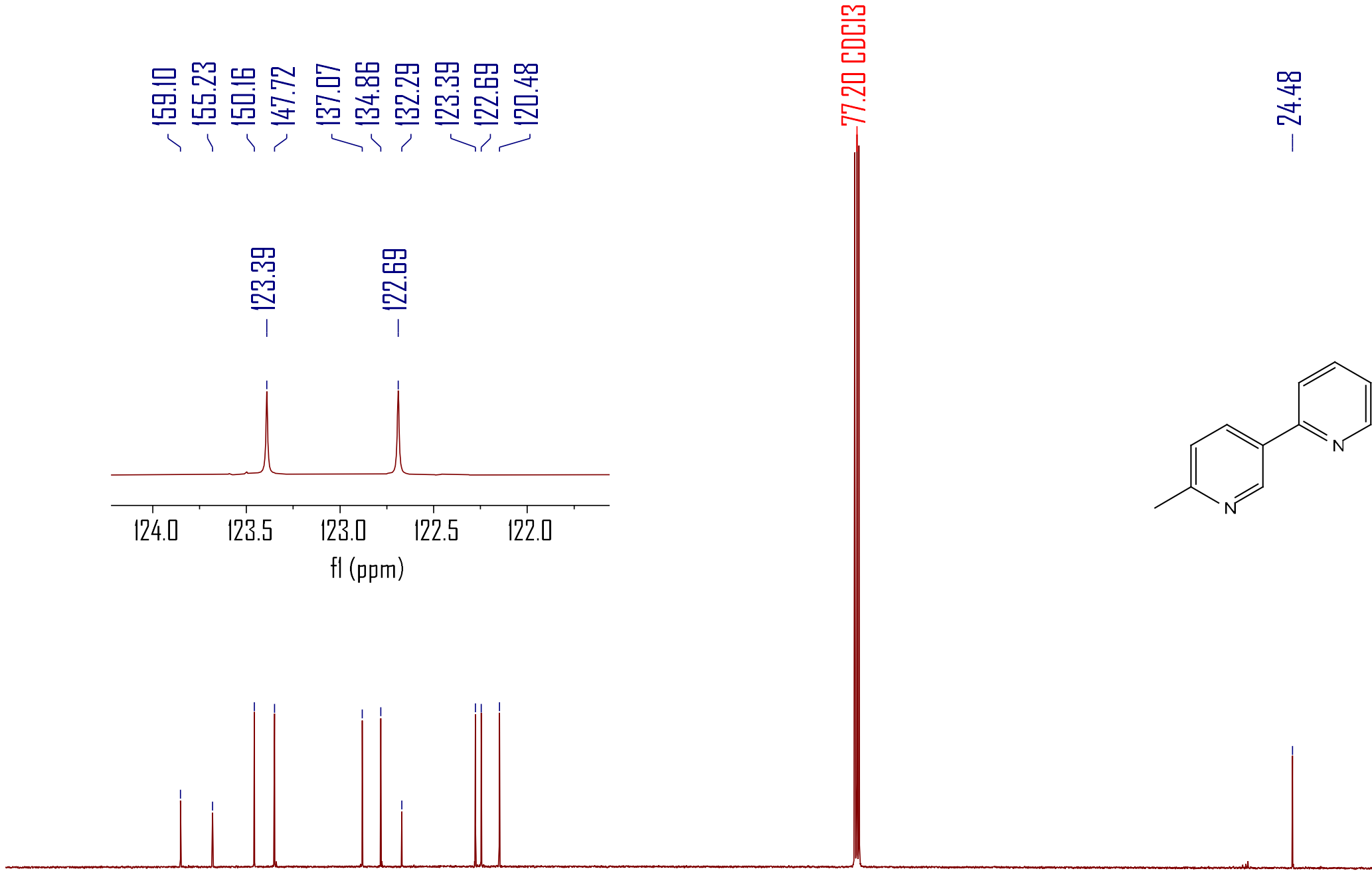

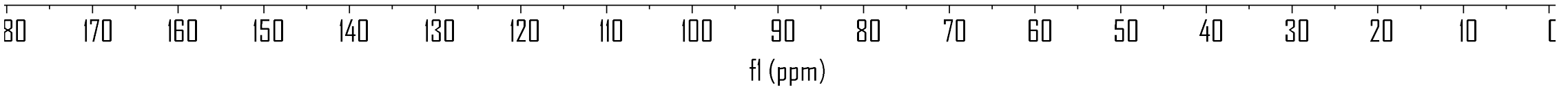




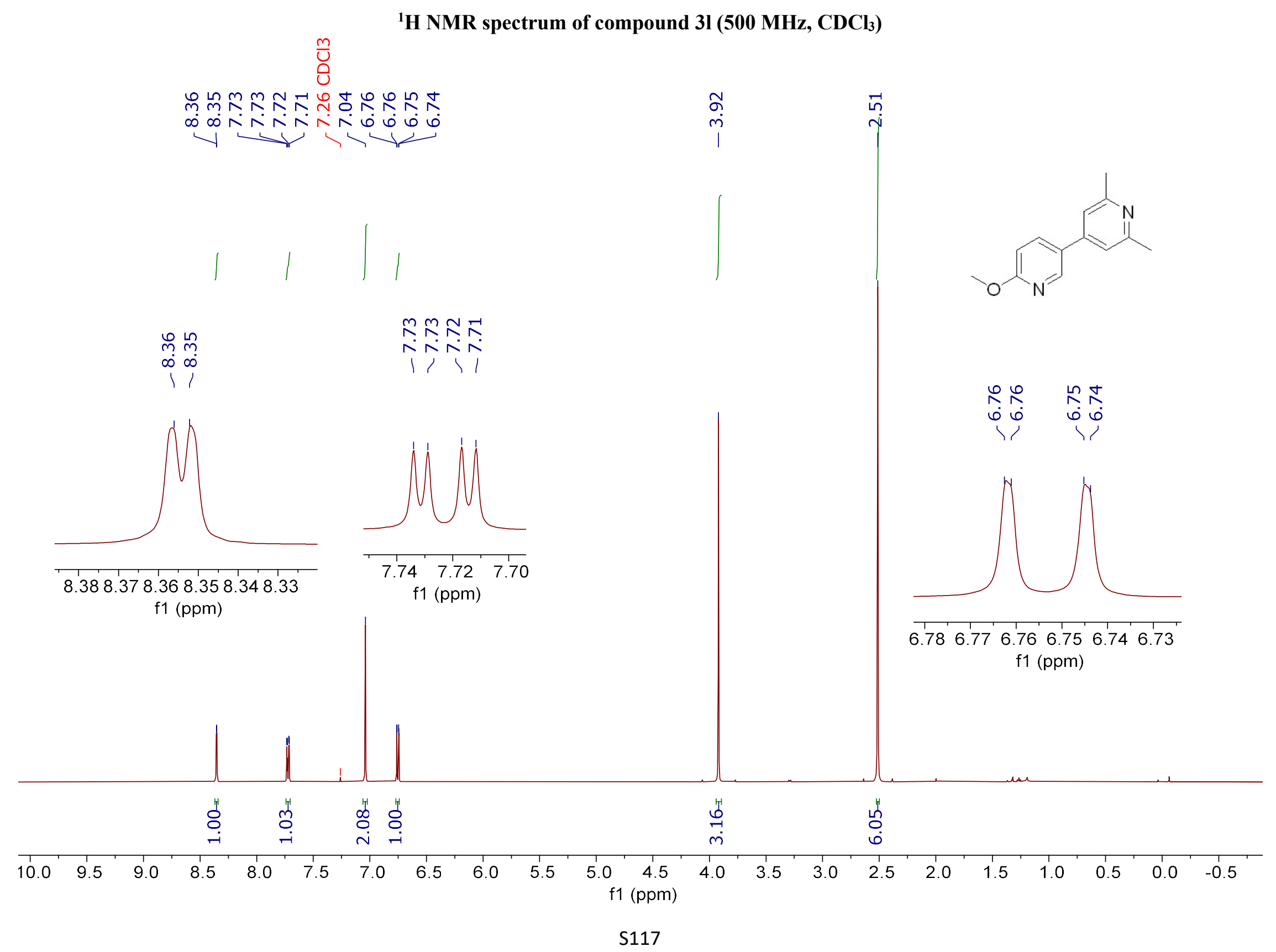




\section{${ }^{13} \mathrm{C}\left\{{ }^{1} \mathrm{H}\right\}$ NMR spectrum of compound $31\left(126 \mathrm{MHz}, \mathrm{CDCl}_{3}\right)$}

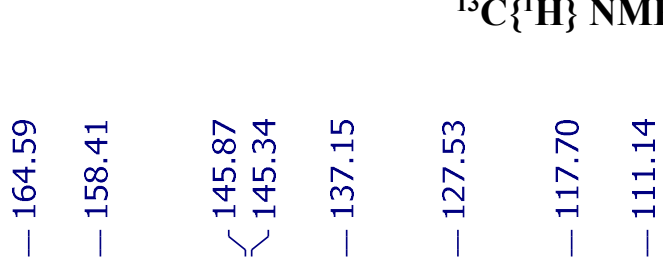

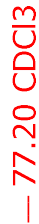

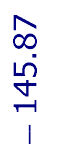

$\stackrel{?}{\stackrel{p}{m}}$

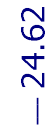
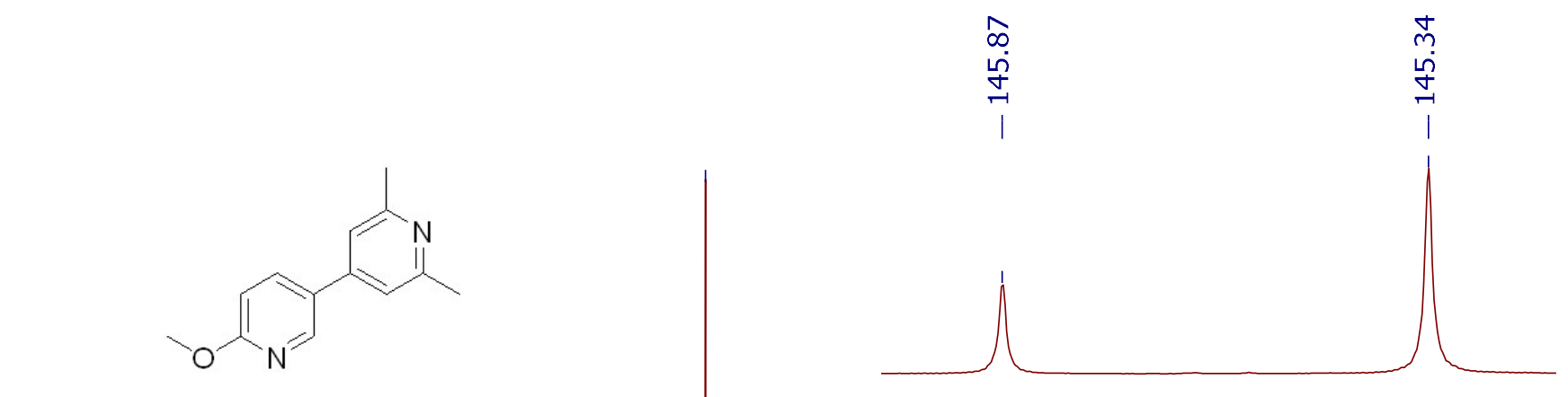

$\begin{array}{lllllllll}146.0 & 145.9 & 145.8 & 145.7 & 145.6 & 145.5 & 145.4 & 145.3 & 145.2\end{array}$ f1 (ppm)
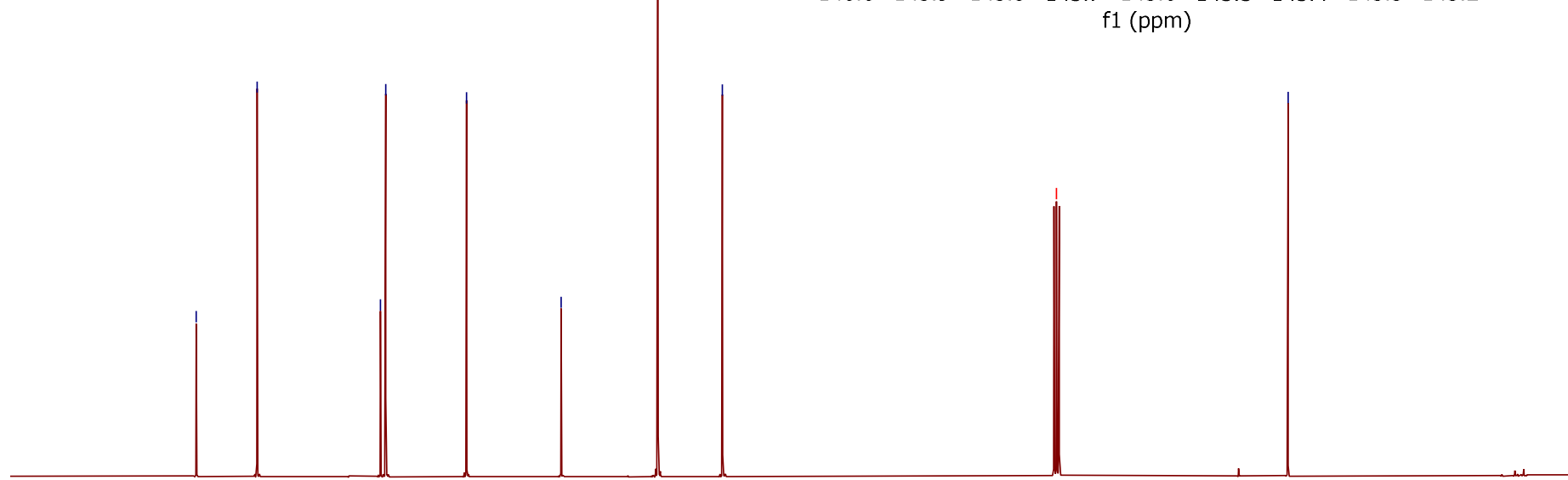

$180 \quad 170 \quad 160$

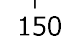

140

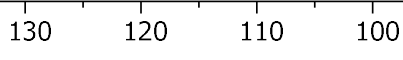

$90 \quad 80$

60

50

40

30

S118 
${ }^{1} \mathrm{H}$ NMR spectrum of compound $3 \mathrm{~m}\left(500 \mathrm{MHz}, \mathrm{CDCl}_{3}\right)$

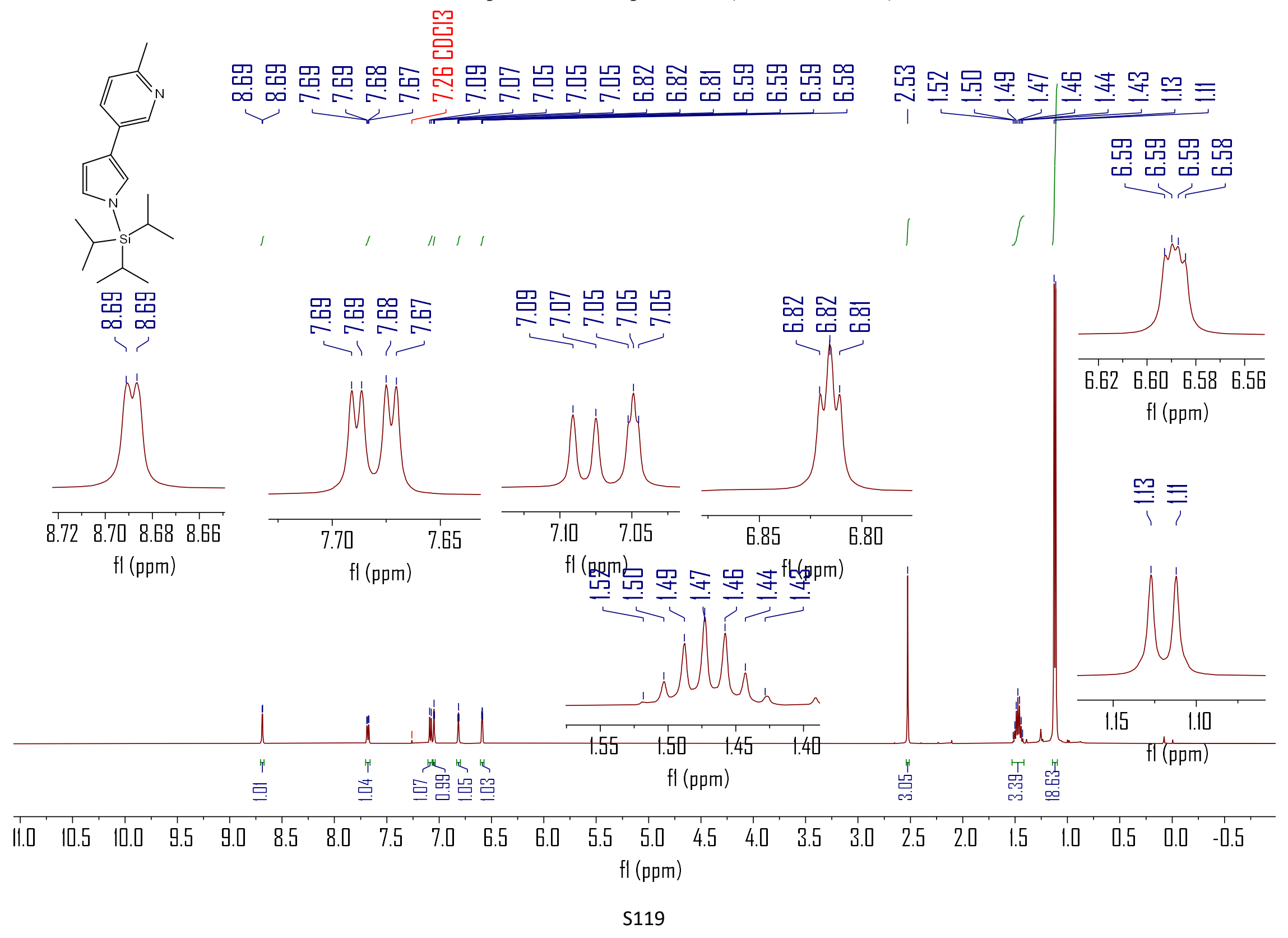


${ }^{13} \mathrm{C}\left\{{ }^{1} \mathrm{H}\right\}$ NMR spectrum of compound $3 \mathrm{~m}\left(126 \mathrm{MHz}, \mathrm{CDCl}_{3}\right)$
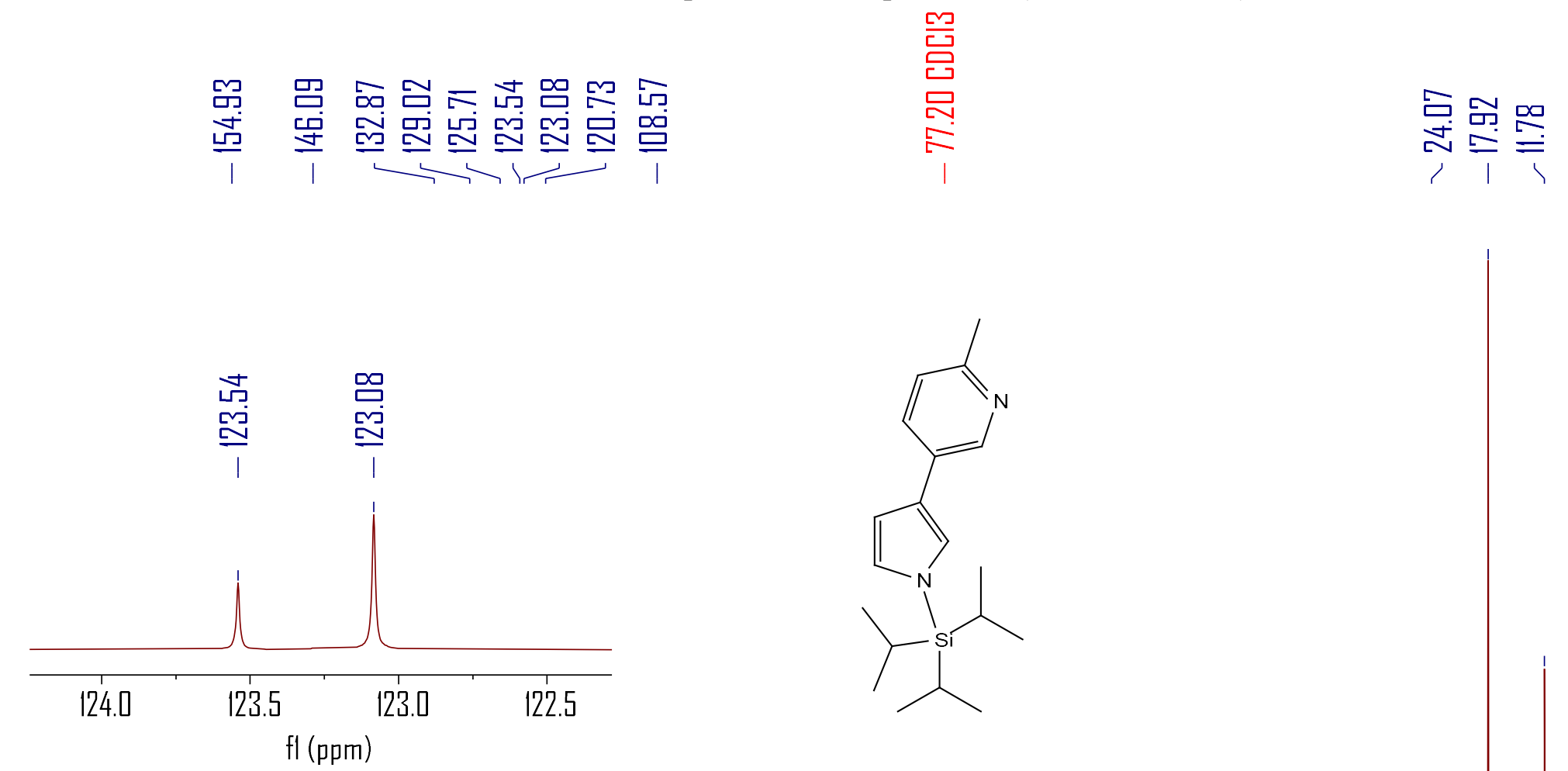

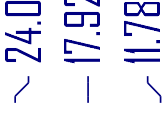
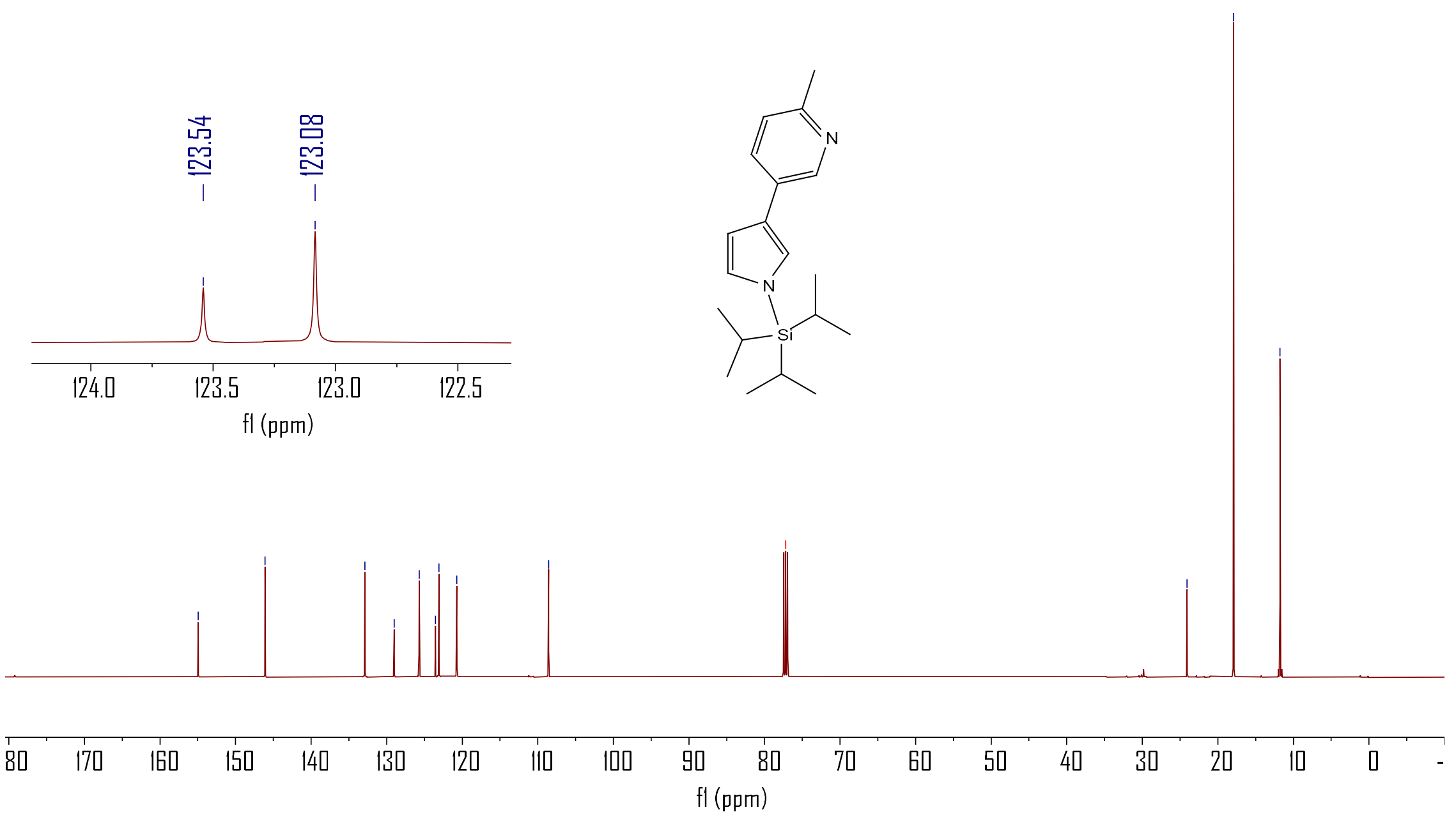
${ }^{1} \mathrm{H}$ NMR spectrum of compound 3n (500 $\left.\mathrm{MHz}, \mathrm{CDCl}_{3}\right)$
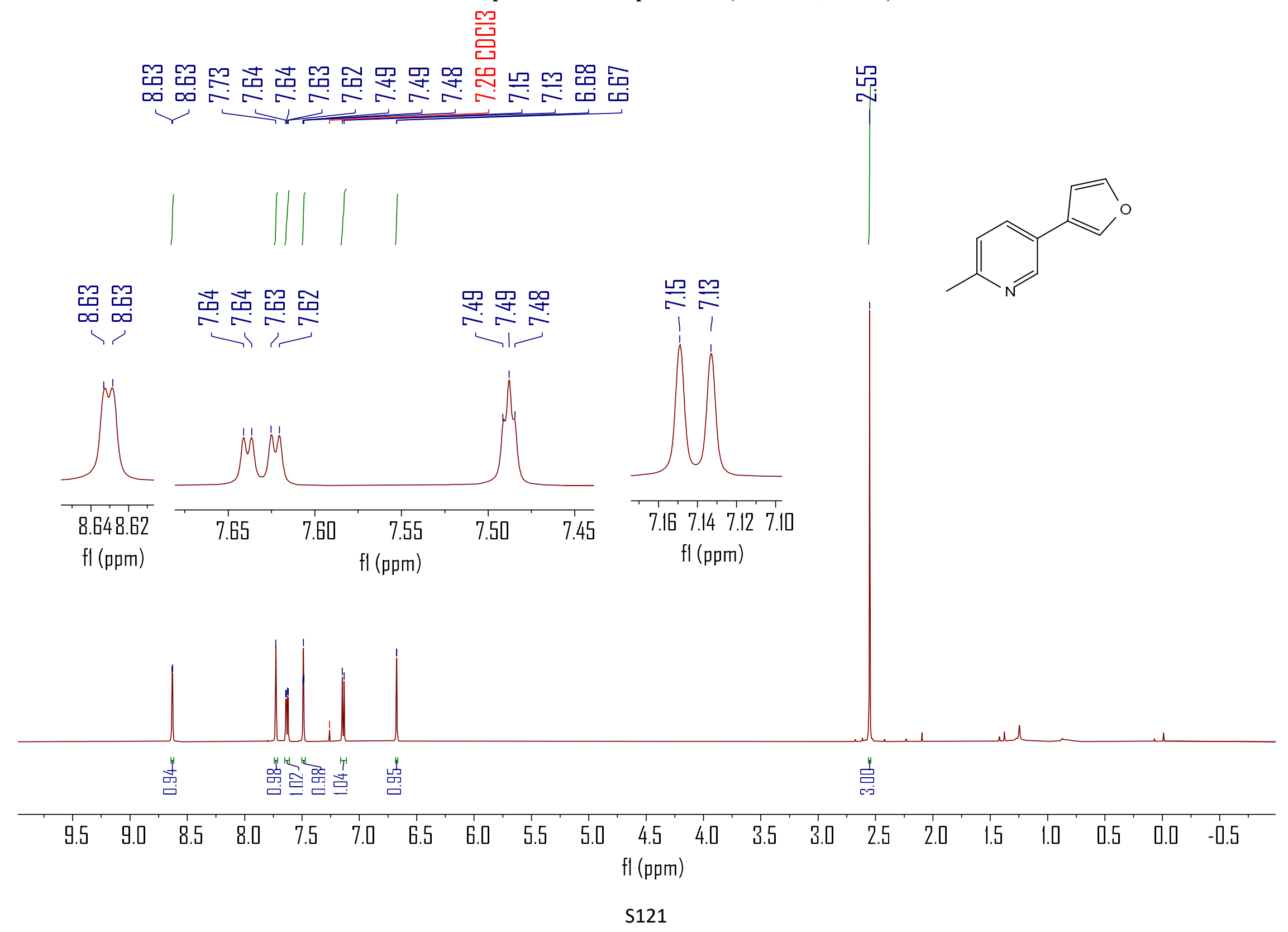


\section{${ }^{13} \mathrm{C}\left\{{ }^{1} \mathrm{H}\right\}$ NMR spectrum of compound 3n (126 MHz, $\left.\mathrm{CDCl}_{3}\right)$}

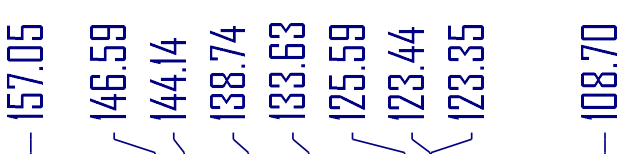

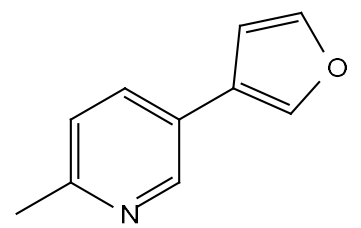

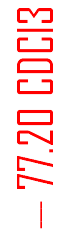

范

뜨ำ

।

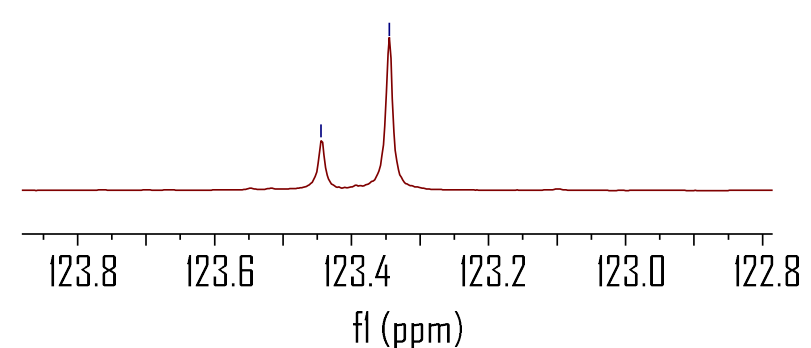

$\stackrel{\text { 号 }}{\stackrel{D}{~}}$

80

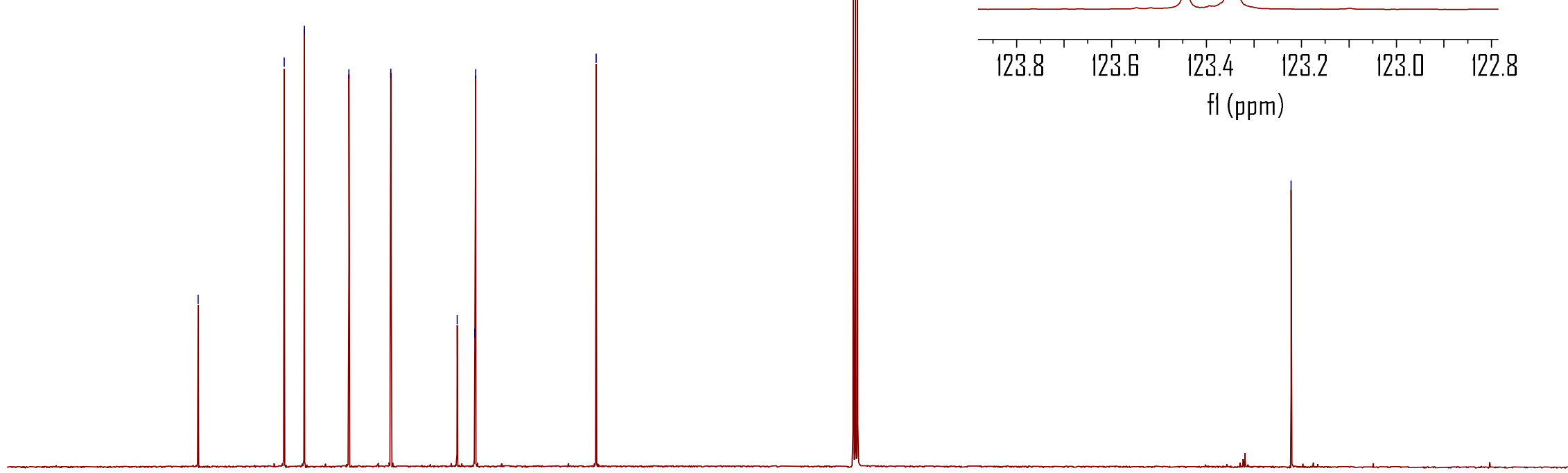

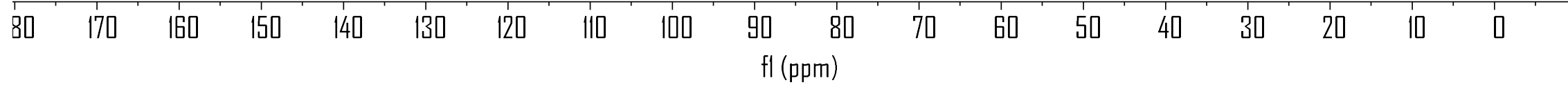




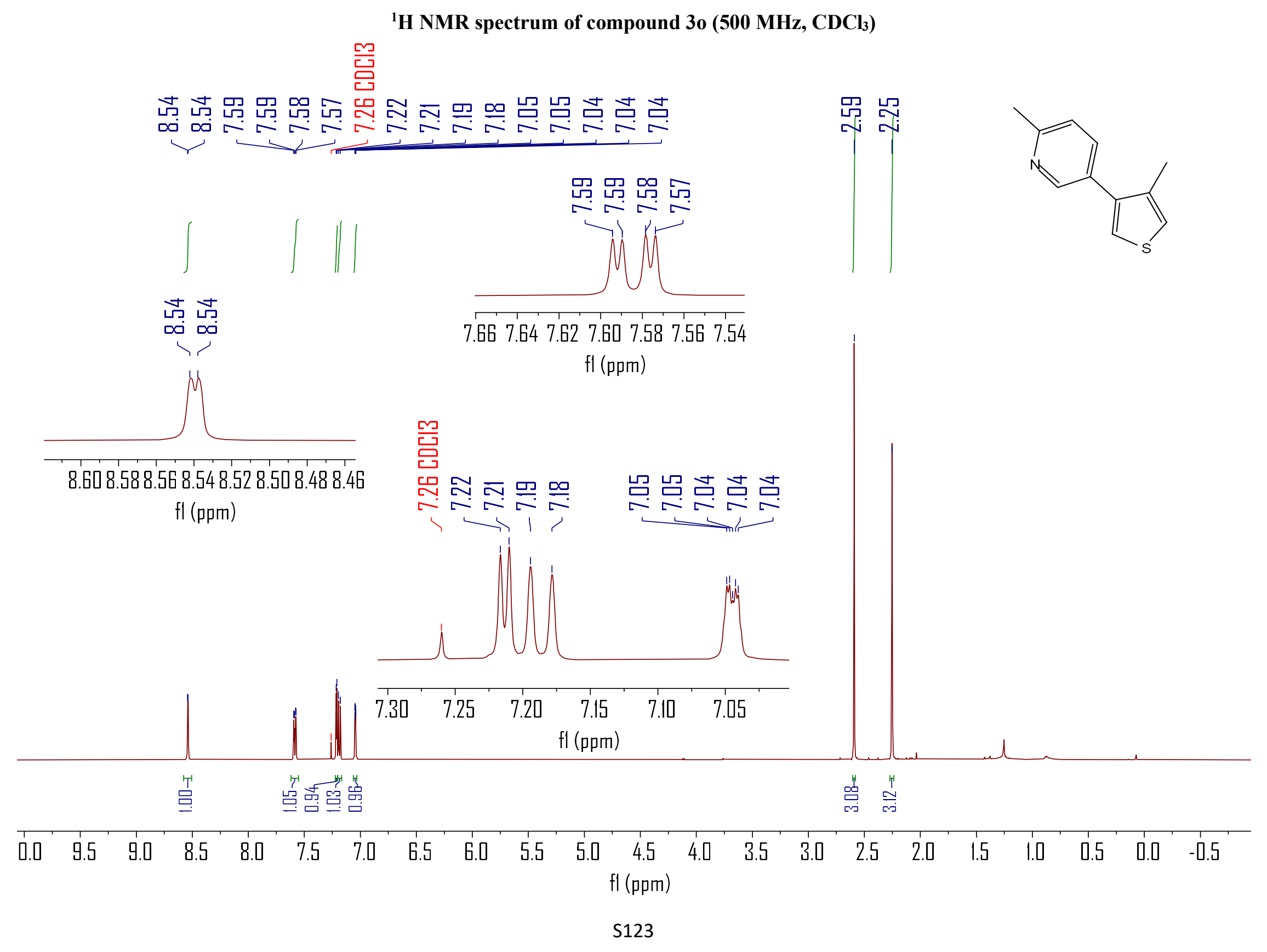


${ }^{13} \mathrm{C}\left\{{ }^{1} \mathrm{H}\right\}$ NMR spectrum of compound $30\left(126 \mathrm{MHz}, \mathrm{CDCl}_{3}\right)$

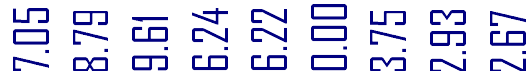

品哭品器品鱼哭导

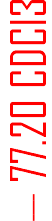
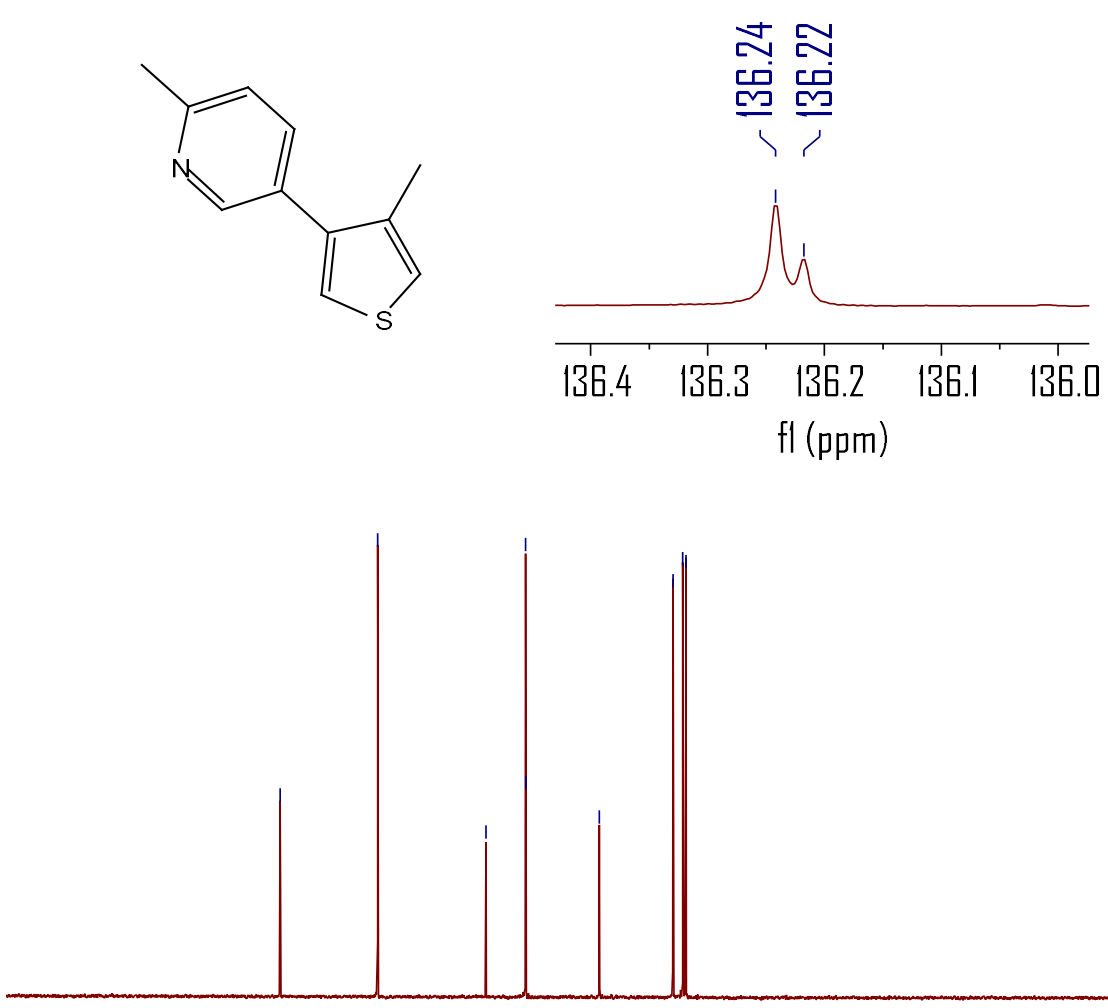

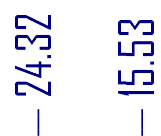
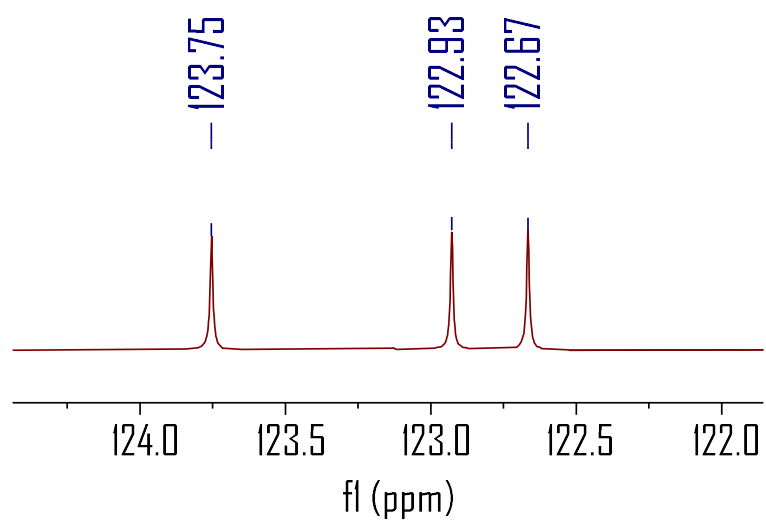

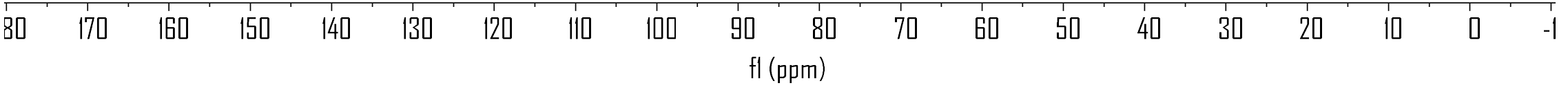




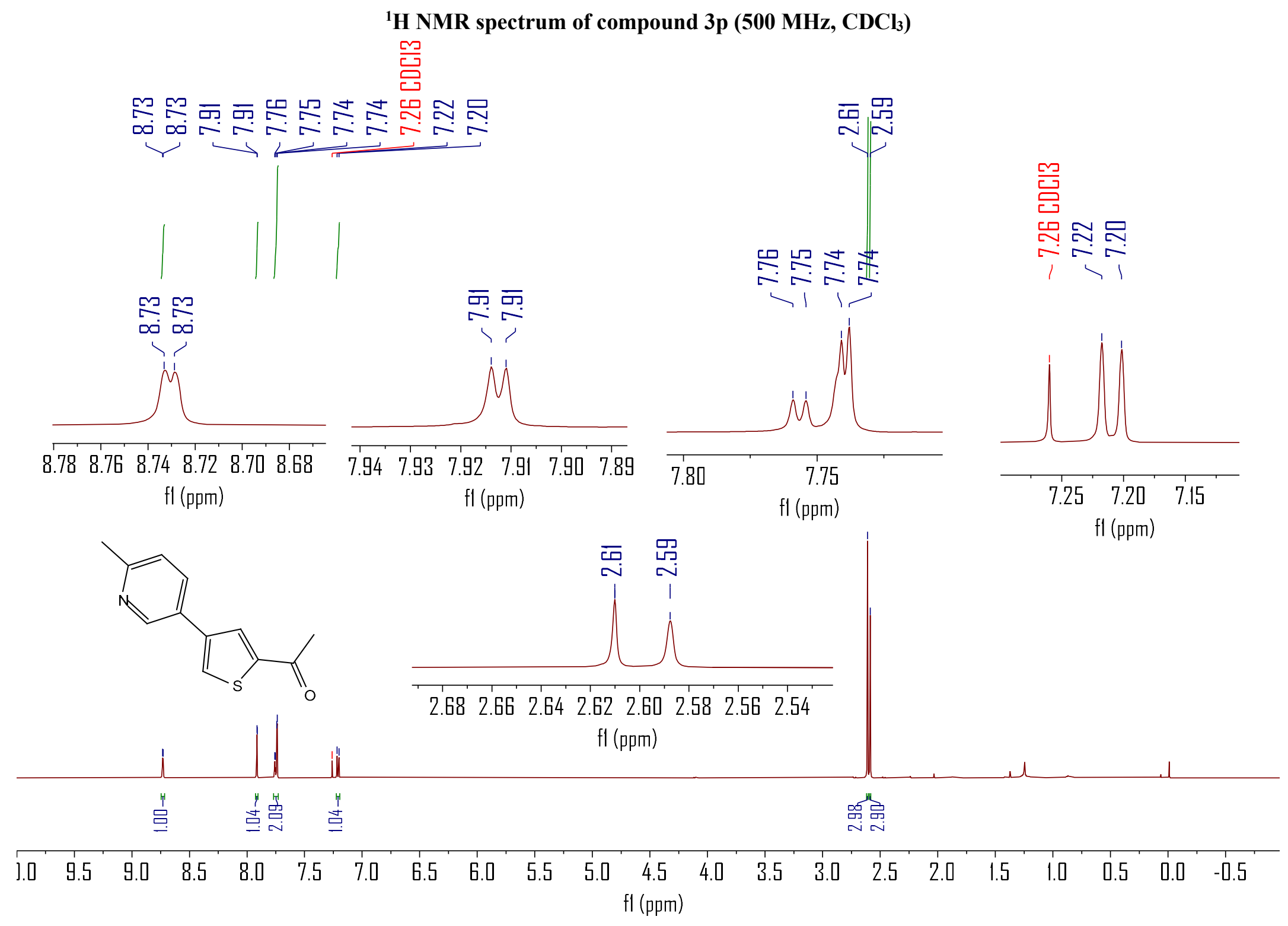




\section{${ }^{13} \mathrm{C}\left\{{ }^{1} \mathrm{H}\right\}$ NMR spectrum of compound $3 p\left(126 \mathrm{MHz}, \mathrm{CDCl}_{3}\right)$}
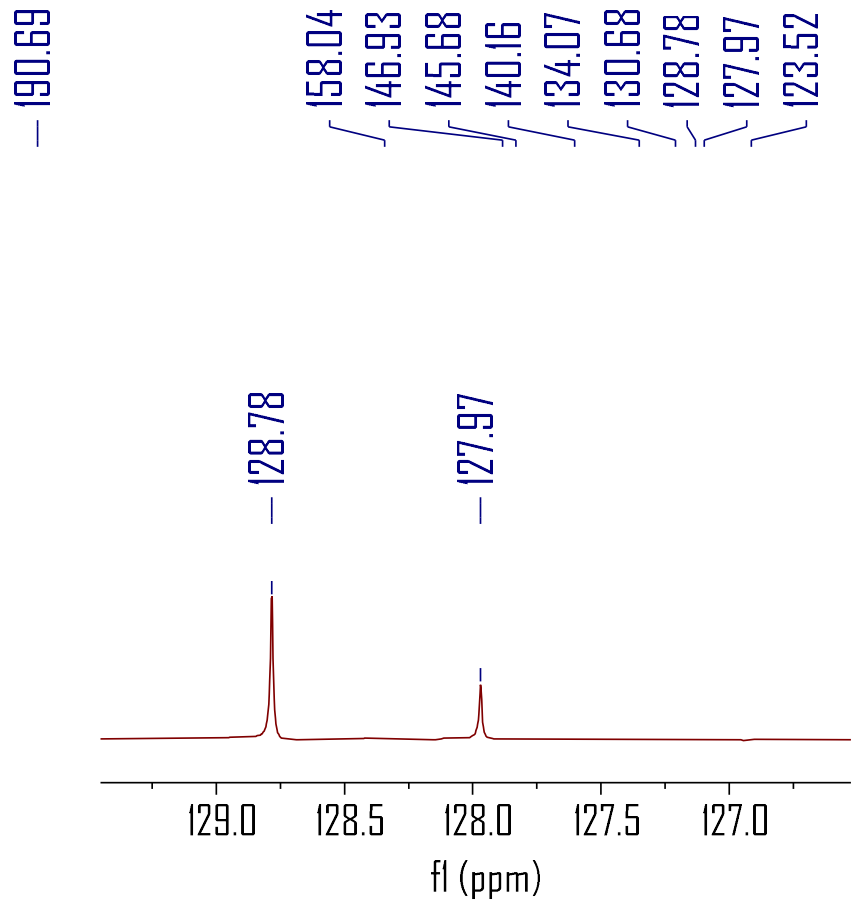

밀

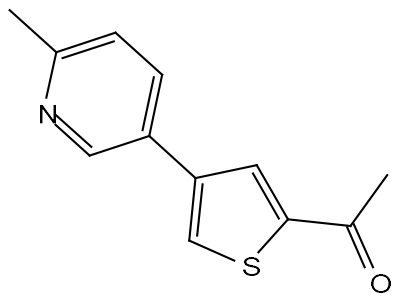

\begin{tabular}{llllllllllllllllllll}
\hline 200 & 190 & 180 & 170 & 160 & 150 & 140 & 130 & 120 & 110 & $\begin{array}{c}100 \\
f 1(\mathrm{pm})\end{array}$ & 80 & 70 & 60 & 50 & 40 & 30 & 20 & 10 & 0
\end{tabular}


${ }^{1} \mathrm{H}$ NMR spectrum of compound 3q (500 $\left.\mathrm{MHz}, \mathrm{CDCl}_{3}\right)$

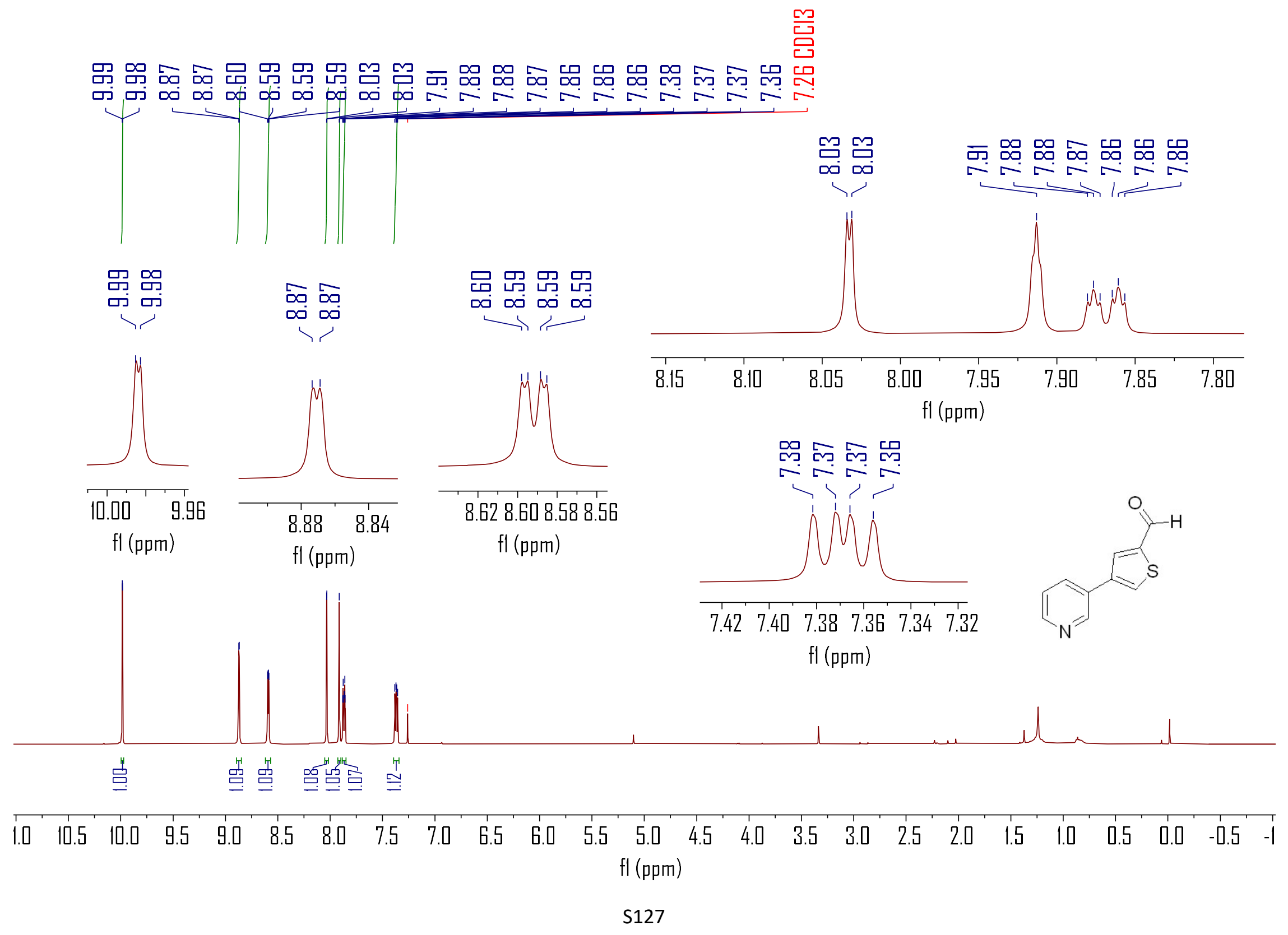




\section{${ }^{13} \mathrm{C}\left\{{ }^{1} \mathrm{H}\right\}$ NMR spectrum of compound $3 q\left(126 \mathrm{MHz}, \mathrm{CDCl}_{3}\right)$}

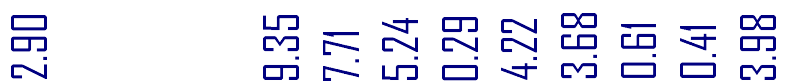

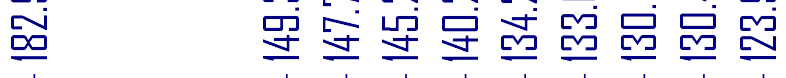

뜸
뭄
뭄
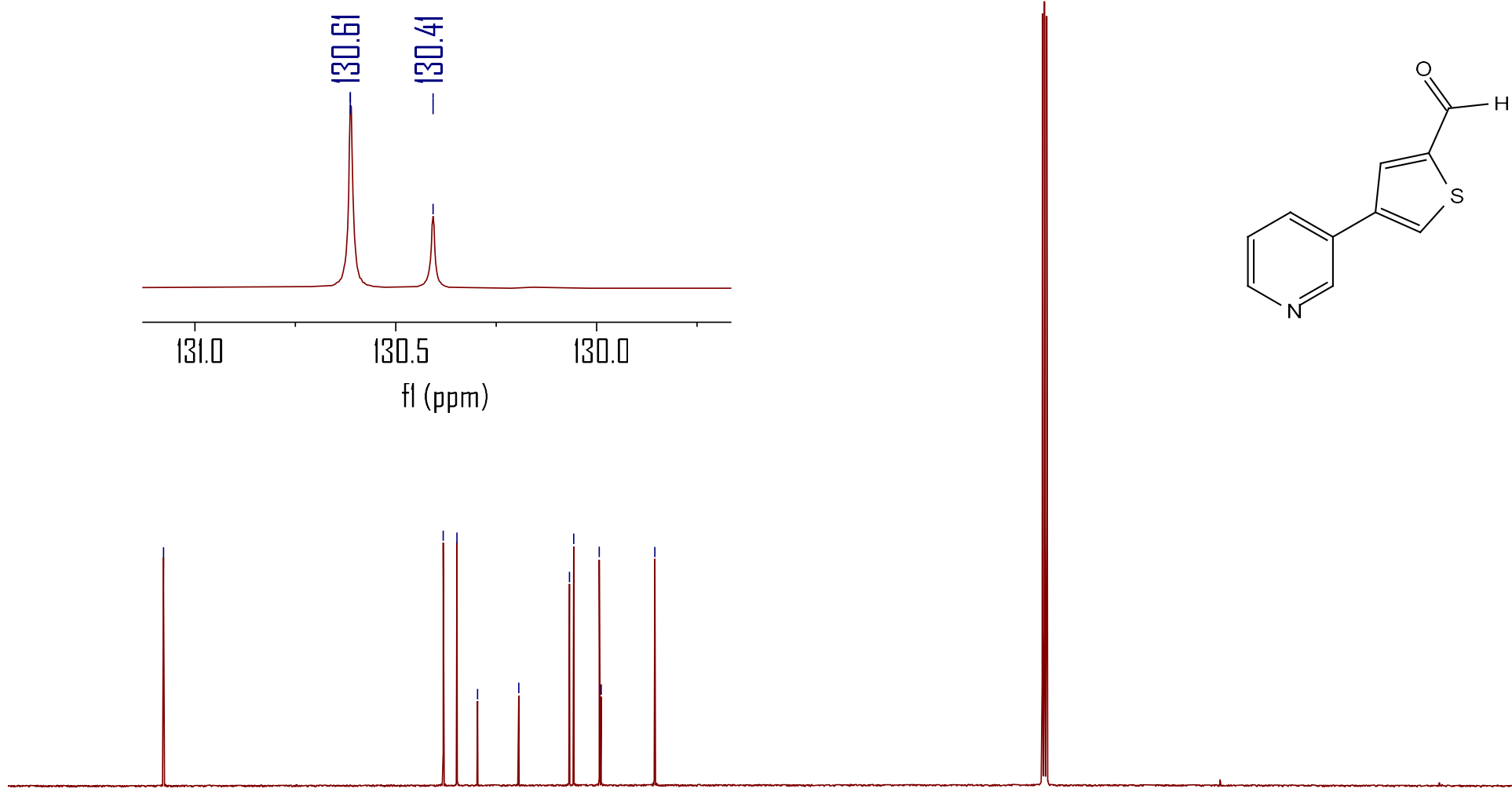

200190180

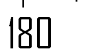

$170 \quad 160$

150 150

$40 \quad 130$

120

110

$\mathrm{fl}(\mathrm{ppm})$ 


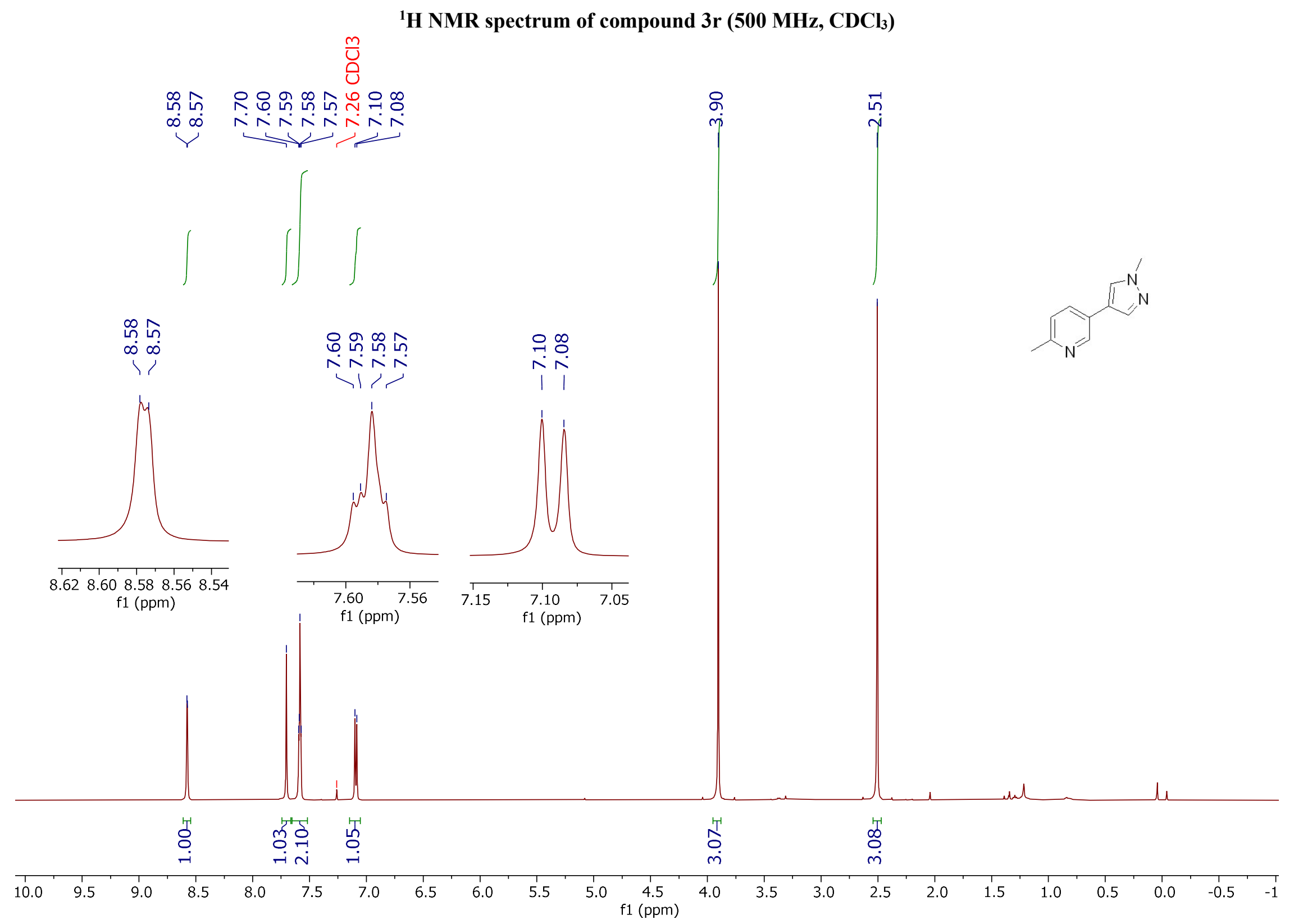

S129 


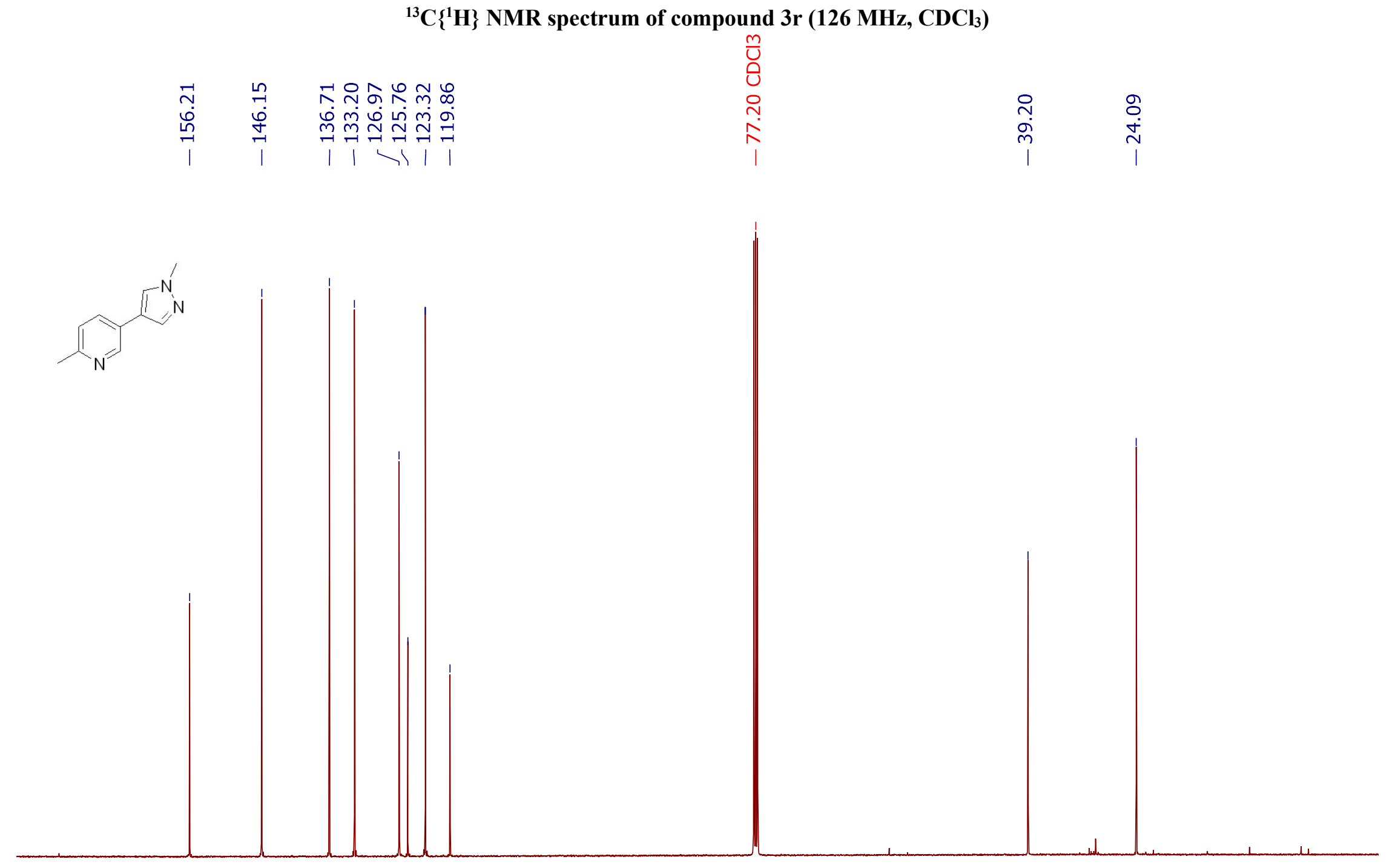

$80 \quad 170 \quad 160$

$150 \quad 140$

130

$120 \quad 110$

100

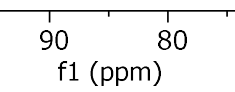

70

60

50

40

30

20 
${ }^{1} \mathrm{H}$ NMR spectrum of compound 3s $\left(500 \mathrm{MHz}, \mathrm{CDCl}_{3}\right)$

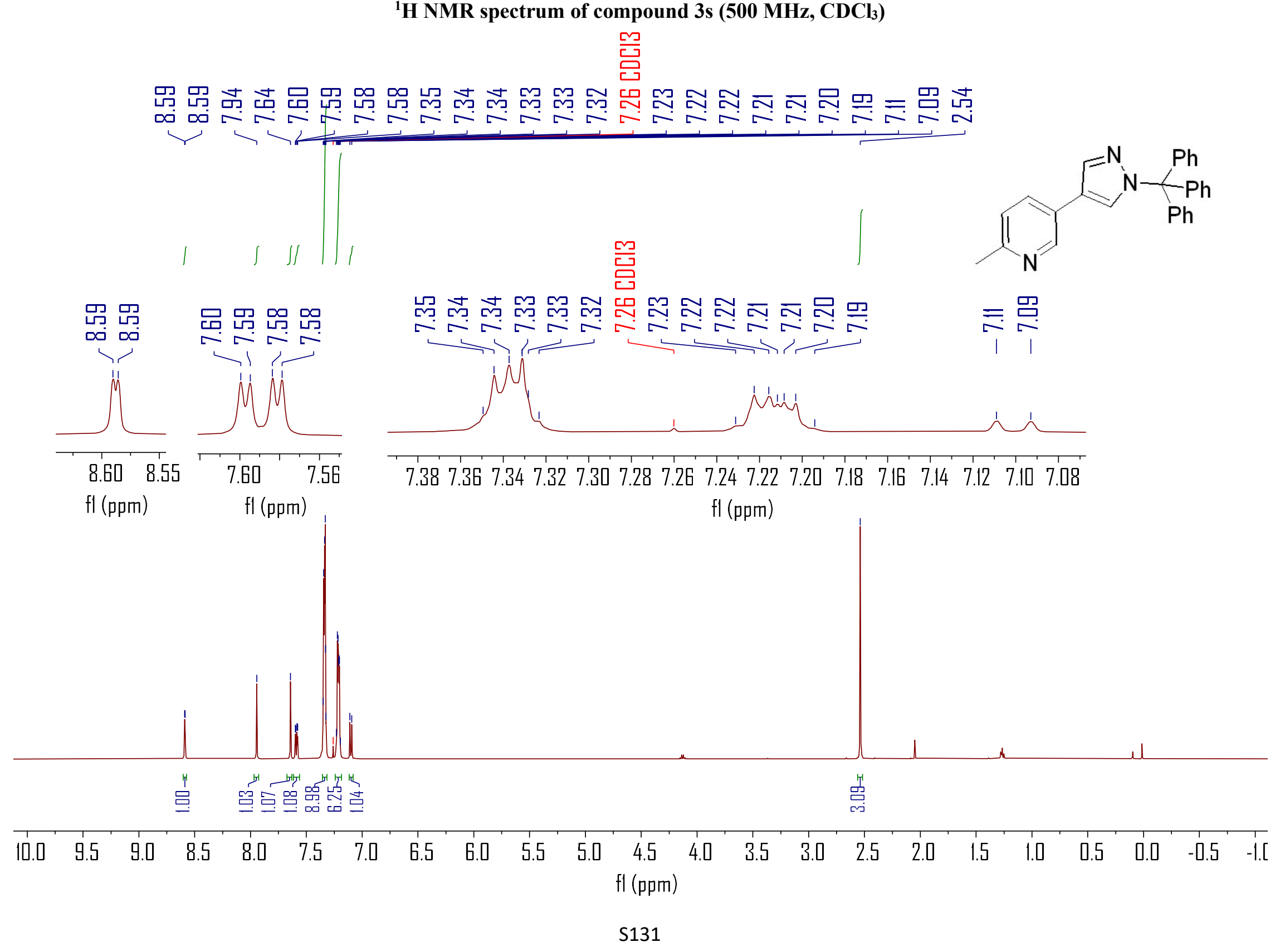




\section{${ }^{13} \mathrm{C}\left\{{ }^{1} \mathrm{H}\right\}$ NMR spectrum of compound 3s $\left(126 \mathrm{MHz}, \mathrm{CDCl}_{3}\right)$}
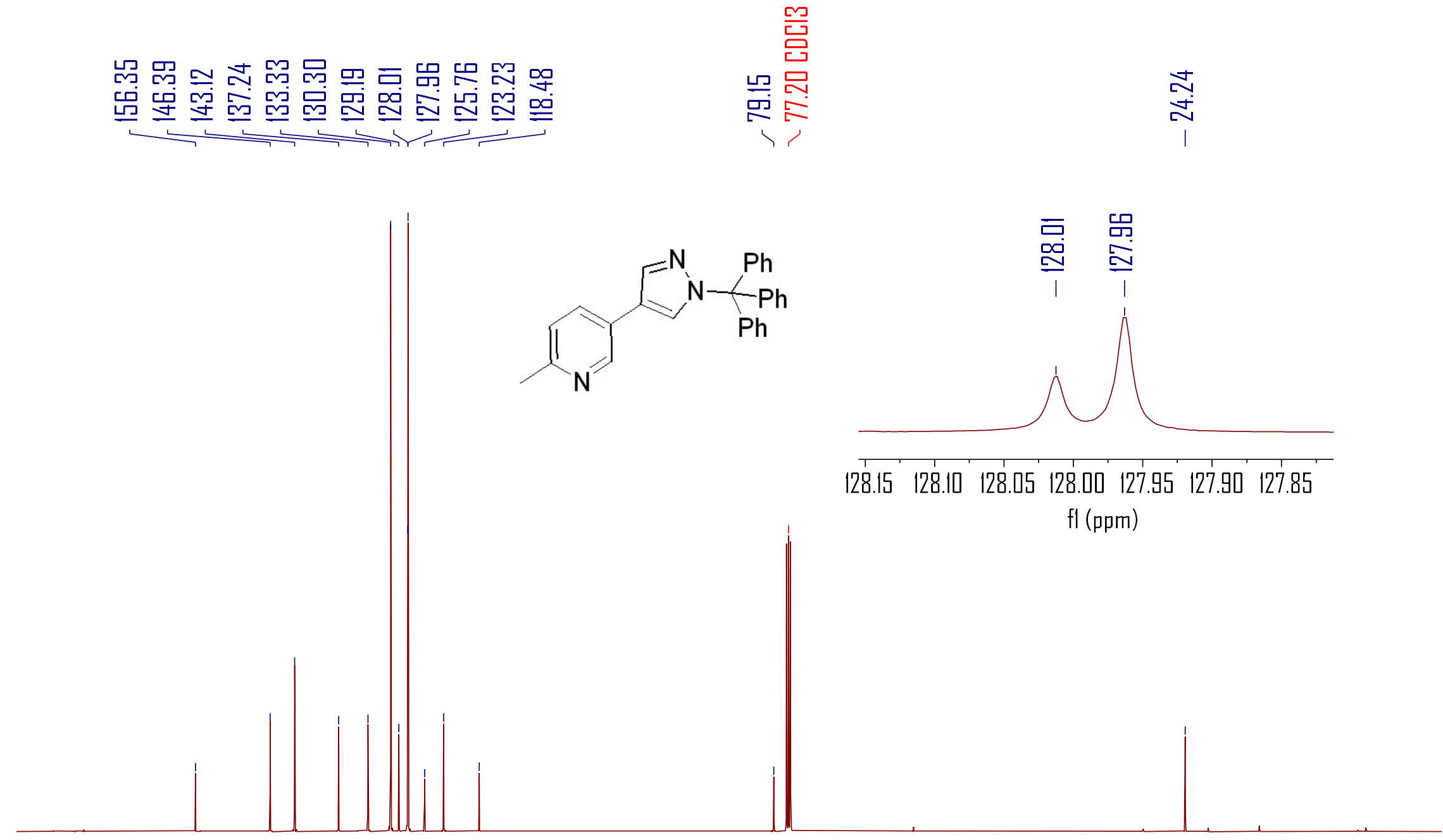

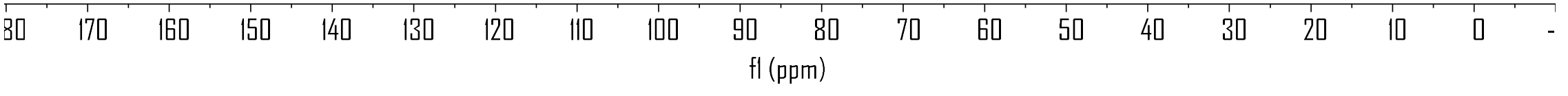




\section{${ }^{1} \mathrm{H}$ NMR spectrum of compound 3t (500 $\left.\mathrm{MHz}, \mathrm{CDCl}_{3}\right)$}

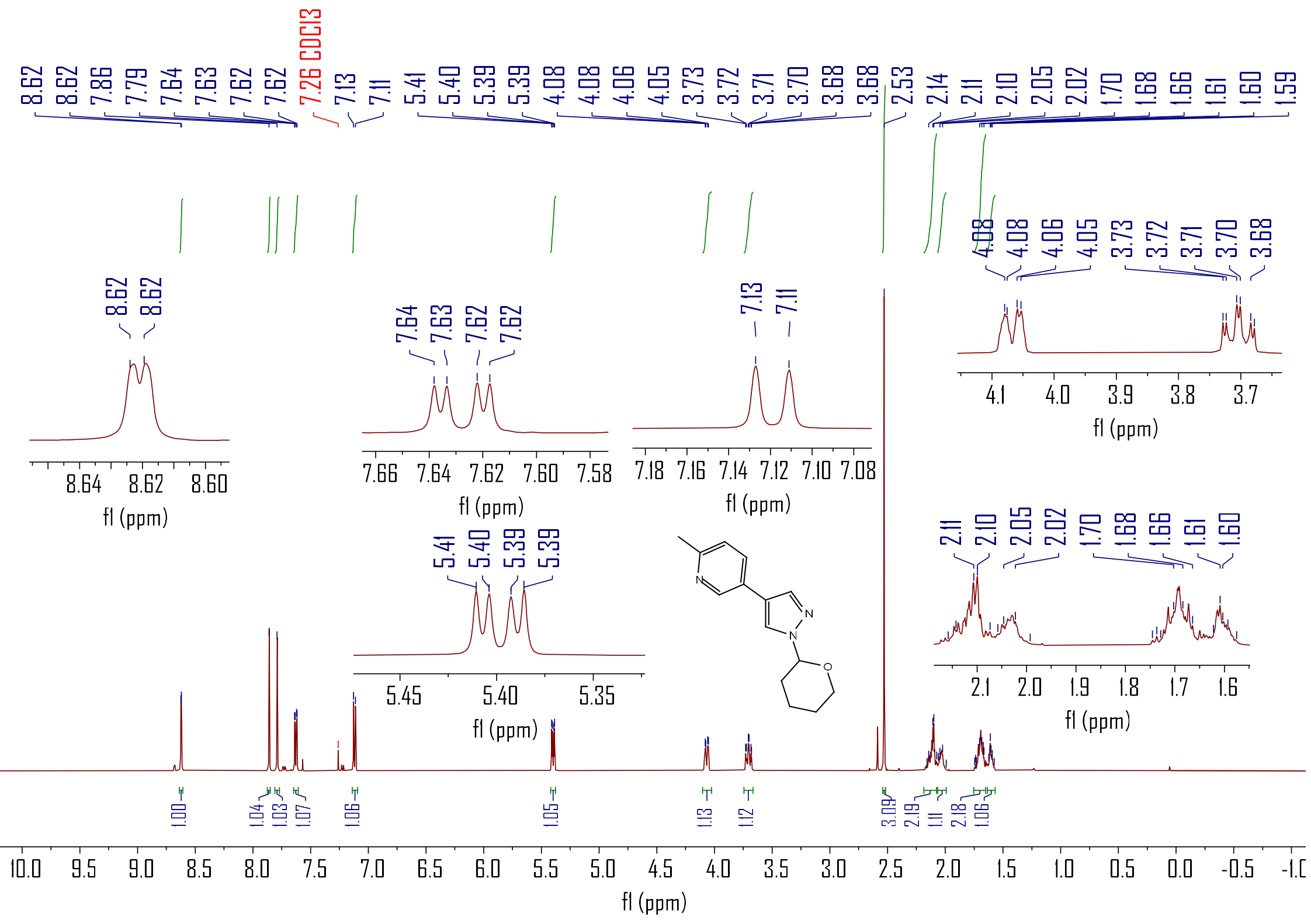


${ }^{13} \mathrm{C}\left\{{ }^{1} \mathrm{H}\right\}$ NMR spectrum of compound $3 \mathrm{t}\left(126 \mathrm{MHz}, \mathrm{CDCl}_{3}\right)$

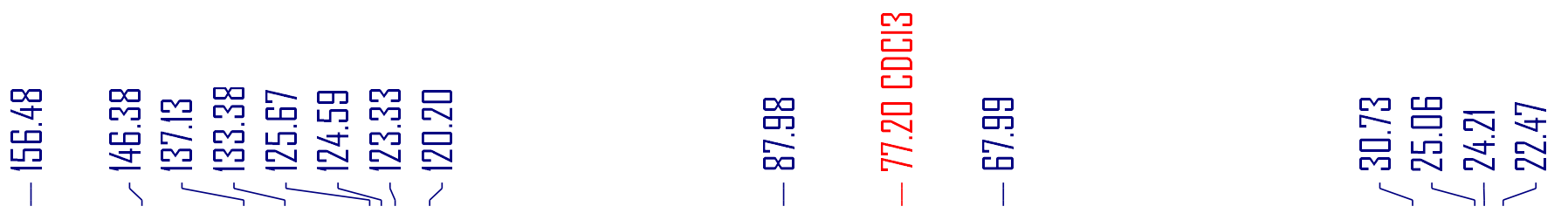

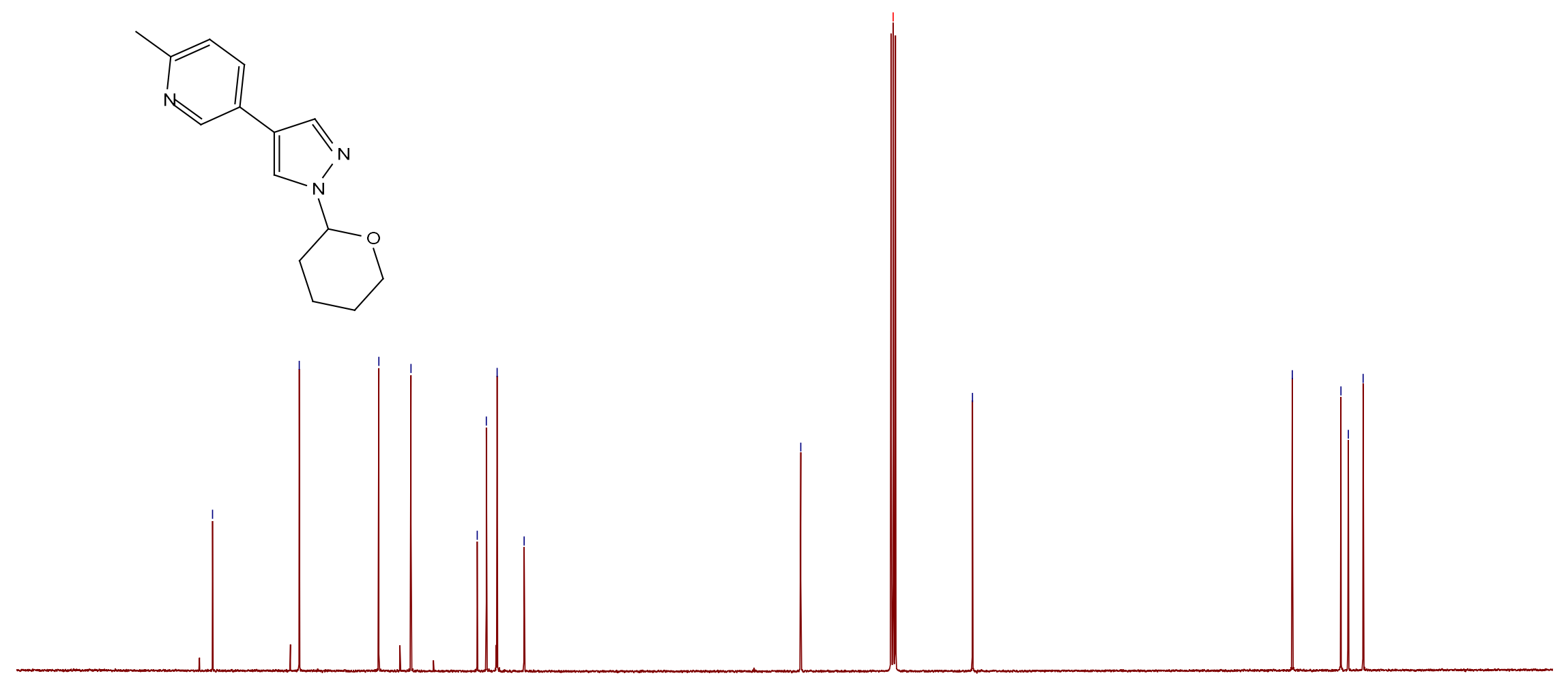

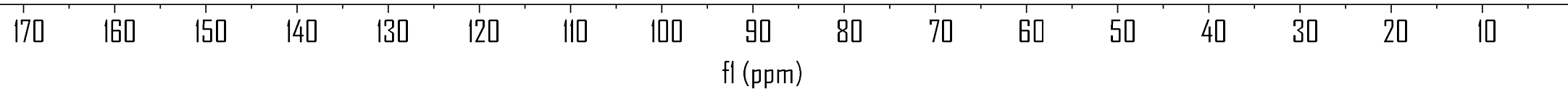




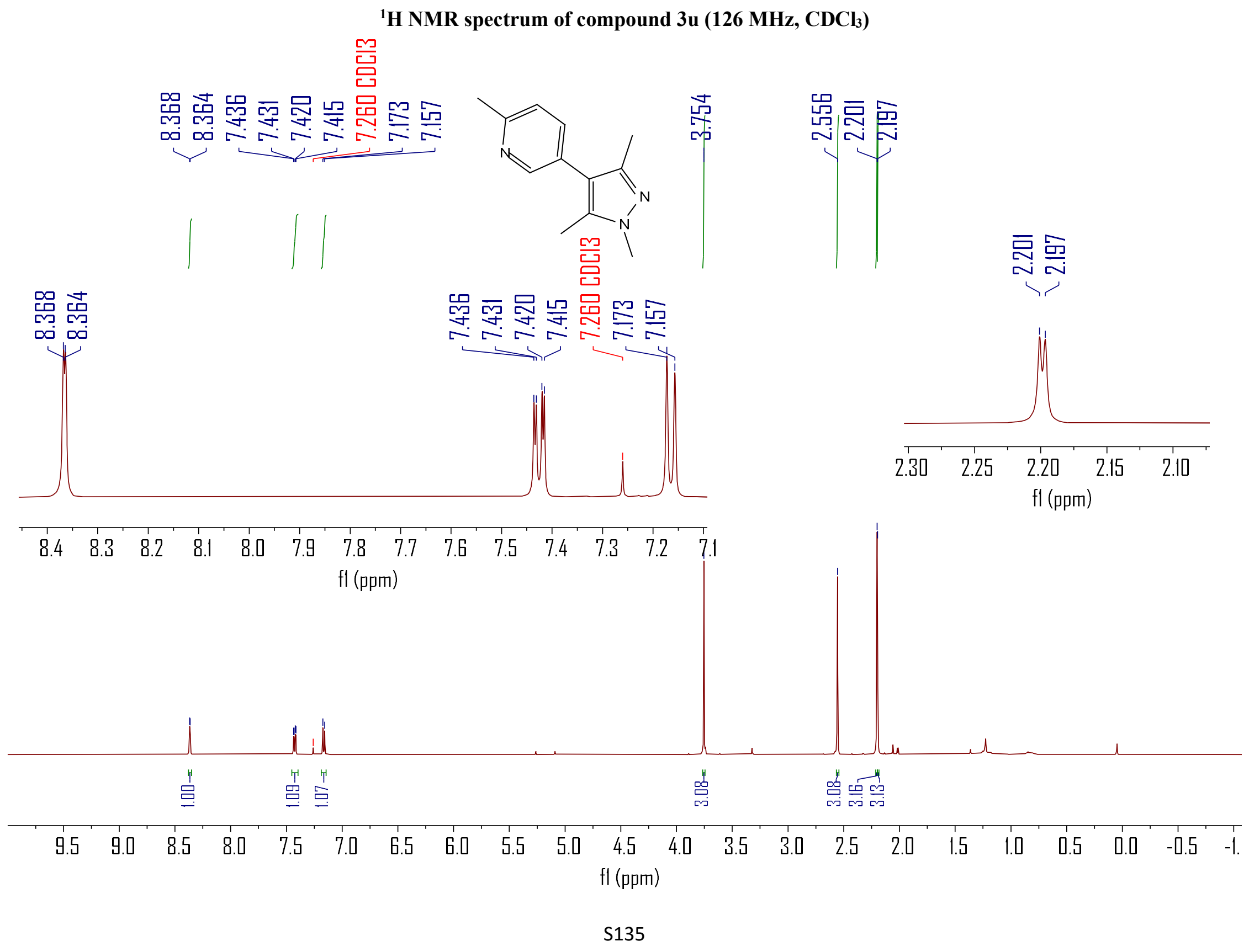




\section{${ }^{13} \mathrm{C}\left\{{ }^{1} \mathrm{H}\right\}$ NMR spectrum of compound $3 \mathrm{u}\left(126 \mathrm{MHz}, \mathrm{CDCl}_{3}\right)$}

氙出品吕哭正响过

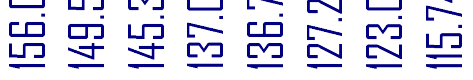

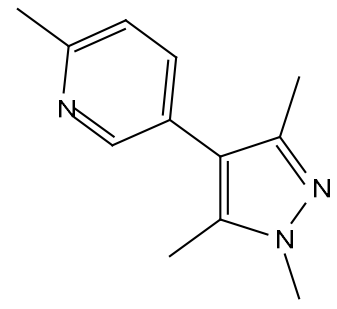

믐
뭄
$ㅁ$

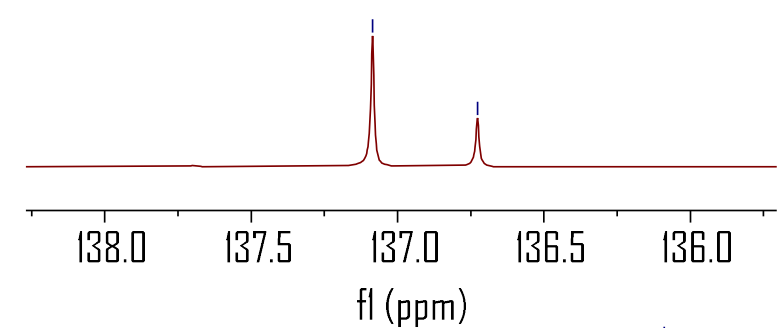

몀 똠

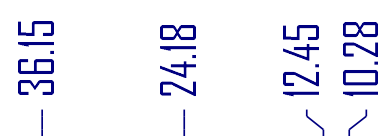

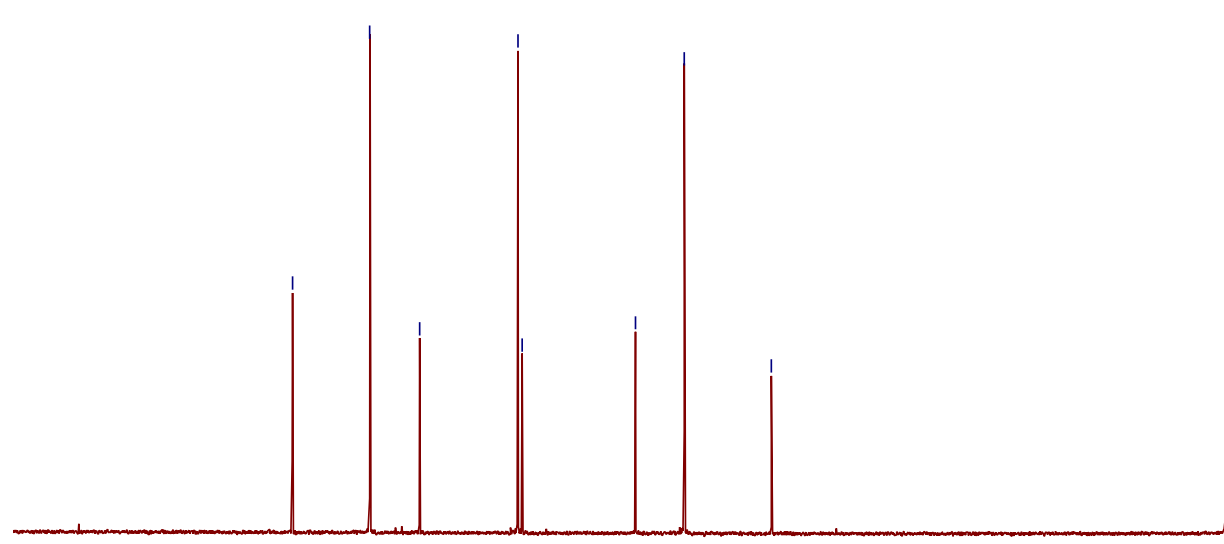

$\begin{array}{lllllllll}170 & 160 & 150 & 140 & 130 & 120 & 110 & 100 & 90\end{array}$ fl (ppm) 
${ }^{1} \mathrm{H}$ NMR spectrum of compound 3v (500 $\left.\mathrm{MHz}, \mathrm{CDCl}_{3}\right)$

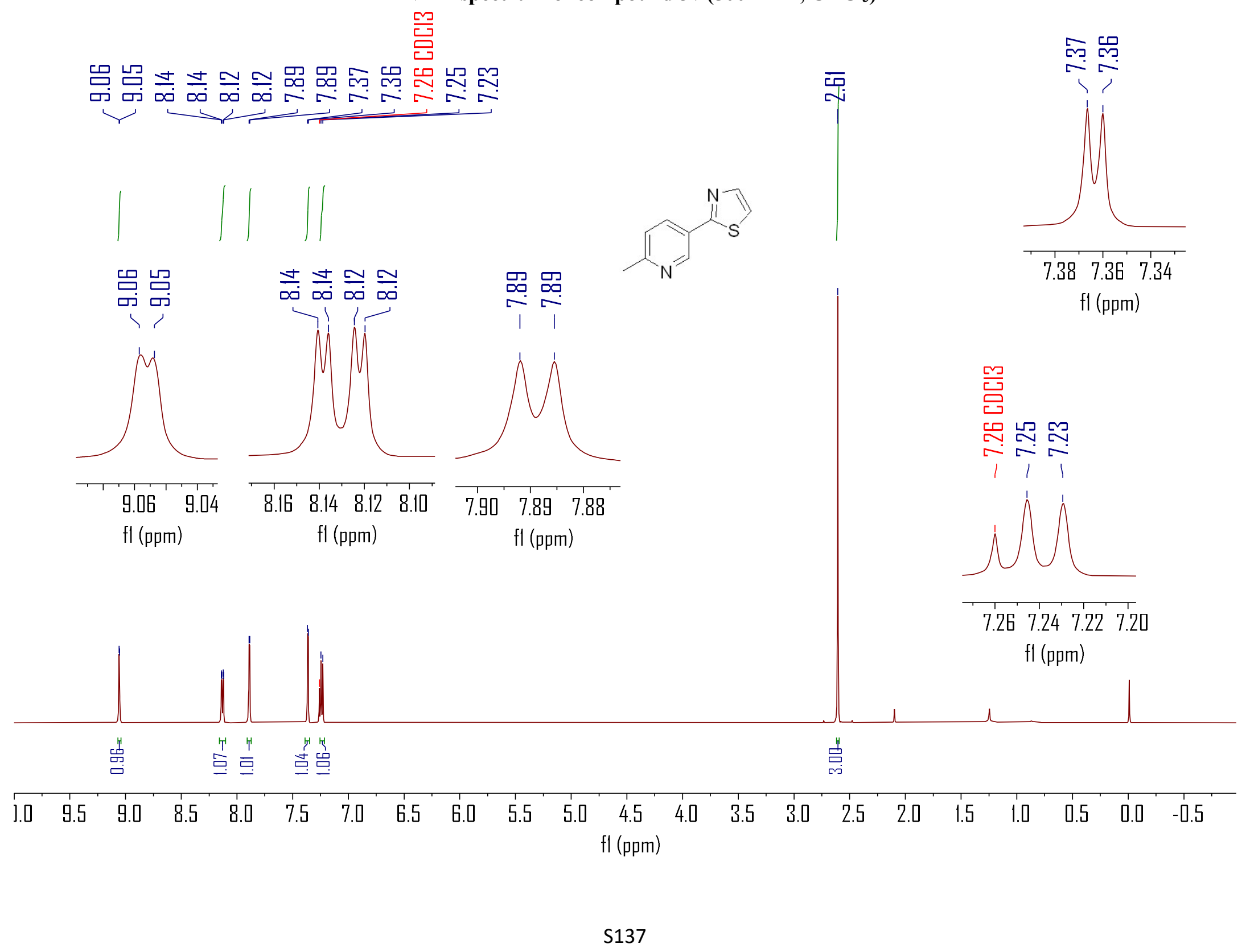


${ }^{13} \mathrm{C}\left\{{ }^{1} \mathrm{H}\right\}$ NMR spectrum of compound 3v (126 MHz, $\left.\mathrm{CDCl}_{3}\right)$

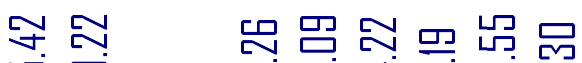

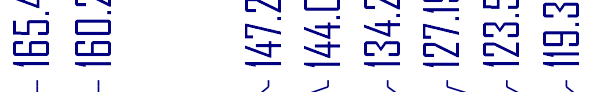

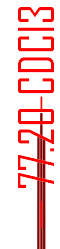

品
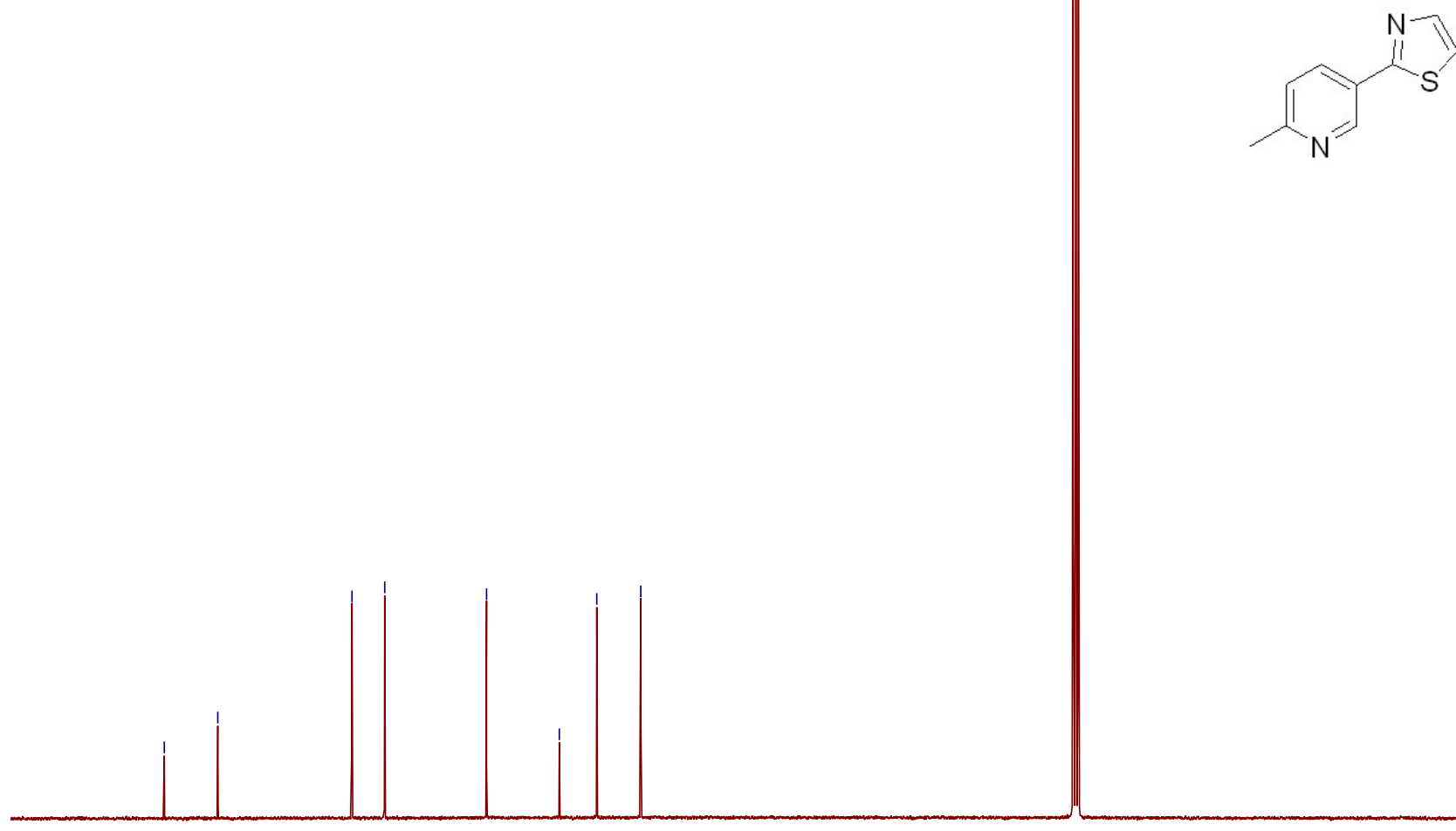

80
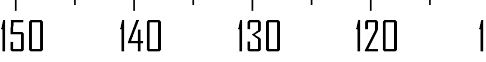

$10 \quad 100$

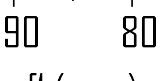

70

605




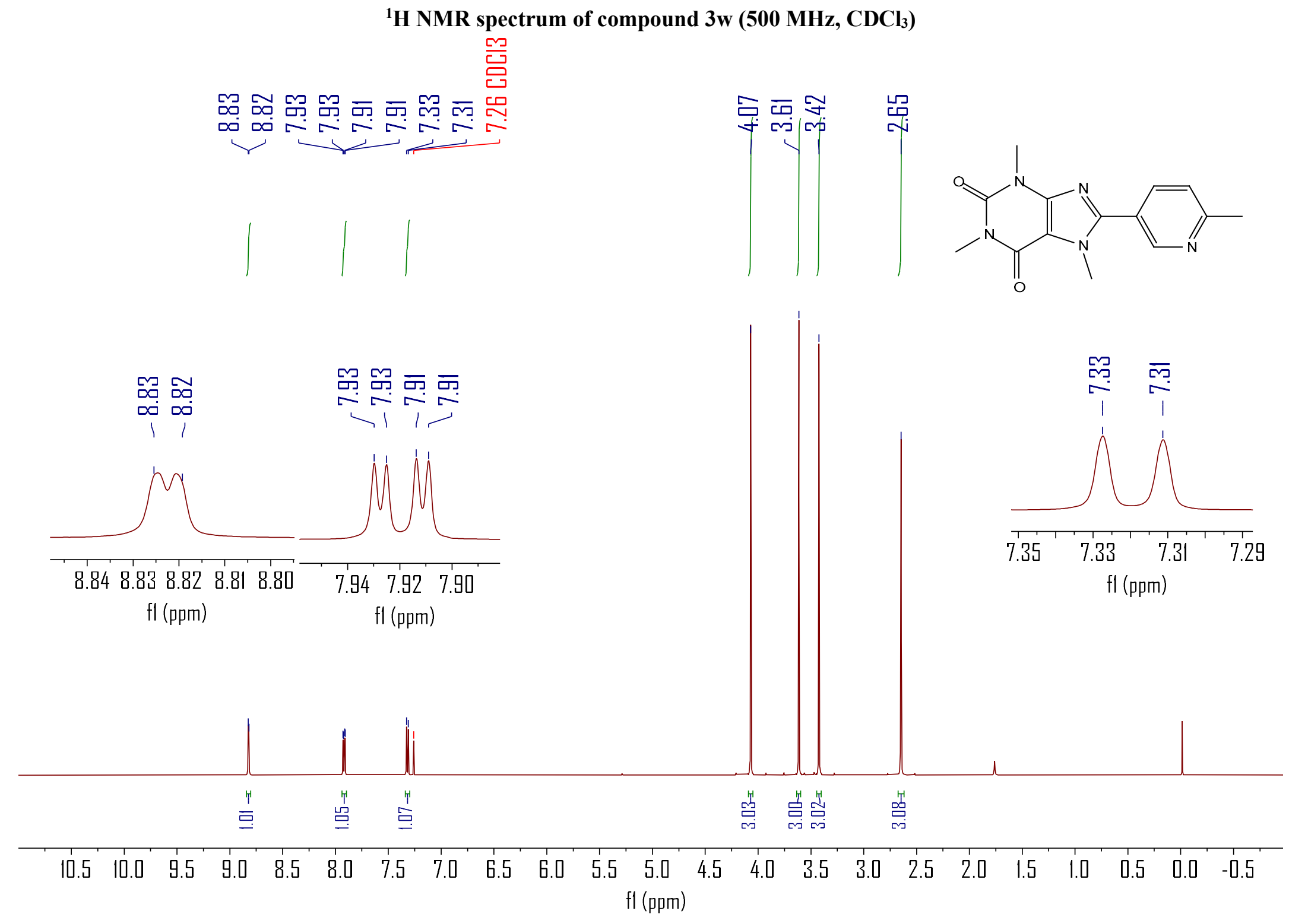




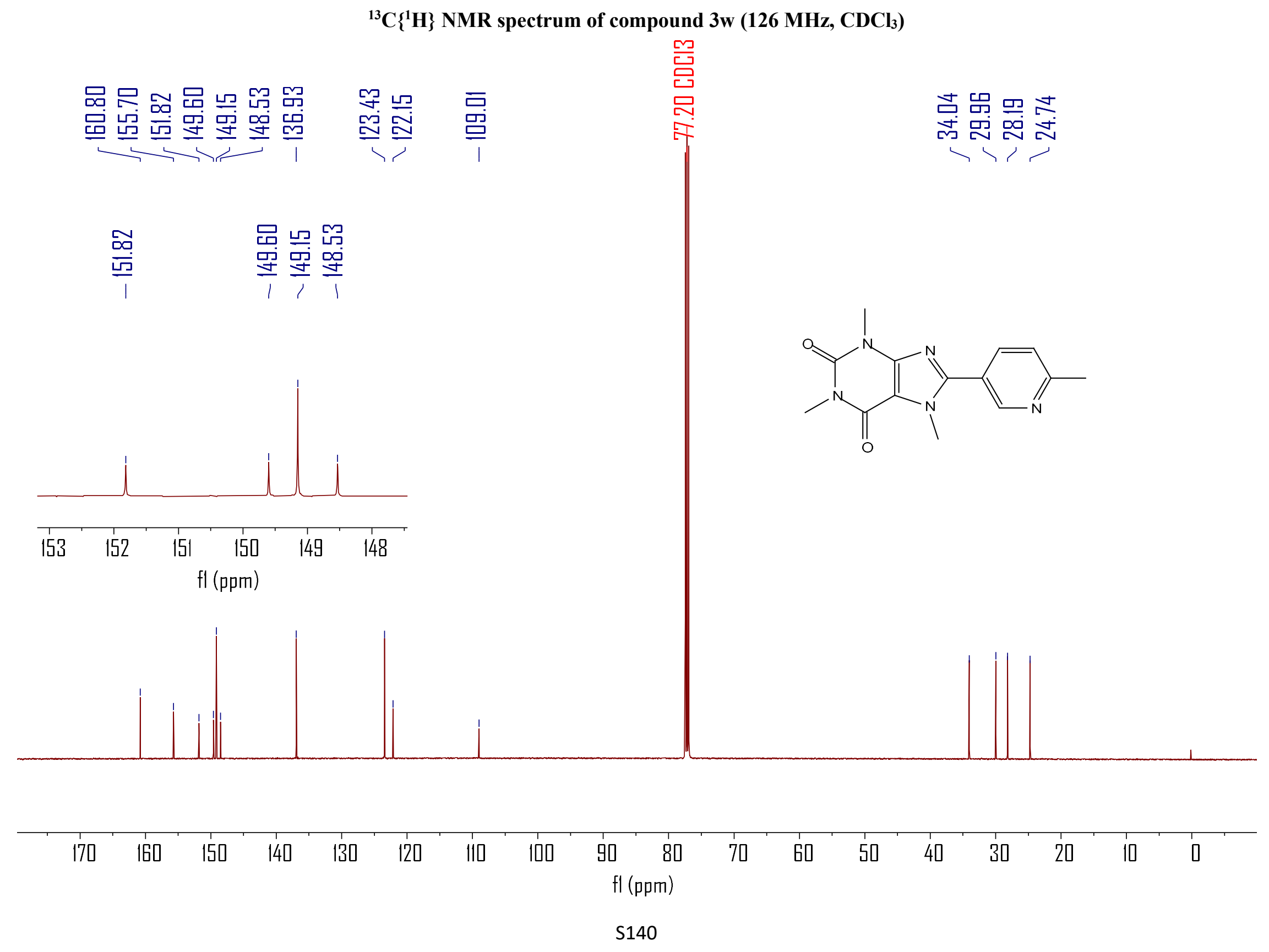


${ }^{1} \mathrm{H}$ NMR spectrum of compound 3x (500 $\left.\mathrm{MHz}, \mathrm{CDCl}_{3}\right)$

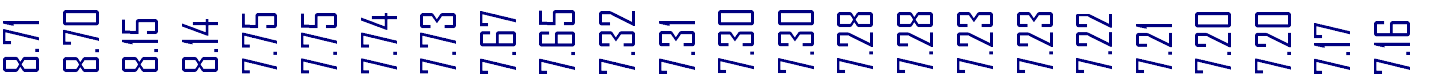
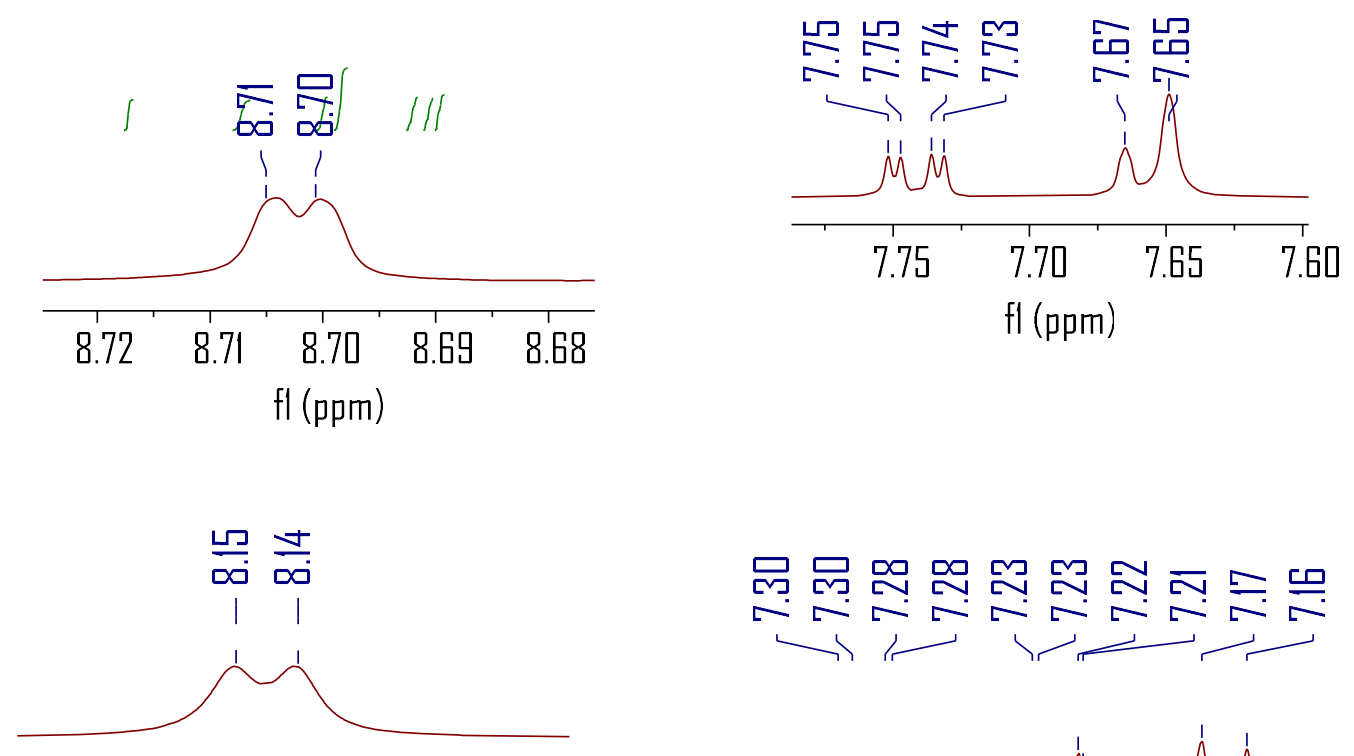

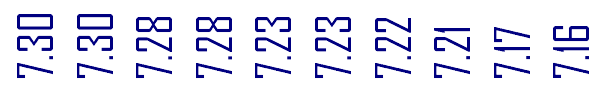

(15250

$8.208 .18 \quad 8.16 \quad 8.148 .128 .108 .08$

$\mathrm{fl}(\mathrm{ppm})$

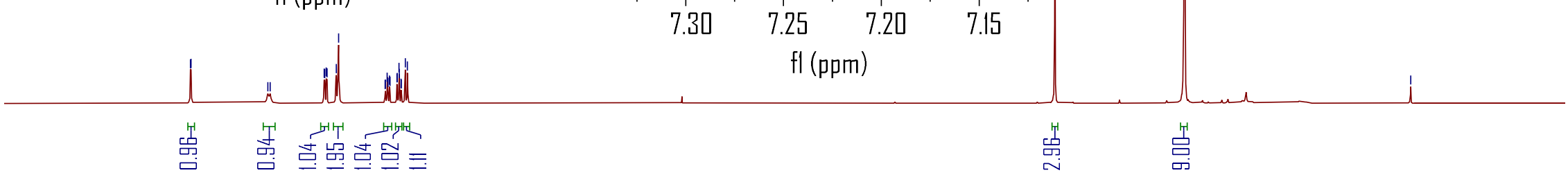

\begin{tabular}{llllllllllllllllllllllll}
\hline .0 & 9.5 & 9.0 & 8.5 & 8.0 & 7.5 & 7.0 & 6.5 & 6.0 & 5.5 & 5.0 & 4.5 & 4.0 & 3.5 & 3.0 & 2.5 & 2.0 & 1.5 & 1.0 & 0.5 & 0.0 & -0.5 & -1.5
\end{tabular} 
${ }^{13} \mathrm{C}\left\{{ }^{1} \mathrm{H}\right\}$ NMR spectrum of compound $3 \times\left(126 \mathrm{MHz}, \mathrm{CDCl}_{3}\right)$

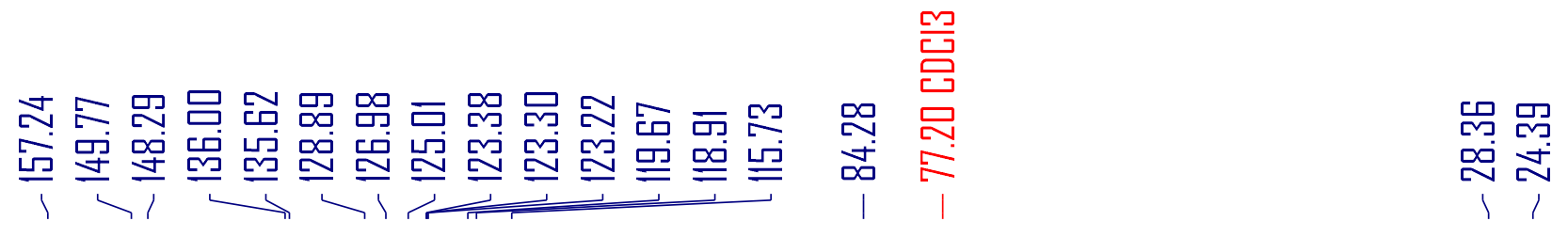
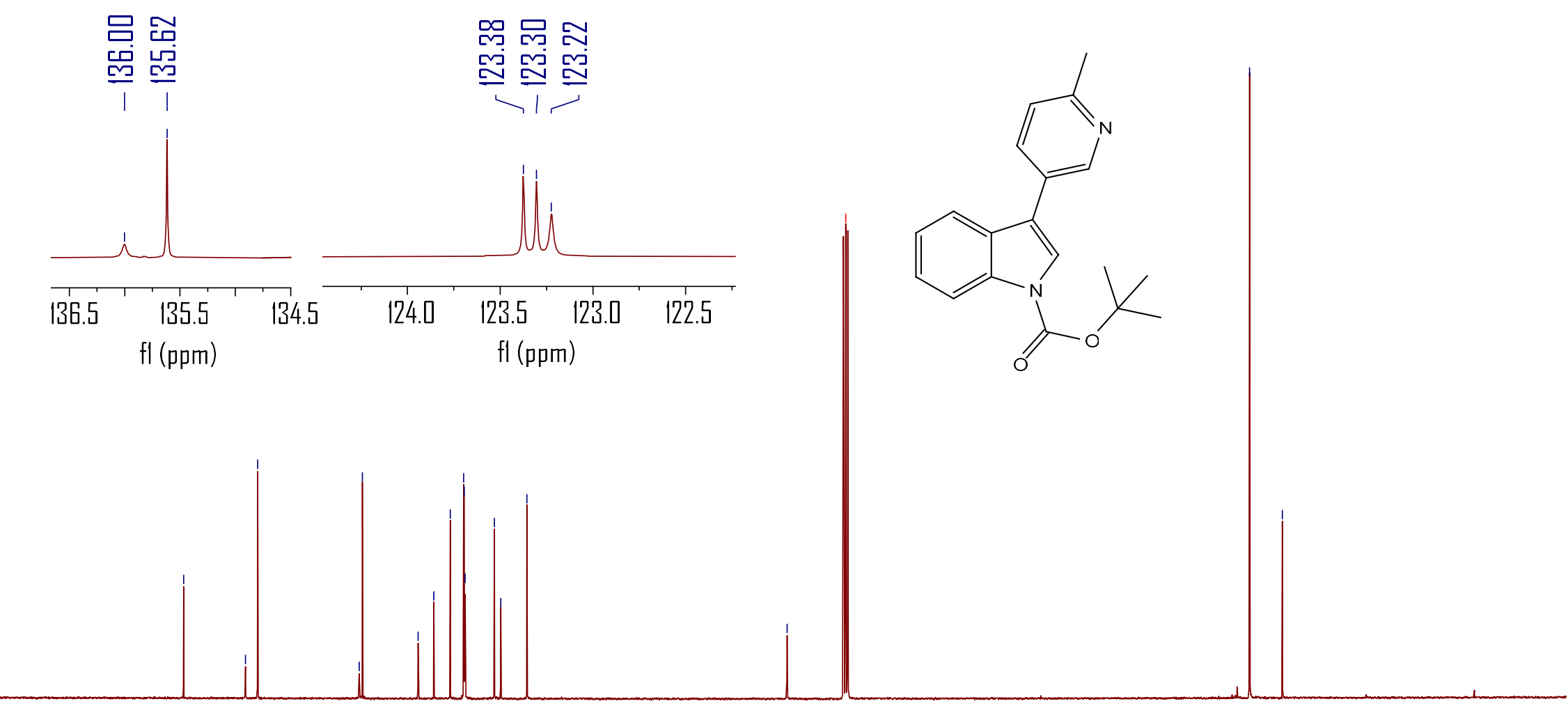

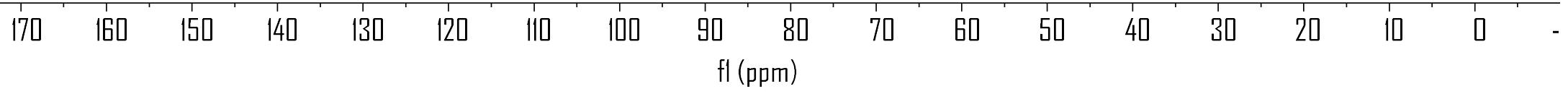


${ }^{1} \mathrm{H}$ NMR spectrum of compound $3 \mathrm{y}\left(500 \mathrm{MHz}, \mathrm{CDCl}_{3}\right)$
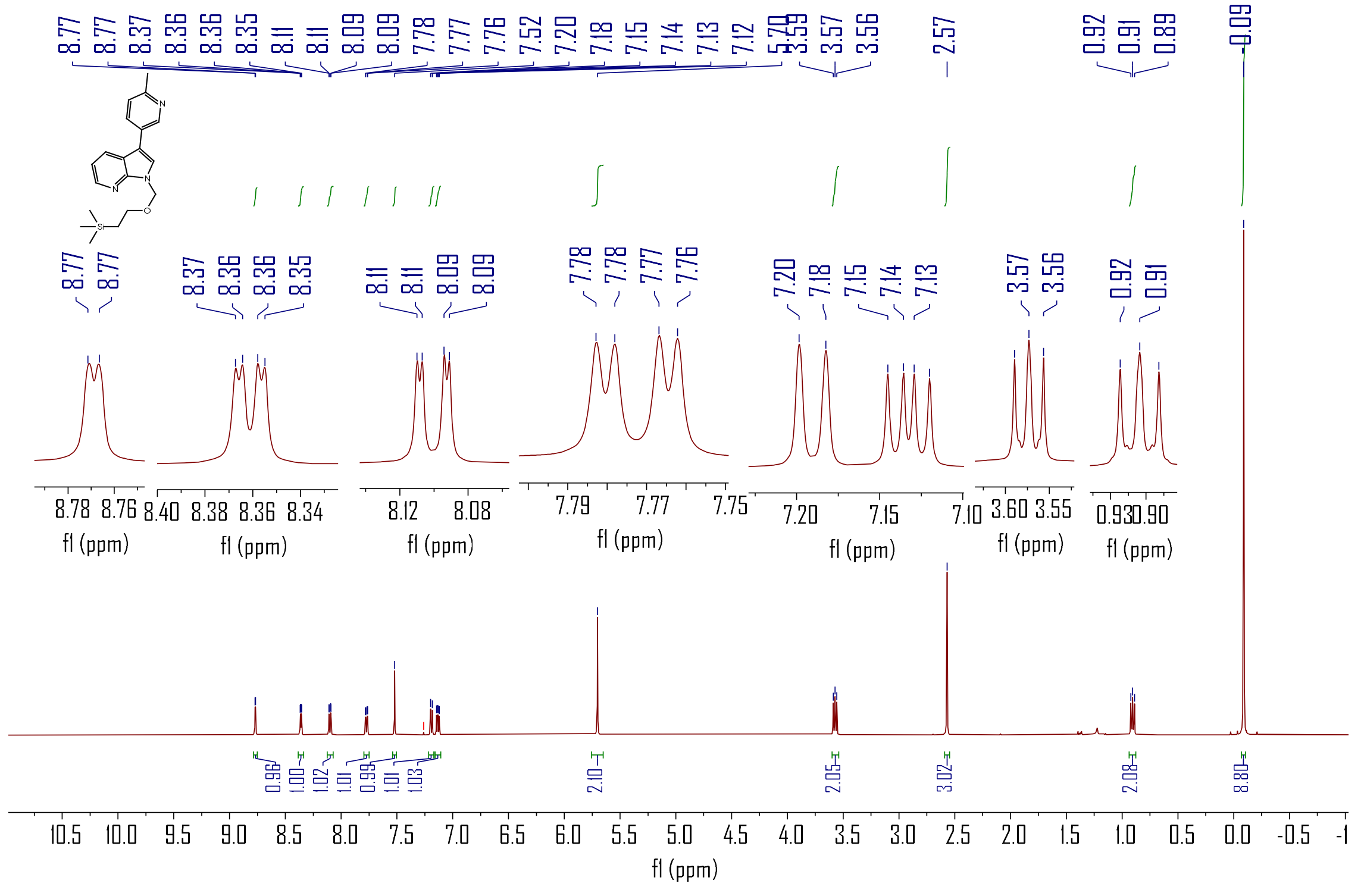
${ }^{13} \mathrm{C}\left\{{ }^{1} \mathrm{H}\right\}$ NMR spectrum of compound $3 \mathrm{y}\left(126 \mathrm{MHz}, \mathrm{CDCl}_{3}\right)$

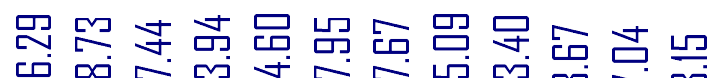

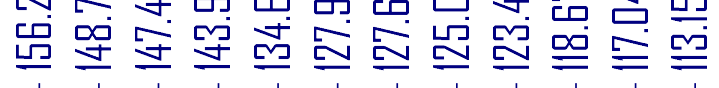

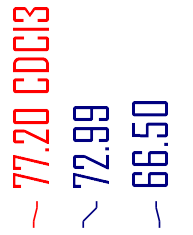

包

모ํ

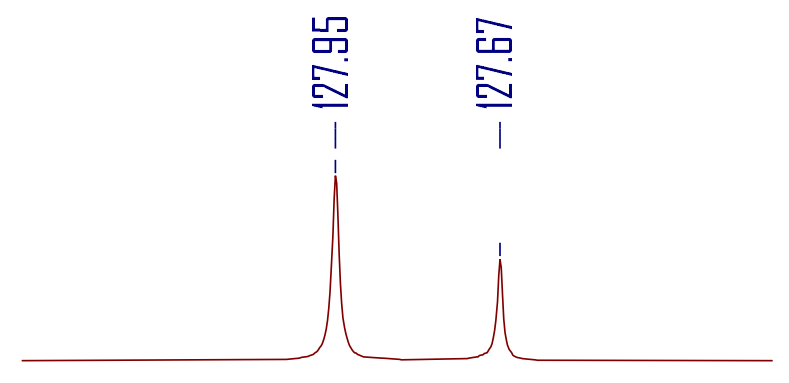

$\begin{array}{llllll}128.3 & 128.1 & 127.9 & 127.7 & 127.5 & 127.3\end{array}$

$\mathrm{fl}$ (ppm)
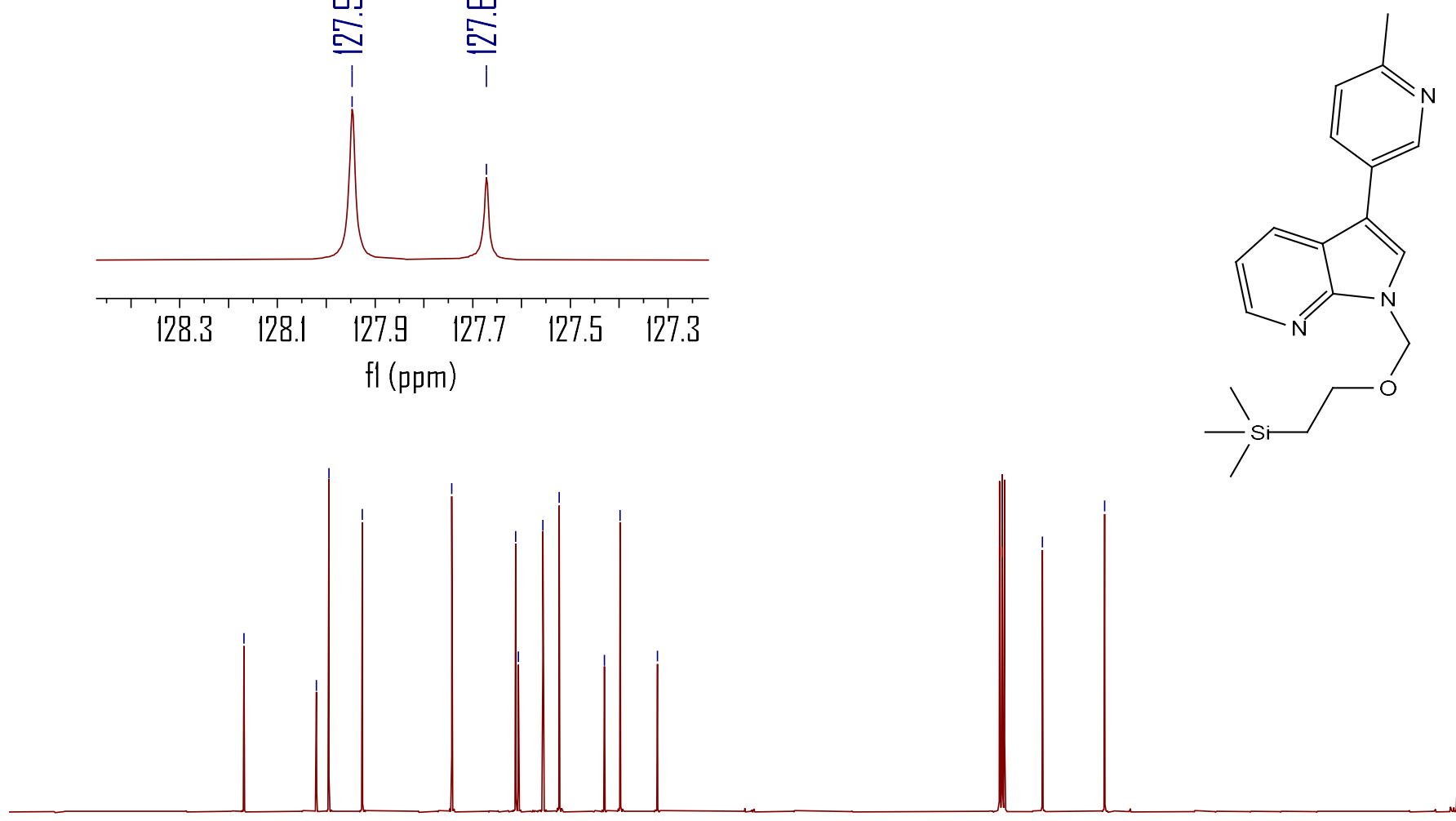

$80 \quad 170 \quad 16$

150

140

130

$20 \quad 110$

100

fl (ppm) 


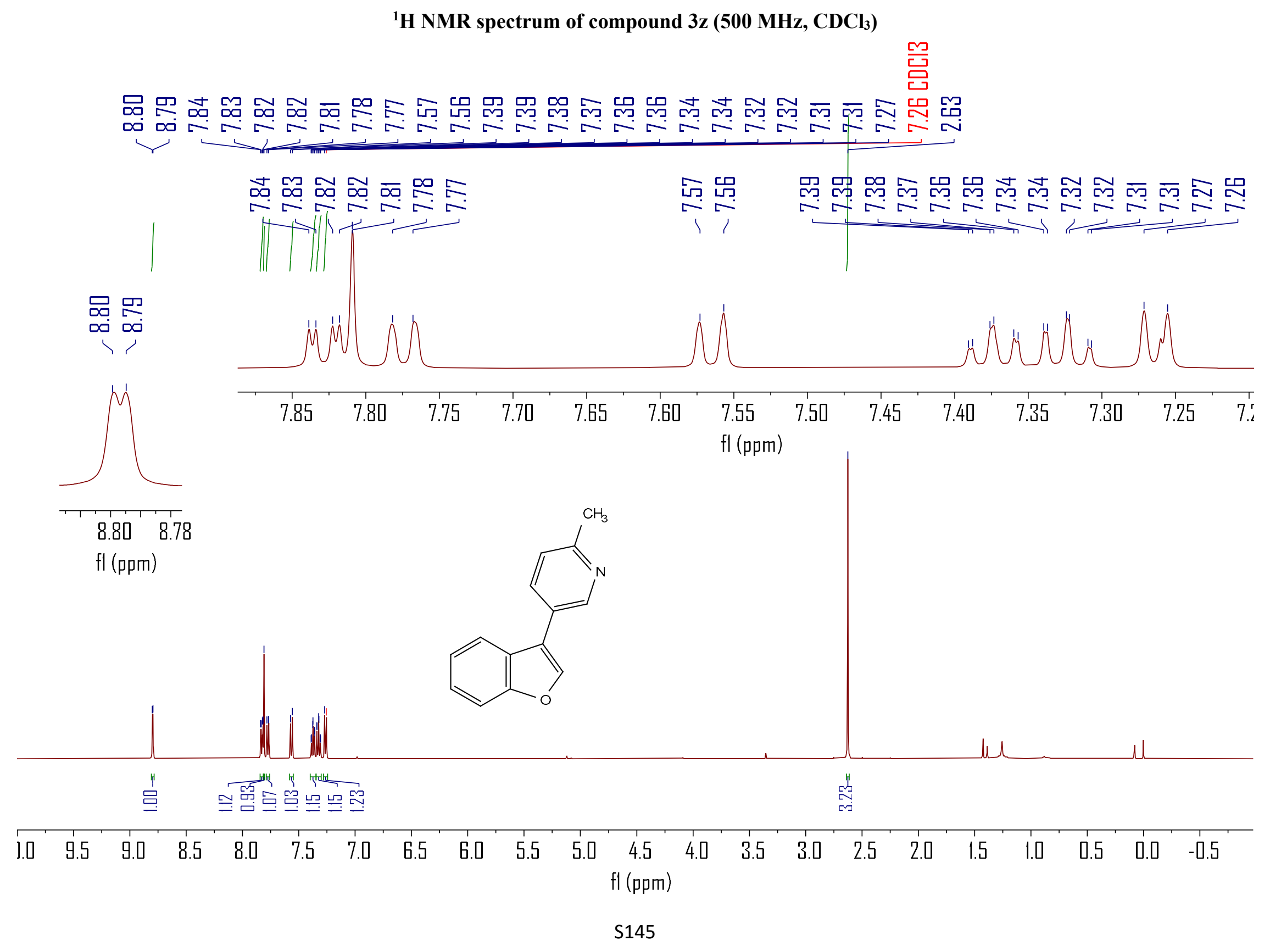


${ }^{13} \mathrm{C}\left\{{ }^{1} \mathrm{H}\right\}$ NMR spectrum of compound $3 \mathrm{z}\left(126 \mathrm{MHz}, \mathrm{CDCl}_{3}\right)$

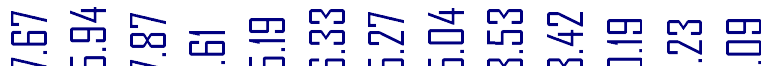

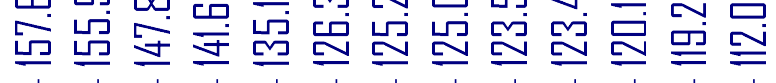

믈

$\stackrel{\text { I }}{\stackrel{N}{*}}$
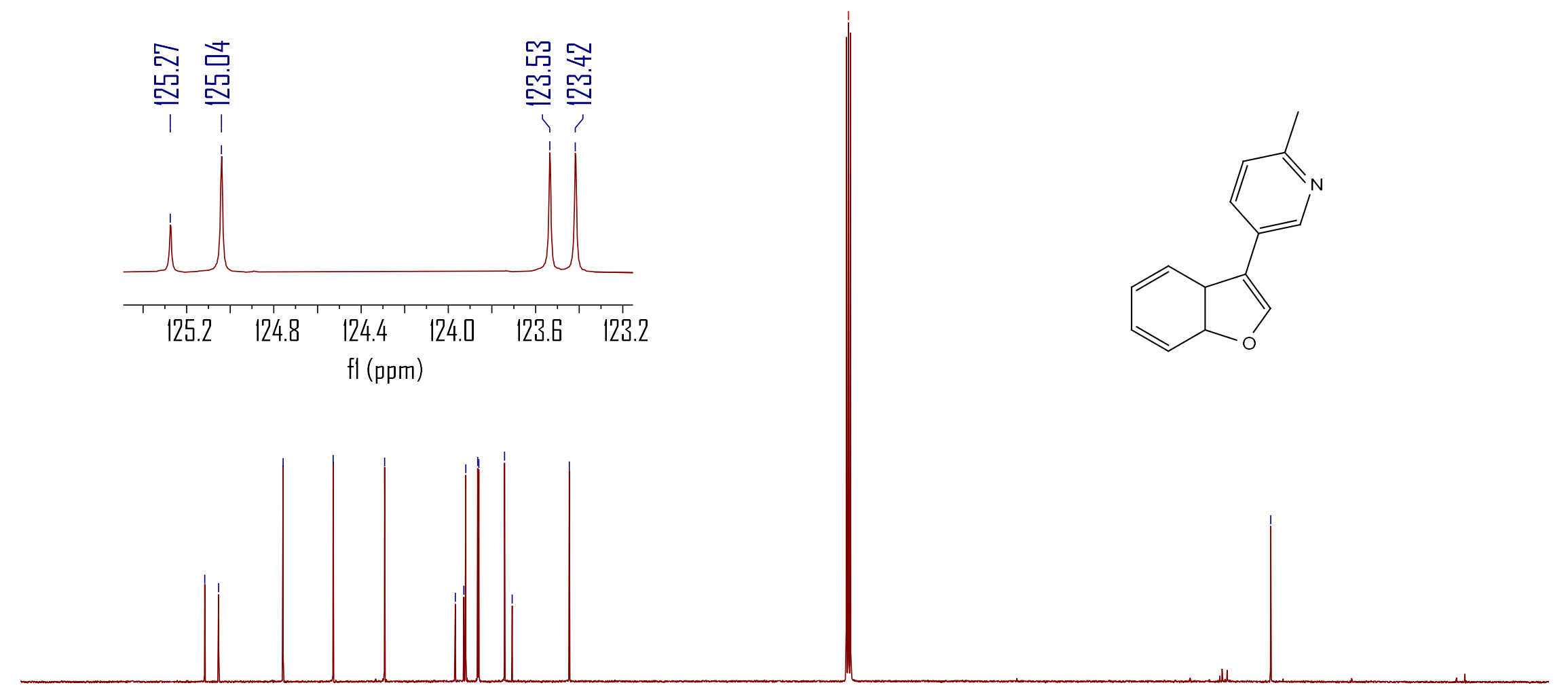

罗

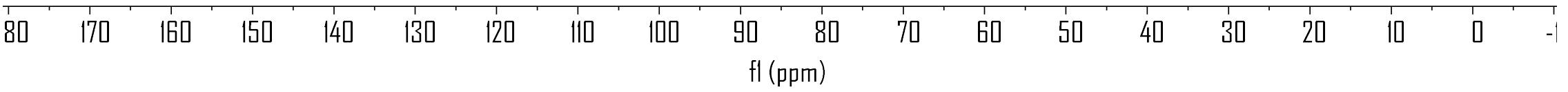


${ }^{1} \mathrm{H}$ NMR spectrum of compound 3aa (400 $\mathrm{MHz}, \mathrm{CDCl}_{3}$ )

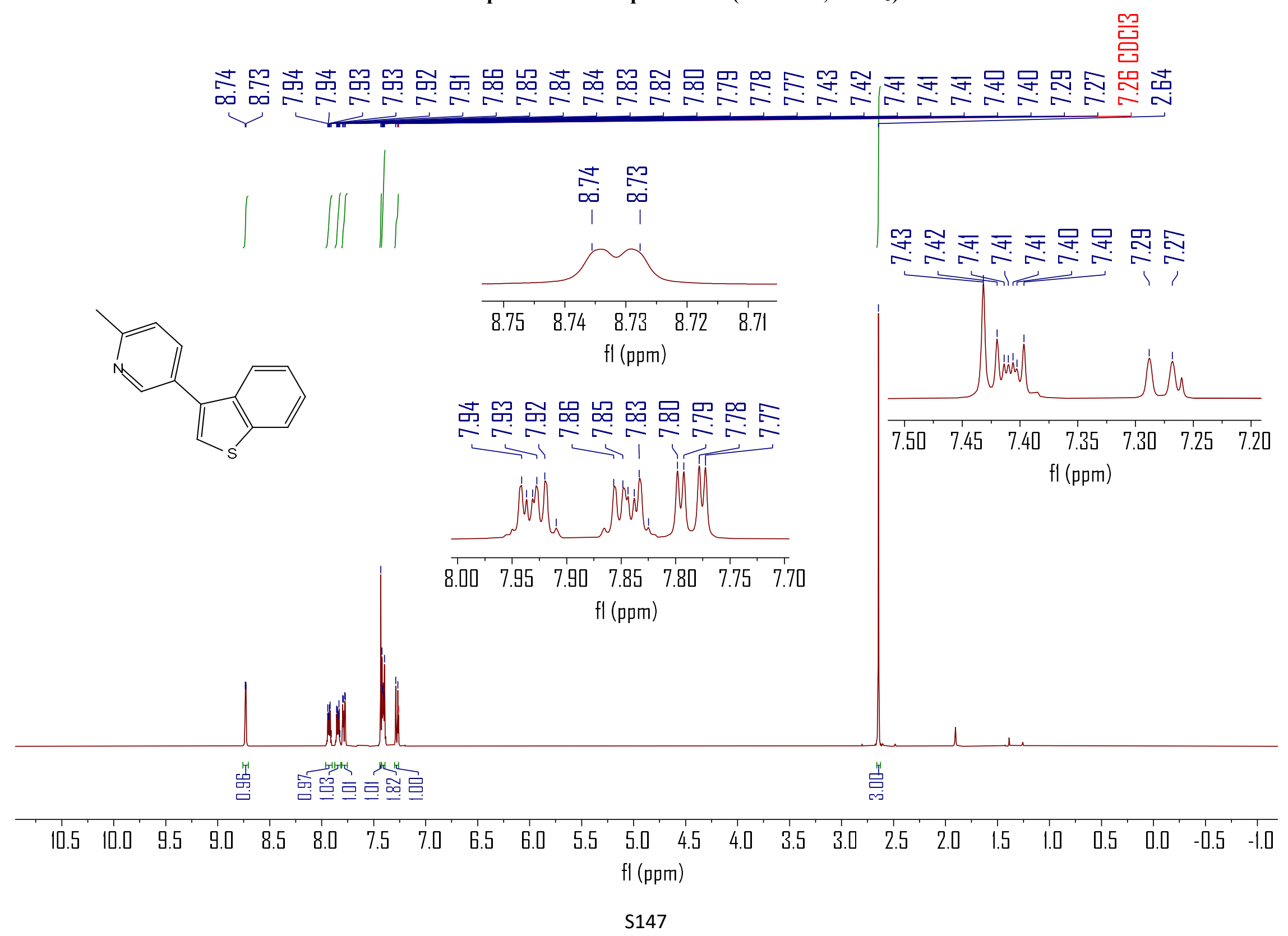


${ }^{13} \mathrm{C}\left\{{ }^{1} \mathrm{H}\right\}$ NMR spectrum of compound 3aa (101 $\left.\mathrm{MHz}, \mathrm{CDCl}_{3}\right)$

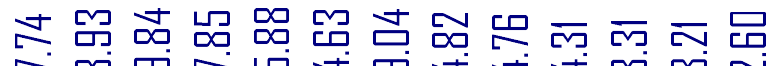

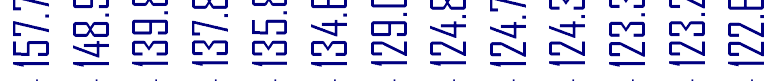

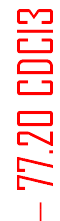

$\underset{\substack{\text { 足 } \\ \text { I }}}{ }$
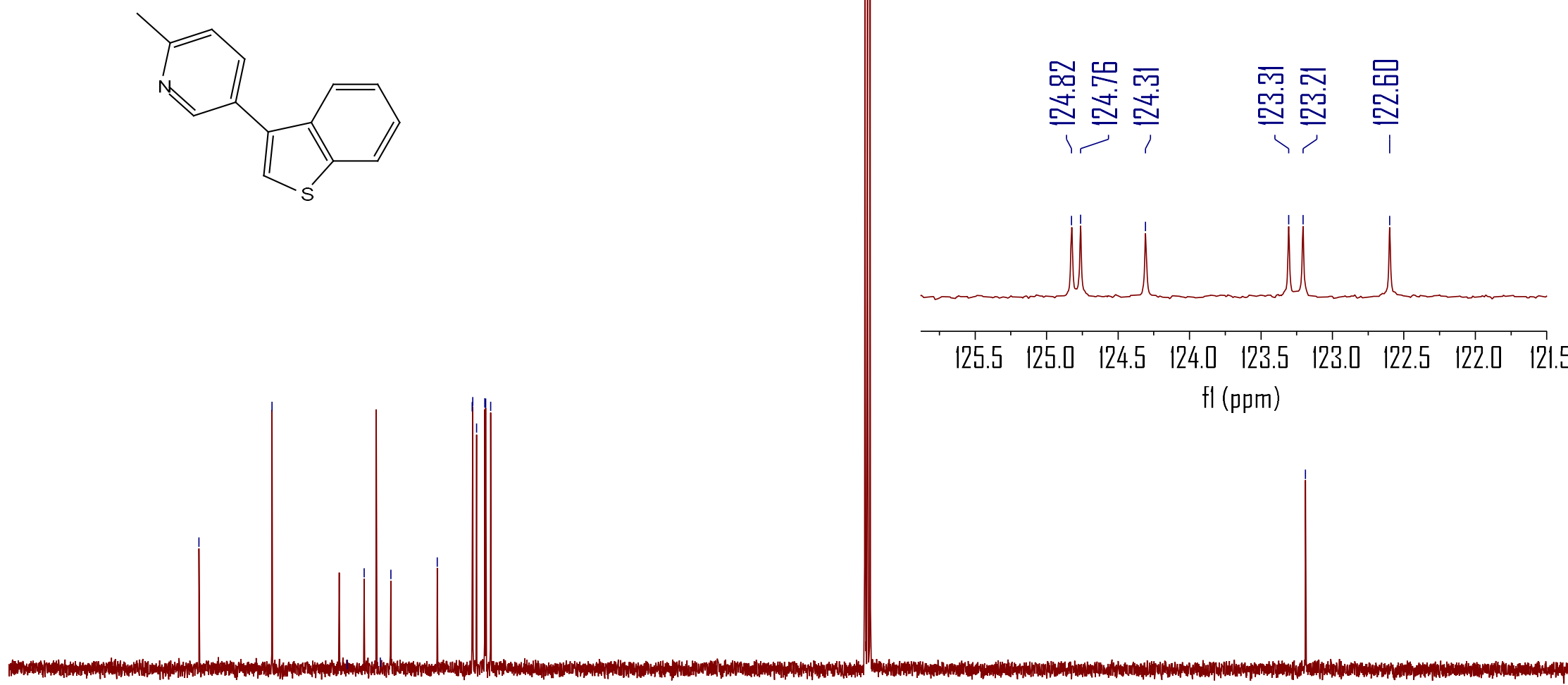

$\mathrm{fl}$ (ppm)

\begin{tabular}{|c|c|c|c|c|c|c|c|c|c|c|c|c|c|c|c|c|}
\hline $80 \quad 170$ & 160 & 150 & 140 & 130 & 120 & 110 & 100 & $\begin{array}{ll}\mathrm{fl}(\mathrm{ppm}) \\
\mathrm{c}\end{array}$ & 70 & 60 & 50 & 40 & 30 & 20 & 10 & 0 \\
\hline
\end{tabular}


${ }^{1} \mathrm{H}$ NMR spectrum of compound 3ab (500 $\left.\mathrm{MHz}, \mathrm{CDCl}_{3}\right)$

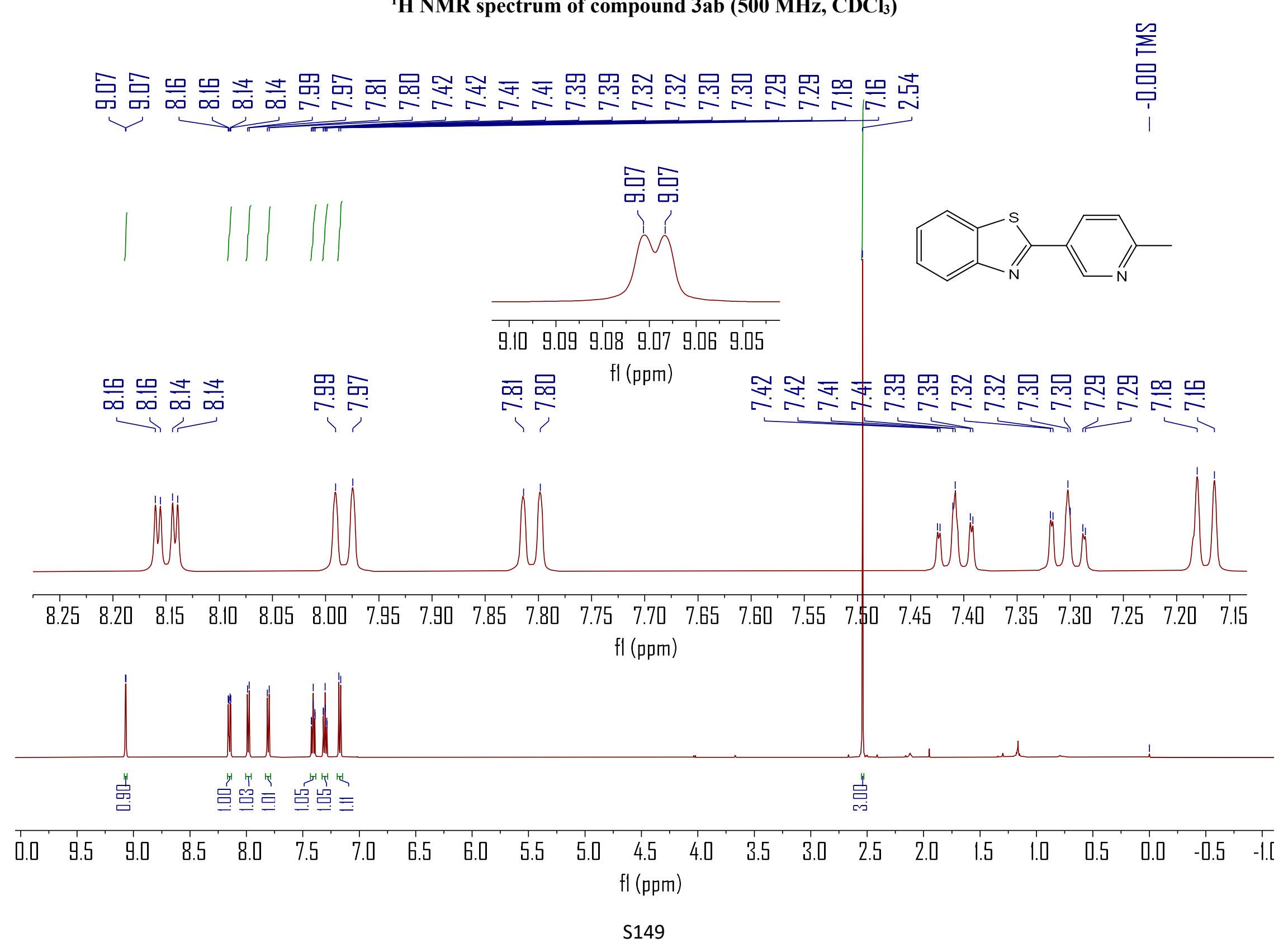




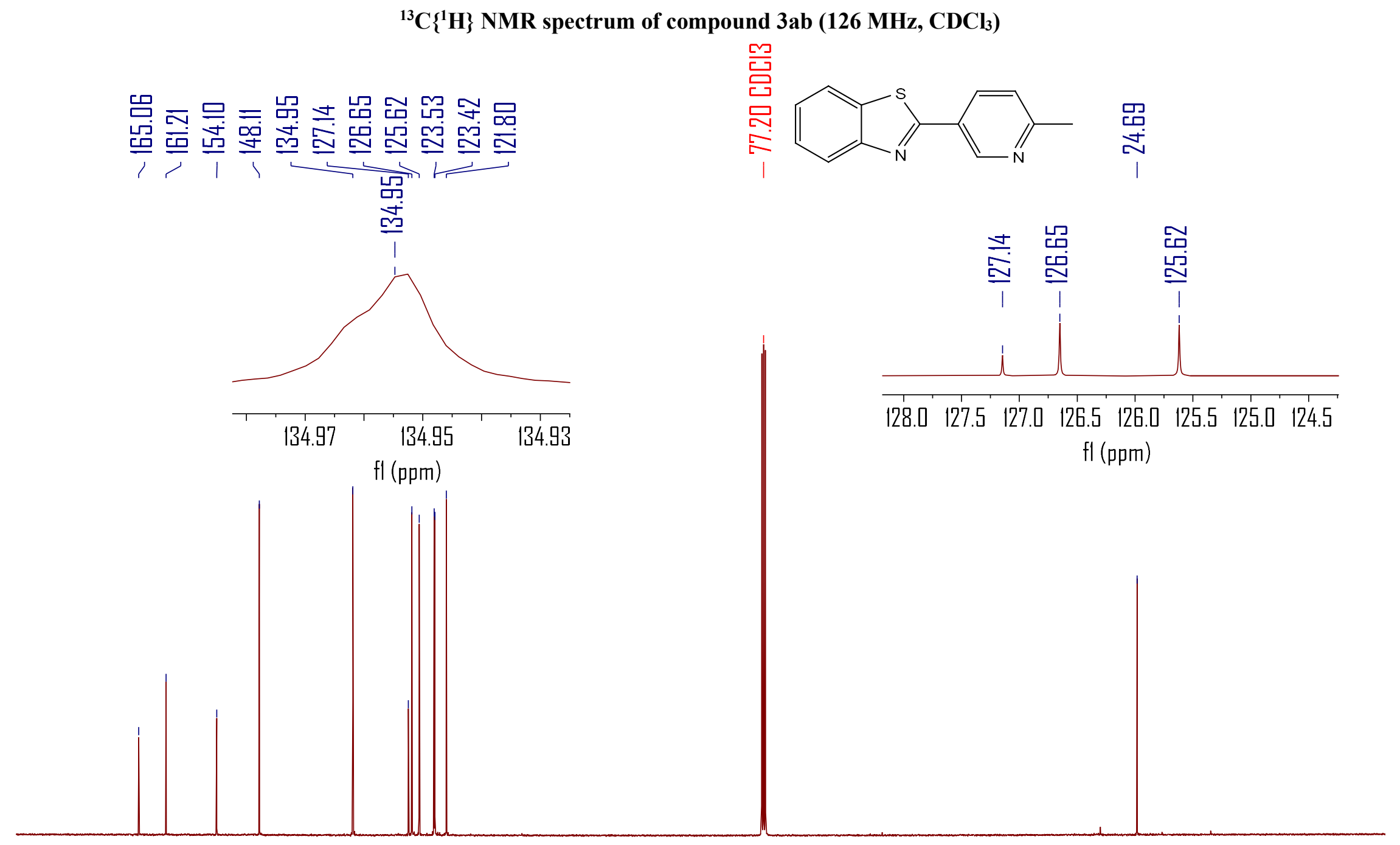

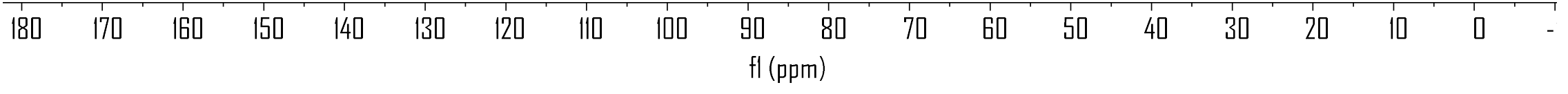


${ }^{1} \mathrm{H}$ NMR spectrum of compound $3 \mathrm{ac}\left(500 \mathrm{MHz}, \mathrm{CDCl}_{3}\right)$
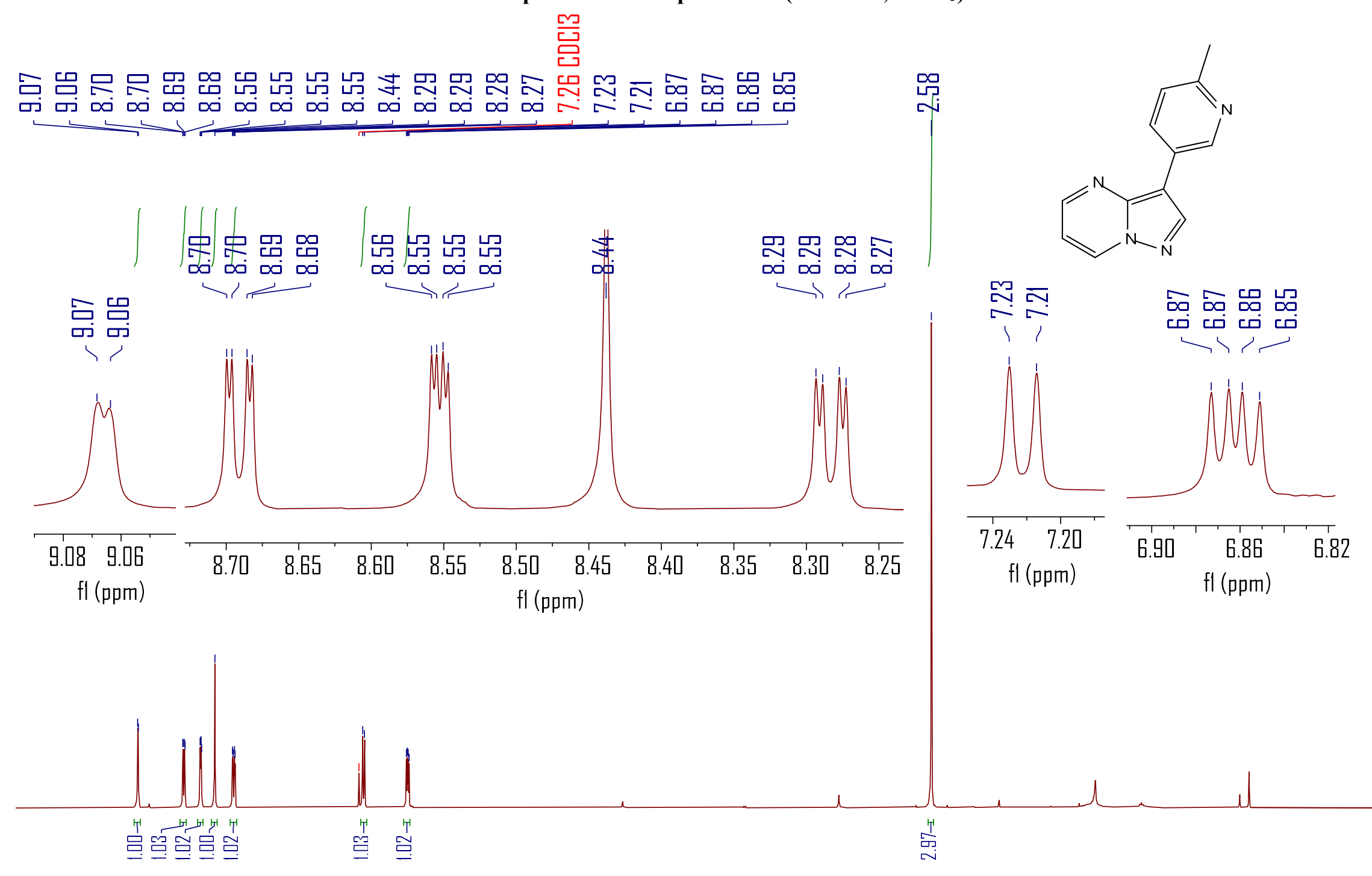

$\begin{array}{llllllllllllllllllllllllll}0.0 & 9.5 & 9.0 & 8.5 & 8.0 & 7.5 & 7.0 & 6.5 & 6.0 & 5.5 & 5.0 & \begin{array}{c}1.5 \\ \mathrm{fl}(\mathrm{pm})\end{array} & 4.0 & 3.5 & 3.0 & 2.5 & 2.0 & 1.5 & 1.0 & 0.5 & 0.0 & -0.5 & -1\end{array}$ 


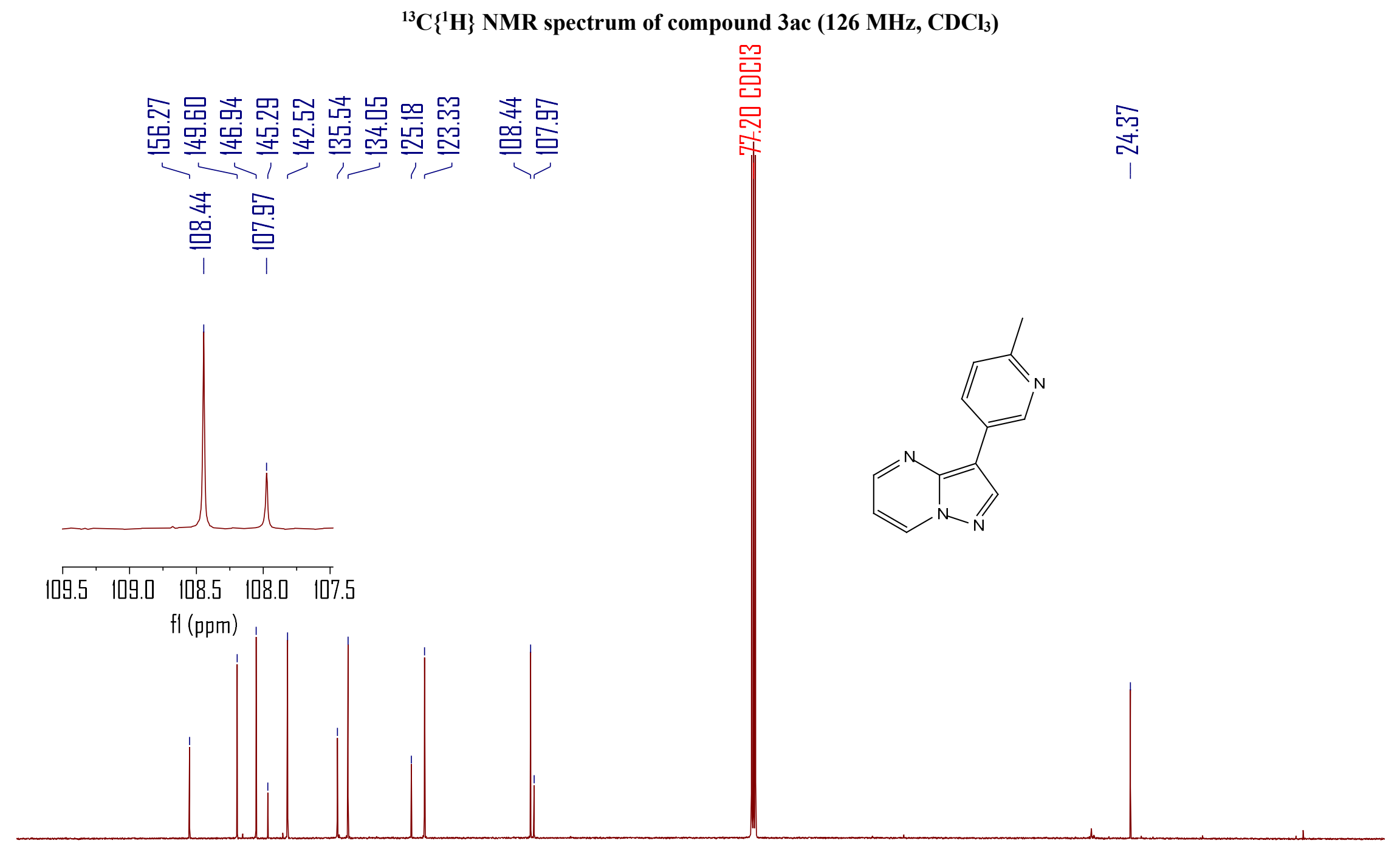

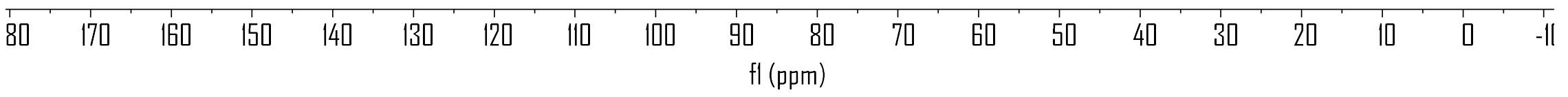


${ }^{1} \mathrm{H}$ NMR spectrum of compound $3 \mathrm{ad}\left(500 \mathrm{MHz}, \mathrm{CDCl}_{3}\right)$

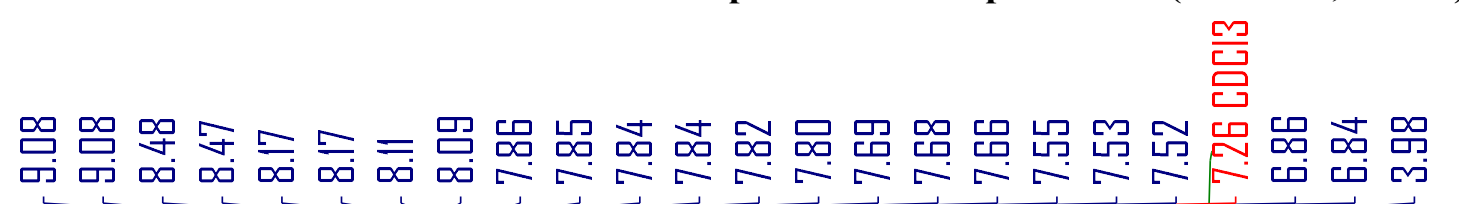
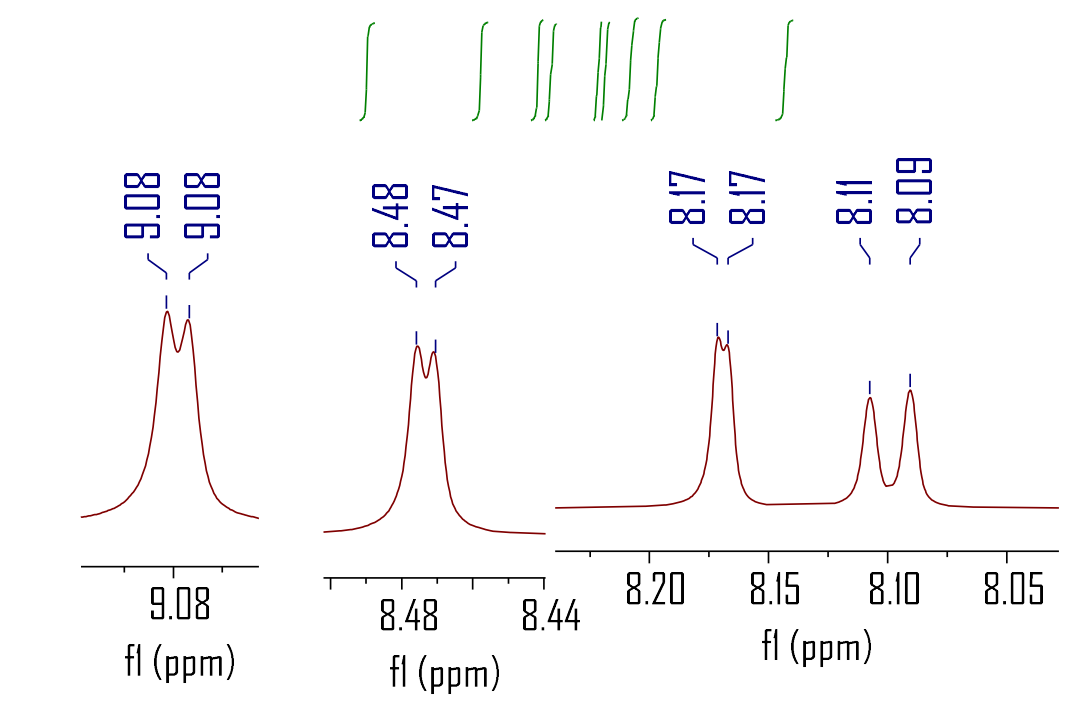

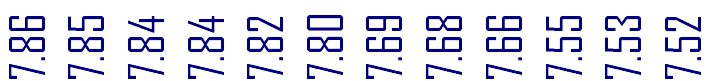

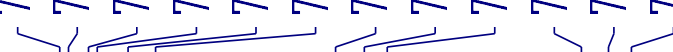

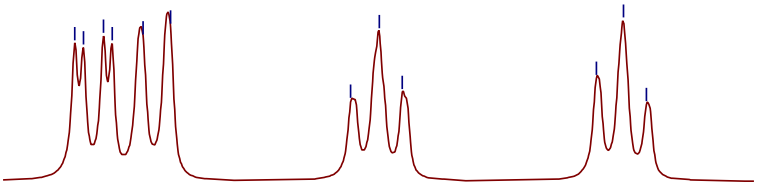

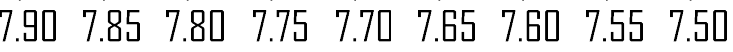

$\mathrm{fl}(\mathrm{ppm})$

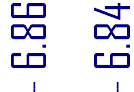

$\mathrm{fl}(\mathrm{ppm})$

$\mathrm{fl}(\mathrm{ppm})$

$\mathrm{fl}(\mathrm{ppm})$

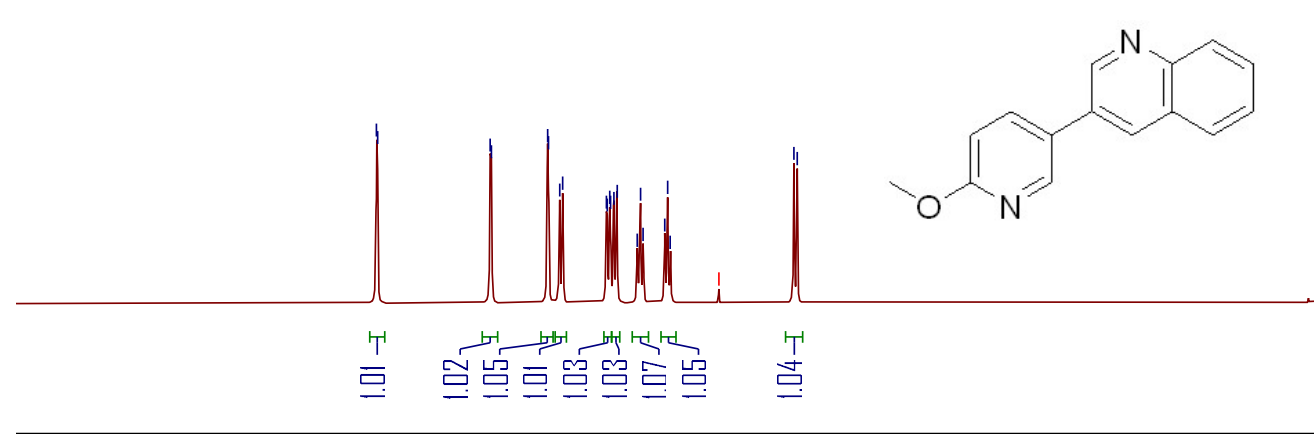

\begin{tabular}{llllllllllllllllllllllll}
\hline .0 & 10.5 & 10.0 & 9.5 & 9.0 & 8.5 & 8.0 & 7.5 & 7.0 & 6.5 & 6.0 & 5.5 & 5.0 & 4.5 & 4.0 & 3.5 & 3.0 & 2.5 & 2.0 & 1.5 & 1.0 & 0.5 & 0.0 & -0.5
\end{tabular} fl (ppm) 


\section{${ }^{13} \mathrm{C}\left\{{ }^{1} \mathrm{H}\right\}$ NMR spectrum of compound $3 \mathrm{ad}\left(126 \mathrm{MHz}, \mathrm{CDCl}_{3}\right)$}

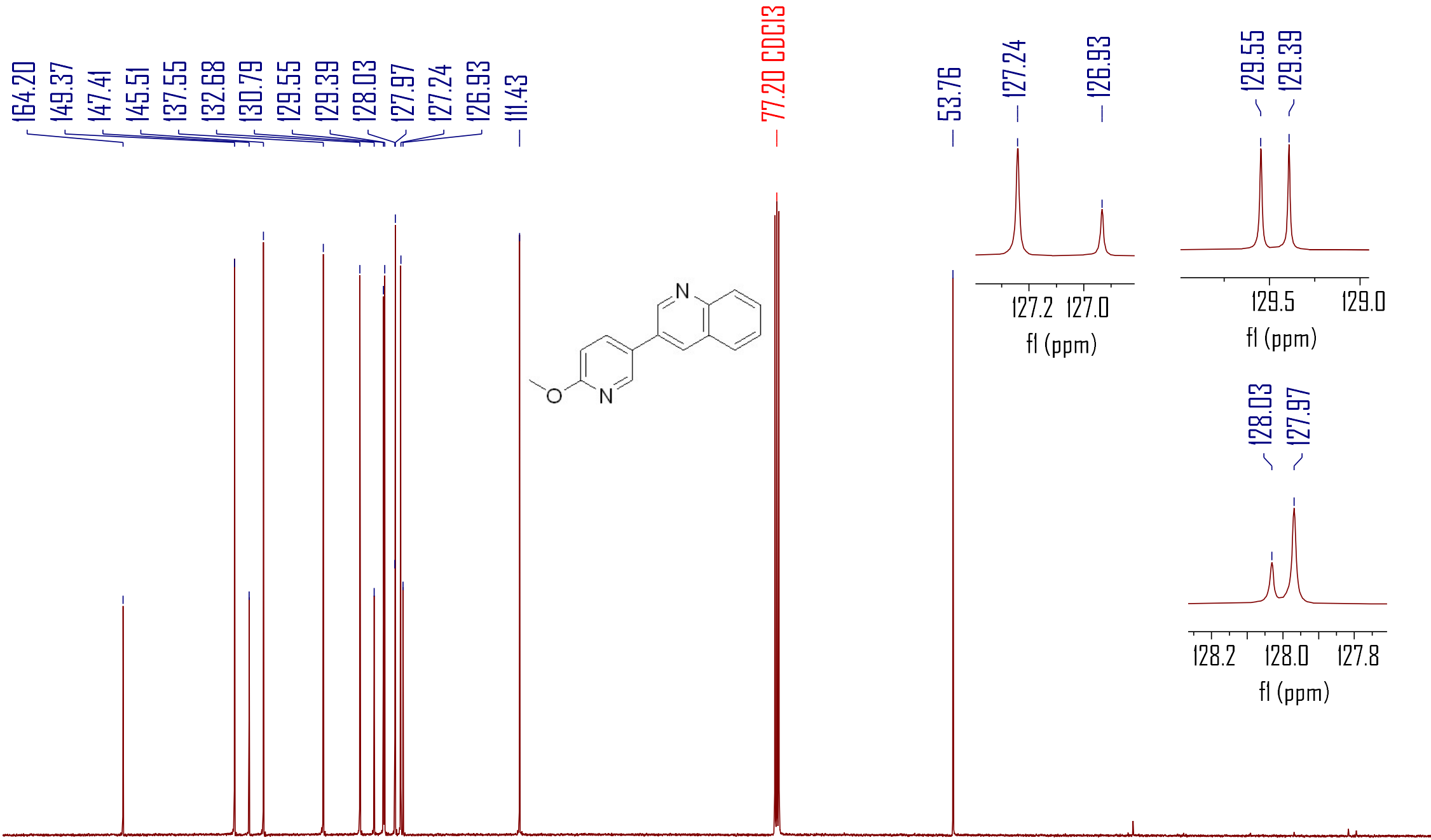

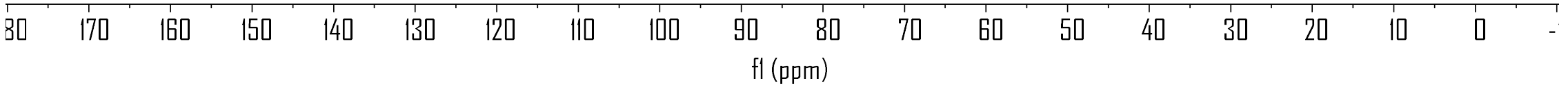


${ }^{1} \mathrm{H}$ NMR spectrum of compound 3ae (500 $\left.\mathrm{MHz}, \mathrm{CDCl}_{3}\right)$

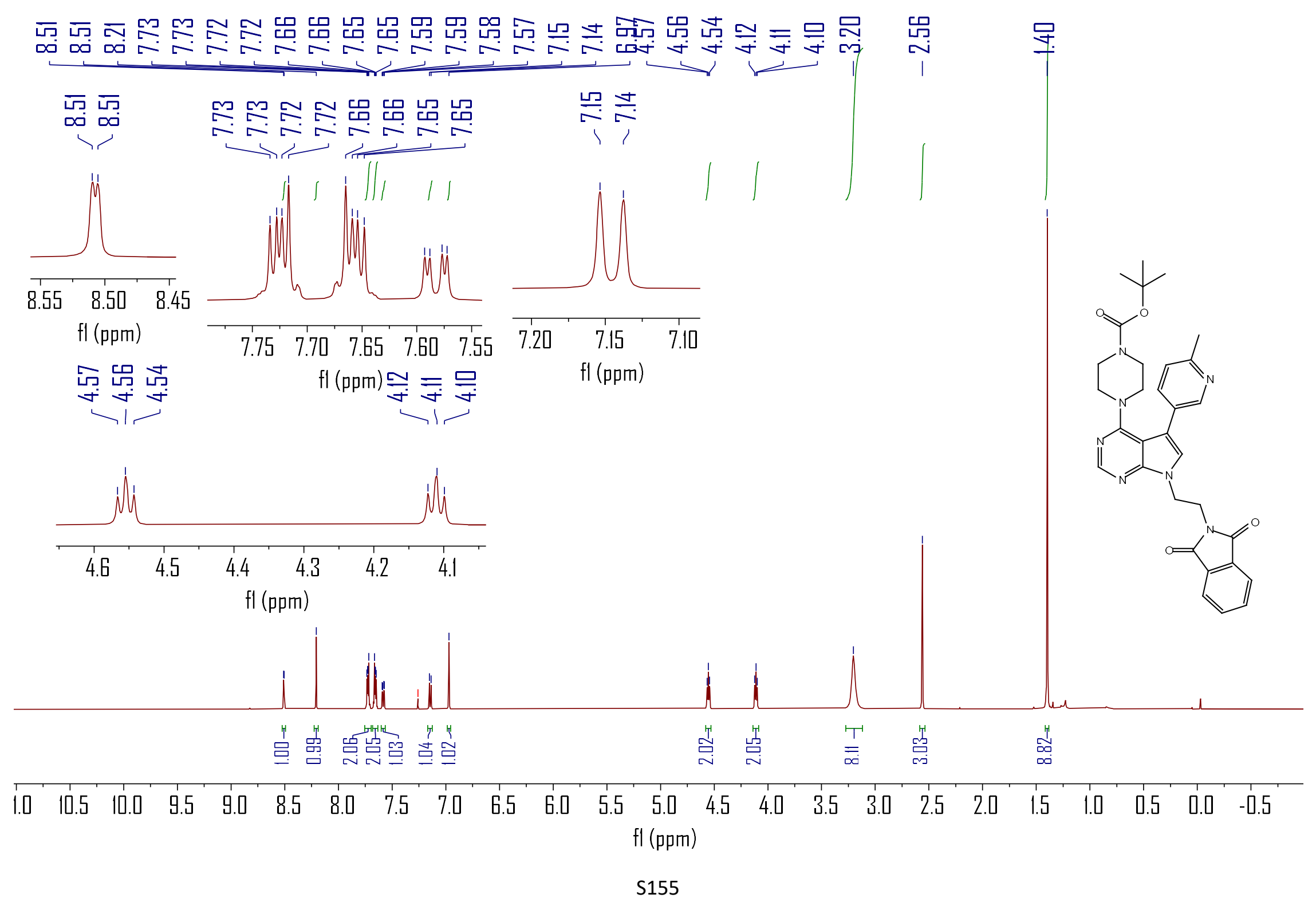


${ }^{13} \mathrm{C}\left\{{ }^{1} \mathrm{H}\right\} \mathrm{NMR}$ spectrum of compound 3ae (126 $\left.\mathrm{MHz}, \mathrm{CDCl}_{3}\right)$

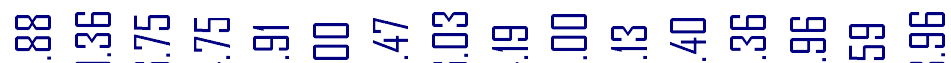

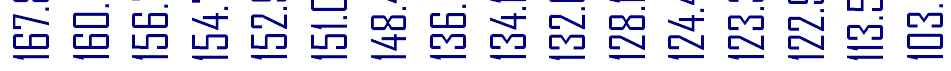

肙 둡

㫛

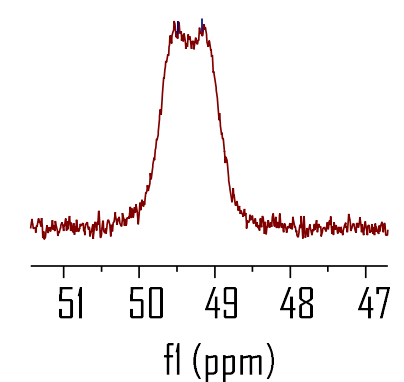

喿訨 먼
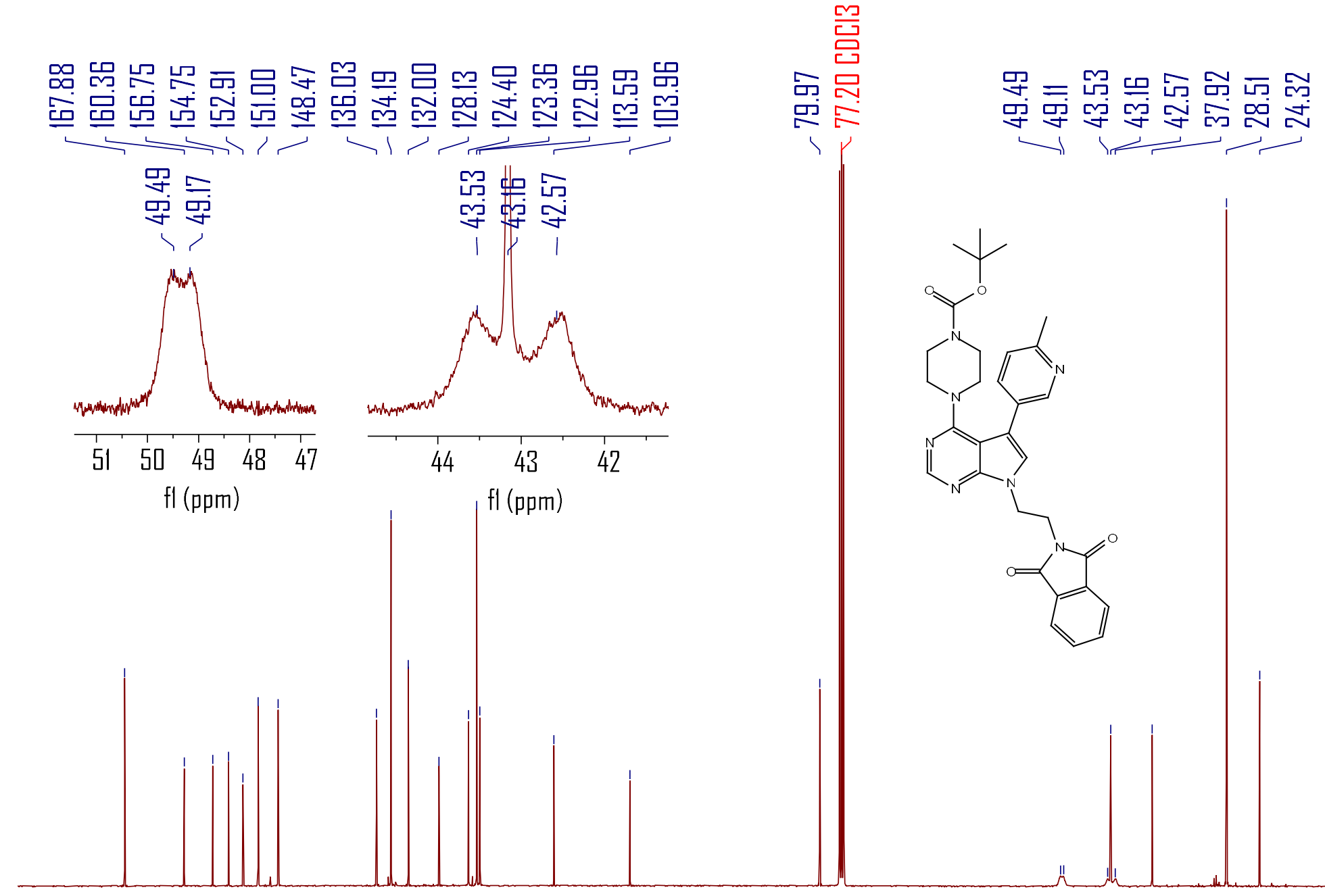

$180 \quad 170$

I60

150

$140 \quad 130 \quad 12$

III IOD

$\mathrm{fl}(\mathrm{ppm})$

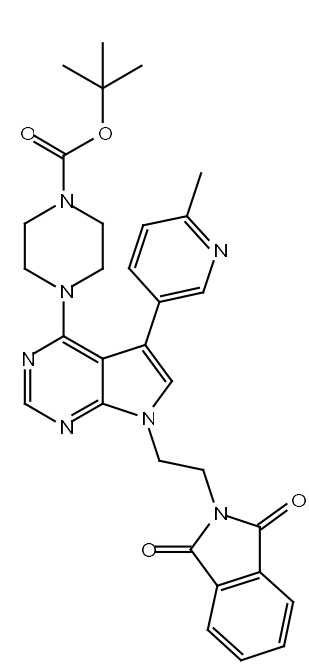


${ }^{1} \mathrm{H}$ NMR spectrum of compound 3af (500 $\left.\mathrm{MHz}, \mathrm{CDCl}_{3}\right)$

든

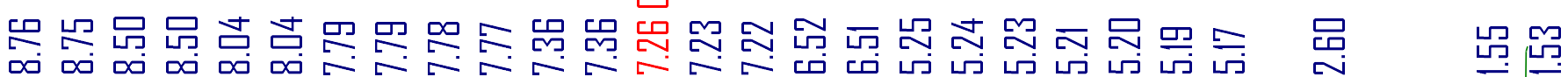

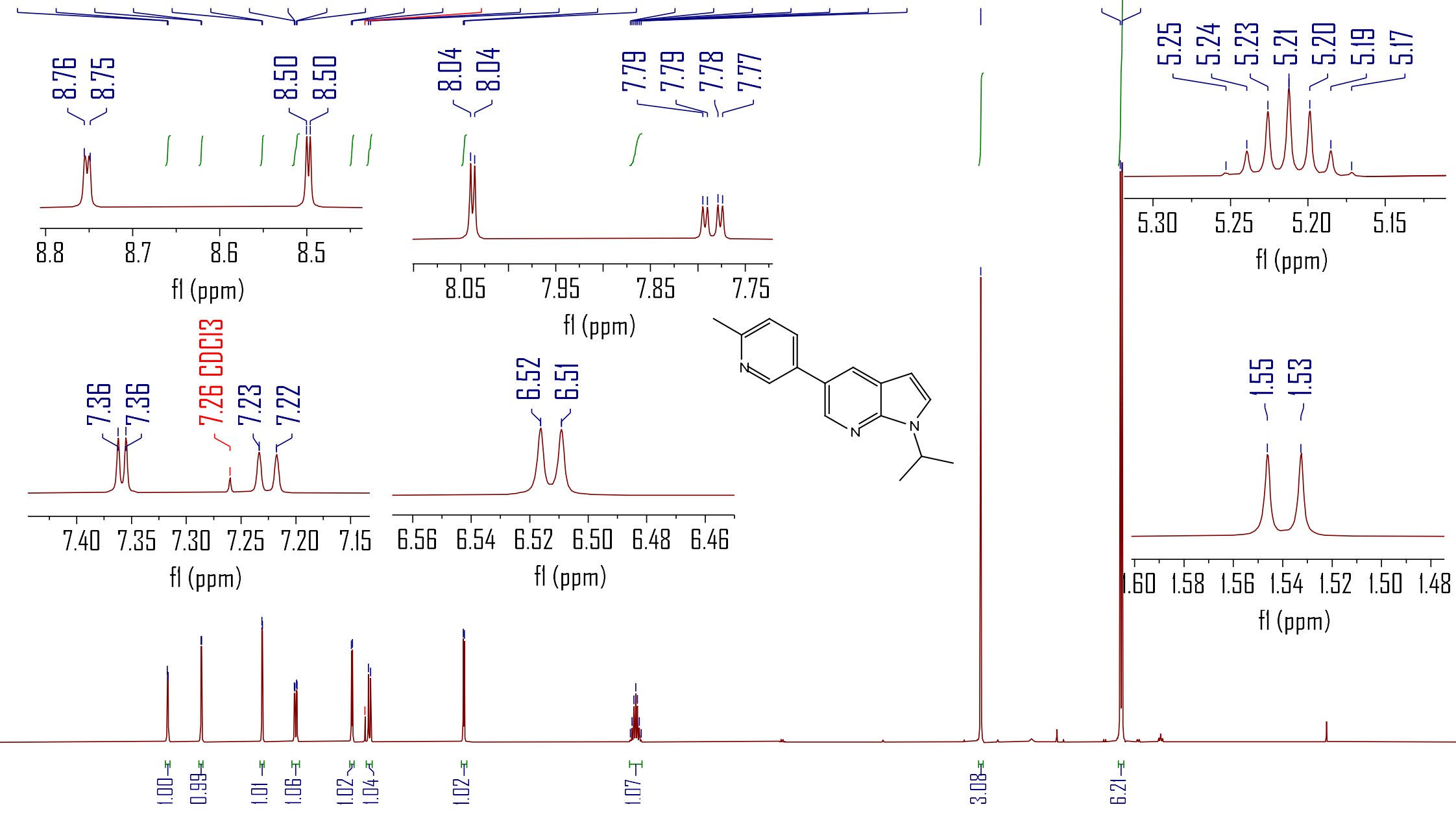

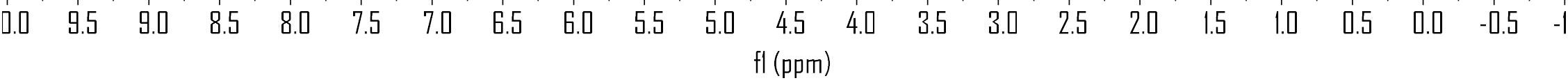


${ }^{13} \mathrm{C}\left\{{ }^{1} \mathrm{H}\right\} \mathrm{NMR}$ spectrum of compound 3af (126 $\left.\mathrm{MHz}, \mathrm{CDCl}_{3}\right)$

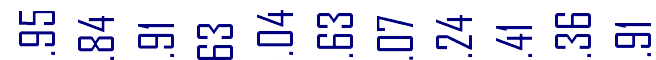

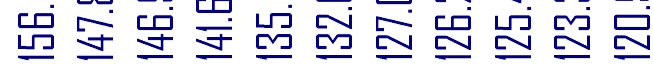

$\mathrm{C}^{\mathrm{N}} \mathrm{C}$

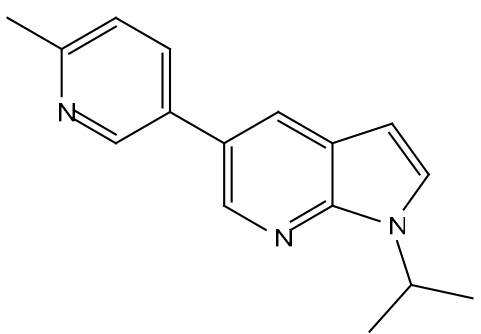

吕

品
몰

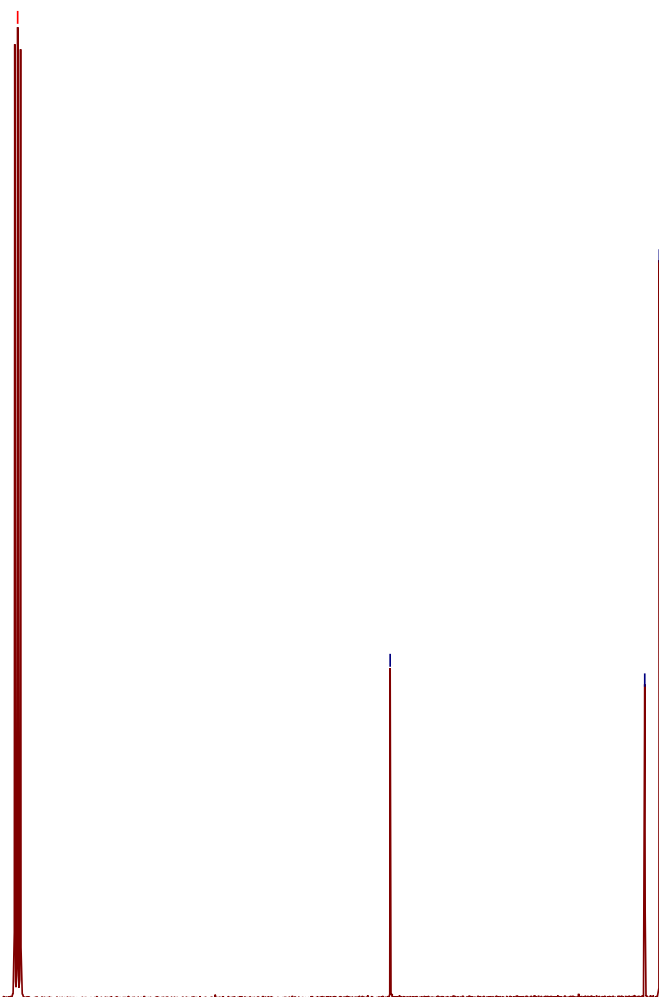

志方

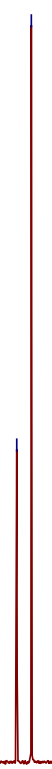




\title{
${ }^{1} \mathrm{H}$ NMR spectrum of compound 3ag (500 $\left.\mathrm{MHz}, \mathrm{CDCl}_{3}\right)$
}

\author{
들
}

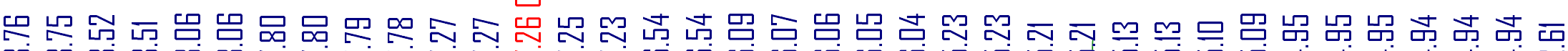
ه

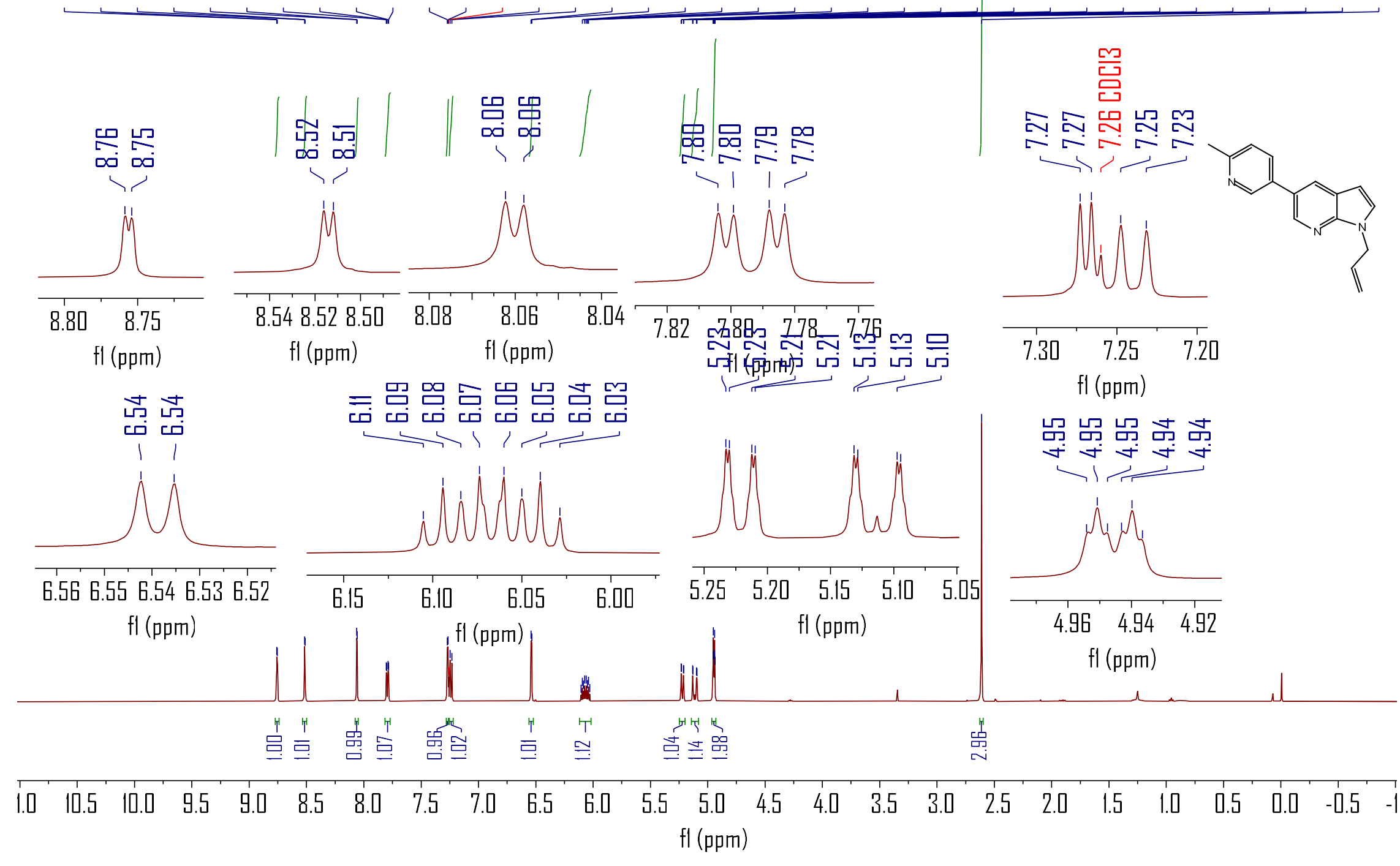


${ }^{13} \mathrm{C}\left\{{ }^{1} \mathrm{H}\right\}$ NMR spectrum of compound $3 \mathrm{ag}\left(126 \mathrm{MHz}^{\mathrm{CDCl}}{ }_{3}\right)$

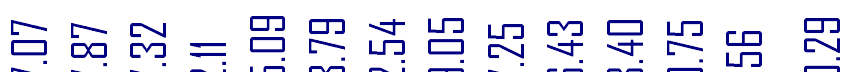

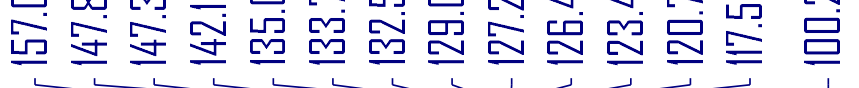
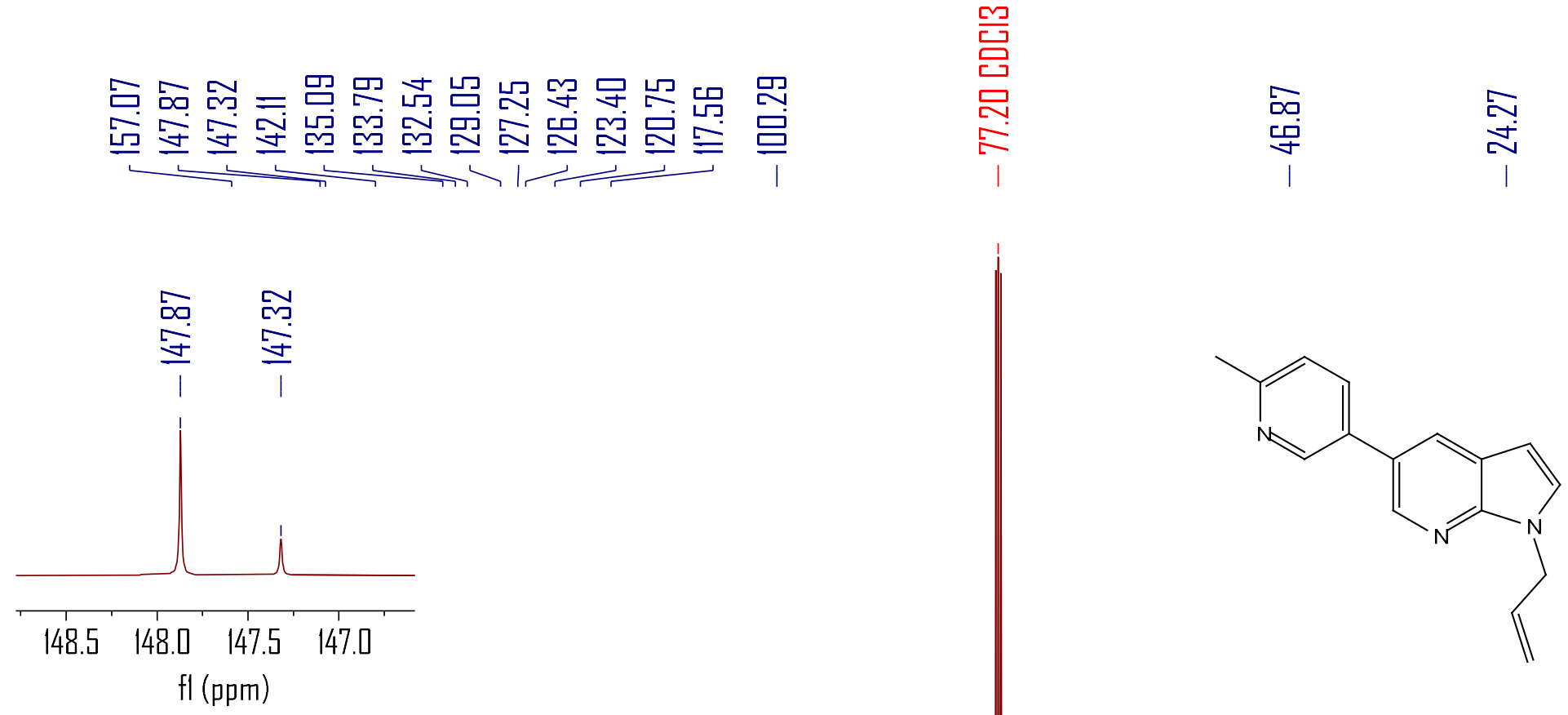

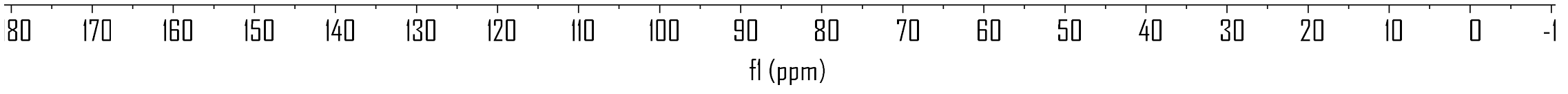


${ }^{1} \mathrm{H}$ NMR spectrum of compound 3ah (500 $\left.\mathrm{MHz}, \mathrm{CDCl}_{3}\right)$

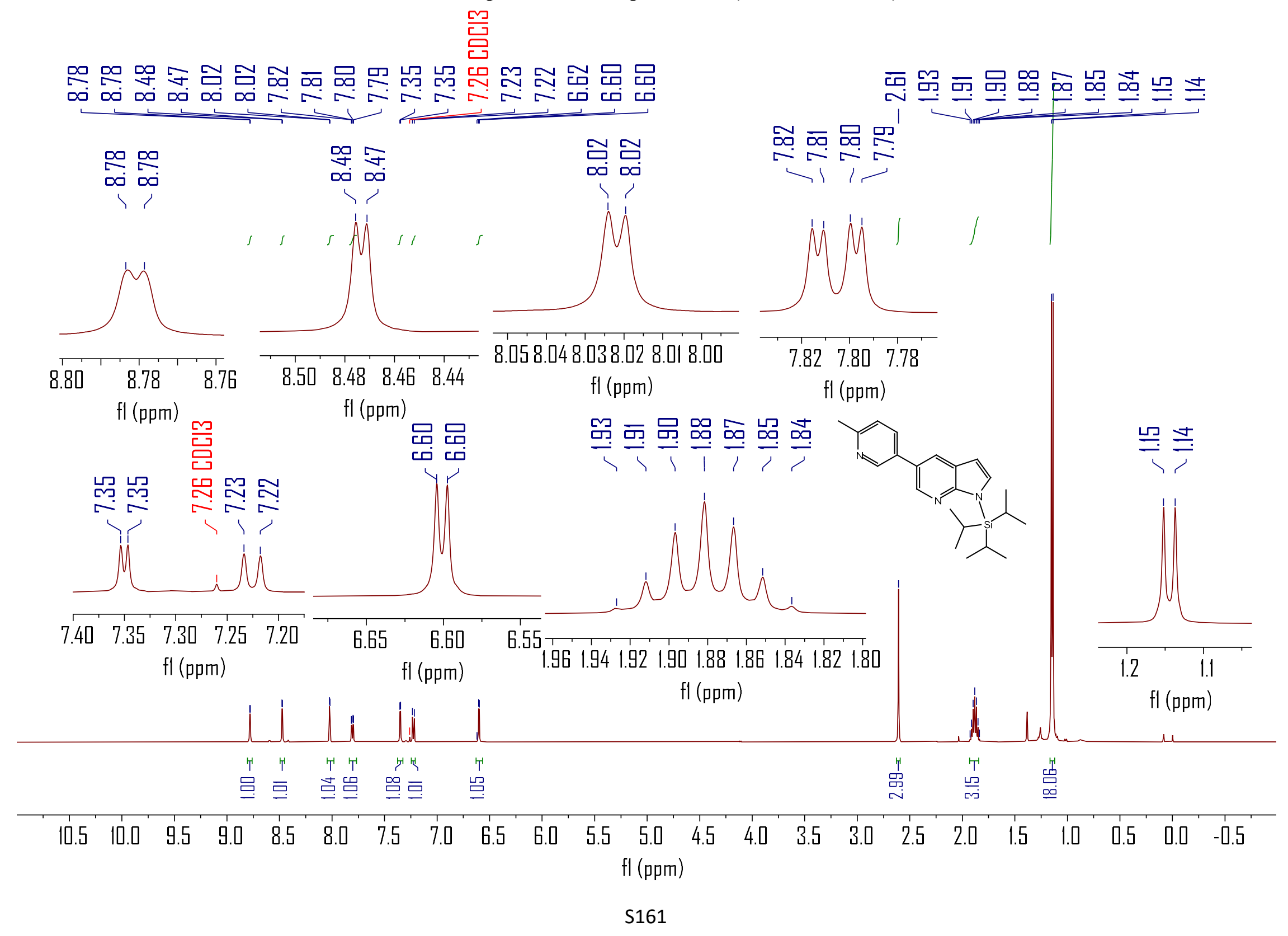


${ }^{13} \mathrm{C}\left\{{ }^{1} \mathrm{H}\right\}$ NMR spectrum of compound 3ah (126 $\left.\mathrm{MHz}, \mathrm{CDCl}_{3}\right)$
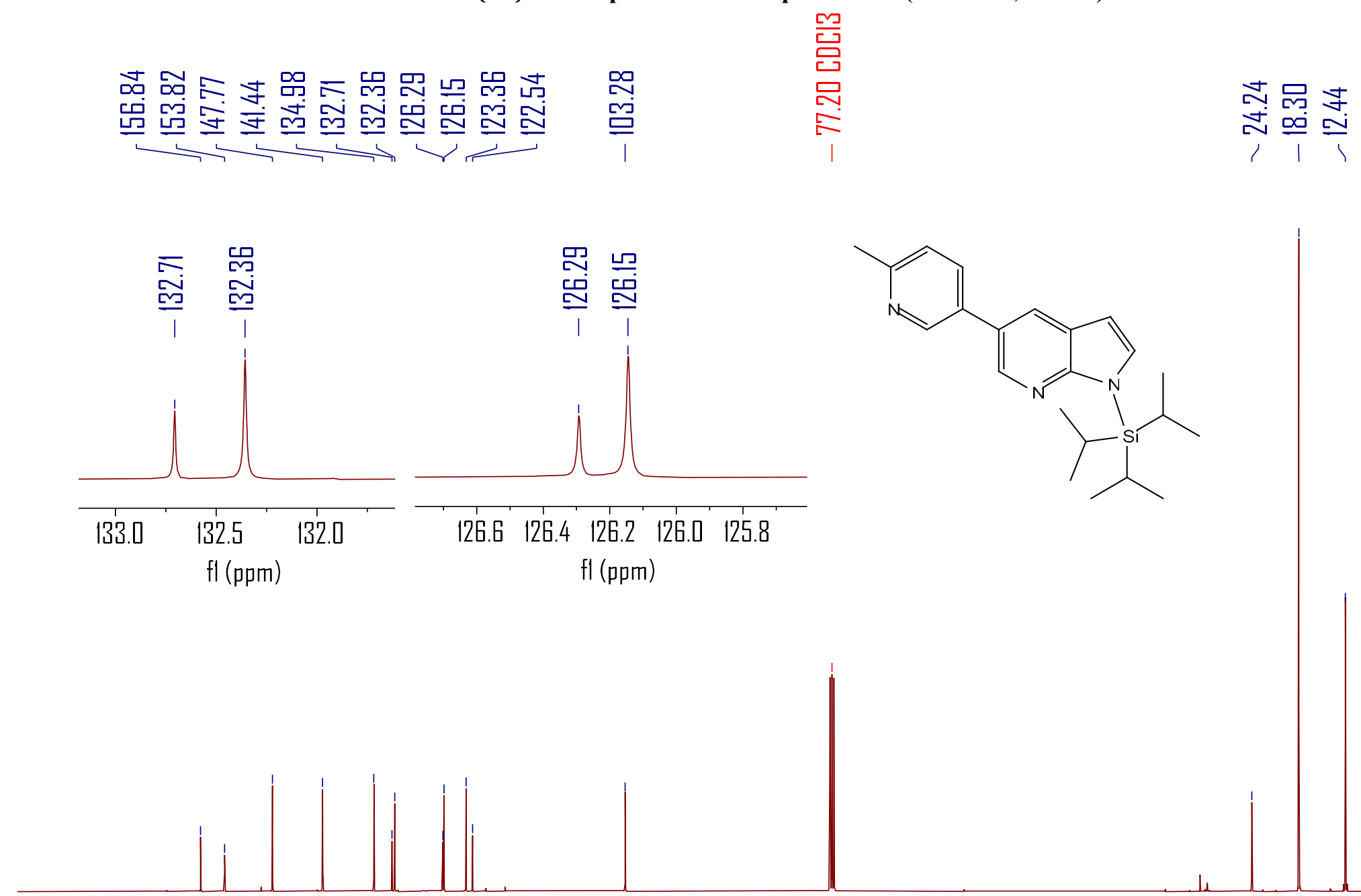

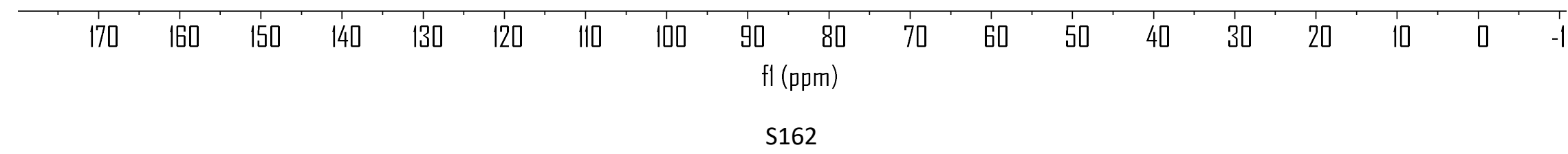


${ }^{1} \mathrm{H}$ NMR spectrum of compound 3ai (500 $\left.\mathrm{MHz}, \mathrm{CDCl}_{3}\right)$

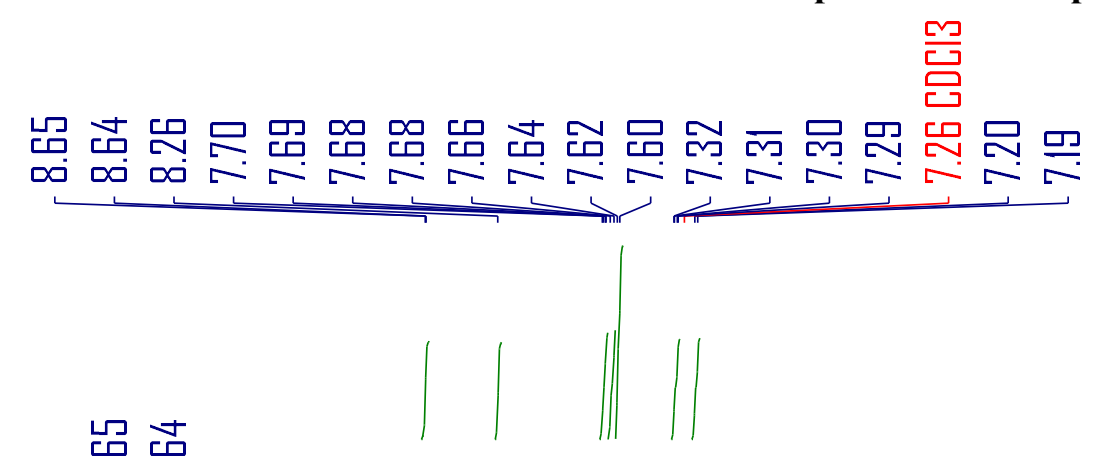

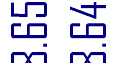
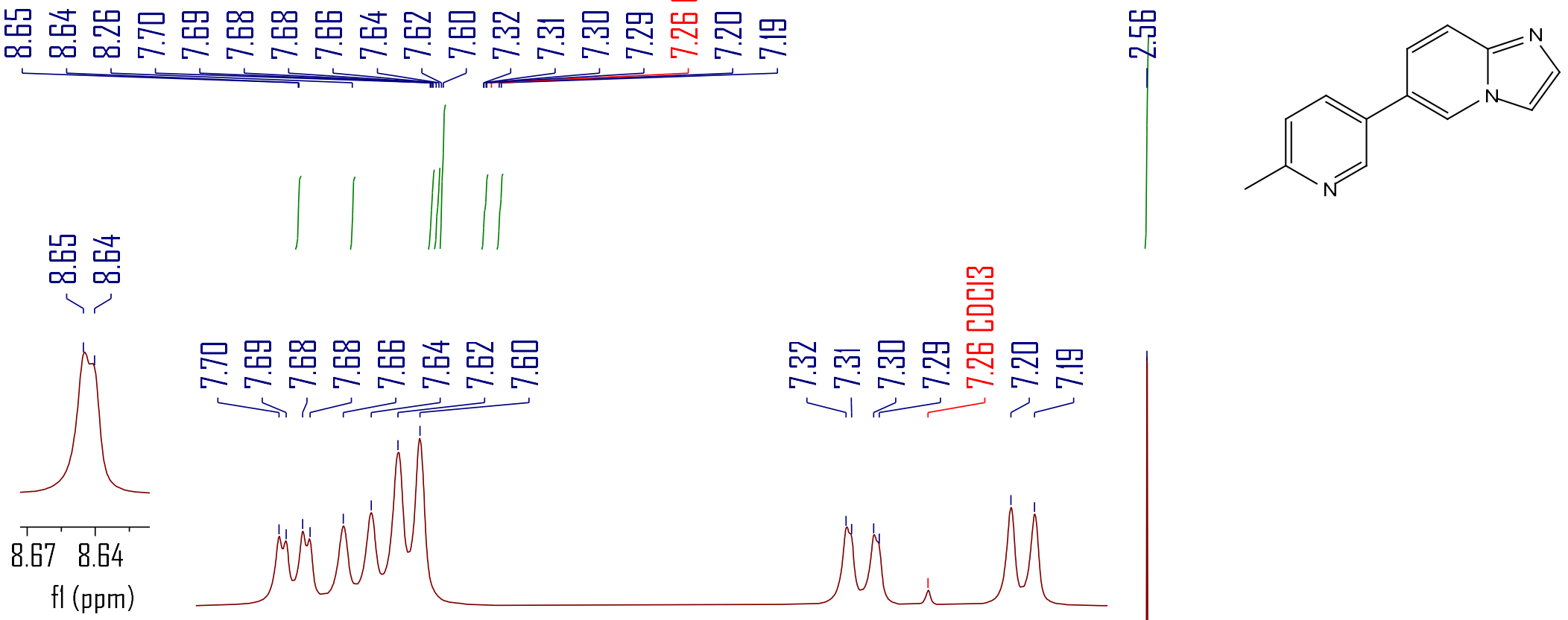

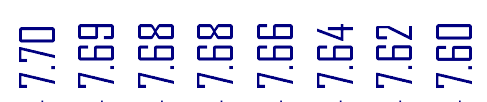

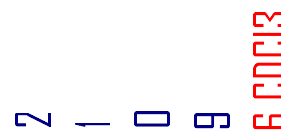

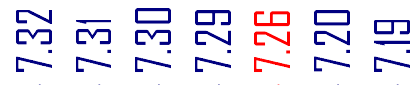

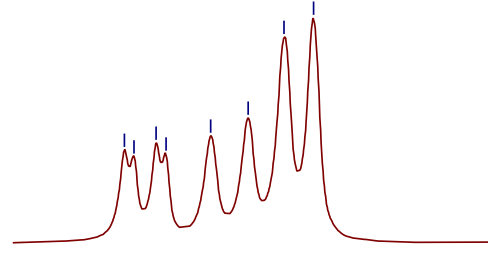

$\longrightarrow 1$
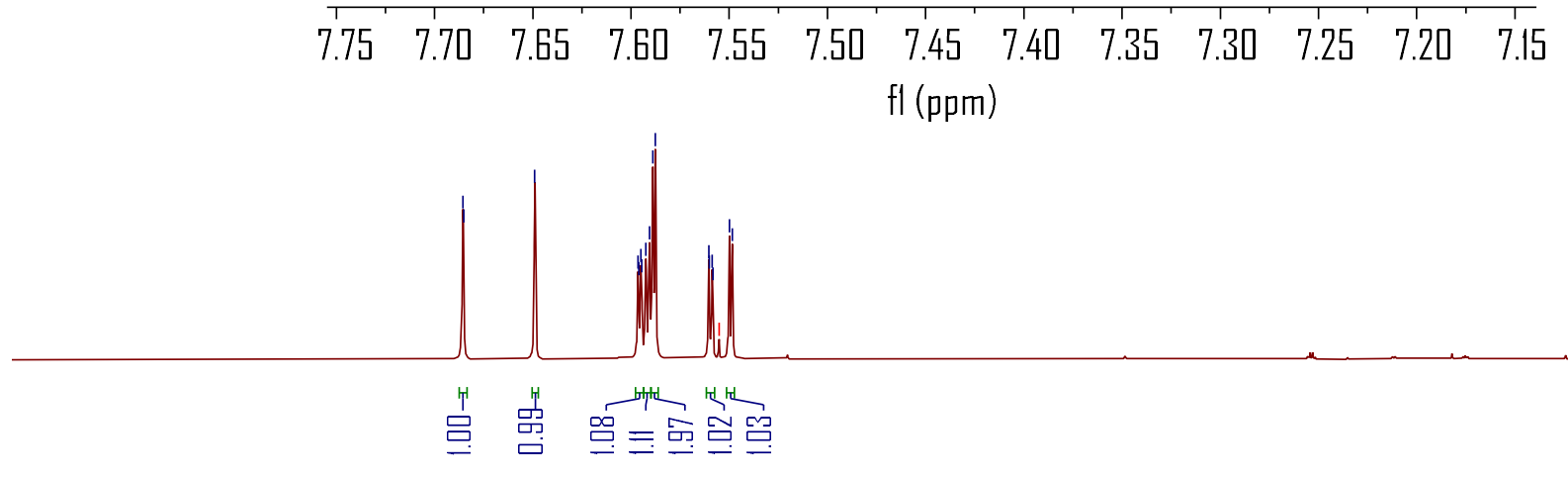

$\begin{array}{llllllllllllllllllllllllll}11.0 & 10.5 & 10.0 & 9.5 & 9.0 & 8.5 & 8.0 & 7.5 & 7.0 & 6.5 & 6.0 & 5.5 & 5.0 & 4.5 & 4.0 & 3.5 & 3.0 & 2.5 & 2.0 & 1.5 & 1.0 & 0.5 & 0.0 & -0.5 \\ \mathrm{fl}(\mathrm{ppm}) & & & & & \end{array}$ 
${ }^{13} \mathrm{C}\left\{{ }^{1} \mathrm{H}\right\}$ NMR spectrum of compound 3ai (126 $\left.\mathrm{MHz}, \mathrm{CDCl}_{3}\right)$

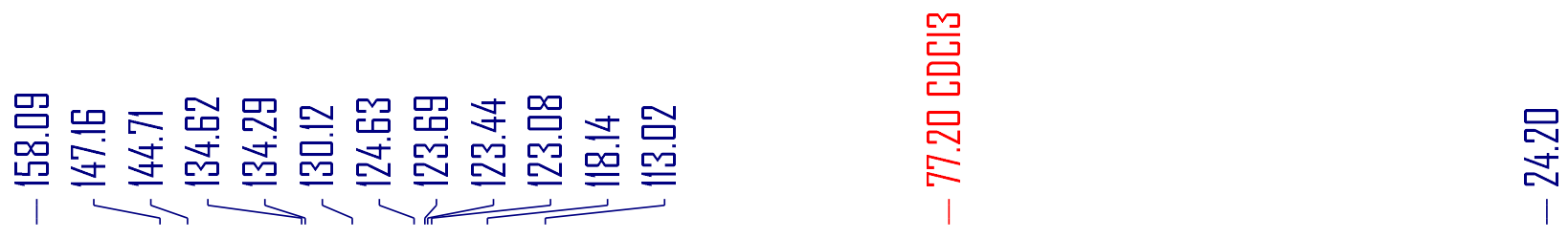

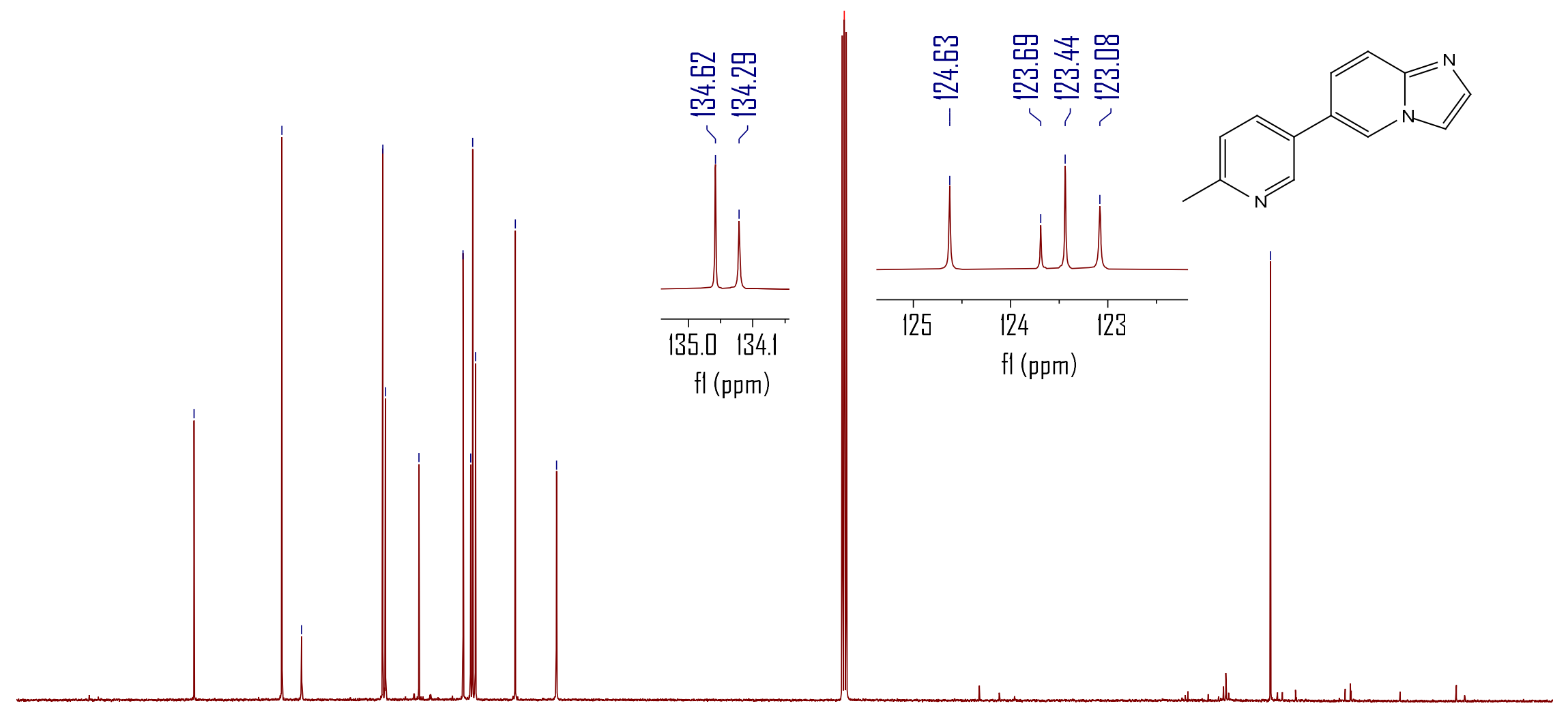

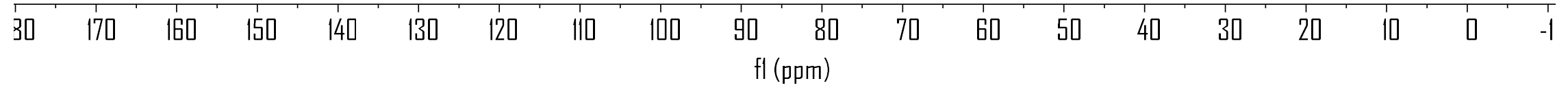


${ }^{1} \mathrm{H}$ NMR spectrum of compound 3aj (500 $\left.\mathrm{MHz}, \mathrm{CDCl}_{3}\right)$

岳

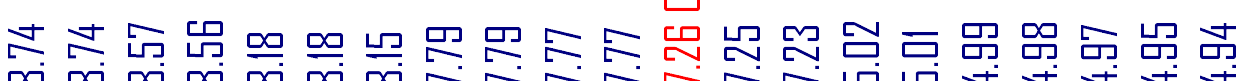

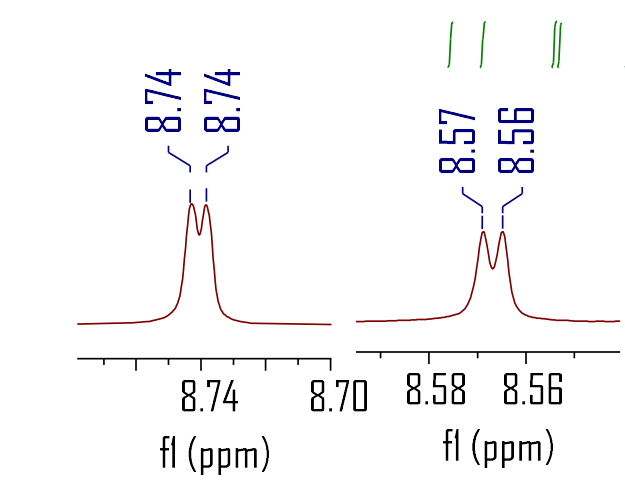

$\operatorname{lng} \frac{\infty}{\infty}$
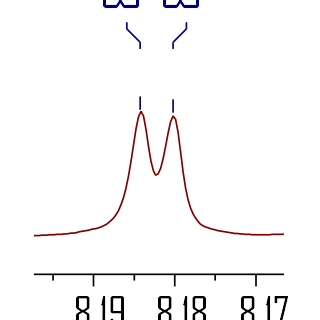

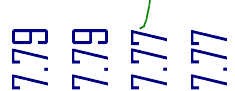

$\sqrt{1} 5$

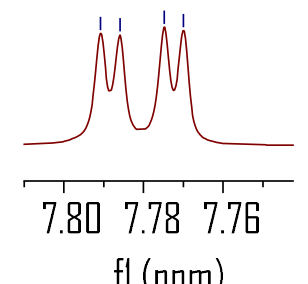

fl (ppm)

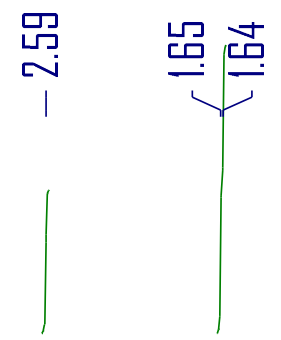

믈

员荝器

$\sqrt{5} 5$

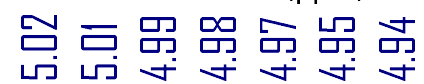
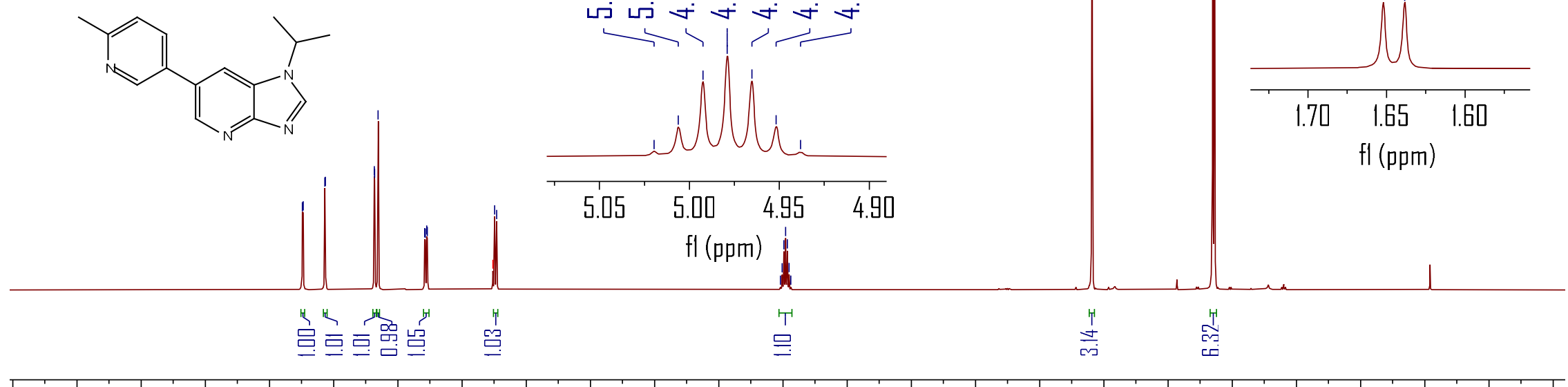

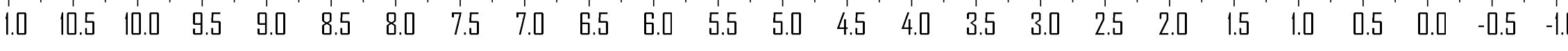
fl (ppm) 
${ }^{13} \mathrm{C}\left\{{ }^{1} \mathrm{H}\right\}$ NMR spectrum of compound 3aj (126 $\left.\mathrm{MHz}, \mathrm{CDCl}_{3}\right)$

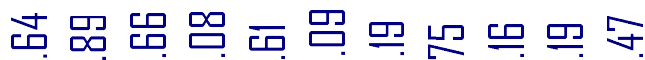

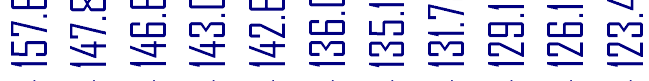
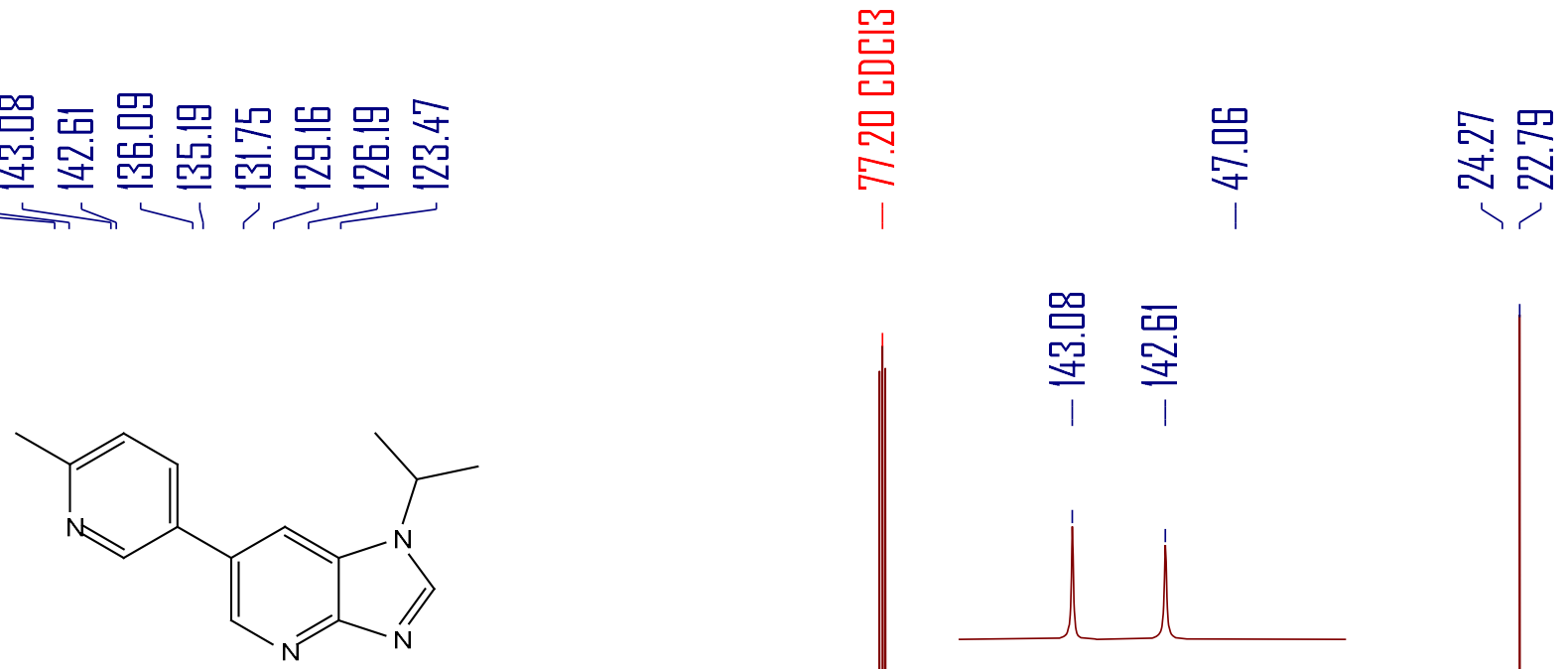

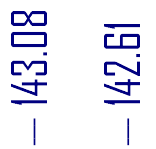
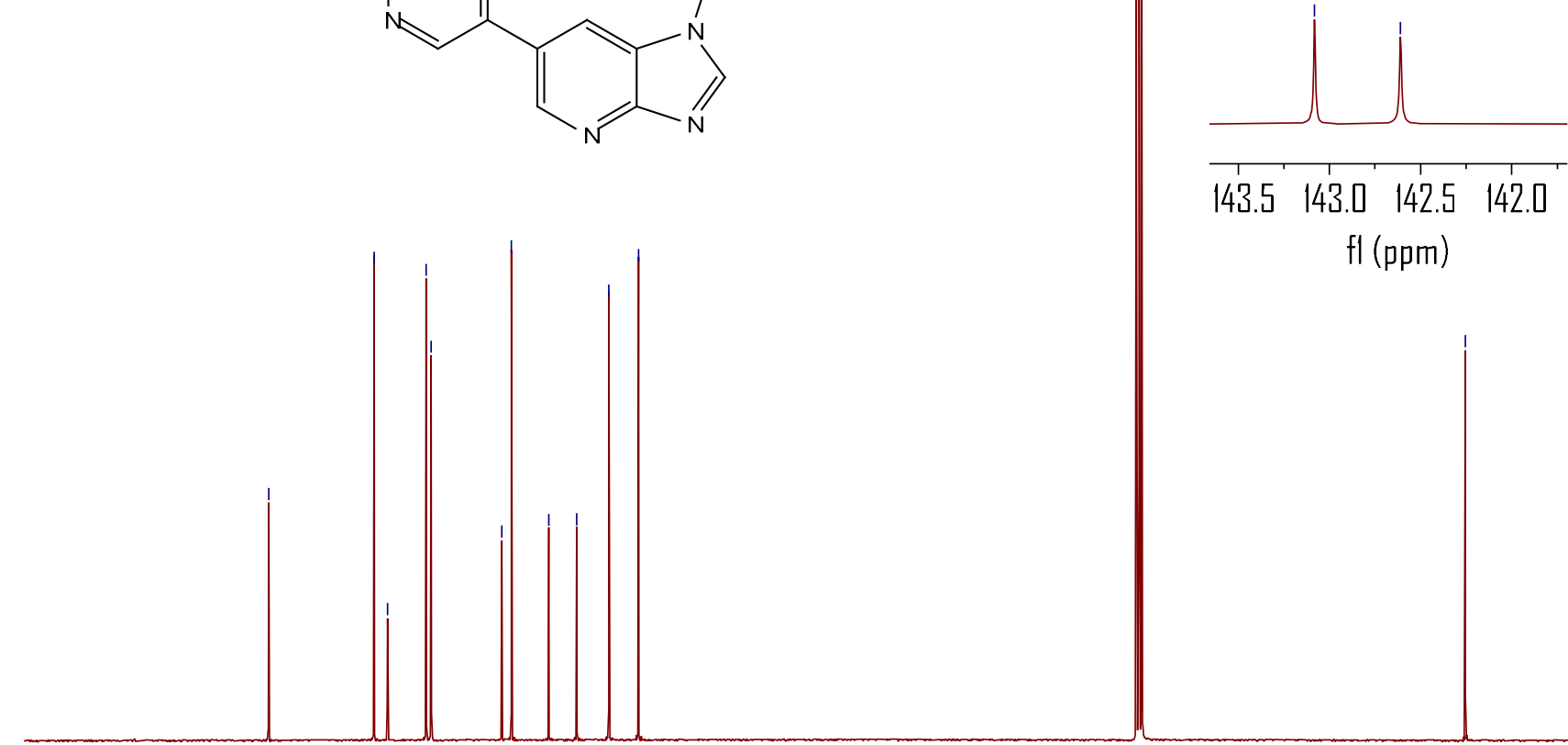

$\mathrm{fl}$ (ppm)

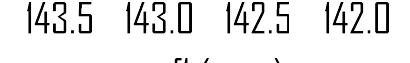

$$
\text { (ppm) }
$$

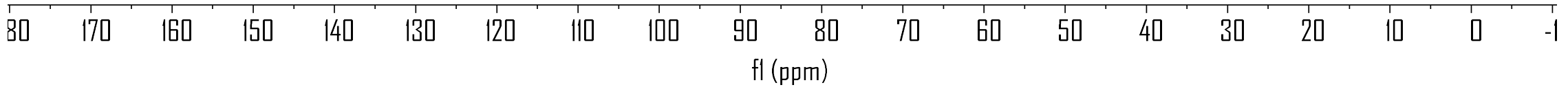


${ }^{1} \mathrm{H}$ NMR spectrum of compound 3ak (500 $\left.\mathrm{MHz}, \mathrm{CDCl}_{3}\right)$

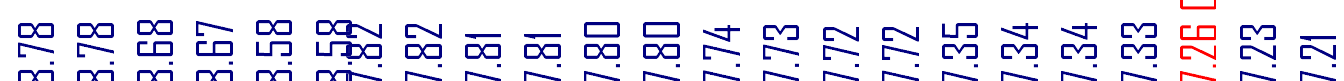

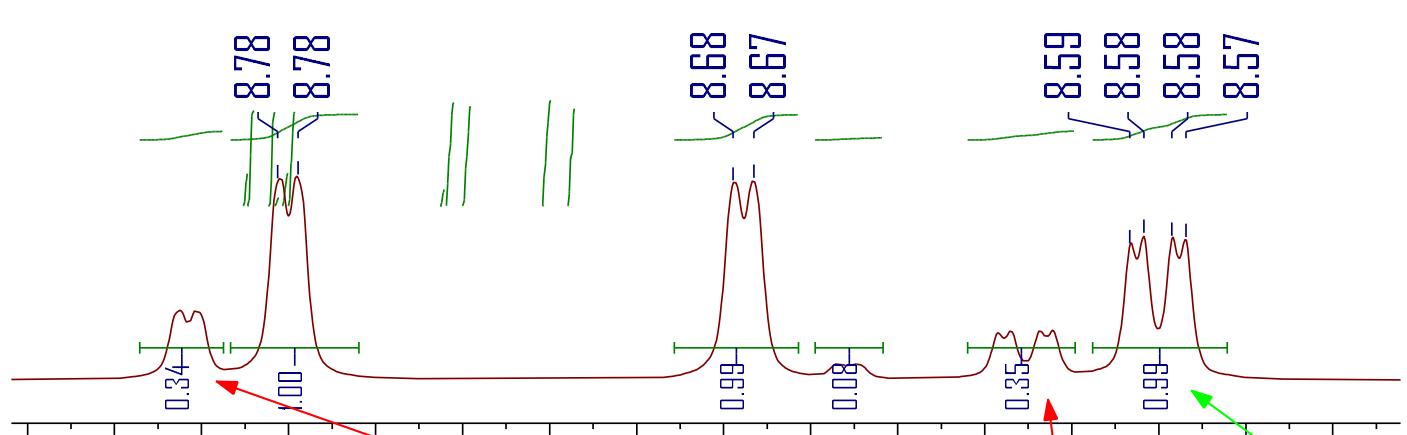

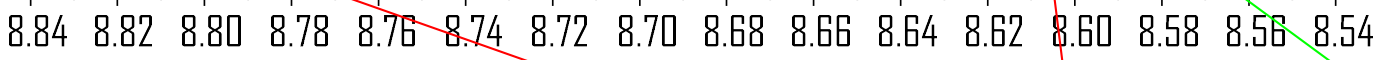

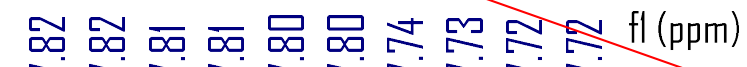
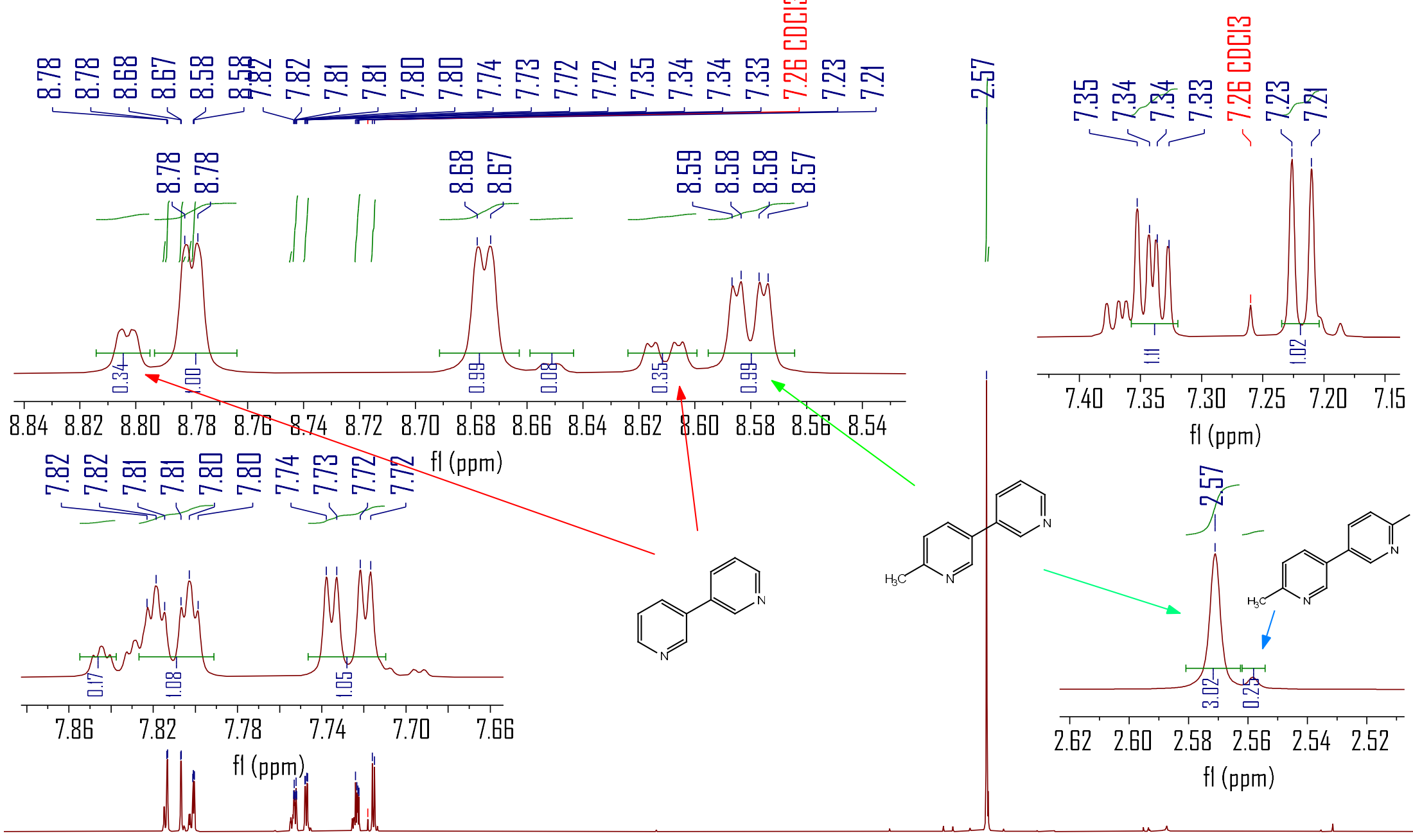

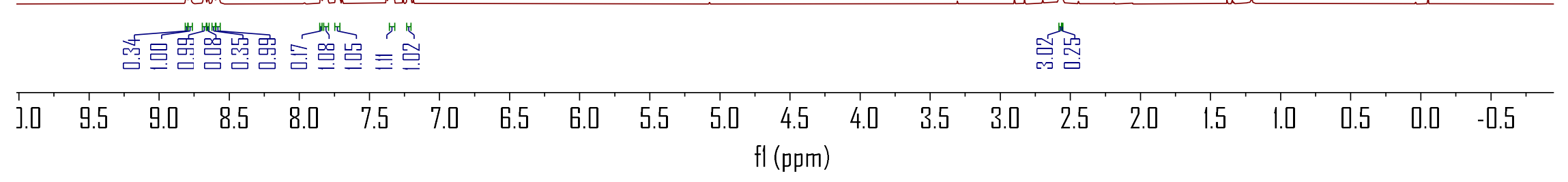


${ }^{1} \mathrm{H}$ NMR spectrum of compound 3al (500 $\left.\mathrm{MHz}, \mathrm{CDCl}_{3}\right)$

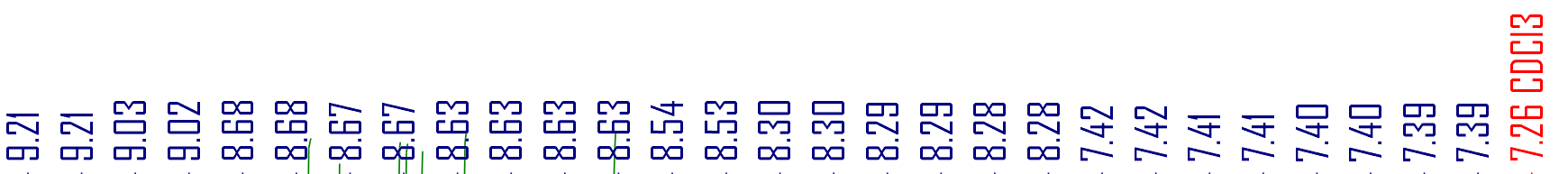

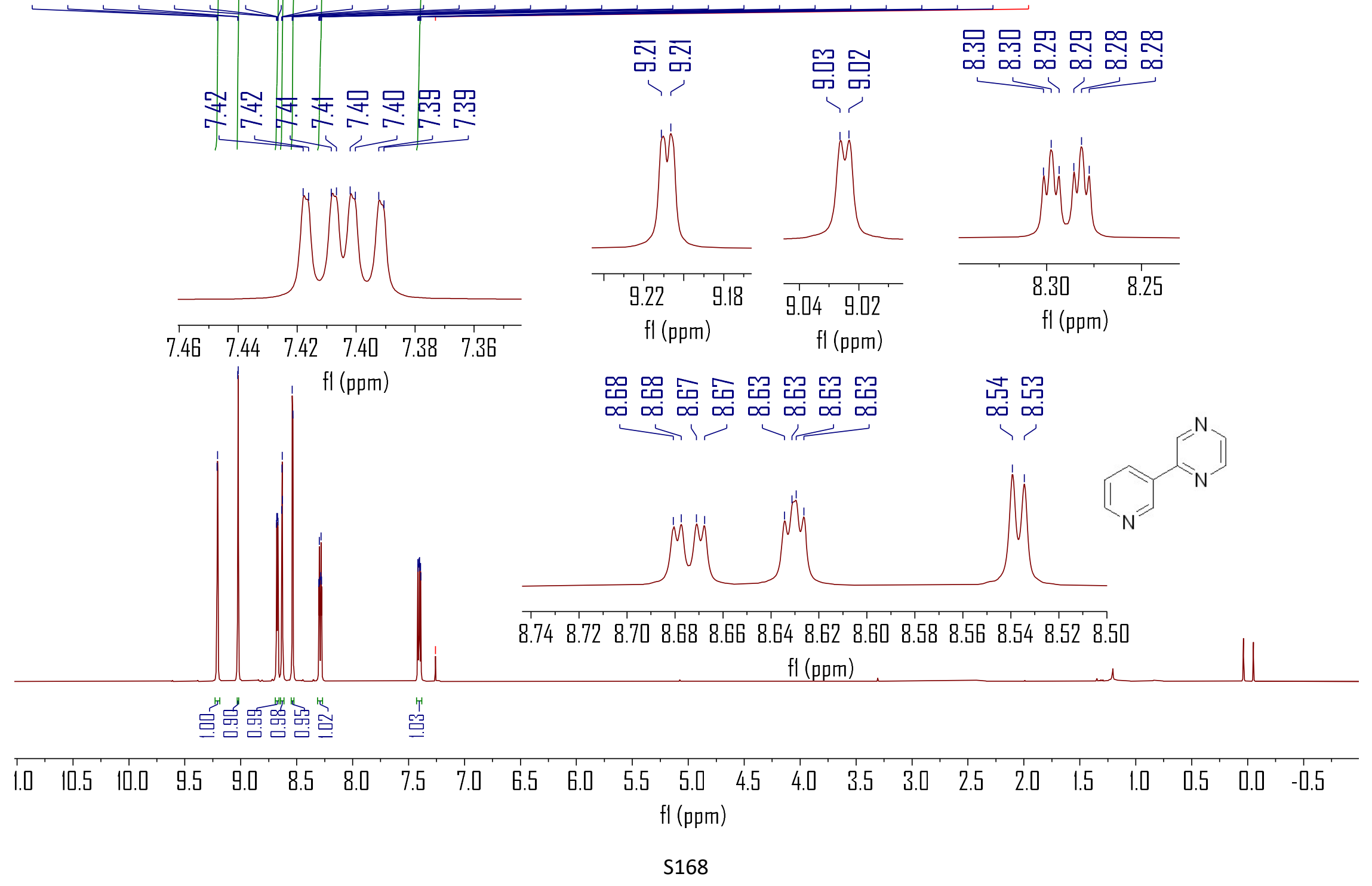




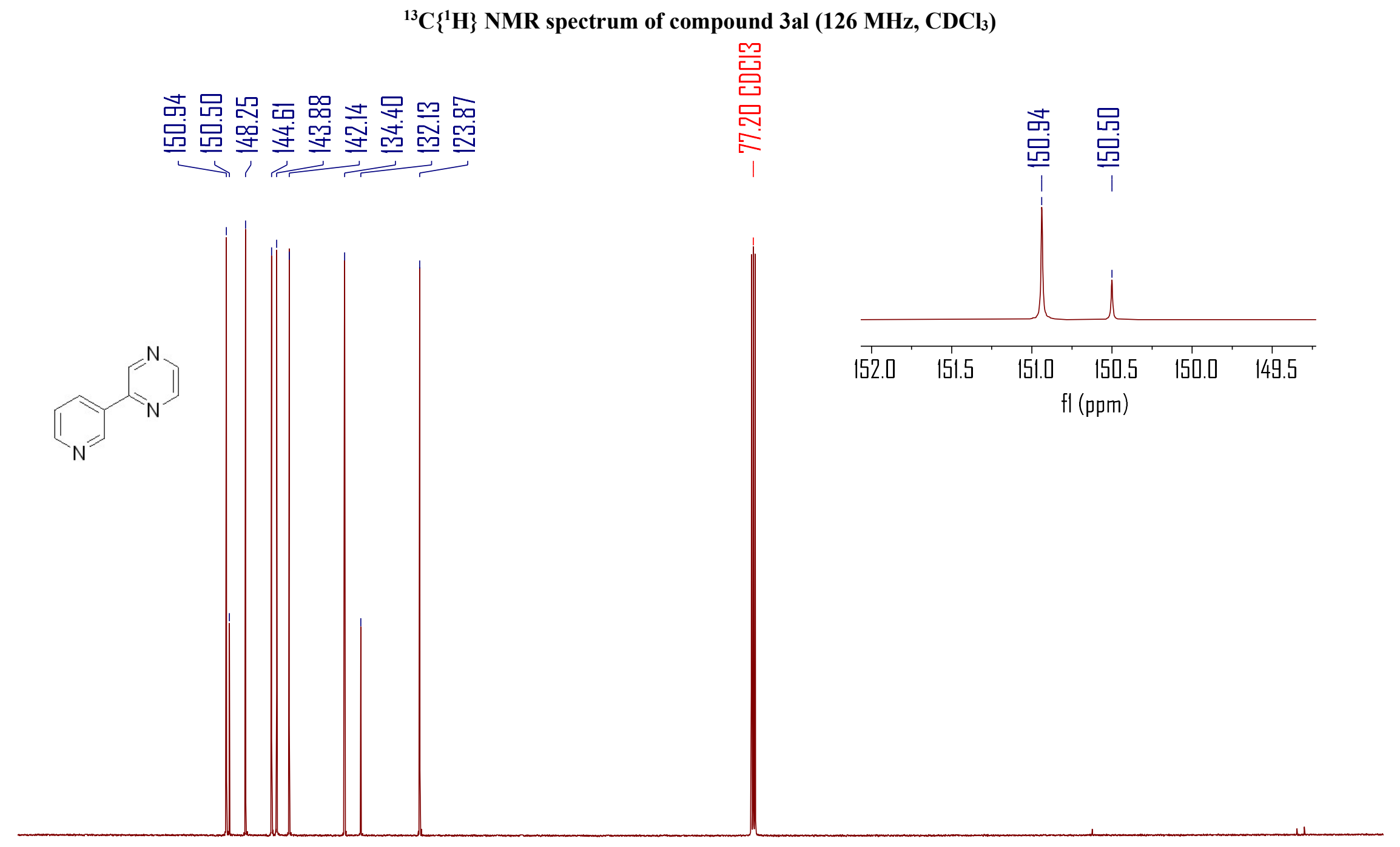

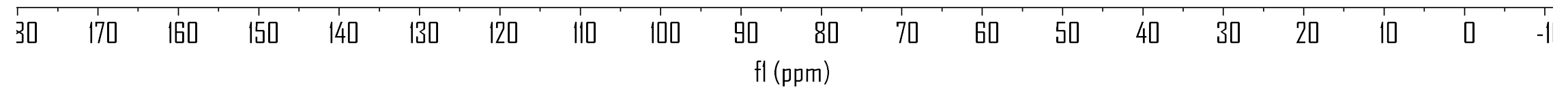




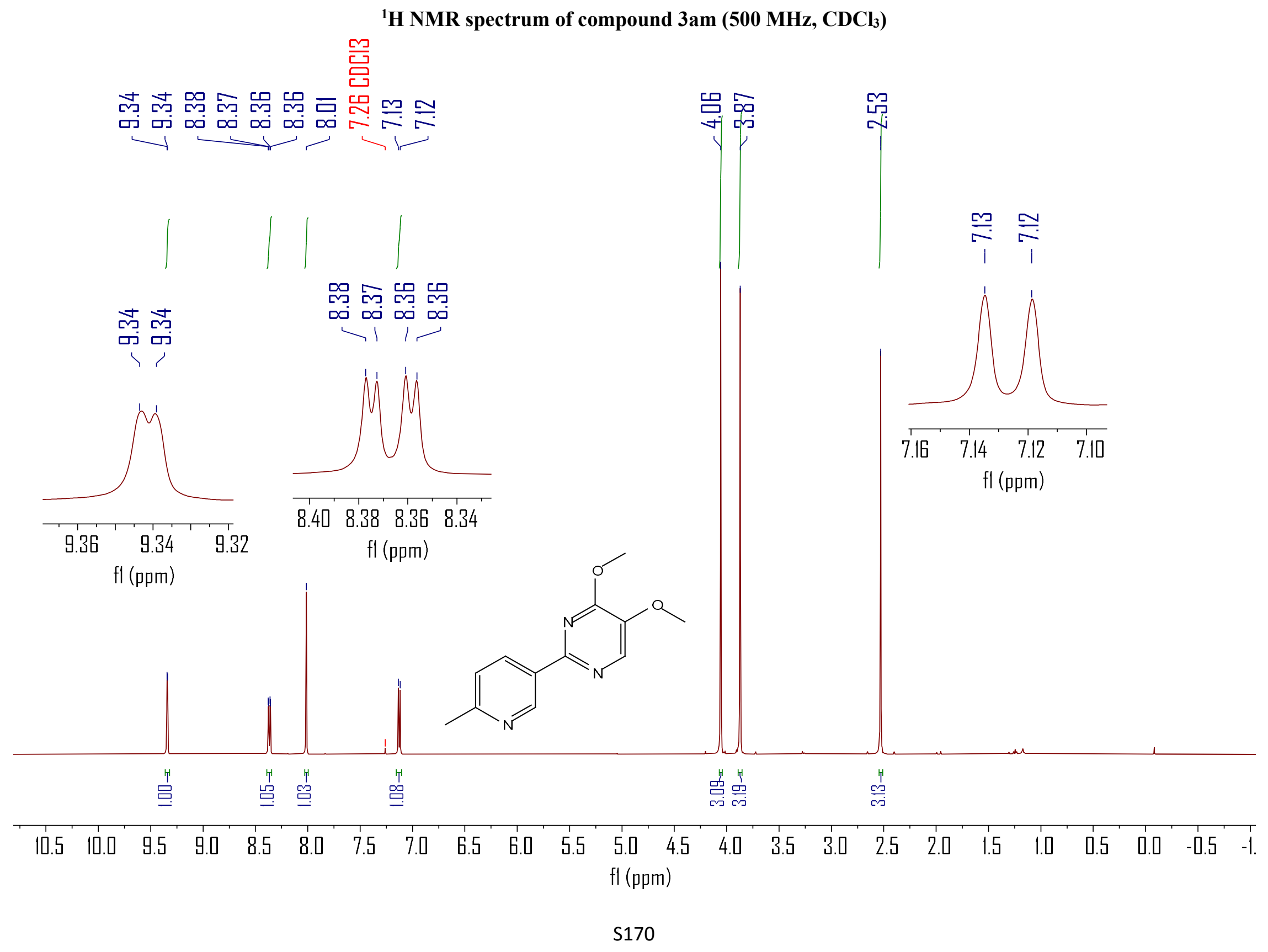




\section{${ }^{13} \mathrm{C}\left\{{ }^{1} \mathrm{H}\right\}$ NMR spectrum of compound 3am $\left(126 \mathrm{MHz}, \mathrm{CDCl}_{3}\right)$}
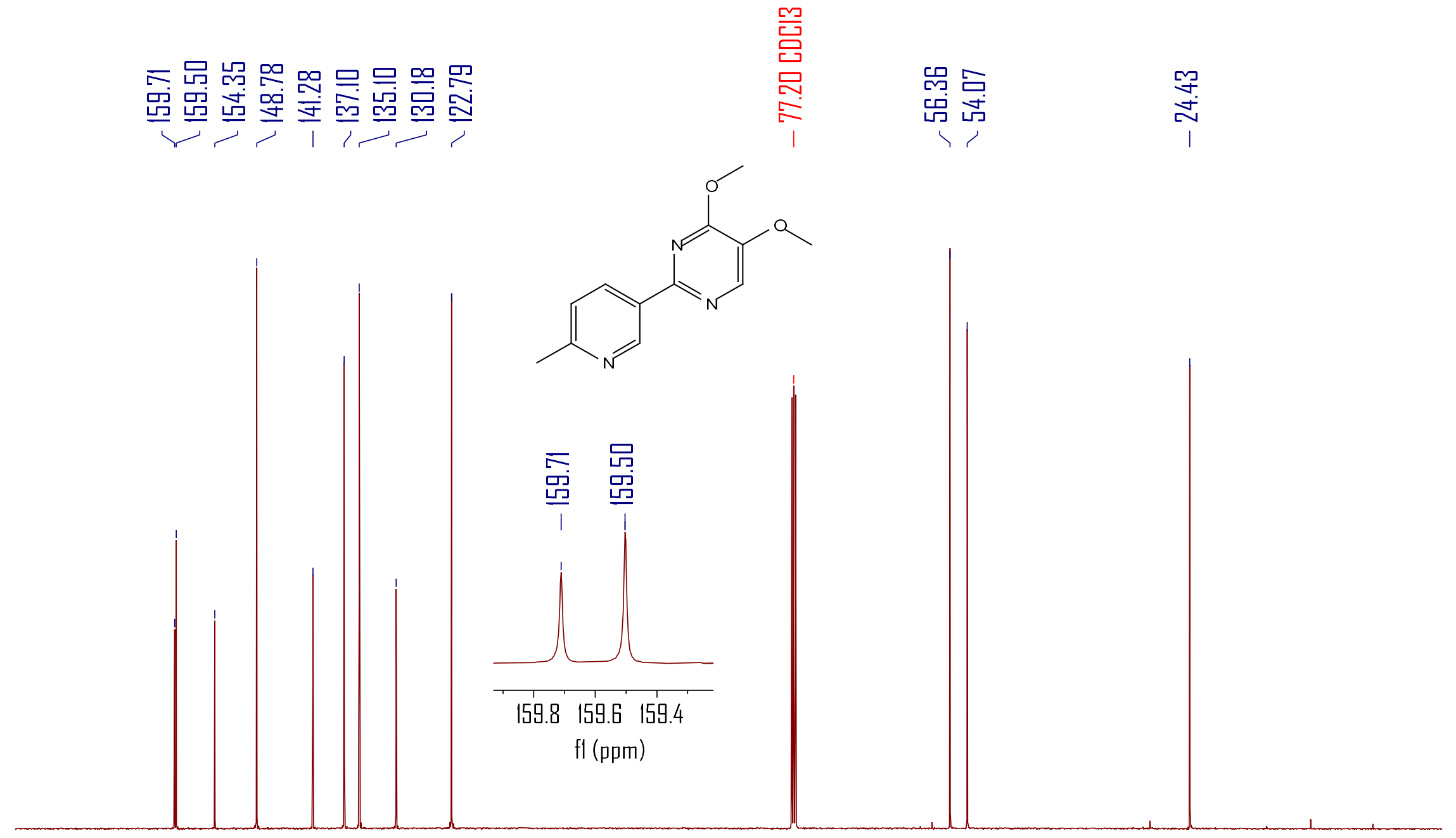

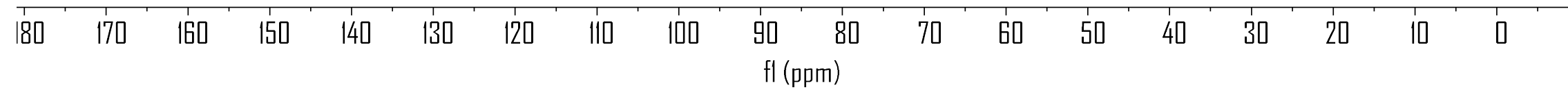


${ }^{1} \mathrm{H}$ NMR spectrum of compound $3 \mathrm{an}\left(500 \mathrm{MHz}, \mathrm{CDCl}_{3}\right)$

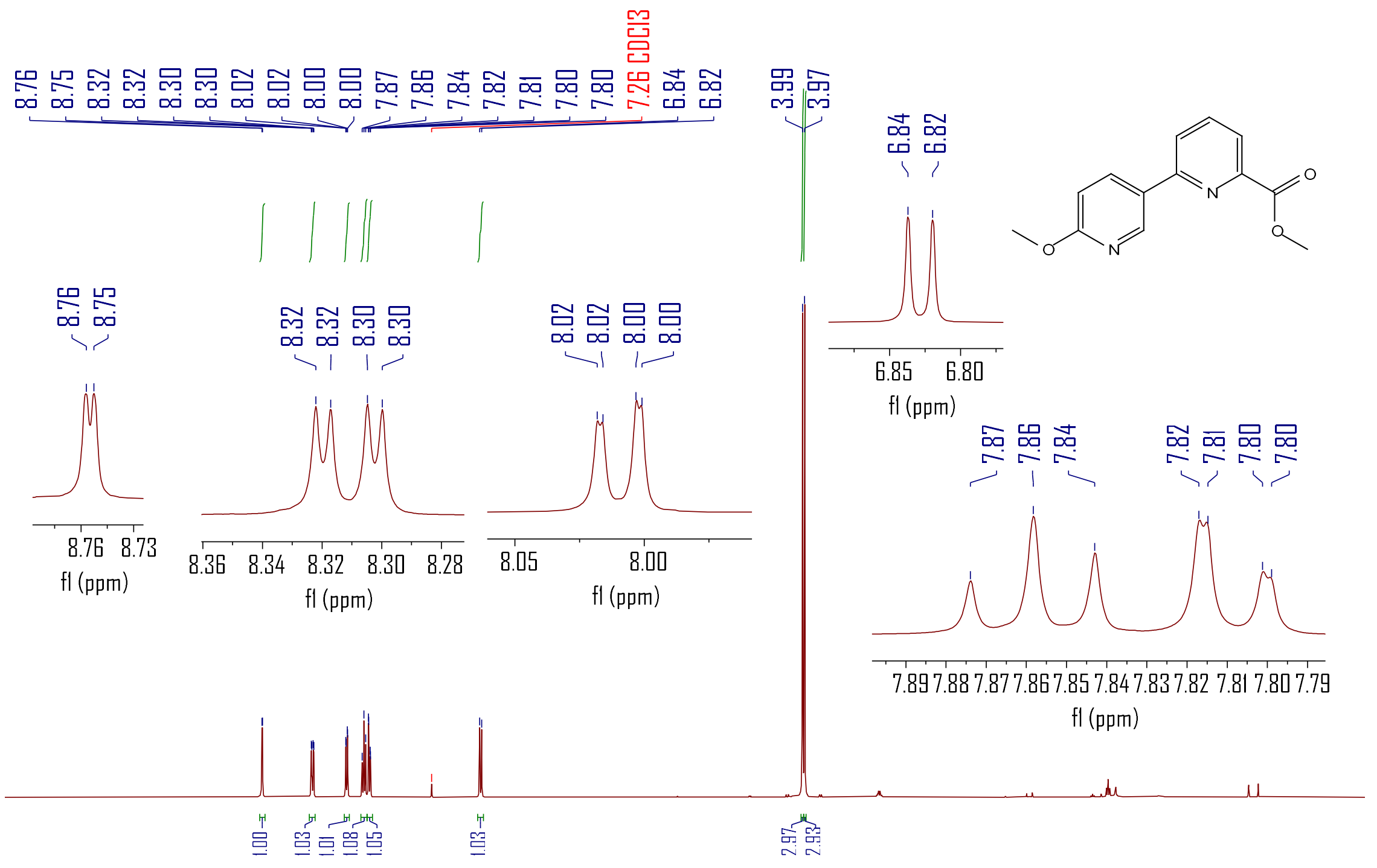

\begin{tabular}{lllllllllllllllllllllllll}
\hline .0 & 10.5 & 10.0 & 9.5 & 9.0 & 8.5 & 8.0 & 7.5 & 7.0 & 6.5 & 6.0 & 5.5 & 5.0 & 4.5 & 4.0 & 3.5 & 3.0 & 2.5 & 2.0 & 1.5 & 1.0 & 0.5 & 0.0 & -0.5
\end{tabular} 
${ }^{13} \mathrm{C}\left\{{ }^{1} \mathrm{H}\right\}$ NMR spectrum of compound 3an (126 $\left.\mathrm{MHz}, \mathrm{CDCl}_{3}\right)$

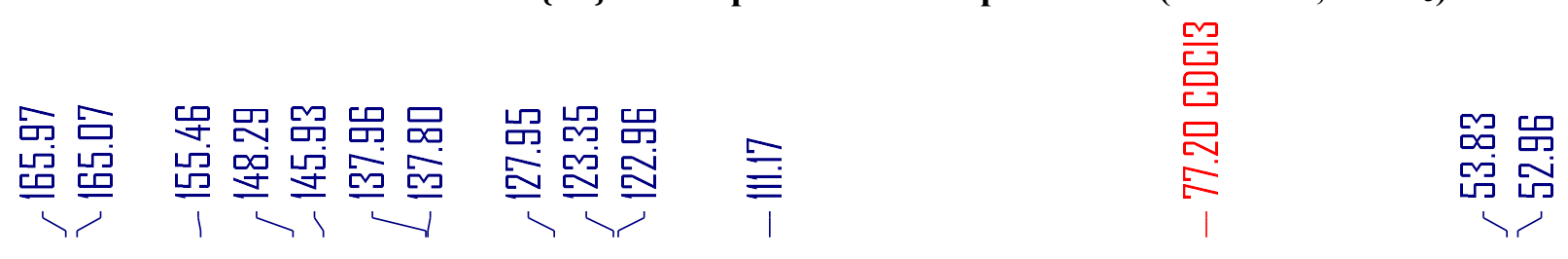

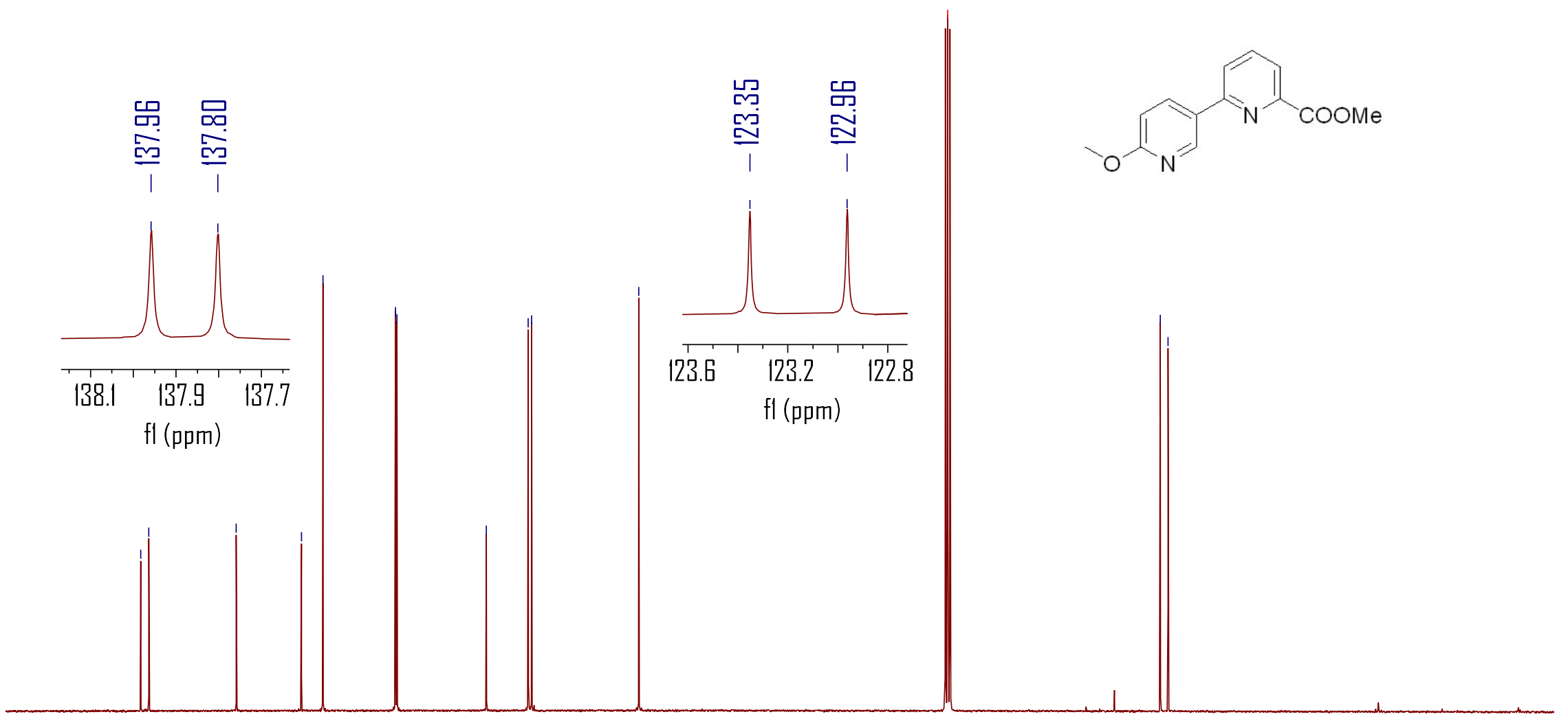




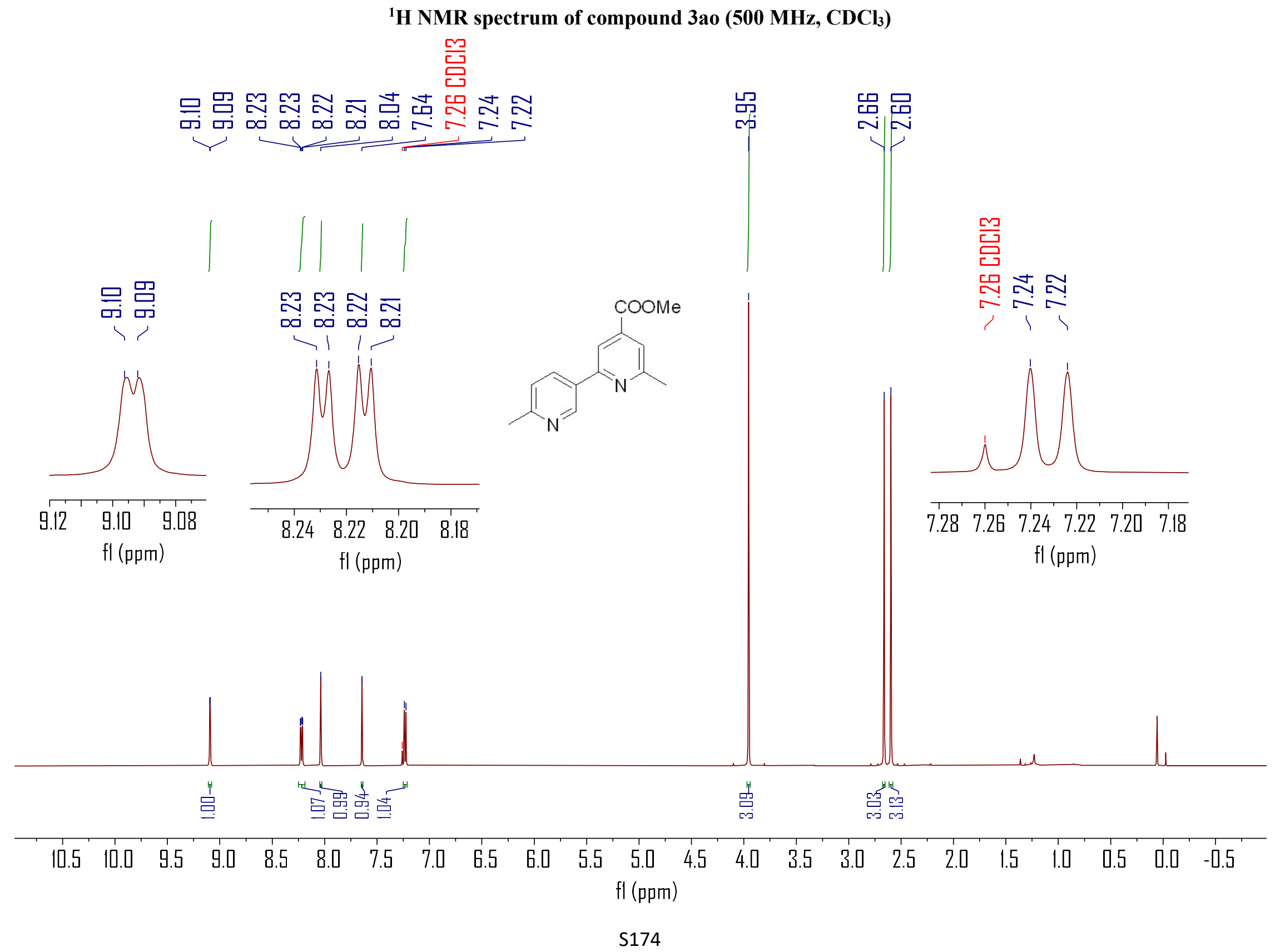


${ }^{13} \mathrm{C}\left\{{ }^{1} \mathrm{H}\right\}$ NMR spectrum of compound $3 \mathrm{ao}\left(126 \mathrm{MHz}, \mathrm{CDCl}_{3}\right)$

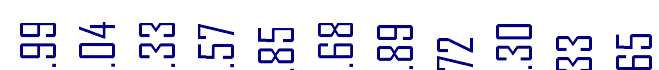

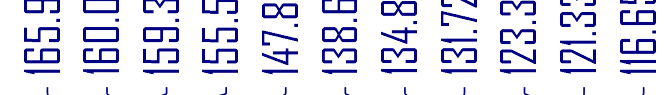

$$
\text { 몯 }
$$

南

吕 号

过

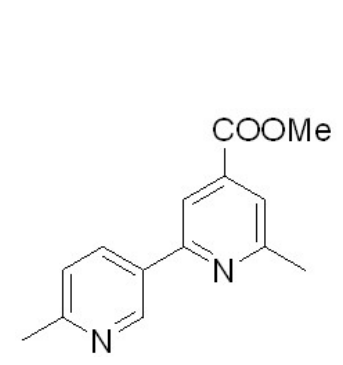

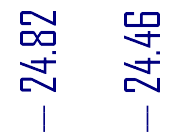
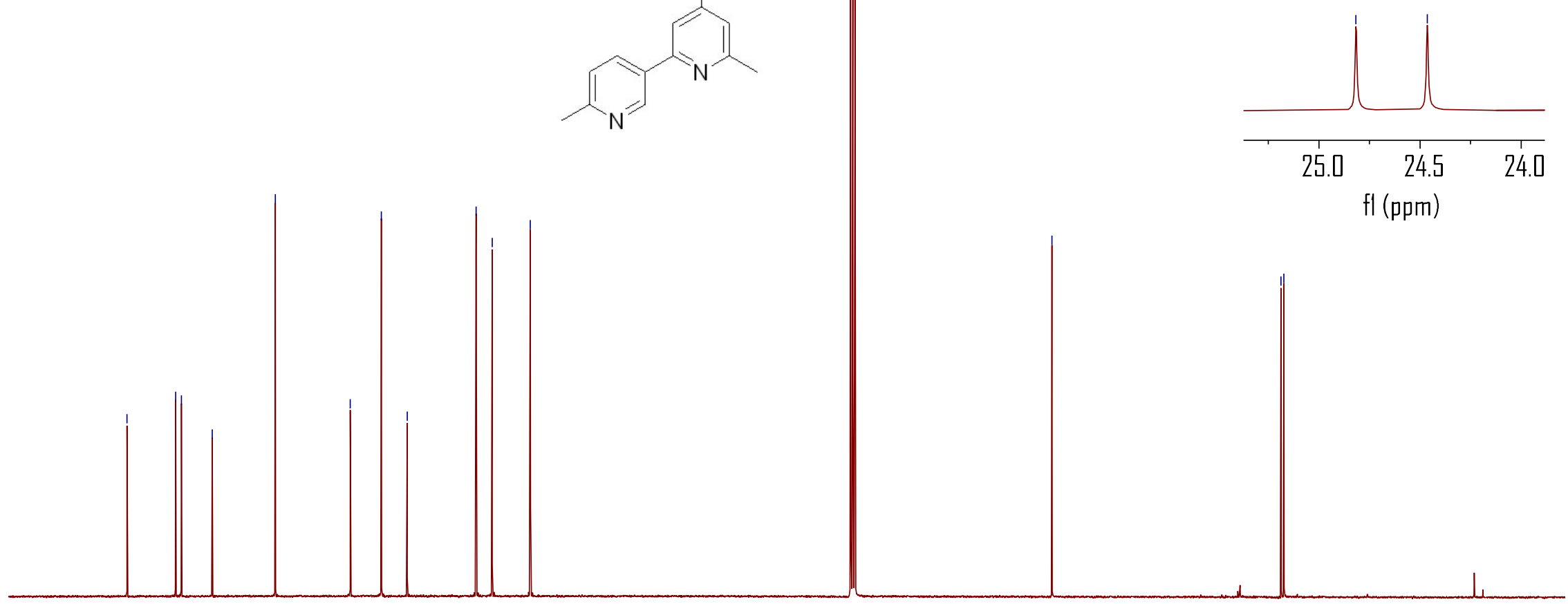

\section{0}

$170 \quad 160$

150

140

$130 \quad 12$

III I0

$\begin{array}{ll}90 & \\ \mathrm{fl}(\mathrm{ppm})\end{array}$

吅

$50 \quad 40$

30

20 10

S175 
${ }^{1} \mathrm{H}$ NMR spectrum of compound 3ap (500 $\left.\mathrm{MHz}, \mathrm{CDCl}_{3}\right)$

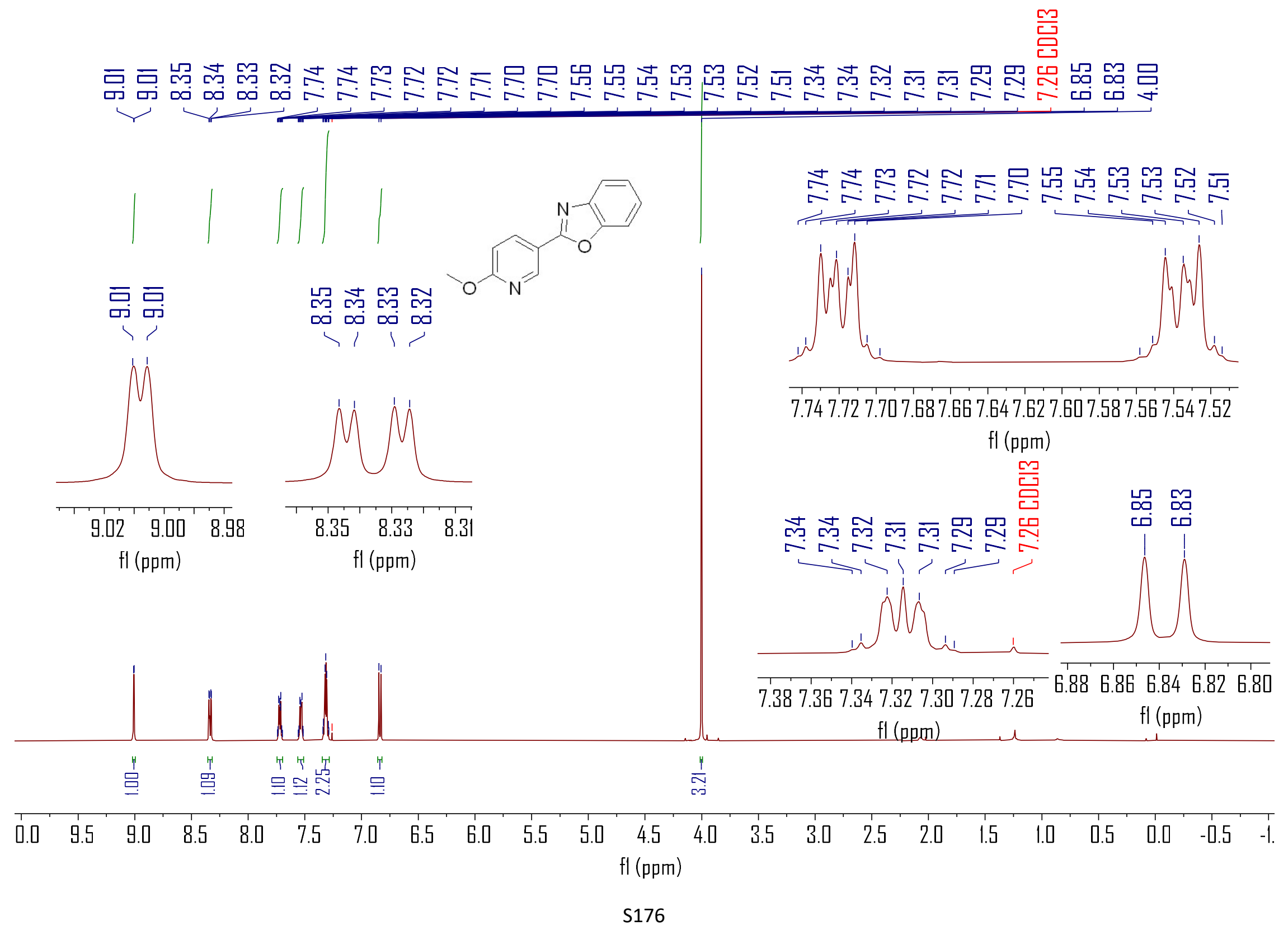


${ }^{13} \mathrm{C}\left\{{ }^{1} \mathrm{H}\right\}$ NMR spectrum of compound $3 \mathrm{ap}\left(126 \mathrm{MHz}, \mathrm{CDCl}_{3}\right)$

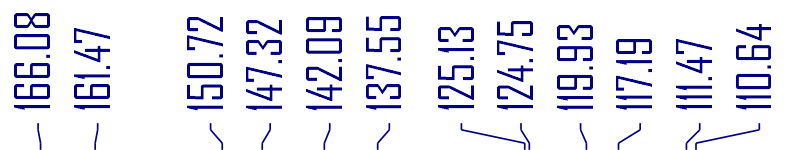
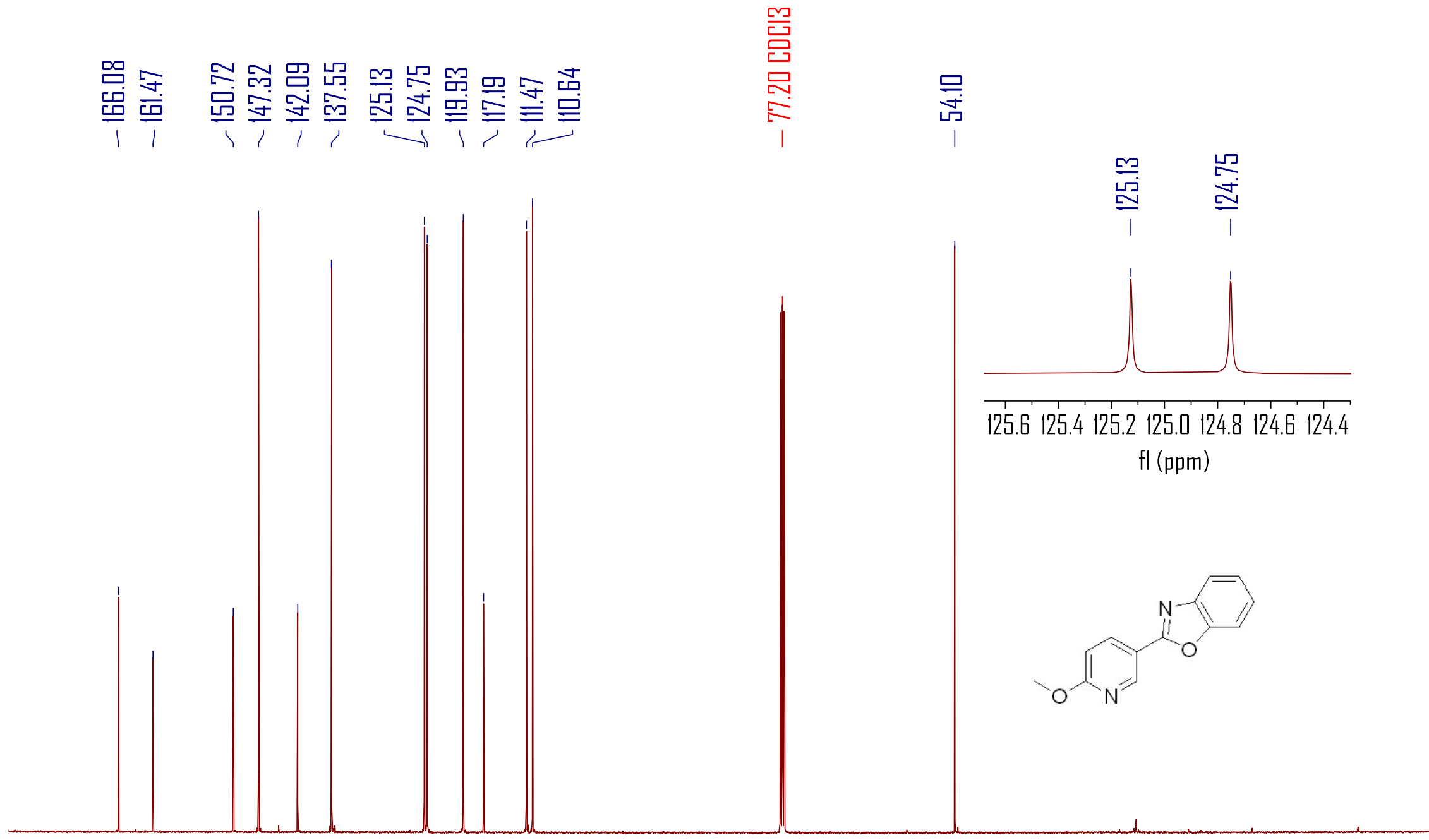

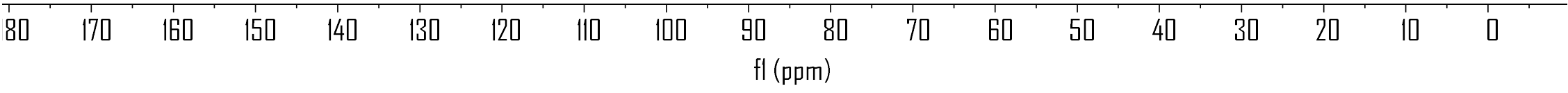



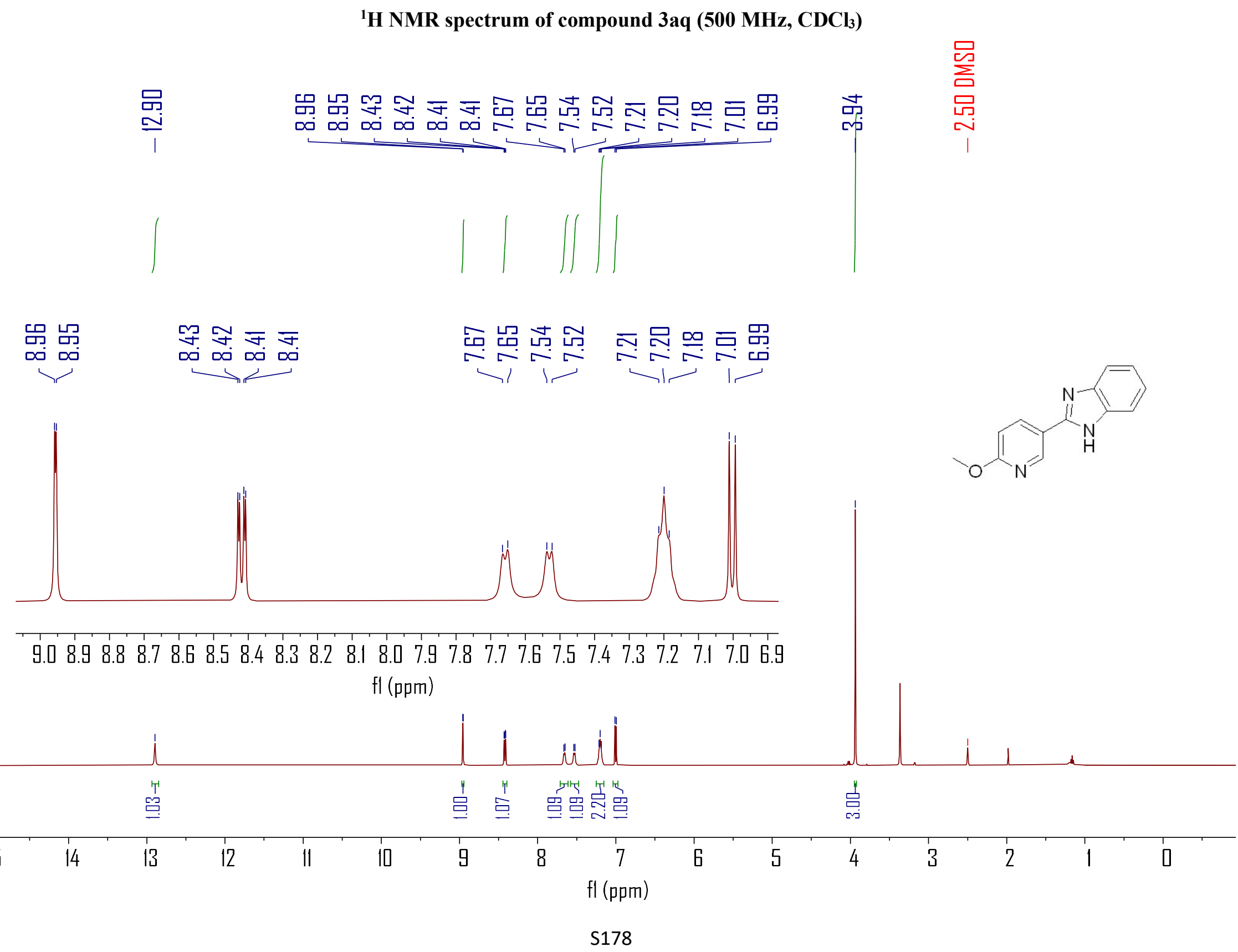
${ }^{1} \mathrm{H}$ NMR spectrum of compound $3 \mathrm{ar}\left(500 \mathrm{MHz}, \mathrm{CDCl}_{3}\right)$

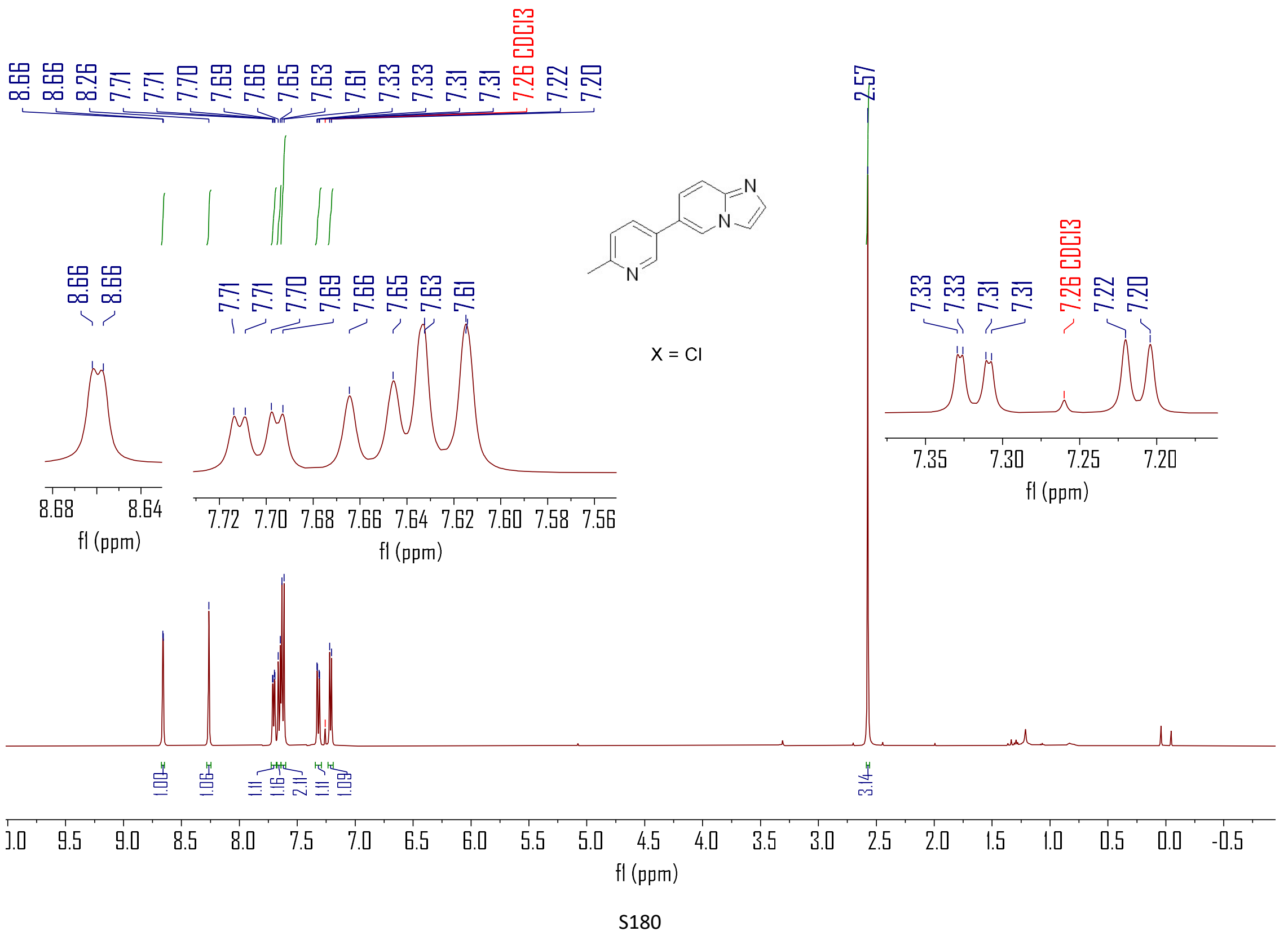


${ }^{13} \mathrm{C}\left\{{ }^{1} \mathrm{H}\right\} \mathrm{NMR}$ spectrum of compound 3ar (126 $\left.\mathrm{MHz}, \mathrm{CDCl}_{3}\right)$

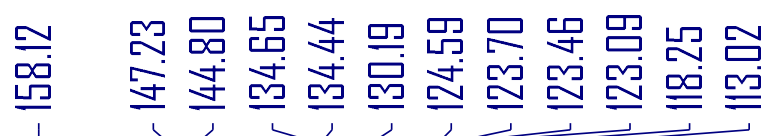

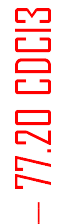

$\stackrel{\text { 峁 }}{\stackrel{ \pm}{N}}$

品穿
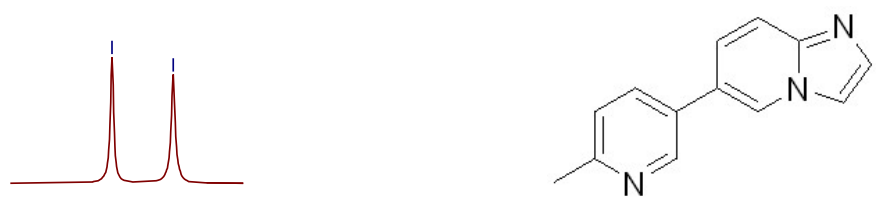

\begin{tabular}{lll}
\hline 134.8 & 134.4
\end{tabular}

$\mathrm{fl}(\mathrm{ppm})$

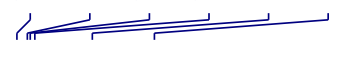

.
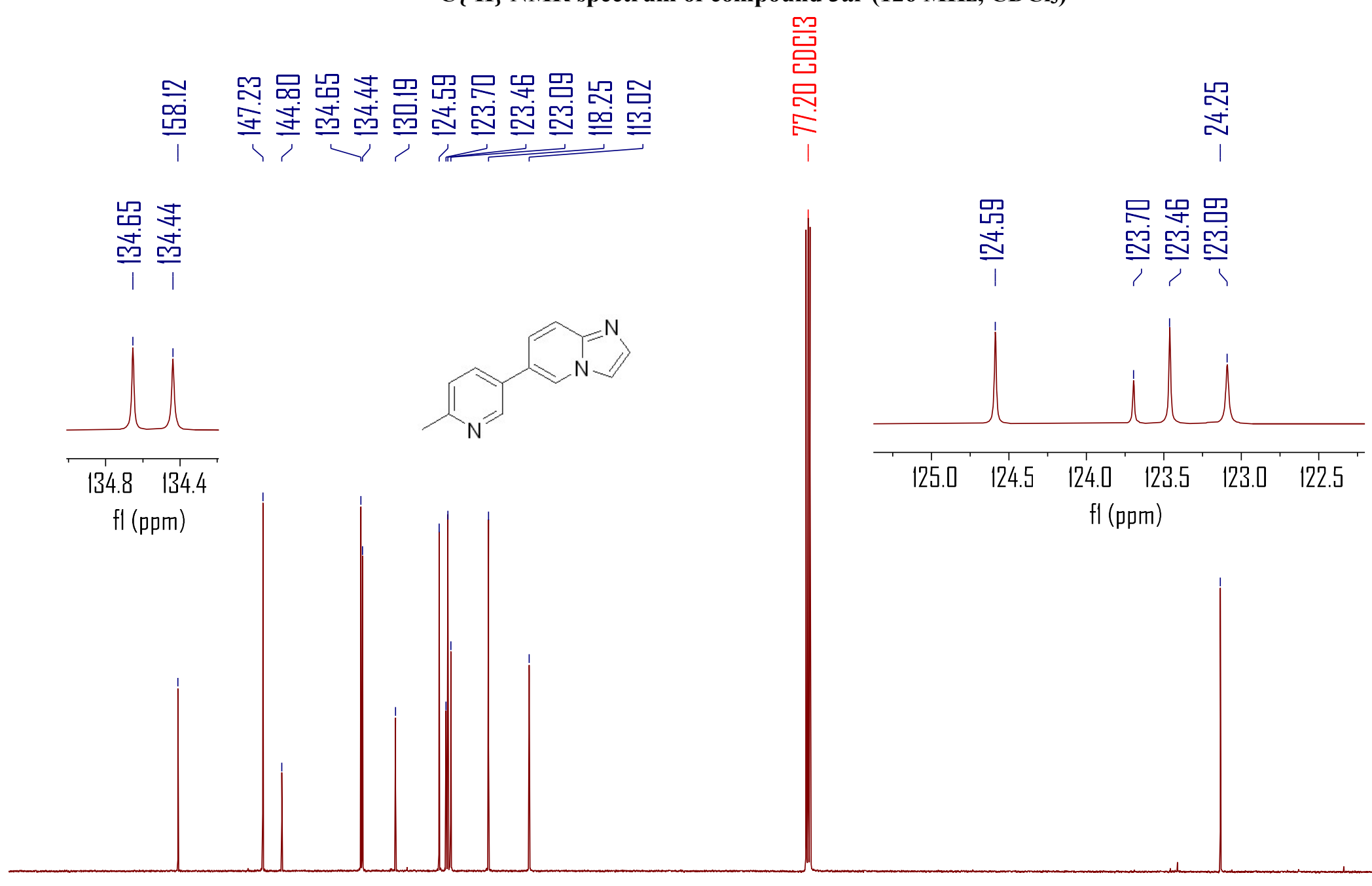

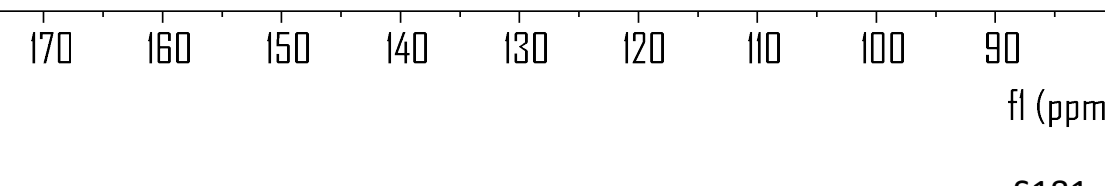


${ }^{1} \mathrm{H}$ NMR spectrum of compound $3 \mathrm{as}\left(500 \mathrm{MHz}, \mathrm{CDCl}_{3}\right)$

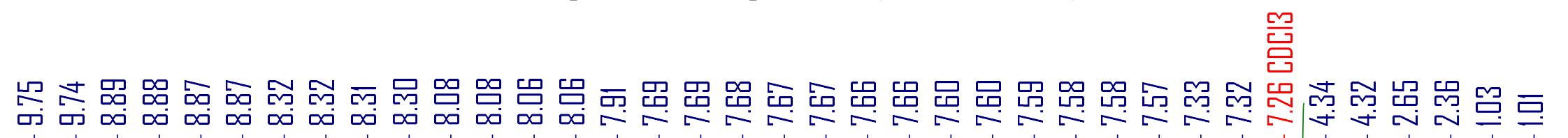

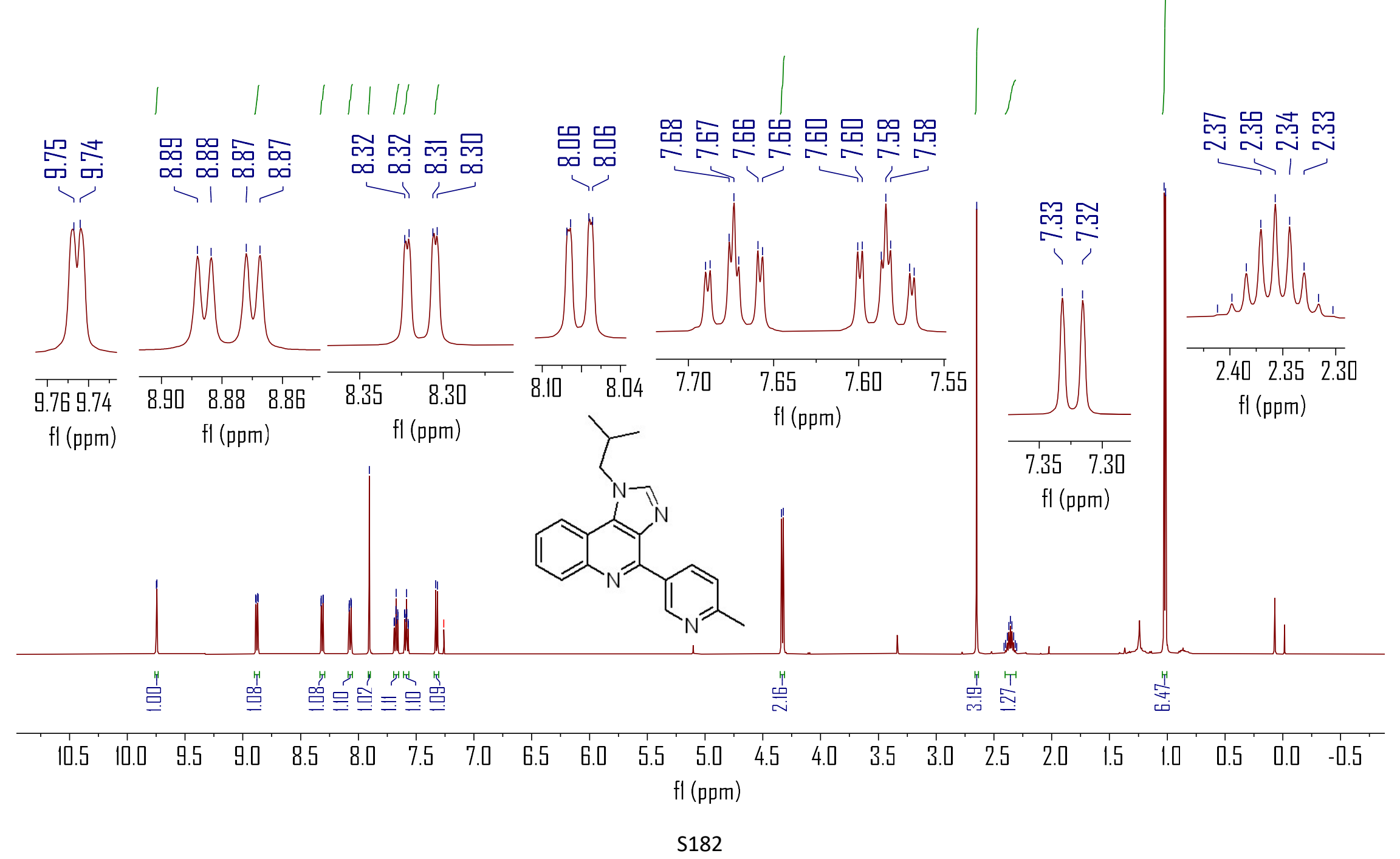


${ }^{13} \mathrm{C}\left\{{ }^{1} \mathrm{H}\right\}$ NMR spectrum of compound 3as (126 $\left.\mathrm{MHz}, \mathrm{CDCl}_{3}\right)$

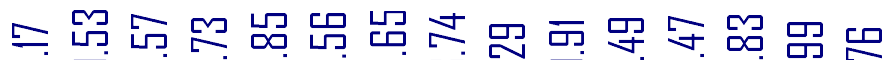

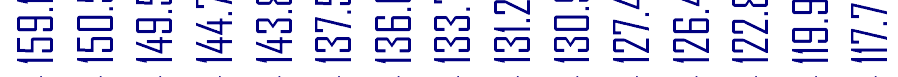
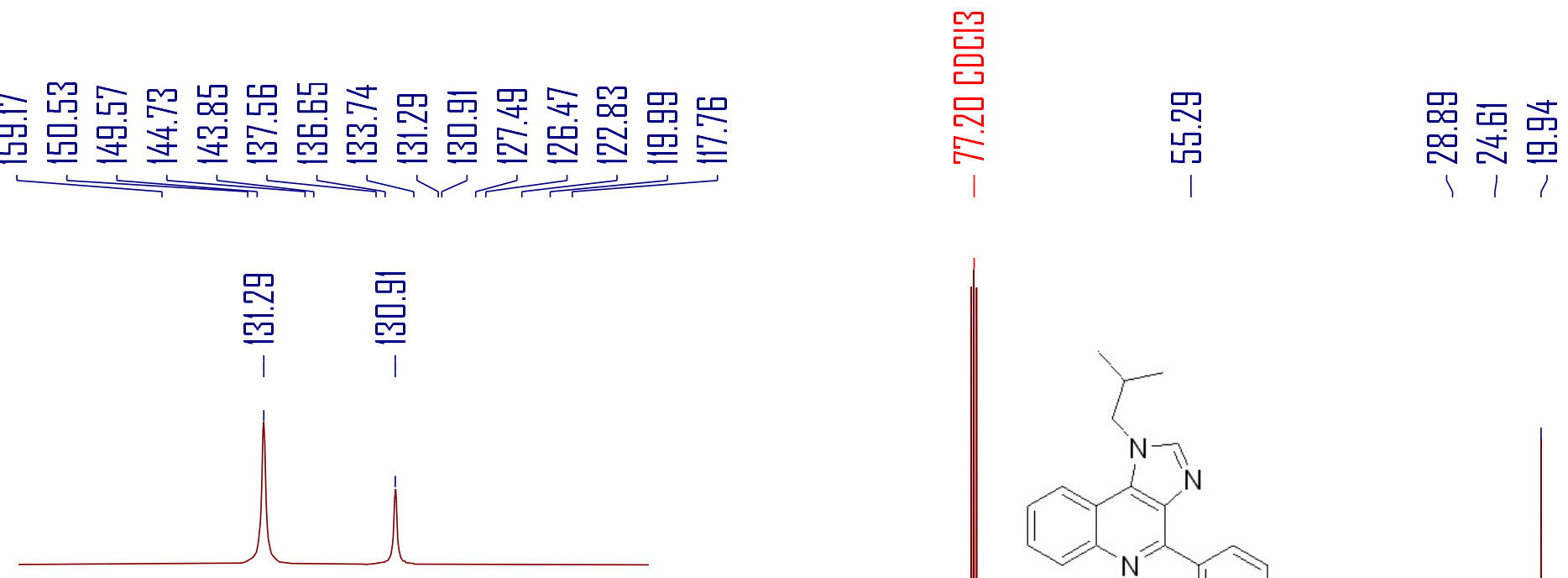

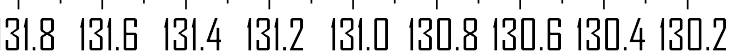
$\mathrm{fl}(\mathrm{ppm})$
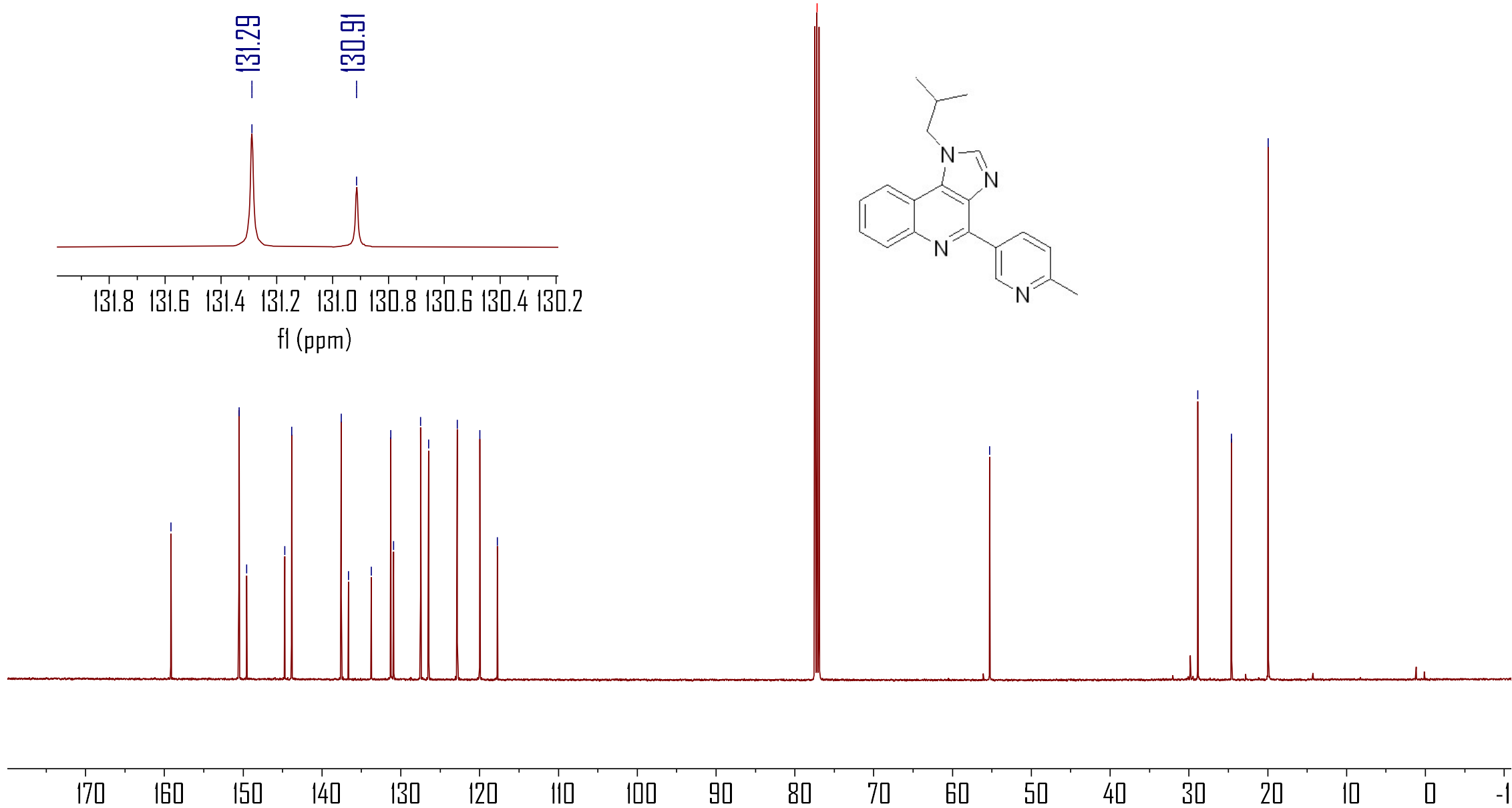

130

$120 \quad 110$

100

90 80


${ }^{1} \mathrm{H}$ NMR spectrum of compound 3at (500 $\mathrm{MHz}, \mathrm{CDCl}_{3}$ )

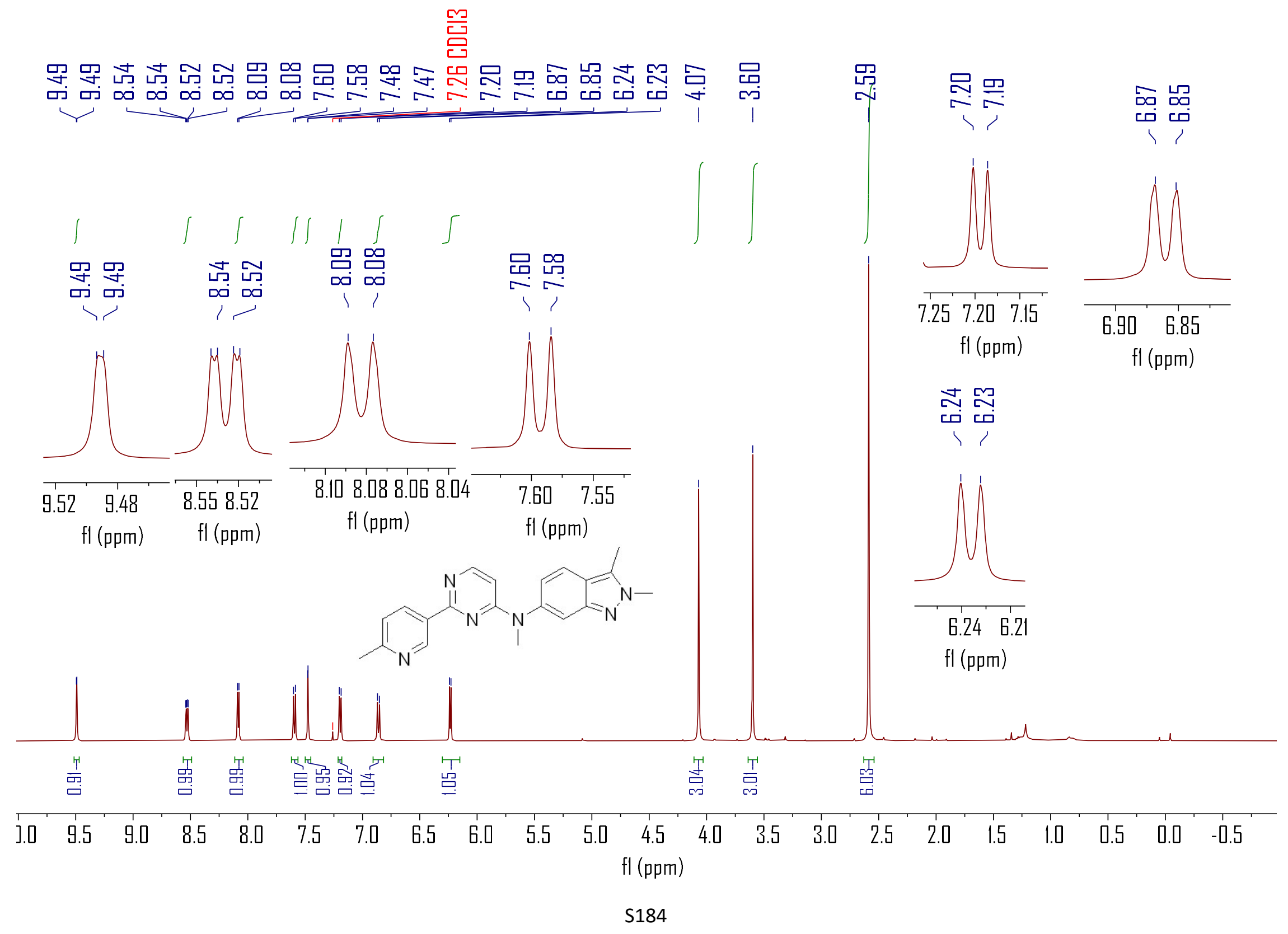


${ }^{13} \mathrm{C}\left\{{ }^{1} \mathrm{H}\right\} \mathrm{NMR}$ spectrum of compound 3at (126 $\left.\mathrm{MHz}, \mathrm{CDCl}_{3}\right)$

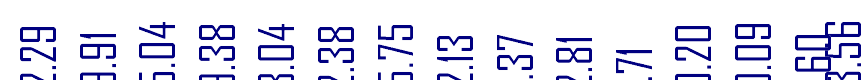

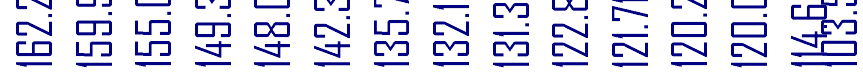
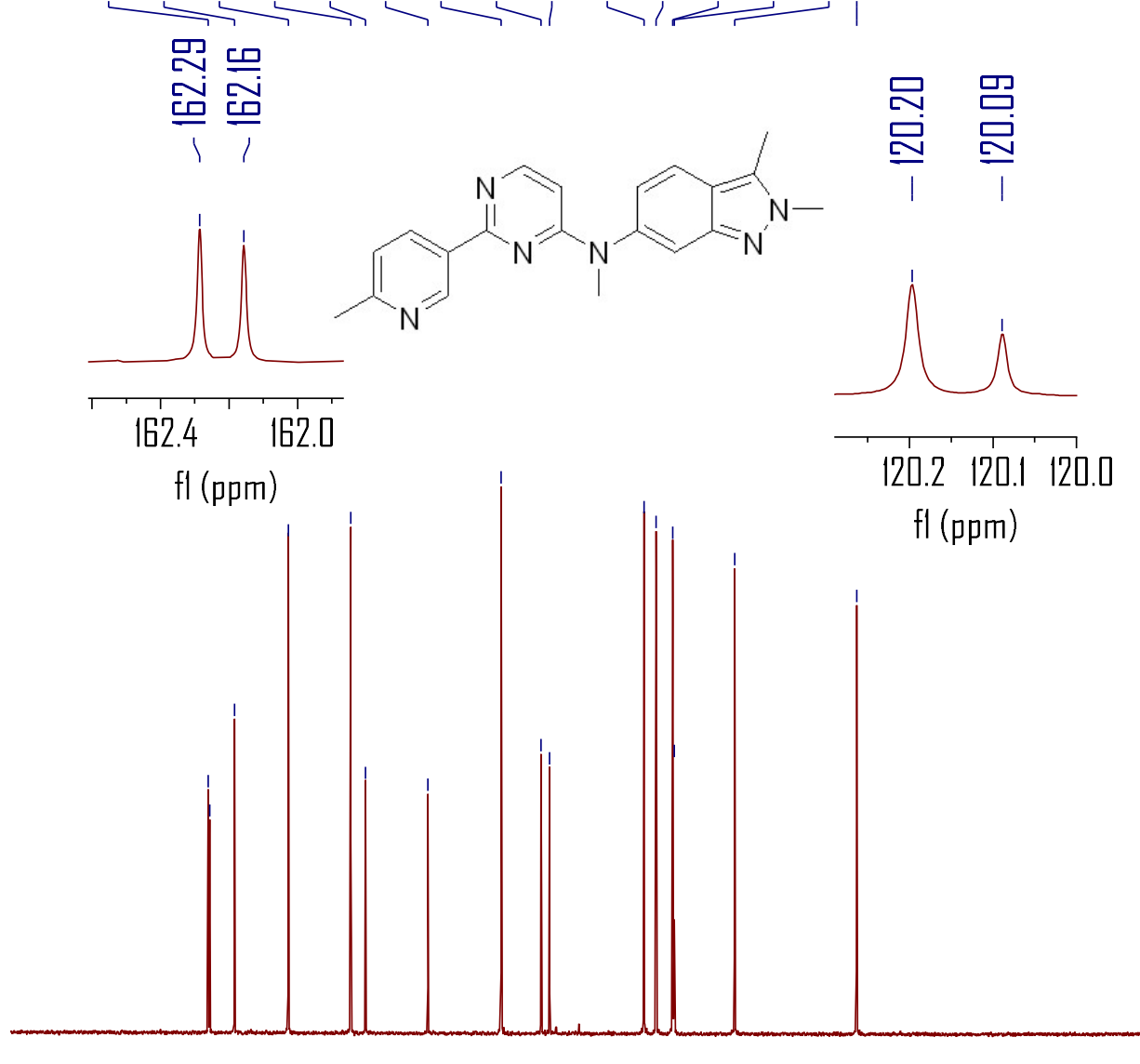

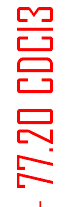

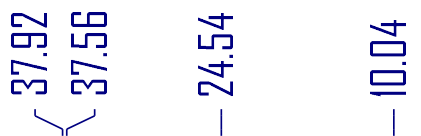

员吕

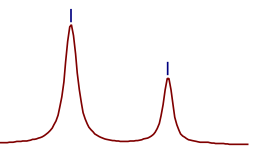

.

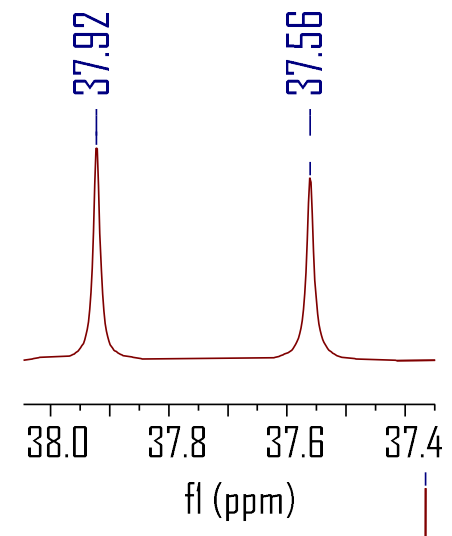

$37.8 \quad 37.6 \quad 37.4$

$\mathrm{fl}(\mathrm{ppm})$

80

150

140

$130 \quad 120 \quad 110$

100

$\mathrm{fl}(\mathrm{ppm})$

$70 \quad 60$

S185 
${ }^{1} \mathrm{H}$ NMR spectrum of compound 3au (500 $\left.\mathrm{MHz}, \mathrm{CDCl}_{3}\right)$

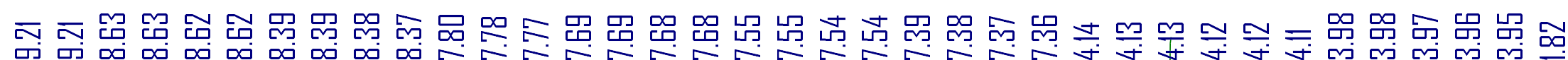
$\rightarrow 4$

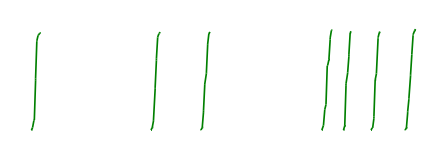

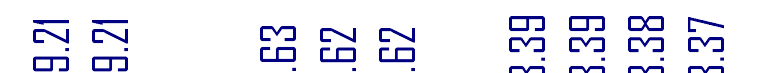

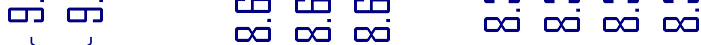

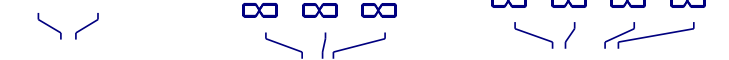
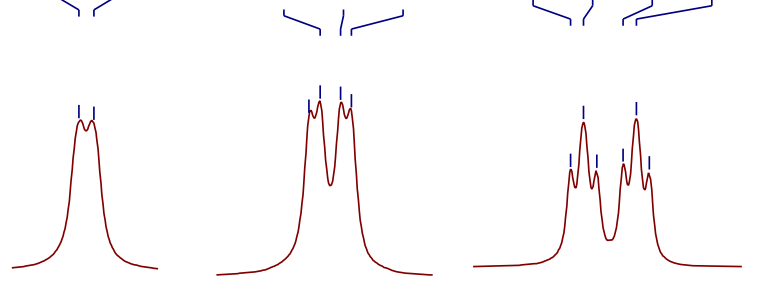

8.42

9.229 .20

$\mathrm{fl}(\mathrm{ppm})$

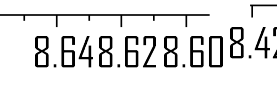

$\mathrm{fl}(\mathrm{ppm})$

$8.38 \quad 834$

$\mathrm{Fl}(\mathrm{ppm})$

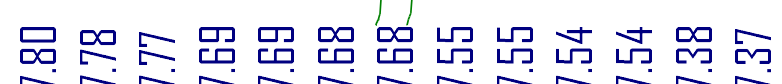

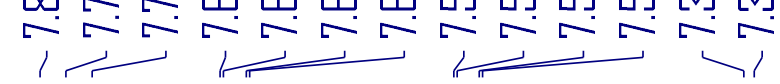
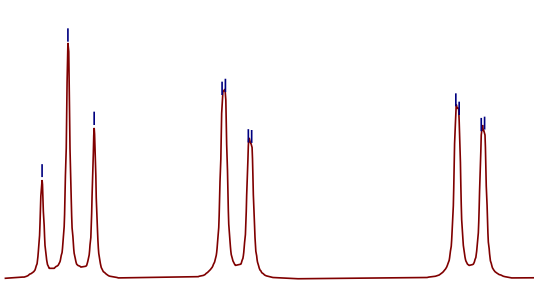

$34 \overline{7.8}$

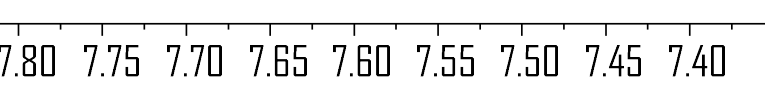

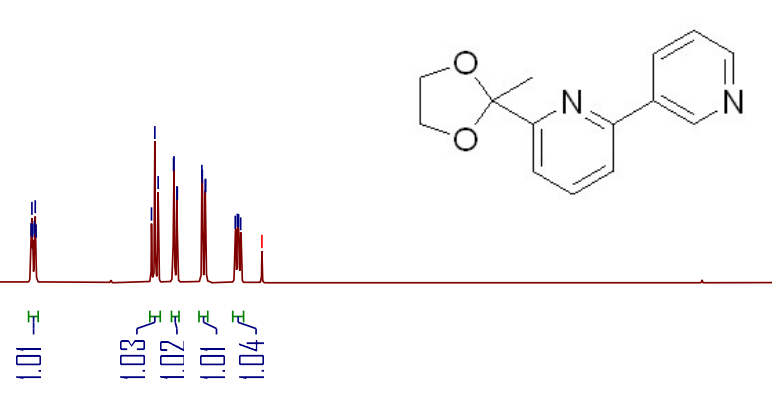

$\mathrm{fl}(\mathrm{ppm})$
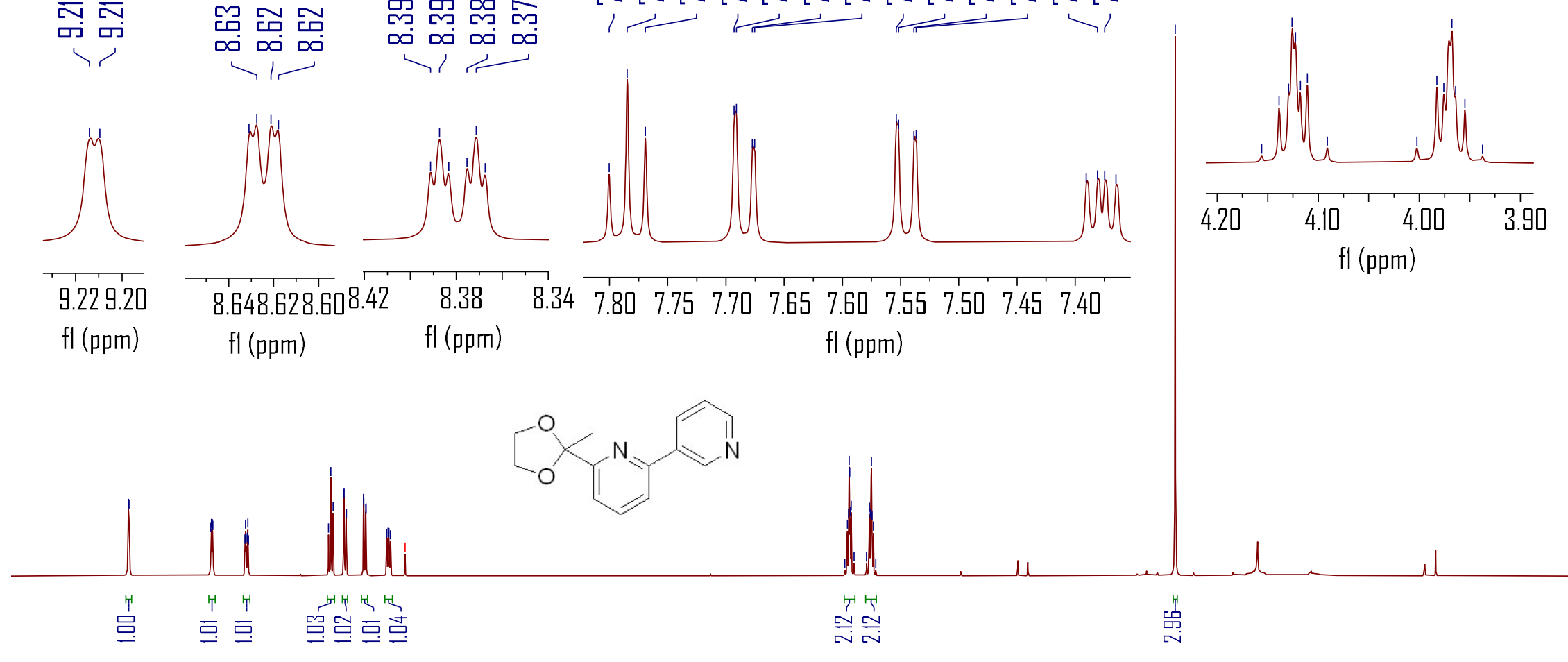

\begin{tabular}{lllllllllll}
\hline 0.0 & 9.5 & 9.0 & 8.5 & 8.0 & 7.5 & 7.0 & 6.5 & 6.0 & 5.5 & 5.0
\end{tabular}

$\mathrm{fl}$ (ppm) 
${ }^{13} \mathrm{C}\left\{{ }^{1} \mathrm{H}\right\}$ NMR spectrum of compound 3au (126 $\left.\mathrm{MHz}, \mathrm{CDCl}_{3}\right)$

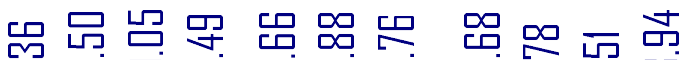

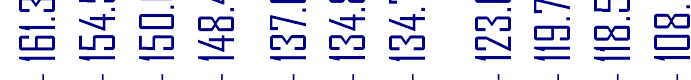

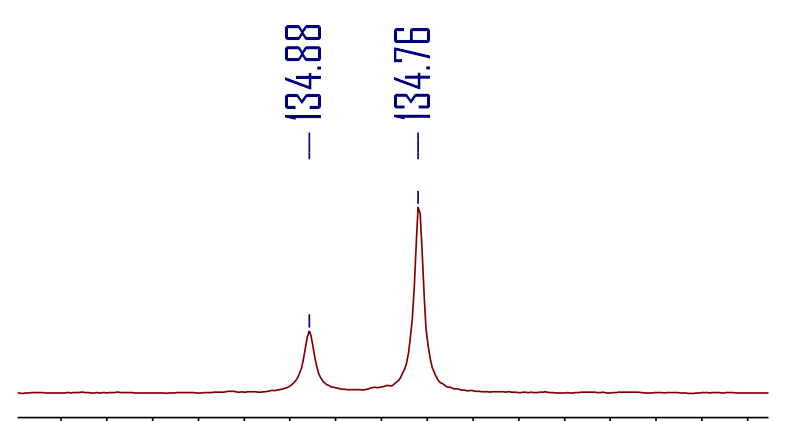

$\begin{array}{lllllllll}135.1 & 135.0 & 134.9 & 134.8 & 134.7 & 134.6 & 134.5 & 134.4\end{array}$ $\mathrm{fl}$ (ppm)
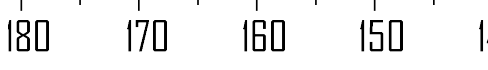

$40 \quad 130 \quad 12$

110 IDO

$\mathrm{fl}(\mathrm{ppm})$

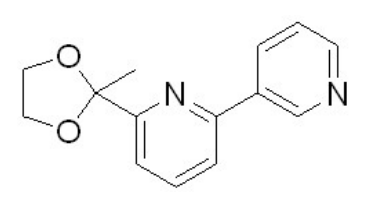




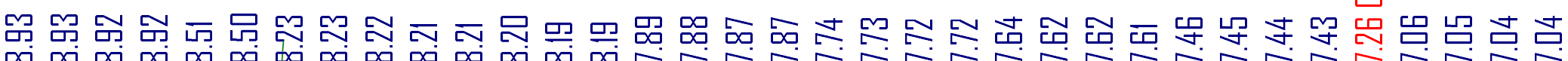

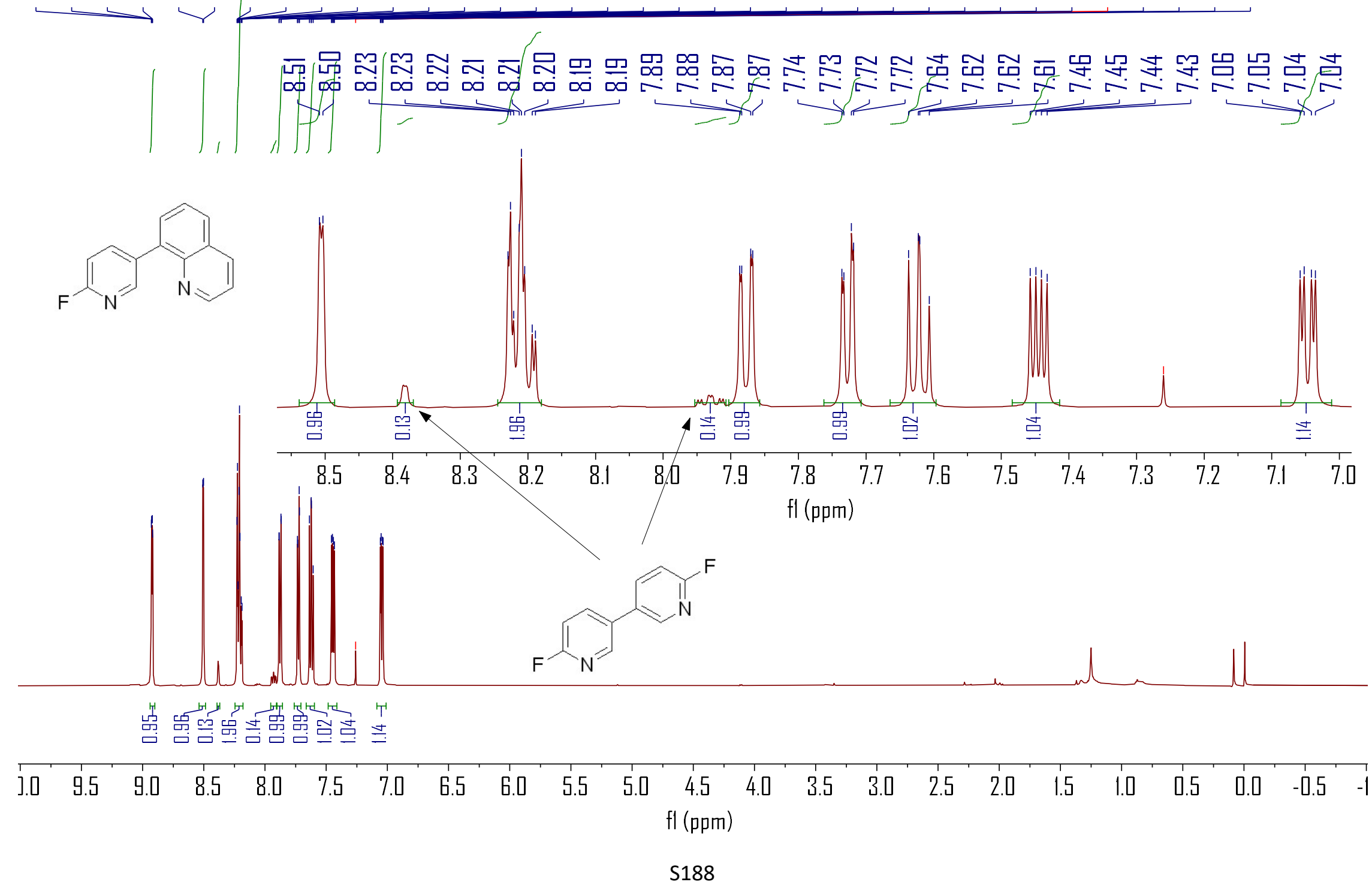


${ }^{19} \mathrm{~F}\left\{{ }^{1} \mathrm{H}\right\}$ NMR spectrum of compound $3 \mathrm{av}\left(376 \mathrm{MHz}, \mathrm{CDCl}_{3}\right)$

品骂

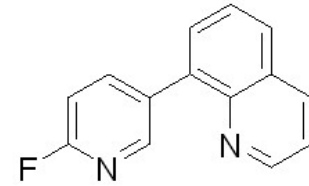

$i$

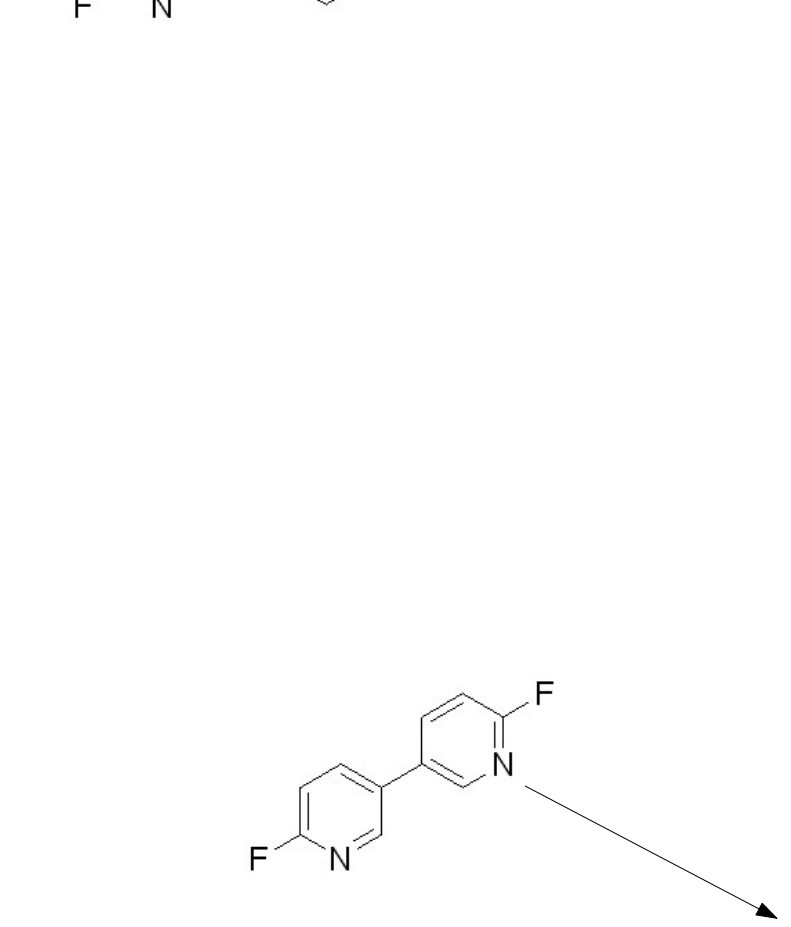

$$
\text { 험를 }
$$

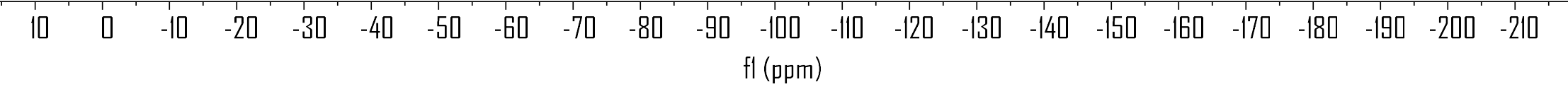


${ }^{13} \mathrm{C}\left\{{ }^{1} \mathrm{H}\right\}$ NMR spectrum of compound $3 \mathrm{av}\left(126 \mathrm{MHz} \mathrm{CDCl}_{3}\right)$

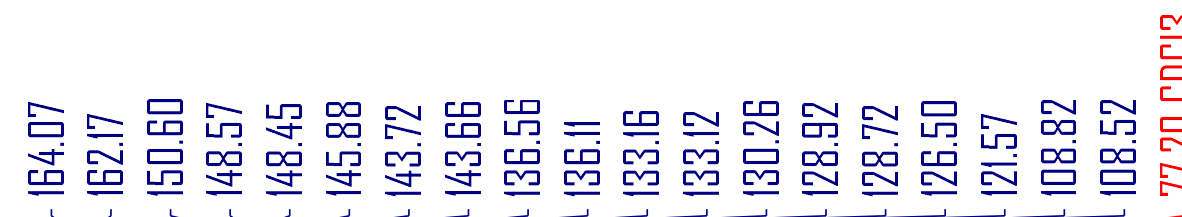

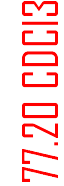

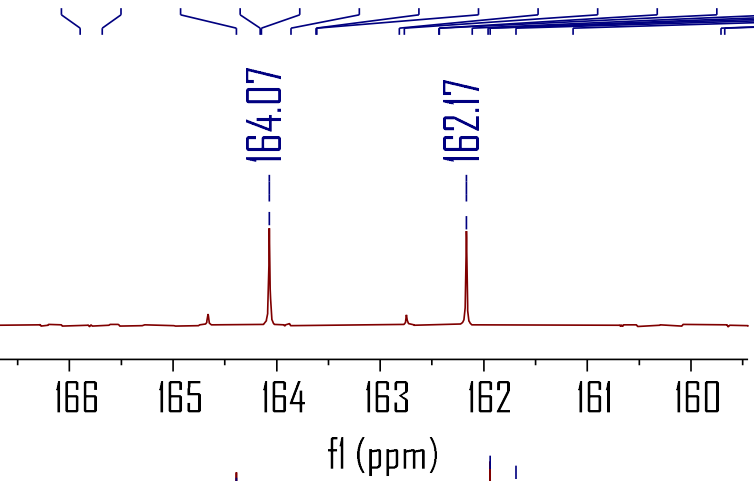

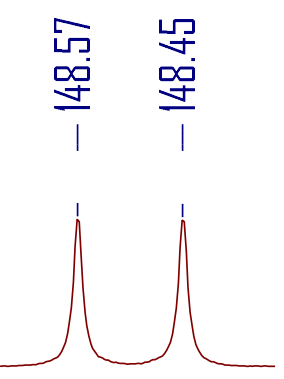

$148.6 \quad 148.5 \quad 148.4$

$\mathrm{fl}(\mathrm{ppm})$

들.

ㅁำ

11

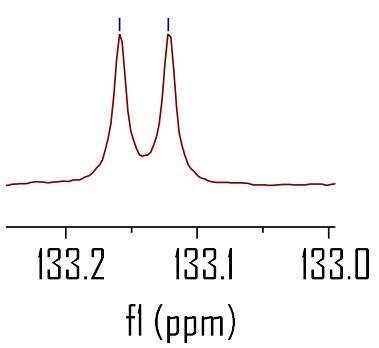

뚬

哭 哭

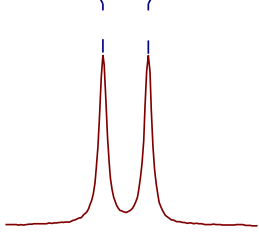

143.8143 .7143 .6

$\mathrm{fl}$ (ppm)

올 뭄

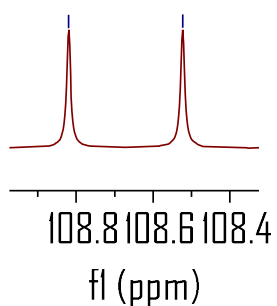

\section{0}

170

160

$150 \quad 140 \quad 130$

$120 \quad 110$

100

9080

$\mathrm{fl}$ (ppm) 


\section{${ }^{1} \mathrm{H}$ NMR spectrum of compound 3aw (500 $\left.\mathrm{MHz}, \mathrm{CDCl}_{3}\right)$}

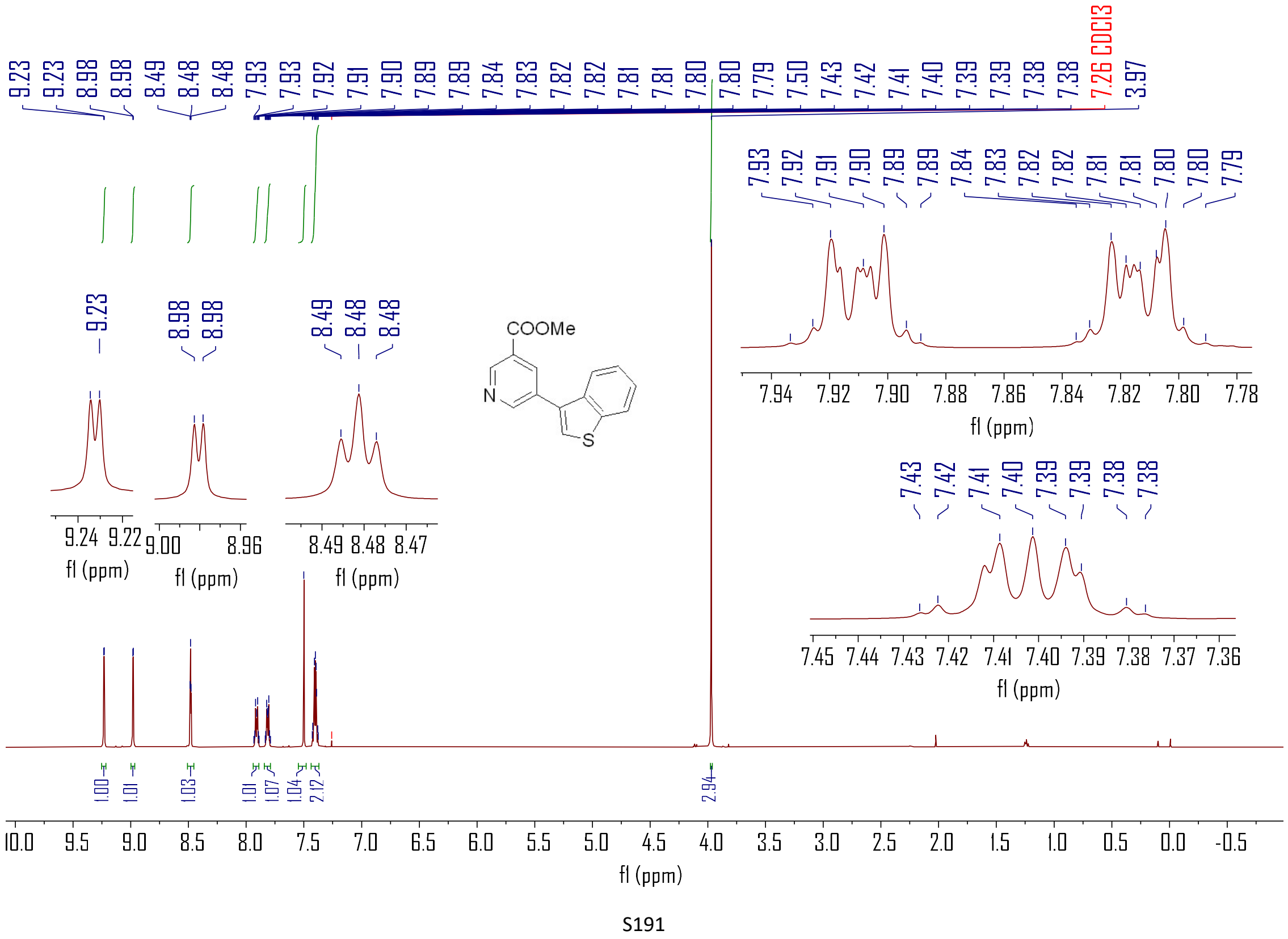


${ }^{13} \mathrm{C}\left\{{ }^{1} \mathrm{H}\right\}$ NMR spectrum of compound 3aw (126 $\left.\mathrm{MHz}, \mathrm{CDCl}_{3}\right)$

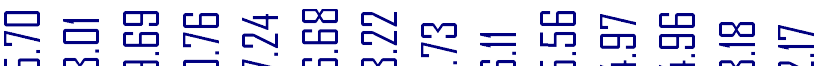

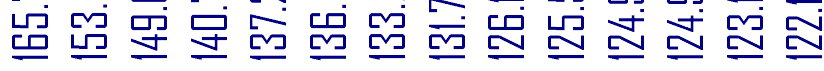
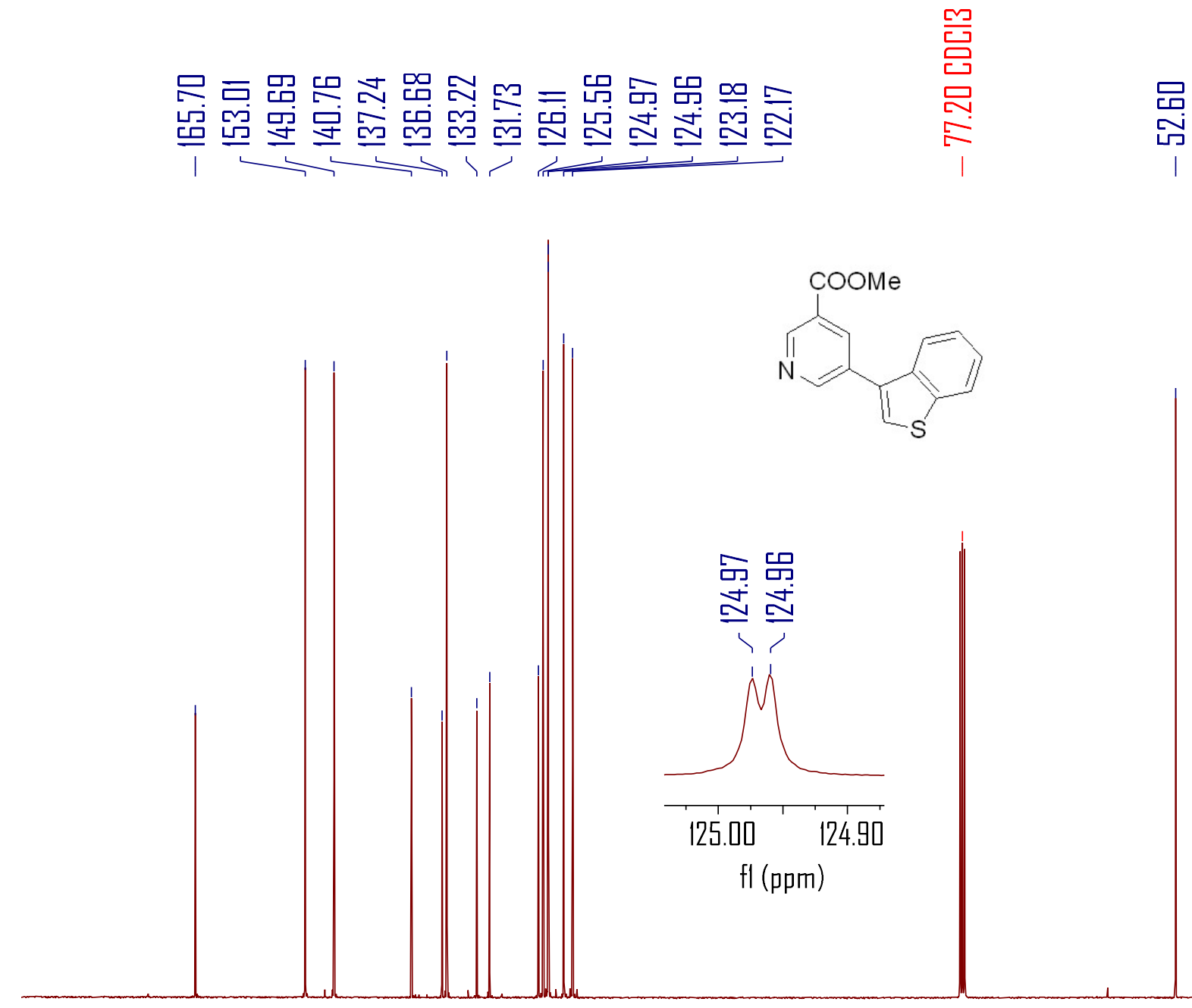

虽

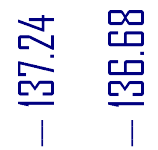
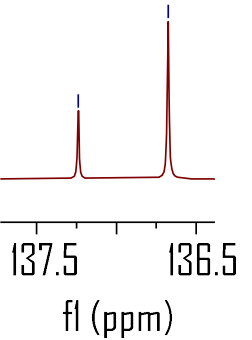

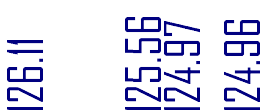
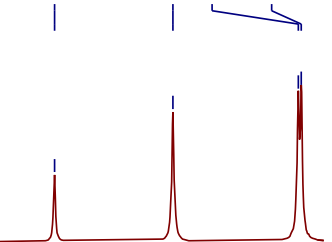

126.0 125.5 125.0

$\mathrm{fl}(\mathrm{ppm})$

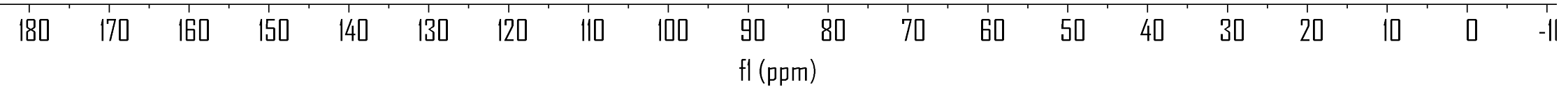


${ }^{1} \mathrm{H}$ NMR spectrum of compound $3 \mathrm{ax}\left(500 \mathrm{MHz}, \mathrm{CDCl}_{3}\right)$
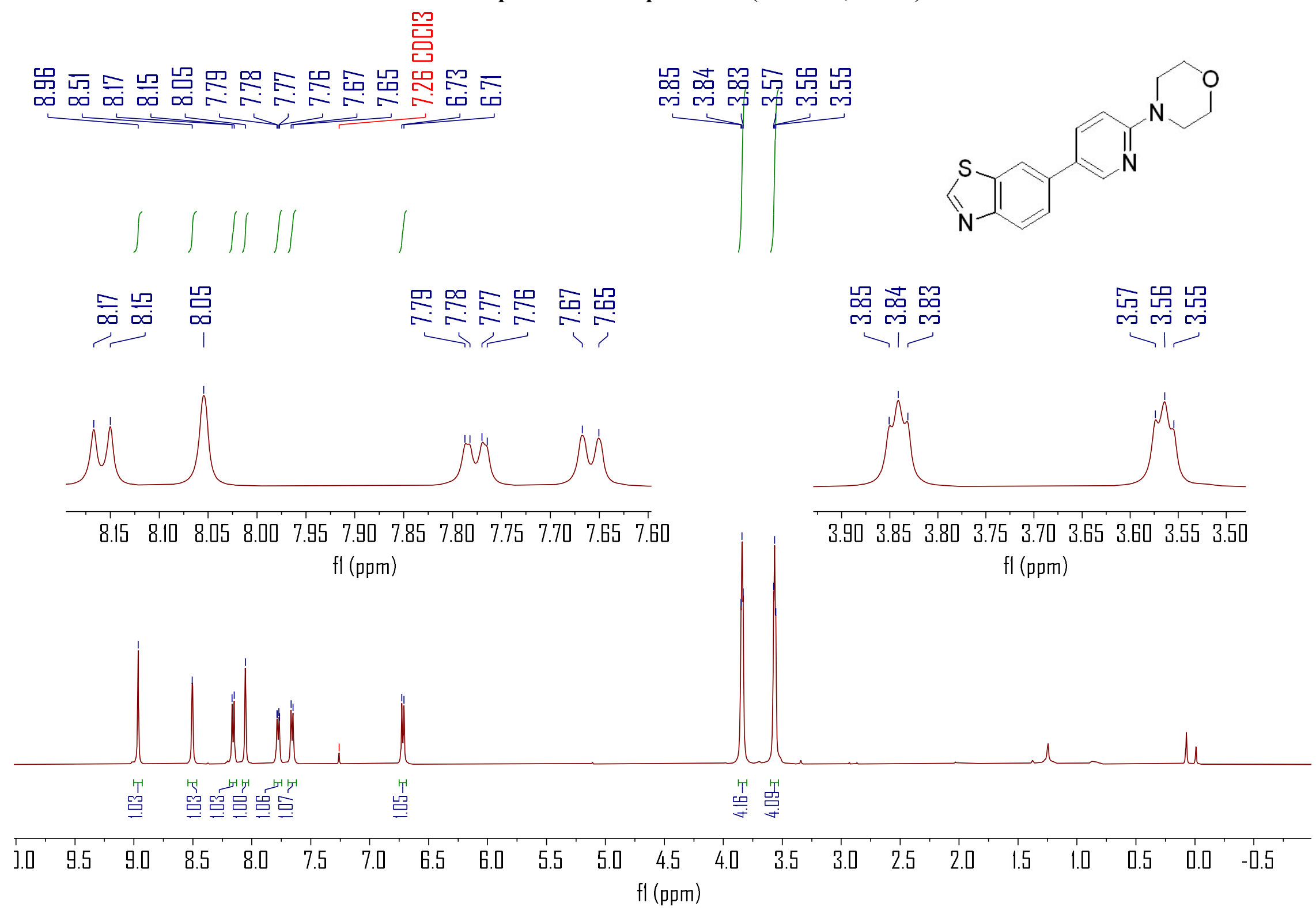
${ }^{13} \mathrm{C}\left\{{ }^{1} \mathrm{H}\right\} \mathrm{NMR}$ spectrum of compound $3 \mathrm{ax}\left(126 \mathrm{MHz}, \mathrm{CDCl}_{3}\right)$

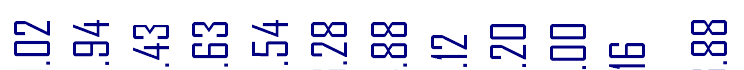

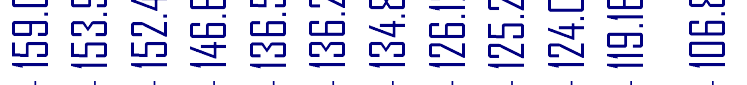

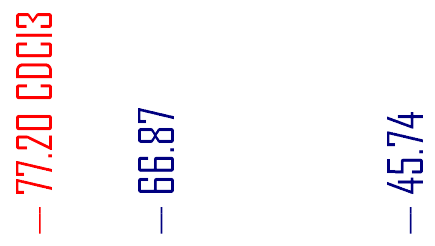
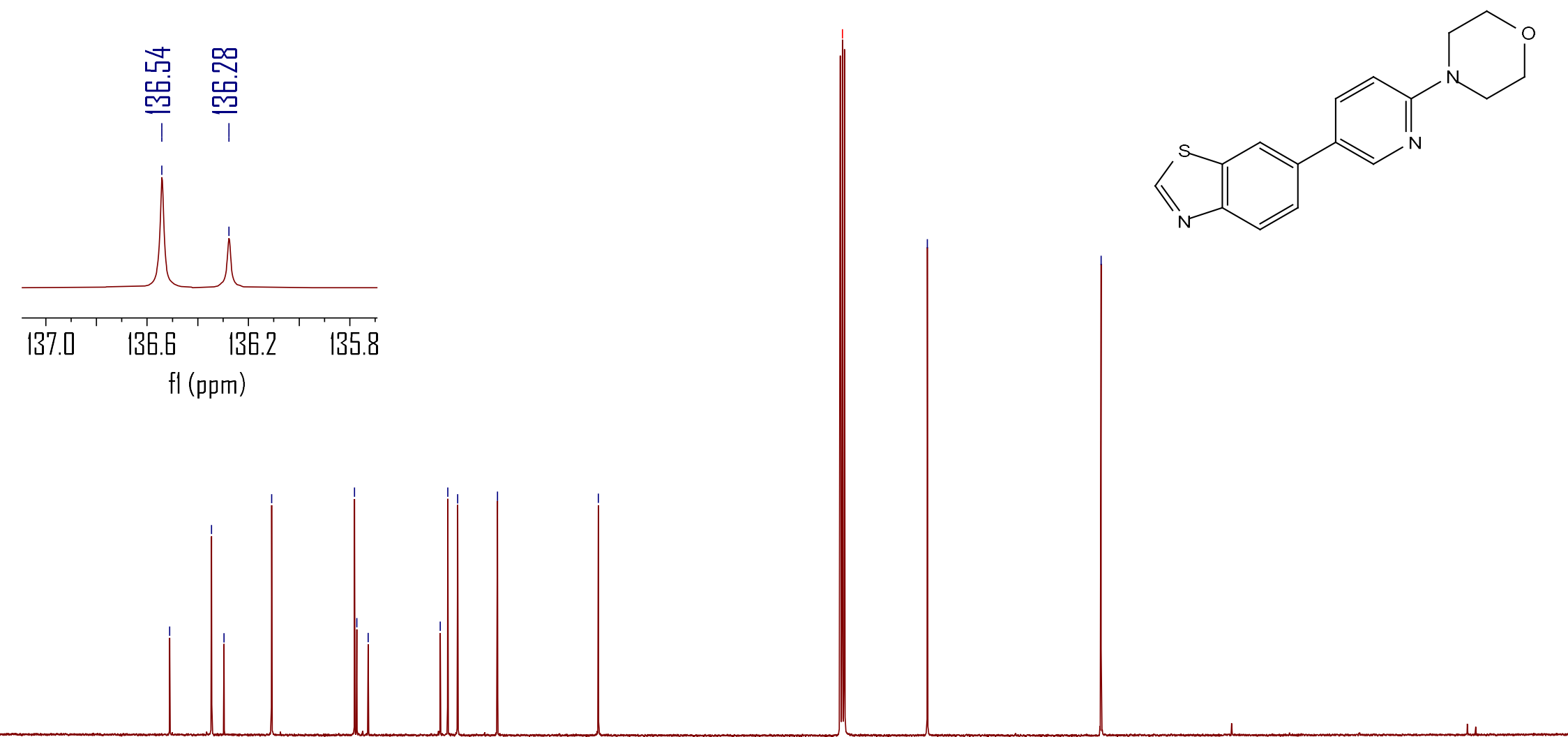

$\begin{array}{llll}170 & 160 & 150 & 140\end{array}$

$130 \quad 120$

III I I

90

$\mathrm{fl}$ (ppm)

S194 


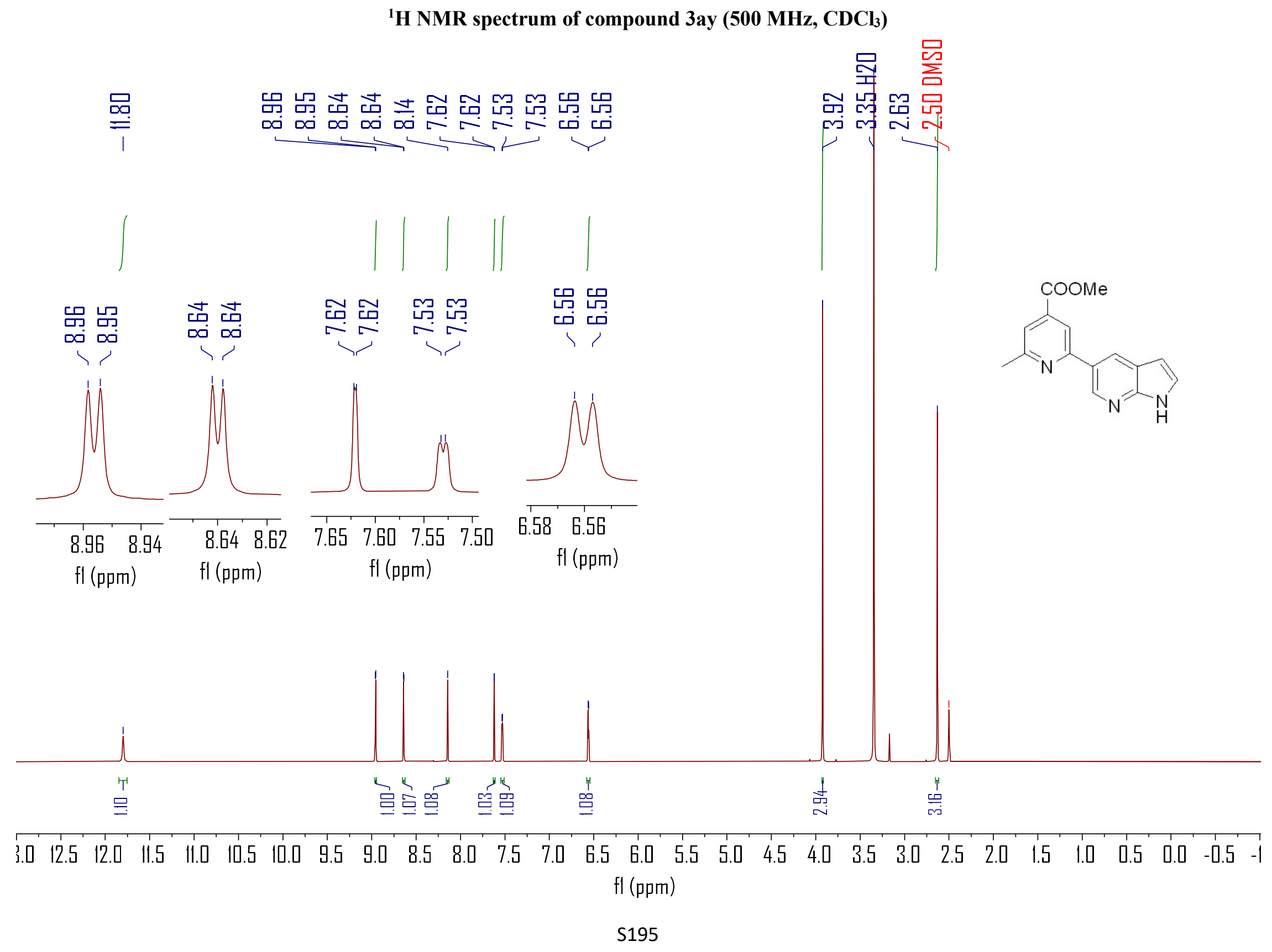


${ }^{13} \mathrm{C}\left\{{ }^{1} \mathrm{H}\right\} \mathrm{NMR}$ spectrum of compound 3ay (126 $\left.\mathrm{MHz}, \mathrm{CDCl}_{3}\right)$

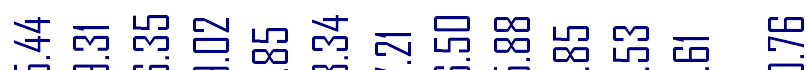

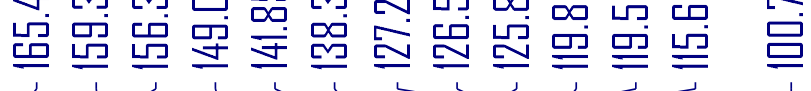

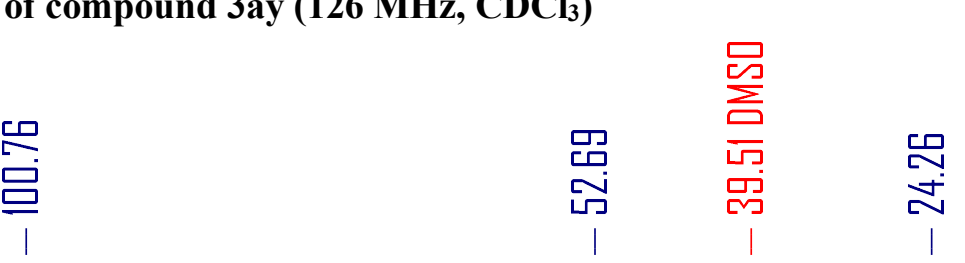

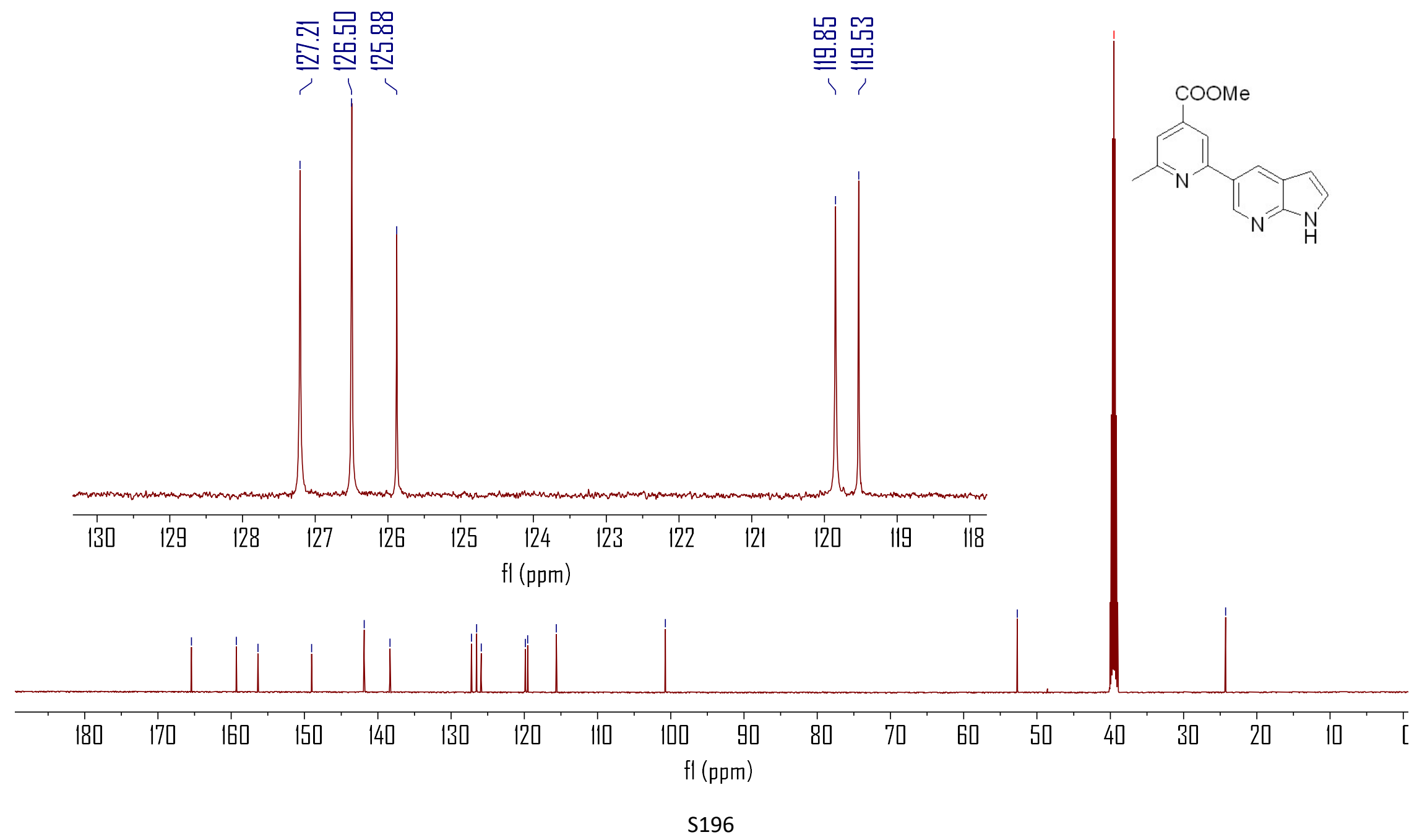


${ }^{1} \mathrm{H}$ NMR spectrum of compound $3 \mathrm{az}\left(500 \mathrm{MHz}, \mathrm{CDCl}_{3}\right)$

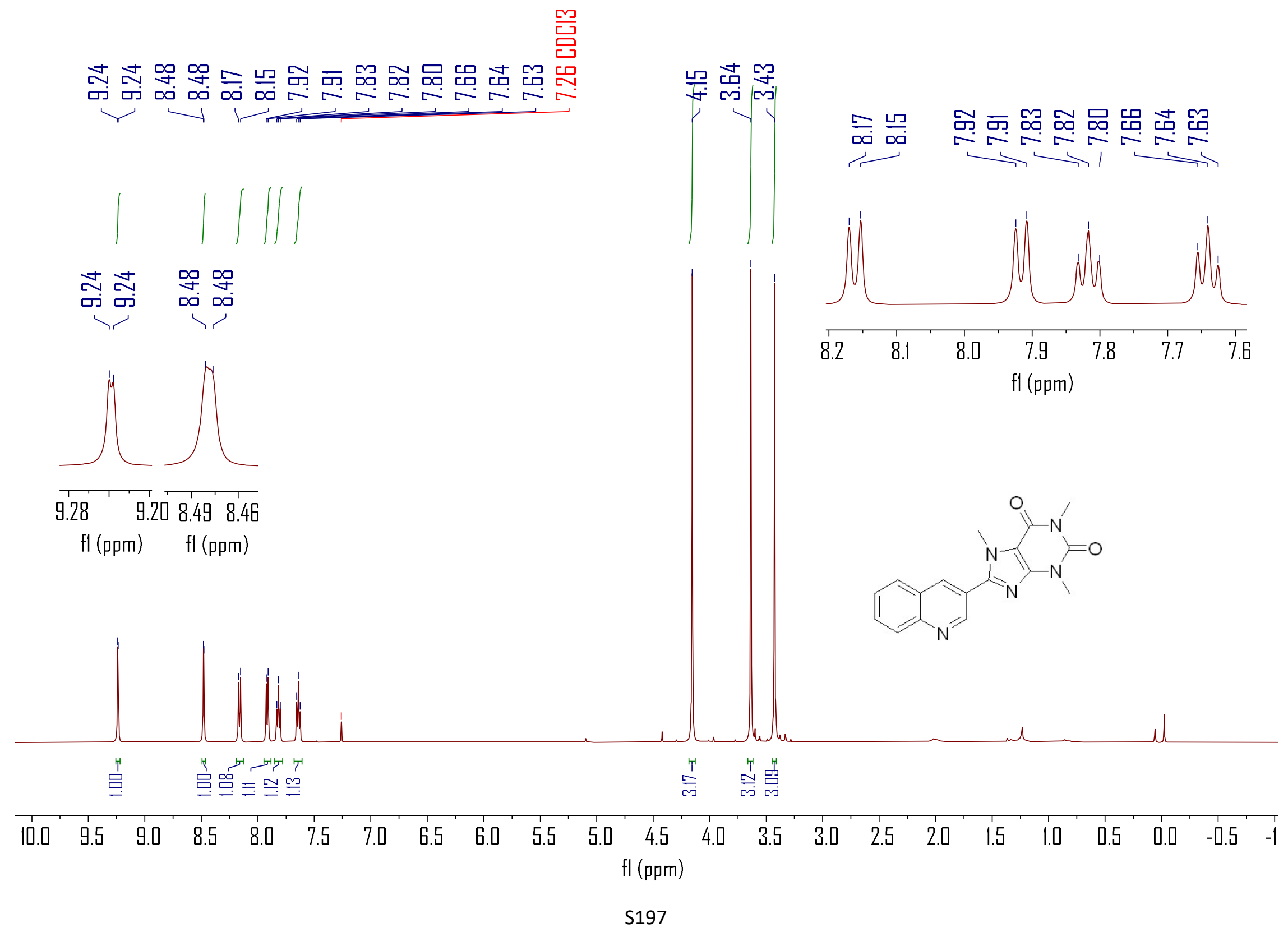




\section{${ }^{13} \mathrm{C}\left\{{ }^{1} \mathrm{H}\right\}$ NMR spectrum of compound $3 \mathrm{az}\left(126 \mathrm{MHz}, \mathrm{CDCl}_{3}\right)$}

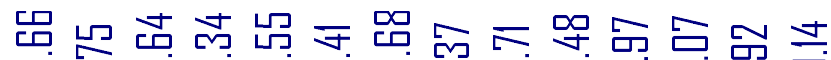

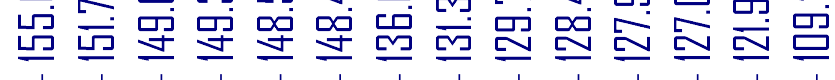

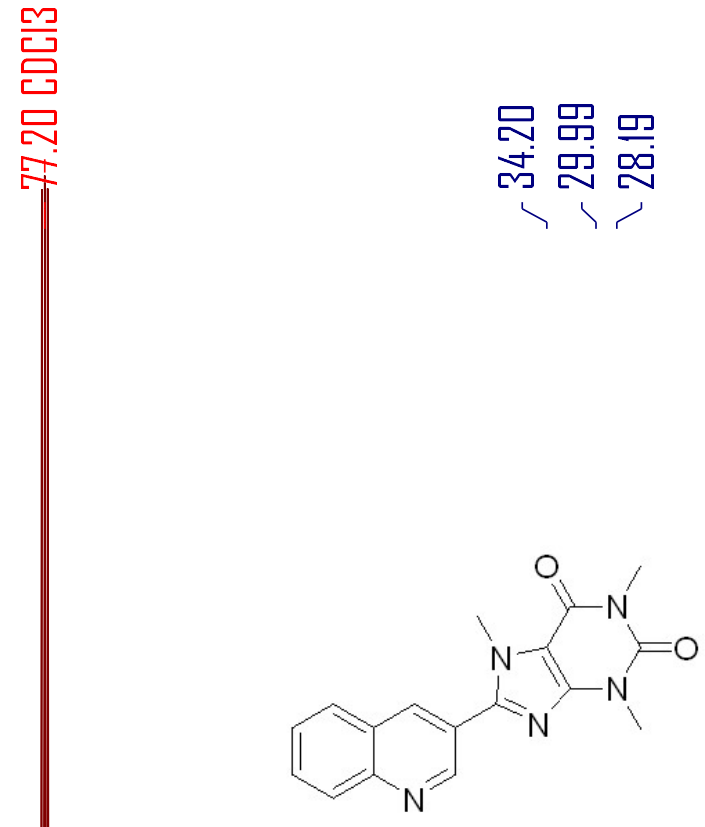

$\begin{array}{lllllllllllll}150.0 & 149.8 & 149.6 & 149.4 & 149.2 & 149.0 & 148.8 & 148.6 & 148.4 & 148.2 & 148.0\end{array}$

fl (ppm)

㟧
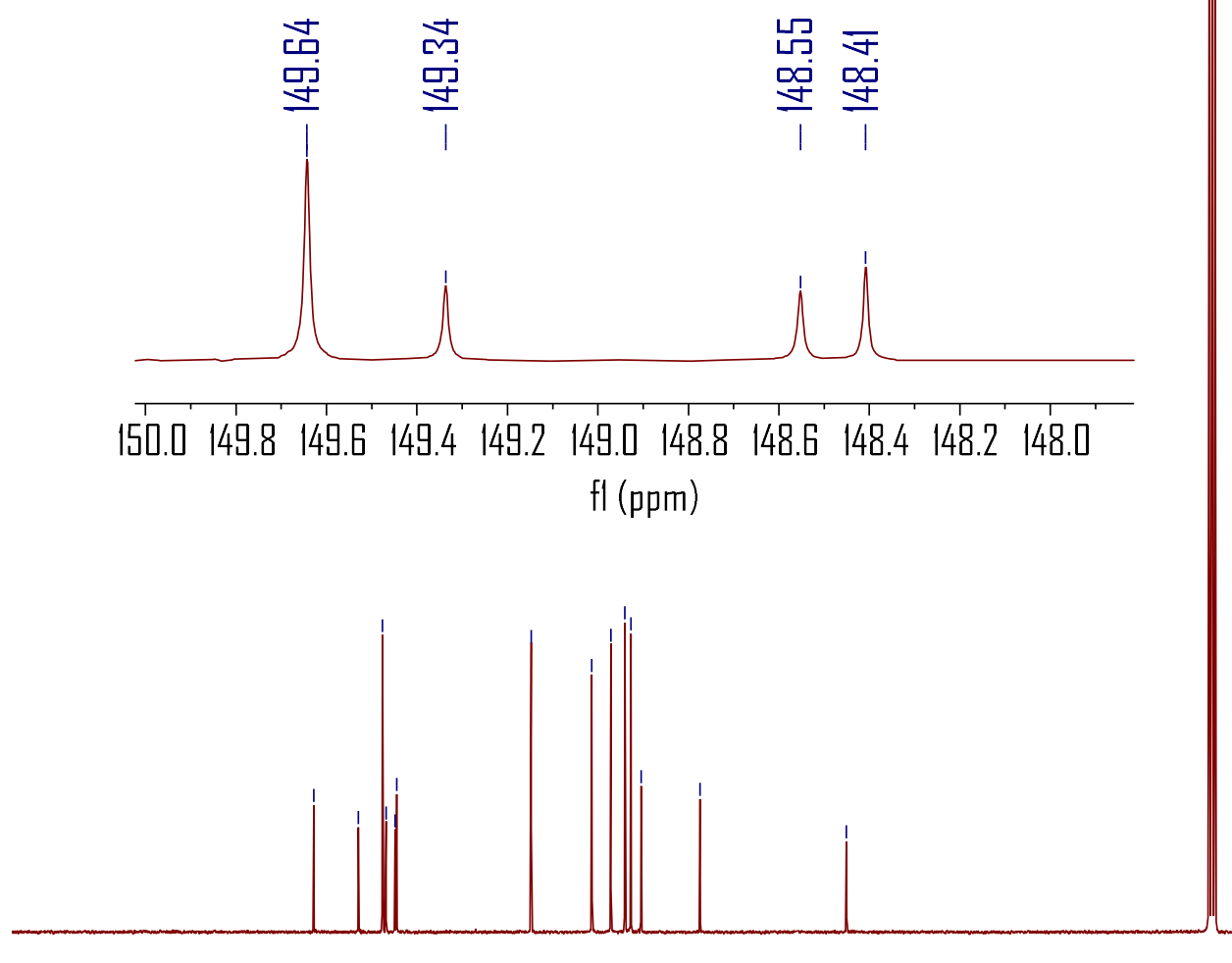

180

$170 \quad 160$

$150 \quad 140 \quad 130$

$120 \quad 110$

100

90 80

$70 \quad 60$

$\mathrm{fl}(\mathrm{ppm})$ 
${ }^{1} \mathrm{H}$ NMR spectrum of compound $3 \mathrm{aaa}\left(500 \mathrm{MHz}, \mathrm{CDCl}_{3}\right)$

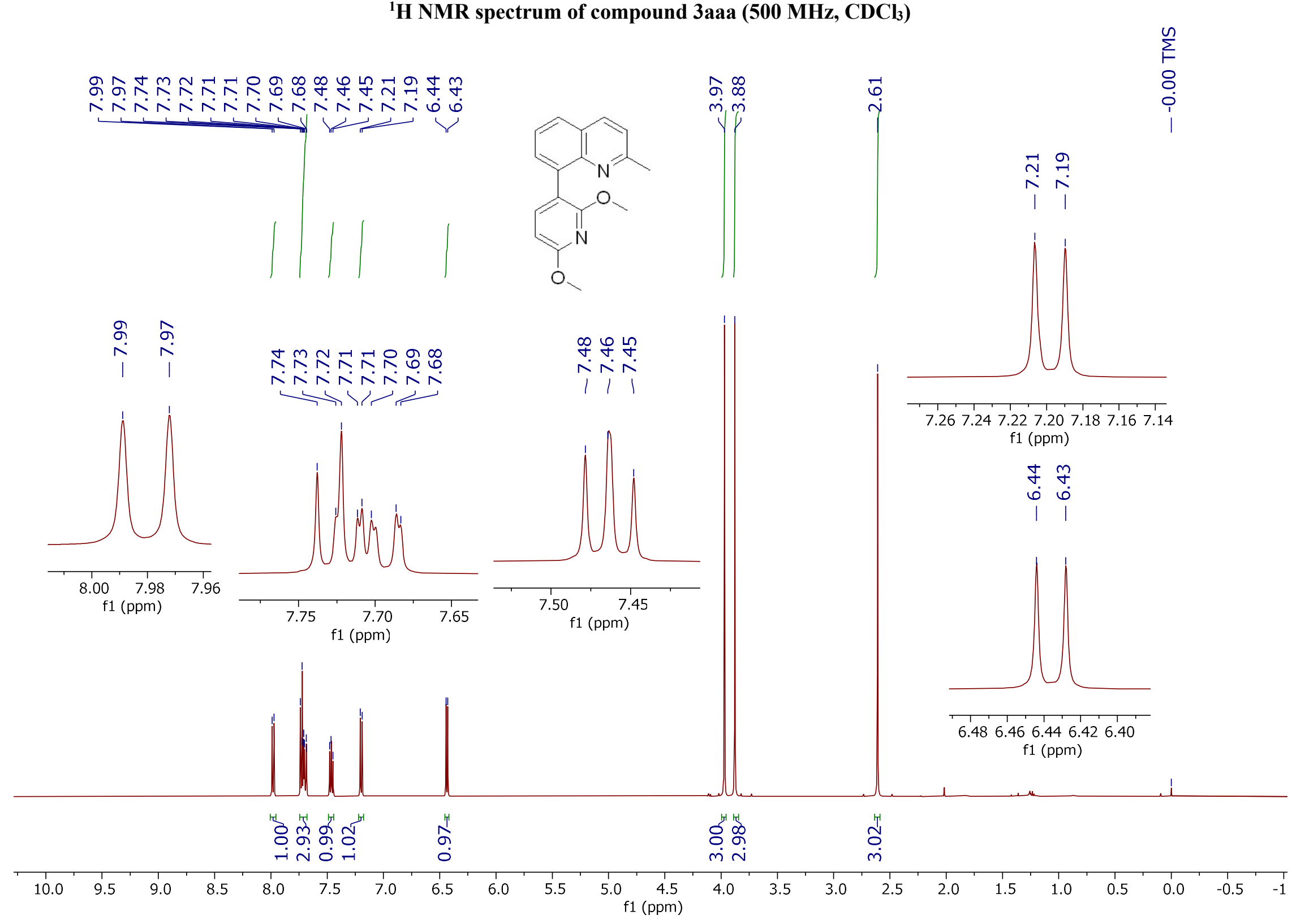

S199 


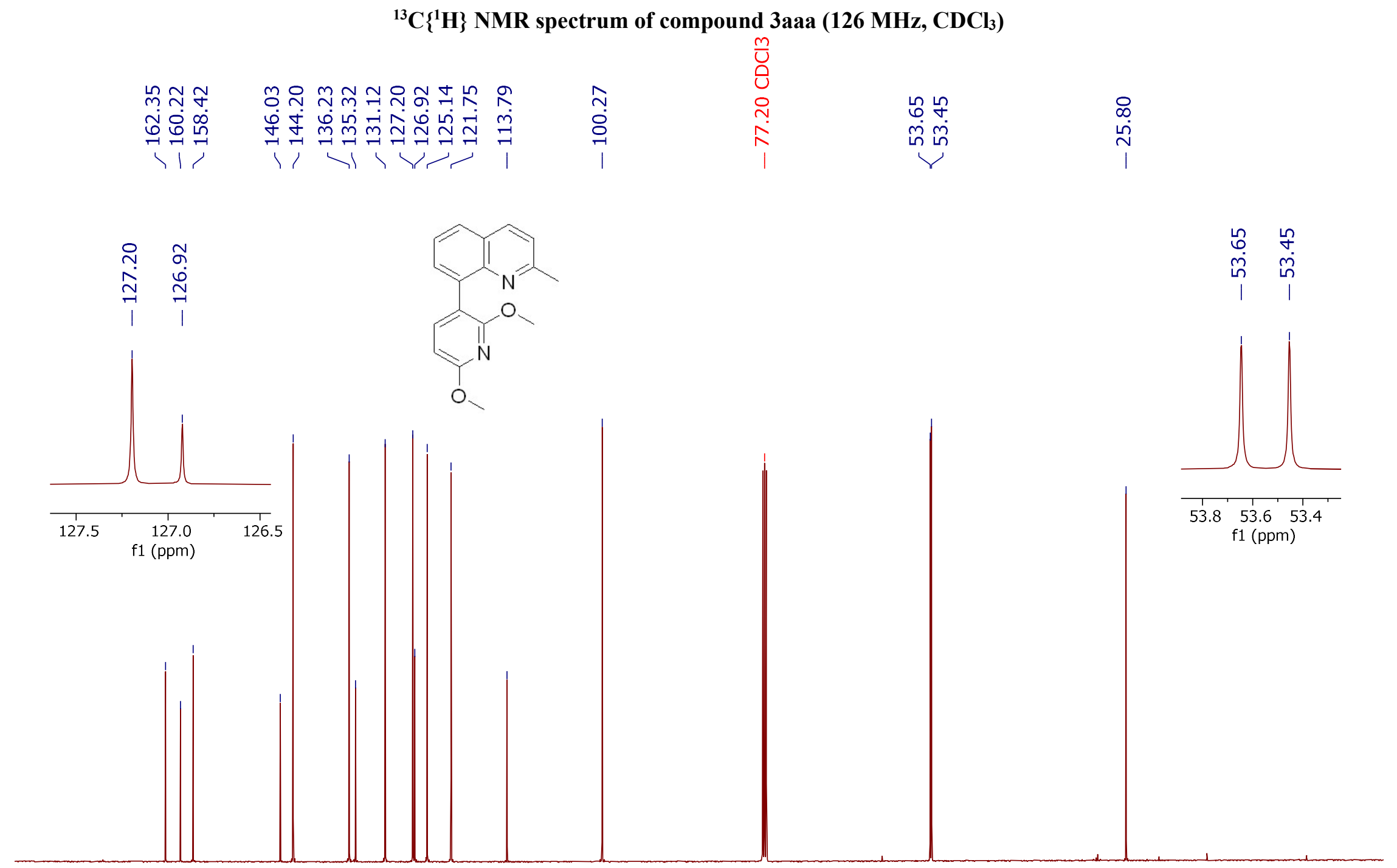

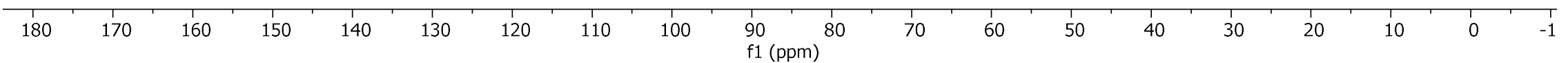




\section{${ }^{1} \mathrm{H}$ NMR spectrum of compound 3aab (500 $\left.\mathrm{MHz}, \mathrm{CDCl}_{3}\right)$}

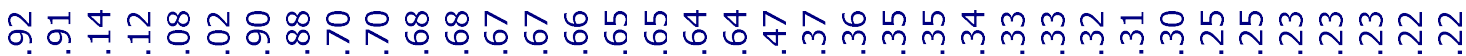

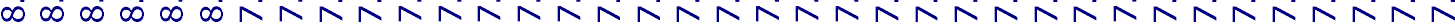

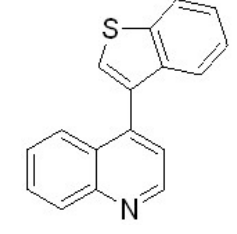

年

$1 \quad \infty \quad \infty \quad \infty$

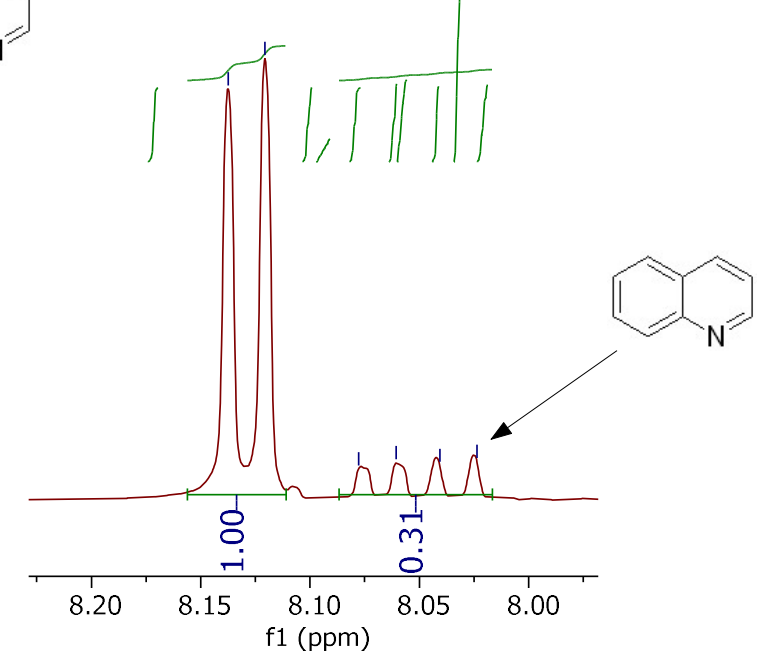

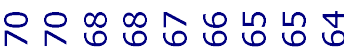
ivivivinj

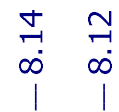

官 $\stackrel{\infty}{\infty}$

సे ने
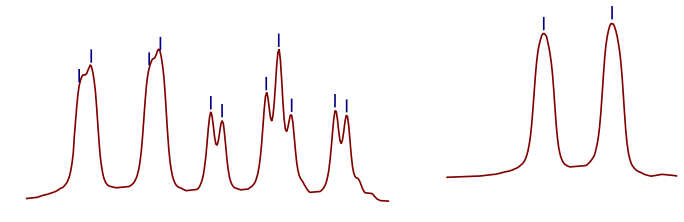

$\begin{array}{llll}7.70 & 7.68 & 7.66 & 7.64\end{array}$ \begin{tabular}{lll}
\hline 8.16 & 8.14 & 8.12
\end{tabular} $\mathrm{f} 1(\mathrm{ppm})$ f1 (ppm)

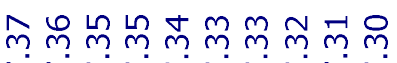

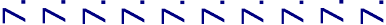
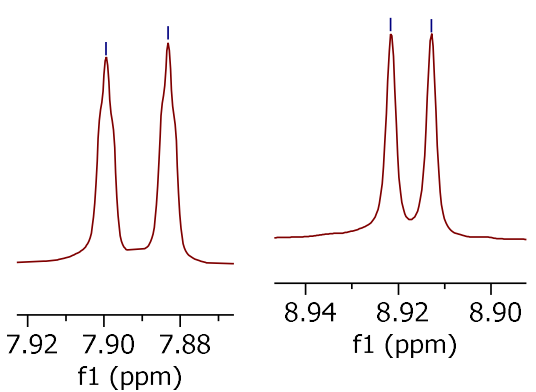

$\stackrel{\llcorner}{N} \stackrel{M}{N} \stackrel{m}{N} \underset{N}{N} N$ Niñกำ

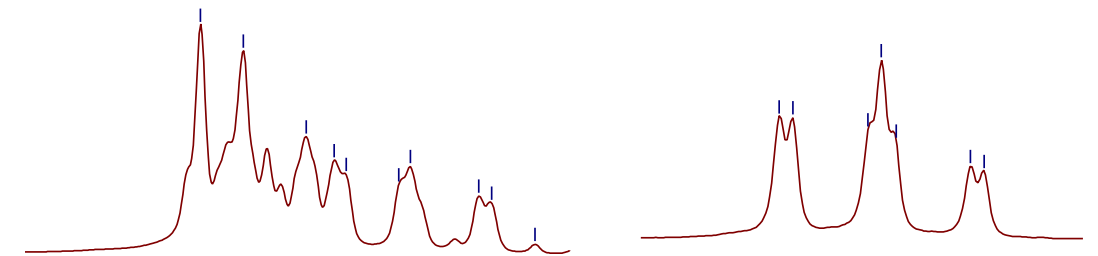

7.277 .267 .257 .247 .237 .227 .21

7.40

7.38 f1 (ppm)

f1 (ppm)

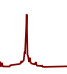

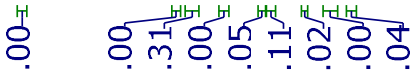
i

$10.0 \quad 9.5$ $\begin{array}{lllll}8.5 & 8.0 & 7.5 & 7.0 & 6.5\end{array}$ $6.5 \quad 6.0 \quad 5.5$ $\begin{array}{rr}5.0 & 4.5 \\ f 1(p p m)\end{array}$ \begin{tabular}{lllllllllll}
\hline .0 & 3.5 & 3.0 & 2.5 & 2.0 & 1.5 & 1.0 & 0.5 & 0.0 & -0.5 & -1.0
\end{tabular} 


\section{${ }^{13} \mathrm{C}\left\{{ }^{1} \mathrm{H}\right\}$ NMR spectrum of compound 3aab (126 $\left.\mathrm{MHz}, \mathrm{CDCl}_{3}\right)$}

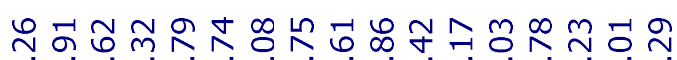

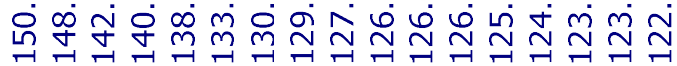

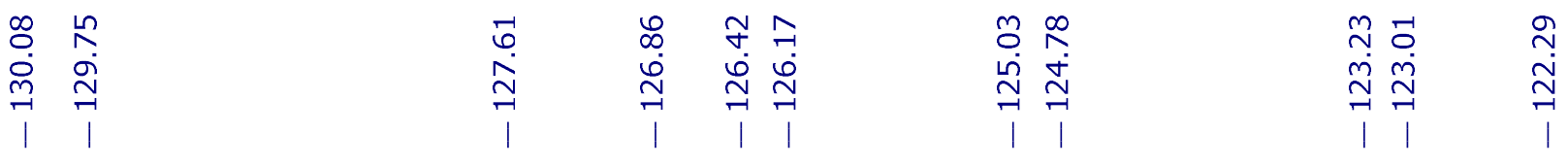
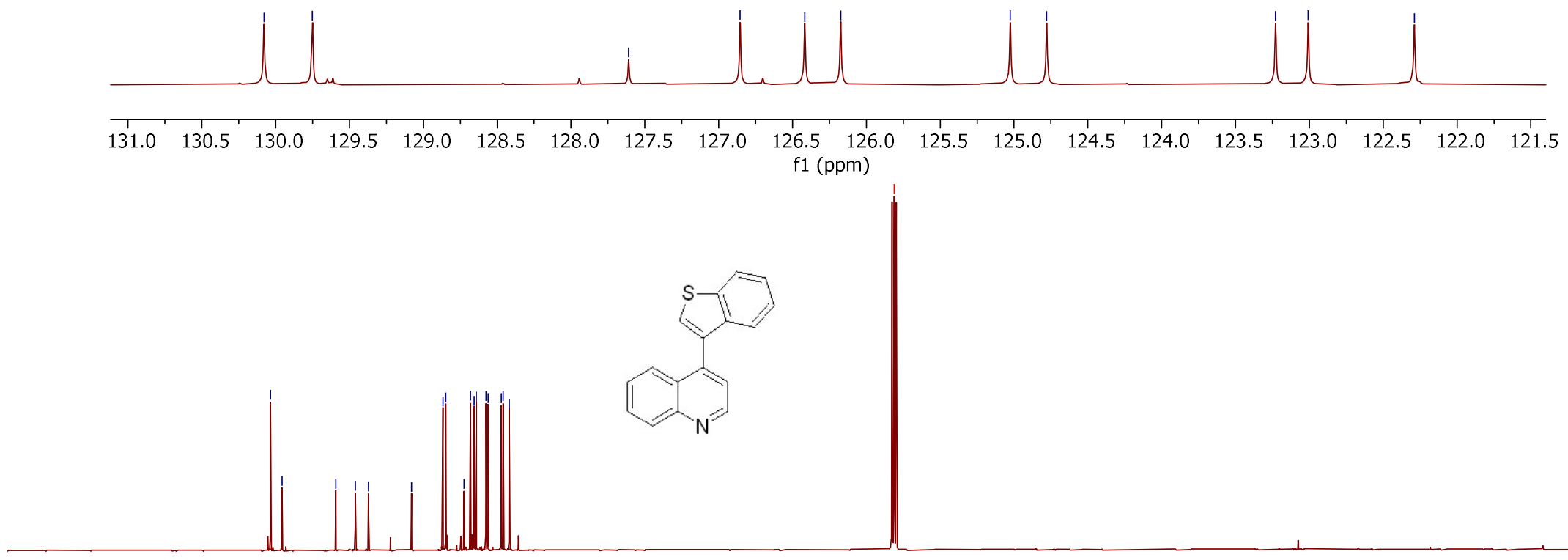

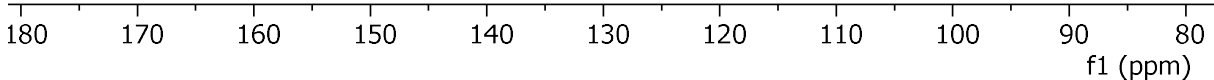


${ }^{1} \mathrm{H}$ NMR spectrum of compound 3aac (500 $\left.\mathrm{MHz}, \mathrm{CDCl}_{3}\right)$

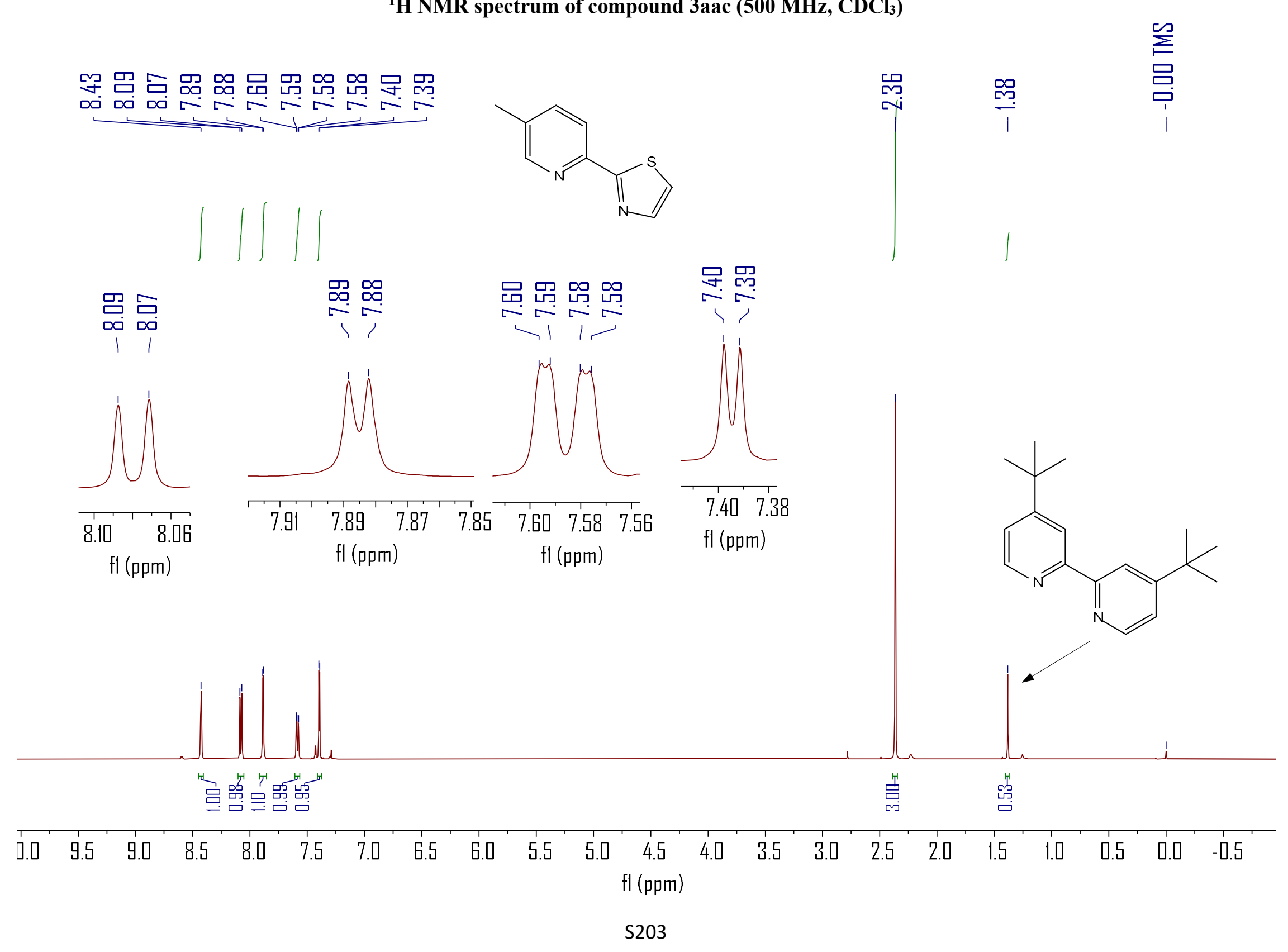




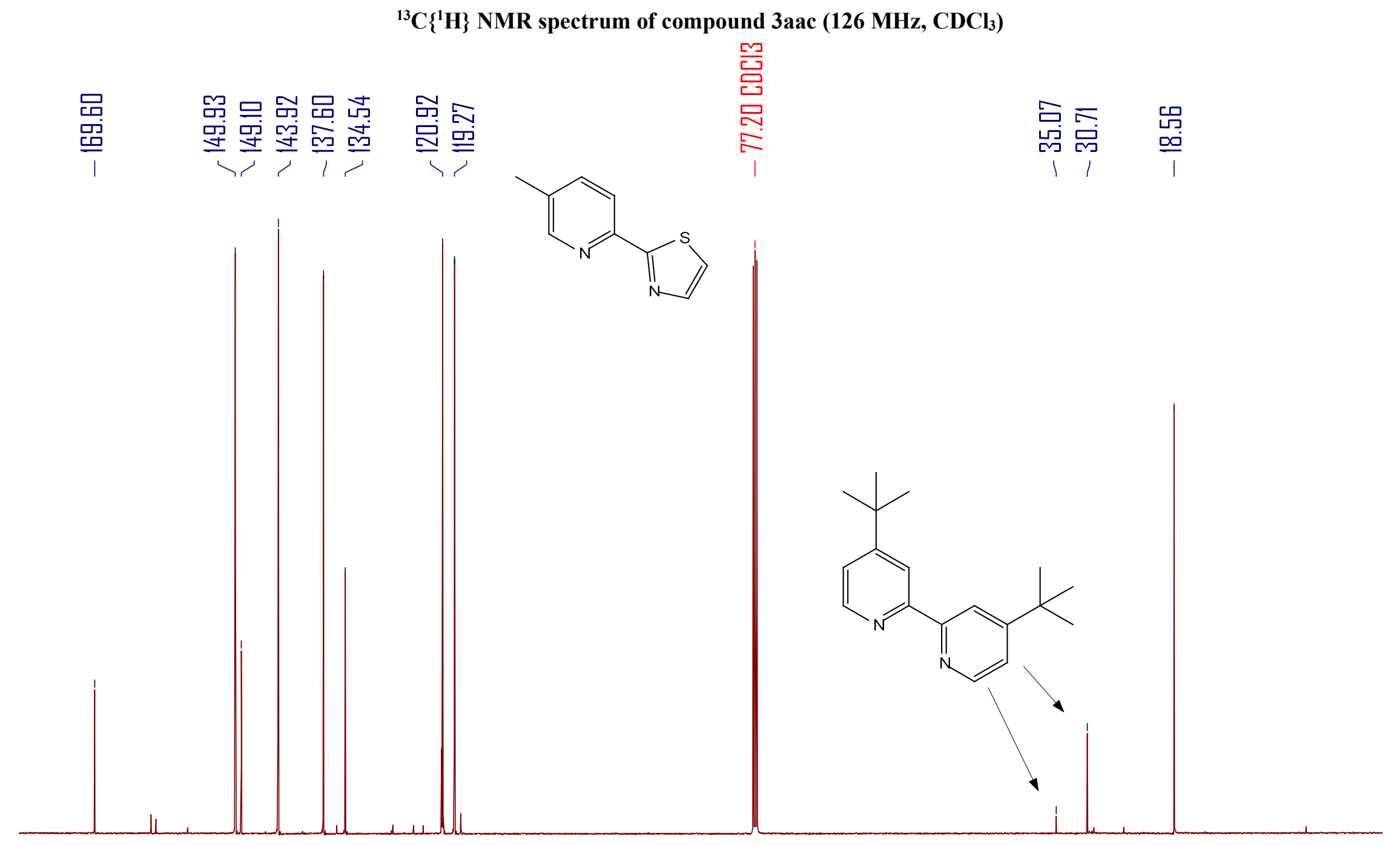

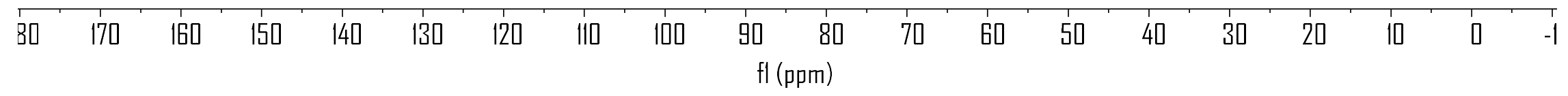




\section{${ }^{1} \mathrm{H}$ NMR spectrum of compound 3aad (500 $\left.\mathrm{MHz}, \mathrm{CDCl}_{3}\right)$}
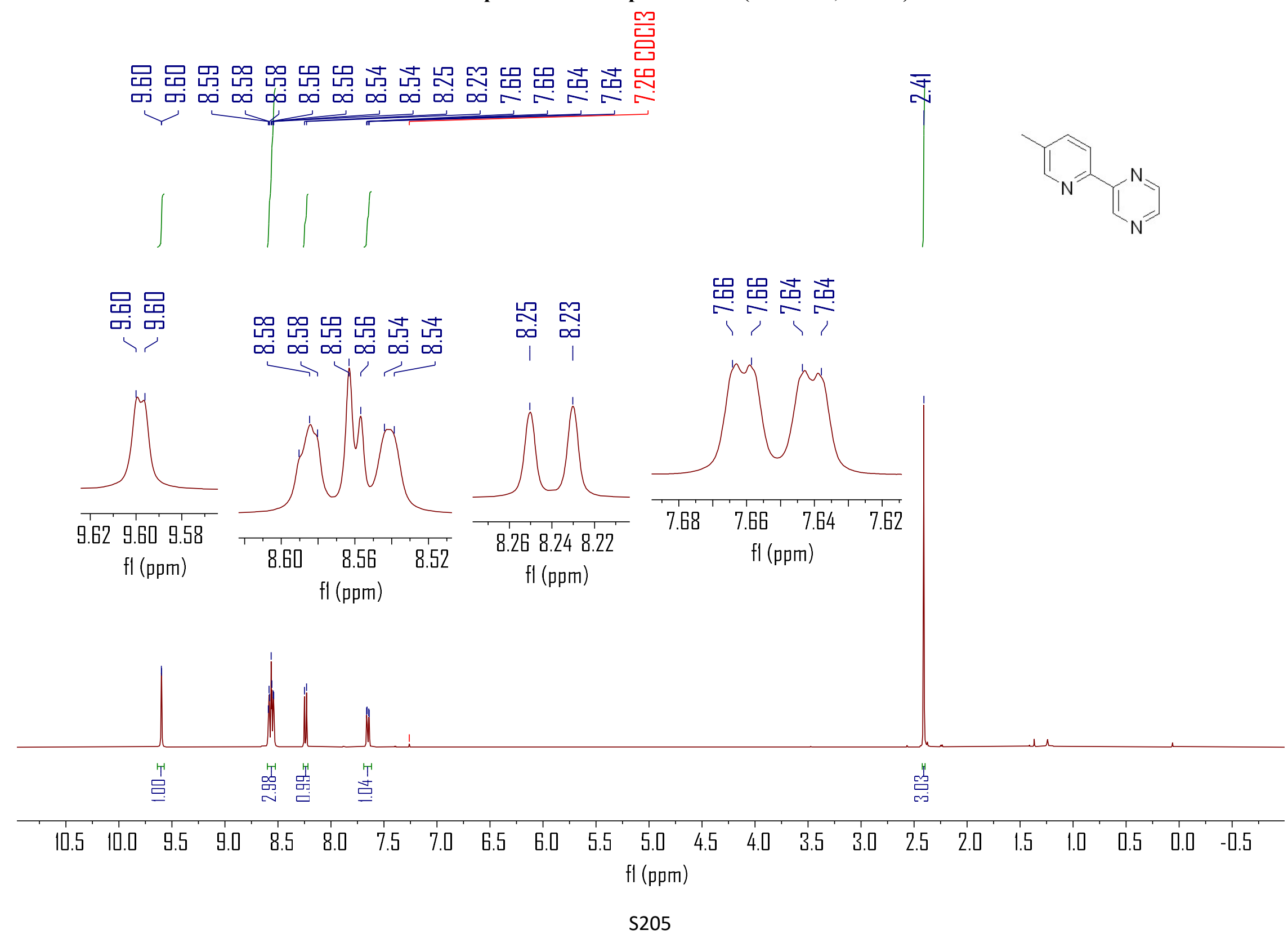
${ }^{13} \mathrm{C}\left\{{ }^{1} \mathrm{H}\right\}$ NMR spectrum of compound 3aad (126 $\left.\mathrm{MHz}, \mathrm{CDCl}_{3}\right)$

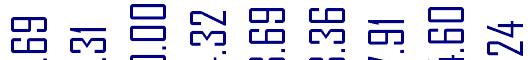

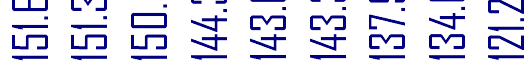

$\longrightarrow<$
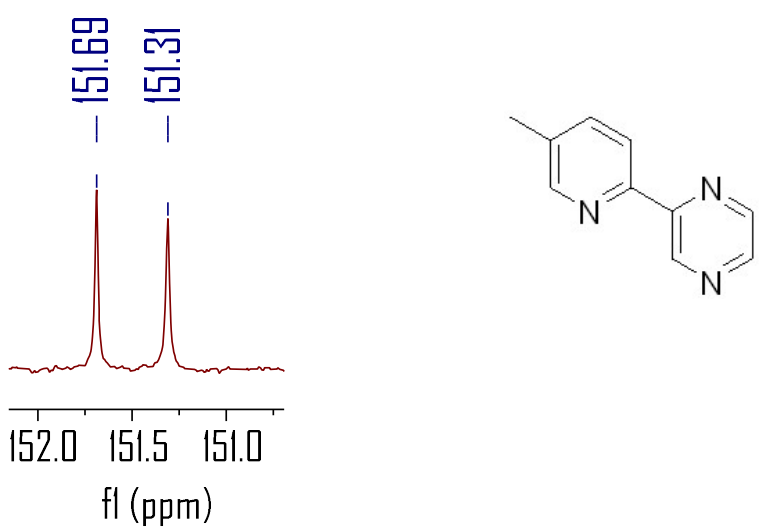

fl (ppm)
믈

또ำ

1

144.6144 .4144 .2144 .0143 .8143 .6143 .4143 .2143 .0

$\mathrm{fl}(\mathrm{ppm})$
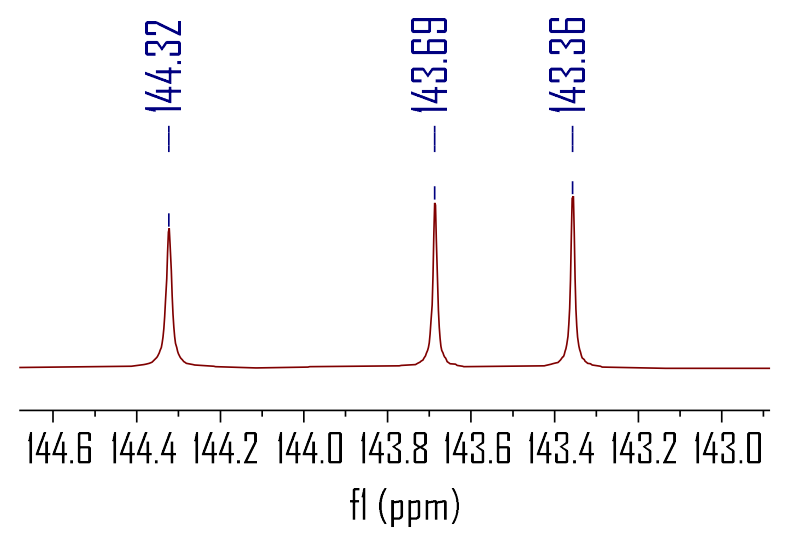

$180 \quad 170$

160

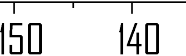

$130 \quad 120$

110

100

fl (ppm)

S206 
${ }^{1} \mathrm{H}$ NMR spectrum of compound 3aae (400 $\left.\mathrm{MHz}, \mathrm{CDCl}_{3}\right)$

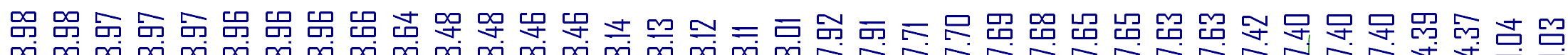

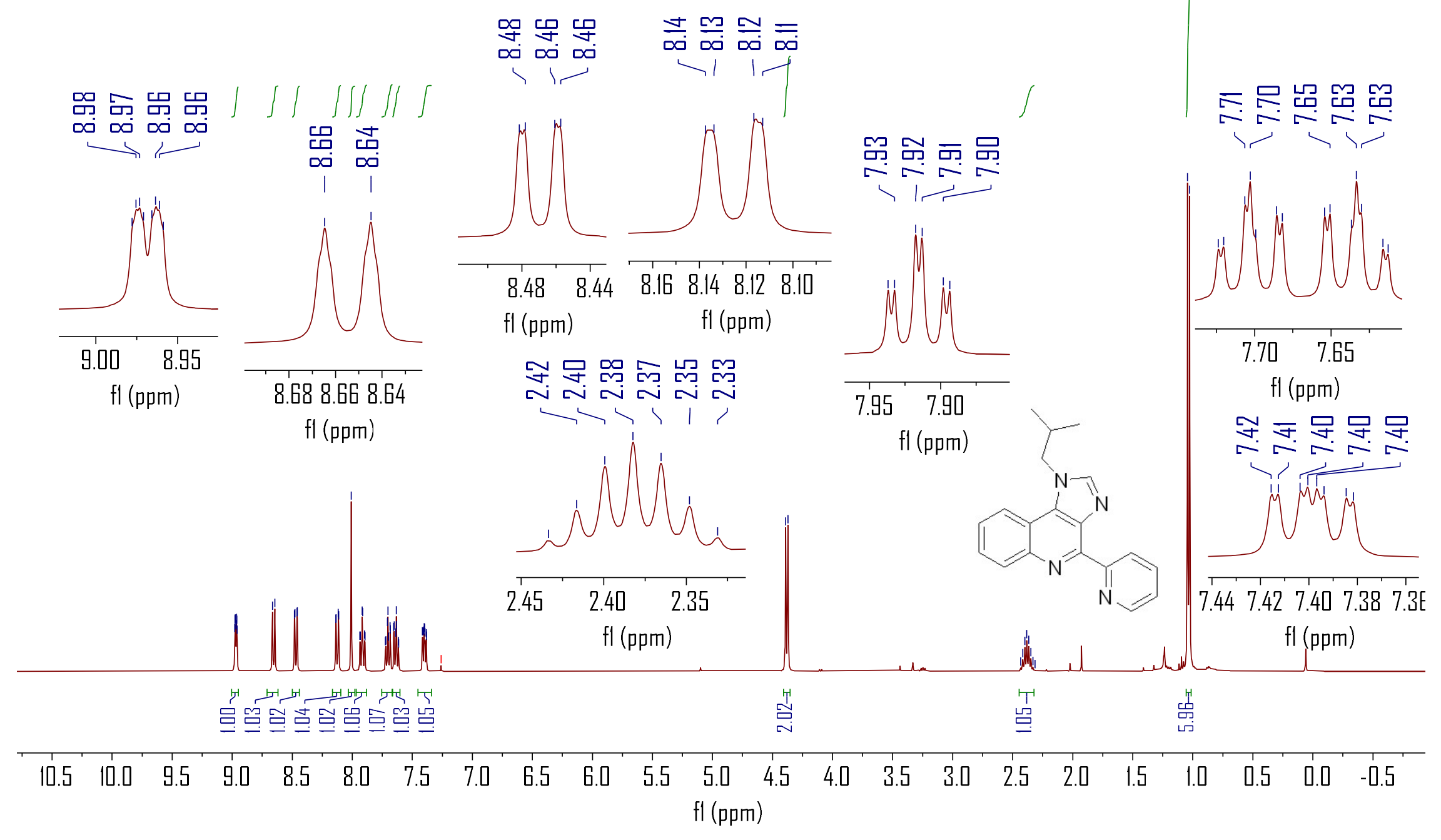




\section{${ }^{13} \mathrm{C}\left\{{ }^{1} \mathrm{H}\right\}$ NMR spectrum of compound 3aae $\left(101 \mathrm{MHz}, \mathrm{CDCl}_{3}\right)$}

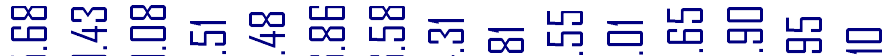

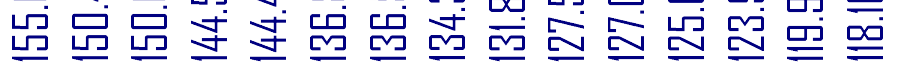

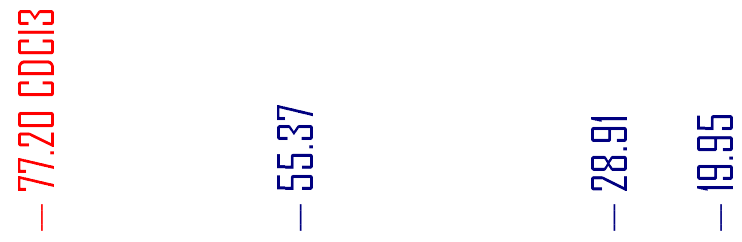

品
肙

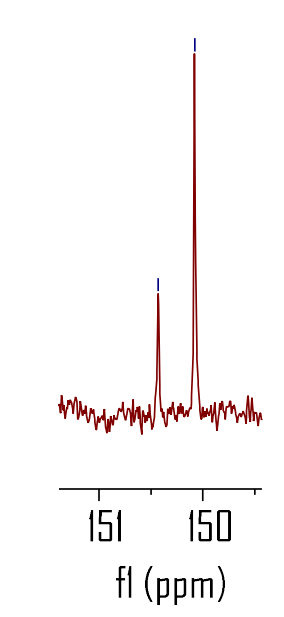

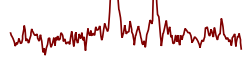

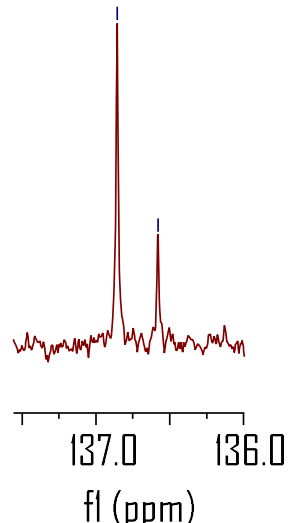

fl (ppm)

$\mathrm{fl}(\mathrm{ppm})$

fl (ppm)
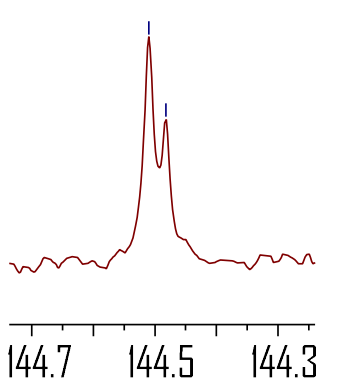

$170 \quad 160$

$150 \quad 140$

130

120

10

100

fl (ppm)

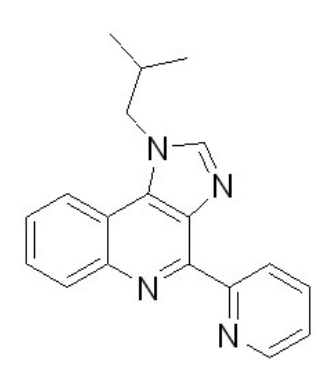


${ }^{1} \mathrm{H}$ NMR spectrum of compound $3 \mathrm{aaf}\left(500 \mathrm{MHz}, \mathrm{CDCl}_{3}\right)$

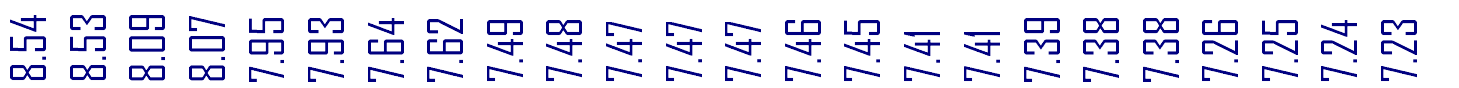

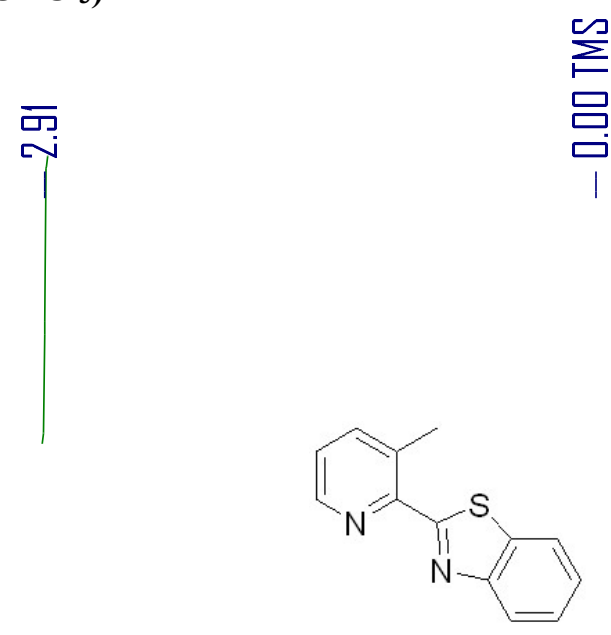

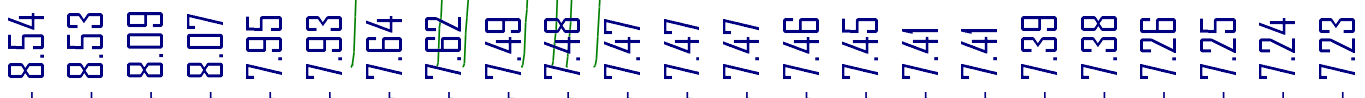
$\stackrel{1}{\longleftarrow}$
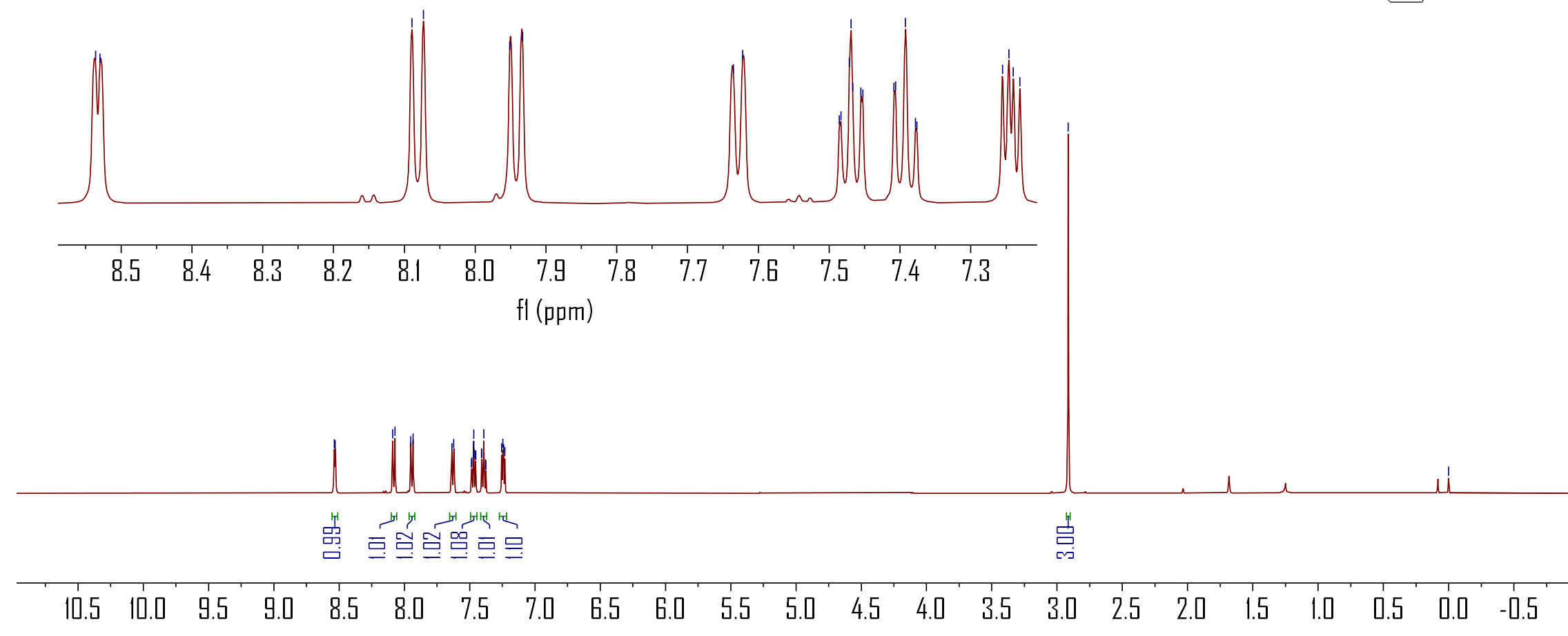

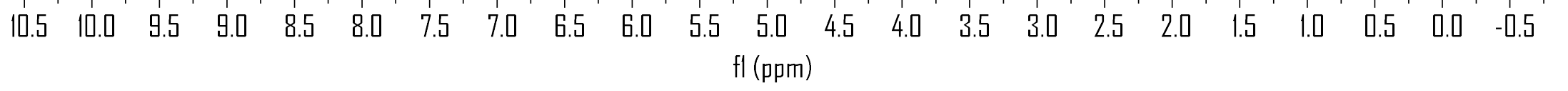


${ }^{13} \mathrm{C}\left\{{ }^{1} \mathrm{H}\right\}$ NMR spectrum of compound 3aaf (126 $\left.\mathrm{MHz}, \mathrm{CDCl}_{3}\right)$

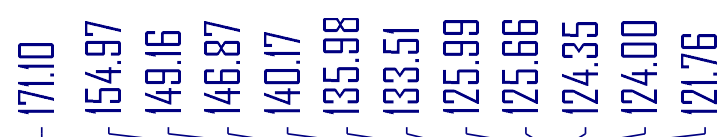

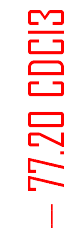

ำ

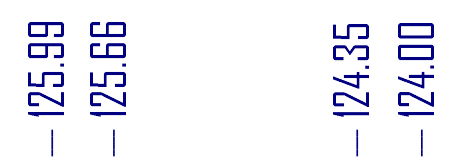

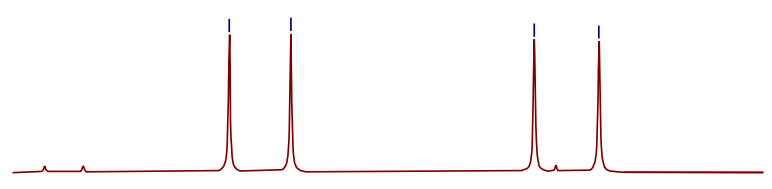

$\begin{array}{lllllllll}127.0 & 126.5 & 126.0 & 125.5 & 125.0 & 124.5 & 124.0 & 123.5\end{array}$

$\mathrm{fl}(\mathrm{ppm})$
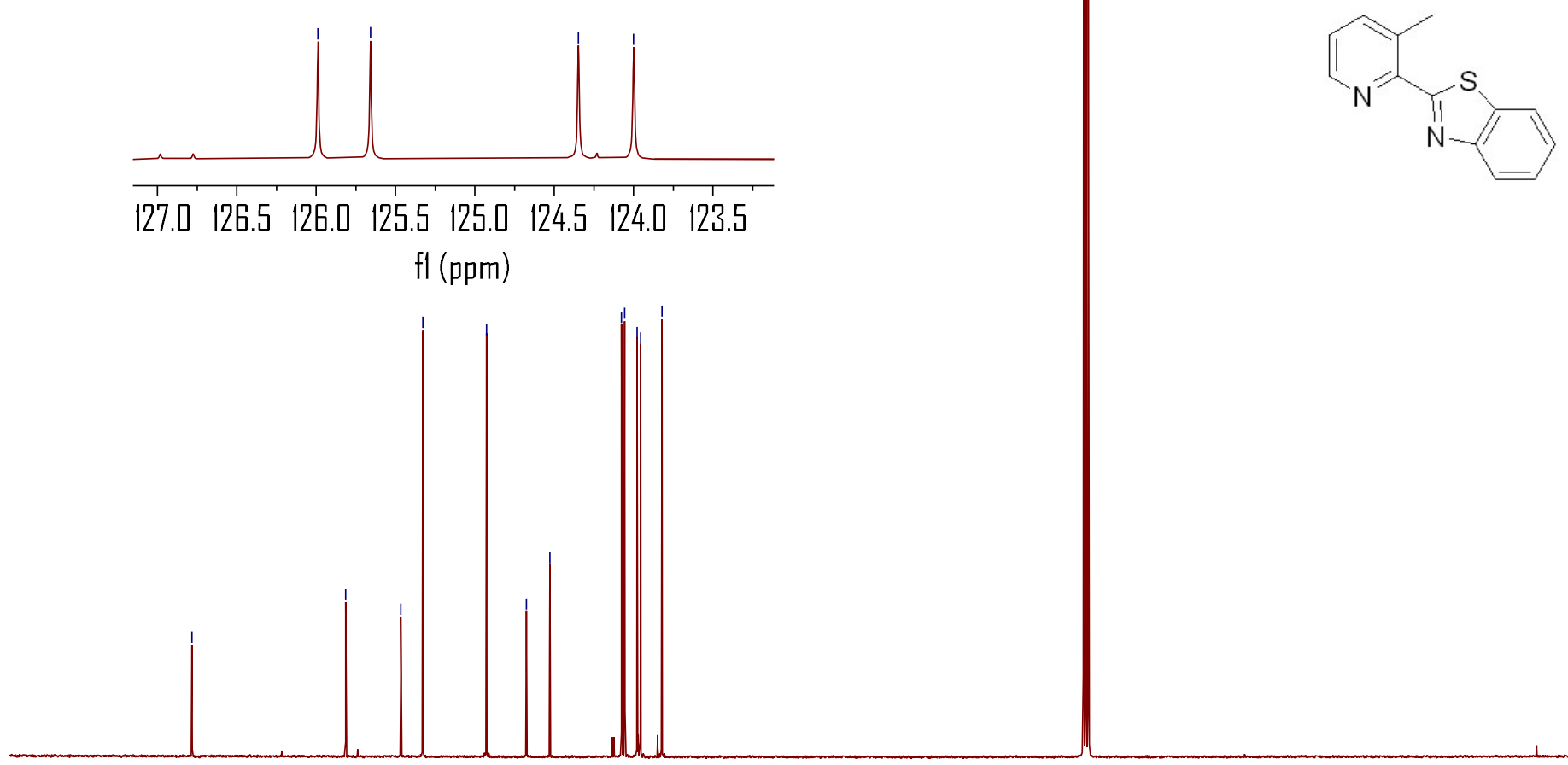

90

$180 \quad 170 \quad 160$

$160 \quad 150$

140

130

$120 \quad 110$

III $\mathrm{fl}$

$\mathrm{fl}$ (ppm)

S210 


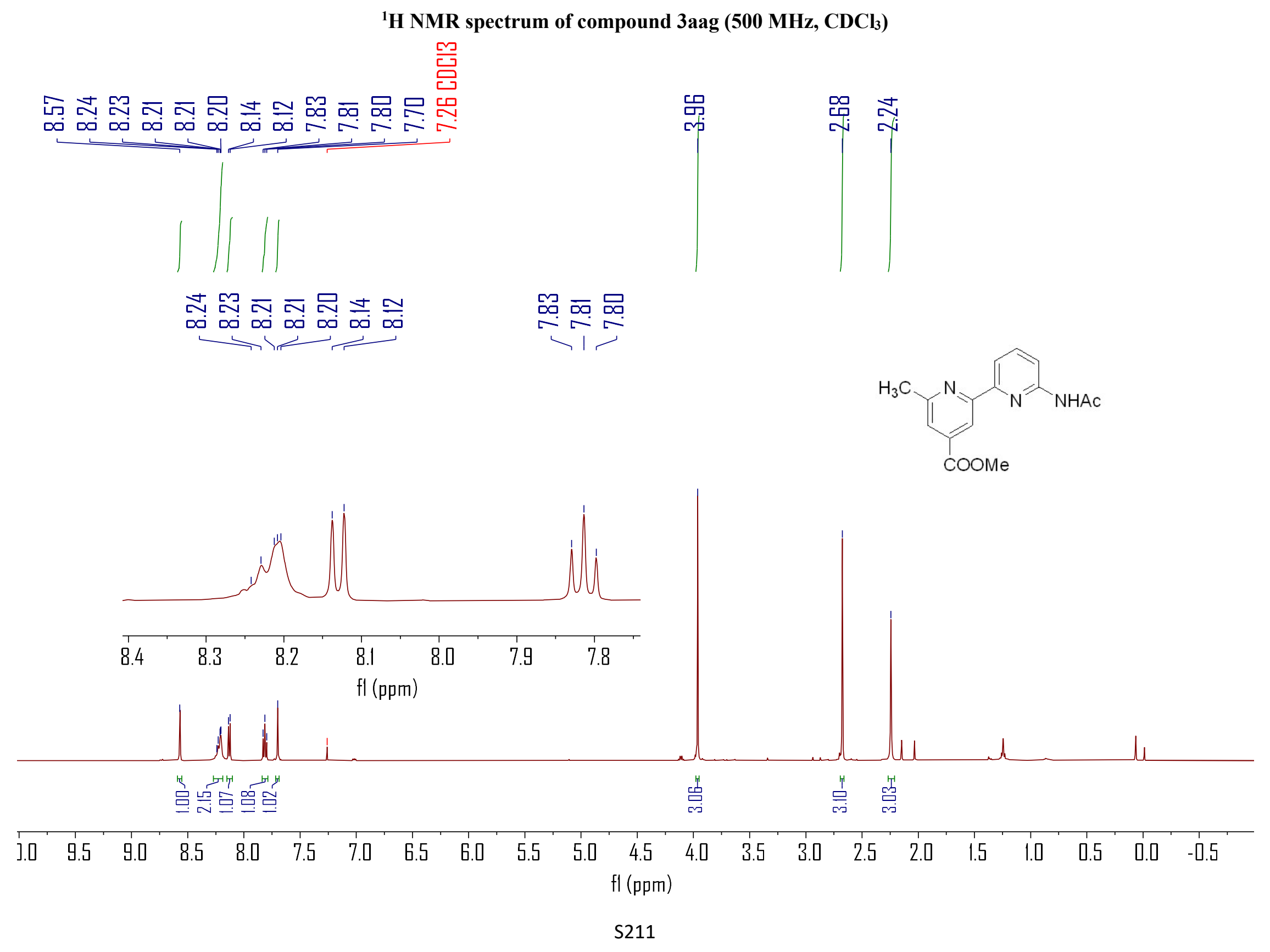


${ }^{13} \mathrm{C}\left\{{ }^{1} \mathrm{H}\right\}$ NMR spectrum of compound 3aag (126 $\left.\mathrm{MHz}, \mathrm{CDCl}_{3}\right)$

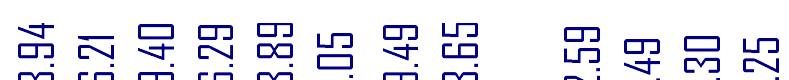

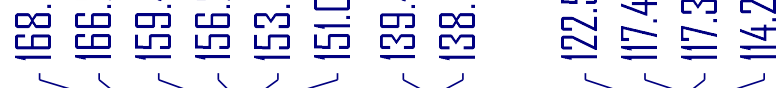

$$
\stackrel{\text { 导 }}{\stackrel{\text { 品 }}{\equiv}}
$$

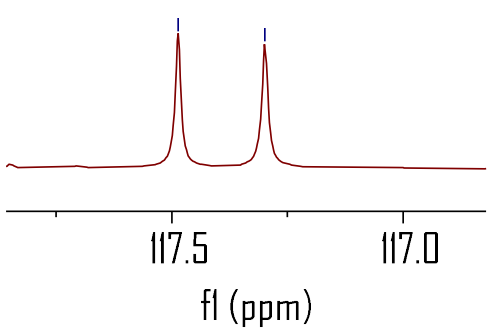

몰

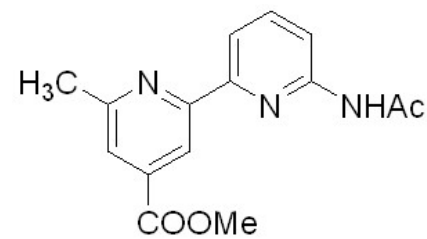

品
哭哭

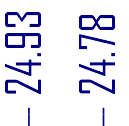

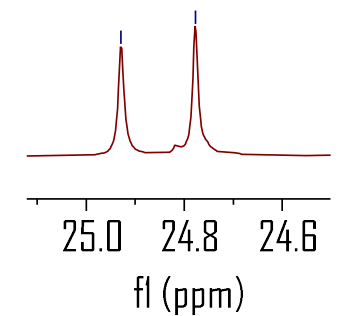

$180 \quad 17$

160

150

140

$30 \quad 120 \quad 110$

IDO

fl (ppm)

60

504

S212 


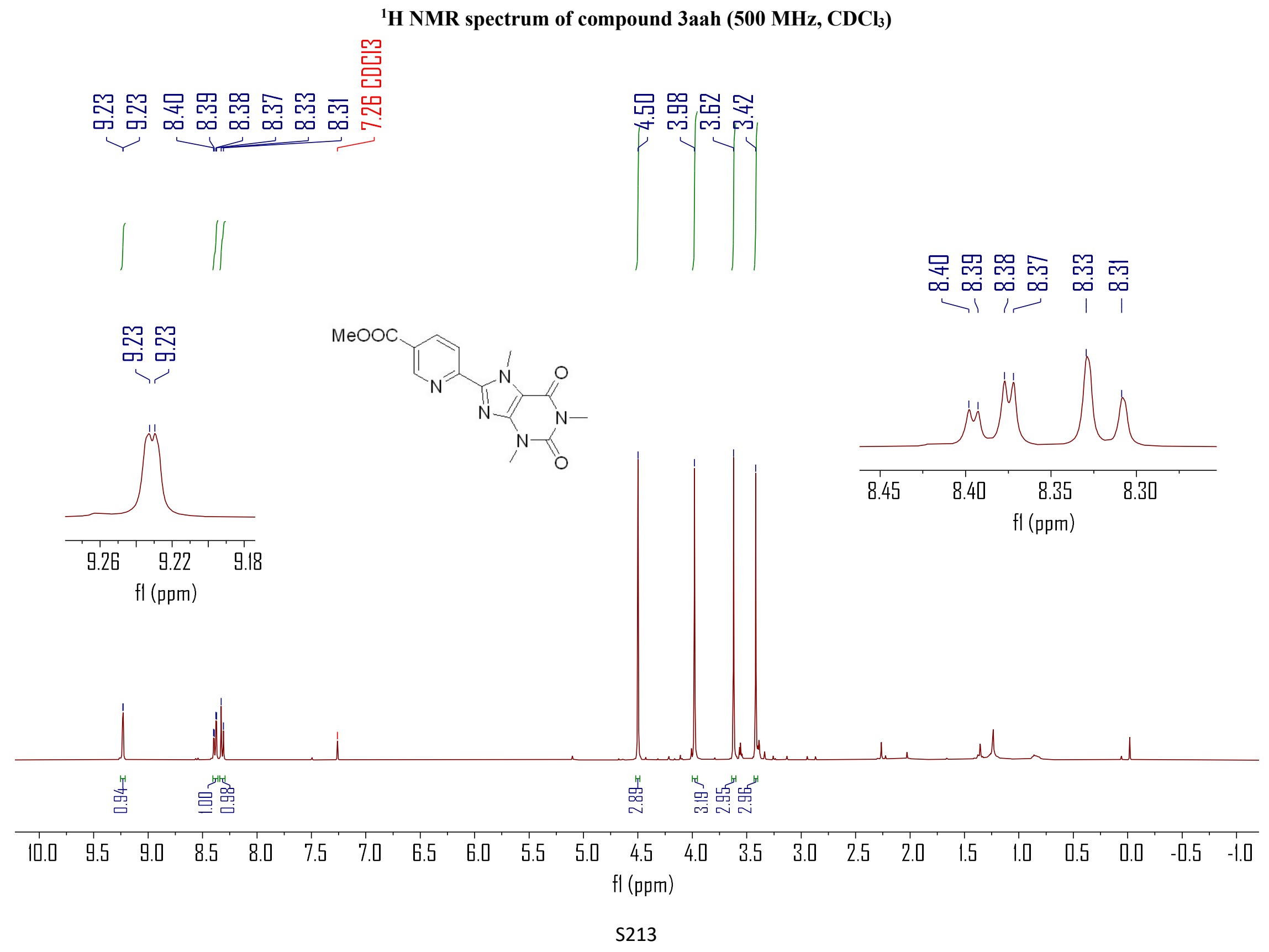


${ }^{13} \mathrm{C}\left\{{ }^{1} \mathrm{H}\right\}$ NMR spectrum of compound 3aah (126 $\left.\mathrm{MHz}, \mathrm{CDCl}_{3}\right)$

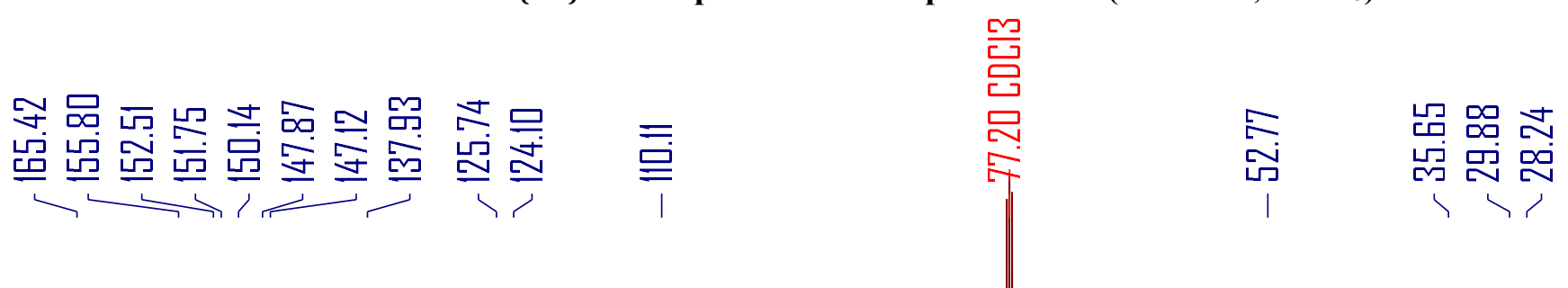

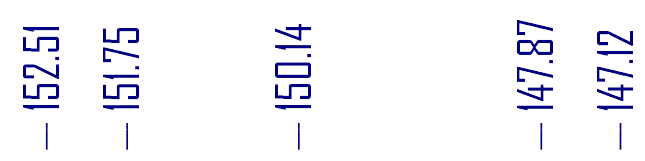
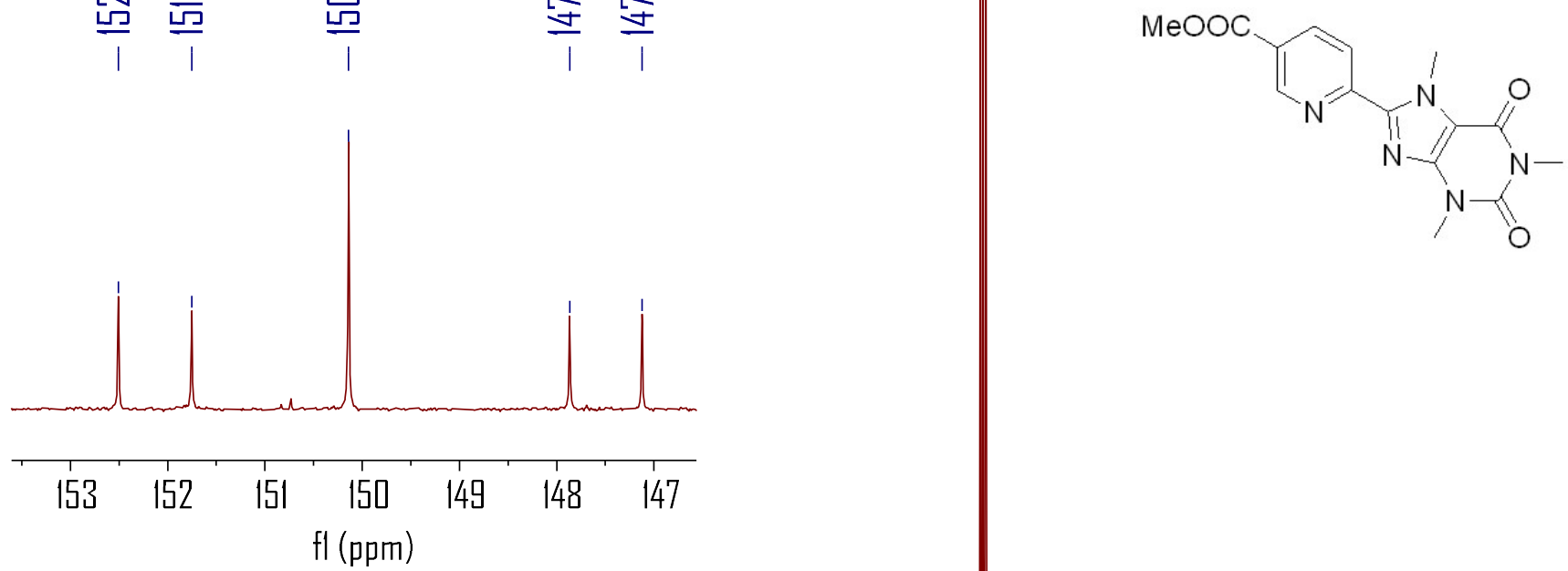

$180 \quad 17$

160

14

110

100

$\mathrm{fl}(\mathrm{ppm})$

S214 


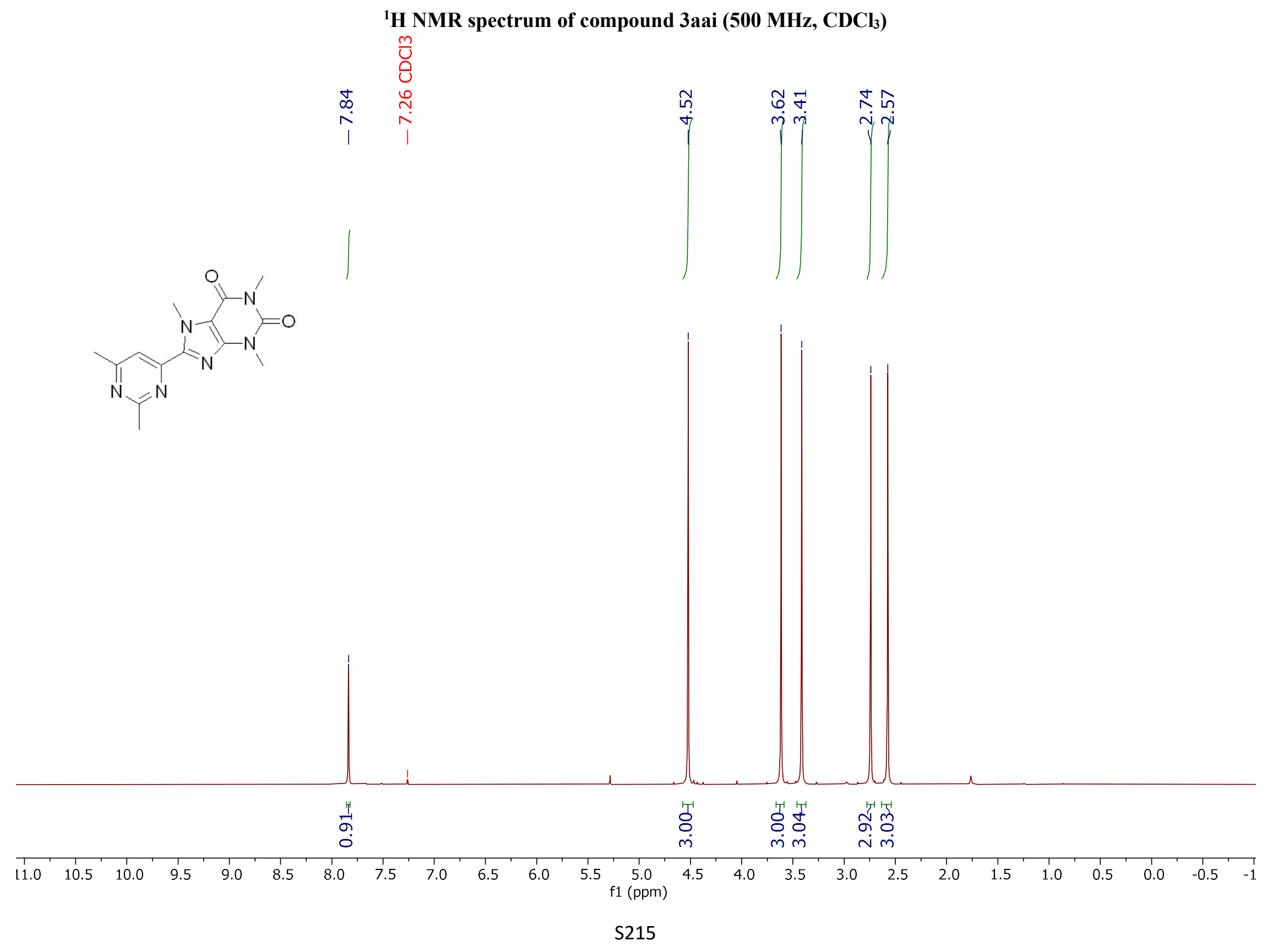




\section{${ }^{13} \mathrm{C}\left\{{ }^{1} \mathrm{H}\right\}$ NMR spectrum of compound 3aai $\left(126 \mathrm{MHz}, \mathrm{CDCl}_{3}\right)$}

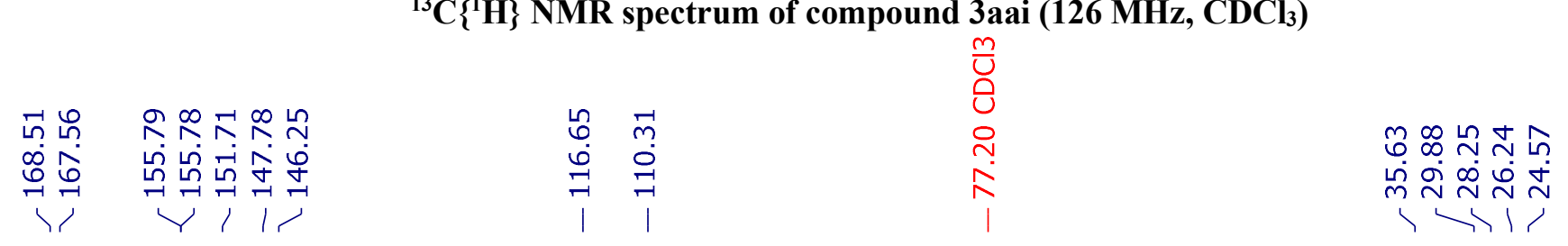

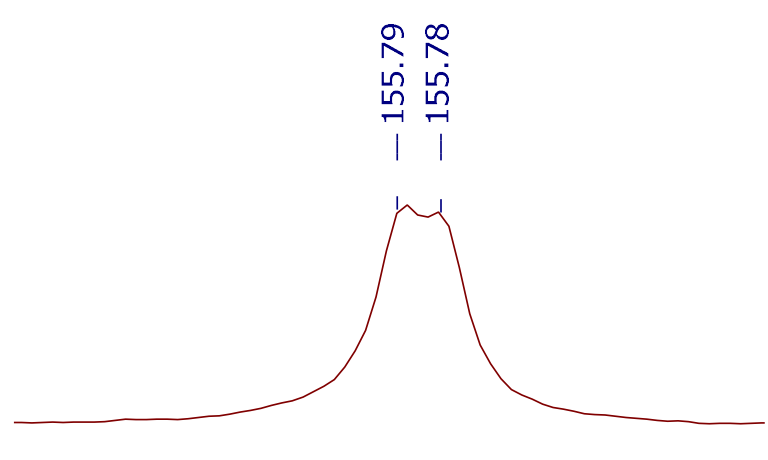

$\begin{array}{llllllll}155.86 & 155.84 & 155.82 & 155.80 & 155.78 & 155.76 & 155.74 & 155.72\end{array}$ f1 (ppm) 
${ }^{1} \mathrm{H}$ NMR spectrum of compound 3aaj (500 $\left.\mathrm{MHz}, \mathrm{CDCl}_{3}\right)$

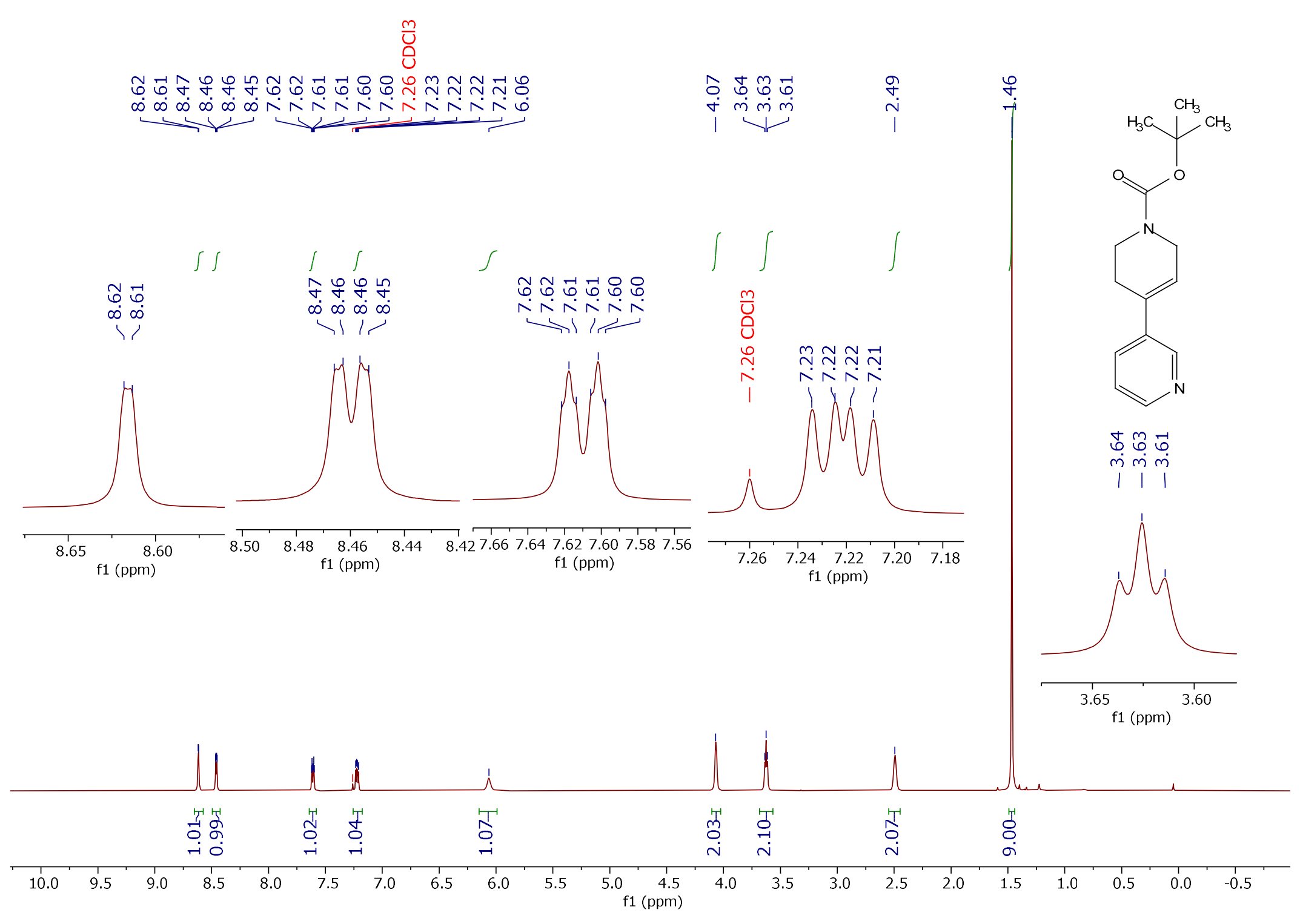




\section{${ }^{1} \mathrm{H}$ NMR spectrum of compound 3aak (500 $\left.\mathrm{MHz}, \mathrm{CDCl}_{3}\right)$}

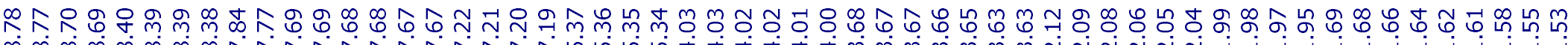

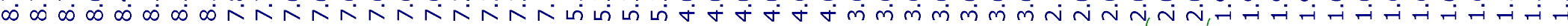

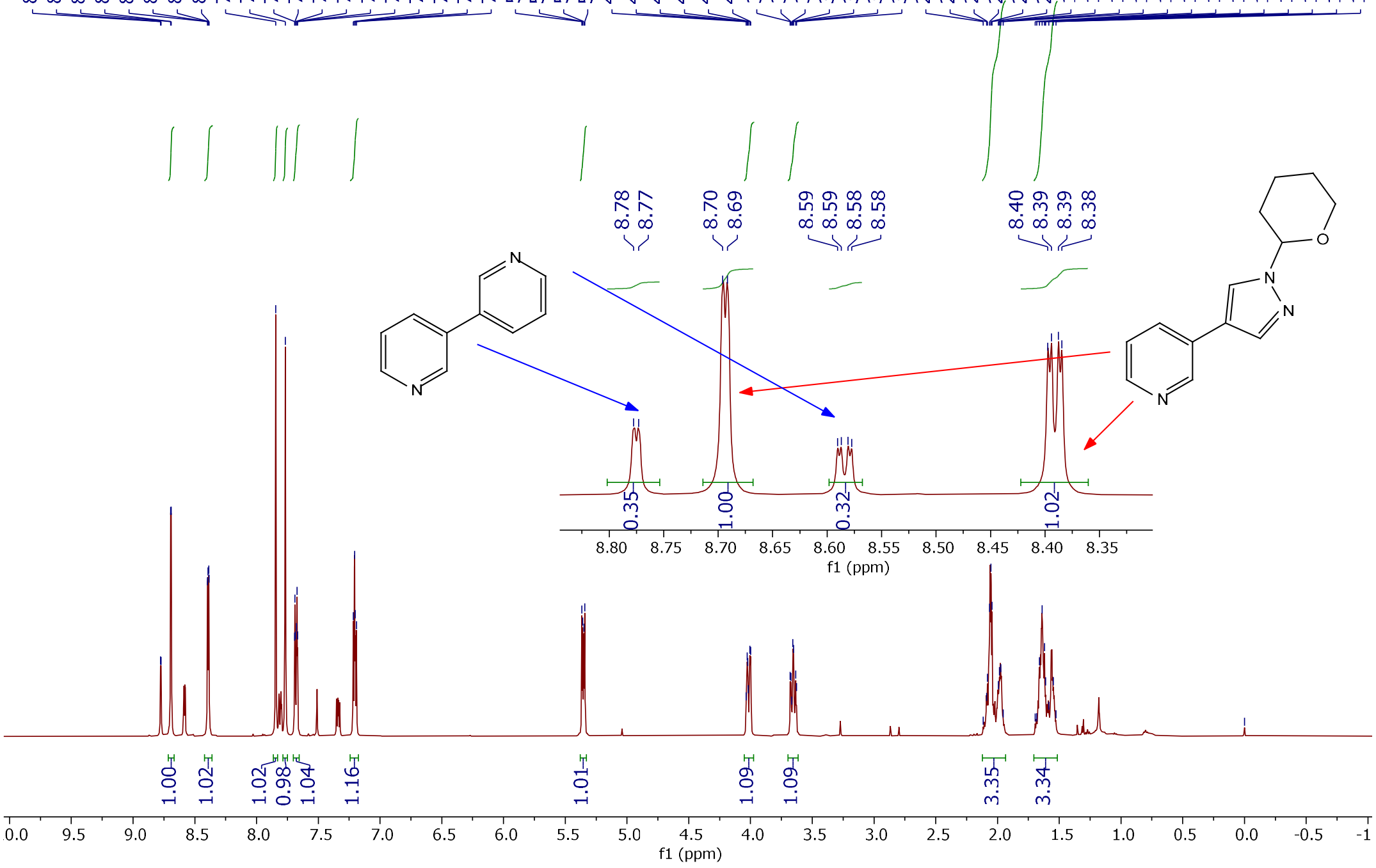


${ }^{13} \mathrm{C}\left\{{ }^{1} \mathrm{H}\right\}$ NMR spectrum of compound 3aak (126 $\left.\mathrm{MHz}, \mathrm{CDCl}_{3}\right)$

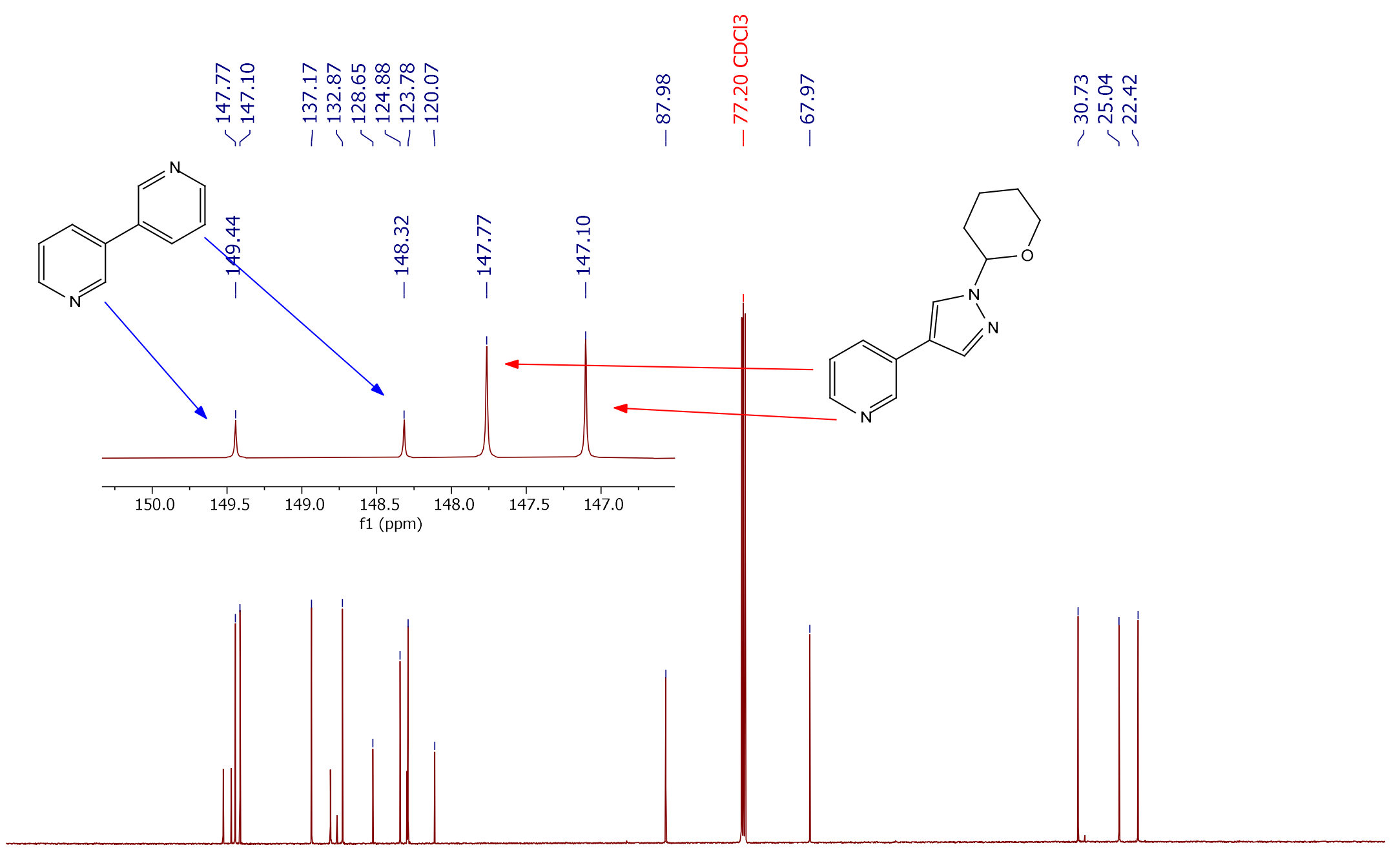

\begin{tabular}{rllllllllllllllllll}
1 & 1 \\
\hline 170 & 160 & 150 & 140 & 130 & 120 & 110 & 100 & 90 & 80 & 70 & 60 & 50 & 40 & 30 & 20 & 10 & 0 & -10
\end{tabular} 


\section{${ }^{1} \mathrm{H}$ NMR spectrum of compound 3aal (500 $\mathrm{MHz}, \mathrm{CDCl}_{3}$ )}

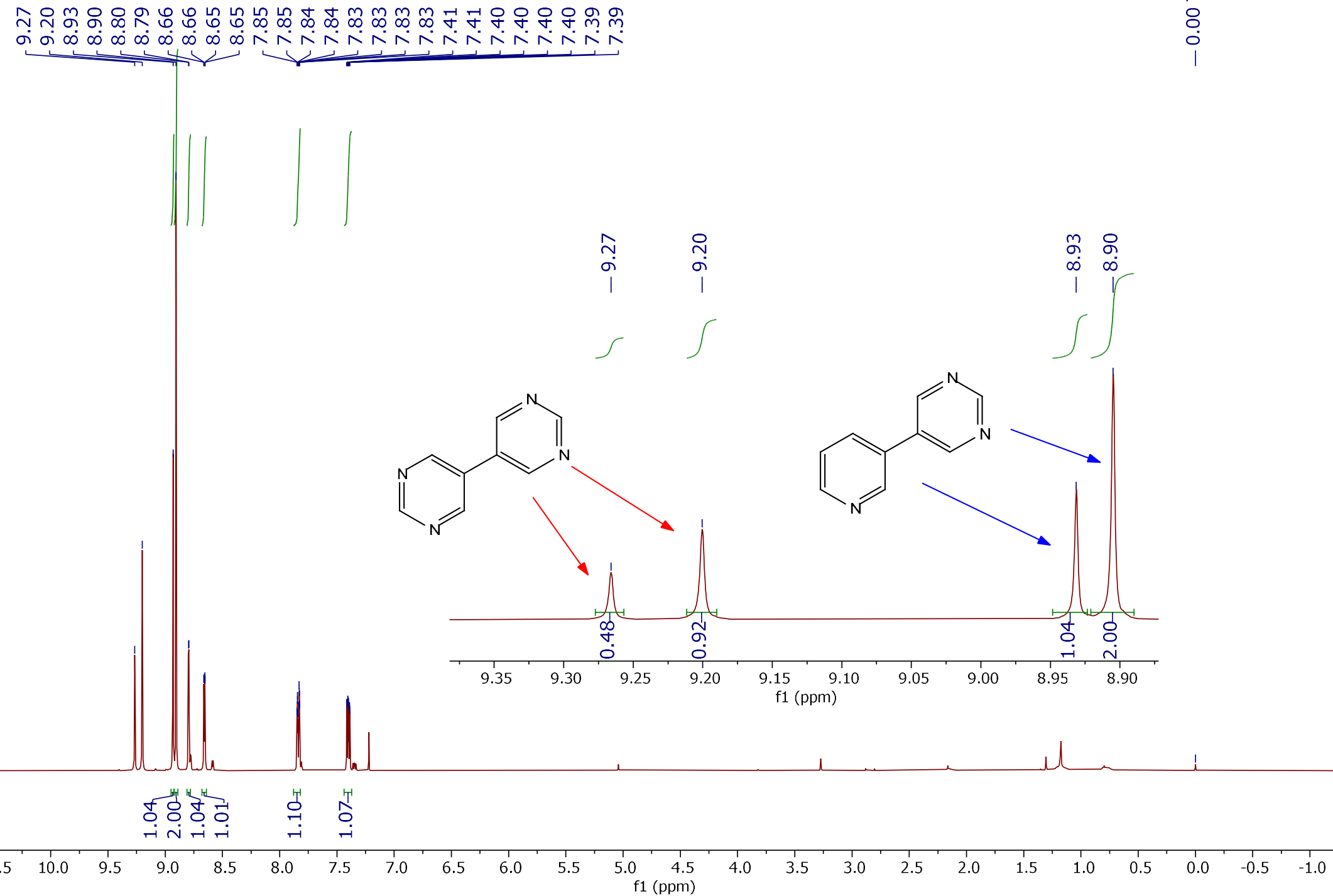




\section{${ }^{13} \mathrm{C}\left\{{ }^{1} \mathrm{H}\right\}$ NMR spectrum of compound 3aal (126 MHz, $\left.\mathrm{CDCl}_{3}\right)$}

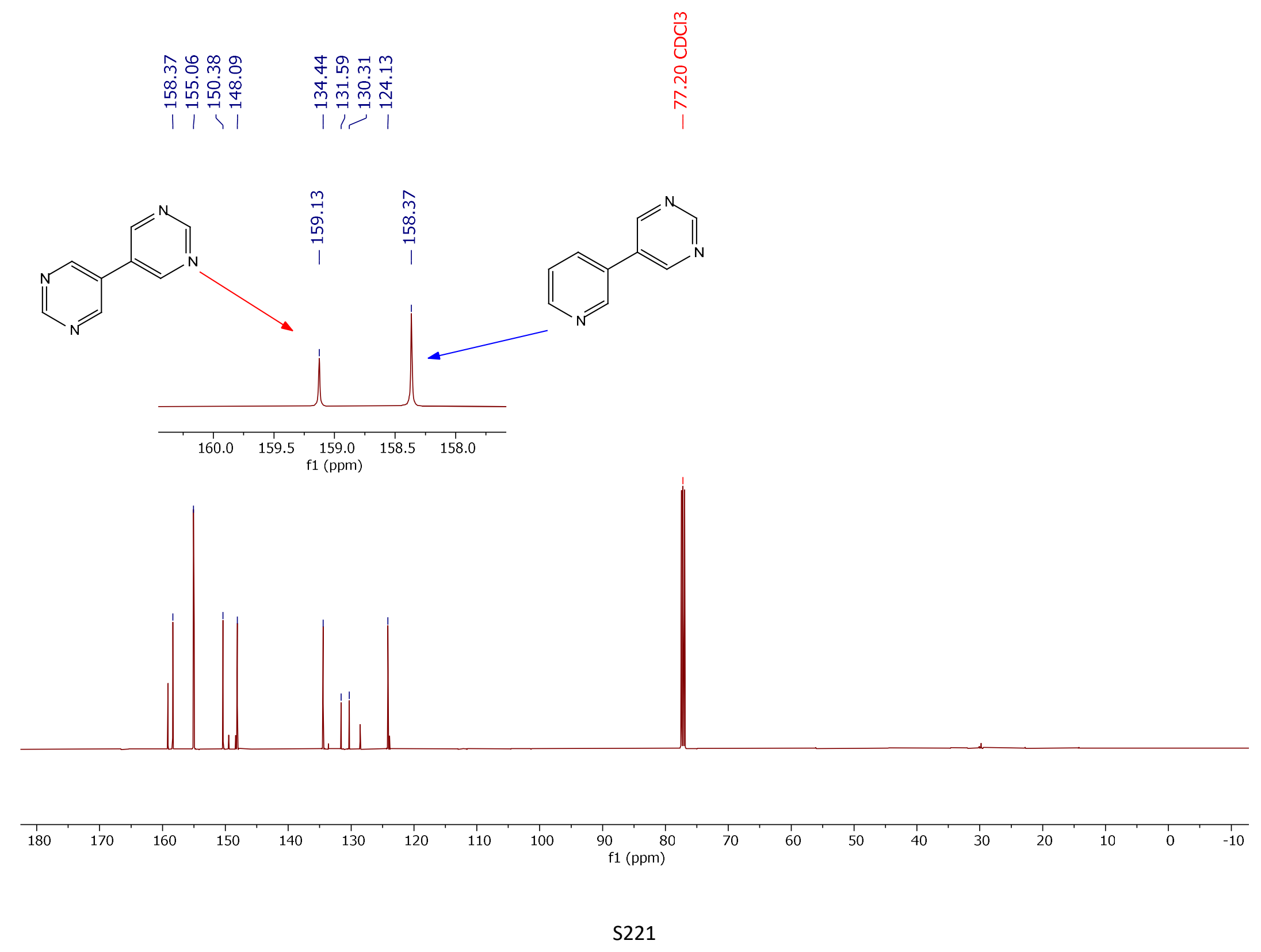




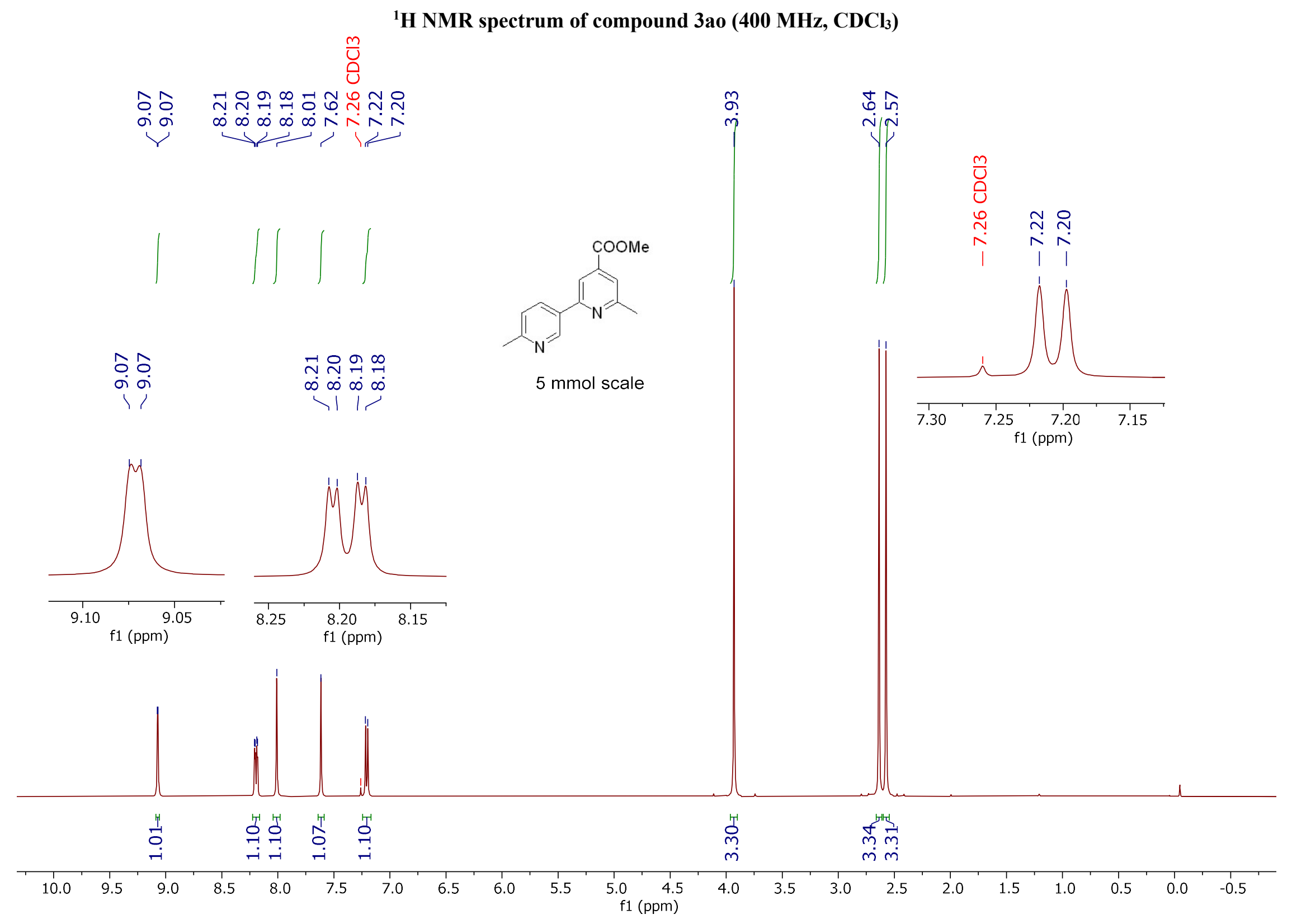


${ }^{13} \mathrm{C}\left\{{ }^{1} \mathrm{H}\right\}$ NMR spectrum of compound 3ao (101 $\left.\mathrm{MHz}, \mathrm{CDCl}_{3}\right)$

\begin{tabular}{|c|c|c|}
\hline 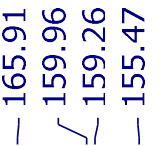 & 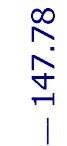 & 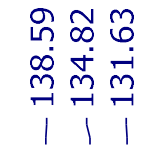 \\
\hline
\end{tabular}

$\infty$

กิ

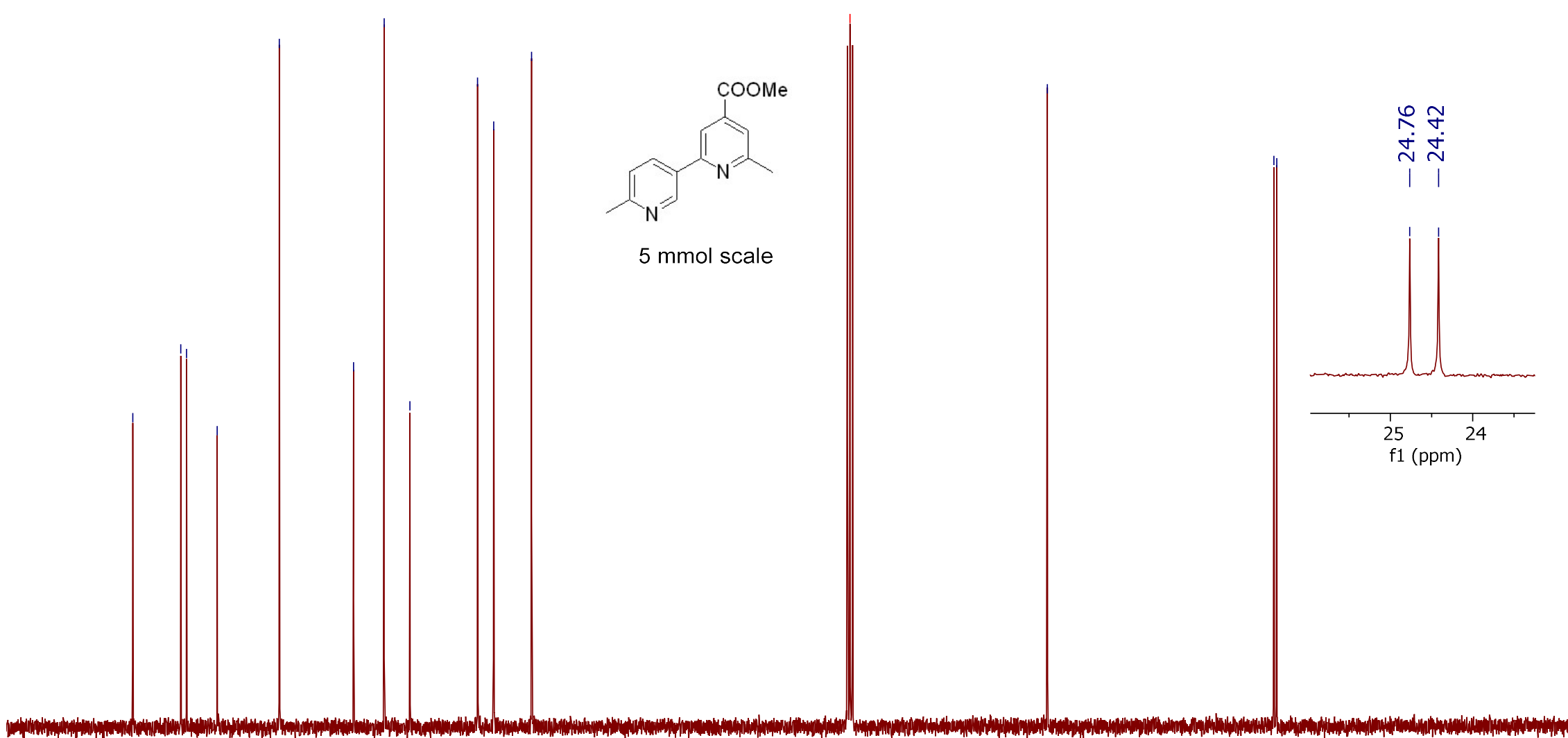


${ }^{1} \mathrm{H}$ NMR spectrum of compound $\mathrm{B3}\left(500 \mathrm{MHz}, \mathrm{CDCl}_{3}\right)$

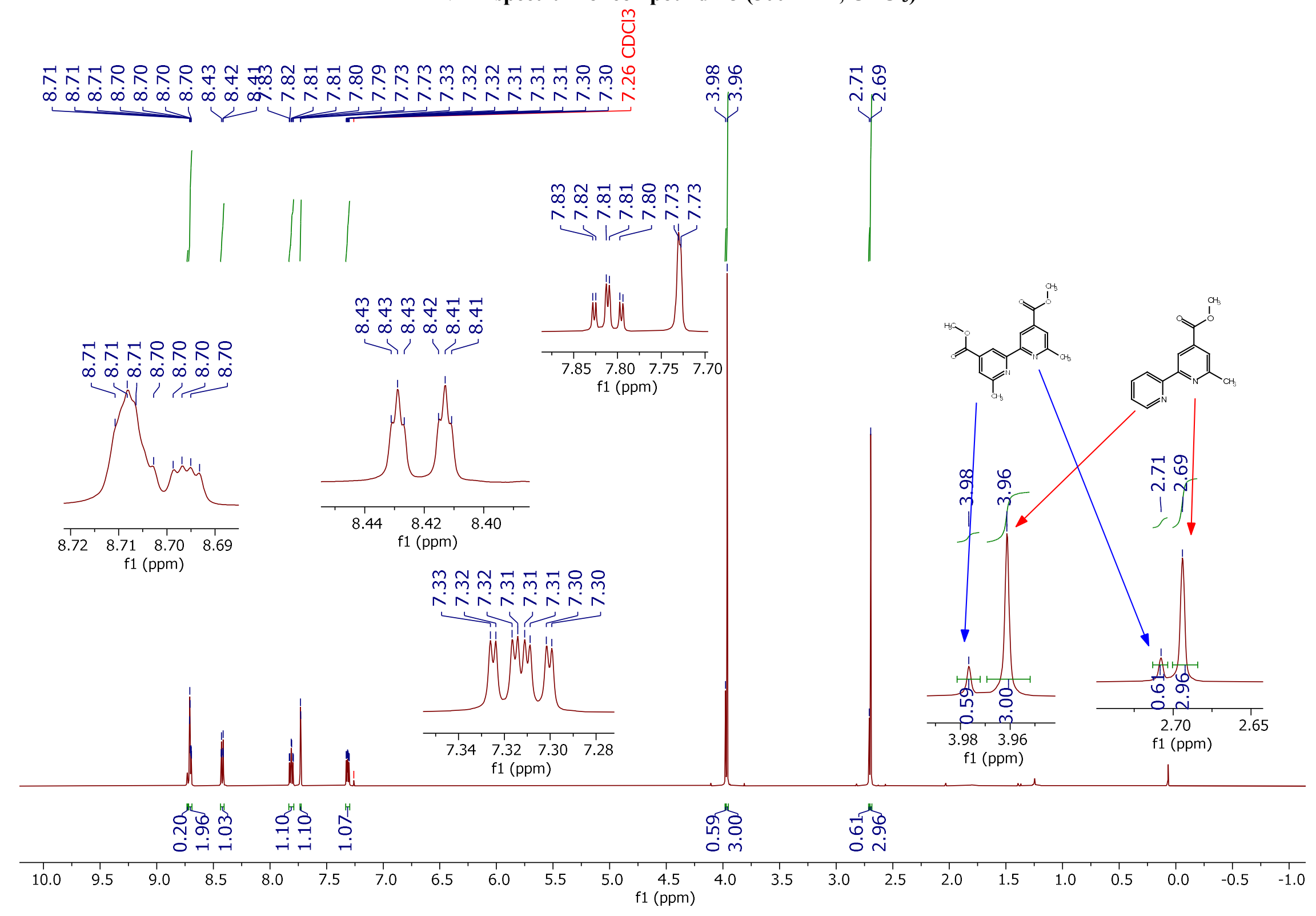


${ }^{13} \mathrm{C}\left\{{ }^{1} \mathrm{H}\right\}$ NMR spectrum of compound $\mathrm{B3}\left(126 \mathrm{MHz}, \mathrm{CDCl}_{3}\right)$

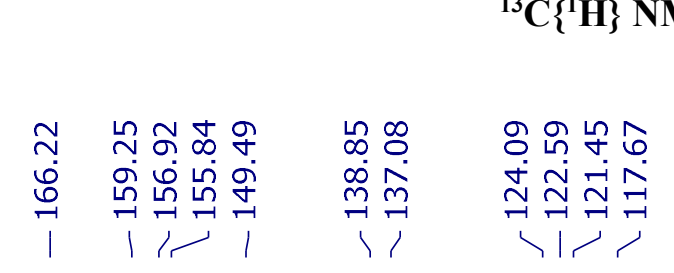

$\stackrel{m}{u}$
Ù
$\stackrel{N}{i}$
$i$
$i$

N

กิ

$\stackrel{\substack{N \\ \stackrel{n}{n}}}{1}$

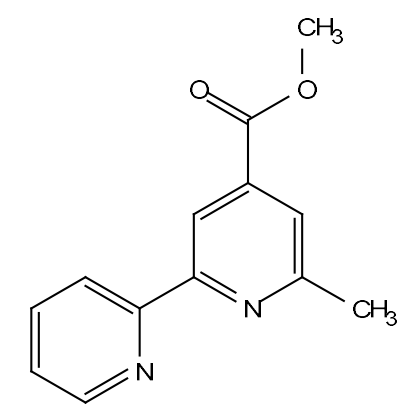




\section{${ }^{1} \mathrm{H}$ NMR spectrum of compound $\mathrm{C6}\left(500 \mathrm{MHz}, \mathrm{C}_{6} \mathrm{D}_{6}\right)$}

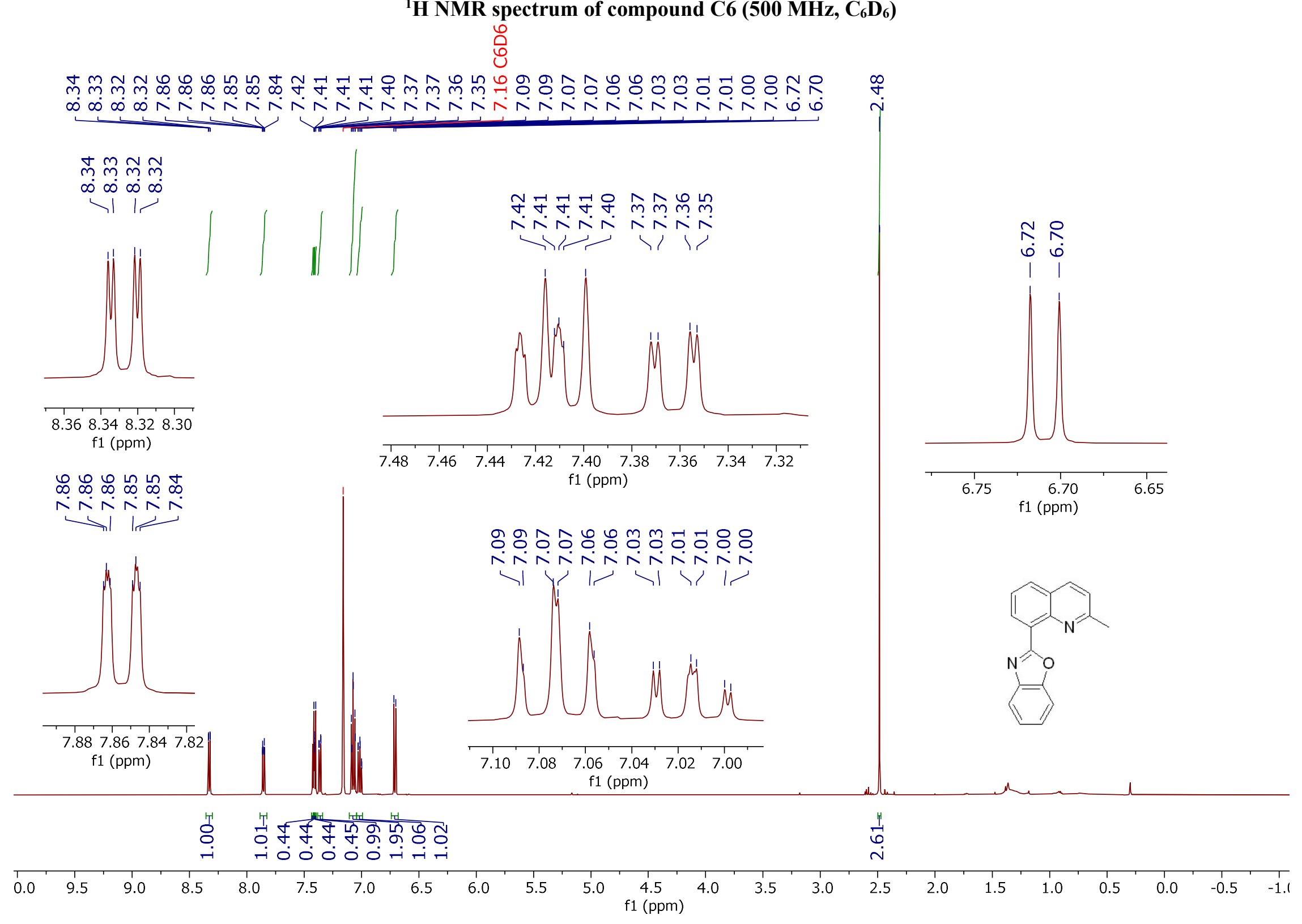




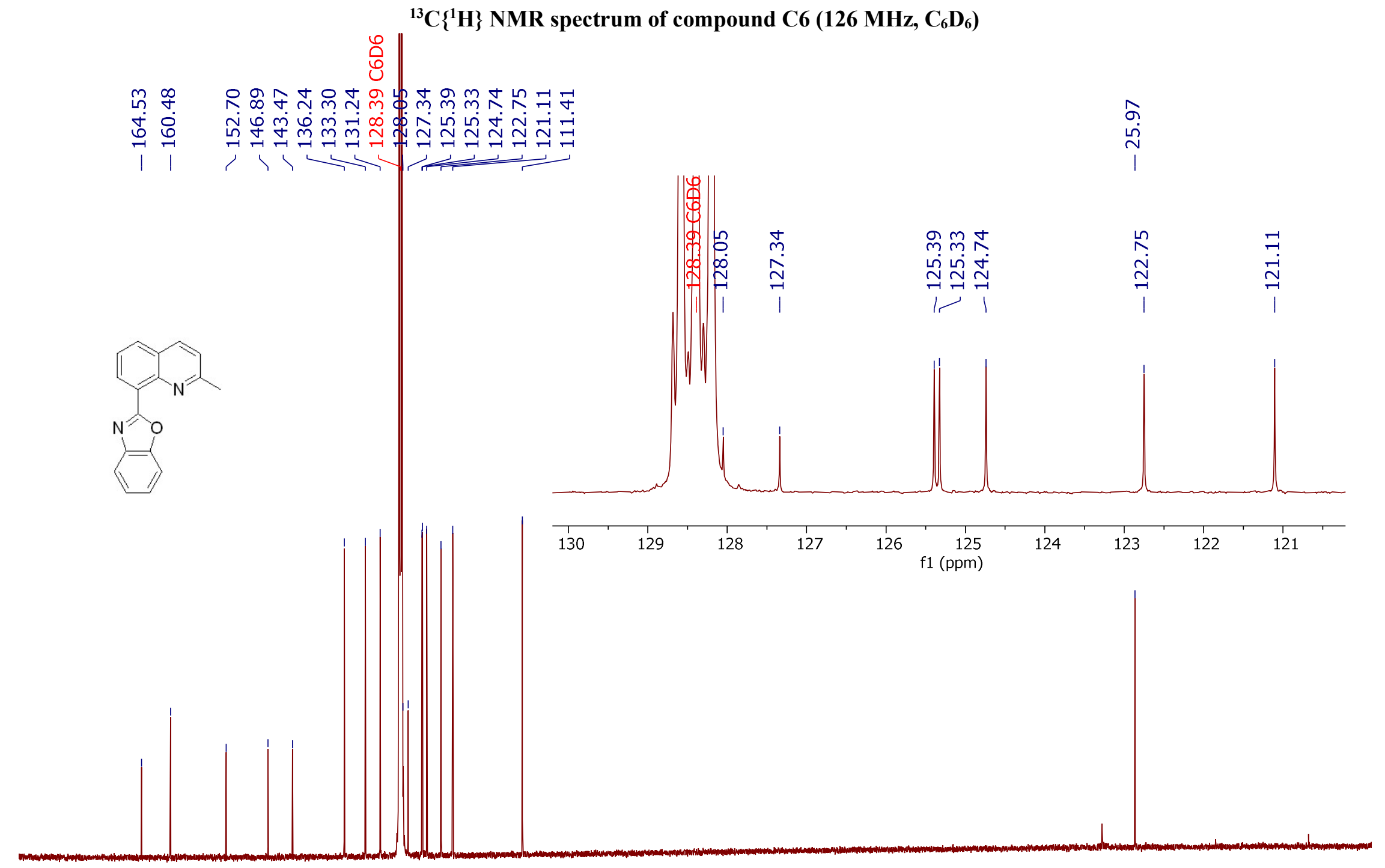

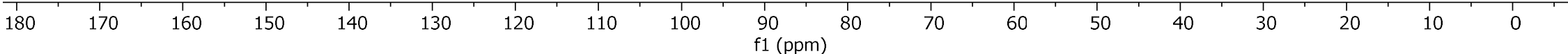


${ }^{1} \mathrm{H}$ NMR spectrum of compound $\mathrm{H} 2\left(600 \mathrm{MHz}, \mathrm{CDCl}_{3}\right)$

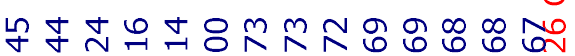
$\infty \infty \infty \infty \infty \infty \wedge$

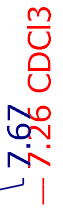

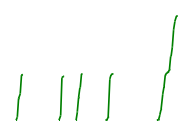

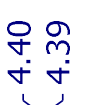

ने ถู

i

نำ

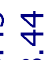

$\infty \infty$

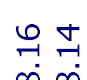

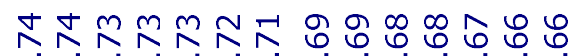

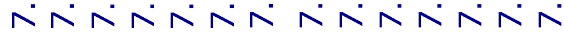

rot

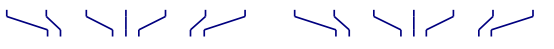
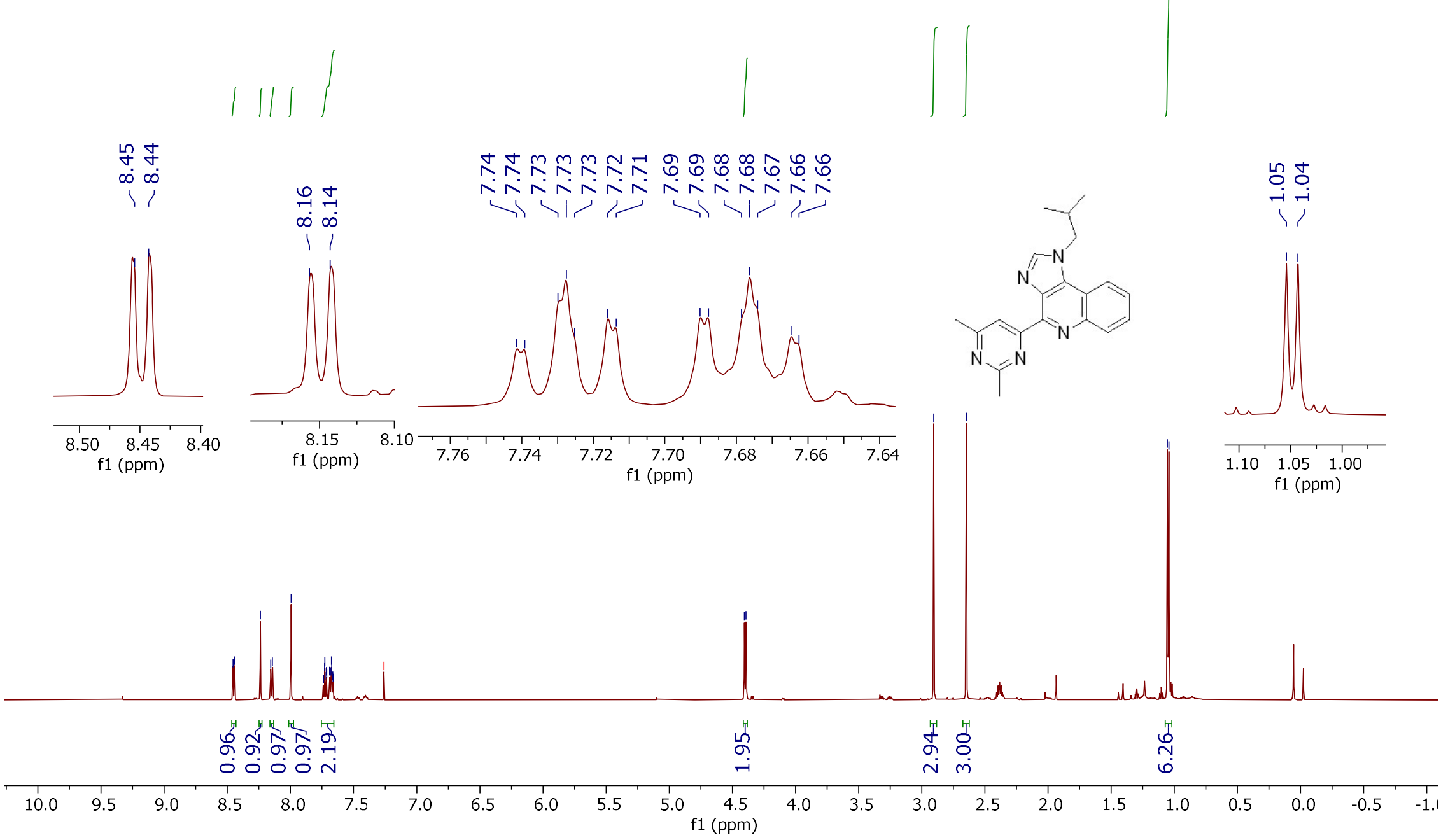
${ }^{13} \mathrm{C}\left\{{ }^{1} \mathrm{H}\right\}$ NMR spectrum of compound $\mathrm{H} 2\left(151 \mathrm{MHz}, \mathrm{CDCl}_{3}\right)$

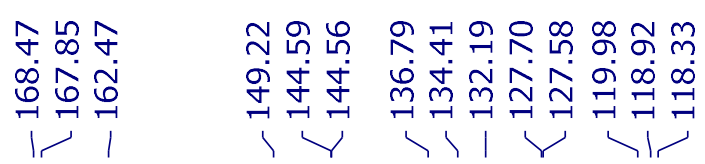

Ù
0
$\stackrel{0}{0}$
$\stackrel{2}{N}$
।

윢옹

岗
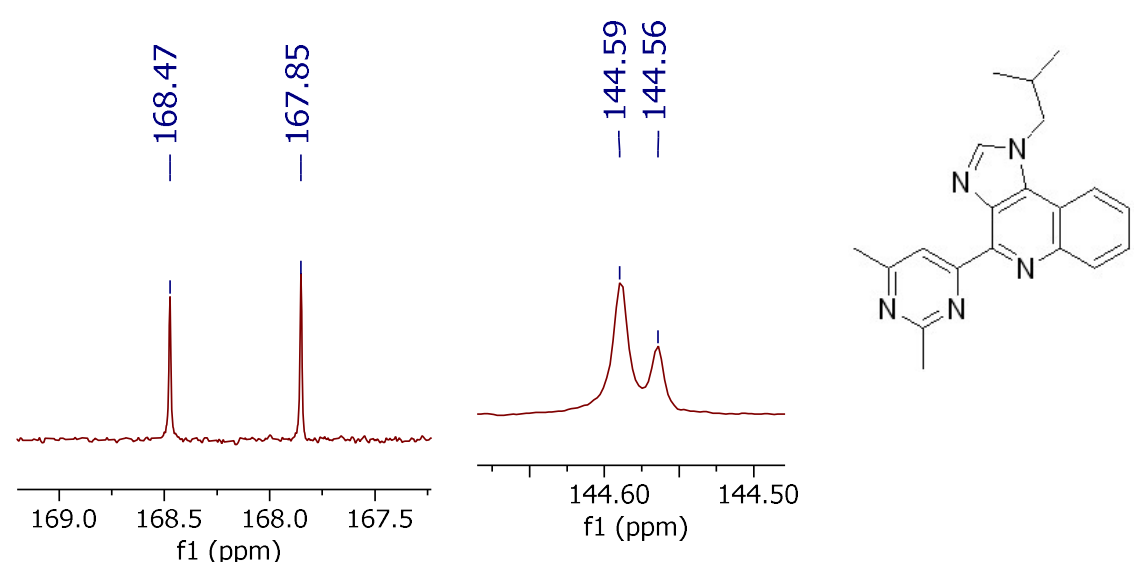

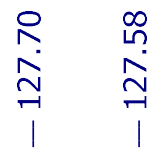

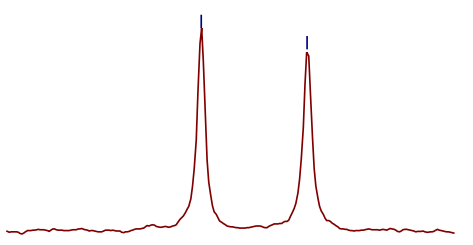

$\begin{array}{llllll}127.9 & 127.8 & 127.7 & 127.6 & 127.5\end{array}$

f1 (ppm)

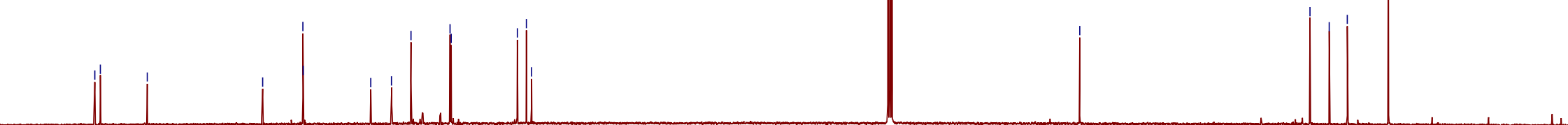

$170 \quad 160$

150

$140 \quad 130$

120

$110 \quad 100$

90

80

60

50

40

30

S229 